\title{
Total Synthesis of $( \pm)$ Maoecrystal V
}

\section{Feng Peng}

Submitted in partial fulfillment of the

Requirements for the degree of

Doctor of Philosophy

in the Graduate School of Arts and Sciences

COLUMBIA UNIVERSITY

2012 
(C) 2012

Feng Peng

All Right Reverved 


\section{Abstract \\ Total Synthesis of Maoecrystal V}

\section{Feng Peng}

This thesis describes in detail our journey toward Maoecrystal V, a potential anticancer diterpenoid. Maoecrsytal V was isolated in 2004 from a Chinese herbal medicine. It has a highly congested structure, in which there are three continuous quaternary carbon center embedded within a rigid pentacyclic scaffold.

The first chapter of this thesis covers the isolation, bioactivity, and synthetic efforts from other groups. The second chapter discusses our first generation strategy toward Maoecrystal V. During our early studies, we successfully found the conditions for the key intramolecular Diels-Alder reaction. We also identified that the facial selectivity of this Diels-Alder reaction is a big challenge for our synthesis plan. The third chapter describes our solution to the core structure of Maoecrystal V. The exploration resulted in the discovery of a novel exo-glycal epoxide rearrangement. The last chapter describes the total synthesis of Maoecrystal V. The difficulties we met and our solution is discussed. 
1.1 Isolation 1

1.3 Synthetic Studies toward Maoecrystal V 3

1.3.1 Professor Baran's Strategy toward Maoecrystal V 4

1.3.2 Professor Nicolaou and Professor Chen 's Strategy toward Maoecrystal V 5

1.3.3 Professor Thomson's Strategy toward Maoecrystal V 7

1.3.4 Professor Trauner's Strategy toward Maoecrystal V 9

1.3.5 Professor Zakarian's Strategy toward Maoecrystal V 10

1.3.6 Professor Yang's Total Synthesis of Maoecrystal V in 2010

$\begin{array}{ll}1.4 \text { References } & 14\end{array}$

CHAPTER 2 FIRST-GENERATION SYNTHETIC APPROACH 17

$\begin{array}{ll}2.1 \text { Introduction } & 17\end{array}$

2.2 Synthetic Plan: Intramolecular Diels-Alder Reaction 17

$\begin{array}{ll}2.3 \text { Results and Discussion } & 20\end{array}$

$\begin{array}{ll}2.4 \text { Experimental } & 37\end{array}$

$\begin{array}{ll}2.5 \text { References } & 65\end{array}$ 
CHAPTER 3 SYNTHESIS OF MAOECRYSTAL V PENTACYCLIC CORE

$\begin{array}{ll}\text { STRUCTRE } & 106\end{array}$

3.1 IMDA Using a Symmetric Precursor 106

3.2 Results and Discussions 109

3. 3 Experimental 119

$\begin{array}{ll}3.4 \text { References } & 133\end{array}$

CHAPTER 4 TOTAL SYNTHESIS OF MAOECRYSTAL V 218

4.1 Early Exploration toward the Total Synthesis of Maoecrystal V 218

4.2 Total Synthesis of Maoecrystal V 233

$\begin{array}{ll}4.3 \text { Experimental } & 244\end{array}$

$\begin{array}{ll}4.4 \text { References } & 253\end{array}$ 


\section{LIST OF ABBREVIATIONS}

\begin{tabular}{|c|c|}
\hline Ac & acetyl \\
\hline AIBN & 2, 2'-azo bisisobutyronitrile \\
\hline $\mathrm{Al}$ & aluminum \\
\hline aq. & Aqueous \\
\hline $\mathrm{B}$ & boron \\
\hline BINAP & 2,2'-Bis(diphenylphosphino)-1,1'-binaphthalene \\
\hline BINOL & 1, 1'-Bi-2-Naphthol \\
\hline $\mathrm{Bn}$ & benzyl \\
\hline$n$-BuLi & $n$-butyl lithium \\
\hline Calcd. & calculated \\
\hline CSA & camphor sulfonic acid \\
\hline${ }^{13} \mathrm{C}$ NMR & carbon-13 magnetic resonance \\
\hline${ }^{\circ} \mathrm{C}$ & degree Celcius \\
\hline $\mathrm{DA}$ & Diels-Alder \\
\hline DBU & 1,8-diazabicyclo[5.4.0]undec-7-ene \\
\hline $\mathrm{DCM}$ & dichloromethane \\
\hline DIBAL-H & diisobutylaluminum hydride \\
\hline DMAP & 4-dimethylaminopyridine \\
\hline DMDO & 2, 2-dimethyldioxirane \\
\hline DME & 1, 2-Dimethoxyethane \\
\hline
\end{tabular}




\begin{tabular}{|c|c|}
\hline DMF & dimethylformamide \\
\hline DMP & Dess-Martin periodinane \\
\hline DMSO & methyl sulfoxide \\
\hline $\mathrm{E}$ & Entgegen \\
\hline $\mathrm{Ee}$ & enantiomeric excess \\
\hline EI & electron impact \\
\hline equiv. & equivalents \\
\hline ES & electrospray \\
\hline EtOH & ethanol \\
\hline $\mathrm{Et}_{2} \mathrm{O}$ & diethyl ether \\
\hline Et & Ethyl \\
\hline EtOAc & ethyl acetate \\
\hline $\mathrm{g}$ & gram \\
\hline $\mathrm{h}$ & hour \\
\hline${ }^{1} \mathrm{H}$ NMR & Proton magnetic resonance \\
\hline HRMS & high resolution mass spectrometry \\
\hline $\mathrm{IC}_{50}$ & half maximal inhibitory concentration \\
\hline IMDA & intramolecular Diels-Alder \\
\hline$i$-Pr & Isopropyl \\
\hline IR & infrared spectroscopy \\
\hline $\operatorname{Ir}$ & Iridium \\
\hline $\mathrm{J}$ & coupling constant \\
\hline KHMDS & potassium bis(trimethylsilyl)amide \\
\hline
\end{tabular}




$\begin{array}{ll}\text { L.A. } & \text { Lewis acid } \\ \text { LDA } & \text { lithium diisopropylamide } \\ \text { LHMDS } & \text { lithium bis(trimethylsilyl)amide } \\ \text { Li } & \text { Lithium } \\ \text { Me } & \text { methyl group } \\ \text { MeOH } & \text { methanol } \\ \text { m-CPBA } & \text { meta-chloroperbenzoic acid } \\ \text { min } & \text { minutes } \\ \text { mL } & \text { milliliters } \\ \text { MOE } & \text { ethoxylmethyl } \\ \text { MOM } & \text { methoxylmethyl } \\ \text { Ms } & \text { mesyl (methanesulfonyl) } \\ \text { NIS } & N \text {-iodosuccinimide } \\ \text { OTf } & \text { fluoromethanesulfonate } \\ \text { Pd } & \text { Palladium } \\ \text { Ph } & \text { Phenyl } \\ \text { PIDA } & \text { phenyliodo(III) diacetate } \\ \text { PIFA } & \text { [bis(trifluoroacetoxy)iodo]benzene } \\ \text { PivCl } & \text { pivaloyl chloride } \\ \text { PPh } & \text { triphenylphosphine } \\ \text { Pt } & \text { Platinum } \\ \text { pyridine } \\ \text { Rhodium }\end{array}$




\begin{tabular}{|c|c|}
\hline r.t. & room temperature \\
\hline $\mathrm{Si}$ & Silicon \\
\hline $\mathrm{Sn}$ & Tin \\
\hline $\mathrm{T}$ & temperature \\
\hline TASF & tris(dimethylamino)sulfonium difluorotrimethylsilicate \\
\hline TBAF & tetrabutylammonium fluoride \\
\hline TBDPS & tert-butylphenylsilyl \\
\hline TBS & tert-butyldimethylsilyl \\
\hline TBSOTf & tert-butyldimethylsilylrifluoromethanesufonate \\
\hline TEA & triethyl amine \\
\hline TFA & trifluoroacetic acid \\
\hline TFDO & Methyl(trifluoromethyl)dioxirane \\
\hline THF & tetrahydrofuran \\
\hline $\mathrm{Ti}$ & Titanium \\
\hline TMS & trimethylsilyl \\
\hline TROSY & Transverse relaxation-optimized spectroscopy \\
\hline $\mathrm{W}$ & Tungsten \\
\hline $\mathrm{Zn}$ & Zinc \\
\hline $\mathrm{Z}$ & Zusammen \\
\hline$\delta$ & chemical shift downfield from TMS \\
\hline
\end{tabular}




\section{Acknowledgements}

I would like to thank my mentor, Professor Samuel J. Danishefsky, for his close supervision, continuous encouragement, and kind support during my Ph.D education. His rich wisdom about chemistry and philosophy has greatly inspired me during the past five years. I feel it is my great honor to work in Sam's group and I deeply appreciate Sam's teaching.

I am deeply grateful to Professor James Leighton, Scott A. Snyder, Tristan Lambert and Erik Sorensen for serving on my defense committee. In addition, I would like to thank Professor Leighton and Snyder for the suggestions on my research and jobhunting, which helped me a lot during my study at Columbia.

It is my great honor to study organic synthesis at Columbia University, which provided me a chance to learn chemistry from other great chemists here. I am in deep debt to Professor Stork and Professor Norton for their help suggestions on my research. I would also like to thanks Professor Breslow, Nakanishi, Katz, Nuckolls and Sames for their beautiful ideas and insightful thinking during the seminars and the Thursday Night Problem Sections.

Working in Sam's lab provided me a great opportunity to collaborate with a group of talent people. I would like to thank Dr. Benjamin J. Wright and Mr. John Hartung for 
the collaboration on pluraflavin A project and for their friendship. They are very nice and patient with me, which help me a lot to learn English and culture in this country. Dr. Maolin $\mathrm{Yu}$ is thanked for his early exploration on Maoecrystal V project, which is very insightful for the further progress on this project. I would also like to thank Dr. Mingji Dai and Dr. Angie R. Angeles for the collaboration on the silyl enol ether isomerization project. I learned a lot from you guys. Dr. Robin E. Grote is thanked for her wonderful contribution to the Iso-Diels-Alder reaction project.

Working in Sam's lab also provided me a great opportunity to learn from other highly motivated chemists. I would like to thank Dr. Shengping Zheng, for teaching me the crazy mechanisms, for valuable suggestions on my research projects and for the friendship. I would like to thank Dr. Xuechen Li for the insightful discussion on chemistry and also on career development. I would like to thank Dr. Xiangyang Wu for the encouragement when I met difficulties in my research. I would like to thank Dr. Yandong Zhang for sharing with me about his beautiful chemistry and also his excellent professional skills. I would like to thank Dr. Zhang Wang for the insightful suggestions on my research projects. I would like to thank Dr. Suwei Dong for the help during my job hunting. I also want to express my gratitude to Dr. Alesandra Polara, Ms. Audrey Ross, Mr. Jordi Rull and Ms. Jessie Joussot, Dr. Jin Chen, Dr. Yu Yuan, Dr. Jianglong Zhu, Dr. Xiaohua Li, Dr. Ping Wang, Dr. Peter Park, Dr. Neeraj Sane, Dr. Karthik Iyer and all other MSKCC group members for the friendship and for the helps to my research. 
I would like to thank Fay, Dana, and Laura for their management of the lab. Special thank goes to Rebecca for her wonderful work on manuscripts editing. I would also like to thank Professor William Berkowitz for his valuable help for literature searching.

During the past five years at Columbia, I am lucky to get help and support from a lot of great people. I would like to thank Alix and Dani who make my study smoothly here. I would also like to thanks John, Micheal and Yasuhiro for the NMR and Mass spectrum service. Without their support, a lot of important results would be missed. Special thanks goes to Aaron and Wes for their valuable help on the X-ray experiments. I would like to thank my classmates for the discussion and friendship. I would like to express my gratitude to Eli Lilly for a graduate fellowship.

Without support from my previous professors, I could not have the opportunity to study at Columbia. I would like to thank my Canada supervisor, Professor Dennis G. Hall, for his training and friendship. I would also like to thank Professor Zhen Yang and Professor Jiahua Chen at Peking University, for provided me an opportunity to study the frontier organic chemistry and for the guidance of my research. I would like to thank Professor $\mathrm{He} \mathrm{Li}$ and Professor Ying Chen at Beijing Normal University for igniting my interest in organic chemistry.

Lastly, I would like to thank my family for endless support of my study. I thank my parents and my parents in-law for their love and encouragement. I especially thank 
my wife, Ms. Yanqing Pan, for her love, understanding, and care. Without her support, I could not move forward in my career. I thank my son, Victor Peng, for all the happiness he brings to my family. 


\section{Chapter 1 Introduction}

\subsection{Isolation}

Located in southern China, Yunnan province is considered as China's Botany Kingdom due to its rich plants resource. According to 2006's data, there were about 14590 different kinds of plants in Yunnan province, accounting for $48 \%$ of that in the whole China. ${ }^{1}$ Most of these plants have been used as fork medicines for a long time. ${ }^{1}$ With the development of natural product identification technologies in China, nowadays significant attention has been paid to the bioactive components of these herb medicines. Several research groups have already been engaged in the investigation of these plants. Among them, Professor Sun Han-Dong's team, who is from the Kunming Institute of Botany in the Yunnan province, is especially focused in the search for bioactive diterpenoids natural products from the genus Isodon plants of the Laiatae family. ${ }^{2}$ Since 1976, his team has isolated and characterized more than 400 new diterpenoids. Most of these natural products share an ent-kaurane skeleton. ${ }^{3}$

Maoecrystal V was first isolated in 1994 from Isodon eriocalyx Hara, which is widely distributed in southern China. Isodon eriocalyx Hara has been used as a herbal medicine for a long time in Yunnan province. It has been used to treat sore throat, inflammation, and hypertension, etc. Since the X-ray structure was not obtained at that time, Professor Sun's group did not report Maoecrystal V. It was in 2004 that the group successfully acquired a single crystal of Maoecrystal V and its X-ray structure was reported on Org. Lett. ${ }^{4}$ As shown (Figure 1-1), Maoecrystal V (1.1) featured two 
contiguous quaternary carbon stereocenters and a tertiary alcohol, embedded within a rigid pentacyclic scaffold. The isolation chemists mentioned in the isolation paper, "Maoecrystal $\mathrm{V}$ is by far the most modified naturally occurring ent-kauranoid from Isodon species."4 These factors make Maoecrystal $\mathrm{V}$ a very challenging target for total synthesis.
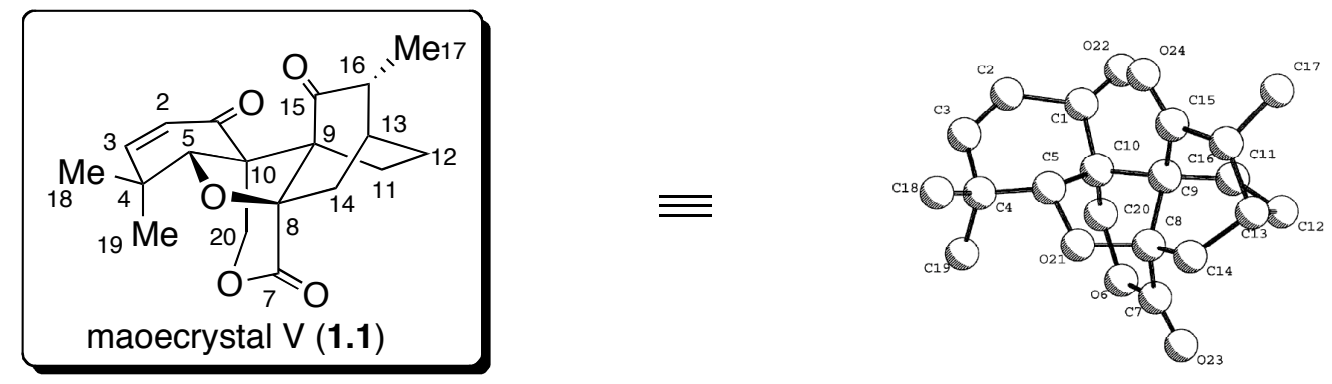

Figure 1-1 Structure of Maoecrystal V

\subsection{Biosynthesis Hypothesis and Biological Activity}

The isolation chemists proposed an interesting pathway accounting for the formation of Maoecrystal V from eriocalyxin B (Scheme 1-1). ${ }^{5}$ An oxidative cleavage and selective reduction of eriocalyxin B would provide epi-eriocalyxin A(1.3). A BaeyerVillager-type degradation of the aldehyde functional group of epi-eriocalyxin $\mathrm{A}(\mathbf{1 . 3})$ would generate the C-5 hydroxyl group with the retention of the stereochemistry (1.4). An enzymatic 1,2 carbon shift followed by tetrahedronfuran ring formation would afford Maoecrystal V. 

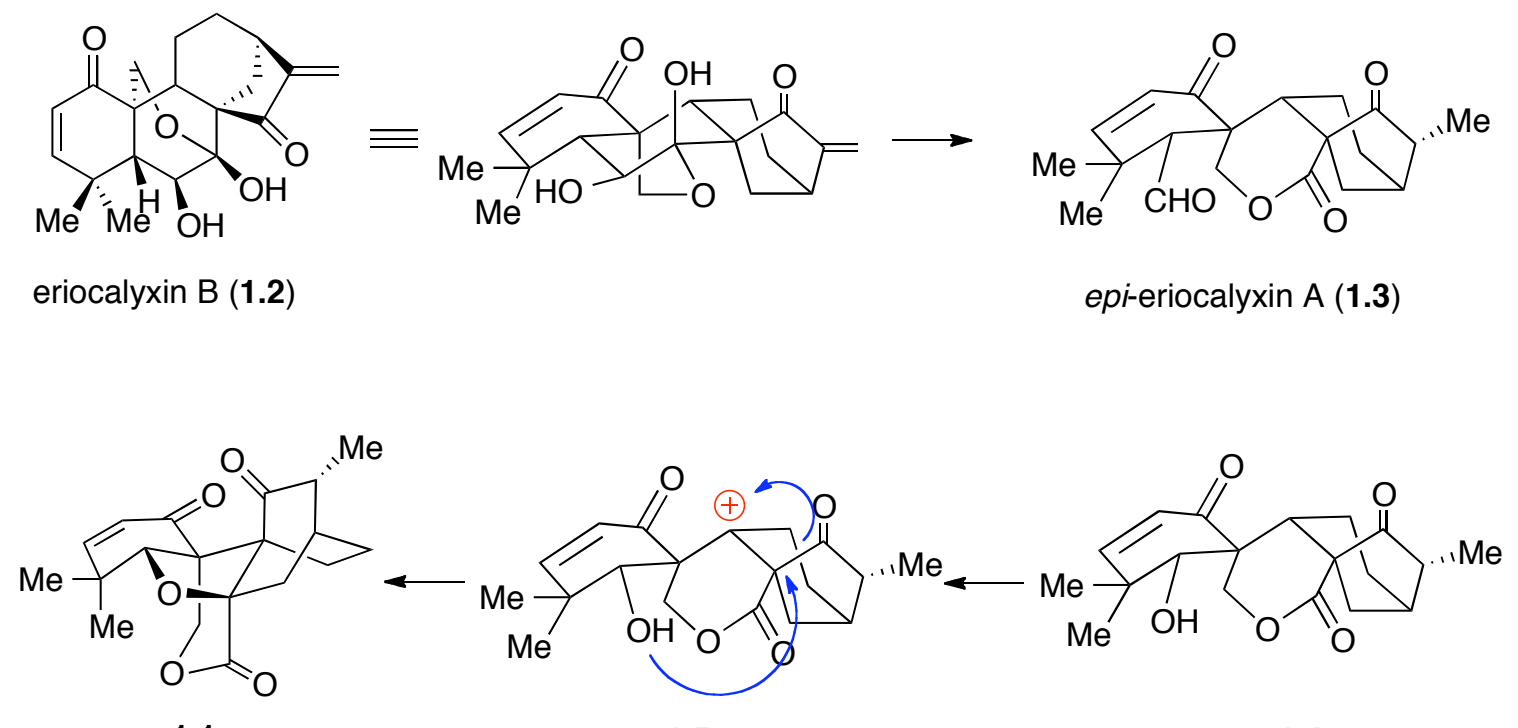

1.1

1.5

1.4

Scheme 1-1 Biosynthesis of Maeocrystal V from Eriocalyxin B

Maoecrystal V was evaluated for its cytotoxicity against five human tumor cell lines. ${ }^{4}$ Maoecrystal V showed high selective activity against the HeLa cell line with $\mathrm{IC}_{50}$ values as low as $20 \mathrm{ng} / \mathrm{mL}$, while there is no significant cytotoxicity toward other four cell lines. These interesting results indicate that Maoecrystal $\mathrm{V}$ and its derivatives could potentially be lead anticancer agents.

\subsection{Synthetic Studies toward Maoecrystal V}

With a promising biological profile and an interesting structure, Maoecrystal V has gained significant attention from the synthetic organic community since its structure elucidation. More than 10 groups $^{6}$ have initiated synthetic programs toward this natural product with one total synthesis reported on $2010^{22}$ from Professor Zhen Yang's group in 
China. The proceeding section will highlight the chemistry involving the synthetic efforts towards Maoecrystal V by the synthetic community.

\subsubsection{Professor Baran's Strategy toward Maoecrystal $V^{7}$}

Baran's group reported a creative approach toward Maoecrystal V based on their understanding of the problems in the maoecrystal V's synthesis in 2009. Their key reaction is an intramolecular Diels-Alder cyclization (1.10 to 1.11) to establish the skeleton. Within the DA strategy, there are three issues to be addressed: 1) what kind of diene and dienophile would work? 2) What kind of DA precursor is the best for this target? 3) How to control the stereoselectivity of this Diels-Alder reaction?

Baran's synthesis commenced with a Barton arylation reaction ${ }^{8}$ between keto aldehyde 1.6 and an aryl bismuth compound 1.7, which afforded 1.8 with the requisite quaternary carbon center. Reduction of the aldehyde and acylation of the resultant

alcohol provided intermediate 1.9. Unmasking of the phenol with TFA and subsequent Wessely oxidation with $\mathrm{Pb}(\mathrm{OAc})_{4}$ gave the key Diels-Alder reaction precursor 1.10. The thermal-induced IMDA proceeded smoothly to produce the desired endo-adduct $\mathbf{1 . 1 1}$. This advanced intermediate was further transformed to compound $\mathbf{1 . 1 2}$ by hydrogenation and $\mathrm{SmI}_{2}$ reduction. Baran's exploration on maoecrystal $\mathrm{V}$ provided a nice answer to the questions related to a Diels-Alder strategy toward Maoecrystal V considering their diene, DA precursor, and stereoselectivity. The chemistry left for a total synthesis is how to prepare the THF ring. 


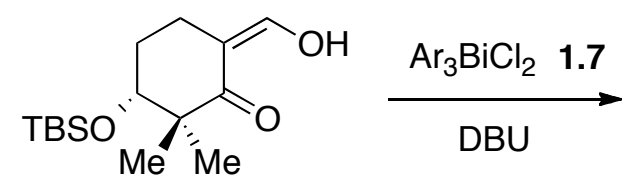

1.6<smiles>COc1c(C)cccc1[C@]1(C=O)CCC[C@](C)(OC(C)(C)C)C1=O</smiles>

1.8
1) $\mathrm{Li}(t-\mathrm{BuO})_{3} \mathrm{AlH}$

2)<smiles>C=CC(=O)Cl</smiles><smiles>C=CC(=O)OC[C@]1(c2cccc(C)c2OC)CC[C@@H](C(C)(C)C)C(C)(C)C1=O</smiles>

1.9 $\underset{\text { 2) } \mathrm{Pb(OAc})_{4}}{\stackrel{\text { TFA }}{\longrightarrow}}$<smiles>C=CC(=O)OC[C@]1(C2=CC=CC(C)(OC(C)(C)C)C2=O)CCC[C@@](C)(OC(C)(C)C)C1=O</smiles>

1.10

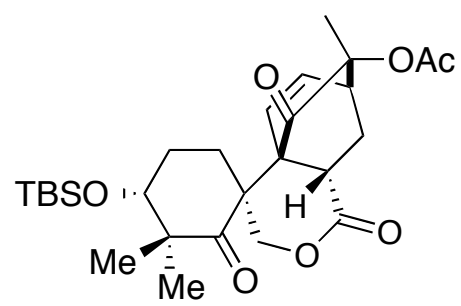

1.11

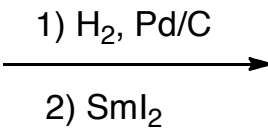

2) $\mathrm{Sml}_{2}$

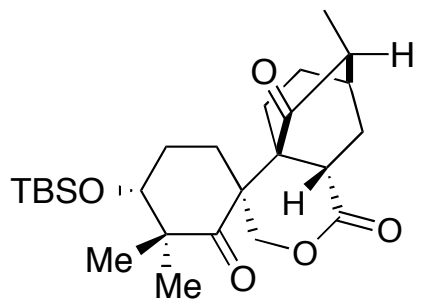

1.12

Scheme 1-2 Baran's Approach Toward Maoecrystal V

\subsubsection{Professor Nicolaou and Professor Chen's Strategy toward Maoecrystal V ${ }^{9,10}$}

Nicolaou and Chen's group also investigated an intramolecular Diels-Alder strategy toward the total synthesis of Maoecrystal V. Their approach employed the ethertethered IMDA precursor 1.18 instead of the lactone-tethered precursor such as Baran's compound 1.10. Accordingly, the core THF ring (compound 1.19) was prepared just after the IMDA reaction, presenting a very creative solution to the core THF ring problem of Maoecrystal V. 

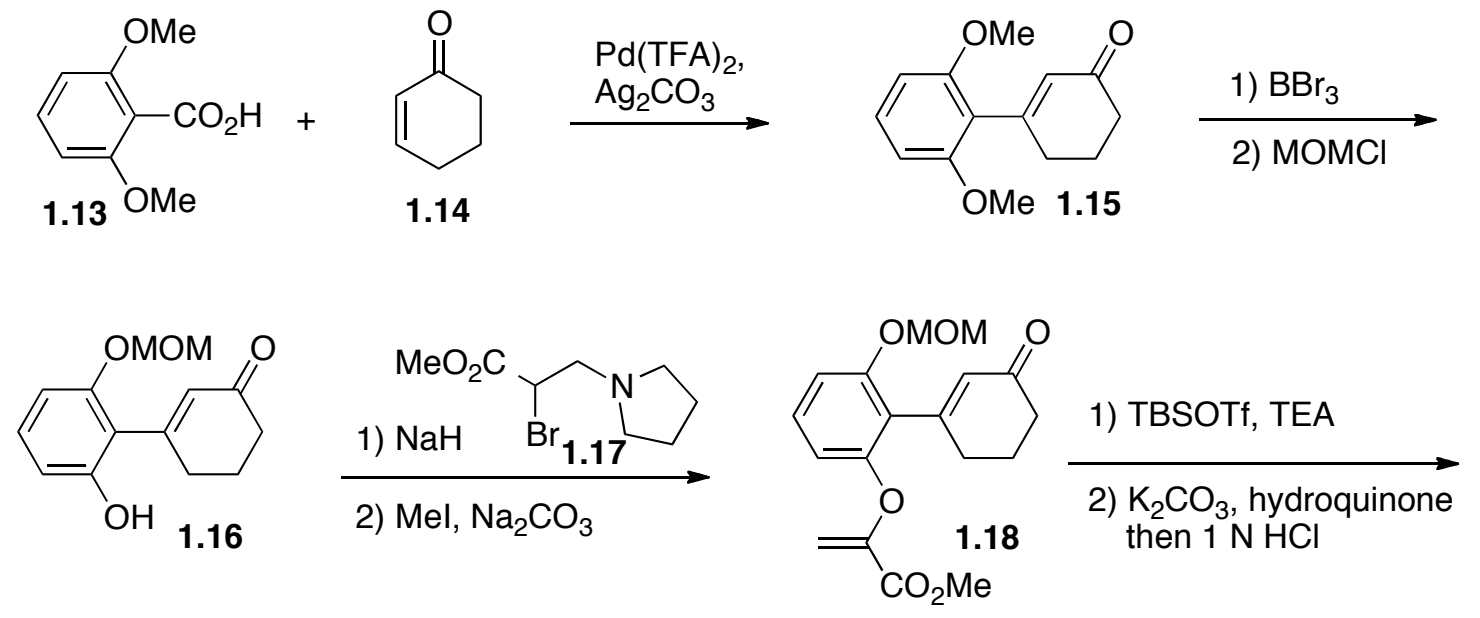<smiles>COC(=O)C1(C(=O)OC)Oc2cccc(OC)c2C12CC(=O)C1C=CC2C1</smiles>

1.19

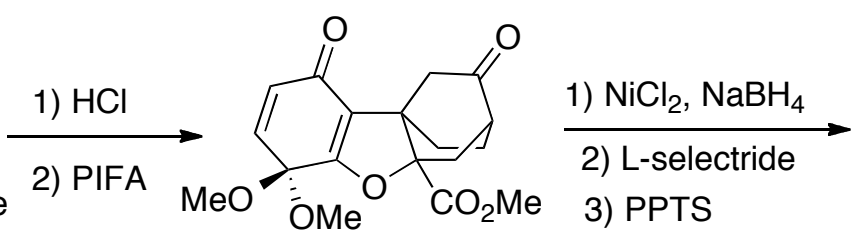

1.20

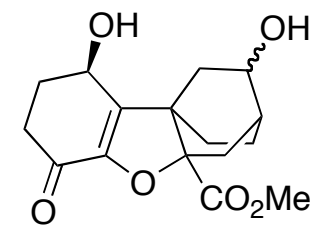

1.21

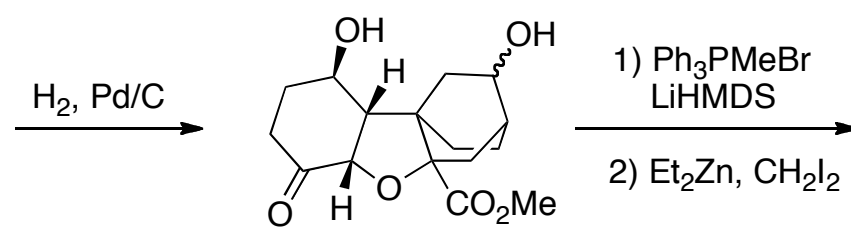

1.22

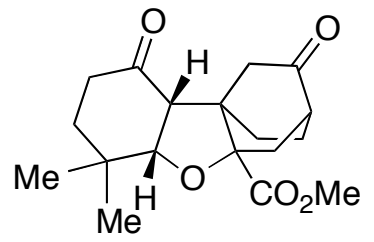

1.24

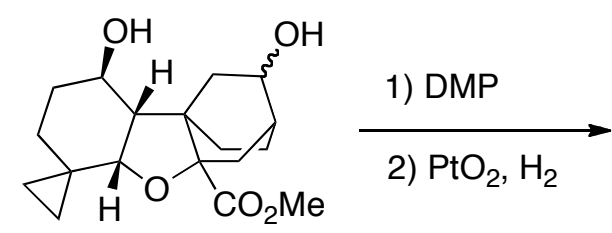

1.23

Scheme 1-3 Nicolaou/Chen's Approach toward Maoecrystal V

The synthesis commenced with a decarboxylative Heck coupling ${ }^{11}$ to provide enone 1.15. Protecting group manipulations of 1.15 afforded mono-phenol 1.16. The mono-phenol 1.16 underwent an alkylation with bromide 1.17, which afforded a 
precursor for the dienophile. The resultant pyrollidine moiety was then alkylated with MeI and subsequent Hofmann elimination reaction furnished compound 1.18. Compound 1.18 was then treated with TBSOTf and the resultant IMDA precursor was directly heated to furnish the desired exo-adduct. Subsequent deprotection gave compound 1.19. This compound was then treated with aqueous $\mathrm{HCl}$ and the resultant phenol was oxidized with PIFA to furnished dearomatized compound 1.20. To synthesize the correct C5 stereogenic center, compound $\mathbf{1 . 2 0}$ was further reduced to compound 1.21 using LSelectride. A hydroxyl-directed hydrogenation with $\mathrm{Pd} / \mathrm{C}$ afforded the compound $\mathbf{1 . 2 2}$ with the desired C5 stereochemistry.

The Nicolaou/Chen group demonstrated their solution to the gem-dimethyl functional group problem. As planned, compound $\mathbf{1 . 2 2}$ underwent Wittig reaction and subsequent Furukawa-modified Simmons-Smith cylopropanation of the exo-methylene provided the desired cyclopropane $\mathbf{1 . 2 3}$. Global oxidation of compound $\mathbf{1 . 2 3}$ followed by hydrolysis indeed afforded the gem-dimethyl compound 1.24. The remaining chemistry required toward a total synthesis of Maoecrystal $\mathrm{V}$ is how to construct the last lactone ring.

\subsubsection{Professor Thomson's Strategy toward Maoecrystal $\mathbf{V}^{12}$}

The Thomson group reported an interesting intermolecular Diels-Alder strategy toward Maoecrystal V (1.30 to 1.31). This strategy reminds me the Corey's synthesis of Antheridic Acid. ${ }^{13}$ The synthesis started with a Rubottom oxidation ${ }^{14}$ of ketone 1.25. The resultant alcohol was protected as a TBS ether. The ketone $\mathbf{1 . 2 6}$ was then subjected to a Horner-Wadsworth-Emmons reaction condition using reagent 1.27. 


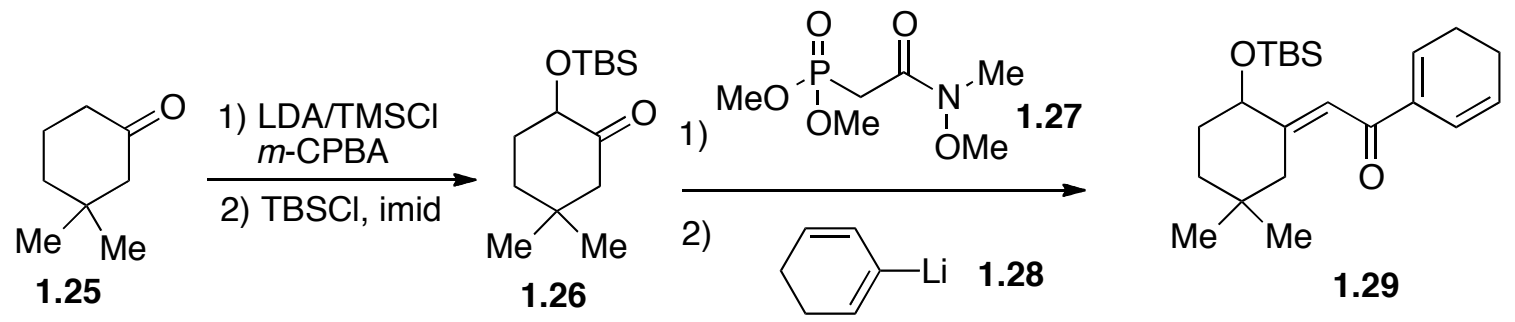

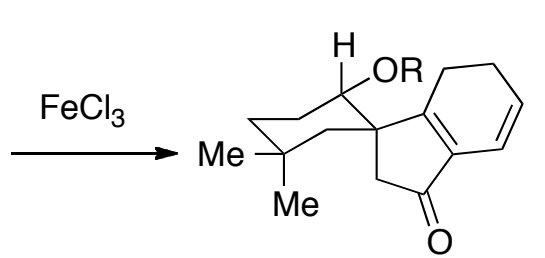

1.30
1) DIBAL-H

2) nitroethene

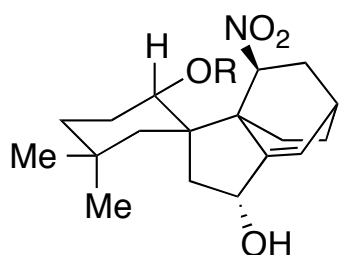

1.31

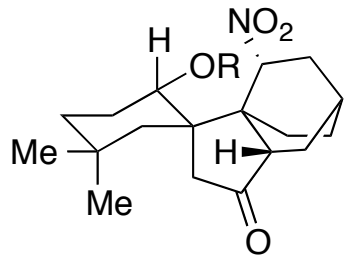

1.32

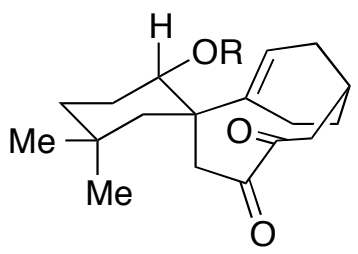

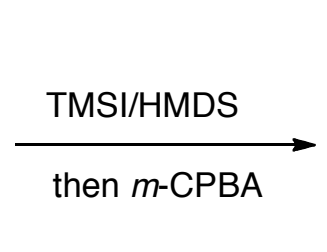

then $m$-CPBA

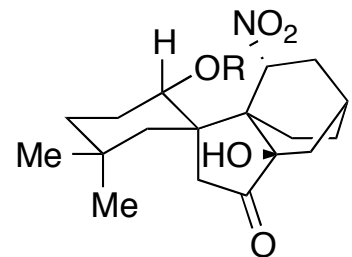

1.33
1) $\mathrm{KOH}$

2) $\mathrm{Pd} / \mathrm{C}, \mathrm{H}_{2}$

3) Jones ox

1.34

Scheme 1-4 Thomson's Approach toward Maoecrystal V

The resulting unsaturated Weinreb amide ${ }^{15}$ was treated with $\mathbf{1 . 2 8}$ to furnish the desired intermediate 1.29. An elegant Lewis-acid-catalyzed Nazarov cyclization ${ }^{16}$ occurred on $^{2}$ compound $\mathbf{1 . 2 9}$ and afforded intermediate $\mathbf{1 . 3 0}$ with the requisite quaternary carbon center. The ketone in compound $\mathbf{1 . 3 0}$ was reduced and the resulting diene was subjected to an intermolecular Diels-Alder reaction with nitroethylene to furnish the desired 
cycloadduct 1.31. Although the designed Nef reaction ${ }^{17}$ with $\mathbf{1 . 3 1}$ did not afford the desired ketone, treatment of $\mathbf{1 . 3 1}$ with $\mathrm{KOH}$ resulted in complete epimerization of the nitro group, which provided a good chance to stereoselectively install the requisite C-8 hydroxyl group. Accordingly, the epimerized nitro compound was further subjected to a hydrogenation and a Jones oxidation to yield compound 1.32. Rubottom oxidation of the ketone $\mathbf{1 . 3 2}$ afforded the desired C-8 hydroxyl compound $\mathbf{1 . 3 3}$, which was poised for a creative oxygen-variant of the Hofmann-Loffler-Freytag reaction. ${ }^{18}$ Toward this end, a remote $\mathrm{C}-\mathrm{H}$ functionalization of $\mathbf{1 . 3 3}$ afforded a Grob fragmentation product $\mathbf{1 . 3 4}$, presumably due to the ring strain.

\subsubsection{Professor Trauner's Strategy toward Maoecrystal $\mathbf{V}^{19}$}

Unlike the previous synthetic approaches, the Trauner group envisaged a non-DA approach toward Maoecrystal V. Their synthesis started with an intramolecular aldol condensation of substituted cyclohexenone $\mathbf{1 . 3 5}$. The resulting secondary alcohol was protected as TBS ether $\mathbf{1 . 3 6}$. This compound was followed by a acetylene anion addition to 1.36 and subsequent hydrogenation afforded the key intermediate (1.37), which contained one quaternary carbon center. At this stage, the plan was to install the second quaternary carbon center by a direct double aldol addition of 1.37 with formaldehyde; however, the second aldol addition proved to be very challenging, probably because it required a concave face addition. To circumvent this issue, the mono-alkylation product was epimerized by an oxidation/reduction sequence (1.38 to $\mathbf{1 . 3 9}$, condition 1 and 2$)$. The

resulting epimerized mono-alkylated product smoothly underwent a second alkylation reaction on the convex face and afforded the desired advanced intermediate 1.39 with 

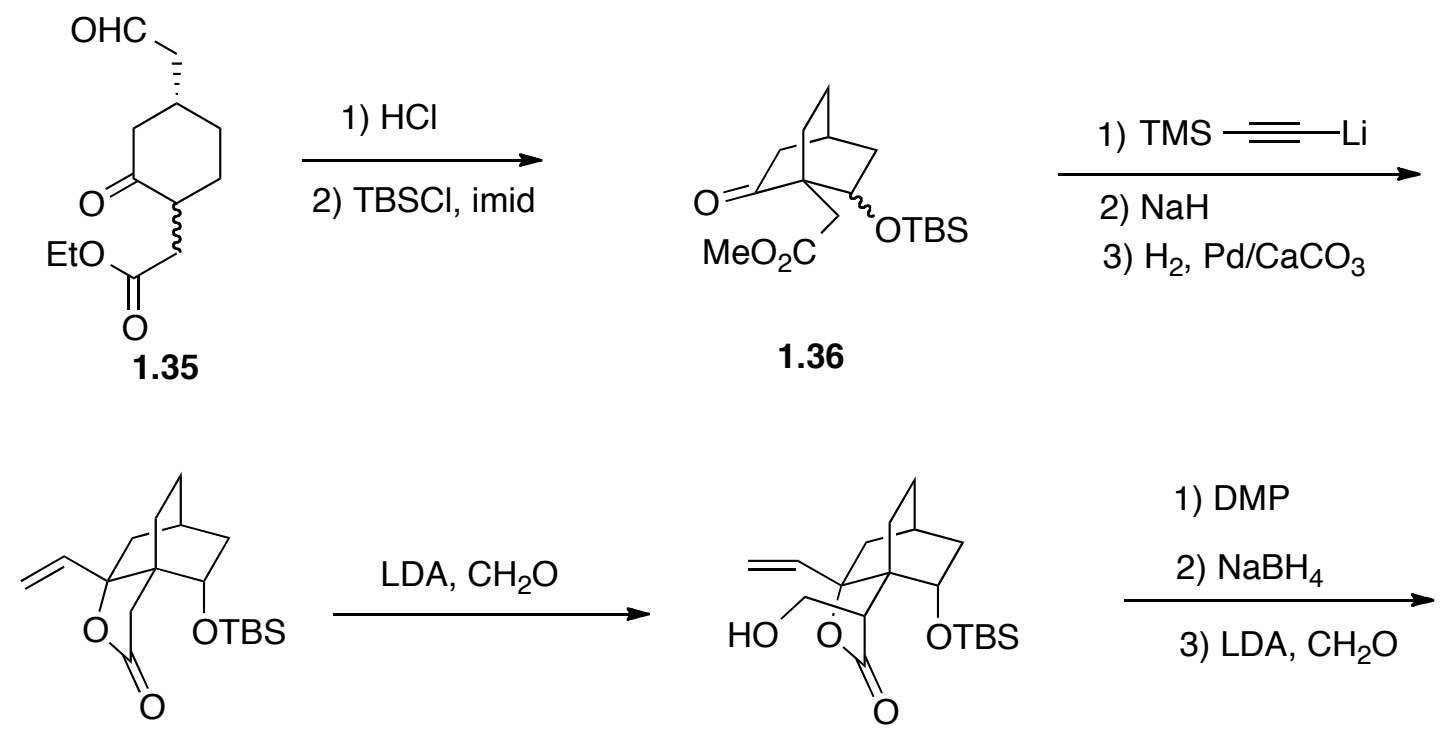

1.37

1.38

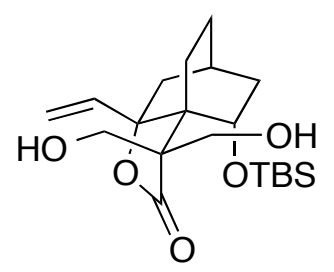

1.39

Scheme 1-5 Trauner's Approach toward Maecrystal V

two continuous quaternary carbon centers. This strategy presented an efficient method to prepare the tetracyclic core with two quaternary centers. The remaining chemistry toward Maoecrystal $\mathrm{V}$ is to synthesize the enone $\mathrm{A}$ ring.

\subsubsection{Professor Zakarian's Strategy toward Maoecrystal $V^{20}$}

The Zakarian group demonstrated an interesting IMDA approach toward Maoecrystal V. 
<smiles>C=CC(C)(C)COc1ccc2c(c1C(=N)COC)OCO2</smiles><smiles>C=C[C@H](C)[C@H](C)[C@H]1Oc2ccc3c(c2[C@@H]1C(=O)OC)OCO3</smiles>

1) LiHMDS, Mel

$\mathrm{Me}$

\subsection{1}<smiles>C=C[C@H](C)[C@H]1Oc2ccc3c(c2[C@@]1(C)CO)OCO3</smiles>

1.42
1) $\mathrm{MeMgBr}$

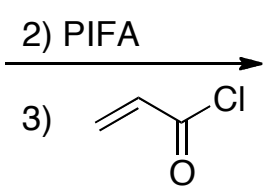

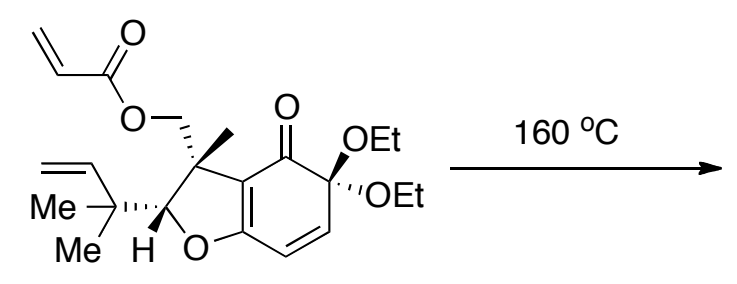

1.43<smiles></smiles>

1.44

Scheme 1-6 Zakarian's Approach toward Maoecrystal V

Their strategy features an early installation of the THF ring. The synthesis commenced with an elegant $\mathrm{C}-\mathrm{H}$ insertion reaction ${ }^{21}(\mathbf{1 . 4 0}$ to $\mathbf{1 4 1})$ to provide a dihydrobenzofuran 1.41. Alkylation of the ester and subsequent reduction of the ester gave alcohol $\mathbf{1 . 4 2}$. Compound 1.42 was then subjected to $\mathrm{MeMgBr}$ to provide a mono ethyl-protected catechol derivative. Treatment with PIFA followed by acylation with acryloyl chloride gave the desired IMDA precursor 1.43. The key IMDA occurred thermally to afford the desired cycloadduct $\mathbf{1 . 4 4}$ in excellent yield. What remains toward the total synthesis are the preparation of the desired lactone and the left A enone ring. 


\subsubsection{Professor Yang's Total Synthesis of Maoecrystal V in $2010{ }^{22}$}

As the first research group published model study toward Maoecrystal V in 2009

${ }^{23}$, Prof. Zhen Yang's group achieved the first total synthesis of Maoecrystal V in 2010. Considering the concise strategy, their synthesis was really a milestone in this research field. Their synthesis started with the preparation of a $\beta$-ketoester 1.46 (1.45 to 1.46). A Barton arylation of this ketoester $\mathbf{1 . 4 6}$ with a lead reagent $\mathbf{1 . 4 7}$ provided the desired key intermediate (1.48), with the requisite quaternary carbon center. The stereoselevtive reduction of the ketone proved to be challenging during their exploration; nevertheless, it was eventually found that $\mathrm{Bu}_{4} \mathrm{NBH}_{4}$ was the right hydride reagent to provide the desired C-5 $\beta$-hydroxyl compound exclusively. A subsequent $\mathrm{LiAlH}_{4}$ reduction of the ester afforded the diol 1.49. Esterification followed by a diazo transfer reaction furnished a diazo compound 1.51. This diazo compound was then subjected to an elegant intramolecular $\mathrm{C}-\mathrm{H}$ insertion ${ }^{24}$ reaction and Horner-Wadsworth-Emmons reaction with paraformylaldehyde to furnish key intermediate 1.52. At this stage the C-5 oxygen and lactone bis-tethered dienophile was ready. The MOM protecting group was removed with TFA and the free phenol was subjected to Wessely oxidation condition to provide the key IMDA precursor. Without isolation, the crude mixture was subjected to thermalpromoted DA condition to provide desired adduct $\mathbf{1 . 5 3}$ as a minor isomer $(36 \% \mathbf{1 . 5 3}$ and $42 \%$ undesired isomer). It would be a perfect solution to Maoecrystal $\mathrm{V}$ if a single diastereoisomer was obtained in this IMDA reaction. Actually, the diastereoselectivity is a challenging issue when IMDA strategy was employed to construct the bicyclo-[2.2.2] octane structure. Now the synthesis went to its endgame stage. 
<smiles>CC1(C)C=CCCC1=O</smiles><smiles>COC(=O)OC</smiles>

1.45<smiles>COc1c(C)cccc1[C@@H]1CC=CC(C)(C)C1=O</smiles>

1.48<smiles>CCOP(=O)(OCC)C(=N)C(=O)OC[C@@]1(c2cccc(C)c2OC)CC=CC(C)(C)[C@@H]1O</smiles>

1.51

1) $\mathrm{Rh}_{2}(\mathrm{OAc})_{4}$
1) $\mathrm{Bu}_{4} \mathrm{NBH}_{4}$

2) $\mathrm{LiAlH}_{4}$

2) ${ }^{\mathrm{B}} \mathrm{BuOK},(\mathrm{HCHO})_{n}$<smiles>CC(=O)C1CC=CC(C)(C)C1=O</smiles>

1.46
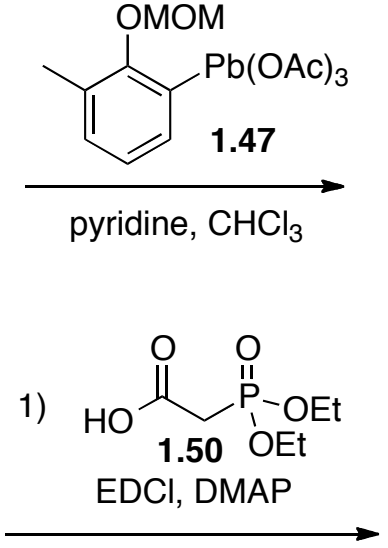

2) $\mathrm{TsN}_{3}, \mathrm{DBU}$

\subsection{9}

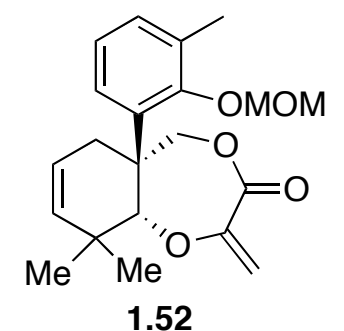

1) $T F A$

2) $\mathrm{Pb}(\mathrm{OAc})_{4}$, then $145^{\circ} \mathrm{C}$

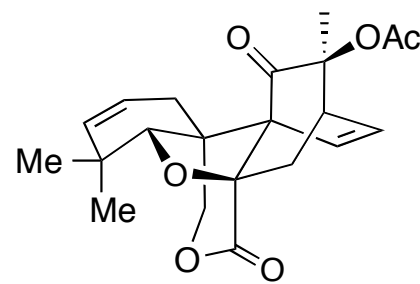

1.53
1) NBS, $\left(\mathrm{PhCO}_{2}\right)_{2}$

2) $\mathrm{Bu}_{3} \mathrm{SnH}, \mathrm{TEMPO}$

3) $\mathrm{Zn}, \mathrm{HOAc}$

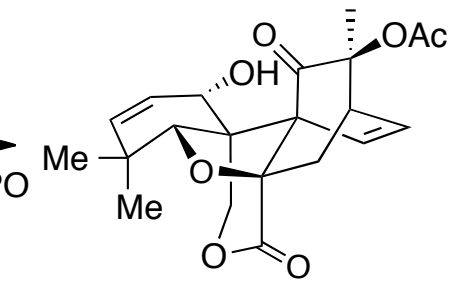

1.54

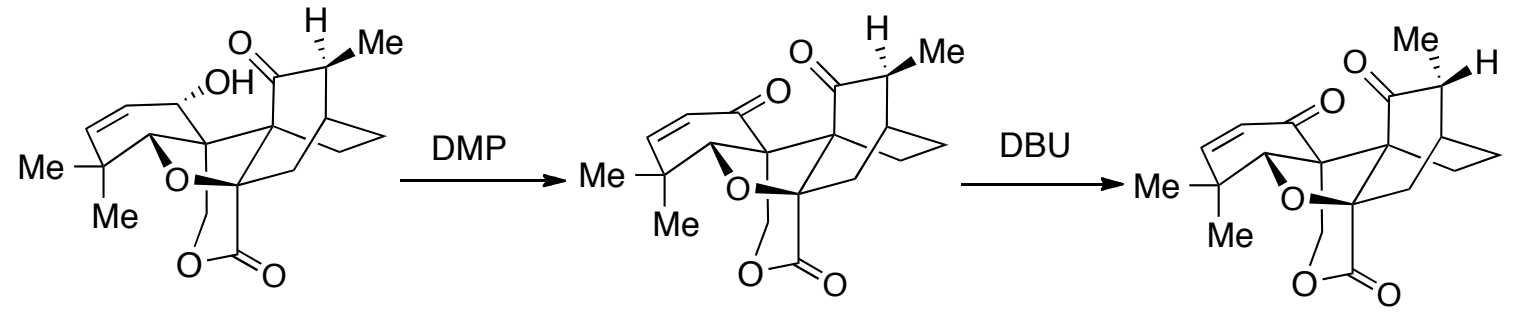

1.55
1) $\mathrm{Sml}_{2}, \mathrm{THF}$

2) Lindlar cat.

Scheme 1-7 Yang's Total Synthesis of Maoecrystal V 
The $\mathrm{C}-1$ oxygen functionality was introduced by an indirect allylic oxidation sequence (1.53 to $\mathbf{1 . 5 4})$ initiated by an allylic bromination reaction. The resulting allylic bromide was then treated with $\mathrm{Bu}_{3} \mathrm{SnH}$ and TEMPO and subsequent $\mathrm{Zn}$ reduction furnished the desired alcohol 1.54. $\mathrm{SmI}_{2}$ reduction and hydrogenation provided a key intermediate 1.55. This intermediate $\mathbf{1 . 5 5}$ was then subjected to DMP oxidation to furnish 16-epi-Maoecrystal V. A base-catalyzed epimerization reaction provided the natural Maoecrystal V. Yang's synthesis featured a concise strategy based on several elegant tranformations such as Barton arylation, Wessely oxidation, intramolecular $\mathrm{OH}$ insertion and subsequent Horner-Wadsworth-Emmons reaction.

\subsection{Reference:}

(1) Wu, Zhengyi. Yunan Zhiwu Zhi; Science Press: Beijing, China, May 2006.

(2) Sun, H. D.; Xu, Y. L.; Jiang, B. Diterpenoids from Isodon Species; Science Press:

Beijing, China, October 2001.

(3) Sun, H. D.; Huang, S. X.; Han, Q. B. Nat. Prod. Rep. 2006, 23, 673-698.

(4) Li, S. H.; Niu, X. M.; Shen, Y. H.; Zhang, H. J.; Sun, H. D.; Li, M. L.; Tian, Q. E.; Lu, Y.; Cao, P.; Zhang, Q. T. Org. Lett. 2004, 6, 4327-4330.

(5) Han, Q. B.; Cheung, S.; Tai, J.; Qiao, C. F.; Song, J. Z.; Tso, T. F.; Sun, H. D.; Xu, H. X. Org. Lett. 2006, 8, 4727-4730.

(6) This data is based on Google search and SCI Finder search

(7) Krawczuk, P. J.; Schone, N.; Baran, P. S. Org. Lett. 2009, 11, 4774-4776. 
(8) Barton, D. H. R.; Lester, D. J.; Motherwell, W. B.; Papoula, M. T. B. J. Chem. Soc., Chem. Commun. 1980, 2246-247.

(9) Nicolaou, K. C.; Dong, L.; Deng, L. J.; Talbot, A. C.; Chen, D. Y. K. Chem. Commun. 2010, 46, 70-72.

(10) Dong, L.; Deng, L.; Lim, Y. H.; Leugn, G. Y. C.; Chen, D. Y.-K. Chem. Eur. J. 2011, 17, 5778-5781.

(11) Tanaka, D.; Myers, A. G. Org. Lett. 2004, 6, 433-436.

(12) Lazarski, K. E.; Hu, D. X.; Sterm, C. L.; Thomson, R. J. Org. Lett. 2010, 12, 30103013.

(13) Corey, E. J.; Myers, A. G. J. Am. Chem. Soc. 1985, 107, 5574-5576.

(14) Rubottom, G. M.; Wazquez, M. A.; Pelegrina, D. R. Tetrahedron Lett. 1974, 15, 4319-4322.

(15) Nahm, S.; Weinreb, S. M. Tetrahedron Lett. 1981, 22, 3815-3818.

(16) Frontier, A. J.; Collison, C. Tetrahedron 2005, 61, 7577-7606.

(17) Ballini, R.; Petrini, M. Tetrahedron 2004, 60, 1017-1047.

(18) Ceccherelli, P.; Curini, M.; Marcotullio, M. C.; Mlari, B. L.; Wenkert, E.J. Org. Chem. 1986, 51, 1505-1509.

(19) Baitinger, I.; Mayer, P.; Trauner, D. Org. Lett. 2010, 12, 5656-5659.

(20) Gu, Z.; Zakarian, A. Org. Lett. 2011, 13, 1080-1082.

(21) Davies, H. M. L.; Manning, J. R. Nature 2008, 451, 417-424.

(22) Gong, J.; Lin, G.; Sun, W.; Li, C.; Yang, Z. J. Am. Chem. Soc. 2010, 132, $16745-$ 16746.

(23) Gong, J.; Lin, G.; Li, C. C.; Yang, Z. Org. Lett. 2009, 11, 4770. 
(24) Ye, T.; Mckervey, A. Chem. Rev. 1994, 94, 1091-1123. 


\section{Chapter 2 First Generation Synthetic Approach}

\subsection{Introduction}

Total synthesis and biological evaluation of structurally challenging natural products continue to be a main focus in our laboratory. ${ }^{1}$ In this context, our attention was attracted by a recent isolated anti-cancer terpenoid, Maoecrystal $\mathrm{V}^{2}$. Maeocrystal $\mathrm{V}$ is a densely functionalized diterpene, which possesses a novel rearranged ent-kaurane skeleton with a bicyclo-[2.2.2] octane motif. There are three continuous quaternary carbon centers imbedded within a pentacyclic ring system, and, at the same time, these three quaternary centers are spiro centers.

\subsection{Synthetic Plan: Intramolecular Diels-Alder Reaction}

We started with a close pattern-analysis of the preparation of the bicyclo-[2.2.2] octane moiety. ${ }^{3}$ Generally speaking, there are three known approaches to build a bicyclo[2.2.2] octane structure. The first method is a Diels-Alder reaction using 1,3cyclohexadiene $\mathbf{2 . 2}$ or its derivatives as dienes. ${ }^{4}$ In this Diels-Alder reaction, two C-C bonds and a ring are formed at the same time, which is highly efficient. The second method is intramolecular C-C bond formation (eg: aldol, 1, 4-addition) from a substituted cyclohexane derivative 2.5. ${ }^{5}$ The stereochemistry of the final bicyclo-[2.2.2] structure is usually controlled by the precursor 2.5 . The third method is the ring-closing metathesis of 
the 1,4-syn-di-vinyl substituted cylohexane derivatives $2.6^{6}$ This approach requires preparation of a diene precursor 2.7, which is not straightforward from simple starting materials.

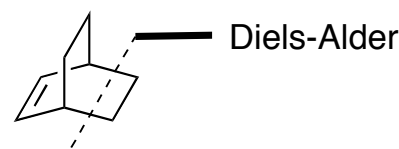

2.1

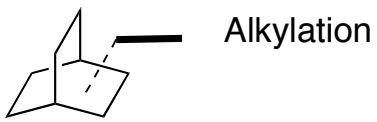

2.4

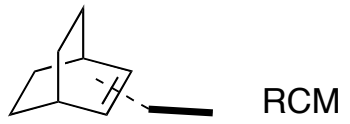

2.6

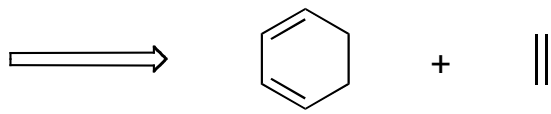

2.2

2.3

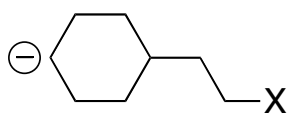

2.5

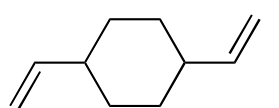

2.7

Figure 2-1 Methods to prepare bicyclic-[2.2.2] octane structure

Based on this pattern analysis and the structure evaluation of Maoecrystal $\mathrm{V}$, we designed our first generation synthesis plan. The key design of this strategy is an intramolecular Diels-Alder reaction (IMDA) ${ }^{7}$ of substrate 2.11 . 


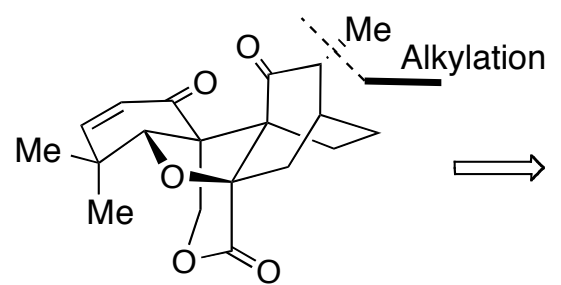

2.8

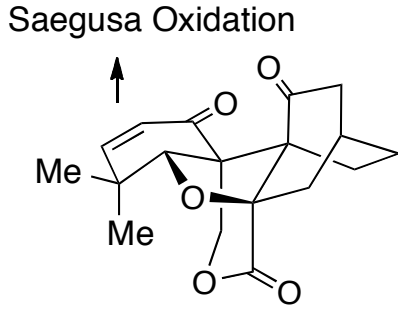

2.9

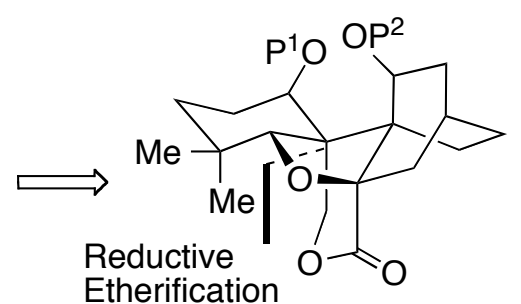

2.10

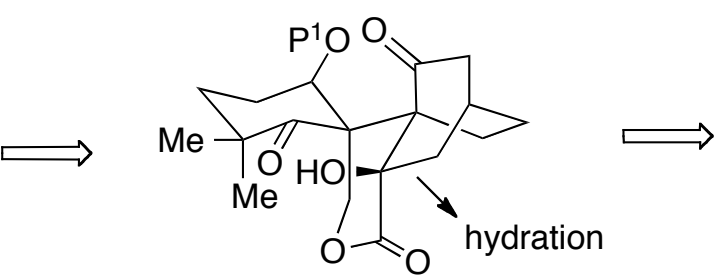

2.11

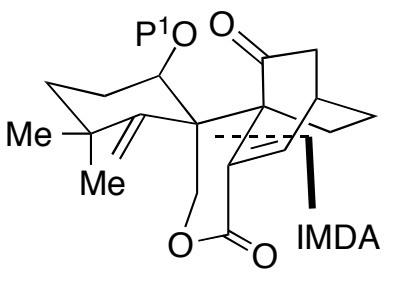

2.12
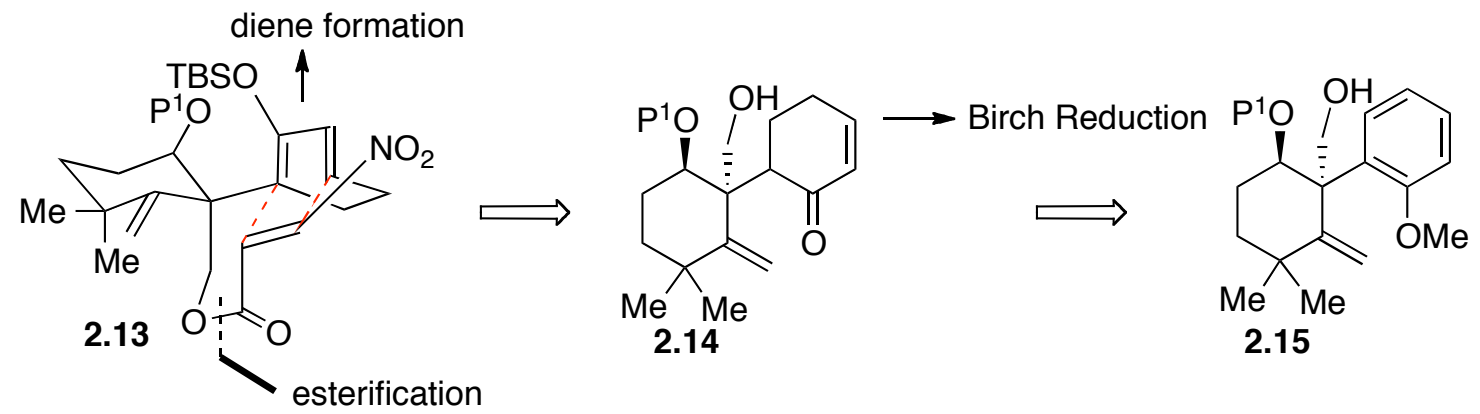

Scheme 2-1 Retrosynthesis of Maoecrystal V

We envisioned that Maoecrystal V could be synthesized from a stereoselective alkylation of substrate 2.9. The enone moiety could be prepared using Saegusa oxidation ${ }^{8}$ from compound 2.10 in a forward sense. At this stage, we further envisioned that the core THF ring could be closed through a reductive etherification reaction from substrate $\mathbf{2 . 1 1}$, in which C-5 is a ketone and C-8 is a tertiary alcohol. We hoped that the axial hydride attack to the corresponding oxonium would afford the desired stereochemistry. The requisite C-8 tertiary alcohol could be synthesized through a selective hydration of the corresponding enone alkene in compound $\mathbf{2 . 1 2}$ in the forward sense. The stereoselectivity 
of this hydration process would be a potential problem since the spatial differences of the $\alpha$ and $\beta$ faces in a bicyclo-[2.2.2] octane structure is subtle. We rationalized that the $\mathrm{C}-15$ ketone function group would help to afford the desired $\beta$-hydroxyl group due to stereoelectronic effect (Cieplak effect ${ }^{9}$ ). Key intermediate $\mathbf{2 . 1 2}$ was anticipated to be synthesized from compound 2.13 through an intramolecular Diels-Alder reaction. The IMDA precursor 2.13 was anticipated to be prepared from compound 2.14 through an esterification reaction using $\beta$-nitro acrylate. We anticipated that the nitro group would increase the reactivity of the dienophile and would also serve as a leaving group after DA reaction to generate an alkene. The net result was a DA from propiolate; however, we concerned that a retro-DA would give an aromatic ring if propiolate was used directly here. The enone moiety in compound $\mathbf{2 . 1 4}$ was envisioned to be prepared from the corresponding functionalized aromatic ring from compound 2.15. Our synthetic plan required the preparation of a substituted cyclohexane derivative 2.15 .

\subsection{Results and Discussion}

Our first objective was to synthesize of the key cyclohexane derivative 2.15. This goal was eventually achieved by our previous postdoctoral researcher, Dr. Maolin Yu. Maolin developed an elegant route to stereoselectively synthesize compound 2.27 and his route is shown in Scheme 2-2.

As outlined in Scheme 2-2, the synthesis commenced with a Carl-Johnson iodination ${ }^{10}$ of cycloalkenone $\mathbf{2 . 1 6}$. The resultant $\alpha$-iodo cyclohexenone was subjected to the Suzuki-Miyaura coupling, with boronic acid 2.18, to afford $\alpha$-arylcyclohexenone $\mathbf{2 . 1 9}$ 


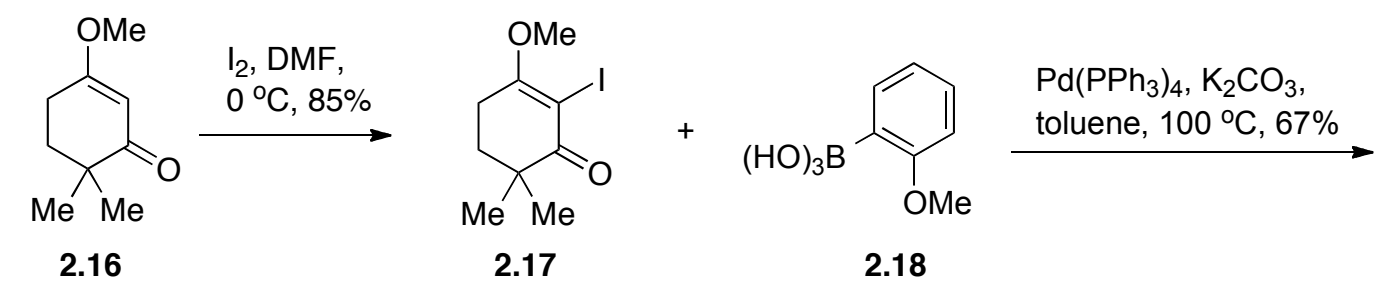<smiles>COC1=C(c2ccccc2OC)C(=O)CCC(C)(C)C(OC)=C1c1ccccc1OC</smiles>

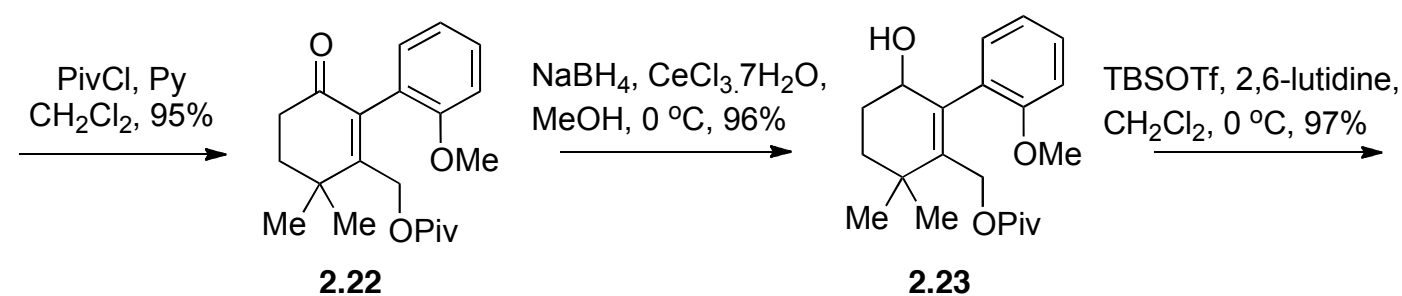

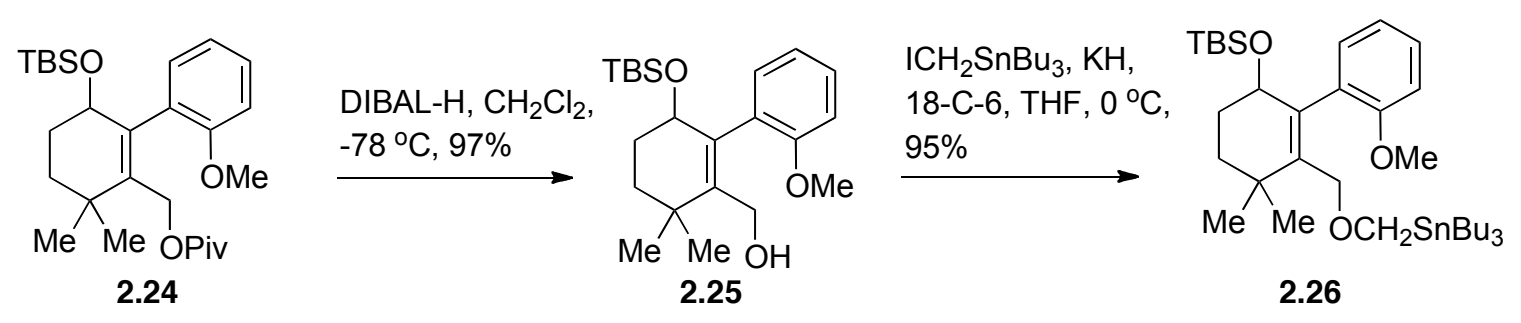
\begin{tabular}{l} 
BuLi, THF, \\
$\stackrel{-78^{\circ} \mathrm{C}, 94 \%}{\longrightarrow}$ \\
\hline
\end{tabular}<smiles>C=C1[C](c2ccccc2OC)[C@H](OC(C)(C)C)CCC1(C)C</smiles>

2.27

Scheme 2-2 Preparation of compound 2.27

in $67 \%$ yield. This intermediate $\mathbf{2 . 1 9}$ was then transformed to the 1,2-disubstituted cyclohexenone 2.20 using Stork-Danheiser protocol. ${ }^{11}$ Following replacement of MOM ether with a pivaloate ester, compound $\mathbf{2 . 2 2}$ was in hand. Luche reduction of $\mathbf{2 . 2 2}$ 
provided an allylic alcohol, which was protected as its TBS ether (2.24). DIBAL-H reduction of $\mathbf{2 . 2 4}$ freed the pivaloate-protected hydroxyl group. The resulting allylic alcohol (2.25) was then alkylated with $\mathrm{ICH}_{2} \mathrm{SnBu}_{3}$, and Wittig-Still [2,3] rearrangement ${ }^{12}$ did occur as planned and afforded the desired $\mathbf{2 . 2 7}$ in 94\% yield as a single stereoisomer.

The relative chemistry of compound $\mathbf{2 . 1 7}$ was unambiguously assigned based on a derivative $\mathbf{2 . 2 8}$ by X-ray crystallography. X-ray crystallography clearly presented that the axial C-18 methyl resides above the phenyl ring, which makes the chemical shift of this methyl group in ${ }^{1} \mathrm{H}$ NMR to be 0.64 while that of the equatorial C-19 methyl group is 1.1 .

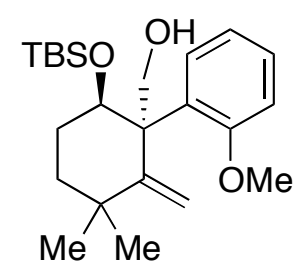

2.27

(1)

$95 \%$
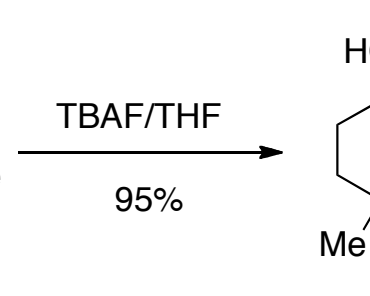

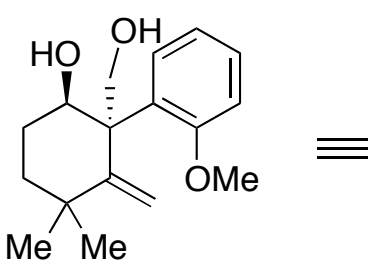

2.28

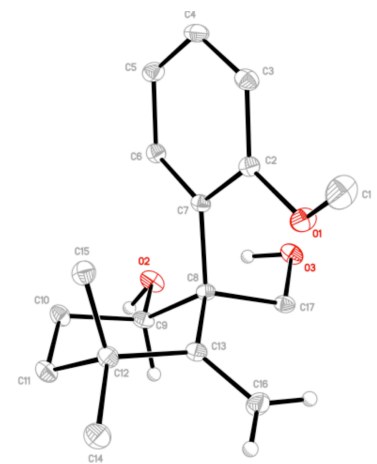

Scheme 2-3 Preparation of 2.28

Based on the analysis above, we proposed that the Wittig-Still [2,3] rearrangement took the transition structure $\mathbf{2 . 2 9}$, to afford our desired product. With compound 2.27 in hand, Maolin then explored the Birch reduction ${ }^{13}$ on it and its derivatives. After extensive study, Maolin eventually found that the Birch reduction of compound 2.30 afforded mono alkene 2.3 instead of the desired para-diene 2.34 Compound 2.32, however, did not undergo dehydrogenation to provide the desired enone 


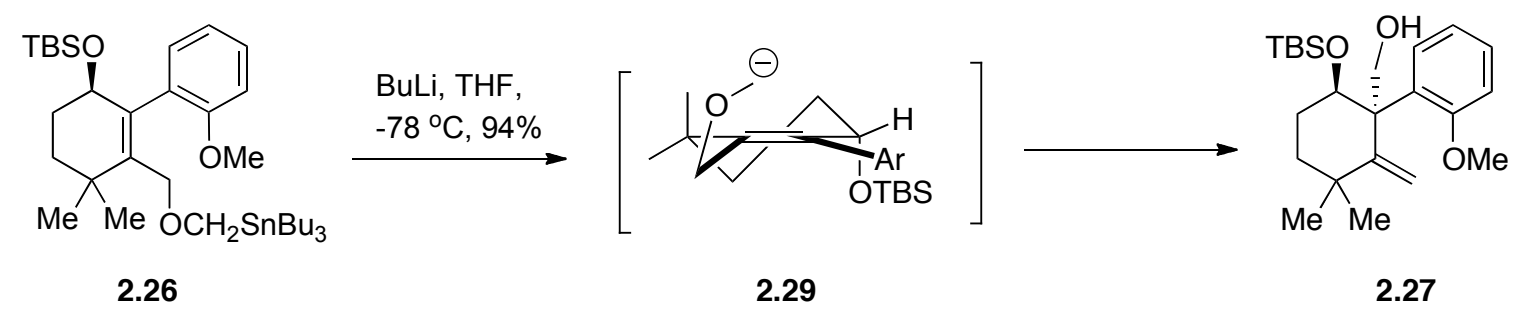

Scheme 2-4 Wittig-Still [2,3]-rearrangement

2.33. At this stage, Maolin then planned to use an alternative diene 2.35, which could be prepared through an oxidative dearomatization reaction ${ }^{14}$, to explore the IMDA reaction.<smiles>C=C1[C@@H](c2ccccc2OC(F)(F)F)[C@H](O)CCC1(C)C</smiles>

2.30
1) $\mathrm{Li}, \mathrm{NH}_{3}(\mathrm{l})$<smiles>C1CCCC1</smiles><smiles>C=C1[C](C2CC=CC(=O)C2=O)[C@H](OCC(C)(C)C)CCC1(C)C</smiles>

2.33<smiles>C=C1C(C)(C)CC[C@@H](OC(C)(C)C)[C@@]1(O)c1ccccc1OC</smiles>

2.27

.<smiles>C=C1C(C)(C)CC[C@@H](O)[C@@]1(C)C1=C(OC)CCCC1</smiles>

2.31

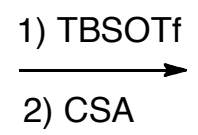

2) CSA

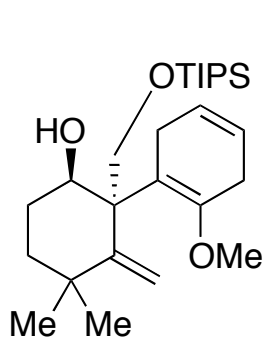

2.34

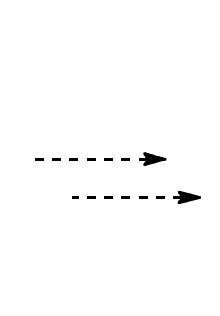

Scheme 2-5 Summary of Birch reduction 
This goal was eventually achieved by Maolin and the successful route was outlined as the following scheme (Scheme 2-6). The synthesis started with a protection of the primary alcohol $\mathbf{2 . 2 7}$ as a TBS ether $\mathbf{2 . 3 6}$. Direct lithiation followed by quenching with DMF furnished the aldehyde 2.37. Global deprotection of the silyl protecting group and selective reprotection of the primary alcohol using acetic anhydride afforded a secondary alcohol $\mathbf{2 . 3 8}$, which was further protected as a MOM ether. This protected aromatic aldehyde was subjected to a Dakin oxidation protocol, furnishing a formyl ester 2.39. During this oxidation, the exo-methylene was also oxidized to an epoxide. The formyl ester $\mathbf{2 . 3 9}$ was then hydrolyzed, and the resultant free phenol was protected as a TES ether to provide intermediate $\mathbf{2 . 4 0}$. DIBAL-H reduction served to free the primary alcohol and the resultant primary alcohol underwent an esterification with $\mathbf{2 . 4 1}$ to furnish compound 2.42. At this stage, the TES protecting group was removed using HF and PIDA oxidation of the free phenol afforded our key IMDA precursor 2.43. With this intermediate $\mathbf{2 . 4 3}$ in hand, Maolin tested the key IMDA reaction, but under the conditions tested, thermo and Lewis-acid-catalyzed conditions did not provide the DA adduct. Only decomposition of starting material was observed.

Then, Maolin was heading for his new place: Boehringer Ingelheim, and I took over this project. Since decomposition was observed during the exploration of IMDA of compound 2.43, this result indicated that the diene 2.46 might be unstable during the IMDA condition. In order to further explore the stability of the diene, an intermolecular Diels-Alder reaction using $\mathbf{2 . 4 4}$ and maleic anhydride or methyl acrylate as dienophile was explored. The thermo-promoted DA reaction afforded a dienone-phenol rearrangement product $\mathbf{2 . 4 5}$, and the desired DA adduct was not observed. 

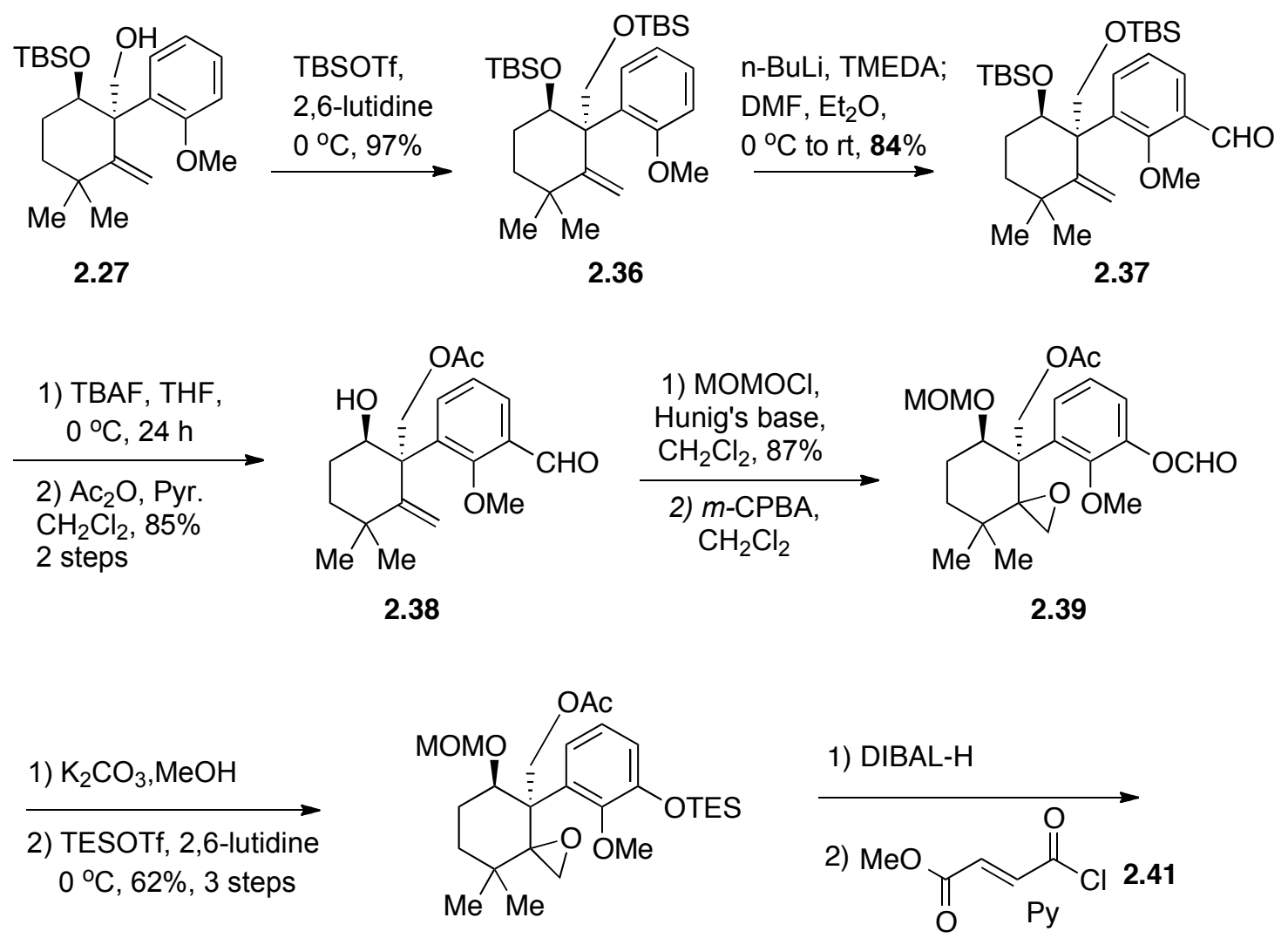

1) DIBAL-H

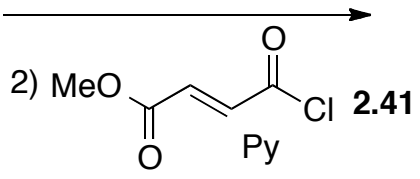

2.40

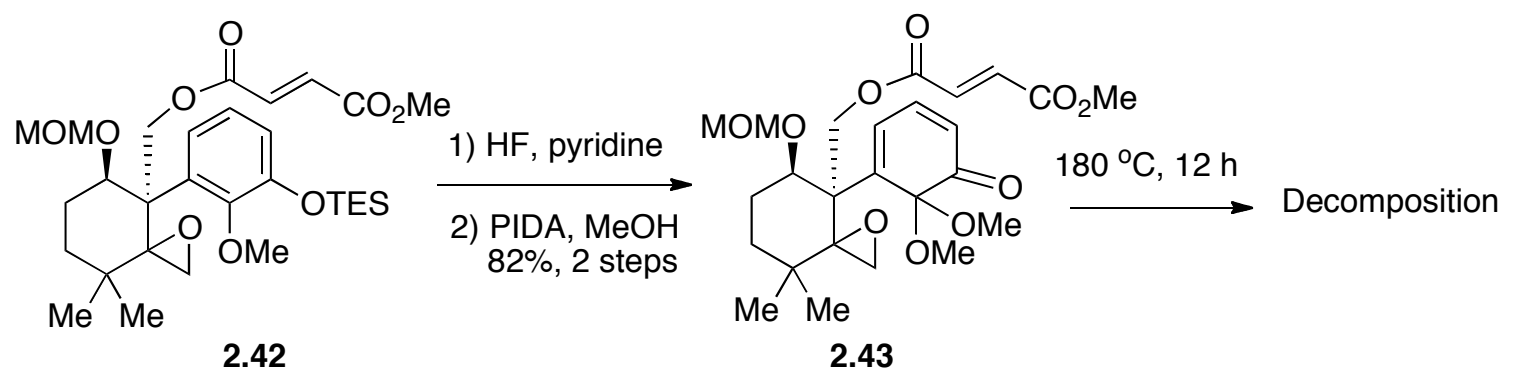

Scheme 2-6 Preparation of compound 2.43 


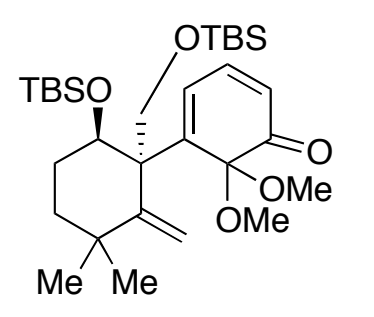

2.44

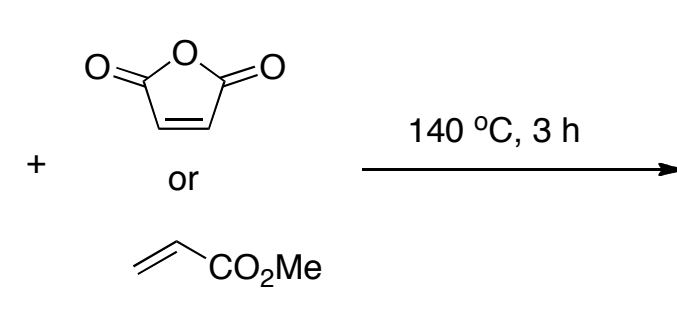

Scheme 3-7 Intermolecular DA reaction of 2.44

We also thought about other dienes that could be used as an alternative in our forward synthesis, such as diene 2.48. This is a Danishefsky-type diene and should be very reactive. It could be prepared from a para-methoxyl phenyl ring through a Birch reduction and thus I could use Maolin's route to prepare our designed compound 2.52.

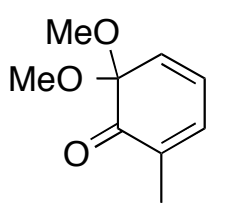

2.46

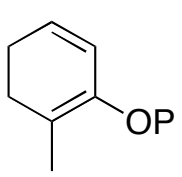

2.47

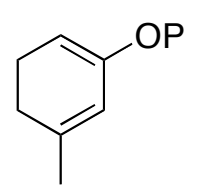

2.48<smiles>CC1=CCCC=C1C</smiles>

2.49

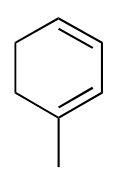

2.50<smiles>CC1=CC([OH2+])=CCC1</smiles>

2.48<smiles>COc1cccc(C)c1</smiles>

2.51<smiles>C=C1C(C)(C)CC[C@@H](P)[C@@]1(O)c1cccc(OC)c1</smiles>

2.52

Figure 2-2 Summary of dienes 
Similar to Maolin's route, the synthesis of compound 2.52 started with a palladium-catalyzed ketone $\alpha$-arylation ${ }^{15}$ reaction of $\mathbf{2 . 5 3}$, and after etherification with $\mathrm{TMSCHN}_{2}$, compound $\mathbf{2 . 5 5}$ was obtained in $90 \%$ overall yield. The $\beta$-methoxylenone was subjected to the Stork-Danheiser protocol using tributyl[(methoxymethoxy)methyl]stannane, to provide compound $\mathbf{2 . 5 6}$ in $75 \%$ yield. The MOM ether protecting group was cleaved under acidic conditions, and the resulting alcohol was protected as its pivaloate ester 2.57 . Luche reduction of 2.57 provided an allylic alcohol, which was immediately protected as the MOM ether $\mathbf{2 . 5 8}$. Hydrolysis of 2.58 liberated a hydroxymethyl intermediate that was alkylated with $\alpha$-iodomethyl tributylstannane to furnish the Wittig-Still [2,3]-rearrangement precursor 2.59. By careful monitoring of the reaction temperature, we were pleased to find that the Wittig-Still $[2,3]$ rearrangement proceeded smoothly to provide alcohol $\mathbf{2 . 6 0}$ in $88 \%$ yield. It is noteworthy that this transformation established the requisite quaternary carbon center in a highly stereo-controlled fashion. Enantiopure 2.60, in principle, could be prepared from an enantiopure compound 2.58, which could be accessed through an enantioselective reduction of ketone $\mathbf{2 . 5 7}$. 

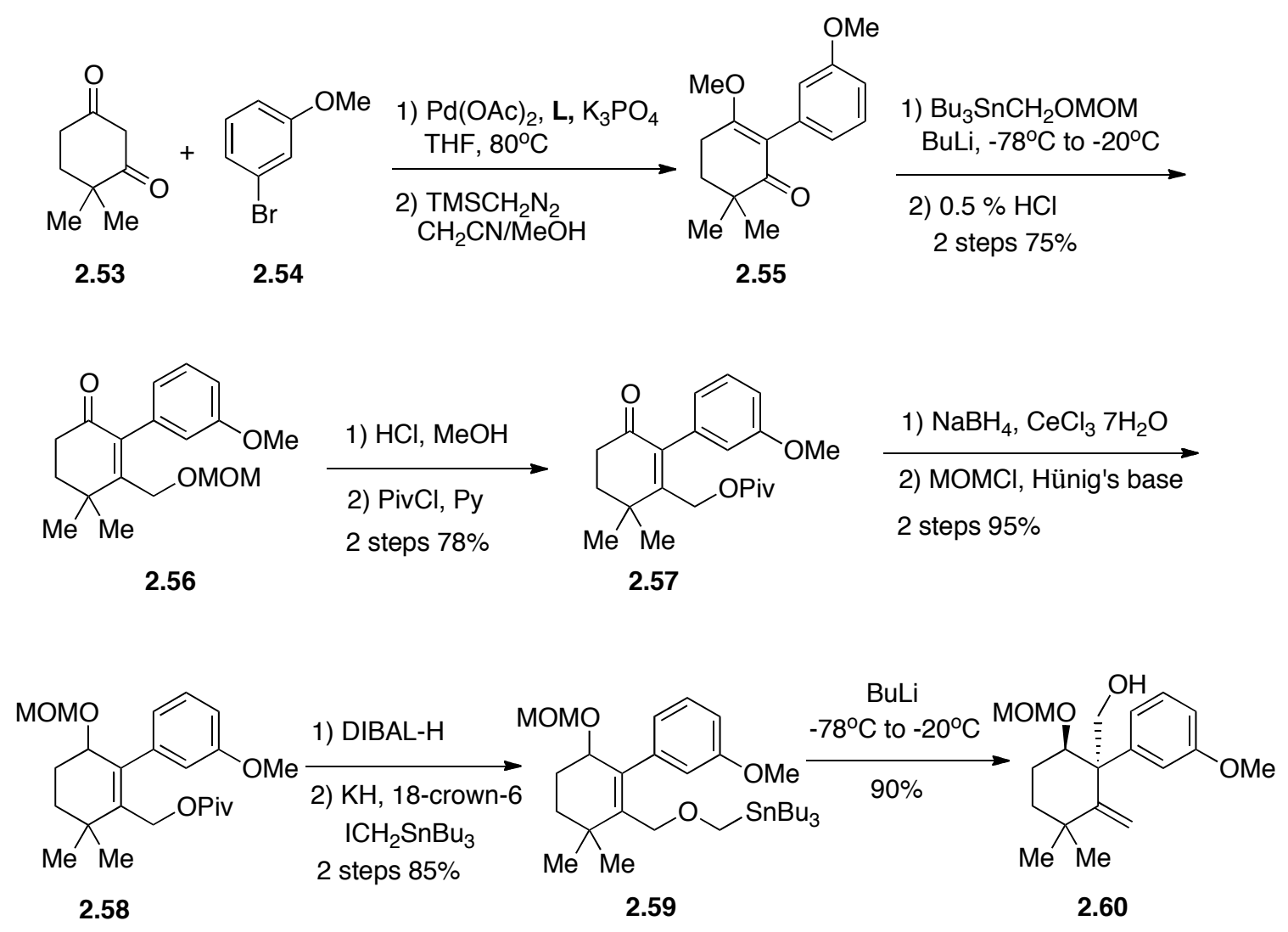

Scheme 2-8 Preparation of compound 2.60

With compound $\mathbf{2 . 6 0}$ in hand, we then explored the Birch reduction reaction on this substrate. We found that when the reaction proceeded for 1 hour, the isolated product was 2.61, as an 1:3 $\alpha / \beta$ mixture of methyl epimers, in which the exo-methylene was reduced instead of the phenyl ring. Continuation of the reaction time to 4 hour resulted the reduction of both exo-methylene and the phenyl ring. These results indicated that the exo-methylene was reduced faster than phenyl ring on compound $\mathbf{2 . 6 2}$, which would cause problem for our forward synthesis since the exo-methylene group would be used as a ketone precursor. 


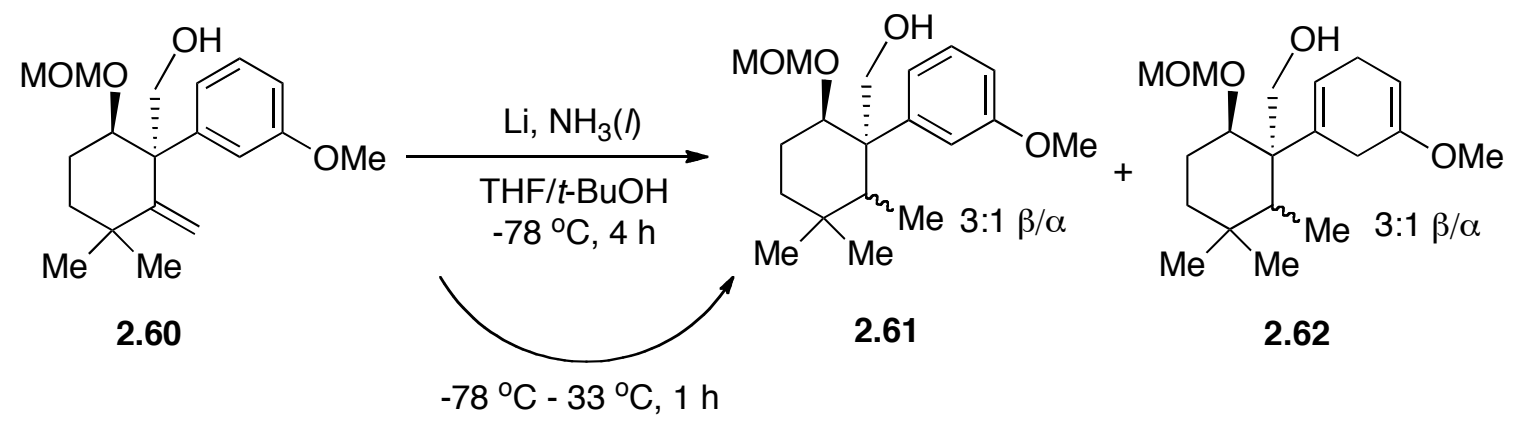

Scheme 2-9 Birch reduction of compound 3.60

To circumvent this problem, we thought that we could transfer compound $\mathbf{2 . 6 0}$ to substrate $\mathbf{2 . 6 3}$ first and carry the Birch reduction on this substrate secondly.<smiles>C=C1[C](c2cccc(OC)c2)[C@H](OC)CCC1(C)C</smiles>

2.60<smiles>COc1cccc([C@H]2[C@@H](O)[C@H](OC)CC[C@]2(C)C(C)(C)C)c1</smiles>

2.63

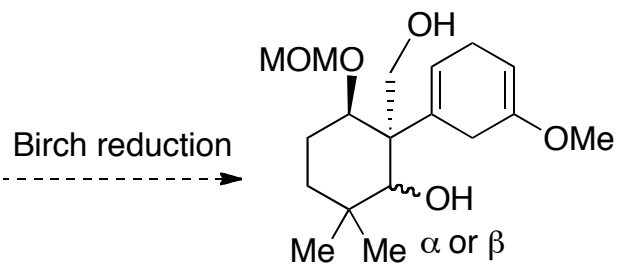

2.64

Scheme 2-10 New plan for Birch reduction

Compound 2.60 was then subjected to ozonolysis reaction, and the intermediate was then treated with $\mathrm{PPh}_{3}$ to afford the desire ketone 2.65 in excellent yield. Further reduction of the ketone $\mathbf{2 . 6 5}$ with $\mathrm{LiAlH}_{4}$ gave a diol 2.66. We noticed that the stereochemistry of the C-5 hydroxyl group is opposite as required by the natural product, presumably due to an axial hydride reduction directed by the primary alcohol (Figure 23). 


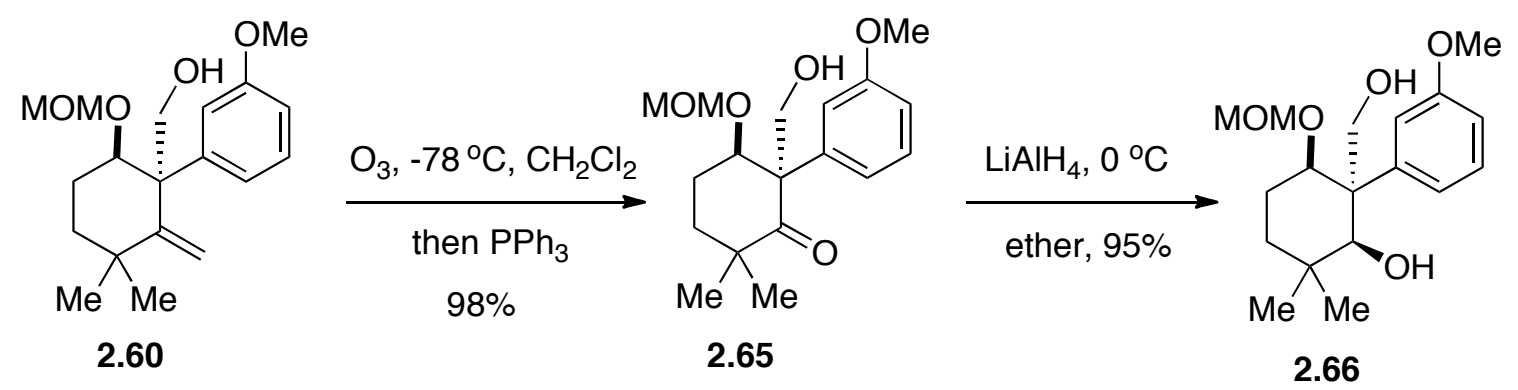

Scheme 2-11 Preparation of compound 2.66

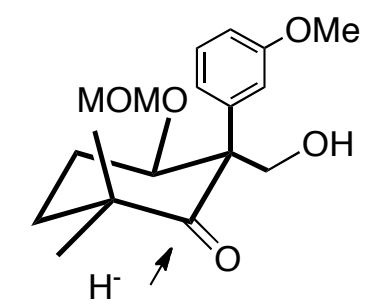

Figure 2-3 Axial Hydride attack of $\mathbf{2 . 6 5}$

We then did further study on this ketone reduction, hoping to obtain a C-5 $\alpha$ hydroxyl compound 2.63. While DIBAL-H and $\mathrm{NaBH}_{4}$ gave the same results as $\mathrm{LiAlH}_{4}$, L-Selectride reduction gave a decomposed compound 2.67. At this stage, we recalled the stereochemistry of the exo-methylene reduction of compound $\mathbf{2 . 6 0}$ during the Birch reduction, in which $\alpha$-methyl epimer was obtained as a minor isomer. This result suggested that during the single electron transfer condition, a $\beta$-hydrogen delivery process would occur. To our delight, when ketone 2.65 was subjected to the $\mathrm{Li} / \mathrm{NH}_{3}(1)$ reduction protocol, the C-5 $\beta$-hydroxyl compound $\mathbf{2 . 7 0}$ was observed indeed. 

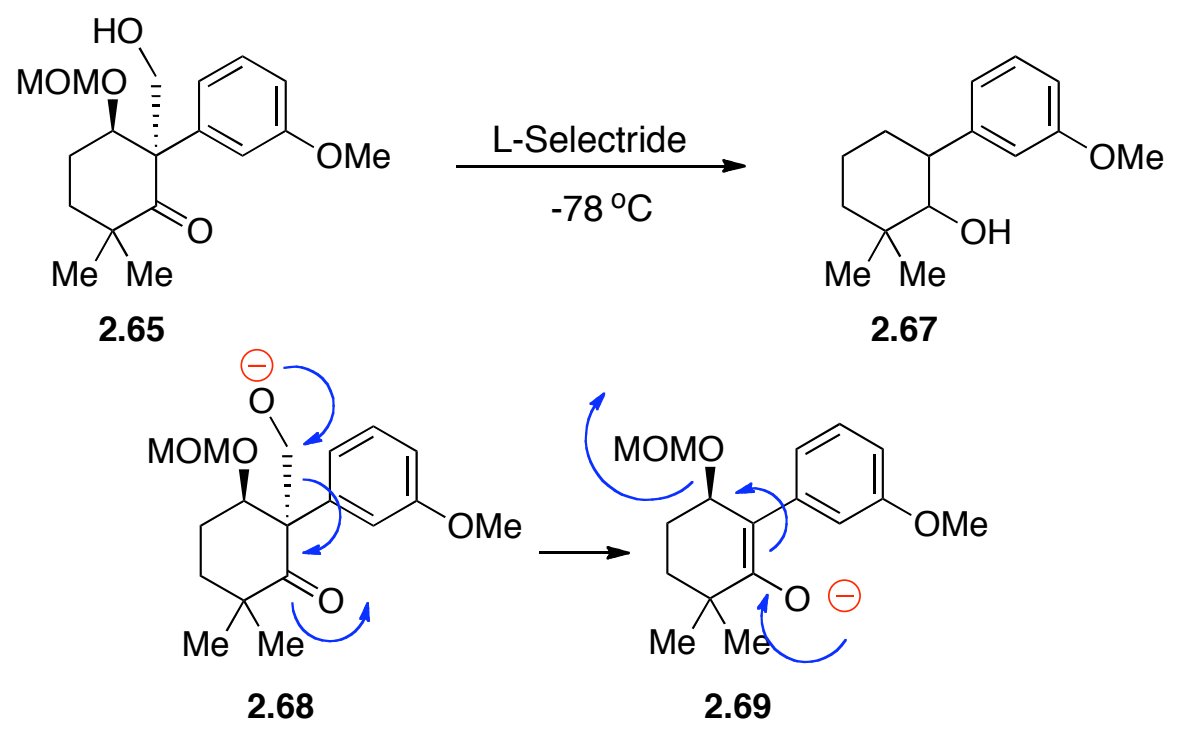

Scheme 2-12 L-Selectride reduction of $\mathbf{2 . 6 5}$

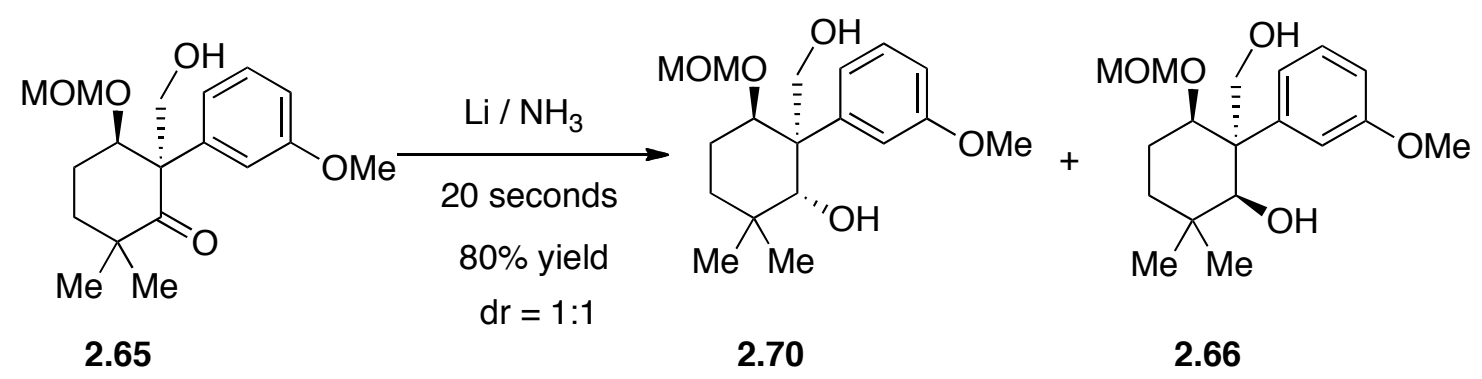

Scheme 2-13 Preparation of 2.70

With compound 2.66 in hand, we carried out Birch reduction on this compound 2.66. Luckily, the Birch reduction went smoothly and afforded the desired diene. Upon treatment with dilute $\mathrm{HCl}$, enone $\mathbf{2 . 7 1}$ was obtained in good yield. The protection of the C-5 secondary alcohol turned out to be problematic, since it is a bis-neopentyl secondary alcohol. At a highly congested situation, an intramolecular reaction would be more 
possible to occur than the corresponding intermolecular reaction. Based on this concept, we developed an "intramolecular protecting group delivery" method to achieve a selective protection of the C 5 secondary alcohol in the presence of the primary alcohol. As outlined in the Scheme 2-14, diol 2.71 was treated with anisaldehyde dimethyl acetal under acidic condition, which afforded acetal 2.72. Upon treatment with DIBAL-H, the PMB protecting group was transferred to the C-5 hydroxyl group. The resulting allylic alcohol $\mathbf{2 . 7 2}$ was selectively re-oxidized to enone $\mathbf{2 . 7 4}$.

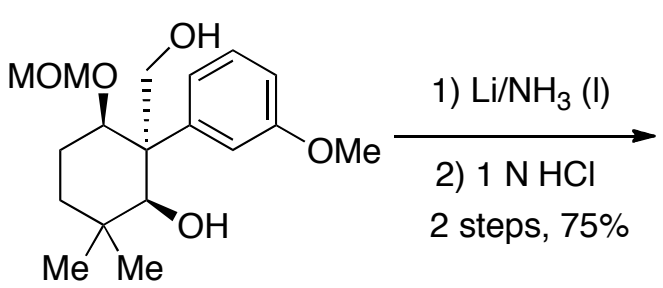

2.66

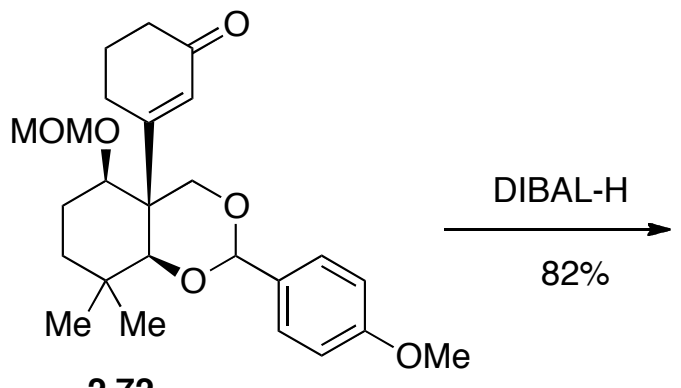

2.72<smiles>CCO[C@H]1[C@@H](C2=CC(=O)CCC2)[C@@H](OC)CCC1(C)C</smiles>

2.74<smiles>CO[C@H]1CCC(C)(C)[C@H](O)[C@H]1C1=CC(=O)CCC1</smiles><smiles>COc1ccc(C(OC)OC)cc1</smiles>

2.71

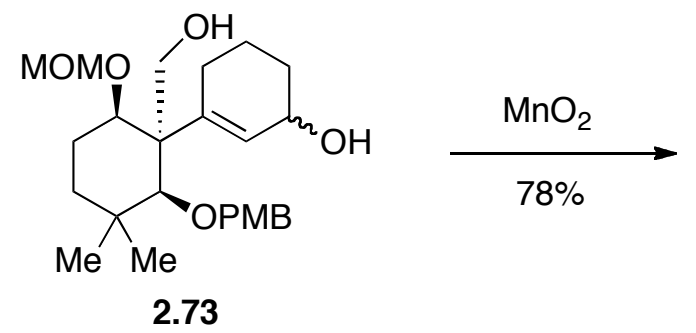

Scheme 2-14 Preparation of compound 2.74 
After solving the exo-methylene reduction on substrate 2.62, we then focused on the exploration of the key intramolecular Diels-Alder reaction, hoping to find the right dienophiles and factors controlling the stereoselectivity. With plenty of compound $\mathbf{2 . 6 2}$ in hand, I felt that this compound could serve as a valuable model substrate to probe the viability of the proposed IMDA reaction. Accordingly, acidic hydrolysis of $\mathbf{2 . 6 2}$ furnished enone 2.75, which was then subjected to esterification with acryloyl chloride, to provide acrylate 2.76. Since there are several reported intermolecular Diels-Alder reaction using 2-siloxyl-1,3-cyclohexadiene as diene and acrylate as dienophile ${ }^{16}$, we were confident that our intramolecular Diels-Alder would work.

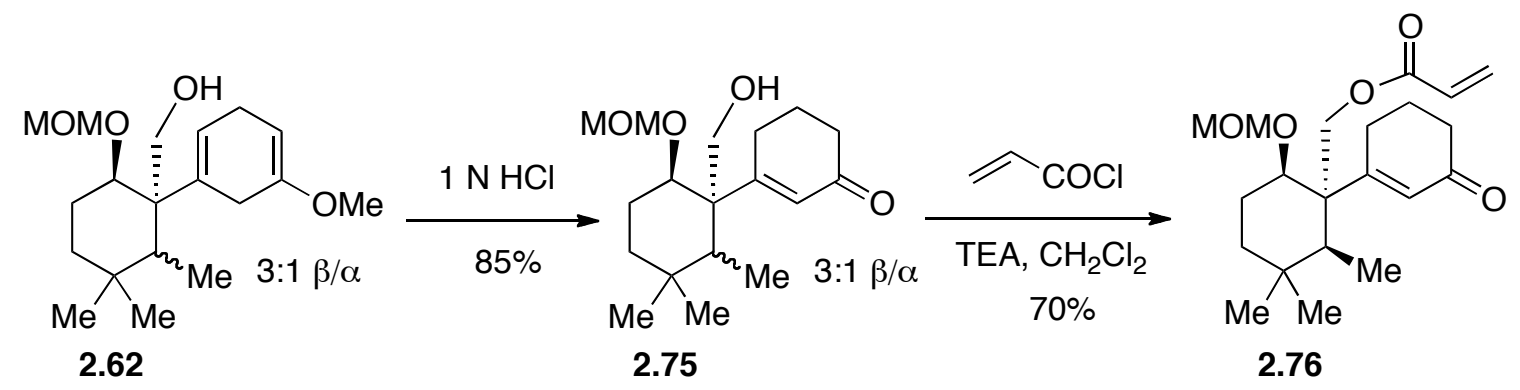

Scheme 2-15 Preparation of compound 2.76

We first explored a double-Michael approach ${ }^{17}$ (i.e, an anionic Diels-Alder reaction). Thus substrate $\mathbf{2 . 7 6}$ was treated with strong base such as LDA, LHMDS. To our disappointment, we only observed decomposition of $\mathbf{2 . 7 6}$ and no DA adduct was obtained. This suggested that our substrate $\mathbf{2 . 7 6}$ might be unstable toward basic condition and we need to carry on a neutral DA reaction. 


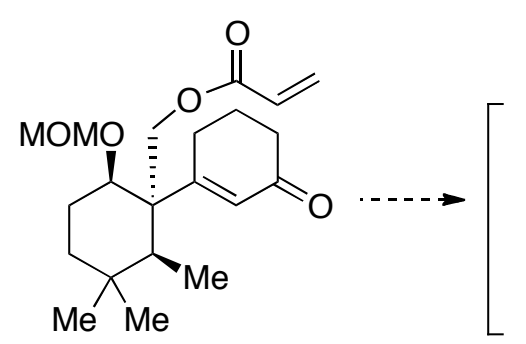

2.76

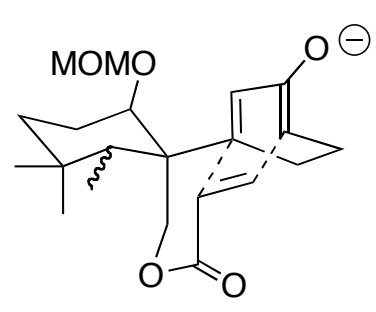

2.77

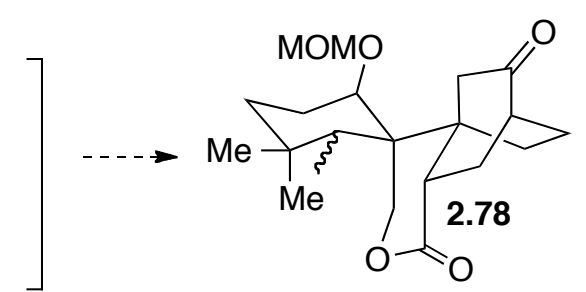

not observed

Scheme 2-16 Double-Michael approach

We then prepared the corresponding siloxyl diene $\mathbf{2 . 7 9}$ and were ready to explore the thermo DA reaction. After extensive study of different temperature factors, we did not observe any cycloadduct, but only isomerized diene $\mathbf{2 . 8 0}$.

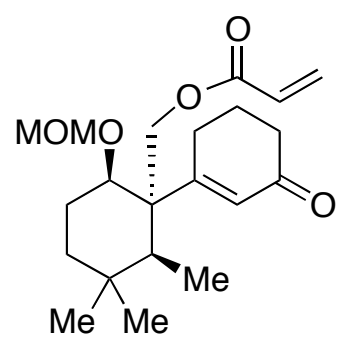

2.76

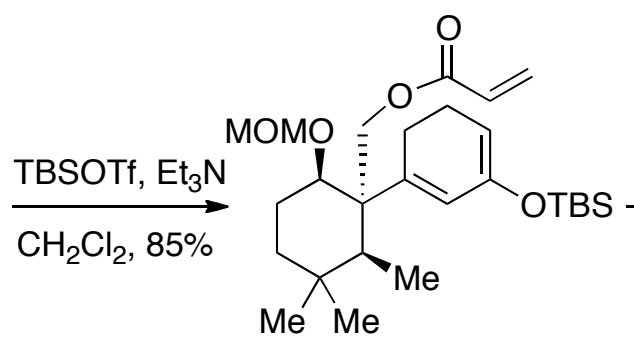

2.79

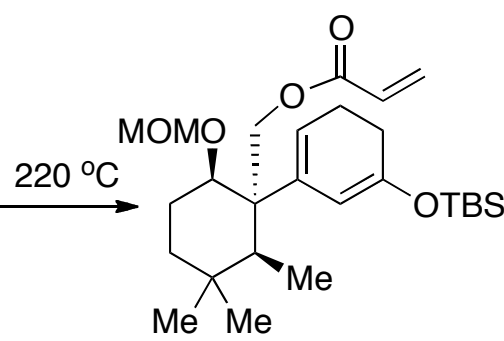

2.80

Scheme 2-17 Thermo Diels-Alder reaction of 2.79

We then switched the dienophile to fumaric ester, which is a more reactive dienophile. After heating the reaction vessel at $180{ }^{\circ} \mathrm{C}$ for 12 hour, we obtained the desired cycloadduct. Upon treatment of TBAF, ketone $\mathbf{2 . 8 2}$ was obtained, in which the stereochemistry was tentatively assigned based on NMR techniques. 


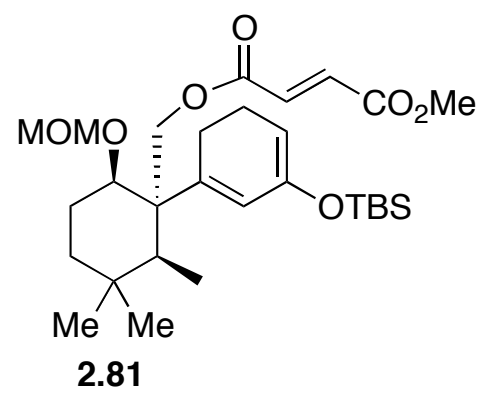

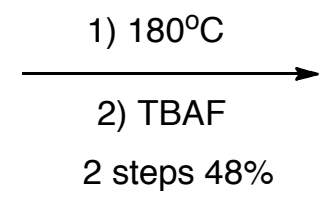

Scheme 2-18 IMDA of compound 2.81

To our disappointment, a key NOESY experiment revealed that the IMDA cycloaddition afforded the opposite facial adduct than that required for the synthetic plan. To analyze the reason for this result, we first listed all the four possible cycloadducts in a non-stereoselective situation. These four diastereoisomers were formed due to the rotation of diene and endo-/exo-adducts of each diene rotamer. In our case, adduct $\mathbf{2 . 8 2}$ formed predominantly, which suggested the possible transition structure would be $2.83 \mathrm{~A}$. 


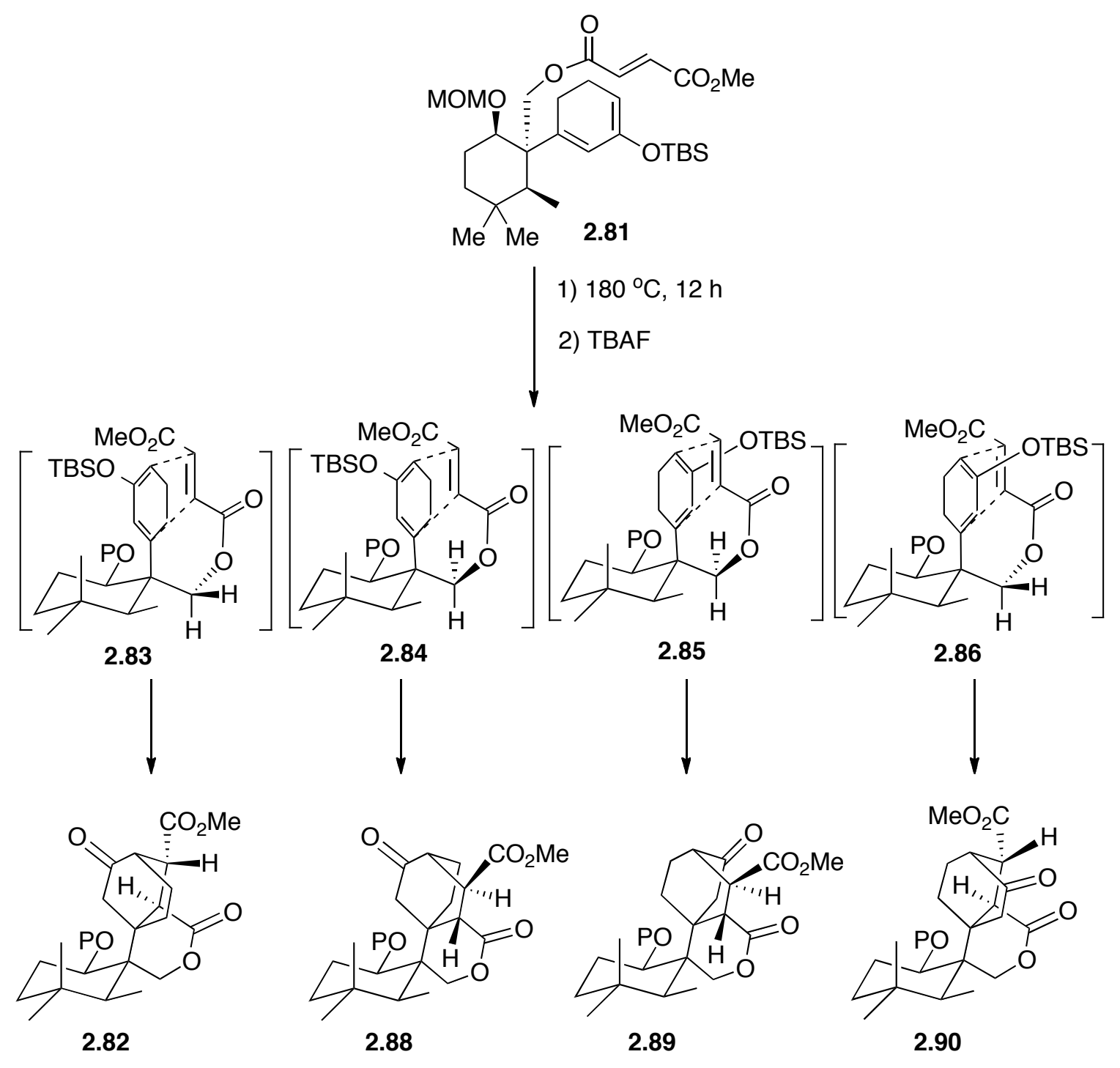

Scheme 2-19 Transition structure of IMDA of $\mathbf{2 . 8 1}$

We also tried a $\beta$-sulfone acrylate 2.91 as an IMDA precursor. We used this dienophile for particular two reasons: (1) changing an IMDA precursor might provide us the desired facial product. (2) sulfone, still activated the dienophile but could serve also as a leaving group after DA reaction. ${ }^{18}$ As predicted, this IMDA reaction proceeded smoothly and afforded us the desired adduct 2.92 and 2.93 as a diastereoisomer at 
sulfone-substituted carbon center. Upon treatment with DBU, compound 2.94 was obtained as a single isomer, whose structure was confirmed by X-ray. Again, the IMDA proceeded with the opposite sense of facial selectivity than that required for our synthetic plan.<smiles>CCOC[C@H]1[C@@H](OC)CCC(C)(C)[C@@H]1COC(=O)/C=C/CS(=O)(=O)O</smiles>

1) $196{ }^{\circ} \mathrm{C}, 3 \mathrm{~h}$

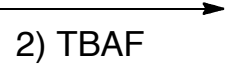

2 steps $65 \%$
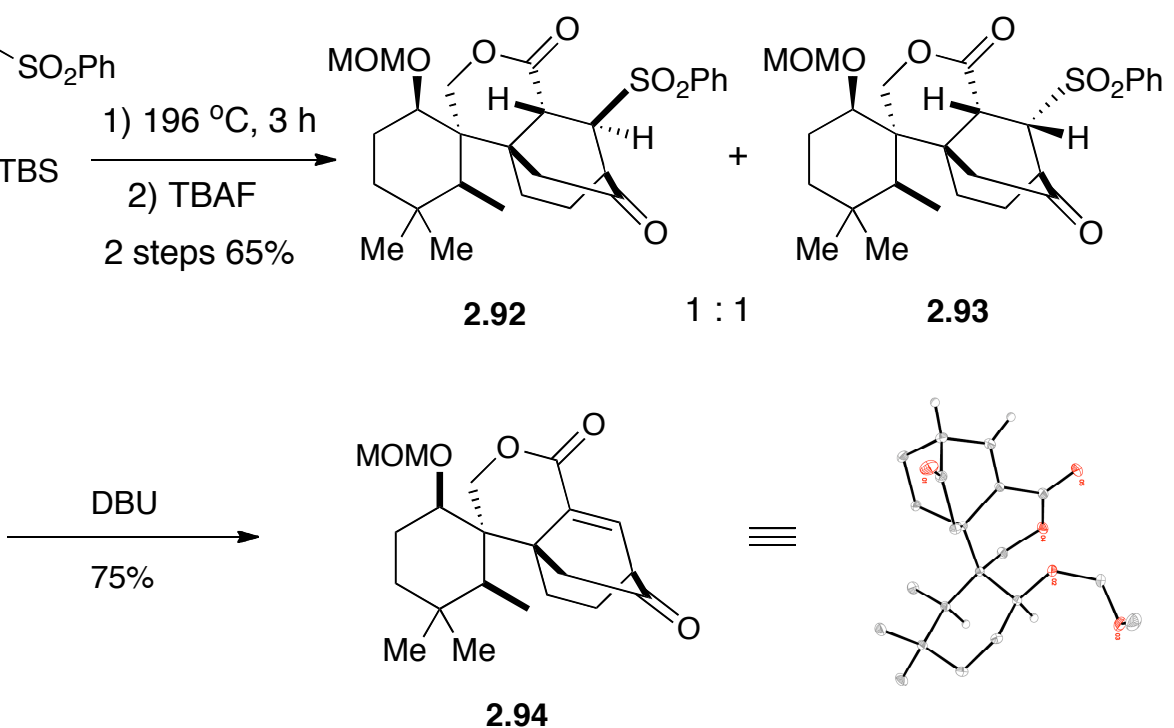

Scheme 2-20 Preparation of $\mathbf{2 . 9 4}$

At this stage, the synthesis seemed to reach a dead end, because none of these cycloadducts could be used to elaborate to the natural product, Maoecrystal V.

\subsection{Experimental}




\section{General information}

Unless otherwise noted, all reactions were performed under an argon atmosphere using flame-dried glassware. Toluene, hexanes and $\mathrm{CH}_{2} \mathrm{Cl}_{2}$ were distilled over $\mathrm{CaH}_{2}$. THF and $\mathrm{Et}_{2} \mathrm{O}$ were distilled over sodium/benzophenone ketyl. All reagents were commercially available and used without further purification unless indicated otherwise. Thin layer chromatography (TLC) was performed on Silica Gel 60 F254 plates and was visualized with UV light and $\mathrm{KMnO}_{4}$ stain. Preparative thin layer chromatography was performed with Merck silica gel 60-F254 coated $0.50 \mathrm{~mm}$ plates. Flash chromatography was performed with Sorbent Tech. silica gel 60 . Yields are for isolated, spectroscopically pure compounds. NMR spectra were recorded on 300,400 or $500 \mathrm{MHz}$ instruments. The residual solvent protons $\left({ }^{1} \mathrm{H}\right)$ or the solvent carbons $\left({ }^{13} \mathrm{C}\right)$ were used as internal standards. ${ }^{1} \mathrm{H}$ NMR data are presented as follows: chemical shift in ppm downfield from tetramethylsilane (multiplicity, coupling constant, integration). The following abbreviations are used in reporting NMR data: s, singlet; br s, broad singlet; d, doublet; $t$, triplet; q, quartet; qt, quartet of triplets; dd, doublet of doublets; dt, doublet of triplets; $\mathrm{AB}, \mathrm{AB}$ quartet; $\mathrm{m}$, multiplet. High-resolution mass spectra were recorded by the Columbia University Mass Spectrometry Core facility on a JEOL HX110 spectrometer.

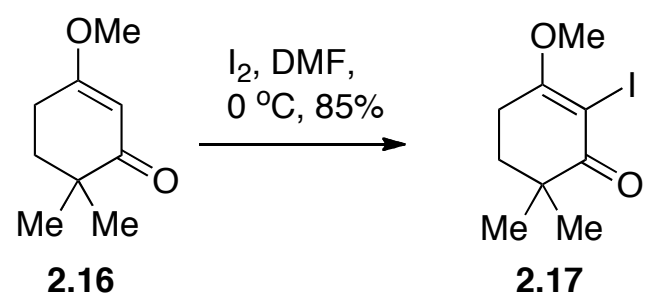


To a stirred and cooled $\left(0{ }^{\circ} \mathrm{C}\right)$ solution of enone 2.16 (120 mg, $\left.0.78 \mathrm{mmol}\right)$ in DMF (4 $\mathrm{mL}$ ) was added $\mathrm{I}_{2}(396 \mathrm{mg}, 1.56 \mathrm{mmol})$. The solution was stirred at this temperature for 30 min before quenched with $\mathrm{Na}_{2} \mathrm{~S}_{2} \mathrm{O}_{3}(2 \mathrm{~mL})$ and $\mathrm{NaHCO}_{3}(2 \mathrm{~mL})$. The mixture was extracted with EtOAc $(3 \times 20 \mathrm{~mL})$. The combined extracts were successively washed with $\mathrm{H}_{2} \mathrm{O}(10 \mathrm{~mL})$, brine $(10 \mathrm{~mL})$ and dried $\left(\mathrm{Na}_{2} \mathrm{SO}_{4}\right)$. Flash chromatography of the residue over silica ( 3 X $18 \mathrm{~cm}$ ), using 5:1 to 3:1 hexane: EtOAc, gave iodide (185 mg, 85\%) as semi-solid: ${ }^{1} \mathrm{H}$ NMR (400 MHz, $\left.\mathrm{CDCl}_{3}\right) \delta 1.11(\mathrm{~s}, 6 \mathrm{H}), 1.84(\mathrm{t}, J=6.0 \mathrm{~Hz}, 2$ H), $2.70(\mathrm{t}, J=6.0 \mathrm{~Hz}, 2 \mathrm{H}), 3.92(\mathrm{~s}, 3 \mathrm{H}) ;{ }^{13} \mathrm{C} \mathrm{NMR}\left(100 \mathrm{MHz}, \mathrm{CDCl}_{3}\right) \delta$ 24.3, 24.7, 34.2, 40.7, 56.2, 81.8, 174.9, 197.1; HRMS (FAB) $\mathrm{m} / z$ calcd for $\mathrm{C}_{9} \mathrm{H}_{14} \mathrm{O}_{2} \mathrm{I}[\mathrm{M}+\mathrm{H}]^{+}$ 281.0039 , found 281.0051 .

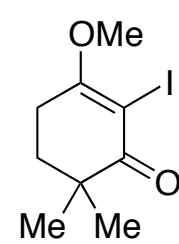

2.17<smiles>[InH]</smiles>

(HO)<smiles></smiles>

2.18
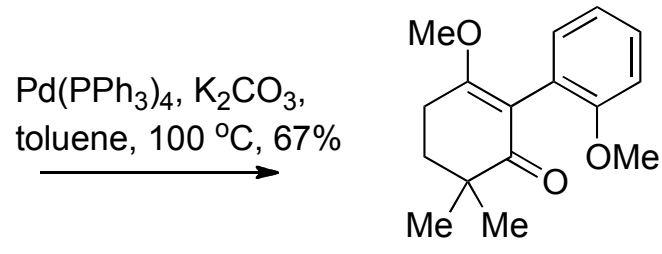

2.19

To a solution of iodide $2.17(50.0 \mathrm{mg}, 0.18 \mathrm{mmol})$ in toluene $(2.5 \mathrm{~mL})$ were added successively 2-methoxyphenylboronic acid 2.18 (54 mg, $0.36 \mathrm{mmol}), \mathrm{K}_{2} \mathrm{CO}_{3}(99.0 \mathrm{mg}$, $0.71 \mathrm{mmol})$ and $\mathrm{Pd}\left(\mathrm{PPh}_{3}\right)_{4}(21.0 \mathrm{mg}, 0.018 \mathrm{mmol})$ under argon. The mixture was stirred at $100{ }^{\circ} \mathrm{C}$ for $18 \mathrm{~h}$ and then cooled to room temperature. Solvent was evaporated. Flash chromatography of the residue over silica $(2.5 \times 18 \mathrm{~cm})$, using 4:1 to 2:1 hexane: EtOAc, gave enone 2.19 (31.0mg, 67\%) as white solid: FTIR $\left(\mathrm{CH}_{2} \mathrm{Cl}_{2}\right.$, cast) $2961,1651,1617$, 1592, 1367, $1250 \mathrm{~cm}^{-1} ;{ }^{1} \mathrm{H}$ NMR (300 MHz, $\left.\mathrm{CDCl}_{3}\right) \delta 1.16(\mathrm{~s}, 3 \mathrm{H}), 1.20(\mathrm{~s}, 3 \mathrm{H}), 1.94(\mathrm{t}$, $J=6.0 \mathrm{~Hz}, 2 \mathrm{H}), 2.61-2.76(\mathrm{~m}, 2 \mathrm{H}), 3.65(\mathrm{~s}, 3 \mathrm{H}), 3.72(\mathrm{~s}, 3 \mathrm{H}), 6.87-7.02(\mathrm{~m}, 3 \mathrm{H})$, 
7.22-7.27 (m, $1 \mathrm{H}) ;{ }^{13} \mathrm{C}$ NMR (100 MHz, $\left.\mathrm{CDCl}_{3}\right) \delta 23.1,24.3,24.7,34.1,39.8,55.6$, $55.7,110.9,115.2,120.1,123.7,128.3,132.0,157.2,169.8,201.7$; HRMS (FAB) $\mathrm{m} / \mathrm{z}$ calcd for $\mathrm{C}_{16} \mathrm{H}_{21} \mathrm{O}_{3}[\mathrm{M}+\mathrm{H}]^{+}$261.1491, found 261.1482.

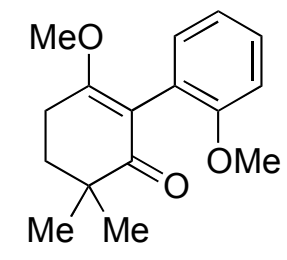

2.19

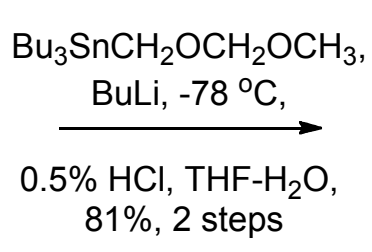

$81 \%, 2$ steps

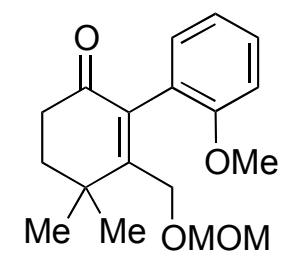

2.20

To a stirred and cooled $\left(-78^{\circ} \mathrm{C}\right)$ solution of stannane $(3.9 \mathrm{~g}, 10.7 \mathrm{mmol})$ in THF $(30 \mathrm{~mL})$ was added dropwise BuLi (2.5 M, $4.3 \mathrm{~mL}, 10.7 \mathrm{mmol})$. After 5 min stirring at this temperature, a solution of enone $2.19(1.63 \mathrm{~g}, 6.27 \mathrm{mmol})$ in THF $(10 \mathrm{~mL})$ was quickly introduced to the above anion solution and stirring was continued for $30 \mathrm{~min}$. The reaction was quenched by successively adding $\mathrm{MeOH}(5 \mathrm{~mL})$ and $\mathrm{NH}_{4} \mathrm{Cl}(20 \mathrm{~mL})$. The mixture was extracted with EtOAc ( 3 X $100 \mathrm{~mL})$. The combined extracts were washed with brine $(50 \mathrm{~mL})$ and dried $\left(\mathrm{Na}_{2} \mathrm{SO}_{4}\right)$. Flash chromatography of the residue over silica (3 X $18 \mathrm{~cm}$ ), using 4:1 hexane:EtOAc, gave crude enone 2.20, which was used directly in the next step without further purification. The above crude (ca. $2 \mathrm{~g}$ ) was dissolved in THF $(20 \mathrm{~mL})$ and the resultant solution was cooled to $\left(0{ }^{\circ} \mathrm{C}\right)$. To this solution were successively added $\mathrm{H}_{2} \mathrm{O}(5 \mathrm{~mL})$ and $0.5 \mathrm{~N} \mathrm{HCl}(1.5 \mathrm{~mL})$. After stirring at $0{ }^{\circ} \mathrm{C}$ for $2 \mathrm{~h}$, the reaction was quenched by the addition of saturated aqueous $\mathrm{NaHCO}_{3}(20 \mathrm{~mL})$. The mixture was extracted with EtOAc $(3 \times 20 \mathrm{~mL})$. The combined extracts were successively washed with $\mathrm{H}_{2} \mathrm{O}(20 \mathrm{~mL})$, brine $(30 \mathrm{~mL})$ and dried $\left(\mathrm{Na}_{2} \mathrm{SO}_{4}\right)$. Flash chromatography of the residue over silica $(3 \times 20 \mathrm{~cm})$, using 6:1 to 3:1 hexane:EtOAc, gave enone 2.20 ( $1.54 \mathrm{~g}, 81 \%$, over two steps) as colorless oil: FTIR $\left(\mathrm{CH}_{2} \mathrm{Cl}_{2}\right.$, cast $) 2940$, 
$1678,1492,1247,1150,1035 \mathrm{~cm}^{-1} ;{ }^{1} \mathrm{H}$ NMR $\left(300 \mathrm{MHz}, \mathrm{CDCl}_{3}\right) \delta 1.36(\mathrm{~s}, 6 \mathrm{H}), 1.97-$ $2.02(\mathrm{~m}, 2 \mathrm{H}), 2.55-2.78(\mathrm{~m}, 2 \mathrm{H}), 3.20(\mathrm{~s}, 3 \mathrm{H}), 3.73(\mathrm{~s}, 3 \mathrm{H}), 3.87(\mathrm{~d}, J=10.5 \mathrm{~Hz}, 1 \mathrm{H})$, $4.06(\mathrm{~d}, J=10.5 \mathrm{~Hz}, 1 \mathrm{H}), 4.43(\mathrm{ABq}, \Delta v=20.1, J=6.6,2 \mathrm{H}), 6.89-6.97(\mathrm{~m}, 2 \mathrm{H}), 7.02$ (dd, $J=7.5,2.1 \mathrm{~Hz}, 1 \mathrm{H}), 7.27-7.33(\mathrm{~m}, 1 \mathrm{H}) ;{ }^{13} \mathrm{C} \mathrm{NMR}\left(100 \mathrm{MHz}, \mathrm{CDCl}_{3}\right) \delta 26.4,27.5$, 34.7, 35.3, 37.9, 55.3, 55.5, 66.0, 96.4, 110.6, 120.1, 125.0, 129.0, 131.0, 136.9, 156.8, 159.4, 198.0; HRMS (FAB) $\mathrm{m} / z$ calcd for $\mathrm{C}_{18} \mathrm{H}_{24} \mathrm{O}_{4}[\mathrm{M}]^{+}$304.1675, found 304.1676.

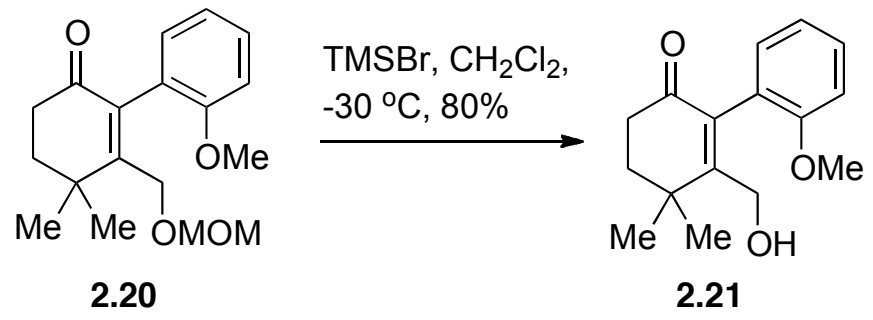

To a stirred and cooled $\left(-30{ }^{\circ} \mathrm{C}\right)$ solution of MOM ether $2.20(75.0 \mathrm{mg}, 0.25 \mathrm{mmol})$ in $\mathrm{CH}_{2} \mathrm{Cl}_{2}(2 \mathrm{~mL})$ was added TMSBr $(0.33 \mathrm{~mL}, 2.46 \mathrm{mmol})$ dropwise. After stirring at this temperature for $30 \mathrm{~min}$, the reaction was quenched by the addition of saturated aqueous $\mathrm{Na}_{2} \mathrm{CO}_{3}(2 \mathrm{~mL})$. The mixture was extracted with EtOAc $(2 \mathrm{X} 15 \mathrm{~mL})$. The combined extracts were washed with brine $(5 \mathrm{~mL})$ and dried $\left(\mathrm{Na}_{2} \mathrm{SO}_{4}\right)$. Flash chromatography of the residue over silica $(2 \times 20 \mathrm{~cm})$, using 3:1 to 1:1, hexane:EtOAc, gave alcohol 2.21 (51.5 mg, $80 \%$ ) as colorless oil: FTIR $\left(\mathrm{CH}_{2} \mathrm{Cl}_{2}\right.$, cast $) 3434,2960,1669,1593,1491,1246 \mathrm{~cm}^{-1}$; ${ }^{1} \mathrm{H}$ NMR (400 MHz, $\left.\mathrm{CDCl}_{3}\right) \delta 1.29(\mathrm{~s}, 3 \mathrm{H}), 1.39$ (s, $\left.3 \mathrm{H}\right), 1.95-2.00$ (m, $\left.2 \mathrm{H}\right), 2.26(\mathrm{dd}, J$ $=7.6,5.6 \mathrm{~Hz}, 1 \mathrm{H}), 2.60-2.66(\mathrm{~m}, 2 \mathrm{H}), 3.74(\mathrm{~s}, 3 \mathrm{H}), 3.98-4.00(\mathrm{~m}, 2 \mathrm{H}), 6.91-7.00(\mathrm{~m}, 3$ H), 7.29-7.33 (m, $1 \mathrm{H}) ;{ }^{13} \mathrm{C} \mathrm{NMR}\left(100 \mathrm{MHz}, \mathrm{CDCl}_{3}\right) \delta 27.0,34.8,35.3,37.5,55.9,61.1$, 
111.6, 121.0, 124.8, 129.2, 131.1, 134.8, 156.3, 163.1, 198.5; HRMS (FAB) $m / z$ calcd for $\mathrm{C}_{16} \mathrm{H}_{20} \mathrm{O}_{3}[\mathrm{M}]^{+}$260.1412, found 260.1418.

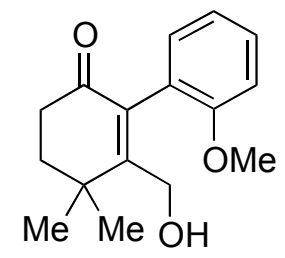

2.21

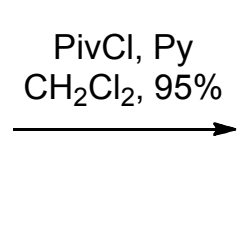

PivCl, Py
$\mathrm{CH}_{2} \mathrm{Cl}_{2}, 95 \%$

To a stirred and cooled $\left(0{ }^{\circ} \mathrm{C}\right)$ solution of alcohol $2.21(1.10 \mathrm{~g}, 4.2 \mathrm{mmol})$ in $\mathrm{CH}_{2} \mathrm{Cl}_{2}(10$ $\mathrm{mL}$ ) were added successively pyridine $(2 \mathrm{~mL})$ and $\mathrm{PivCl}(2.1 \mathrm{~mL}, 16.9 \mathrm{mmol})$. The stirring was continued at $0{ }^{\circ} \mathrm{C}$ for $5 \mathrm{~h}$ and room temperature for $3 \mathrm{~h}$. The reaction was quenched by the addition of saturated aqueous $\mathrm{NaHCO}_{3}(20 \mathrm{~mL})$. The mixture was extracted with EtOAc (3 X $50 \mathrm{~mL})$. The combined extracts were washed with brine (20 $\mathrm{mL})$ and dried $\left(\mathrm{Na}_{2} \mathrm{SO}_{4}\right)$. Flash chromatography of the residue over silica $(2.5 \mathrm{X} 18 \mathrm{~cm})$, using 4:1 hexane:EtOAc, gave Piv ester $2.22(1.41 \mathrm{~g}, 97 \%)$ as white solid: FTIR $\left(\mathrm{CH}_{2} \mathrm{Cl}_{2}\right.$, cast) $2968,1729,1681,1276,1248,1149 \mathrm{~cm}^{-1} ;{ }^{1} \mathrm{H}$ NMR (400 MHz, $\left.\mathrm{CDCl}_{3}\right) \delta 1.19$ (s, 9 H), 1.28 (s, $3 \mathrm{H}), 1.34$ (s, $3 \mathrm{H}), 2.00-2.05$ (m, $2 \mathrm{H}), 2.59-2.65$ (m, $1 \mathrm{H}), 2.71-2.75$ (m, 1 H), $3.72(\mathrm{~s}, 3 \mathrm{H}) 4.24$ (d, $J=11.6 \mathrm{~Hz}, 1 \mathrm{H}), 4.64$ ( d, $J=11.6 \mathrm{~Hz}, 1 \mathrm{H}), 6.88-6.93$ (m, 2 $\mathrm{H}), 6.97-7.00(\mathrm{~m}, 1 \mathrm{H}), 7.28-7.32(\mathrm{~m}, 1 \mathrm{H}) ;{ }^{13} \mathrm{C} \mathrm{NMR}\left(100 \mathrm{MHz}, \mathrm{CDCl}_{3}\right) \delta$ 26.1, 27.0, $27.5,34.6,35.3,37.7,38.6,55.4,62.5,110.6,120.0,124.3,129.4,131.0,138.6,156.6$, 157.0, 177.7, 197.7; HRMS (FAB) $m / z$ calcd for $\mathrm{C}_{21} \mathrm{H}_{28} \mathrm{O}_{3}[\mathrm{M}]^{+}$344.1988, found 344.1971. 


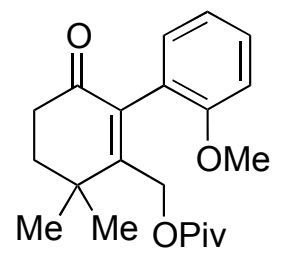

2.22
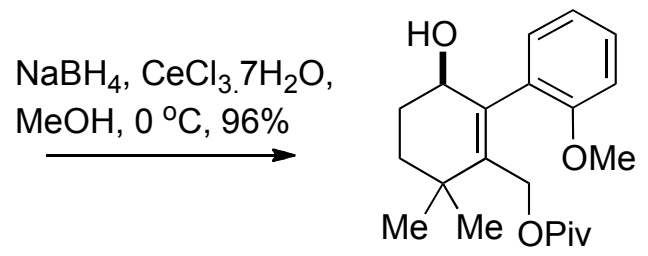

2.23

To a stirred and cooled $\left(0{ }^{\circ} \mathrm{C}\right)$ solution of enone $2.22(1.41 \mathrm{~g}, 4.10 \mathrm{mmol})$ in $\mathrm{MeOH}(30$ $\mathrm{mL})$ was added $\mathrm{CeCl}_{3}-7 \mathrm{H}_{2} \mathrm{O}(3.05 \mathrm{~g}, 8.20 \mathrm{mmol})$. After stirring for $10 \mathrm{~min}, \mathrm{NaBH}_{4}(465$ $\mathrm{mg}, 12.3 \mathrm{mmol}$ ) was added piece by piece. Stirring was continued for $15 \mathrm{~min}$ and the reaction was quenched by the addition of saturated aqueous $\mathrm{NH}_{4} \mathrm{Cl}(20 \mathrm{~mL})$. The mixture was extracted with EtOAc ( 3 X $50 \mathrm{~mL})$. The combined extracts were washed with brine $(20 \mathrm{~mL})$ and dried $\left(\mathrm{Na}_{2} \mathrm{SO}_{4}\right)$. Flash chromatography of the residue over silica (3 X 18 $\mathrm{cm})$, using 4:1 hexane:EtOAc, gave alcohols $2.23(1.36 \mathrm{~g}, 96 \%)$ as a mixture of ca 1:1 two rotomers, which both are colorless oil: FTIR $\left(\mathrm{CH}_{2} \mathrm{Cl}_{2}\right.$, cast $) 3459,2961,1723,1480$, 1284, 1244, $1157 \mathrm{~cm}^{-1} ;{ }^{1} \mathrm{H}$ NMR $\left(400 \mathrm{MHz}, \mathrm{CDCl}_{3}\right)$ (mixture of $1: 1$ two rotomers) $\delta$ 1.05- $1.21(\mathrm{~m}, 30 \mathrm{H}), 1.50-1.56(\mathrm{~m}, 1 \mathrm{H}), 1.79-1.91(\mathrm{~m}, 2 \mathrm{H}), 1.95-2.05(\mathrm{~m}, 1 \mathrm{H}), 3.76(\mathrm{~s}$, $3 \mathrm{H}), 3.80$ (s, $3 \mathrm{H}), 3.99$ (d, $J=11.6 \mathrm{~Hz}, 1 \mathrm{H}), 4.09$ (d, $J=11.6 \mathrm{~Hz}, 1 \mathrm{H}), 4.16(\mathrm{t}, J=4.8$ $\mathrm{Hz}, 1 \mathrm{H}), 4.37-4.40$ (m, $2 \mathrm{H}), 4.48(\mathrm{~d}, J=11.6 \mathrm{~Hz}, 1 \mathrm{H}), 6.85-6.95$ (m, $4 \mathrm{H}), 7.01$ (dd, $J=$ 7.2, $1.6 \mathrm{~Hz}, 1 \mathrm{H}), 7.10(\mathrm{dd}, J=8.0,1.6 \mathrm{~Hz}, 1 \mathrm{H}), 7.23-7.28(\mathrm{~m}, 2 \mathrm{H}) ;{ }^{13} \mathrm{C}$ NMR $(100$ $\mathrm{MHz}, \mathrm{CDCl}_{3}$ )(mixture of $1: 1$ two rotomers) $\delta 27.0,27.2,27.5,28.2,28.3,28.4,31.3$, $34.2,34.41,34.44,34.7,38.4,55.2,55.5,62.0,62.1,66.9,70.4,110.5,110.6,119.8$, 121.0, 127.6, 128.7, 128.9, 129.1, 130.5, 132.0, 138.3, 139.1, 139.9, 141.2, 156.0, 156.3, 178.05, 178.10; HRMS (FAB) $m / z$ calcd for $\mathrm{C}_{21} \mathrm{H}_{30} \mathrm{O}_{4}[\mathrm{M}]^{+}$346.2144, found 346.2159. 


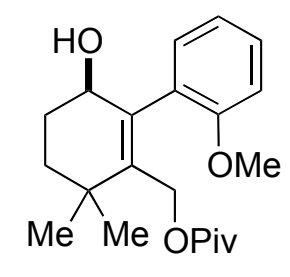

2.23
TBSOTf, 2,6-lutidine,

$\mathrm{CH}_{2} \mathrm{Cl}_{2}, 0^{\circ} \mathrm{C}, 97 \%$

2.24

To a stirred and cooled $\left(0{ }^{\circ} \mathrm{C}\right)$ solution of alcohol $2.23(1.50 \mathrm{~g}, 4.3 \mathrm{mmol})$ in $\mathrm{CH}_{2} \mathrm{Cl}_{2}(20$ $\mathrm{mL})$ were added successively 2,6 -lutidine $(3.0 \mathrm{~mL}, 26.0 \mathrm{mmol})$ and TBSOTf $(2.0 \mathrm{~mL}, 8.6$ mmol). Stirring was continued at this temperature for $3 \mathrm{~h}$. The reaction was quenched by the addition of saturated aqueous $\mathrm{NaHCO}_{3}(20 \mathrm{~mL})$. The mixture was extracted with EtOAc $(3 \times 50 \mathrm{~mL})$. The combined extracts were washed with brine $(20 \mathrm{~mL})$, dried $\left(\mathrm{Na}_{2} \mathrm{SO}_{4}\right)$ and concentrated. Flash chromatography of the residue over silica $(3 \mathrm{X} 18 \mathrm{~cm})$, using 10:1 hexane:EtOAc, gave silyl ethers $2.24(1.93 \mathrm{~g}, 97 \%)$ as mixture of ca 10:1 two rotomers, which are colorless oil: FTIR $\left(\mathrm{CH}_{2} \mathrm{Cl}_{2}\right.$, cast $) 2957,2857,1725,1474,1249$, $1156,1048 \mathrm{~cm}^{-1} ;{ }^{1} \mathrm{H} \mathrm{NMR}\left(400 \mathrm{MHz}, \mathrm{CDCl}_{3}\right)($ major isomer) $\delta-0.55(\mathrm{~s}, 3 \mathrm{H}),-0.21$ (s, 3 H), $0.72(\mathrm{~s}, 9 \mathrm{H}), 1.04-1.17(\mathrm{~m}, 18 \mathrm{H}), 1.42-1.46(\mathrm{~m}, 1 \mathrm{H}), 1.70-1.74(\mathrm{~m}, 1 \mathrm{H}), 1.82-1.88$ (m, $1 \mathrm{H}), 1.92-1.98(\mathrm{~m}, 1 \mathrm{H}), 3.73(\mathrm{~s}, 3 \mathrm{H}), 4.00(\mathrm{~d}, J=11.6 \mathrm{~Hz}, 1 \mathrm{H}), 4.33(\mathrm{t}, J=4.4 \mathrm{~Hz}$, $1 \mathrm{H}), 4.44(\mathrm{~d}, J=11.6 \mathrm{~Hz}, 1 \mathrm{H}), 6.76-6.83(\mathrm{~m}, 2 \mathrm{H}), 7.00(\mathrm{dd}, J=7.2,1.6 \mathrm{~Hz}, 1 \mathrm{H}), 7.15-$ $7.19(\mathrm{~m}, 1 \mathrm{H}) ;{ }^{13} \mathrm{C} \mathrm{NMR}\left(100 \mathrm{MHz}, \mathrm{CDCl}_{3}\right)($ major rotomer) $\delta-5.8,-5.2,17.9,25.7,27.1$, $28.5,28.9,34.3,34.4,38.4,55.2,62.4,67.4,110.1,119.6,128.2,128.8,133.1,137.1$, 142.1, 156.3, 178.2; HRMS (FAB) $m / z$ calcd for $\mathrm{C}_{27} \mathrm{H}_{43} \mathrm{O}_{4} \mathrm{Si}[\mathrm{M}-\mathrm{H}]^{+}$459.2931, found 459.2917. 


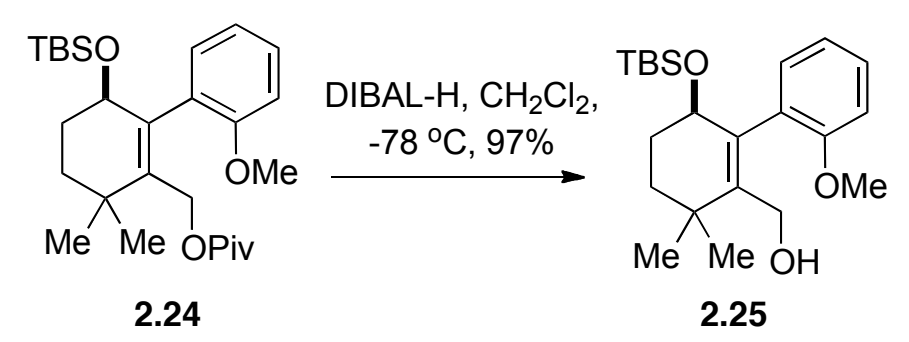

To a stirred and cooled $\left(-78^{\circ} \mathrm{C}\right)$ solution of ester $(1.93 \mathrm{~g}, 4.20 \mathrm{mmol})$ in $\mathrm{CH}_{2} \mathrm{Cl}_{2}(30 \mathrm{~mL})$ was added dropwise a solution of DIBAL-H $(21.0 \mathrm{~mL}, 21.0 \mathrm{mmol})$. After stirring at this temperature for $30 \mathrm{~min}$, the reaction was quenched by the addition of $\mathrm{MeOH}(5 \mathrm{~mL})$ and then $\mathrm{Na}_{2} \mathrm{SO}_{4}-10 \mathrm{H}_{2} \mathrm{O}(15 \mathrm{~g})$. Stirring was continued at room temperature for $3 \mathrm{~h}$. The mixture was filtered through a pad of celite $(5 \times 6 \mathrm{~cm})$, rinsed with EtOAc $(4 \times 20 \mathrm{~mL})$. Solvent was evaporated. Flash chromatography of the residue over silica $(3 \mathrm{X} 18 \mathrm{~cm})$, using 10:1 hexane:EtOAc, gave alcohols $2.25(1.50 \mathrm{~g}, 97 \%)$ as a mixture of ca. 2:1 two rotamers, which are colorless oil: FTIR $\left(\mathrm{CH}_{2} \mathrm{Cl}_{2}\right.$, cast $) 3469,2932,2856,1598,1580$, $1490,1463,1435,1249,1048,836 \mathrm{~cm}^{-1} ;{ }^{1} \mathrm{H}$ NMR (400 MHz, $\left.\mathrm{CDCl}_{3}\right)$ (mixture of 2:1 two rotomers) $\delta-0.53(\mathrm{~s}, 1.5 \mathrm{H}),-0.52(\mathrm{~s}, 3 \mathrm{H}),-0.20(\mathrm{~s}, 3 \mathrm{H}),-0.13(\mathrm{~s}, 1.5 \mathrm{H}), 0.71(\mathrm{~s}, 4.5 \mathrm{H})$, 0.77 (s, 9 H), 1.09 (s, $1.5 \mathrm{H}), 1.17$ (s, $3 \mathrm{H}), 1.20(\mathrm{~s}, 3 \mathrm{H}), 1.31(\mathrm{~s}, 1.5 \mathrm{H}), 1.47-1.56$ (m, $1.5 \mathrm{H}), 1.67$ (dd, $J=8.4,3.2 \mathrm{~Hz}, 1 \mathrm{H}), 1.74-1.86(\mathrm{~m}, 3 \mathrm{H}), 1.91-1.94(\mathrm{~m}, 1.5 \mathrm{H}), 2.86$ (dd, $J=10.4,2.0 \mathrm{~Hz}, 0.5 \mathrm{H}), 3.71-3.91(\mathrm{~m}, 7.5 \mathrm{H}), 4.19(\mathrm{t}, J=5.2 \mathrm{~Hz}, 1 \mathrm{H}), 4.24(\mathrm{t}, J=5.2$ $\mathrm{Hz}, 0.5 \mathrm{H}), 6.89-6.95(\mathrm{~m}, 2.5 \mathrm{H}), 6.97-6.99(\mathrm{~m}, 1 \mathrm{H}), 7.06(\mathrm{dd}, J=7.2,1.6 \mathrm{~Hz}, 1 \mathrm{H})$, 7.21-7.29 (m, 1.5 H); ${ }^{13} \mathrm{C}$ NMR $\left(100 \mathrm{MHz}, \mathrm{CDCl}_{3}\right)$ (mixture of 2:1 two rotomers) $\delta$-5.82, $-5.76,-5.3,-5.1,17.7,17.9,25.5,25.7,27.56,27.64,28.4,28.6,29.3,29.8,34.27,34.3$, $34.96,35.0,55.4,55.9,60.3,60.9,68.6,71.3,110.4,111.9,120.3,121.2,127.9,128.1$, 129.0, 129.5, 131.2, 132.6, 135.8, 137.6, 143.3, 143.5, 155.9, 157.1; HRMS (FAB) $\mathrm{m} / \mathrm{z}$ calcd for $\mathrm{C}_{22} \mathrm{H}_{35} \mathrm{O}_{3} \mathrm{Si}[\mathrm{M}-\mathrm{H}]^{+}$375.2355, found 375.2339. 


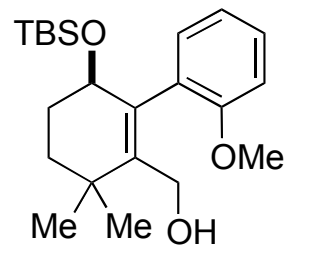

2.25

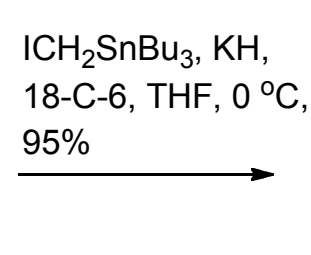

$18-\mathrm{C}-6$, THF $0^{\circ} \mathrm{C}$

$95 \%$

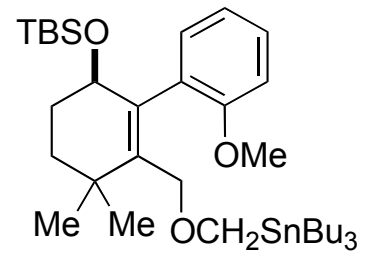

2.26

To a stirred and cooled $\left(0{ }^{\circ} \mathrm{C}\right)$ solution of aocohol $2.25(30 \mathrm{mg}, 0.08 \mathrm{mmol})$ in THF (2 $\mathrm{mL})$ were added successively 18 -Crown-6 (10.0 mg, $0.04 \mathrm{mmol})$ and $\mathrm{KH}(10.0 \mathrm{mg}, 0.24$ mmol). After stirring at this temperature for $30 \mathrm{~min}, \mathrm{Bu}_{3} \mathrm{SnCH}_{2} \mathrm{I}(105.0 \mathrm{mg}, 0.24 \mathrm{mmol})$ was added. Stirring was continued for $12 \mathrm{~h}$ at $0{ }^{\circ} \mathrm{C}$. The reaction was quenched by the addition of saturated aqueous $\mathrm{NH}_{4} \mathrm{Cl}(1 \mathrm{~mL})$. The mixture was extracted with EtOAc (3 X $5 \mathrm{~mL})$. The combined extracts were dried $\left(\mathrm{Na}_{2} \mathrm{SO}_{4}\right)$ and the solvent was evaporated. Flash chromatography of the residue over silica $(1.5 \times 18 \mathrm{~cm})$, using hexane to $10: 1$ hexane:EtOAc, gave stannane $\mathbf{2 . 2 6}$ ( $52.0 \mathrm{mg}$, 95\%) as colorless oil, which was used directly in the next step.

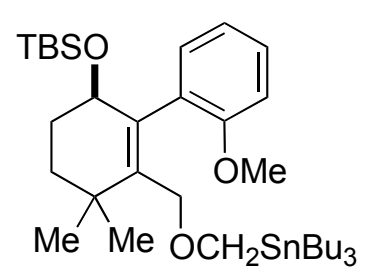

2.26
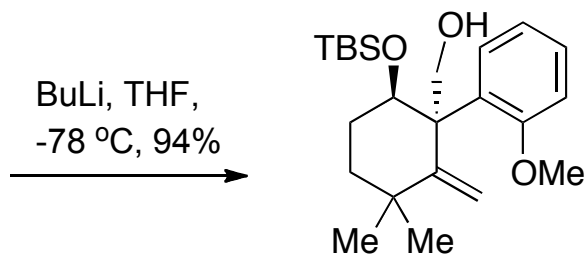

2.27

To a stirred and cooled $\left(-78^{\circ} \mathrm{C}\right)$ solution of stannane $2.26(640.0 \mathrm{mg}, 0.95 \mathrm{mmol})$ in THF $(10 \mathrm{~mL})$ was added dropwise a solution of BuLi $(1.6 \mathrm{M}, 2.4 \mathrm{~mL}, 3.81 \mathrm{mmol})$. The resultant solution was slowly warmed to $-20{ }^{\circ} \mathrm{C}$ over $6 \mathrm{~h}$ and the reaction was quenched by the addition of $\mathrm{MeOH}(2 \mathrm{~mL})$ and then $\mathrm{NH}_{4} \mathrm{Cl}(3 \mathrm{~mL})$. The mixture was extracted 
with EtOAc (2 X $15 \mathrm{~mL})$. The combined extracts were dried $\left(\mathrm{Na}_{2} \mathrm{SO}_{4}\right)$ and the solvent was evaporated. Flash chromatography of the residue over silica $(2.5 \times 18 \mathrm{~cm})$, using 10:1 hexane:EtOAc, gave olefin $(350.0 \mathrm{mg}, 94 \%)$ as colorless oil: : FTIR $\left(\mathrm{CH}_{2} \mathrm{Cl}_{2}\right.$, cast) 3512, 2954, 1626, 1594, 1462, 1249, 1100, $1036 \mathrm{~cm}^{-1} ;{ }^{1} \mathrm{H}$ NMR (400 MHz, $\left.\mathrm{CDCl}_{3}\right) \delta$ $0.043(\mathrm{~s}, 3 \mathrm{H}), 0.12(\mathrm{~s}, 3 \mathrm{H}), 0.59(\mathrm{~s}, 3 \mathrm{H}), 0.87$ (s, $9 \mathrm{H}), 1.08(\mathrm{~s}, 3 \mathrm{H}), 1.24-1.34(\mathrm{~m}, 1 \mathrm{H})$, $1.48(\mathrm{dt}, J=4.0,13.6 \mathrm{~Hz}, 1 \mathrm{H}), 1.73-1.80(\mathrm{~m}, 1 \mathrm{H}), 2.33-2.43(\mathrm{~m}, 1 \mathrm{H}), 2.65(\mathrm{dd}, J=8.0$, $3.6 \mathrm{~Hz}, 1 \mathrm{H}), 3.66(\mathrm{~s}, 3 \mathrm{H}), 3.88(\mathrm{dd}, J=4.0,11.2 \mathrm{~Hz}, 1 \mathrm{H}), 4.03(\mathrm{dd}, J=10.4,8.4 \mathrm{~Hz}, 1$ H), $4.16(\mathrm{dd}, J=11.2,4.0 \mathrm{~Hz}, 1 \mathrm{H}), 4.96(\mathrm{~s}, 1 \mathrm{H}), 5.17(\mathrm{~s}, 1 \mathrm{H}), 6.76(\mathrm{~d}, J=8.0 \mathrm{~Hz}, 1 \mathrm{H})$, $6.88(\mathrm{t}, J=7.6 \mathrm{~Hz}, 1 \mathrm{H}), 7.17(\mathrm{t}, J=7.2 \mathrm{~Hz}, 1 \mathrm{H}), 8.07(\mathrm{~d}, J=8.0 \mathrm{~Hz}, 1 \mathrm{H}) ;{ }^{13} \mathrm{C} \mathrm{NMR}$ $\left(100 \mathrm{MHz}, \mathrm{CDCl}_{3}\right) \delta-4.7,-3.8,18.4,26.3,29.0,32.5,37.5,39.1,54.9,55.1,67.4,79.3$, $110.2,112.1,120.3,127.8,130.9,131.4,155.8,159.4$; HRMS (FAB) $m / z$ calcd for $\mathrm{C}_{23} \mathrm{H}_{39} \mathrm{O}_{3} \mathrm{Si}[\mathrm{M}+\mathrm{H}]^{+}$491.2668, found 491.2668.

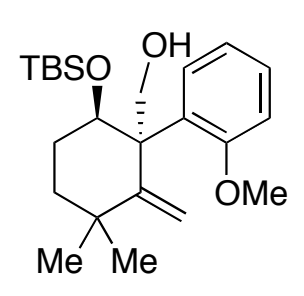

2.27

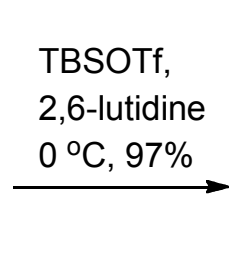

2,6-lutidine

$0^{\circ} \mathrm{C}, 97 \%$

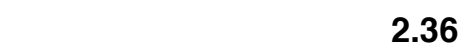

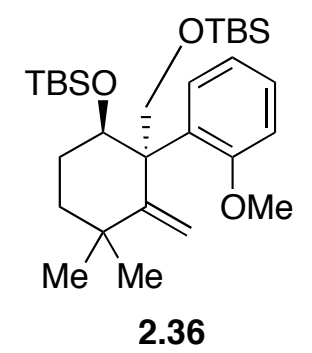

To a stirred and cooled $\left(0{ }^{\circ} \mathrm{C}\right)$ solution of alcohol 2.27 (135.0 $\left.\mathrm{mg}, 0.35 \mathrm{mmol}\right)$ in $\mathrm{CH}_{2} \mathrm{Cl}_{2}$ $(5 \mathrm{~mL})$ were added successively 2,6 -lutidine $(0.16 \mathrm{~mL}, 1.38 \mathrm{mmol})$ and TBSOTf $(0.16$ $\mathrm{mL}, 0.69 \mathrm{mmol})$. Stirring was continued at this temperature for $10 \mathrm{~h}$. The reaction was quenched by the addition of saturated aqueous $\mathrm{NaHCO}_{3}(3 \mathrm{~mL})$. The mixture was extracted with EtOAc (3 X $15 \mathrm{~mL})$. The combined extracts were washed with brine (10 
$\mathrm{mL})$, dried $\left(\mathrm{Na}_{2} \mathrm{SO}_{4}\right)$ and concentrated. Flash chromatography of the residue over silica (2.5 X $16 \mathrm{~cm})$, using 5\% EtOAc in hexane, gave silyl ethers 2.36 (169 mg, 97\%) as colorless oil: FTIR $\left(\mathrm{CH}_{2} \mathrm{Cl}_{2}\right.$, cast) 2954, 2859, 1465, 1251, $1084 \mathrm{~cm}^{-1} ;{ }^{1} \mathrm{H}$ NMR (400 $\left.\mathrm{MHz}, \mathrm{CDCl}_{3}\right) \delta-0.24(\mathrm{~s}, 3 \mathrm{H}),-0.010(\mathrm{~s}, 3 \mathrm{H}), 0.082(\mathrm{~s}, 3 \mathrm{H}), 0.12(\mathrm{~s}, 3 \mathrm{H}), 0.75(\mathrm{~s}, 9 \mathrm{H})$, $0.94(\mathrm{~s}, 12 \mathrm{H}), 1.13(\mathrm{~s}, 3 \mathrm{H}), 1.32-1.38(\mathrm{~m}, 1 \mathrm{H}), 1.61-1.67(\mathrm{~m}, 1 \mathrm{H}), 1.77-1.81(\mathrm{~m}, 1 \mathrm{H})$, 2.04-2.12 (m, $1 \mathrm{H}), 3.66(\mathrm{~s}, 3 \mathrm{H}), 3.88(\mathrm{~d}, J=10.0 \mathrm{~Hz}, 1 \mathrm{H}), 4.00(\mathrm{~d}, J=10.0 \mathrm{~Hz}, 1 \mathrm{H})$, 4.21-4.24 (m, $1 \mathrm{H}), 4.74(\mathrm{~s}, 3 \mathrm{H}), 4.99(\mathrm{~s}, 1 \mathrm{H}), 6.74(\mathrm{~d}, J=8.0 \mathrm{~Hz}, 1 \mathrm{H}), 6.84(\mathrm{t}, J=7.6$ $\mathrm{Hz}, 1 \mathrm{H}), 7.13(\mathrm{t}, J=8.0 \mathrm{~Hz}, 1 \mathrm{H}), 7.85(\mathrm{~d}, J=7.6 \mathrm{~Hz}, 1 \mathrm{H}) ;{ }^{13} \mathrm{C} \mathrm{NMR}(100 \mathrm{MHz}$, $\left.\mathrm{CDCl}_{3}\right) \delta-5.5,-5.07,-5.03,-4.54,17.9,18.4,25.8,26.1,28.6,30.9,33.4,35.9,36.0$, $54.4,55.1,68.4,72.3,110.9,111.6,119.4,126.8,129.6,134.1,156.9,158.8$; HRMS (FAB) $m / z$ calcd for $\mathrm{C}_{29} \mathrm{H}_{51} \mathrm{O}_{3} \mathrm{Si}_{2}[\mathrm{M}-\mathrm{H}]^{+}$503.3377, found 503.3377.

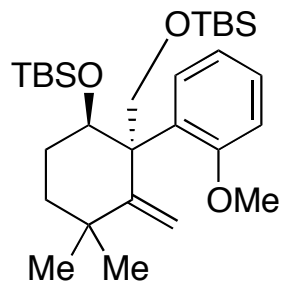

2.36
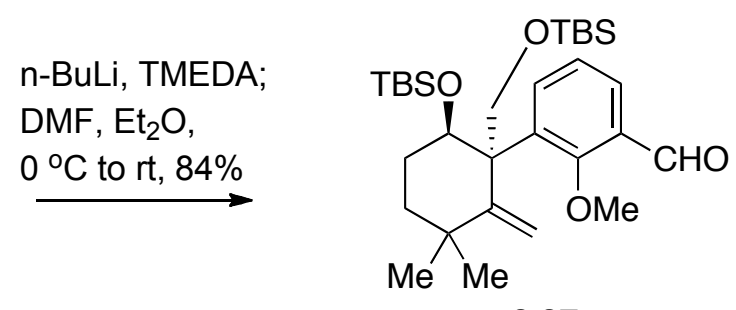

To a stirred and cooled $\left(0{ }^{\circ} \mathrm{C}\right)$ solution of methyl aryl ether $\mathbf{2 . 3 6}(600.0 \mathrm{mg}, 1.19 \mathrm{mmol})$ in $\mathrm{Et}_{2} \mathrm{O}(10 \mathrm{~mL})$ were added successively TMEDA $(0.54 \mathrm{~mL}, 3.57 \mathrm{mmol})$ and a solution of $\operatorname{BuLi}(2.5 \mathrm{M}, 1.43 \mathrm{~mL}, 3.57 \mathrm{mmol})$. The resultant solution was stirred at $0{ }^{\circ} \mathrm{C}$ for $2 \mathrm{~h}$ and then DMF $(0.92 \mathrm{~mL}, 11.9 \mathrm{mmol})$ was added in one portion. Stirring was continued at this temperature for another $3 \mathrm{~h}$ and the reaction was quenched by the addition of saturated aqueous $\mathrm{NH}_{4} \mathrm{Cl}(3 \mathrm{~mL})$. The mixture was extracted with EtOAc $(3 \mathrm{X} 20 \mathrm{~mL})$. 
The combined extracts were dried $\left(\mathrm{Na}_{2} \mathrm{SO}_{4}\right)$ and the solvent was evaporated. Flash chromatography of the residue over silica $(2.5 \times 18 \mathrm{~cm})$, using $1 \%$ to $2 \%$ EtOAc in hexane, gave aldehyde $2.37(76.0 \mathrm{mg}, 84 \%$ based on recovered starting material) as colorless oil: FTIR $\left(\mathrm{CH}_{2} \mathrm{Cl}_{2}\right.$, cast) 2955, 2857, 1689, 1581, 1471, 1256, $1094 \mathrm{~cm}^{-1} ;{ }^{1} \mathrm{H}$ NMR (400 MHz, $\left.\mathrm{CDCl}_{3}\right) \delta-0.21(\mathrm{~s}, 3 \mathrm{H}), 0.014$ (s, $\left.3 \mathrm{H}\right), 0.049$ (s, $\left.3 \mathrm{H}\right), 0.085$ (s, $\left.3 \mathrm{H}\right)$, $0.78(\mathrm{~s}, 9 \mathrm{H}), 0.89(\mathrm{~s}, 9 \mathrm{H}), 1.00(\mathrm{~s}, 3 \mathrm{H}), 1.15(\mathrm{~s}, 3 \mathrm{H}), 1.28-1.33$ (m, $1 \mathrm{H}), 1.63-1.69$ (m, $1 \mathrm{H}), 1.82-1.87(\mathrm{~m}, 1 \mathrm{H}), 1.95-2.00(\mathrm{~m}, 1 \mathrm{H}), 3.79(\mathrm{~s}, 3 \mathrm{H}), 3.82(\mathrm{~d}, J=10.4 \mathrm{~Hz}, 1 \mathrm{H})$, $4.10(\mathrm{~d}, J=10.4 \mathrm{~Hz}, 1 \mathrm{H}), 4.18(\mathrm{dd}, J=9.6,4.8 \mathrm{~Hz}, 1 \mathrm{H}), 4.95(\mathrm{~s}, 1 \mathrm{H}), 5.15(\mathrm{~s}, 1 \mathrm{H})$, $7.07(\mathrm{t}, J=8.0 \mathrm{~Hz}, 1 \mathrm{H}), 7.68(\mathrm{t}, J=7.6 \mathrm{~Hz}, 1 \mathrm{H}), 8.29$ (d, $J=8.0 \mathrm{~Hz}, 1 \mathrm{H}), 10.28(\mathrm{~s}, 1$ $\mathrm{H}) ;{ }^{13} \mathrm{C} \mathrm{NMR}\left(100 \mathrm{MHz}, \mathrm{CDCl}_{3}\right) \delta-5.5,-5.1,-5.0,-4.5,18.0,18.3,25.8,26.0,28.3,30.6$, $33.6,35.7,36.2,55.5,65.6,67.9,72.3,111.8,122.6,127.8,129.3,136.9,138.8,156.0$, 164.4, 190.8; HRMS (FAB) $m / z$ calcd for $\mathrm{C}_{30} \mathrm{H}_{51} \mathrm{O}_{4} \mathrm{Si}_{2}[\mathrm{M}-\mathrm{H}]^{+}$531.3326, found 531.3307.

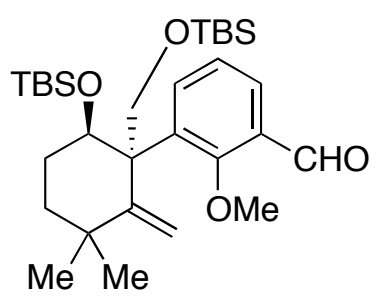

2.37
1) TBAF, THF, $0^{\circ} \mathrm{C}, 24 \mathrm{~h}$

2) $\mathrm{Ac}_{2} \mathrm{O}, \mathrm{Pyr}$. $\mathrm{CH}_{2} \mathrm{Cl}_{2}, 85 \%$ 2 steps

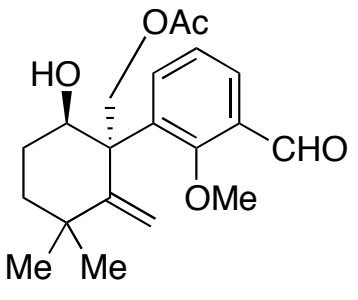

2.38

To a stirred and cooled $\left(0{ }^{\circ} \mathrm{C}\right)$ solution of silyl ether $\mathbf{2 . 3 7}(201.0 \mathrm{mg}, 0.38 \mathrm{mmol})$ in THF (3 mL) was added dropwise a solution of TBAF $(1.0 \mathrm{M}, 1.13 \mathrm{~mL}, 1.13 \mathrm{mmol})$. The resultant solution was stirred at $0{ }^{\circ} \mathrm{C}$ for $24 \mathrm{~h}$. The reaction was quenched by the addition of saturated aqueous $\mathrm{NH}_{4} \mathrm{Cl}(2 \mathrm{~mL})$. The mixture was extracted with EtOAc $(3 \mathrm{X} 10 \mathrm{~mL})$. 
The combined extracts were dried $\left(\mathrm{Na}_{2} \mathrm{SO}_{4}\right)$ and concentrated. The residue was purified by a short silica column $(1.5 \times 16 \mathrm{~cm})$, using 5:1 hexane/EtOAc, gave diol (ca. $120 \mathrm{mg}$ ), which was used directly in the next step. To a stirred and cooled $\left(0{ }^{\circ} \mathrm{C}\right)$ solution of the above diol (ca. $120 \mathrm{mg}$ ) in $\mathrm{CH}_{2} \mathrm{Cl}_{2}(3 \mathrm{~mL})$ were added successively pyridine $(0.193 \mathrm{~mL}$, $2.39 \mathrm{mmol})$ and $\mathrm{Ac}_{2} \mathrm{O}(0.12 \mathrm{~mL}, 1.19 \mathrm{mmol})$. The resulting solution was stirred at $0{ }^{\circ} \mathrm{C}$ for 5 days and the reaction was quenched by the addition of saturated aqueous $\mathrm{NaHCO}_{3}$ $(3 \mathrm{~mL})$. The mixture was extracted with EtOAc $(3 \mathrm{X} 10 \mathrm{~mL})$. The combined extracts were dried $\left(\mathrm{Na}_{2} \mathrm{SO}_{4}\right)$ and the solvent was evaporated. Flash chromatography of the residue over silica $(2 \times 18 \mathrm{~cm}$ ), using 4:1 to 1:1 hexane/EtOAc, gave monoacetate $\mathbf{2 . 3 8}$ (117.0 mg, 85\%) as colorless oil: FTIR $\left(\mathrm{CH}_{2} \mathrm{Cl}_{2}\right.$, cast) 3476, 2959, 1738, 1685, 1581, 1466, 1381, 1240, $1044 \mathrm{~cm}^{-1} ;{ }^{1} \mathrm{H}$ NMR (400 MHz, $\left.\mathrm{CDCl}_{3}\right) \delta 0.72(\mathrm{~s}, 3 \mathrm{H}), 1.15(\mathrm{~s}, 3 \mathrm{H})$, 1.30-1.38 (m, $1 \mathrm{H}), 1.46-1.52(\mathrm{~m}, 1 \mathrm{H}), 1.96-2.03$ (m, including a singlet at 2.03, $4 \mathrm{H}$ in all), 2.14-2.20 (m, $1 \mathrm{H}), 3.76(\mathrm{~d}, J=6.0 \mathrm{~Hz}, 1 \mathrm{H}), 3.82-3.88(\mathrm{~m}$, including a singlet at 3.88, $4 \mathrm{H}$ in all), $4.69(\mathrm{ABq}, \Delta v=13.3 \mathrm{~Hz}, J=12.0 \mathrm{~Hz}, 2 \mathrm{H}), 5.15(\mathrm{~s}, 1 \mathrm{H}), 5.32(\mathrm{~s}, 1 \mathrm{H})$, $7.16(\mathrm{t}, J=8.0 \mathrm{~Hz}, 1 \mathrm{H}), 7.73(\mathrm{~d}, J=7.6 \mathrm{~Hz}, 1 \mathrm{H}), 8.27$ (d, $J=8.0 \mathrm{~Hz}, 1 \mathrm{H}), 10.25(\mathrm{~s}, 1$ $\mathrm{H}) ;{ }^{13} \mathrm{C}$ NMR (100 MHz, $\left.\mathrm{CDCl}_{3}\right) \delta 21.0,27.3,29.2,32.3,37.0,37.7,54.3,65.8,67.0$, 74.1, 112.4, 123.6, 129.7, 134.6, 137.6, 153.8, 162.7, 171.3, 189.9; HRMS (FAB) $\mathrm{m} / \mathrm{z}$ calcd for $\mathrm{C}_{20} \mathrm{H}_{27} \mathrm{O}_{5}[\mathrm{M}+\mathrm{H}]^{+}$347.1858, found 347.1861.

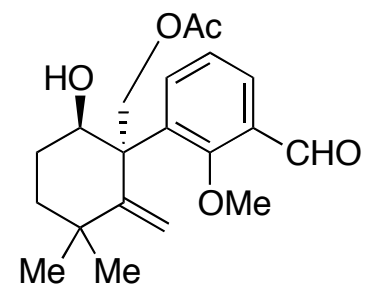

2.38

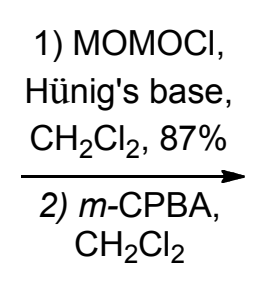

$\mathrm{CH}_{2} \mathrm{Cl}_{2}$

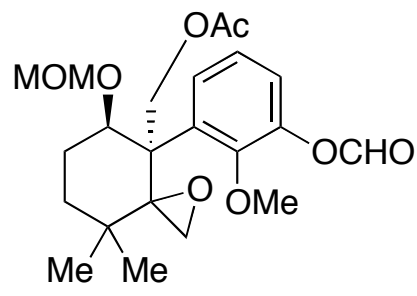

2.39 
To a stirred and cooled $\left(0^{\circ} \mathrm{C}\right)$ solution of alcohol $2.38(117.0 \mathrm{mg}, 0.338 \mathrm{mmol})$ in $\mathrm{CH}_{2} \mathrm{Cl}_{2}$ $(5 \mathrm{~mL})$ were added successively Hunig's base $(1.18 \mathrm{~mL}, 6.76 \mathrm{mmol})$ and $\mathrm{MOMCl}(0.26$ $\mathrm{mL}, 3.38 \mathrm{mmol})$. The resulting solution was stirred at room temperature for 3 days and the reaction was quenched by the addition of saturated aqueous $\mathrm{NaHCO}_{3}(2 \mathrm{~mL})$. The mixture was extracted with EtOAc $(3 \times 10 \mathrm{~mL})$. The combined extracts were dried $\left(\mathrm{Na}_{2} \mathrm{SO}_{4}\right)$ and the solvent was evaporated. Flash chromatography of the residue over silica (1.5 X $18 \mathrm{~cm})$, using 3:1 hexane/EtOAc, gave MOM ether $\mathbf{2 . 3 9}(115.0 \mathrm{mg}, 87 \%)$ as colorless oil: FTIR $\left(\mathrm{CH}_{2} \mathrm{Cl}_{2}\right.$, cast $) 2959,1738,1685,1580,1472,1240 \mathrm{~cm}^{-1} ;{ }^{1} \mathrm{H}$ NMR (400 MHz, $\left.\mathrm{CDCl}_{3}\right) \delta 0.68(\mathrm{~s}, 3 \mathrm{H}), 1.15(\mathrm{~s}, 3 \mathrm{H}), 1.37-1.45(\mathrm{~m}, 1 \mathrm{H}), 1.51-1.56(\mathrm{~m}, 1 \mathrm{H})$, 2.02-2.09 (m, including a singlet at 2.07, $4 \mathrm{H}$ in all), 2.26-2.37 (m, $1 \mathrm{H}), 3.34(\mathrm{~s}, 3 \mathrm{H})$, $3.84(\mathrm{~s}, 3 \mathrm{H}), 3.92(\mathrm{dd}, \mathrm{J}=11.6,4.4 \mathrm{~Hz}, 1 \mathrm{H}), 4.18(\mathrm{~d}, \mathrm{~J}=11.2 \mathrm{~Hz}, 1 \mathrm{H}), 4.63(\mathrm{ABq}, \Delta v$ $=18.0 \mathrm{~Hz}, J=6.8 \mathrm{~Hz}, 2 \mathrm{H}), 5.01(\mathrm{~d}, \mathrm{~J}=11.6 \mathrm{~Hz}, 1 \mathrm{H}), 5.04(\mathrm{~s}, 1 \mathrm{H}), 5.27(\mathrm{~s}, 1 \mathrm{H}), 7.15$ (t, $J=8.0 \mathrm{~Hz}, 1 \mathrm{H}), 7.73(\mathrm{~d}, J=7.6 \mathrm{~Hz}, 1 \mathrm{H}), 8.29$ (d, $J=8.0 \mathrm{~Hz}, 1 \mathrm{H}), 10.27(\mathrm{~s}, 1 \mathrm{H})$; ${ }^{13} \mathrm{C}$ NMR $\left(100 \mathrm{MHz}, \mathrm{CDCl}_{3}\right) \delta 21.1,25.7,28.6,32.2,37.1,38.5,54.0,55.7,65.3,65.9$, 80.4, 97.0, 111.9, 123.3, 129.1, 129.8, 135.4, 137.1, 152.7, 164.1, 170.8, 190.2; HRMS (FAB) $m / z$ calcd for $\mathrm{C}_{22} \mathrm{H}_{31} \mathrm{O}_{6}[\mathrm{M}+\mathrm{H}]^{+}$391.2121, found 391.2139. To a stirred and cooled $\left(0{ }^{\circ} \mathrm{C}\right)$ solution of aldehyde $(115.0 \mathrm{mg}, 0.29 \mathrm{mmol})$ in $\mathrm{CH}_{2} \mathrm{Cl}_{2}(8 \mathrm{~mL})$ was added $m$-CPBA (264.0 mg, $1.18 \mathrm{mmol})$. The resulting solution was stirred at room temperature for $12 \mathrm{~h}$ and the reaction mixture was diluted by the addition of $\mathrm{CH}_{2} \mathrm{Cl}_{2}(100 \mathrm{~mL})$. The mixture was washed with saturated $\mathrm{NaHCO}_{3}\left(4 \mathrm{X} 30 \mathrm{~mL}\right.$ ). The $\mathrm{CH}_{2} \mathrm{Cl}_{2}$ solution was dried $\left(\mathrm{Na}_{2} \mathrm{SO}_{4}\right)$ and concentrated. The residue was used directly in the next step without further purification. 


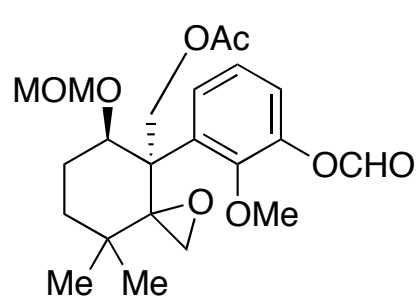

2.39

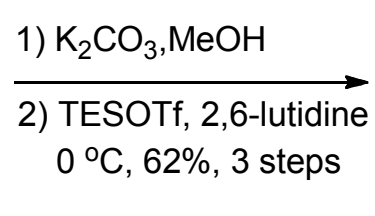

$0{ }^{\circ} \mathrm{C}, 62 \%$, 3 steps

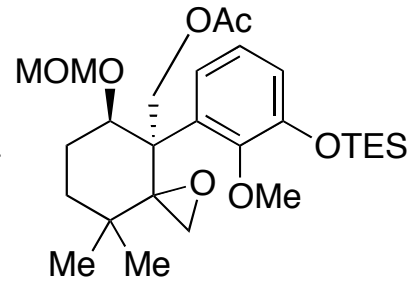

2.40

The above residue $\mathbf{2 . 3 9}$ was dissolved in $\mathrm{MeOH}(5 \mathrm{~mL})$. The solution was then cooled to $0{ }^{\circ} \mathrm{C}$. A $5 \%$ aqueous $\mathrm{K}_{2} \mathrm{CO}_{3}$ solution $(0.5 \mathrm{~mL})$ was added. Stirring was continued at this temperature for $30 \mathrm{~min}$ and the reaction mixture was acidified by the addition of $\mathrm{PH}=6$ buffer $(2 \mathrm{~mL})$. The mixture was extracted with EtOAc $(3 \mathrm{X} 10 \mathrm{~mL})$. The combined organic extracts were dried $\left(\mathrm{Na}_{2} \mathrm{SO}_{4}\right)$ and concentrated. The residue was filtered through a pad of silica (1 X $4 \mathrm{~cm})$, using EtOAc $(10 \mathrm{~mL})$, giving crude phenol, which was dried under high vacuum oil pump and used directly in the next step without further purification. To a stirred and cooled $\left(0{ }^{\circ} \mathrm{C}\right)$ solution of the above crude phenol in $\mathrm{CH}_{2} \mathrm{Cl}_{2}$ $(5 \mathrm{~mL})$ were added successively 2,6 -lutidine $(0.15 \mathrm{~mL}, 1.27 \mathrm{mmol})$ and TBSOTf $(0.17$ $\mathrm{mL}, 0.76 \mathrm{mmol})$. Stirring was continued at this temperature for $30 \mathrm{~min}$. The reaction was quenched by the addition of saturated aqueous $\mathrm{NaHCO}_{3}(3 \mathrm{~mL})$. The mixture was extracted with EtOAc ( 3 X $15 \mathrm{~mL})$. The combined extracts were washed with brine (10 $\mathrm{mL})$, dried $\left(\mathrm{Na}_{2} \mathrm{SO}_{4}\right)$ and concentrated. Flash chromatography of the residue over silica (2 X $18 \mathrm{~cm}$ ), using 6:1 hexane/EtOAc, gave silyl ethers 2.40 (105.0 mg, 62\% over 3 steps) as colorless oil: FTIR $\left(\mathrm{CH}_{2} \mathrm{Cl}_{2}\right.$, cast $) 2956,1740,1465,1284,1237,1050 \mathrm{~cm}^{-1} ;{ }^{1} \mathrm{H}$ NMR $\left(400 \mathrm{MHz}, \mathrm{CDCl}_{3}\right) \delta 0.49(\mathrm{~s}, 3 \mathrm{H}), 0.68(\mathrm{~s}, 3 \mathrm{H}), 0.74(\mathrm{q}, J=8.0 \mathrm{~Hz}, 6 \mathrm{H}), 0.98(\mathrm{t}, J=8.0$ Hz, 9 H), 1.23-1.28 (m, 1 H), 1.68-1.76 (m, 1 H), 2.02 (s, 3 H), 2.04-2.09 (m, 1 H), 2.42$2.53(\mathrm{~m}, 1 \mathrm{H}), 2.97(\mathrm{~d}, J=3.6 \mathrm{~Hz}, 1 \mathrm{H}), 3.40(\mathrm{~d}, J=3.6 \mathrm{~Hz}, 1 \mathrm{H}), 3.45(\mathrm{~s}, 3 \mathrm{H}), 3.72(\mathrm{~d}, J$ 
$=12.0 \mathrm{~Hz}, 1 \mathrm{H}), 3.87(\mathrm{~s}, 3 \mathrm{H}), 4.28(\mathrm{dd}, J=12.8,4.0 \mathrm{~Hz}, 1 \mathrm{H}), 4.80(\mathrm{ABq}, \Delta v=18.7 \mathrm{~Hz}$, $J=6.8 \mathrm{~Hz}, 2 \mathrm{H}), 4.88(\mathrm{~d}, J=12.0 \mathrm{~Hz}, 1 \mathrm{H}), 6.76(\mathrm{dd}, J=8.0,1.6 \mathrm{~Hz}, 1 \mathrm{H}), 6.87(\mathrm{t}, J=$ $8.0 \mathrm{~Hz}, 1 \mathrm{H}), 7.76(\mathrm{dd}, J=8.0,1.6 \mathrm{~Hz}, 1 \mathrm{H}) ;{ }^{13} \mathrm{C} \mathrm{NMR}\left(100 \mathrm{MHz}, \mathrm{CDCl}_{3}\right) \delta 5.1,6.6$, $21.3,24.6,25.8,26.2,36.0,37.1,50.9,52.5,55.7,59.8,63.1,65.2,81.3,97.9,119.7$, 122.6, 123.9, 132.9, 148.9, 151.4, 170.5; HRMS (FAB) $m / z$ calcd for $\mathrm{C}_{27} \mathrm{H}_{44} \mathrm{O}_{7} \mathrm{Si}[\mathrm{M}]^{+}$ 508.2856, found 508.2865.

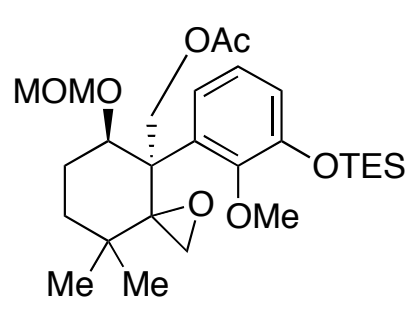

2.40

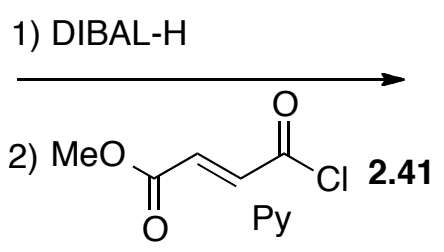

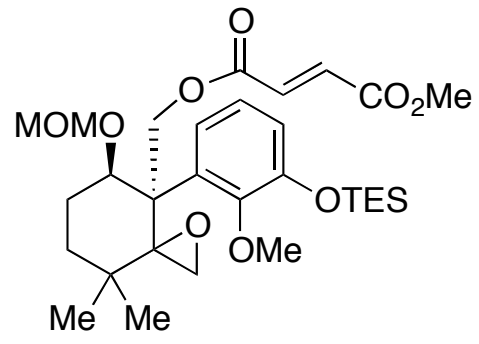

2.42

To a stirred and cooled $\left(-78^{\circ} \mathrm{C}\right)$ solution of actetate $2.40(80.0 \mathrm{mg}, 0.16 \mathrm{mmol})$ in $\mathrm{CH}_{2} \mathrm{Cl}_{2}$ $(5 \mathrm{~mL})$ was added DIBAL-H $(1.0 \mathrm{M}, 0.63 \mathrm{~mL}, 0.63 \mathrm{mmol})$. Stirring was continued at this temperature for $30 \mathrm{~min}$. The reaction was quenched by the addition of $\mathrm{MeOH}(1 \mathrm{~mL})$ and $\mathrm{Na}_{2} \mathrm{SO}_{4} \bullet 10 \mathrm{H}_{2} \mathrm{O}(1 \mathrm{~g})$. After warmed up to room temperature, the mixture was stirred for $1 \mathrm{~h}$ and filtered through a pad of celite $(2 \mathrm{X} 4 \mathrm{~cm})$, using EtOAc $(30 \mathrm{~mL})$ as rinse. After concentration of the filtrate, the residue was purified by flash chromatography over silica (2 X $16 \mathrm{~cm}$ ), using 4:1 hexane/EtOAc, gave alcohol $(67.0 \mathrm{mg}, 90 \%$ ) as colorless oil: FTIR $\left(\mathrm{CH}_{2} \mathrm{Cl}_{2}\right.$, cast) $3468,2956,1464,1424,1284,1049 \mathrm{~cm}^{-1} ;{ }^{1} \mathrm{H}$ NMR $(400 \mathrm{MHz}$, $\left.\mathrm{CDCl}_{3}\right) \delta 0.51(\mathrm{~s}, 3 \mathrm{H}), 0.69(\mathrm{~s}, 3 \mathrm{H}), 0.74(\mathrm{q}, J=8.0 \mathrm{~Hz}, 6 \mathrm{H}), 0.98(\mathrm{t}, J=8.0 \mathrm{~Hz}, 9 \mathrm{H})$, 1.26-1.31 (m, $1 \mathrm{H}), 1.71-1.79(\mathrm{~m}, 1 \mathrm{H}), 2.04-2.09(\mathrm{~m}, 1 \mathrm{H}), 2.43-2.54(\mathrm{~m}, 1 \mathrm{H}), 3.19(\mathrm{~d}, J$ $=2.8 \mathrm{~Hz}, 1 \mathrm{H}), 3.47(\mathrm{~s}, 3 \mathrm{H}), 3.52(\mathrm{~d}, J=10.8 \mathrm{~Hz}, 1 \mathrm{H}), 3.62(\mathrm{~d}, J=2.8 \mathrm{~Hz}, 1 \mathrm{H}), 3.84(\mathrm{~s}$, 
$3 \mathrm{H}), 3.84-3.92(\mathrm{~m}$, including a singlet at 3.84, $4 \mathrm{H}$ in all), $3.94(\mathrm{~d}, J=11.6 \mathrm{~Hz}, 1 \mathrm{H})$, $4.37(\mathrm{dd}, J=12.8,4.0 \mathrm{~Hz}, 1 \mathrm{H}), 4.77(\mathrm{~d}, J=6.8 \mathrm{~Hz}, 1 \mathrm{H}), 5.00(\mathrm{~d}, J=6.8 \mathrm{~Hz}, 1 \mathrm{H}), 6.75$ $(\mathrm{d}, J=8.0 \mathrm{~Hz}, 1 \mathrm{H}), 6.87(\mathrm{t}, J=8.0 \mathrm{~Hz}, 1 \mathrm{H}), 7.77(\mathrm{~d}, J=8.0 \mathrm{~Hz}, 1 \mathrm{H}) ;{ }^{13} \mathrm{C} \mathrm{NMR}(100$ $\left.\mathrm{MHz}_{\mathrm{CDCl}}\right) \delta 5.1,6.6,24.2,25.4,26.3,35.9,37.3,51.3,52.6,55.6,59.8,64.2,68.6$, 79.5, 98.1, 119.6, 122.7, 123.9, 133.1, 148.8, 151.2; HRMS (FAB) $m / z$ calcd for $\mathrm{C}_{25} \mathrm{H}_{42} \mathrm{O}_{6} \mathrm{Si}[\mathrm{M}]^{+}$466.2751, found 466.2732.

The resulting alcohol was then under esterification with 2.41 to afford $\mathbf{2 . 4 2}$. FTIR $\left(\mathrm{CH}_{2} \mathrm{Cl}_{2}\right.$, cast) $2956,1727,1650,1466,1296,1154,1047 \mathrm{~cm}^{-1} ;{ }^{1} \mathrm{H}$ NMR $(400 \mathrm{MHz}$, $\left.\mathrm{CDCl}_{3}\right) \delta 0.51(\mathrm{~s}, 3 \mathrm{H}), 0.68(\mathrm{~s}, 3 \mathrm{H}), 0.75(\mathrm{q}, J=8.0 \mathrm{~Hz}, 6 \mathrm{H}), 0.98(\mathrm{t}, J=8.0 \mathrm{~Hz}, 9 \mathrm{H})$, $1.26-1.30(\mathrm{~m}, 1 \mathrm{H}), 1.70-1.77(\mathrm{~m}, 1 \mathrm{H}), 2.05-2.11(\mathrm{~m}, 1 \mathrm{H}), 2.45-2.54(\mathrm{~m}, 1 \mathrm{H}), 2.99(\mathrm{~d}, J$ $=2.8 \mathrm{~Hz}, 1 \mathrm{H}), 3.40(\mathrm{~d}, J=2.8 \mathrm{~Hz}, 1 \mathrm{H}), 3.42(\mathrm{~s}, 3 \mathrm{H}), 3.38(\mathrm{~s}, 3 \mathrm{H}), 3.79(\mathrm{~s}, 3 \mathrm{H}), 3.88$ $(\mathrm{s}, 3 \mathrm{H}), 3.94(\mathrm{~d}, J=12.0 \mathrm{~Hz}, 1 \mathrm{H}), 4.30(\mathrm{dd}, J=12.4,3.6 \mathrm{~Hz}, 1 \mathrm{H}), 4.80(\mathrm{ABq}, \Delta v=$ $11.7 \mathrm{~Hz}, J=6.4 \mathrm{~Hz}, 2 \mathrm{H}), 4.93(\mathrm{~d}, J=12.0 \mathrm{~Hz}, 1 \mathrm{H}), 6.78(\mathrm{~d}, J=7.6 \mathrm{~Hz}, 1 \mathrm{H}), 6.89$ (t, $J$ $=8.0 \mathrm{~Hz}, 1 \mathrm{H}), 7.77(\mathrm{~d}, J=8.0 \mathrm{~Hz}, 1 \mathrm{H}) ;{ }^{13} \mathrm{C} \mathrm{NMR}\left(100 \mathrm{MHz}, \mathrm{CDCl}_{3}\right) \delta 5.1,6.6,24.6$, $25.8,25.9,36.0,37.0,50.8,52.2,52.4,55.7,59.8,64.0,65.3,81.4,97.7,119.9,122.7$, 124.0, 132.4, 133.1, 134.2, 148.8, 151.3, 164.4, 165.6; HRMS (FAB) $m / z$ calcd for $\mathrm{C}_{30} \mathrm{H}_{46} \mathrm{O}_{9} \mathrm{Si}[\mathrm{M}]^{+}$578.2911, found 578.2900.
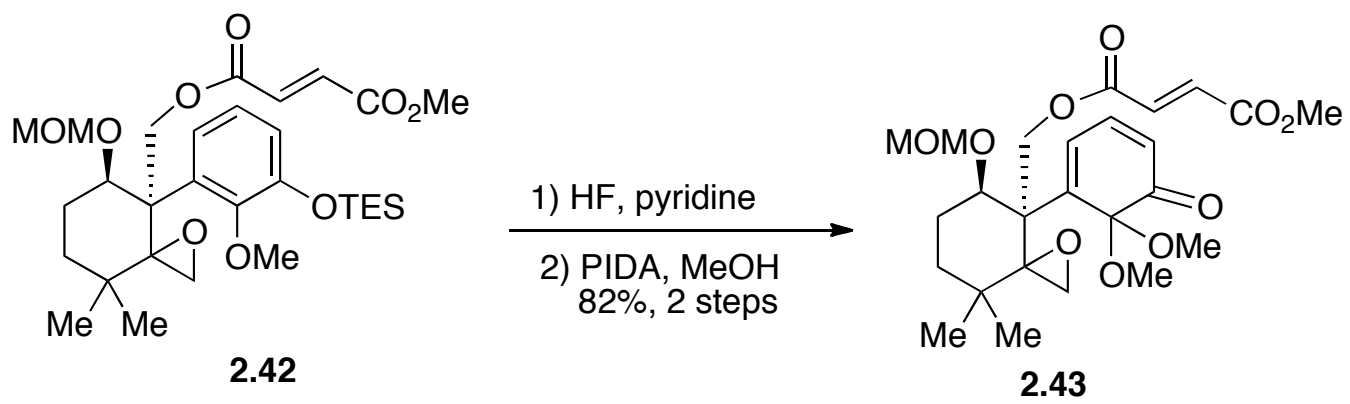
To a stirred and cooled $\left(0{ }^{\circ} \mathrm{C}\right)$ solution of TES silyl ether $\mathbf{2 . 4 2}(10.0 \mathrm{mg}, 0.017 \mathrm{mmol})$ in THF $(1.5 \mathrm{~mL})$ was added pyridine $(0.05 \mathrm{~mL})$ and HF.pyr. $(0.02 \mathrm{~mL})$. Stirring was continued at this temperature for $15 \mathrm{~min}$. The reaction mixture was concentrated and the residue was filtered through a pad of silica $(1 \mathrm{X} 5 \mathrm{~cm})$, using EtOAc $(5 \mathrm{~mL})$ as rinse. After concentration of the filtrate, the residue was dried under oil pump and used directly in the next step without further purification. The above residue was dissolved in $\mathrm{MeOH}$ $(1.5 \mathrm{~mL})$ and the solution was cooled to $0{ }^{\circ} \mathrm{C}$. A solution of PIDA (14.0 mg, $\left.0.043 \mathrm{mmol}\right)$ in $\mathrm{MeOH}(0.5 \mathrm{~mL})$ was added dropwise. After $10 \mathrm{~min}$ at $0{ }^{\circ} \mathrm{C}$, the reaction was quenched by the addition of saturated aqueous $\mathrm{NaHCO}_{3}(1 \mathrm{~mL})$. The mixture was extracted with EtOAc $(3 \times 3 \mathrm{~mL})$. The combined extracts were dried $\left(\mathrm{Na}_{2} \mathrm{SO}_{4}\right)$ and concentrated. The residue was purified by preparative TLC $(500 \mu \mathrm{m}, 20 \quad \mathrm{X} 20 \quad \mathrm{~cm})$, using $2: 1$ hexane/EtOAc, gave $o$-quinoneketal $\mathbf{2 . 4 3}$ (7.0 $\mathrm{mg}, 82 \%$ over 2 steps) as colorless oil: FTIR $\left(\mathrm{CH}_{2} \mathrm{Cl}_{2}\right.$, cast $) 2950,1724,1675,1439,1302,1257,1156,1030 \mathrm{~cm}^{-1} ;{ }^{1} \mathrm{H}$ NMR (400 MHz, $\left.\mathrm{CDCl}_{3}\right) \delta 0.73(\mathrm{~s}, 3 \mathrm{H}), 0.99(\mathrm{~s}, 3 \mathrm{H}), 1.38-1.44(\mathrm{~m}, 1 \mathrm{H}), 1.64-1.72(\mathrm{~m}, 1 \mathrm{H})$, 2.07-2.13 (m, $1 \mathrm{H}), 2.24-2.35(\mathrm{~m}, 1 \mathrm{H}), 2.77(\mathrm{~d}, J=3.2 \mathrm{~Hz}, 1 \mathrm{H}), 3.10(\mathrm{~s}, 3 \mathrm{H}), 3.17$ (d, $J$ $=3.2 \mathrm{~Hz}, 1 \mathrm{H}), 3.38(\mathrm{~s}, 3 \mathrm{H}), 3.45(\mathrm{~s}, 3 \mathrm{H}), 3.80(\mathrm{~s}, 3 \mathrm{H}), 3.90(\mathrm{~d}, J=12.0 \mathrm{~Hz}, 1 \mathrm{H}), 4.28$ (dd, $J=12.4,4.8 \mathrm{~Hz}, 1 \mathrm{H}), 4.72(\mathrm{ABq}, \Delta v=11.8 \mathrm{~Hz}, J=6.8 \mathrm{~Hz}, 2 \mathrm{H}), 5.03(\mathrm{~d}, J=12.0$ $\mathrm{Hz}, 1 \mathrm{H}), 6.03(\mathrm{~d}, J=9.2 \mathrm{~Hz}, 1 \mathrm{H}), 6.86(\mathrm{~s}, 2 \mathrm{H}), 6.98(\mathrm{dd}, J=10.0,7.2 \mathrm{~Hz}, 1 \mathrm{H}), 7.28$ $(\mathrm{d}, J=6.8 \mathrm{~Hz}, 1 \mathrm{H}) ;{ }^{13} \mathrm{C} \mathrm{NMR}\left(100 \mathrm{MHz}, \mathrm{CDCl}_{3}\right) \delta$ 25.5, 25.9, 26.3, 35.7, 37.3, 49.0, $49.4,52.2,53.1,54.2,55.8,64.2,64.6,81.2,95.7,97.6,125.3,128.2,133.0,134.2,141.2$, 147.0, 164.3, 165.5, 196.8; HRMS (FAB) $m / z$ calcd for $\mathrm{C}_{25} \mathrm{H}_{35} \mathrm{O}_{10}[\mathrm{M}+\mathrm{H}]^{+}$495.2230, found 495.2235 . 


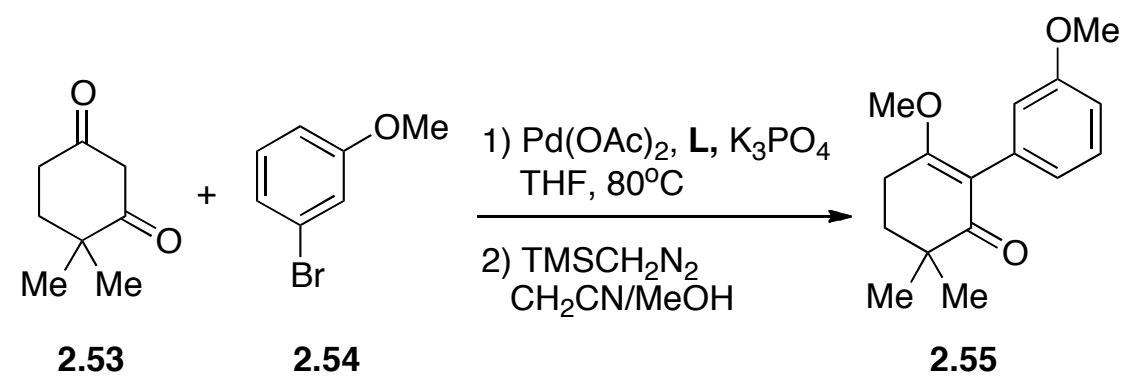

Compound 2.55: An oven-dried, $250 \mathrm{~mL}$ round bottom high-pressure flask containing a stirbar was capped with a rubber septum. This flask was then charged with 1,3-diketone 2.53 (5.1 g, $36 \mathrm{mmol}), \mathrm{Pd}(\mathrm{OAc})_{2}(67 \mathrm{mg}, 0.3 \mathrm{mmol})$, 2-di-t-butylphosphino-2'methylbiphenyl (206 mg, $0.6 \mathrm{mmol})$, and $\mathrm{K}_{3} \mathrm{PO} 4(14.6 \mathrm{~g}, 69 \mathrm{mmol})$. The flask was evacuated and backfilled with argon. $100 \mathrm{~mL}$ of THF and aryl bromide $2.54(3.8 \mathrm{~mL}, 30$ mmol) were sequentially injected, and the septum was replaced with a Teflon screw cap. The flask was sealed and heated at $80^{\circ} \mathrm{C}$ for 12 hours. The reaction mixture was then diluted with $\mathrm{MeOH}$ and filtered. The filtrate was concentrated and dissolved in $27 \mathrm{~mL}$ of $\mathrm{CH}_{3} \mathrm{CN}$ and $3 \mathrm{ml}$ of $\mathrm{MeOH}$ under argon. To this mixture, Hunig's base $(7.5 \mathrm{~mL}, 43$ mmol) and a solution of $\operatorname{TMSCHN}_{2}(21 \mathrm{~mL}, 2 \mathrm{M})$ in hexane were added sequentially at 0 ${ }^{\circ} \mathrm{C}$. The reaction mixture was stirred at room temperature for additional 12 hours. Solvents were removed under reduced pressure using rotavap. The residues were purified by flash chromatography to give a colourless oil 2.55. (7.1g, 91\% yield). ${ }^{1} \mathrm{H}$ NMR $\left(\mathrm{CDCl}_{3}, 300 \mathrm{MHz}\right): \delta 7.24(\mathrm{dd}, J=7.8,7.8 \mathrm{~Hz}, 1 \mathrm{H}), 6.81-6.70(\mathrm{~m}, 3 \mathrm{H}), 3.79(\mathrm{~s}, 3 \mathrm{H}), 3.70$ (s, 3H), $2.70(\mathrm{dd}, J=6.3,6.3 \mathrm{~Hz}, 2 \mathrm{H}), 1.93(\mathrm{dd}, J=6.3,6.3 \mathrm{~Hz}, 2 \mathrm{H}), 1.18(\mathrm{~s}, 6 \mathrm{H}) ;{ }^{13} \mathrm{C}$ NMR ( $\left.\mathrm{CDCl}_{3}, 75 \mathrm{MHz}\right): \delta 201.7,170.0,158.9,135.3,128.4,123.2,118.4,116.3,112.3$, 55.5, 55.1, 39.6, 34.0, 24.5, 22.7; HRMS (FAB, $m / z)$ calcd for $\mathrm{C}_{16} \mathrm{H}_{21} \mathrm{O}_{3}[\mathrm{M}+\mathrm{H}]^{+}$ 
261.1491, found 261.1485.

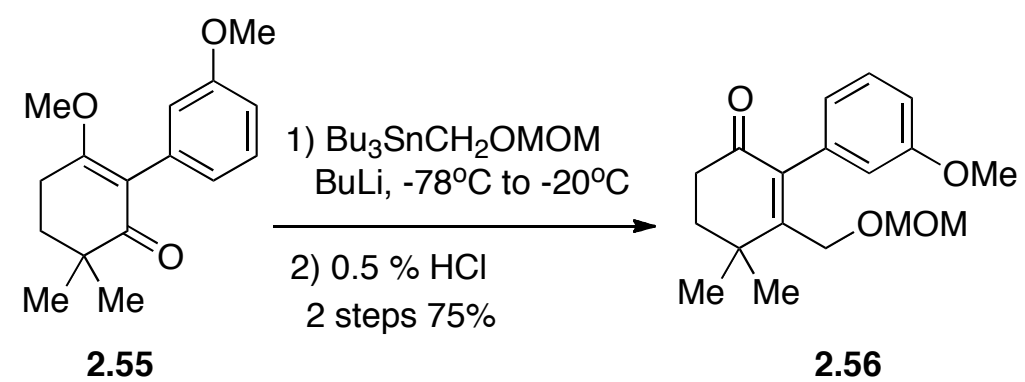

compound 2.56: A solution of $\mathrm{Bu}_{3} \mathrm{SnCH}_{2} \mathrm{OMOM}(5.9 \mathrm{~g}, 16 \mathrm{mmol})$ in $40 \mathrm{~mL}$ of anhydrous THF was cooled to $-78{ }^{\circ} \mathrm{C}$ under argon. BuLi in hexane $(6.45 \mathrm{ml}, 2.5 \mathrm{M})$ was added dropwise in 5 mins. The reaction mixture was stirred at this temperature for additional $10 \mathrm{~min}$ and a solution of $\mathbf{2 . 5 5}(2.6 \mathrm{~g}, 10 \mathrm{mmol})$ in $10 \mathrm{~mL}$ of THF was injected quickly. The reaction mixture was slowly warmed up to $-40{ }^{\circ} \mathrm{C}$ in $30 \mathrm{~min}$ and stirred for additional 1 hour at this temperature. Then the reaction mixture was warmed up to $0{ }^{\circ} \mathrm{C}$ and acidified with $0.5 \% \mathrm{HCl}$ solution until $\mathrm{pH}=3.30$ min later, the reaction mixture was neutralized with saturated $\mathrm{NaHCO}_{3}$ solution, extracted with ethyl acetate $(3 \times 20 \mathrm{~mL})$, and dried over $\mathrm{MgSO}_{4}$. The extract was filtered over cotton and the solvent was removed under reduced pressure. The residue was put on a short silica gel column. The nonpolar impurity was removed by hexane and the polar part was collected using ethyl acetate as eluent. The ethyl acetate was removed under reduced pressure and the residue was dissolved in $50 \mathrm{~mL}$ of $\mathrm{MeOH} .8$ drops of concentrated $\mathrm{HCl}(37 \%)$ was added dropwise and the reaction mixture was stirred at $50{ }^{\circ} \mathrm{C}$ for 3 hours. Then the mixture was cooled to room temperature, neutralized with saturated $\mathrm{NaHCO}_{3}$ solution, and extracted with ethyl acetate $(3 \times 30 \mathrm{~mL})$. The extract was dried over $\mathrm{MgSO}_{4}$, filtered and the solvent was 
removed using rotovap. The residue was purified using flash chromatography to give a colorless oil $2.56\left(1.5 \mathrm{~g}, 56 \%\right.$ for two steps). ${ }^{1} \mathrm{H} \mathrm{NMR}\left(\mathrm{CDCl}_{3}, 400 \mathrm{MHz}\right): \delta 7.26(\mathrm{dd}, J=$ 7.6, 7.6 Hz, 2H), $6.84(\mathrm{dd}, J=8.0,2.4 \mathrm{~Hz}, 1 \mathrm{H}), 6.63$ (d, $J=7.6 \mathrm{~Hz}, 1 \mathrm{H}), 6.60(\mathrm{~s}, 1 \mathrm{H})$, $4.08(\mathrm{~d}, J=5.6 \mathrm{~Hz}, 2 \mathrm{H}), 3.77(\mathrm{~s}, 3 \mathrm{H}), 2.61(\mathrm{dd}, J=6.4,6.4 \mathrm{~Hz}, 2 \mathrm{H}), 1.95(\mathrm{dd}, J=6.3,6.3$ $\mathrm{Hz}, 2 \mathrm{H}), 1.33(\mathrm{~s}, 6 \mathrm{H}) ;{ }^{13} \mathrm{C} \mathrm{NMR}\left(\mathrm{CDCl}_{3}, 100 \mathrm{MHz}\right): \delta 198.5,162.7,159.4,138.5,136.9$, 129.3, 121.7, 115.1, 113.1, 60.4, 55.1, 37.6, 35.3, 34.6, 26.8; IR (neat): $\mathrm{cm}^{-1} 3441,2960$, 2926, 1701, 1670, 1596, 1484, 1466, 1287, 1256, 1047; HRMS (FAB, m/z) calcd for $\mathrm{C}_{16} \mathrm{H}_{20} \mathrm{O}_{3}[\mathrm{M}+\mathrm{H}]^{+}$261.1412, found 261.1417.

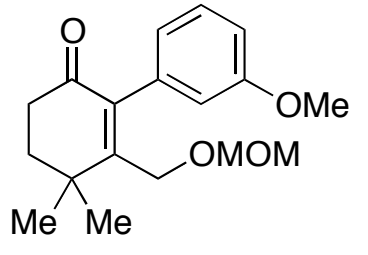

2.56
1) $\mathrm{HCl}, \mathrm{MeOH}$

2) PivCl, Py 2 steps $78 \%$

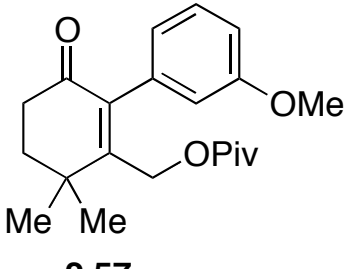

2.57

Compound 2.57: compound 2.56 (1.3 g, $5 \mathrm{mmol})$ was dissolved in $20 \mathrm{~mL}$ of anhydrous DCM under argon at $0{ }^{\circ} \mathrm{C}$. Pyridine $(2.4 \mathrm{~mL}, 30 \mathrm{mmol})$ was injected into this solution and 5 min later pivaloyl chloride $(2.4 \mathrm{~mL}, 20 \mathrm{mmol})$ was injected into the mixture. The reaction mixture was stirred at this temperature for 12 hours, quenched with water, extracted with ether $(3 \times 20 \mathrm{~mL})$ and dried over $\mathrm{MgSO}_{4}$. After filtration of the drying agent, the solvent was removed under reduced pressure and the residue was purified by flash chromatography (1.65 g, 96\%). ${ }^{1} \mathrm{H}$ NMR $\left(\mathrm{CDCl}_{3}, 400 \mathrm{MHz}\right): \delta 7.22(\mathrm{dd}, J=8.0$, $8.0 \mathrm{~Hz}, 1 \mathrm{H}), 6.82(\mathrm{dd}, J=8.4,2.0 \mathrm{~Hz}, 1 \mathrm{H}), 6.61(\mathrm{~d}, J=7.6 \mathrm{~Hz}, 1 \mathrm{H}), 6.58(\mathrm{br} \mathrm{s}, 1 \mathrm{H}), 4.43$ (s, 2H), $3.76(\mathrm{~s}, 3 \mathrm{H}), 2.66(\mathrm{dd}, J=6.4,6.4 \mathrm{~Hz}, 2 \mathrm{H}), 2.13(\mathrm{dd}, J=6.8,6.8 \mathrm{~Hz}, 2 \mathrm{H}), 1.28$ 


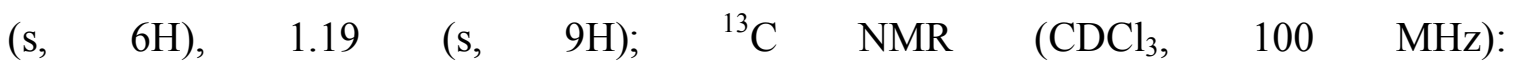
$\delta 197.9,177.6,159.1,157.3,141.4,136.2,128.8,122.0,115.6,113.1,62.3,55.1,38.6,37$

$.5,35.3,34.7,27.1$; IR (neat): $\mathrm{cm}^{-1} 2952,1724,1667,1453,1437,1301,1259,1156$, 1034; HRMS (FAB, $m / z$ ) calcd for $\mathrm{C}_{21} \mathrm{H}_{28} \mathrm{O}_{4}[\mathrm{M}]^{+} 344.1988$, found 344.2001.

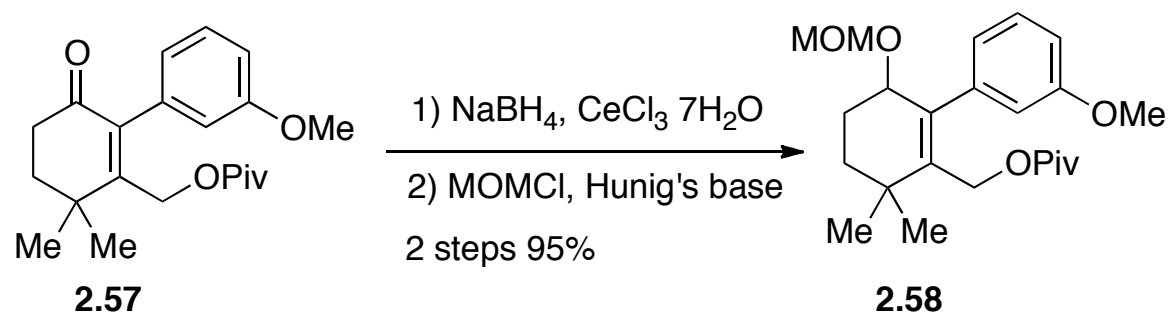

Compound 2.58: compound $2.57(3.4 \mathrm{~g}, 10 \mathrm{mmol})$ and cerium chloride heptahydrate $(5 \mathrm{~g}$, $13 \mathrm{mmol})$ were dissolved in $20 \mathrm{~mL}$ of methanol at $0{ }^{\circ} \mathrm{C} .5 \mathrm{~min}$ later $\mathrm{NaBH}_{4}(600 \mathrm{mg}, 15$ mmol) was added carefully in three portions. The reaction mixture was stirred at $0{ }^{\circ} \mathrm{C}$ for two hours and quenched with water. The product was extracted with ethyl acetate $(3 \times 30$ $\mathrm{mL}$ ) and dried over $\mathrm{MgSO}_{4}$. After filtration of the drying agent, the solvent was removed under reduced pressure and the residue was purified using flash chromatography $(3.22 \mathrm{~g}$, 93\%). ${ }^{1} \mathrm{H} \mathrm{NMR}\left(\mathrm{CDCl}_{3}, 400 \mathrm{MHz}\right): \delta 7.20(\mathrm{dd}, J=7.6,7.6 \mathrm{~Hz}, 2 \mathrm{H}), 6.79(\mathrm{dd}, J=7.6 \mathrm{~Hz}$, 1H), $6.75(\mathrm{~d}, J=7.6 \mathrm{~Hz}, 1 \mathrm{H}), 6.71(\mathrm{~s}, 1 \mathrm{H}), 4.42(\mathrm{~d}, J=11.2 \mathrm{~Hz}, 1 \mathrm{H}), 4.32(\mathrm{~s}, 1 \mathrm{H}), 4.11$ $(\mathrm{d}, J=11.2 \mathrm{~Hz}, 1 \mathrm{H}), 3.77(\mathrm{~s}, 3 \mathrm{H}), 2.02-1.74(\mathrm{~m}, 4 \mathrm{H}), 1.16(\mathrm{~s}, 9 \mathrm{H}), 1.14(\mathrm{~s}, 3 \mathrm{H}), 1.08(\mathrm{~s}$, $3 \mathrm{H}) ;{ }^{13} \mathrm{C} \mathrm{NMR}\left(\mathrm{CDCl}_{3}, 100 \mathrm{MHz}\right): \delta 178.1,159.4,143.1,140.7,138.3,129.2,121.4$, $114.8,112.6,69.1,62.0,55.1,38.5,34.7,34.4,28.3,27.5,27.2,27.1 ; \quad$ IR (neat): $\quad \mathrm{cm}^{-1}$ 3435, 2960, 2936, 2870, 1724, 1597, 1577, 1480, 1285; HRMS (FAB, m/z) calcd for $\mathrm{C}_{21} \mathrm{H}_{30} \mathrm{O}_{4}[\mathrm{M}]^{+}$346.2144, found 346.2158. The above alcohol (1.8 g, $\left.5.2 \mathrm{mmol}\right)$ and 
Hunig's base $(2.72 \mathrm{~mL}, 15.6 \mathrm{mmol})$ were dissolved in anhydrous DCM under argon at room temperature. $\mathrm{MOMCl}(775 \mathrm{~mL}, 11 \mathrm{mmol})$ was injected into the solution and the reaction mixture was stirred at room temperature for 12 hours. Then the reaction was quenched with 2 drops of water. The solvent was removed under reduced pressure and the residue was purified using flash chromatography $(1.93 \mathrm{~g}, 95 \%) .{ }^{1} \mathrm{H} \mathrm{NMR}\left(\mathrm{CDCl}_{3}\right.$, $400 \mathrm{MHz}): \delta 7.16(\mathrm{dd}, J=7.6,7.6 \mathrm{~Hz}, 1 \mathrm{H}), 6.79-6.74(\mathrm{~m}, 3 \mathrm{H}), 4.53(\mathrm{~d}, J=7.2 \mathrm{~Hz}, 1 \mathrm{H})$, $4.46(\mathrm{~d}, J=11.6 \mathrm{~Hz}, 1 \mathrm{H}), 4.27-4.25(\mathrm{~m}, 1 \mathrm{H}), 4.24(\mathrm{~d}, J=7.2 \mathrm{~Hz}, 1 \mathrm{H}), 4.10(\mathrm{~d}, J=11.6$ $\mathrm{Hz}, 1 \mathrm{H}), 3.76$ (s, 3H), 2.85 (s, 3H), 1.94-1.77 (m, 3H), 1.49-1.44 (m, 1H), $1.16(\mathrm{~s}, 9 \mathrm{H})$, $1.13 \quad(\mathrm{~s}, \quad 3 \mathrm{H}), \quad 1.09 \quad(\mathrm{~s}, \quad 3 \mathrm{H}) ; \quad{ }^{13} \mathrm{C} \quad \mathrm{NMR} \quad\left(\mathrm{CDCl}_{3}, \quad 100 \quad \mathrm{MHz}\right)$ $\delta 178.1,159.1,143.8,141.6,139.4,128.6,121.7,114.8,112.3,94.9$,

$73.4,62.0,55.2,54.8,38.5,34.4,34.3,28.4,27.1,26.7,25.1$; IR (neat): $\mathrm{cm}^{-1} 2959,2935$, 1724, 1577, 1480, 1284, 1152, 1032; $\operatorname{HRMS}(\mathrm{FAB}, \mathrm{m} / z)$ calcd for $\mathrm{C}_{23} \mathrm{H}_{34} \mathrm{O}_{5}[\mathrm{M}]^{+}$ 390.2406, found 390.2418.

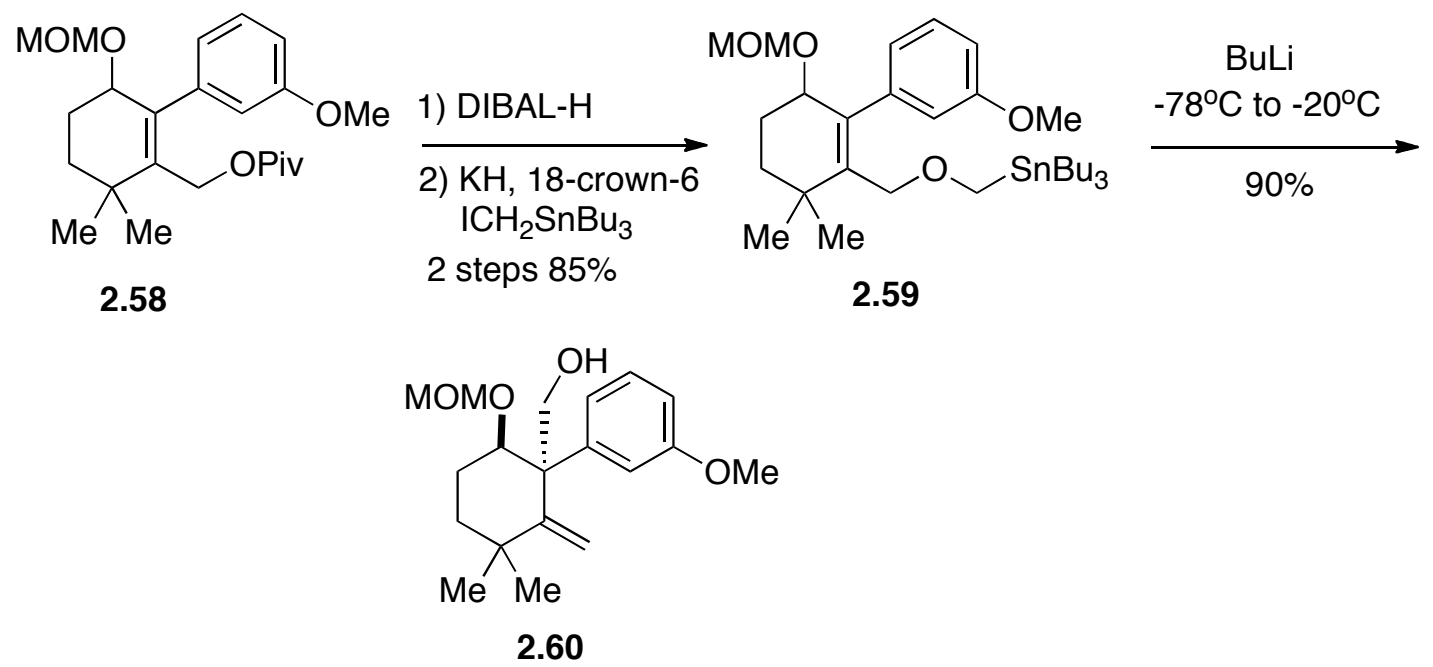


Compound 2.59 : compound 2.58 (1.95 g, $5 \mathrm{mmol}$ ) was dissolved in anhydrous DCM under argon and cooled to $-78{ }^{\circ} \mathrm{C} .7 .5 \mathrm{~mL}$ of DIBAL-H in hexane $(2 \mathrm{M})$ was injected into the reaction mixture slowly and the reaction mixture was stirred for $30 \mathrm{~min}$ at this temperature. Then the reaction was quenched with $0.5 \mathrm{~mL}$ of methanol and $5 \mathrm{~g}$ of sodium sulfate decahydrate. After warming up to room temperature, the mixture was stirred for additional 1 hour and filtered. The solvent was removed using rotovap and the residue was purified with flash chromatography (1.45, 95\%). ${ }^{1} \mathrm{H} \mathrm{NMR}\left(\mathrm{CDCl}_{3}, 400 \mathrm{MHz}\right): \delta 7.22$ $(\mathrm{dd}, J=7.6,7.6 \mathrm{~Hz}, 1 \mathrm{H}), 6.82-6.79(\mathrm{~m}, 3 \mathrm{H}), 4.53(\mathrm{~d}, J=7.2 \mathrm{~Hz}, 1 \mathrm{H}), 4.25(\mathrm{~d}, J=7.2 \mathrm{~Hz}$, $1 \mathrm{H}), 4.21(\mathrm{dd}, J=4.0,4.0 \mathrm{~Hz}, 1 \mathrm{H}), 4.05(\mathrm{dd}, J=12.0,4.4 \mathrm{~Hz}, 1 \mathrm{H}), 3.86(\mathrm{dd}, J=12.0$, $5.6 \mathrm{~Hz}, 1 \mathrm{H}), 3.80(\mathrm{~s}, 3 \mathrm{H}), 2.88(\mathrm{~s}, 3 \mathrm{H}), 1.93-1.76(\mathrm{~m}, 3 \mathrm{H}), 1.48-1.43(\mathrm{~m}, 1 \mathrm{H}), 1.22(\mathrm{~s}$, 3H), $1.14(\mathrm{~s}, 3 \mathrm{H}) ;{ }^{13} \mathrm{C} \mathrm{NMR}\left(\mathrm{CDCl}_{3}, 100 \mathrm{MHz}\right): \delta 159.5,144.7,142.5,138.3,129.2$, $121.3,114.4,112.6,94.9,73.8,59.7,55.2,54.9,34.5,34.4,28.4,26.8,25.2 ; \quad$ IR (neat): $\mathrm{cm}^{-1} 3458,2936,1597,1577,1483,1149,1027$; HRMS (FAB, $\left.m / z\right)$ calcd for $\mathrm{C}_{18} \mathrm{H}_{26} \mathrm{O}_{4}$ $[\mathrm{M}]^{+}$306.1831, found 306.1836. an oven-dried round-bottomed flask was charged with $\mathrm{KH}(360 \mathrm{mg}, 9 \mathrm{mmol})$ and $20 \mathrm{~mL}$ of $\mathrm{THF}$ under argon at $0{ }^{\circ} \mathrm{C}$. A solution of compound 2.59 (920 mg, $3 \mathrm{mmol}$ ) in $5 \mathrm{~mL}$ of THF was injected slowly into the flask. 30 mins later, 18-crown-6 (780 mg, $3 \mathrm{mmol})$ was added. The reaction mixture was stirred for additional $5 \mathrm{~min}$ and then $\alpha$-iodomethyl tributylstanne (3.9 $\mathrm{g}, 9 \mathrm{mmol})$ was injected and the reaction mixture was stirred at this temperature for 6 hours. After quenched with water carefully, the product was extracted with ether $(3 \times 20 \mathrm{~mL})$ and dried over $\mathrm{MgSO}_{4}$. The drying agent was removed by filtration and the solvent was concentrated using rotovap. The residue was purified by flash chromatograph. And the desired product was dissolved in THF under argon. The mixture was cooled to $-78{ }^{\circ} \mathrm{C}$ and BuLi in hexane $(3.6 \mathrm{~mL}, 9$ 
mmol) was injected dropwise. The temperature was slowly warmed up to $-20{ }^{\circ} \mathrm{C}$ in 6 hours and the reaction was quenched with saturated $\mathrm{NH}_{4} \mathrm{Cl}$ solution, extracted with ether ( $3 \times 30 \mathrm{~mL}$ ), and dried over $\mathrm{MgSO}_{4}$. After filtration, the extract was concentrated and the residue was purified by chromatography $(0.78 \mathrm{~g}, 80 \%$ for 2 steps $)$.

${ }^{1} \mathrm{H}$ NMR $\left(\mathrm{CDCl}_{3}, 400 \mathrm{MHz}\right): \delta$ 7.25-7.14 (m, 3H), 6.75-6.71 (m, 1H), 5.37 (s, 1H), 5.34 (s, 1H), $4.78(\mathrm{~d}, J=10.4 \mathrm{~Hz}, 2 \mathrm{H}), 3.96(\mathrm{dd}, J=16.0,6.4 \mathrm{~Hz}, 1 \mathrm{H}), 3.84-3.79(\mathrm{~m}, 2 \mathrm{H})$, $3.78(\mathrm{~s}, 3 \mathrm{H}), 3.45$ (s, 3H), 3.26 (dd, $J=9.6,9.6 \mathrm{~Hz}, 1 \mathrm{H}), 2.27$ (dddd, $J=16.4,16.4,16.4$, $6.8 \mathrm{~Hz}, 1 \mathrm{H}), 1.96$ (dddd, $J=17.6,6.0,6.0,6.0 \mathrm{~Hz}, 1 \mathrm{H}), 1.46-1.31(\mathrm{~m}, 2 \mathrm{H}), 1.10(\mathrm{~s}, 3 \mathrm{H})$, $0.52(\mathrm{~s}, 3 \mathrm{H}) ;{ }^{13} \mathrm{C} \mathrm{NMR}\left(\mathrm{CDCl}_{3}, 100 \mathrm{MHz}\right): \delta 159.0,154.6,143.0,128.5,121.4,115.4$, $110.8,110.7,96.8,80.5,66.5,56.3,55.9,55.0,38.5,36.9,31.3,30.1,24.9$; IR (neat): $\mathrm{cm}^{-1} 3485,2953,1606,1579,1485,1464,1053,1027$; HRMS (FAB, $\left.m / z\right)$ calcd for $\mathrm{C}_{19} \mathrm{H}_{28} \mathrm{O}_{4}[\mathrm{M}+\mathrm{Na}]^{+}$343.1885, found 343.1868.

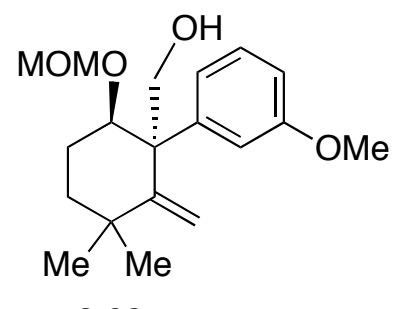

2.60

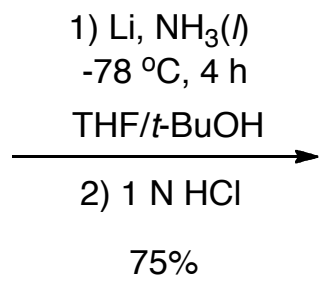

1) $\mathrm{Li}, \mathrm{NH}_{3}($ ( ) ${ }^{\circ} \mathrm{C}, 4 \mathrm{~h}$

2) $1 \mathrm{~N} \mathrm{HCl}$ $75 \%$

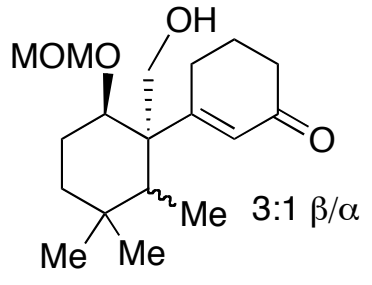

2.75

Compound 2.75: an oven-dried three-neck flash equipped with a dry ice acetone condenser was charged with $30 \mathrm{mg}$ of compound $\mathbf{2 . 6 0}, 1 \mathrm{~mL}$ of THF, $1 \mathrm{~mL}$ of tertbutanol, and $2 \mathrm{~mL}$ of liquid ammonia. Then $20 \mathrm{mg}$ of lithium was added and the reaction mixture was stirred at $-78{ }^{\circ} \mathrm{C}$ for 20 mins and $-33{ }^{\circ} \mathrm{C}$ for 40 mins. Then ammonium chloride solid was added to quench the reaction until the blue color disappeared. The 
reaction mixture was then slowly warmed up to room temperature to vaporize the ammonia. The residue was extracted with ethyl acetate $(3 \times 20 \mathrm{~mL})$ and dried over magnesium sulfate. After filtration of the drying agent, the solvent was removed under the reduced pressure and the residue was dissolved in $\mathrm{THF} / \mathrm{MeOH}$ mixed solvent $(\mathrm{THF} / \mathrm{MeOH}=10: 1)$. The mixture was cooled to $0{ }^{\circ} \mathrm{C}$ and 2 drop of $1 \mathrm{~N} \mathrm{HCl}$ solution was added. The reaction mixture was stirred at $0{ }^{\circ} \mathrm{C}$ for 12 hours and quenched with saturated $\mathrm{NaHCO}_{3}$. The extract was dried over $\mathrm{MgSO}_{4}$. After filtration, the solvent was removed using rotovap and the residue was used without purification in the next step.

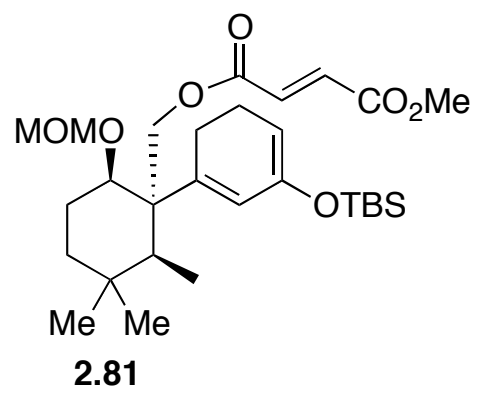

Compound 2.81: 2.75 (28 $\mathrm{mg}$ ) and pyridine (73 vL, $0.9 \mathrm{mmol})$ was dissolved in DCM under argon at $0{ }^{\circ} \mathrm{C}$. Acyl chloride $(65 \mathrm{mg}, 0.4 \mathrm{mmol})$ in $1 \mathrm{~mL}$ of DCM was injected slowly. After addition of acyl chloride solution, the reaction mixture was stirred at this temperature for 12 hours. The reaction was then quenched with methanol and the solvent was removed under the reduced pressure. The residue was purified using preparation TLC. 2.81: ${ }^{1} \mathrm{H}$ NMR (CDCl, $\left.400 \mathrm{MHz}\right): \delta 6.88(\mathrm{~d}, J=15.6 \mathrm{~Hz}, 2 \mathrm{H}), 6.52(\mathrm{~s}, 1 \mathrm{H}), 4.68$ $(\mathrm{d}, J=6.8 \mathrm{~Hz}, 1 \mathrm{H}), 4.52(\mathrm{~d}, J=7.2 \mathrm{~Hz}, 1 \mathrm{H}), 4.45(\mathrm{~d}, J=11.2 \mathrm{~Hz}, 1 \mathrm{H}), 4.22(\mathrm{~d}, J=11.2$ Hz, 1H), $3.82(\mathrm{~s}, 3 \mathrm{H}), 3.74$ (dd, $J=12,4 \mathrm{~Hz}, 1 \mathrm{H}), 3.29(\mathrm{~s}, 3 \mathrm{H}), 2.63-2.56(\mathrm{~m}, 1 \mathrm{H}), 2.52-$ 2.29 (m, 3H), 2.13 (dddd, $J=13.2,13.2,13.2,4 \mathrm{~Hz}, 1 \mathrm{H}), 2.00-1.97$ (m, 1H), 1.92-1.84 (m, 2H), 1.67 (ddd, $J=7.2,7.2,7.2 \mathrm{~Hz}, 1 \mathrm{H}), 1.53(\mathrm{ddd}, J=13.6,3.6,3.6 \mathrm{~Hz}, 1 \mathrm{H}), 1.37$ (ddd, $J=13.2,13.2,4 \mathrm{~Hz}, 1 \mathrm{H}), 1.04$ (d, $J=7.6 \mathrm{~Hz}, 3 \mathrm{H}), 0.89$ (s, 3H), $0.84(\mathrm{~s}, 3 \mathrm{H}) ;{ }^{13} \mathrm{C}$ 


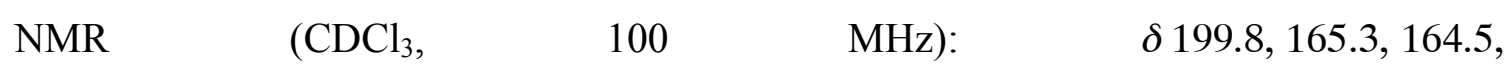
$163.7,133.7,133.3,131.4,96.2,80.1,65.2,55.9,52.4,52.1,45.2,40.1,37.6,34.4,32.0$, 29.1, 25.0, 23.7, 21.2, 12.4 ; IR (neat): $\mathrm{cm}^{-1} 2967,1728,1680,1579,1480,1280,1256$, 1147; HRMS (FAB, $m / z$ ) calcd for $\mathrm{C}_{23} \mathrm{H}_{35} \mathrm{O}_{7}[\mathrm{M}+\mathrm{H}]^{+}$423.2383, found 423.2400. an oven-dried flask was charged with starting material $(22 \mathrm{mg}, 0.05 \mathrm{mmol})$, TEA (22 $\mu \mathrm{L}$, $0.15 \mathrm{mmol})$, and $5 \mathrm{~mL}$ of DCM under argon at $0{ }^{\circ} \mathrm{C}$. TBSOTf $(27 \mu \mathrm{L}, 0.1 \mathrm{mmol})$ was added and the reaction mixture was stirred at this temperature for 12 hours. The product was purified using preparation TLC. ${ }^{1} \mathrm{H} \mathrm{NMR}\left(\mathrm{CDCl}_{3}, 400 \mathrm{MHz}\right): \delta 6.88(\mathrm{~d}, J=15.6 \mathrm{~Hz}$, 2H), $6.18(\mathrm{~s}, 1 \mathrm{H}), 4.91-4.89(\mathrm{~m}, 1 \mathrm{H}), 4.69(\mathrm{~d}, J=6.8 \mathrm{~Hz}, 1 \mathrm{H}), 4.52(\mathrm{~d}, J=6.8 \mathrm{~Hz}, 1 \mathrm{H})$, $4.45(\mathrm{~d}, J=11.6 \mathrm{~Hz}, 1 \mathrm{H}), 4.7(\mathrm{~d}, J=11.2 \mathrm{~Hz}, 1 \mathrm{H}), 3.82(\mathrm{~s}, 3 \mathrm{H}), 3.74(\mathrm{dd}, J=12.4,4 \mathrm{~Hz}$, $1 \mathrm{H}), 3.30(\mathrm{~s}, 3 \mathrm{H}), 2.25-2.01(\mathrm{~m}, 5 \mathrm{H}), 1.89-1.84(\mathrm{~m}, 1 \mathrm{H}), 1.56(\mathrm{ddd}, J=7.2,7.2,7.2 \mathrm{~Hz}$, 1H), 1.49 (ddd, $J=13.6,3.6,3.6 \mathrm{~Hz}, 1 \mathrm{H}), 1.35(\mathrm{ddd}, J=13.2,13.2,4 \mathrm{~Hz}, 1 \mathrm{H}), 1.01(\mathrm{~d}, J$ $=7.6 \mathrm{~Hz}, 3 \mathrm{H}), 0.92(\mathrm{~s}, 9 \mathrm{H}), 0.89(\mathrm{~s}, 3 \mathrm{H}), 0.86(\mathrm{~s}, 3 \mathrm{H}), 0.12(\mathrm{~s}, 6 \mathrm{H}) ;{ }^{13} \mathrm{C} \mathrm{NMR}\left(\mathrm{CDCl}_{3}\right.$, $100 \mathrm{MHz}): \delta 165.4,164.8,148.9,138.7,133.8,133.2,127.4,102.5,96.5,81.0,65.5$, $55.6,52.3,50.8,44.8,40.9,34.3,32.3,26.4,25.7,25.3,22.7,20.6,18.1,12.1 ;$ IR (neat): $\mathrm{cm}^{-1} 2952,2360,1725,1721,1667,1455,1300,1258,1155,1035 ;$ HRMS (FAB, $\left.m / z\right)$ calcd for $\mathrm{C}_{29} \mathrm{H}_{49} \mathrm{O}_{7} \mathrm{Si}[\mathrm{M}+\mathrm{H}]^{+}$537.3248, found 537.3237.
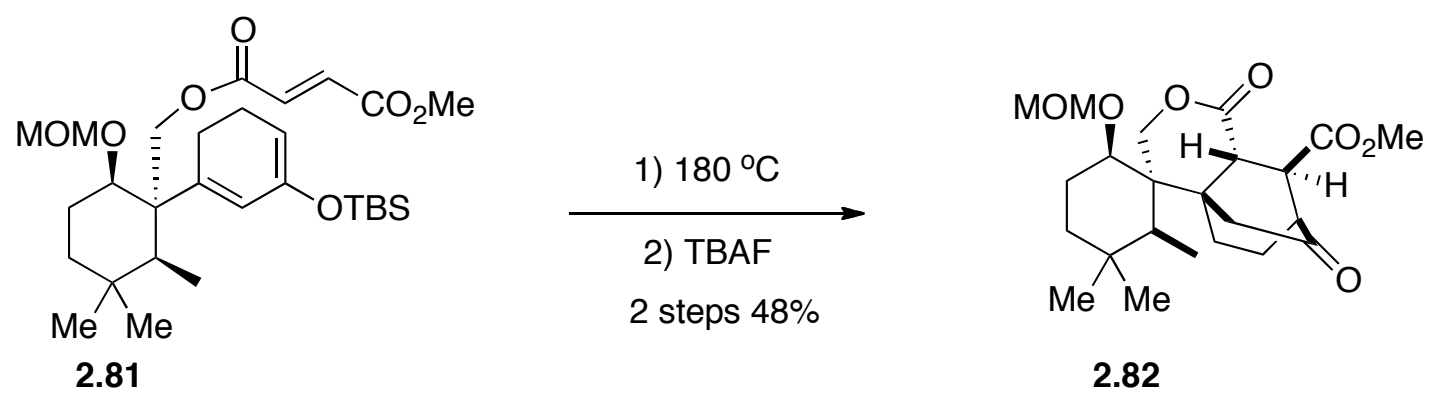
Compound 2.83: compound 2.81 (37 $\mathrm{mg}, 0.07 \mathrm{mmol}$ ) was dissolved in $5 \mathrm{~mL}$ of anhydrous toluene in a high-pressure tube. The reactor was wash with BSA first and dried in oven for 12 hours. The reaction mixture was degassed with argon and then stirred at $180{ }^{\circ} \mathrm{C}$ for 16 hours. After cooling to room temperature and the mixture was transfer to a round bottom flask. The mixture was further cooled to $0{ }^{\circ} \mathrm{C}$ and TBAF in THF $(70 \mu \mathrm{L}$, $0.07 \mathrm{mmol}$ ) was added. 5 mins later the reaction was quenched with saturated ammonium chloride solution. After extracting with ethyl acetate $(3 \times 10 \mathrm{~mL})$, the solution was dried over $\mathrm{MgSO}_{4}$. The drying agent was removed by filtration and the solvent was removed under reduced pressure. The residue was purified using flash chromatography (14 mg, $48 \%){ }^{1} \mathrm{H} \mathrm{NMR}\left(\mathrm{CDCl}_{3}, 400 \mathrm{MHz}\right): \delta 4.76(\mathrm{~d}, J=7.2 \mathrm{~Hz}, 1 \mathrm{H}), 4.68(\mathrm{~d}, J=12.0 \mathrm{~Hz}, 1 \mathrm{H})$, $4.62(\mathrm{~d}, J=6.8 \mathrm{~Hz}, 1 \mathrm{H}), 4.12(\mathrm{br} \mathrm{s}, 1 \mathrm{H}), 3.94(\mathrm{dd}, J=3.2,3.2 \mathrm{~Hz}, 1 \mathrm{H}), 3.88(\mathrm{~d}, J=12.0$ $\mathrm{Hz}, 1 \mathrm{H}), 3.67(\mathrm{~s}, 3 \mathrm{H}), 3.48(\mathrm{dd}, J=18.8,3.2 \mathrm{~Hz}, 1 \mathrm{H}), 3.44(\mathrm{~s}, 3 \mathrm{H}), 3.11(\mathrm{dd}, J=12.4,4.8$ Hz, 1H), 2.93 (d, $J=18.4 \mathrm{~Hz}, 1 \mathrm{H}), 2.74$ (br s, 1H), 2.04-1.72 (m, 5H), 1.60-1.55 (m, 2H), 1.40-1.29 (m, 2H), $1.19(\mathrm{~d}, J=7.6 \mathrm{~Hz}, 3 \mathrm{H}), 0.97(\mathrm{~s}, 3 \mathrm{H}), 0,88(\mathrm{~s}, 3 \mathrm{H}) ;{ }^{13} \mathrm{C} \mathrm{NMR}\left(\mathrm{CDCl}_{3}\right.$, $100 \mathrm{MHz}): \delta 212.5,175.7,173.5,96.0,84.1,78.0,56.8,56.6,52.5,48.7,47.0,44.7$, 43.9, 43.3, 41.6, 40.7, 34.1, 32.7, 27.2, 25.9, 23.1, 21.9, 13.9; IR (neat): $\mathrm{cm}^{-1} 2952,2850$, 1740, 1665, 1461, 1311, 1184, 1023; HRMS (FAB, $m / z)$ calcd for $\mathrm{C}_{23} \mathrm{H}_{35} \mathrm{O}_{7}[\mathrm{M}+\mathrm{H}]^{+}$ 423.2383, found 423.2396 .

\subsection{Reference}


(1) (a) Danishefsky, S. J. Tetrahedron 1997, 53, 8689-8730. (b) Wilson, R. M.;

Danishefsky, S. J. Acc. Chem. Res. 2006, 39, 539-549.

(2) Li, S.; Wang, J.; Niu, X.; Shen, Y.; Zhang, H.; Sun, H.; Li, M.; Tian, Q.; Lu, Y.; Cao, P.; Zheng, Q. Org. Lett. 2004, 6, 4327-4330.

(3) Wilson, R. M.; Danishefsky, S, J. J. Org. Chem. 2007, 72, 4293-4305.

(4) Walborsky, H. M.; Loncrini, D. F. J. Am. Chem. Soc. 1954, 76, 5396-5397.

(5) Nagata, W.; Narisada, M; Sugasuwa, T.; Wakabayashi, T. Chemical \& Pharmaceutical Bulletin 1968, 16, 885-887.

(6) (a) Tiefenbacher, K.; Mulzer, J. Angew. Chem. Int. Ed. 2008, 47, 6199-6200. (b)

Tiefenbacher, K.; Mulzer, J. J. Org. Chem. 2009, 74, 2937-2941.

(7) a) Nicolaou, K. C.; Snyder, S. A.; Montagnon, T.; Vassilikogiannakis, G. Angew.

Chem. Int. Ed. 2002, 41, 1668-1698. b) Liao, C.; Peddinti, R. K. Acc. Chem. Res. 2002, 35, 856-866. c). Ihara, M. Chem. Pharm. Bull. 2006, 54, 765-774.

(8) Ito, Y.; Hirao, T.; Saegusa, T. J. Org. Chem. 1978, 43, 1011-1013.

(9) A. S. Cieplak, J. Am. Chem. Soc. 1981, 105, 4540-4552.

(10) Johnson, C. R.; Adams, J. P.; Braun, M. P.; Senanayake, C. B. W.; Wovkulich, P. M.; Uskokovic, M. R. Tetrahedron Lett. 1992, 33, 917-918.

(11) Stork, G.; Danheiser, R. J. Org. Chem. 1973, 38, 1775.

(12) a) Still, W. C. J. Am. Chem. Soc. 1978, 100, 1481-1487. b) Danheiser, R.; Romines, K.; Koyama, H.; Gee, S.; Johnson, C. R.; Medich, J. R. Organic Synthesis, 1993, 71, 133139.

(13) Rabideau, P. W. Tetrahedron 1989, 45, 1579-1603.

(14) Roche, S. P.; Porco, J. A. Angew. Chem. Int. Ed. 2011, 50, 4068-4093. 
(15) Fox, J. M.; Huang, X.; Chieffi, A.; Buchwald, S. J. Am. Chem. Soc. 2000, 122, $1360-1370$.

(16) Ihara, M.; Ishida, Y.; Abe, M.; Toyota, M.; Fukumoto, K.; Kametani, T. J. Chem.

Soc. Perkin Trans. 1. 1988, 1155-1163.

(17) Lee, R. A. Tetrahedron Lett. 1973, 35, 3333-3336.

(18) (a) Strekowski, L.; Kong, S.; Battiste, M. A. J. Org. Chem. 1988, 53, 901-904; (b) Guan, Z. H.; Zuo, W.; Zhao, L. B.; Ren, Z. H.; Liang, Y. M. Synthesis. 2007, 14651470; (c) Danishefsky, S. J.; Harayama, T.; Singh, R. K. J. Am. Chem. Soc. 1979, 101, 7008-7012; (d) Danishefsky, S. J.; Morris, J.; Mullen, G.; Gammill, R. J. Am. Chem. Soc. 1982, 104, 7591-7599. 


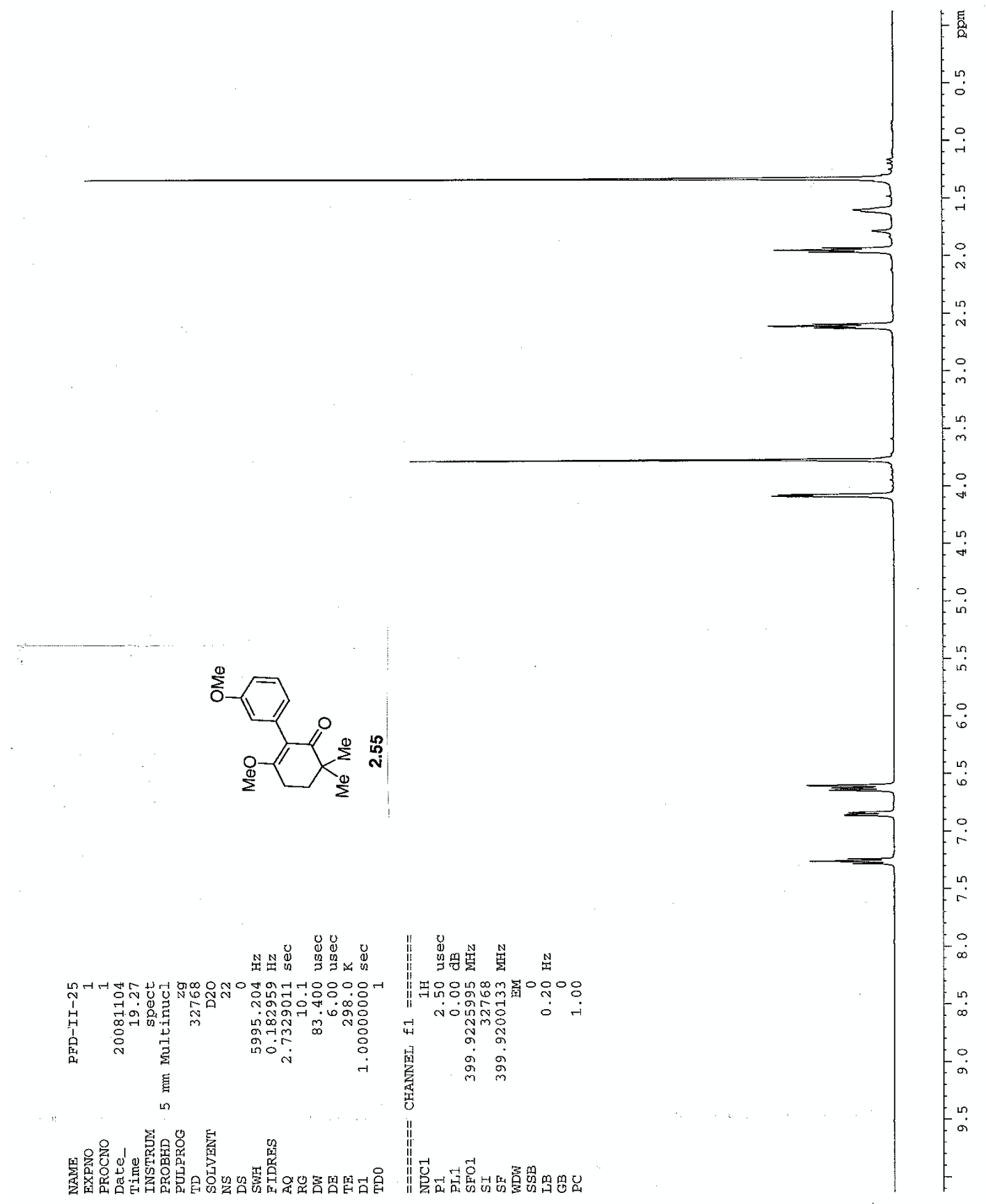



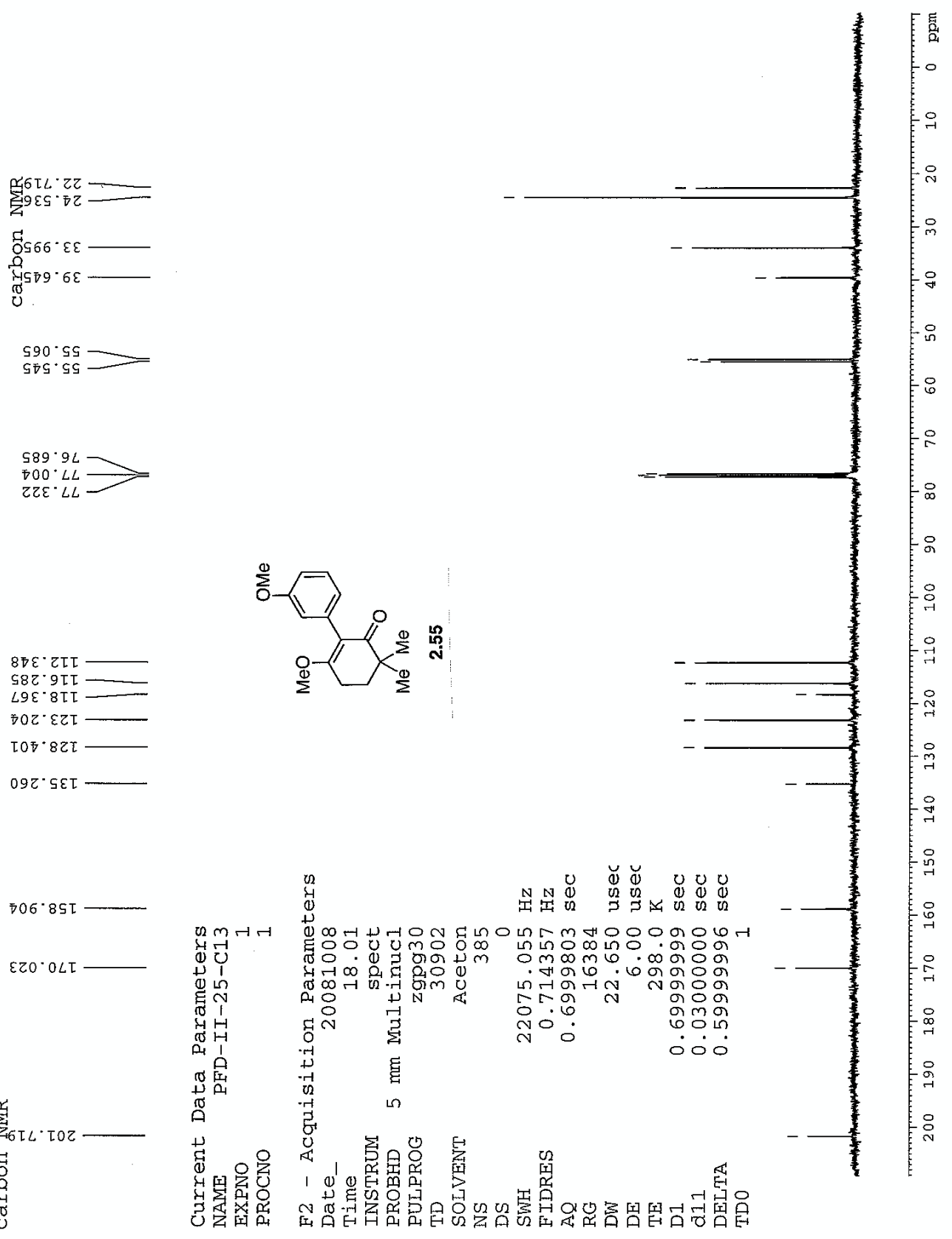


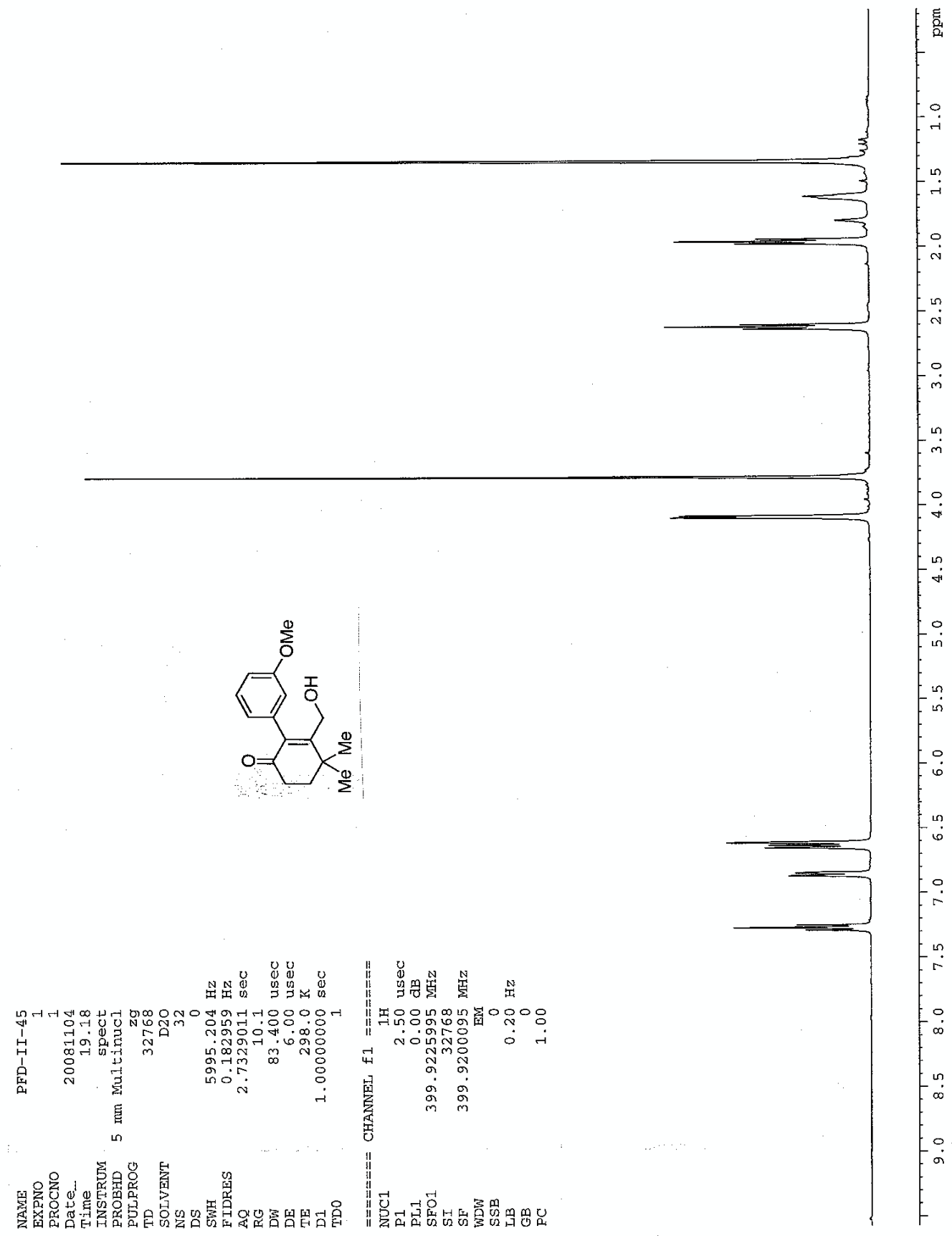




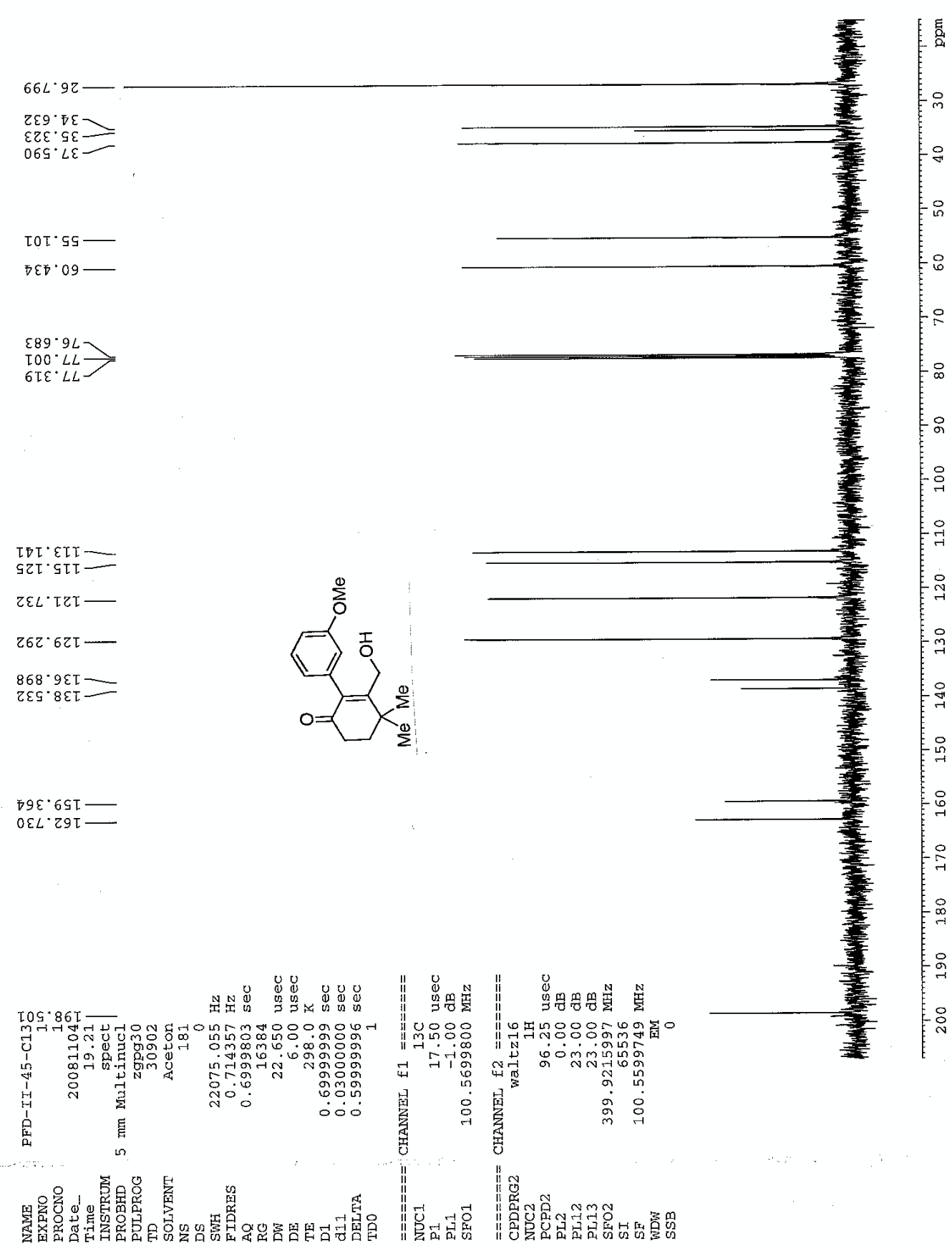




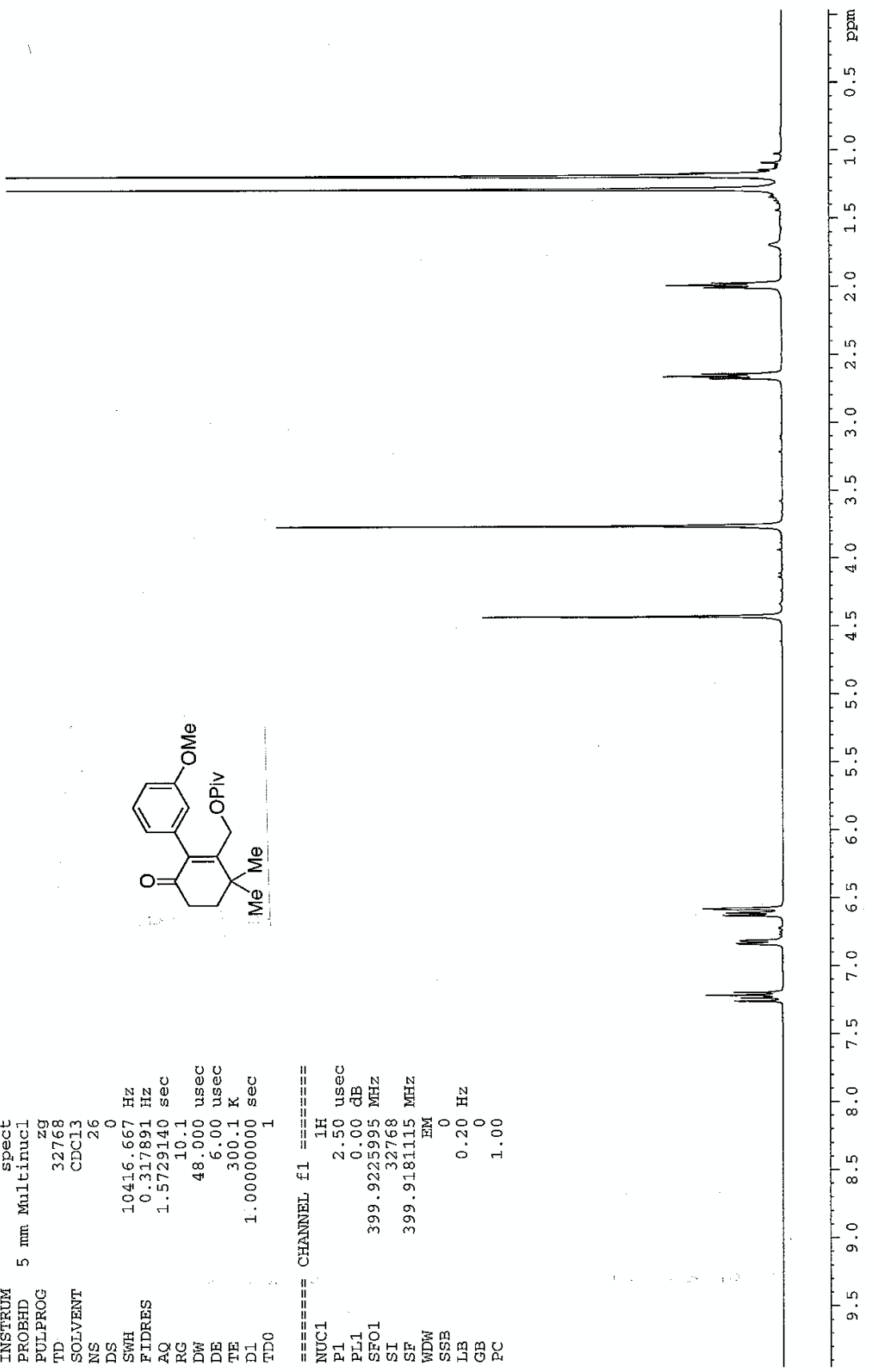




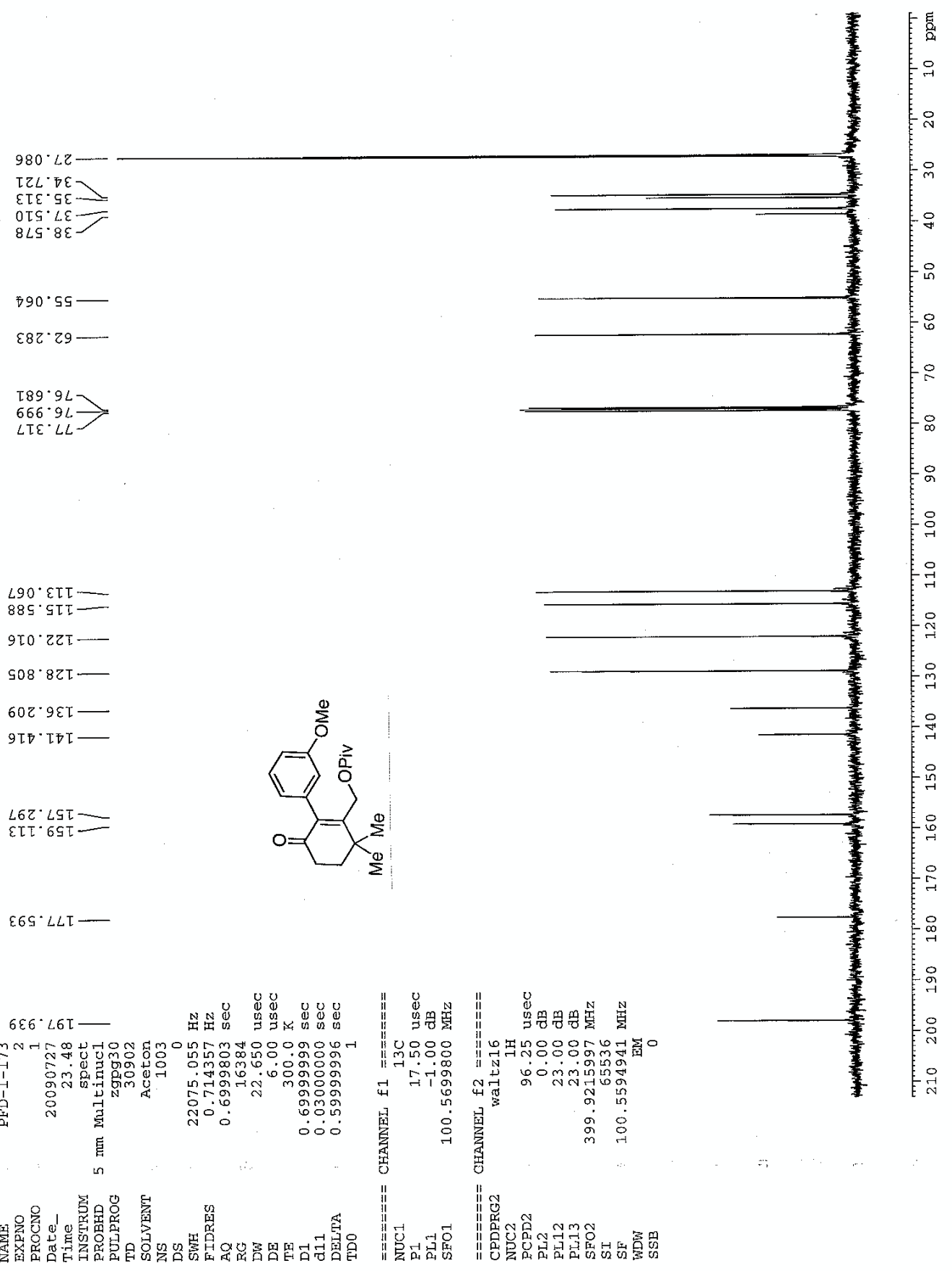




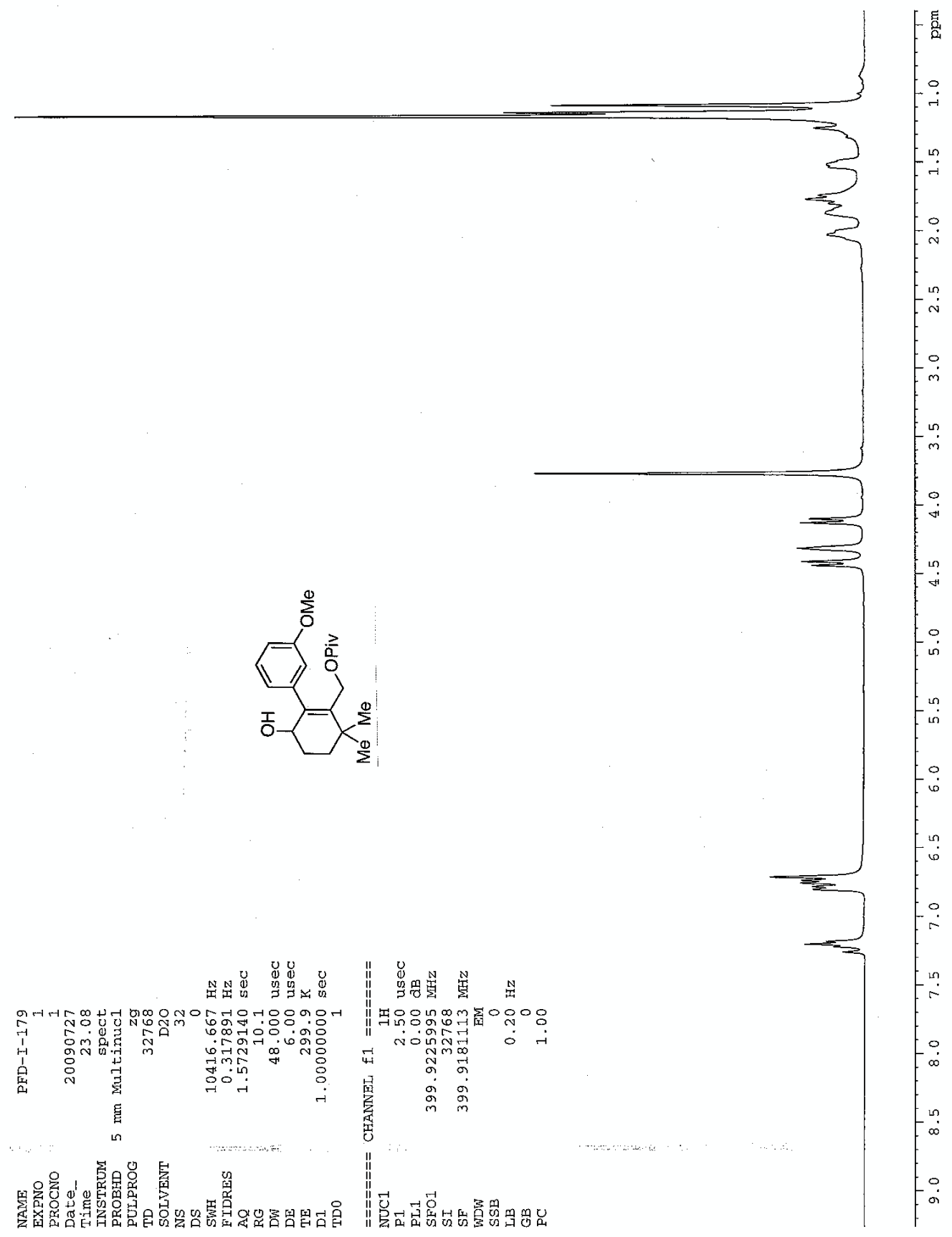




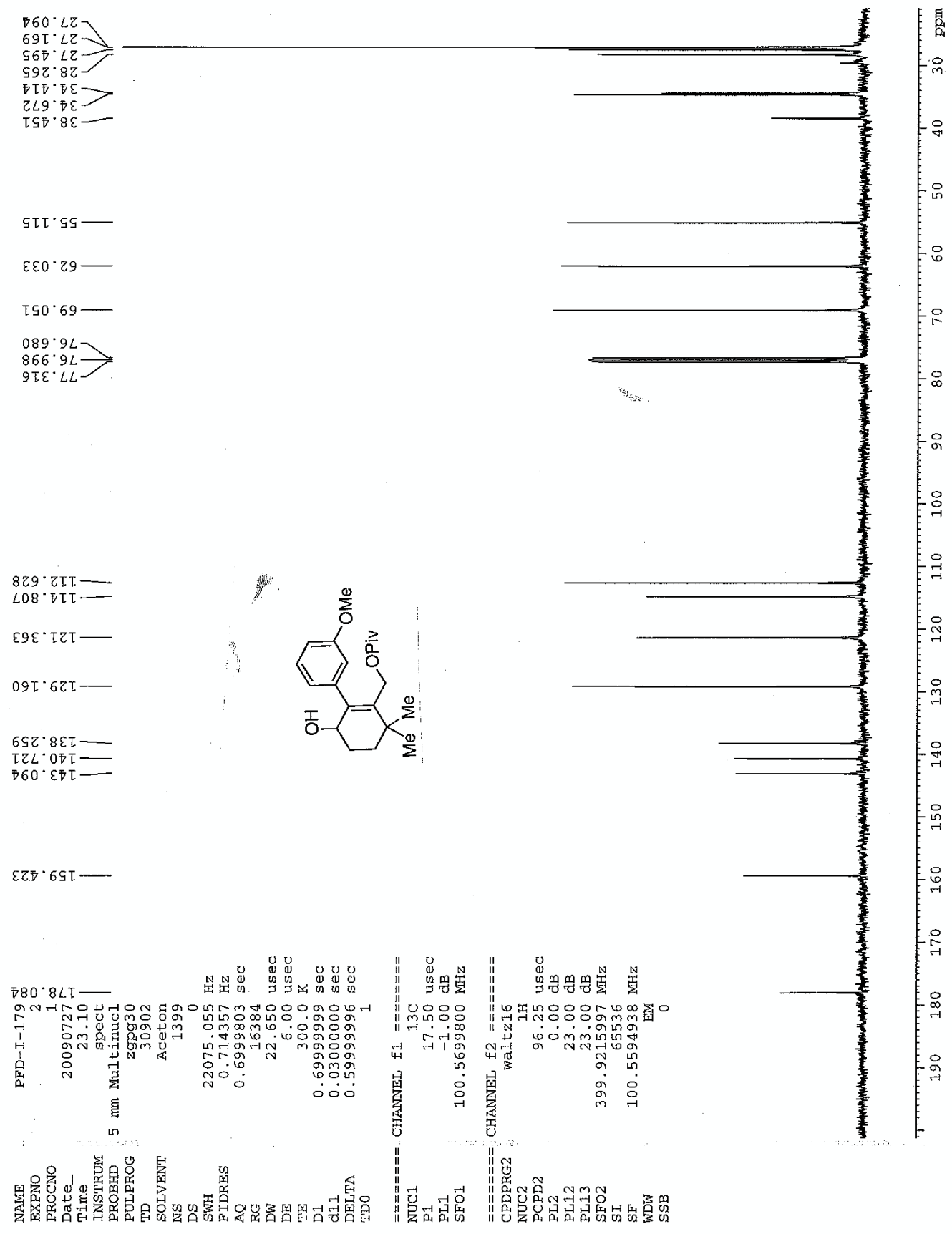




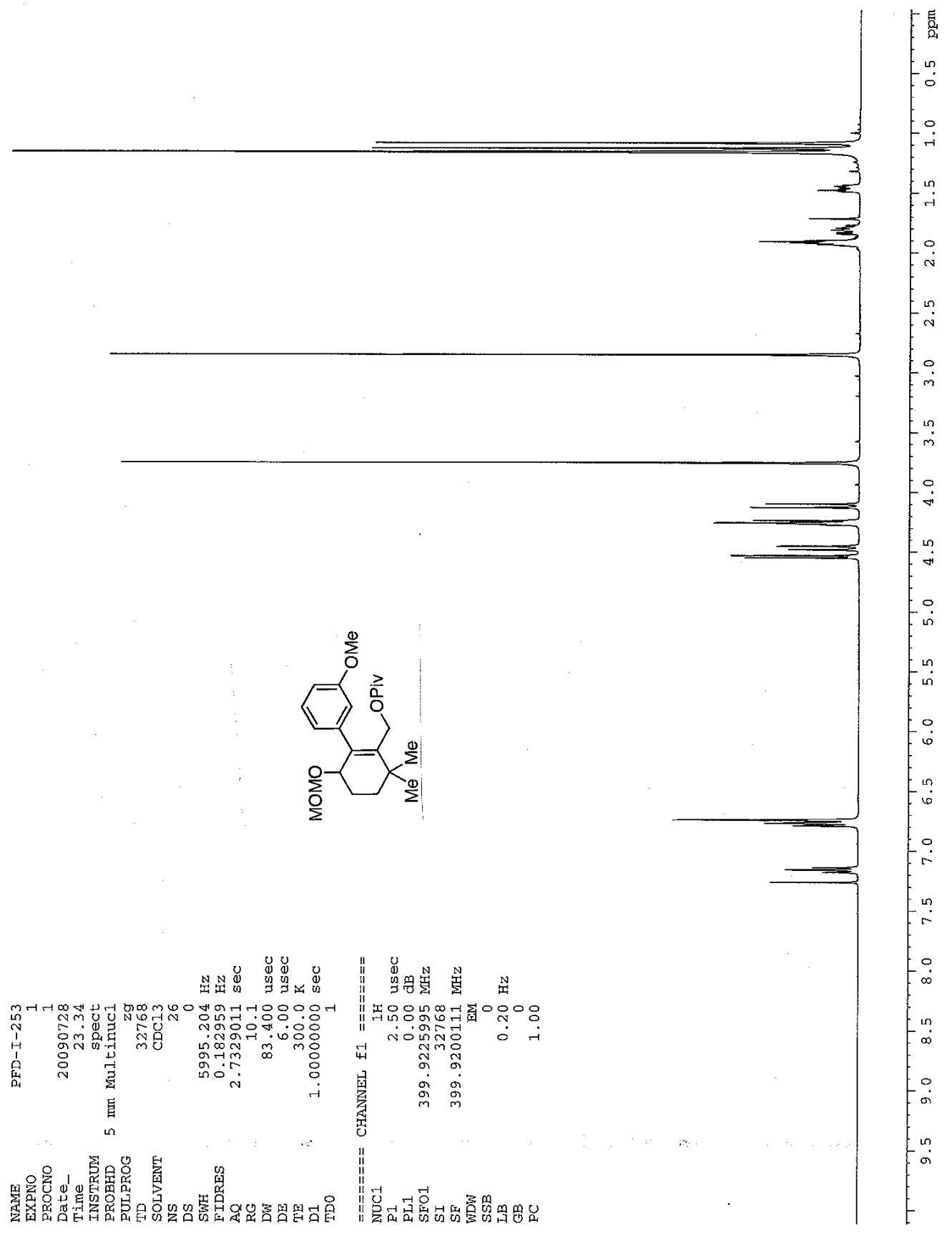




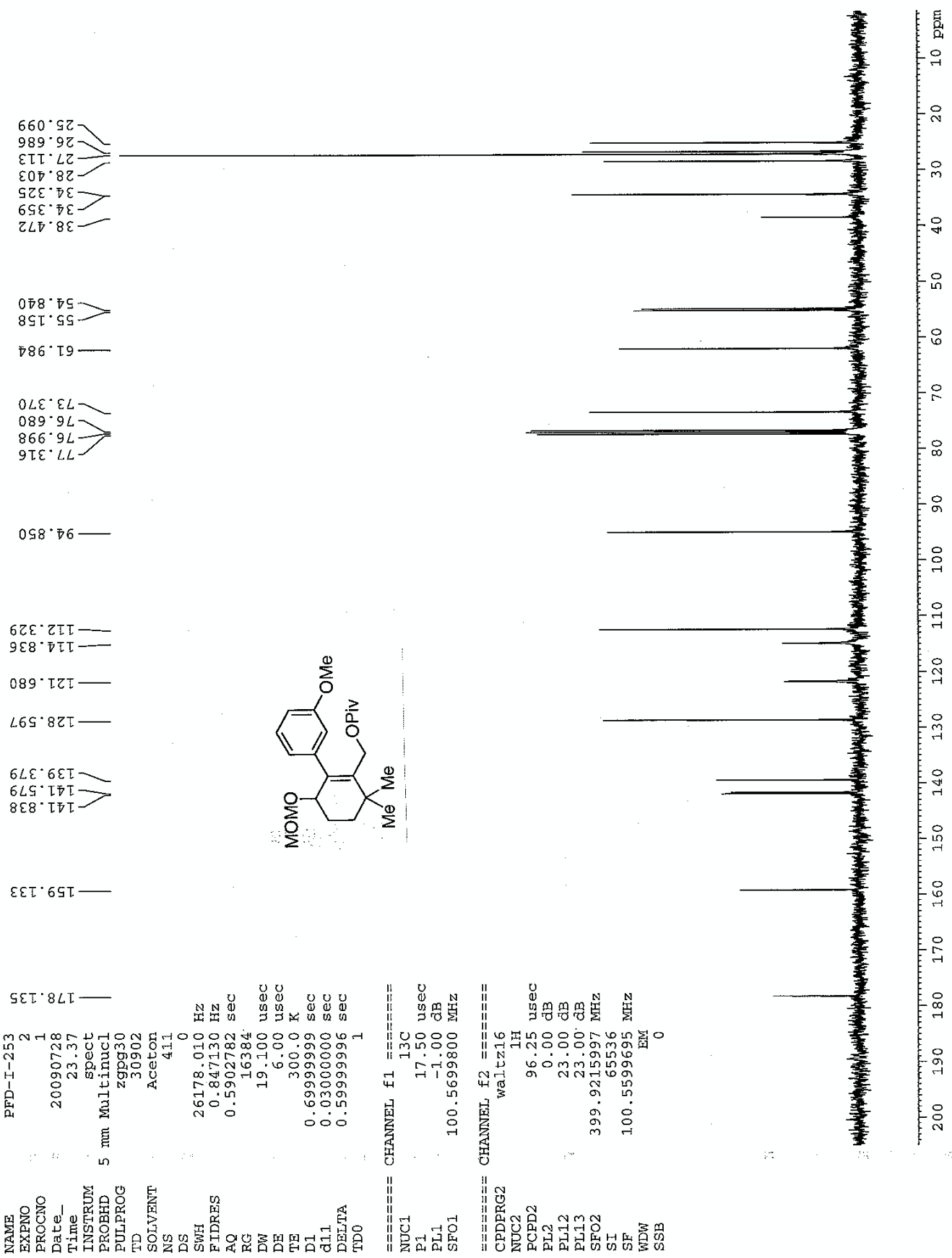




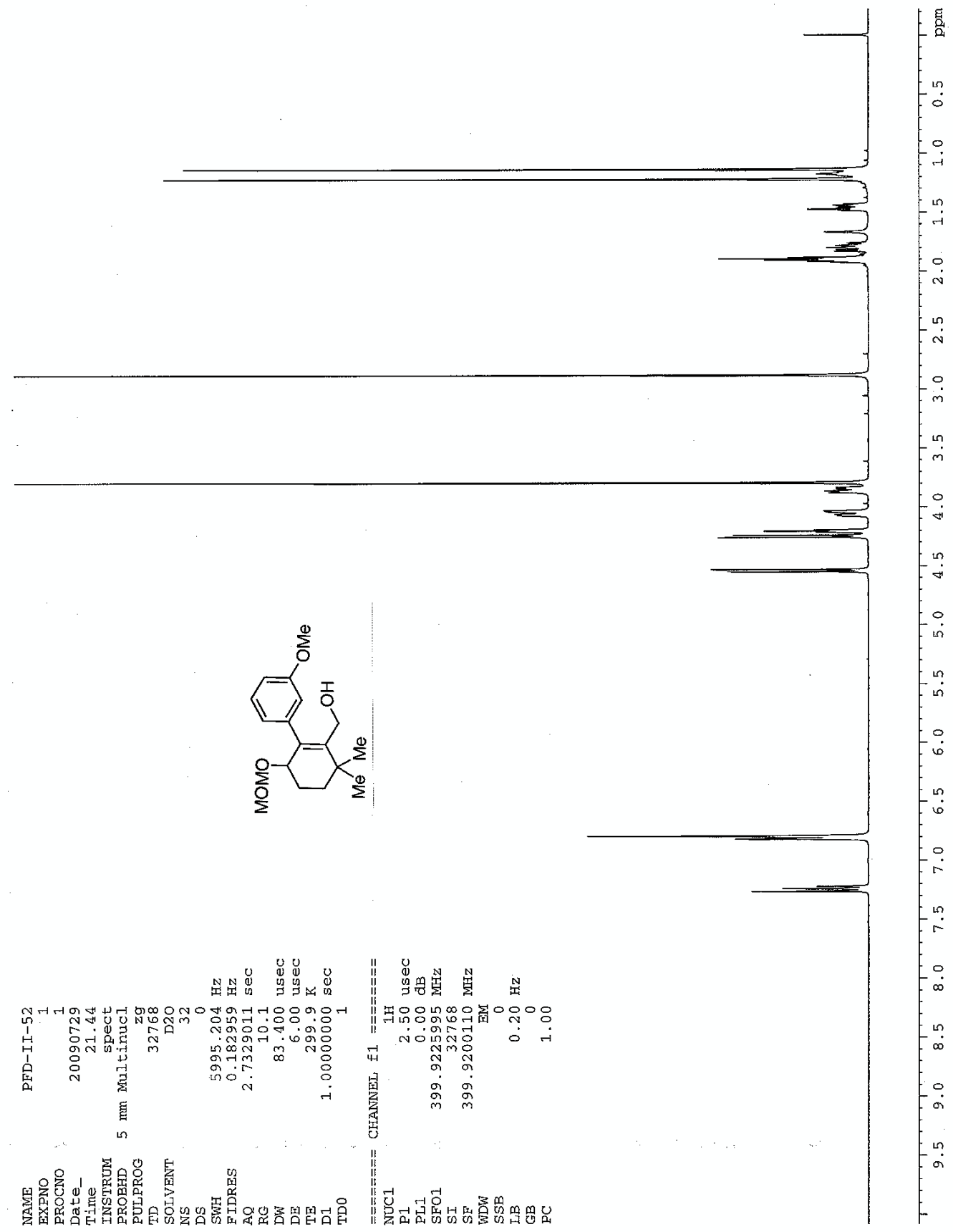


$\tau L \tau \cdot 9 Z=$

518.92

$95 \varepsilon \cdot \nabla \varepsilon>$
$897-7 \varepsilon>$

$272 \cdot 55>$

$\varepsilon \nabla L \cdot 6 S$

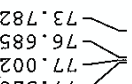

$906^{\circ} 76$

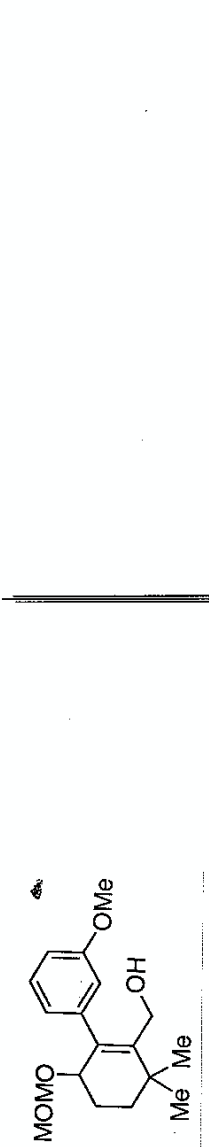

$89 \tau \cdot 8 \varepsilon \tau-$

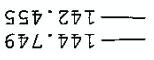

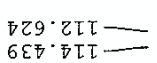

$\varepsilon 6 \tau^{\circ} \tau \tau \tau-$

SOZ'6ZT-

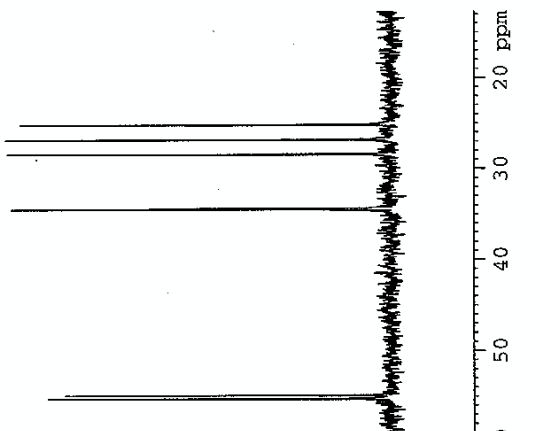

LSF.6ST-

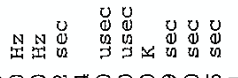

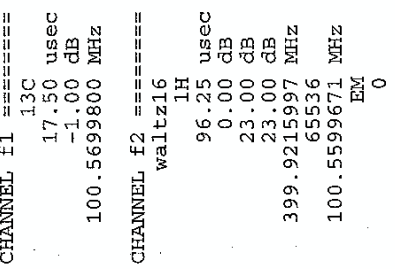

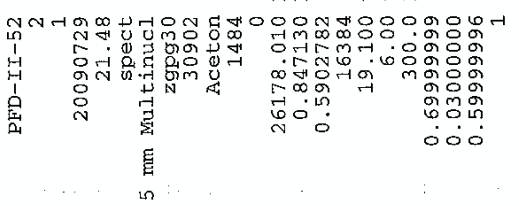

$\|$

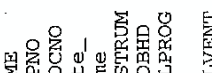

年舅员

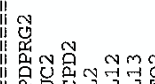

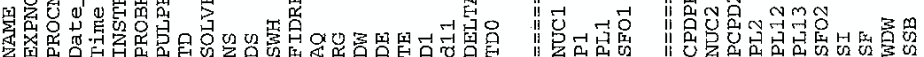

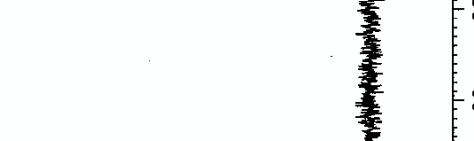




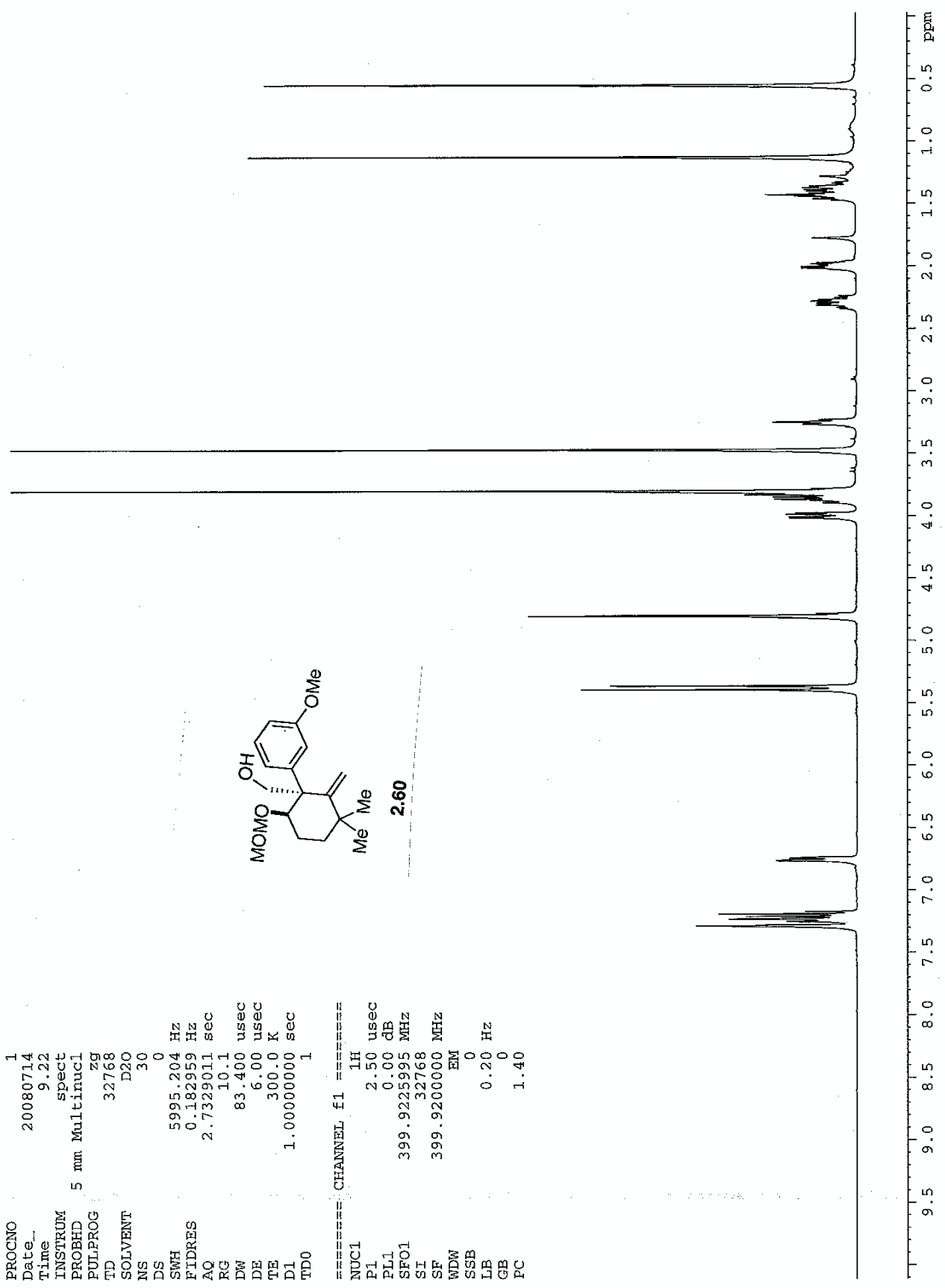



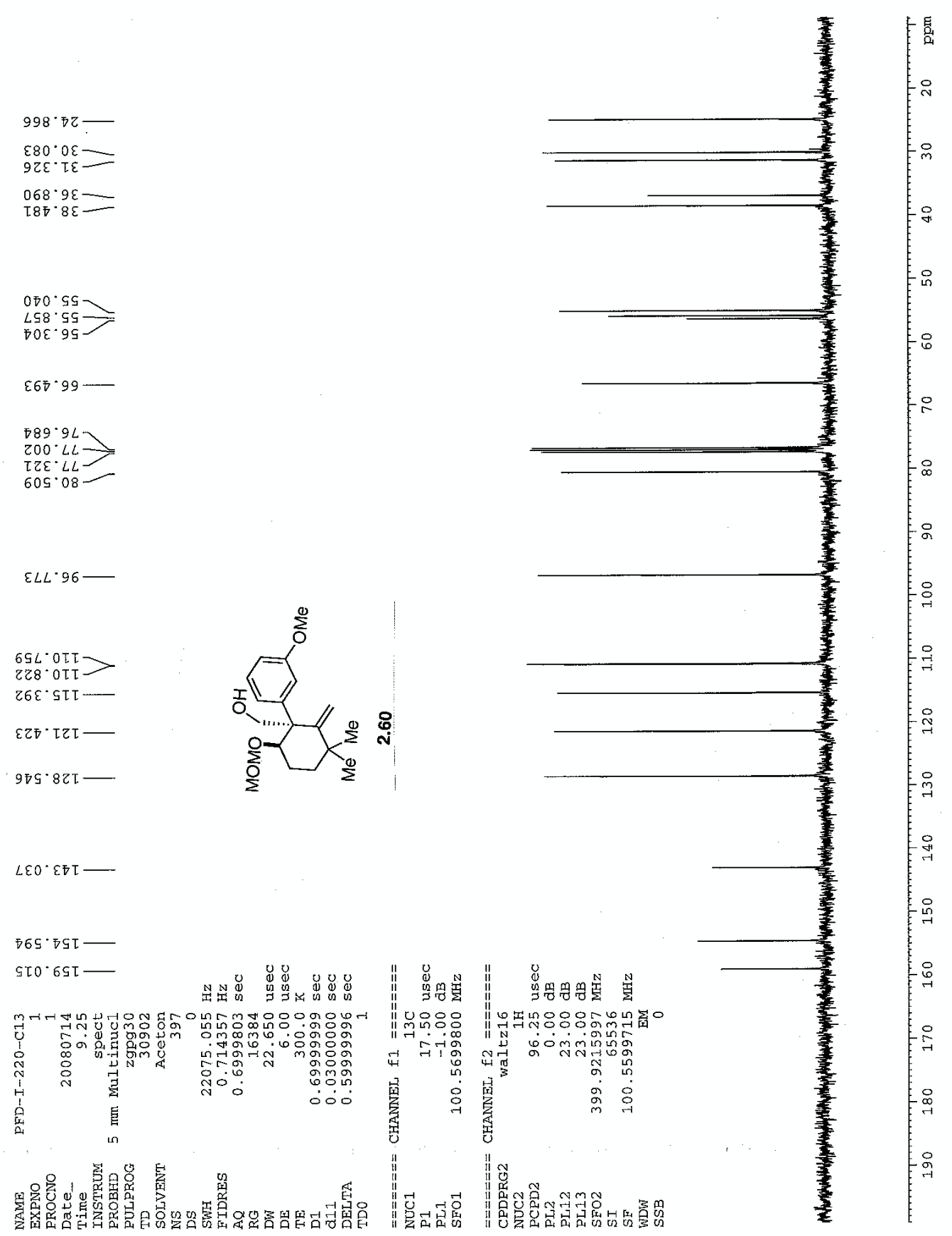

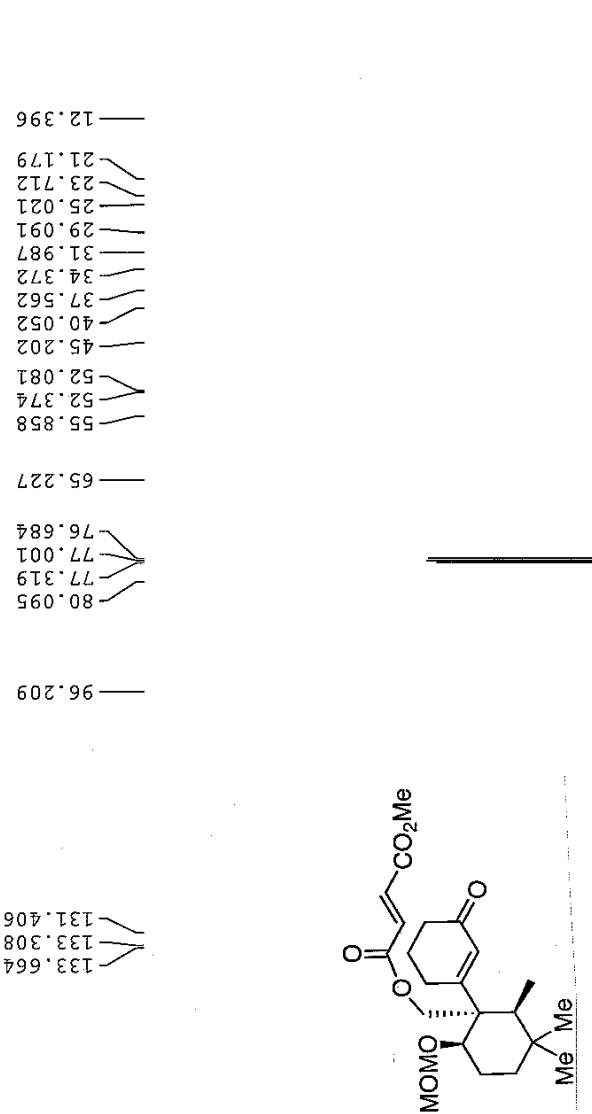

$599^{\circ} \varepsilon 9 \tau$
$9 \varepsilon \mathcal{G}^{\circ}+\mathrm{\nabla 9 \tau}$
$\angle 9 \tau \cdot 59 \tau$

$90 \bar{D}^{\circ} \cdot \tau \varepsilon \tau \longrightarrow$ $80 \varepsilon \cdot \varepsilon \varepsilon \tau=$
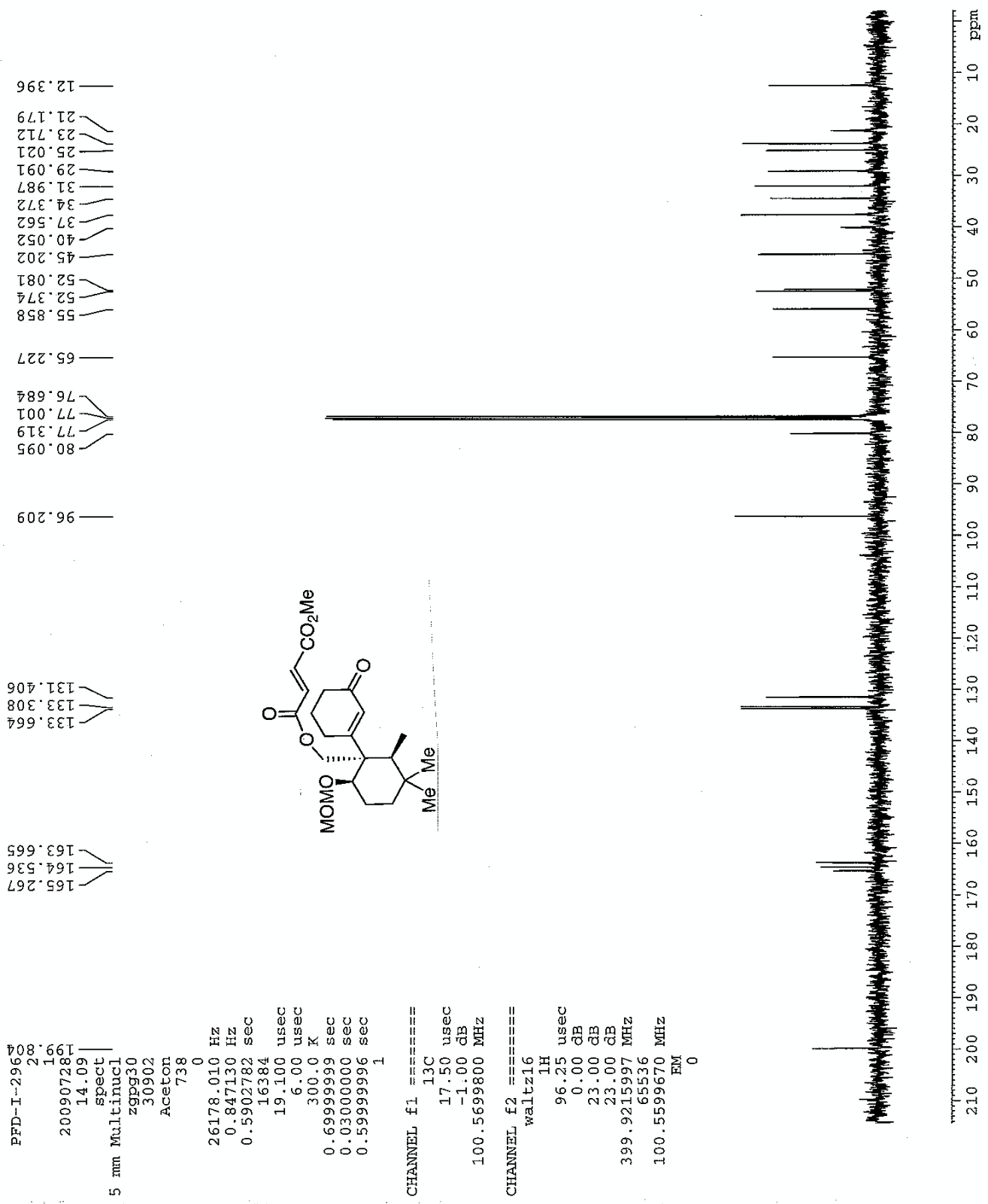

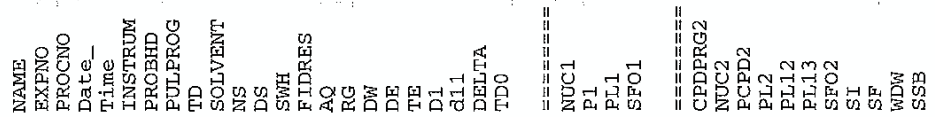




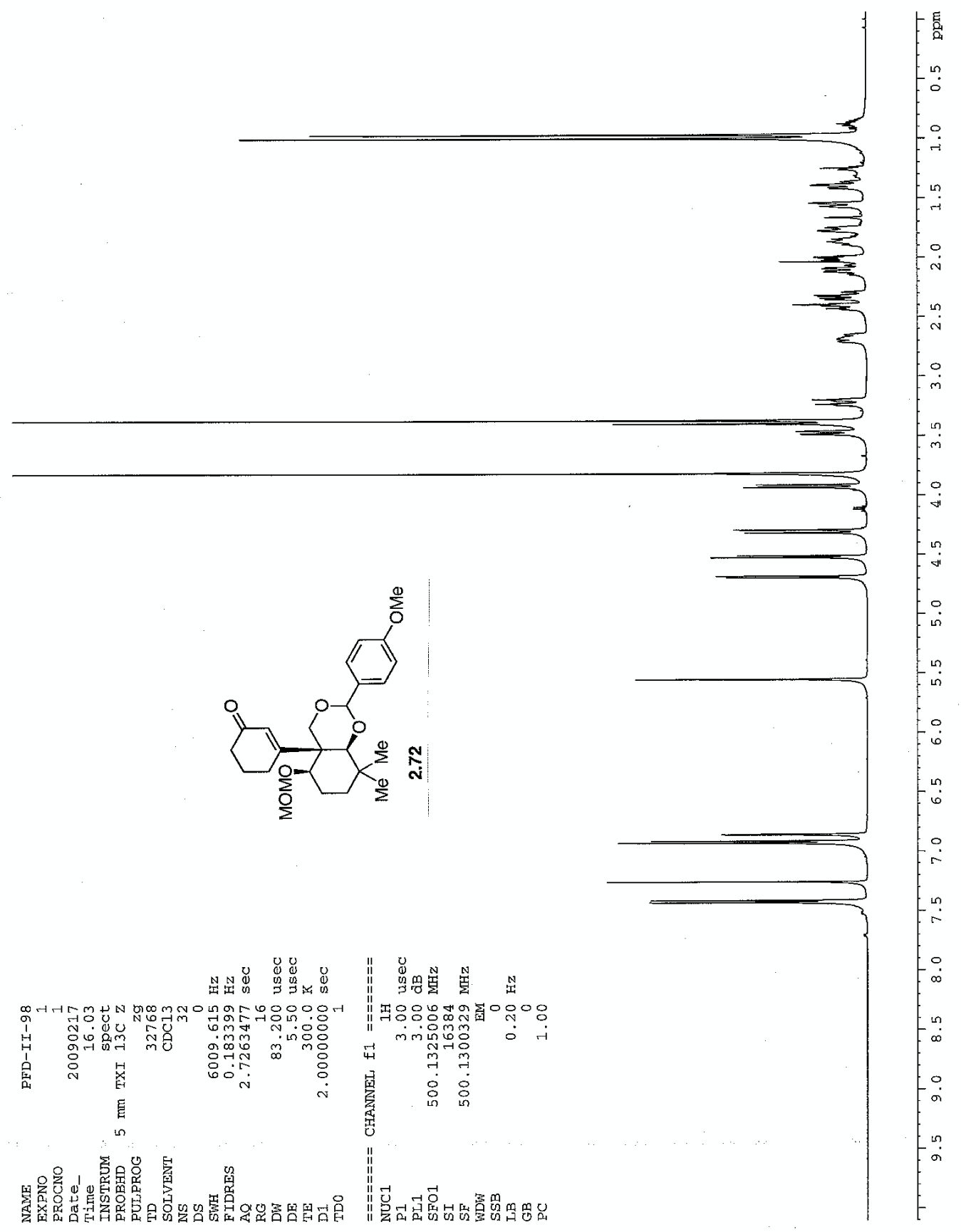




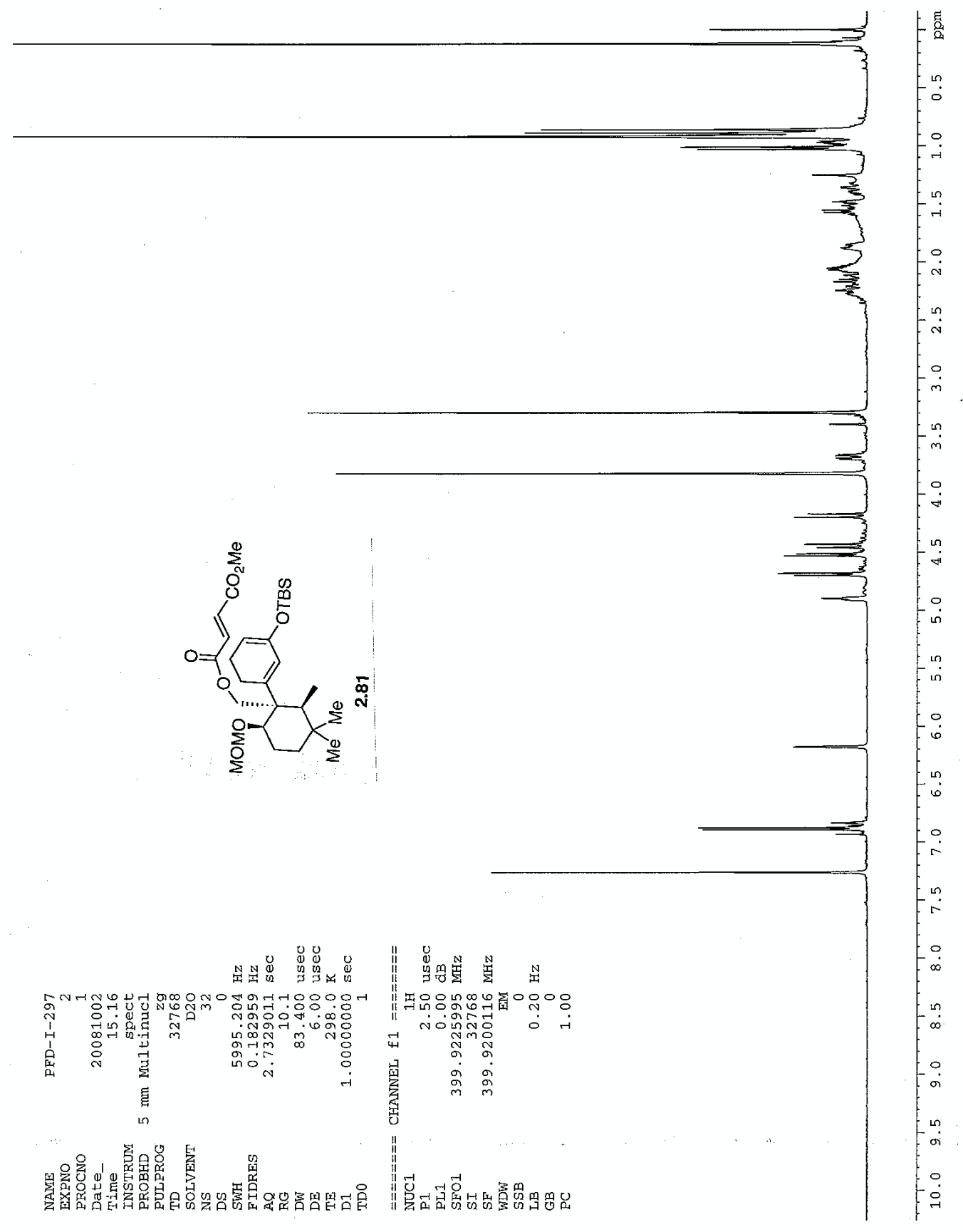




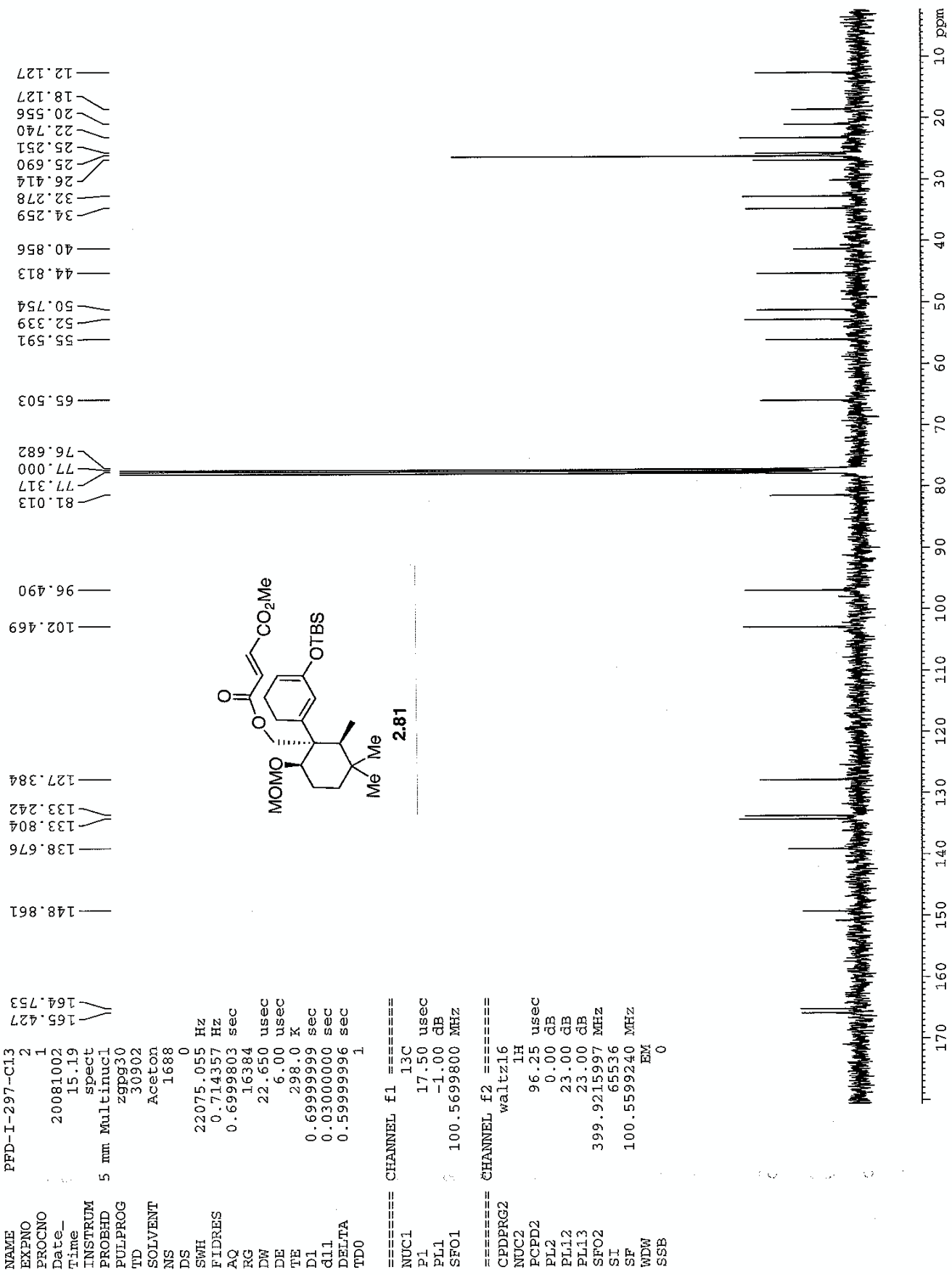




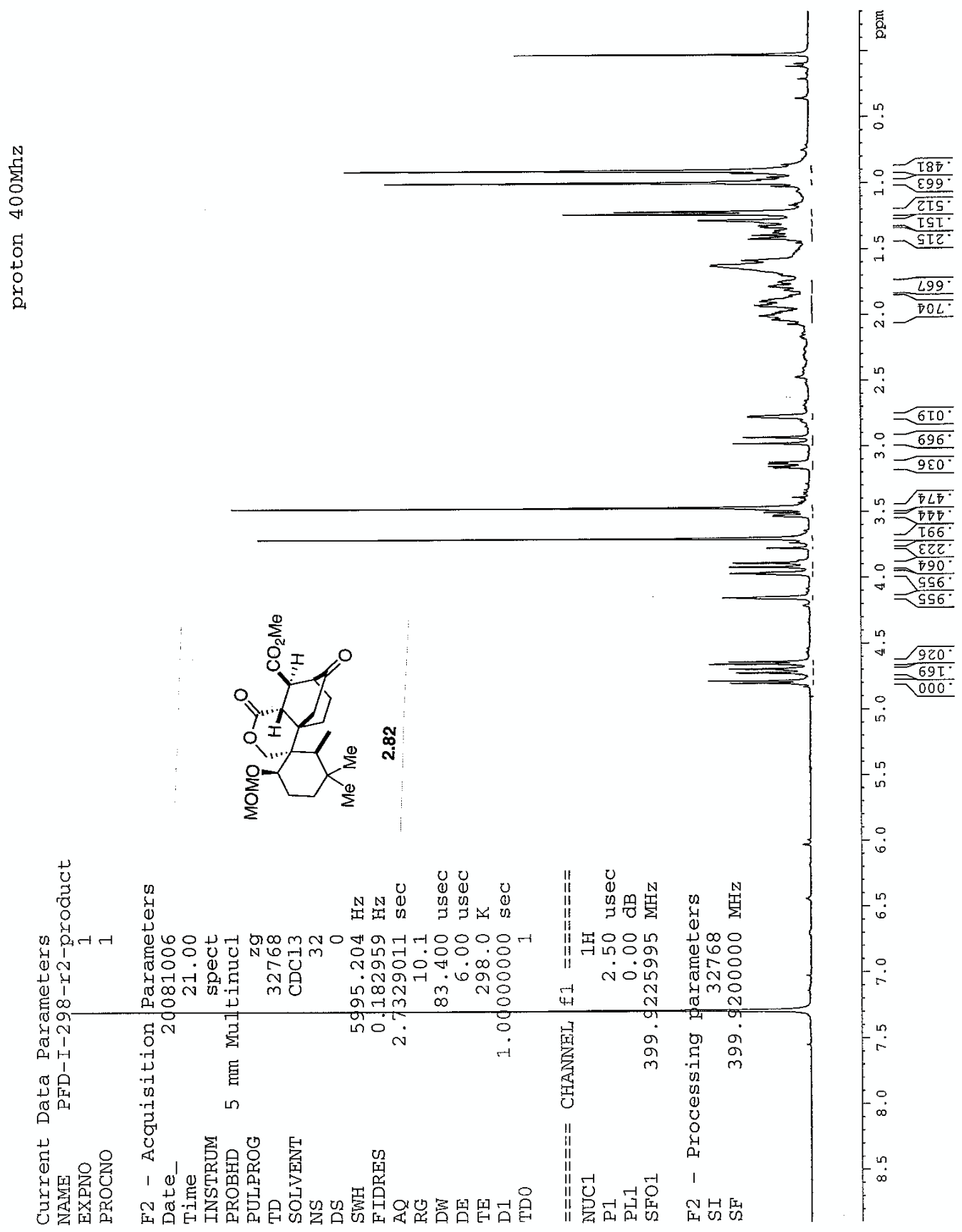




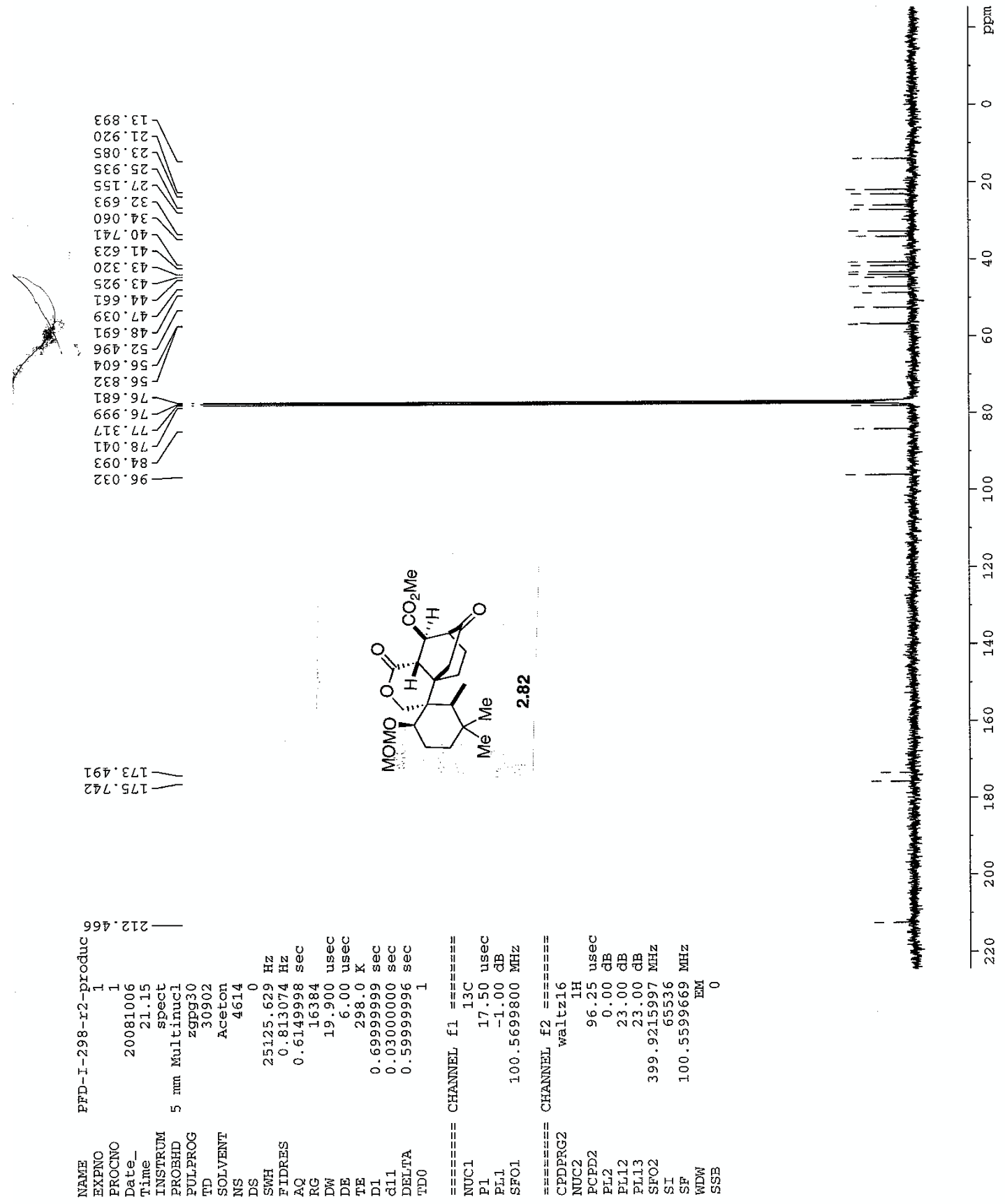




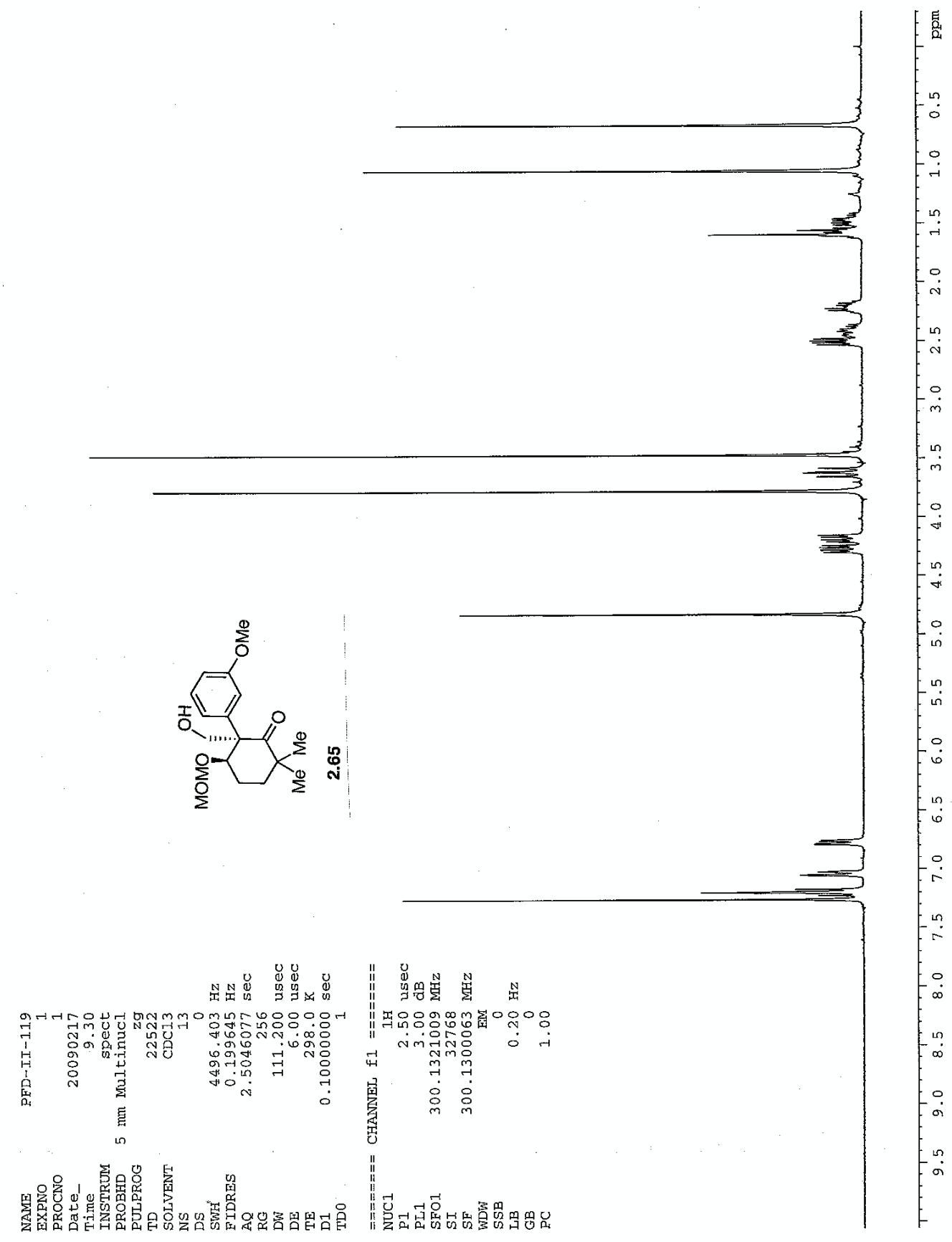




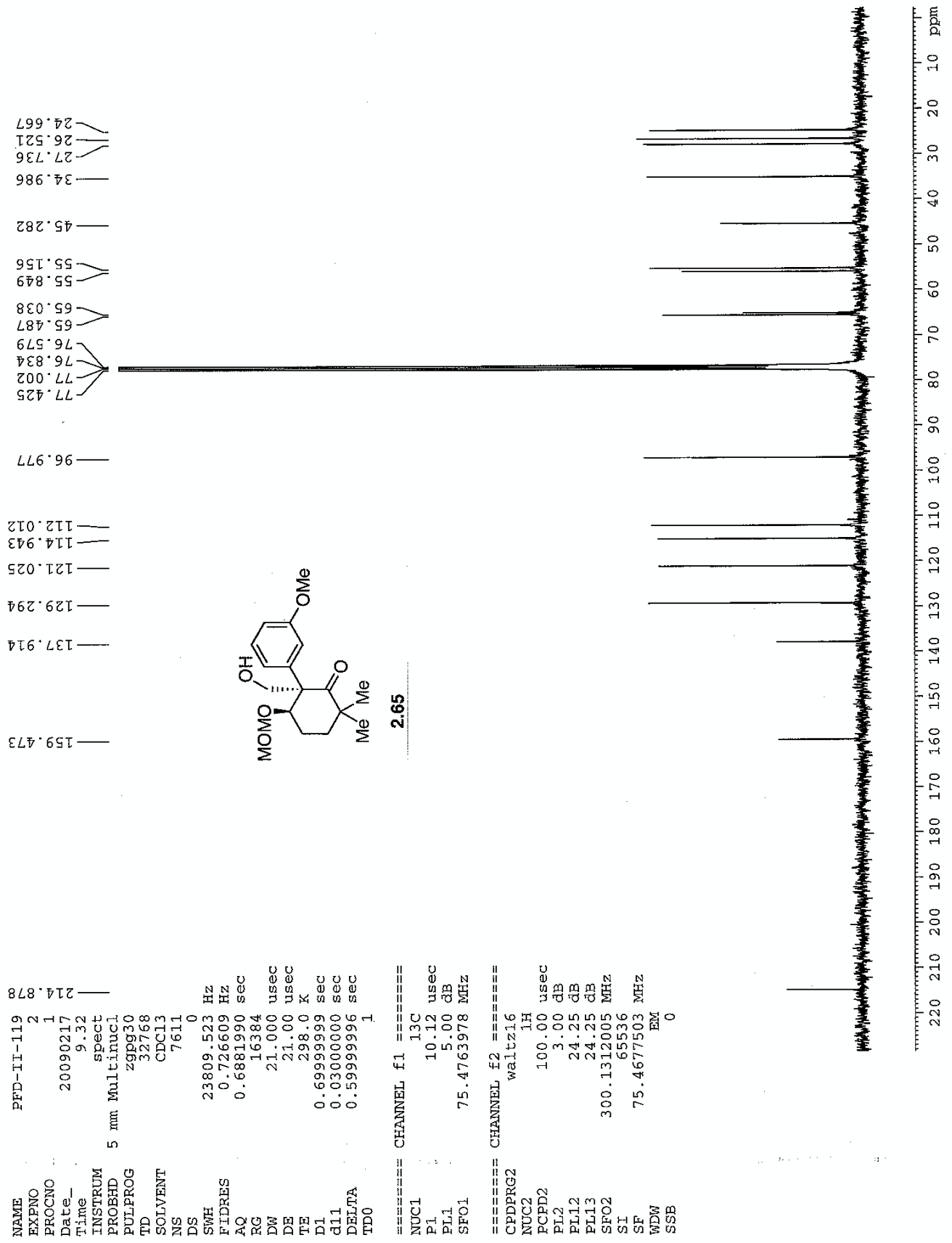




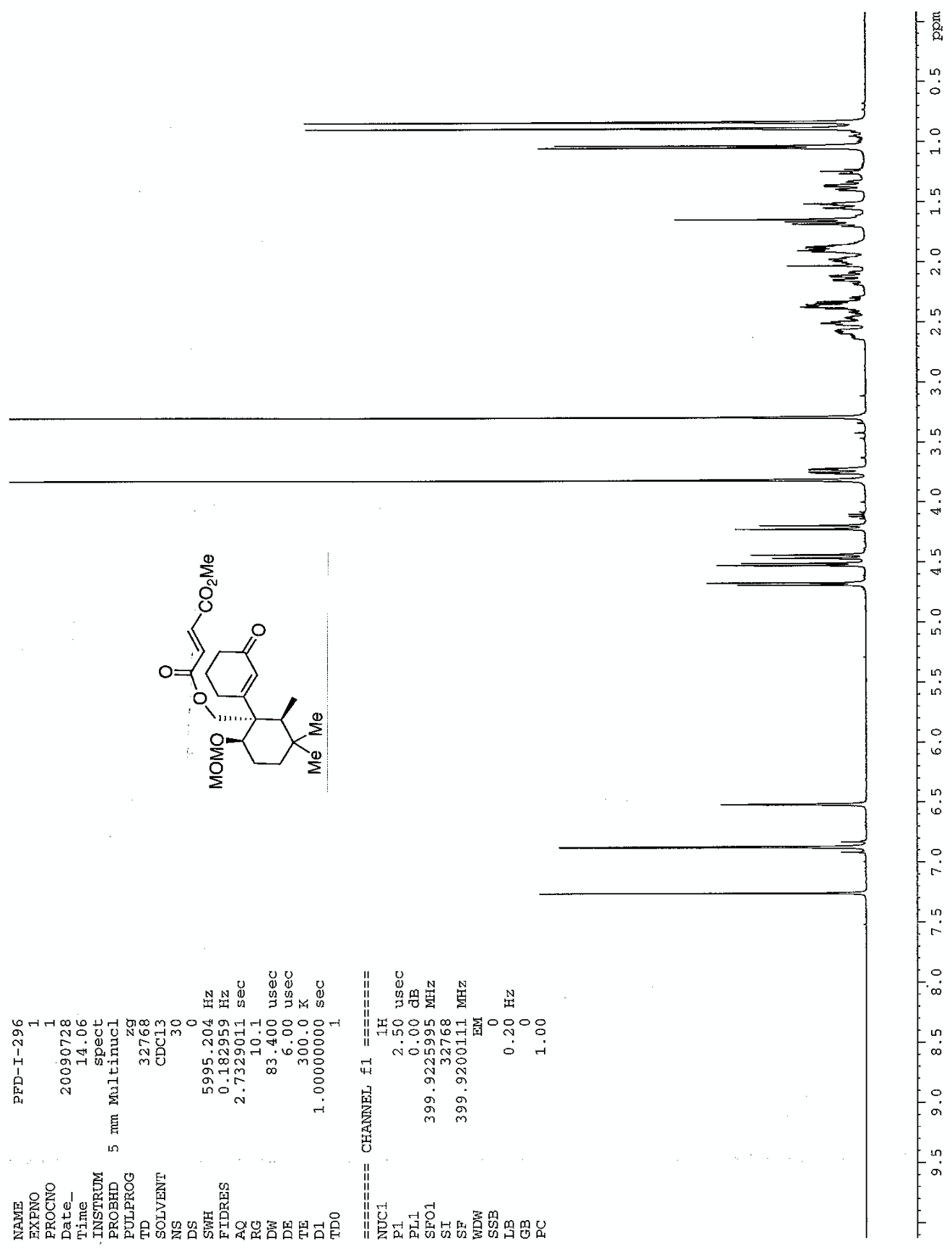




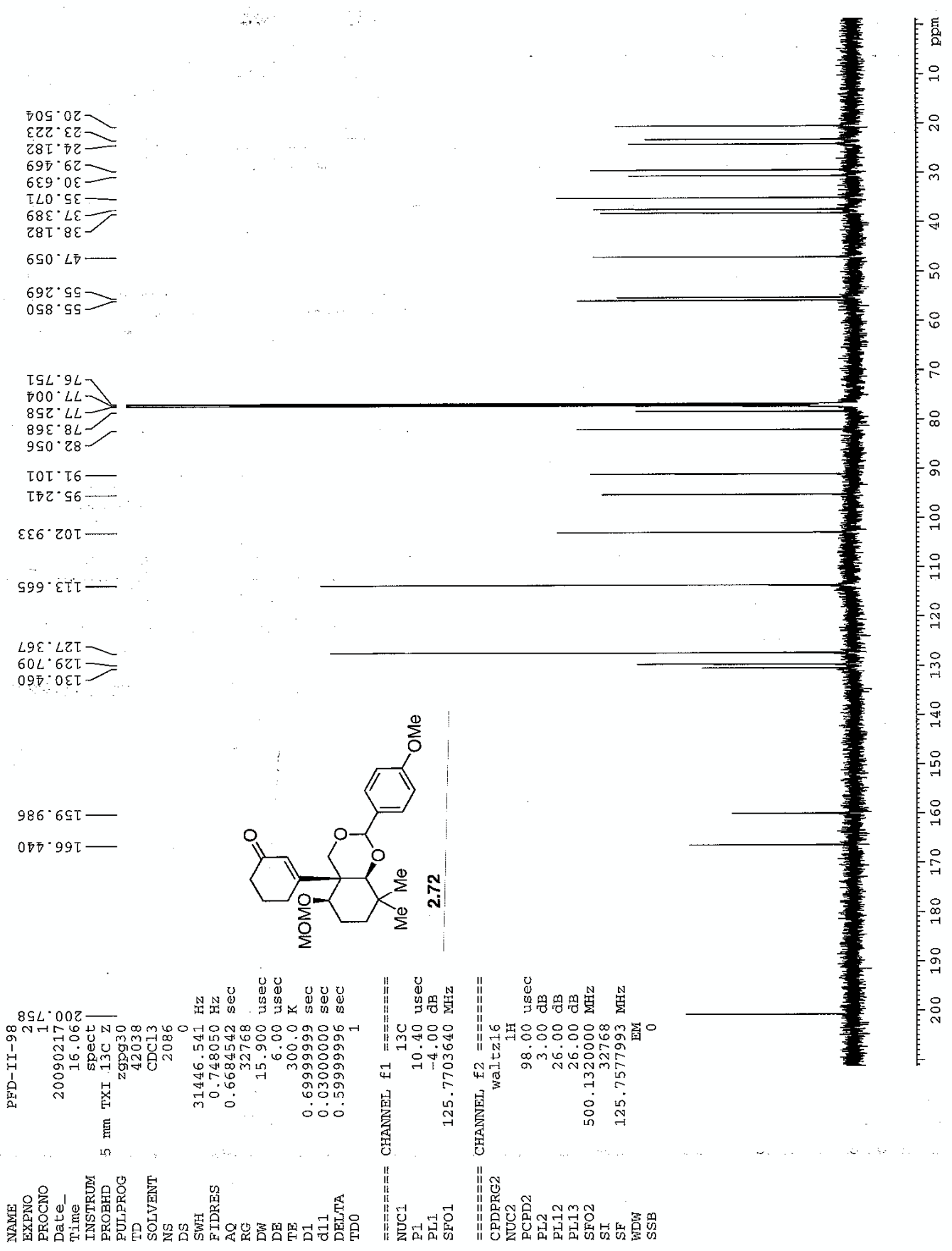




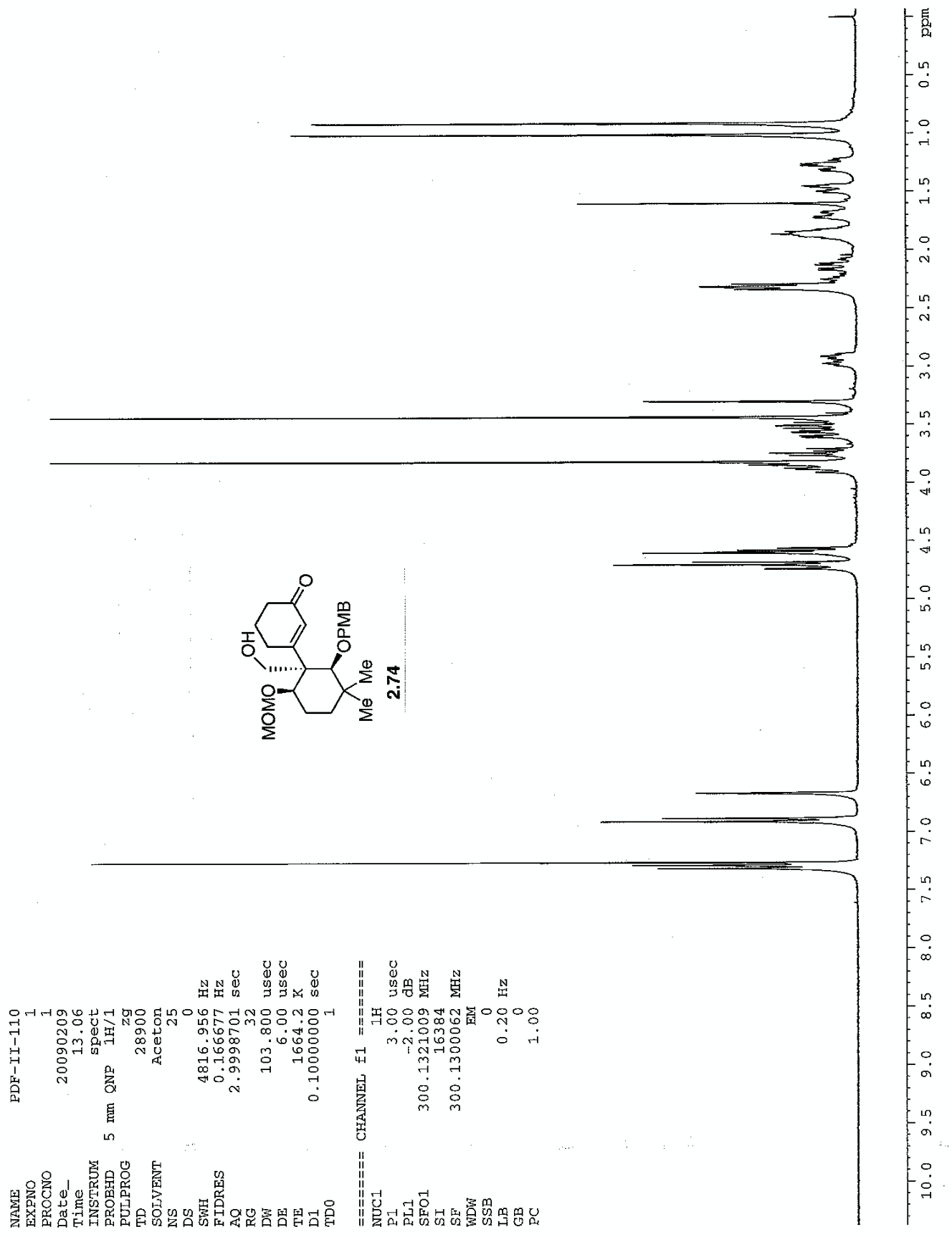




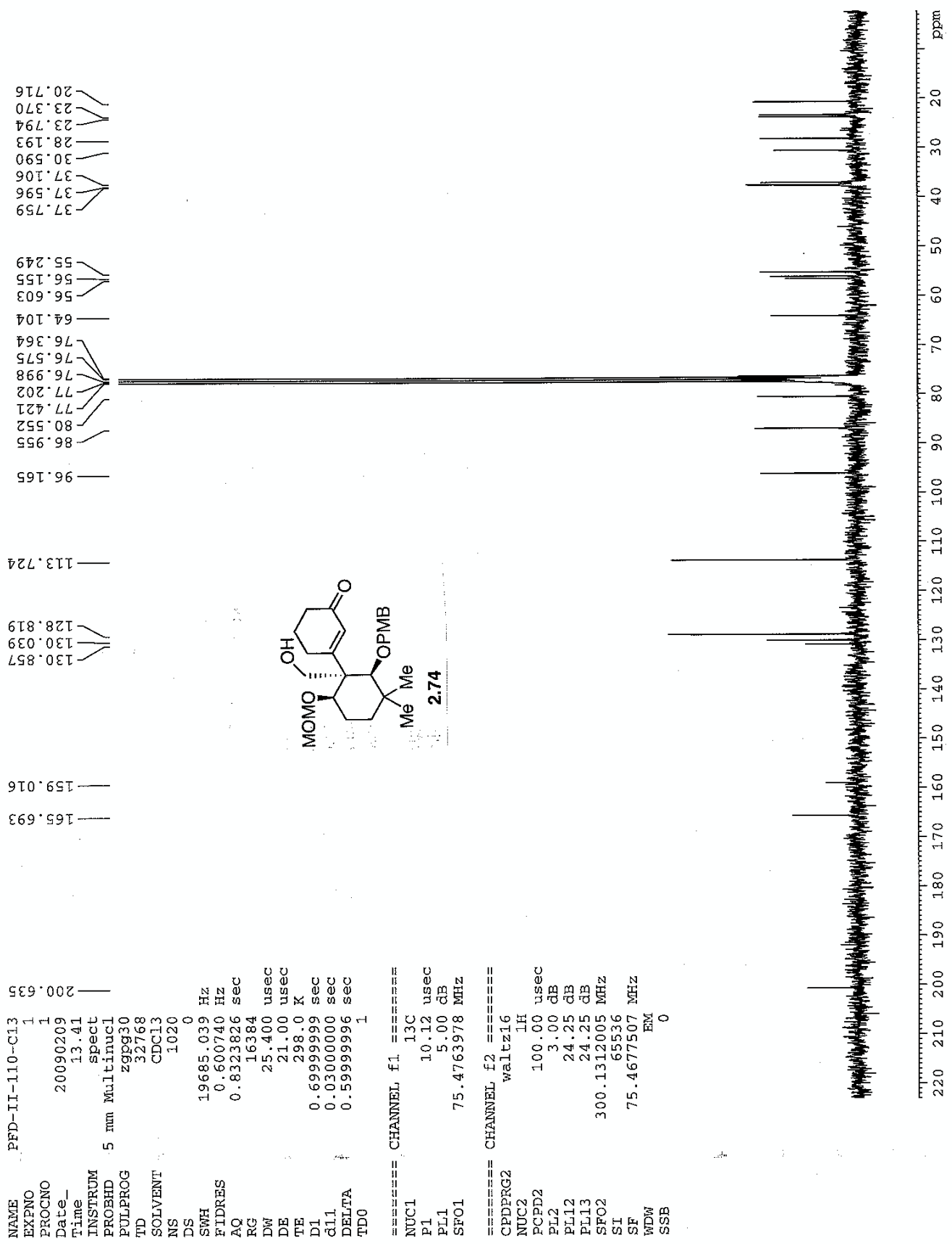




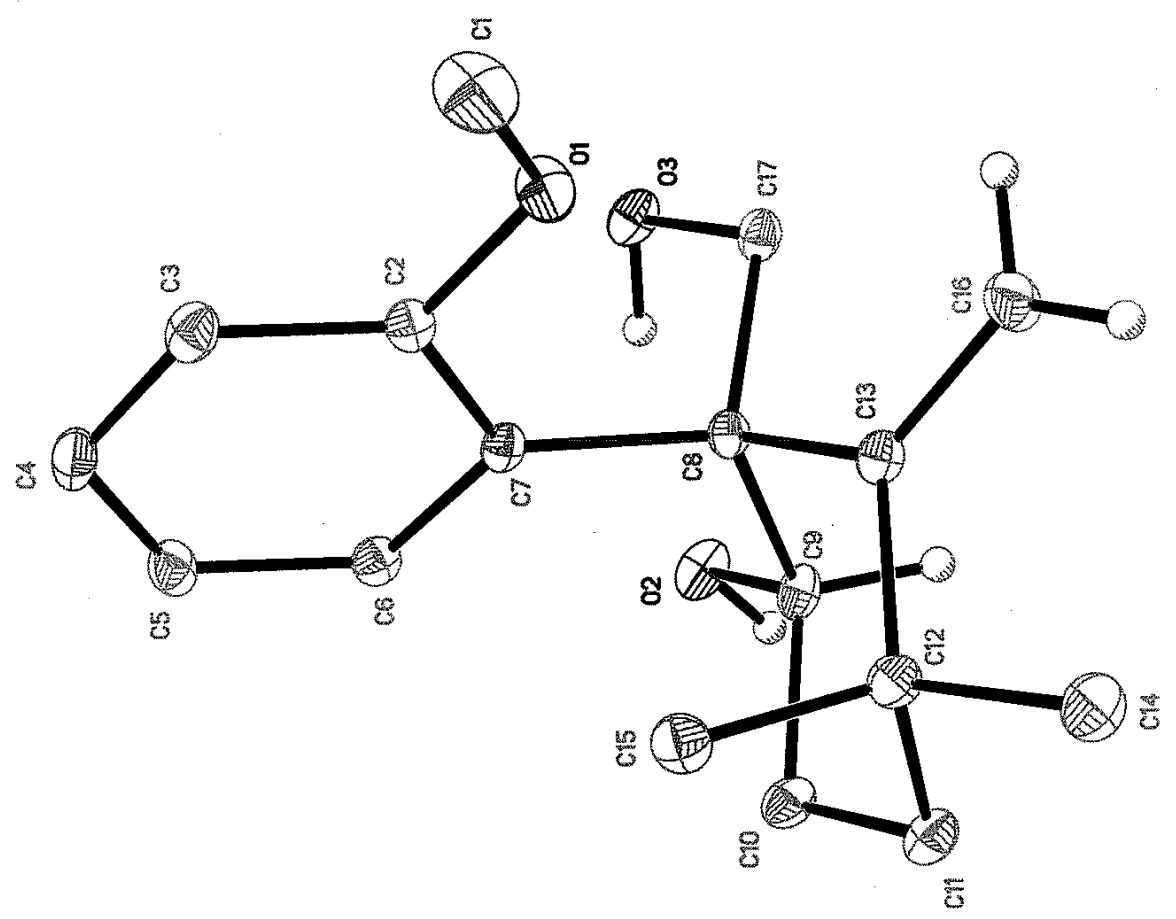


Table 1. Crystal data and structure refinement for fps10.

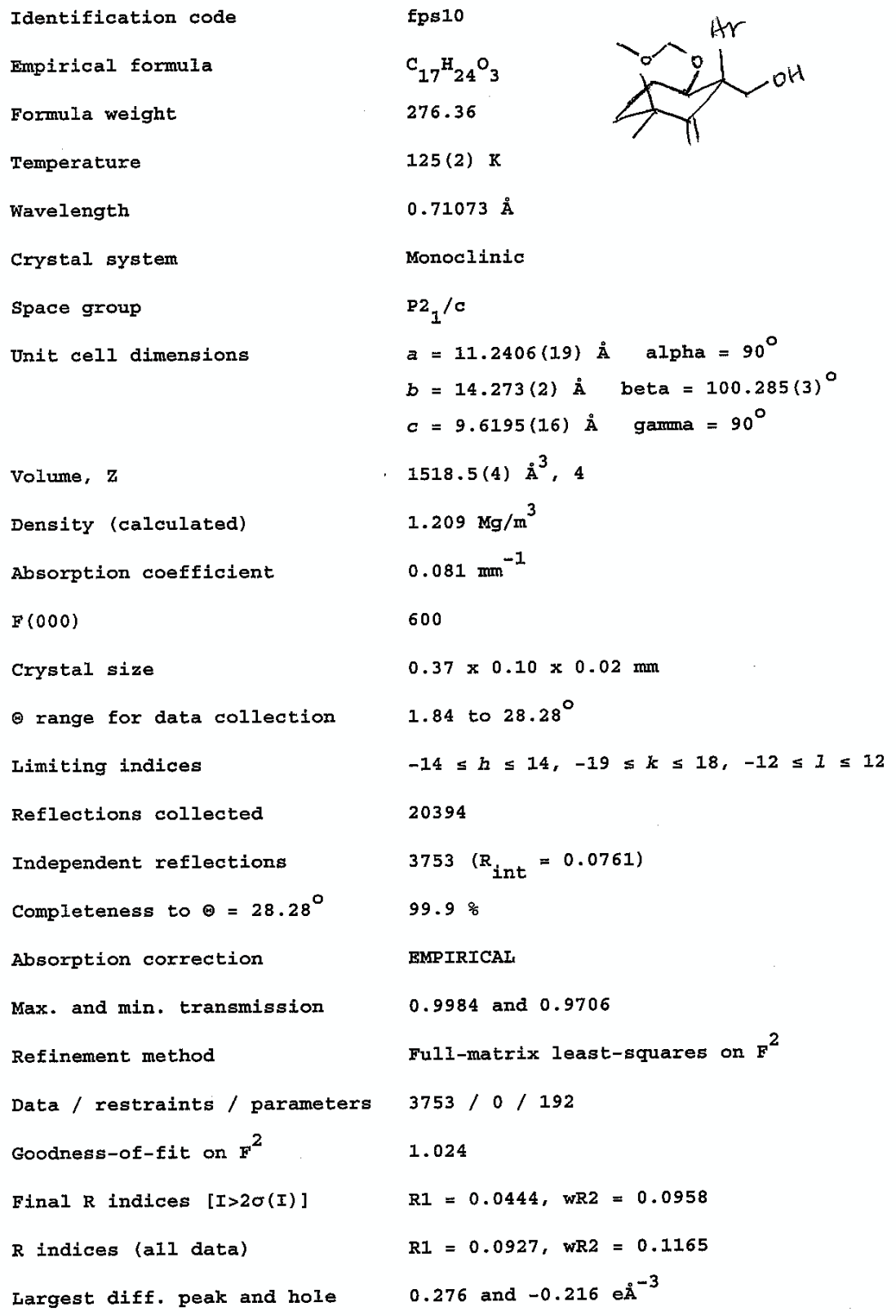


Table 2. Atomic coordinates $\left[\begin{array}{lll}x & 10^{4}\end{array}\right]$ and equivalent isotropic displacement parameters $\left[\AA^{2} \times 10^{3}\right]$ for fps10. $U(e q)$ is defined as one third of the trace of the orthogonalized $U_{i j}$ tensor.

\begin{tabular}{lrrrr}
\hline & & & & \\
& & & & \\
& & & & \\
& & & \\
$O(1)$ & & & & \\
$O(2)$ & $-2253(1)$ & $143(1)$ & $-671(1)$ & $25(1)$ \\
$O(3)$ & $-1604(1)$ & $2196(1)$ & $-4756(1)$ & $25(1)$ \\
$C(1)$ & $-686(1)$ & $2274(1)$ & $-2047(1)$ & $23(1)$ \\
$C(2)$ & $-2629(2)$ & $-462(2)$ & $321(2)$ & $37(1)$ \\
$C(3)$ & $-3057(1)$ & $798(1)$ & $-1327(2)$ & $18(1)$ \\
$C(4)$ & $-4057(2)$ & $1081(1)$ & $-776(2)$ & $22(1)$ \\
$C(5)$ & $-4813(2)$ & $1771(1)$ & $-1446(2)$ & $23(1)$ \\
$C(6)$ & $-4561(2)$ & $2177(1)$ & $-2658(2)$ & $22(1)$ \\
$C(7)$ & $-3569(2)$ & $1880(1)$ & $-3218(2)$ & $19(1)$ \\
$C(8)$ & $-2803(1)$ & $1174(1)$ & $-2589(2)$ & $16(1)$ \\
$C(9)$ & $-1677(1)$ & $854(1)$ & $-3172(2)$ & $15(1)$ \\
$C(10)$ & $-1638(2)$ & $1193(1)$ & $-4702(2)$ & $19(1)$ \\
$C(11)$ & $-2643(2)$ & $786(1)$ & $-5799(2)$ & $22(1)$ \\
$C(12)$ & $-2527(2)$ & $-278(1)$ & $-5822(2)$ & $23(1)$ \\
$C(13)$ & $-2472(2)$ & $-742(1)$ & $-4367(2)$ & $20(1)$ \\
$C(14)$ & $-1538(2)$ & $-225(1)$ & $-3274(2)$ & $18(1)$ \\
$C(15)$ & $-2131(2)$ & $-1773(1)$ & $-4525(2)$ & $28(1)$ \\
$C(16)$ & $-3732(2)$ & $-734(1)$ & $-3952(2)$ & $22(1)$ \\
$C(17)$ & $-601(2)$ & $-669(1)$ & $-2534(2)$ & $26(1)$ \\
& $-571(1)$ & $1284(1)$ & $-2195(2)$ & $19(1)$ \\
\hline
\end{tabular}




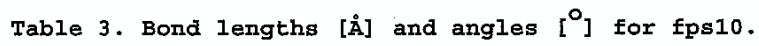

\begin{tabular}{llll}
\hline & & & \\
$O(1)-C(2)$ & $1.373(2)$ & $O(1)-C(1)$ & $1.407(2)$ \\
$O(2)-C(9)$ & $1.435(2)$ & $O(3)-C(17)$ & $1.429(2)$ \\
$C(2)-C(3)$ & $1.386(2)$ & $C(2)-C(7)$ & $1.402(2)$ \\
$C(3)-C(4)$ & $1.383(2)$ & $C(4)-C(5)$ & $1.377(2)$ \\
$C(5)-C(6)$ & $1.389(2)$ & $C(6)-C(7)$ & $1.392(2)$ \\
$C(7)-C(8)$ & $1.542(2)$ & $C(8)-C(17)$ & $1.546(2)$ \\
$C(8)-C(13)$ & $1.553(2)$ & $C(8)-C(9)$ & $1.556(2)$ \\
$C(9)-C(10)$ & $1.517(2)$ & $C(10)-C(11)$ & $1.525(2)$ \\
$C(11)-C(12)$ & $1.540(2)$ & $C(12)-C(14)$ & $1.535(2)$ \\
$C(12)-C(13)$ & $1.536(2)$ & $C(12)-C(15)$ & $1.539(2)$ \\
$C(13)-C(16)$ & $1.322(2)$ & & \\
& & & $122.33(15)$ \\
$C(2)-O(1)-C(1)$ & & & $121.60(15)$ \\
$O(1)-C(2)-C(7)$ & $118.47(14)$ & $O(1)-C(2)-C(3)$ & $119.49(16)$ \\
$C(4)-C(3)-C(2)$ & $120.16(16)$ & $C(3)-C(2)-C(7)$ & $121.91(16)$ \\
$C(4)-C(5)-C(6)$ & $120.12(16)$ & $C(5)-C(6)-C(3)$ & $12.63(14)$ \\
$C(6)-C(7)-C(2)$ & $116.65(15)$ & $C(6)-C(7)-C(8)$ & $106.56(12)$ \\
$C(2)-C(7)-C(8)$ & $120.60(14)$ & $C(7)-C(8)-C(17)$ & $110.62(13)$ \\
$C(7)-C(8)-C(13)$ & $114.50(13)$ & $C(17)-C(8)-C(13)$ & $107.23(13)$ \\
$C(7)-C(8)-C(9)$ & $114.56(13)$ & $C(17)-C(8)-C(9)$ & $112.25(14)$ \\
$C(13)-C(8)-C(9)$ & $103.21(12)$ & $O(2)-C(9)-C(10)$ & $113.32(13)$ \\
$O(2)-C(9)-C(8)$ & $110.51(13)$ & $C(10)-C(9)-C(8)$ & $113.87(14)$ \\
$C(9)-C(10)-C(11)$ & $109.66(14)$ & $C(10)-C(11)-C(12)$ & $106.90(14)$ \\
$C(14)-C(12)-C(13)$ & $112.13(14)$ & $C(14)-C(12)-C(15)$ & $106.99(14)$ \\
$C(13)-C(12)-C(15)$ & $111.53(13)$ & $C(14)-C(12)-C(11)$ & $110.07(14)$ \\
$C(13)-C(12)-C(11)$ & $109.10(13)$ & $C(15)-C(12)-C(11)$ & $121.39(15)$ \\
$C(16)-C(13)-C(12)$ & $121.29(15)$ & $C(16)-C(13)-C(8)$ & \\
$C(12)-C(13)-C(8)$ & $117.09(14)$ & $O(3)-C(17)-C(8)$ & $112.08(13)$ \\
& & & \\
\hline
\end{tabular}

Symmetxy txansformations used to generate equivalent atoms: 
Table 4. Anisotropic displacement parameters $\left[\AA^{2} \times 10^{3}\right]$ for fpsio.

The anisotropic displacement factor exponent takes the form: $-2 \pi^{2}\left[\left(\mathrm{ha}^{*}\right)^{2} \mathrm{U}_{11}+\ldots+2 \mathrm{hka}{ }^{*} \mathrm{~b}^{*} \mathrm{U}_{12} \mathrm{l}\right.$

\begin{tabular}{llllrrr}
\hline & & & & & & \\
& U11 & U22 & U33 & U23 & U13 & U12 \\
\hline & & & & & & \\
$O(1)$ & $24(1)$ & $27(1)$ & $25(1)$ & $11(1)$ & $9(1)$ & $4(1)$ \\
$O(2)$ & $38(1)$ & $16(1)$ & $23(1)$ & $2(1)$ & $13(1)$ & $-3(1)$ \\
$O(3)$ & $28(1)$ & $16(1)$ & $25(1)$ & $-5(1)$ & $9(1)$ & $-6(1)$ \\
$C(1)$ & $36(1)$ & $42(1)$ & $35(1)$ & $22(1)$ & $10(1)$ & $2(1)$ \\
$C(2)$ & $17(1)$ & $15(1)$ & $21(1)$ & $-1(1)$ & $3(1)$ & $-1(1)$ \\
$C(3)$ & $23(1)$ & $23(1)$ & $22(1)$ & $-1(1)$ & $9(1)$ & $-5(1)$ \\
$C(4)$ & $20(1)$ & $24(1)$ & $29(1)$ & $-8(1)$ & $10(1)$ & $-1(1)$ \\
$C(5)$ & $21(1)$ & $18(1)$ & $28(1)$ & $-3(1)$ & $3(1)$ & $4(1)$ \\
$C(6)$ & $21(1)$ & $15(1)$ & $19(1)$ & $-1(1)$ & $3(1)$ & $0(1)$ \\
$C(7)$ & $17(1)$ & $13(1)$ & $18(1)$ & $-3(1)$ & $5(1)$ & $-2(1)$ \\
$C(8)$ & $16(1)$ & $13(1)$ & $18(1)$ & $0(1)$ & $5(1)$ & $1(1)$ \\
$C(9)$ & $24(1)$ & $14(1)$ & $21(1)$ & $0(1)$ & $10(1)$ & $0(1)$ \\
$C(10)$ & $29(1)$ & $22(1)$ & $16(1)$ & $1(1)$ & $6(1)$ & $0(1)$ \\
$C(11)$ & $26(1)$ & $21(1)$ & $22(1)$ & $-5(1)$ & $6(1)$ & $-2(1)$ \\
$C(12)$ & $21(1)$ & $15(1)$ & $23(1)$ & $-3(1)$ & $2(1)$ & $-1(1)$ \\
$C(13)$ & $19(1)$ & $15(1)$ & $20(1)$ & $-1(1)$ & $6(1)$ & $-1(1)$ \\
$C(14)$ & $30(1)$ & $18(1)$ & $35(1)$ & $-7(1)$ & $2(1)$ & $-1(1)$ \\
$C(15)$ & $20(1)$ & $19(1)$ & $26(1)$ & $-2(1)$ & $3(1)$ & $-4(1)$ \\
$C(16)$ & $27(1)$ & $18(1)$ & $32(1)$ & $-4(1)$ & $-2(1)$ & $5(1)$ \\
$C(17)$ & $18(1)$ & $17(1)$ & $23(1)$ & $-3(1)$ & $7(1)$ & $-2(1)$ \\
& & & & & & \\
\hline
\end{tabular}


Table 5. Hydrogen coordinates $\left(x 0^{4}\right)$ and isotropic displacement parameters $\left(\dot{A}^{2} \times 10^{3}\right)$ for fpsio.

\begin{tabular}{|c|c|c|c|c|}
\hline & $\mathbf{x}$ & $y$ & $\mathrm{z}$ & $\mathrm{v}(\mathrm{eq})$ \\
\hline H $(1 \mathrm{~A})$ & -2028 & -959 & 572 & 56 \\
\hline $\mathrm{H}(1 \mathrm{~B})$ & -3410 & -741 & -87 & 56 \\
\hline $\mathrm{H}(1 \mathrm{C})$ & -2713 & -107 & 1171 & 56 \\
\hline H $(3 B)$ & -4224 & 800 & 64 & 27 \\
\hline $\mathrm{H}(4 \mathrm{~A})$ & -5502 & 1963 & -1072 & 28 \\
\hline $\mathrm{H}(5 \mathrm{~A})$ & -5066 & 2662 & -3111 & 27 \\
\hline H $(6 A)$ & -3407 & 2168 & -4056 & 22 \\
\hline H $(9 A)$ & -859 & 963 & -4940 & 23 \\
\hline H $(10 \mathrm{~A})$ & -2592 & 1044 & -6742 & 26 \\
\hline $\mathrm{H}(10 \mathrm{~B})$ & -3438 & 961 & -5569 & 26 \\
\hline $\mathrm{H}(11 \mathrm{~A})$ & -1785 & -443 & -6189 & 27 \\
\hline $\mathrm{H}(11 \mathrm{~B})$ & -3225 & -538 & -6484 & 27 \\
\hline $\mathrm{H}(14 \mathrm{~A})$ & -2124 & -2101 & -3629 & 43 \\
\hline$H(14 B)$ & -1327 & -1809 & -4781 & 43 \\
\hline H $(14 \mathrm{C})$ & -2725 & -2067 & -5267 & 43 \\
\hline $\mathrm{H}(15 \mathrm{~A})$ & -3680 & -1003 & -3005 & 33 \\
\hline H $(15 B)$ & -4292 & -1107 & -4633 & 33 \\
\hline $\mathrm{H}(15 \mathrm{C})$ & -4027 & -88 & -3953 & 33 \\
\hline $\mathrm{H}(16 \mathrm{~A})$ & -495 & -1320 & -2675 & 31 \\
\hline H $(16 B)$ & -33 & -336 & -1863 & 31 \\
\hline H $(17 \mathrm{~A})$ & -478 & 986 & -1252 & 23 \\
\hline H $(17 B)$ & 167 & 1148 & -2588 & 23 \\
\hline $\mathrm{H}(2 \mathrm{~A})$ & $-1330(20)$ & $2354(15)$ & $-5560(20)$ & $44(6)$ \\
\hline$H(3 A)$ & $-950(20)$ & $2470(17)$ & $-2930(30)$ & $51(7)$ \\
\hline
\end{tabular}




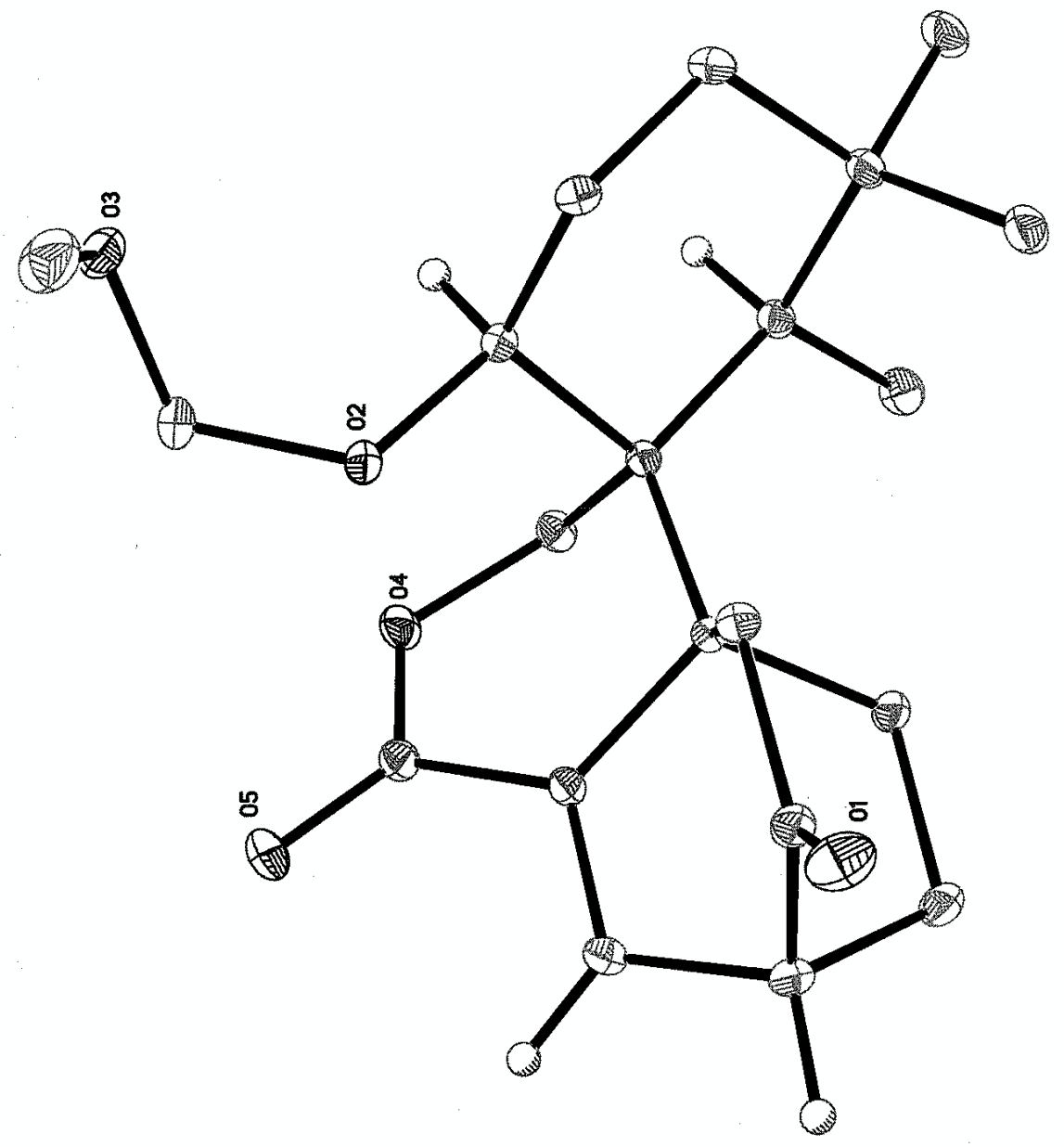


Table 1. Crystal data and structure refinement for FENGWS10.

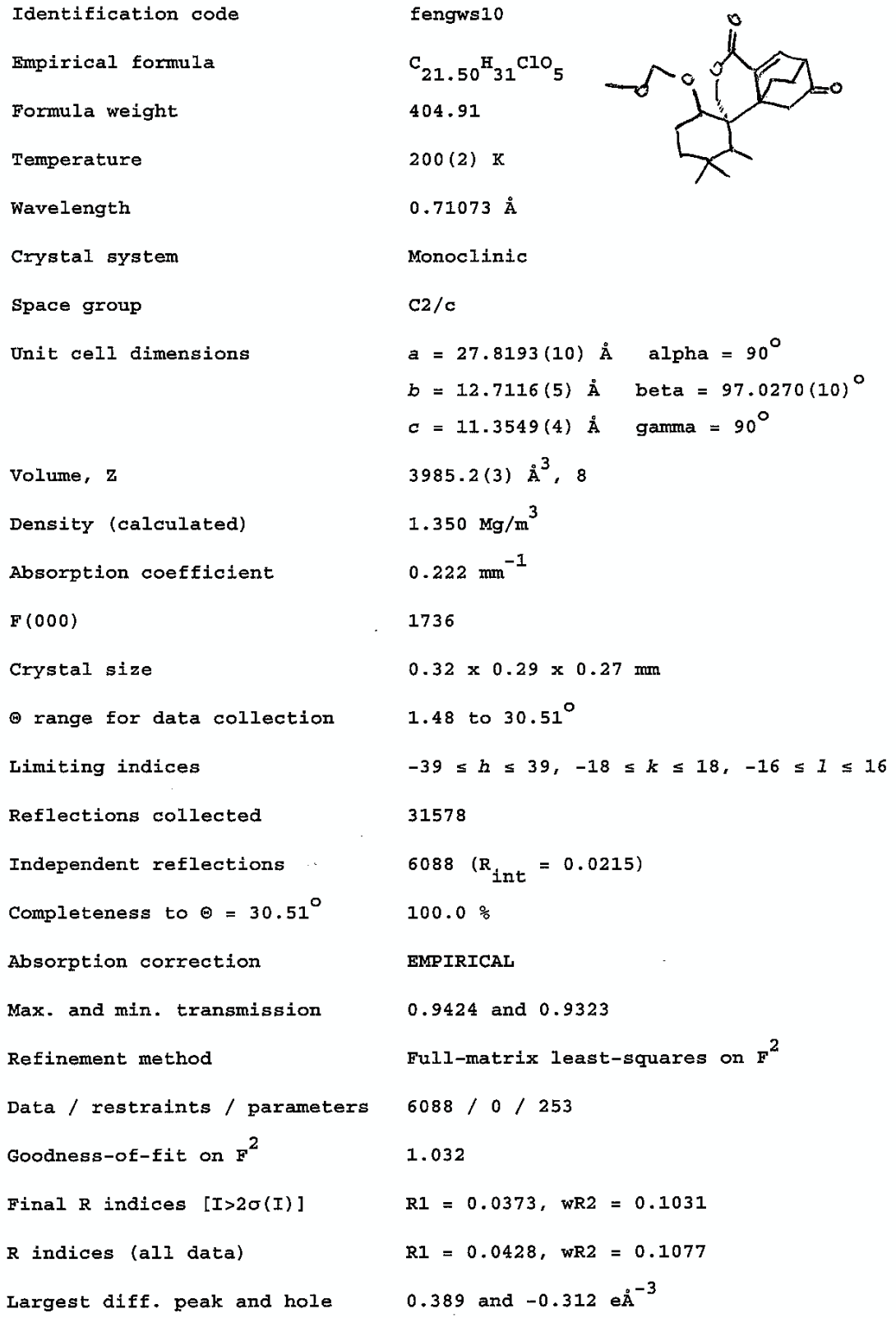


Table 2. Atomic coordinates $\left[x 10^{4}\right]$ and equivalent isotropic displacement parameters $\left[\AA^{2} \times 10^{3}\right]$ for FENGWS10. U(eq) is defined as one third of the trace of the orthogonalized $U_{i j}$ tensor.

\begin{tabular}{|c|c|c|c|c|}
\hline & $\mathbf{x}$ & $y$ & $\mathbf{z}$ & $\mathrm{U}(\mathrm{eq})$ \\
\hline$O(1)$ & $3930(1)$ & $5945(1)$ & $3184(1)$ & $38(1)$ \\
\hline$O(2)$ & $3493(1)$ & $2331(1)$ & $1920(1)$ & $25(1)$ \\
\hline$o(3)$ & $3666(1)$ & $1158(1)$ & 432 (1) & $34(1)$ \\
\hline$O(4)$ & $2656(1)$ & $1938(1)$ & $2967(1)$ & $29(1)$ \\
\hline$o(5)$ & $2351(1)$ & 3148 (1) & $1695(1)$ & $37(1)$ \\
\hline$C(1)$ & $3665(1)$ & $5268(1)$ & $3492(1)$ & $24(1)$ \\
\hline$C(2)$ & $3170(1)$ & $5475(1)$ & $3878(1)$ & $26(1)$ \\
\hline$C(3)$ & $3217(1)$ & $5111(1)$ & $5194(1)$ & 29 (1) \\
\hline$c(4)$ & $3358(1)$ & $3933(1)$ & $5301(1)$ & $23(1)$ \\
\hline$C(5)$ & $3412(1)$ & $3442(1)$ & $4063(1)$ & $18(1)$ \\
\hline$c(6)$ & $3487(1)$ & $2209(1)$ & $4085(1)$ & 19 (1) \\
\hline$C(7)$ & $3775(1)$ & $1609(1)$ & $5185(1)$ & $22(1)$ \\
\hline$C(8)$ & $3579(1)$ & $1737(1)$ & $6391(1)$ & 32 (1) \\
\hline$c(9)$ & $4339(1)$ & $1620(1)$ & $5322(1)$ & $25(1)$ \\
\hline$c(10)$ & $4576(1)$ & $2666(1)$ & $5742(1)$ & $31(1)$ \\
\hline$c(11)$ & $4524(1)$ & $786(1)$ & $6253(1)$ & $36(1)$ \\
\hline$C(12)$ & $4500(1)$ & $1267(1)$ & $4138(1)$ & $29(1)$ \\
\hline$C(13)$ & $4258(1)$ & $1866(1)$ & $3065(1)$ & $25(1)$ \\
\hline$C(14)$ & $3707(1)$ & $1794(1)$ & $2975(1)$ & $21(1)$ \\
\hline$C(15)$ & $3304(1)$ & $1667(1)$ & $976(1)$ & $30(1)$ \\
\hline$C(16)$ & $3913(1)$ & 1852 (1) & $-265(1)$ & $45(1)$ \\
\hline$c(17)$ & $2971(1)$ & $1747(1)$ & $4052(1)$ & $25(1)$ \\
\hline$C(18)$ & 2630 (1) & 2941 (1) & $2569(1)$ & $26(1)$ \\
\hline$c(19)$ & 2944 (1) & $3715(1)$ & $3260(1)$ & $22(1)$ \\
\hline$C(20)$ & 2829 (1) & $4739(1)$ & $3173(1)$ & $26(1)$ \\
\hline$c(21)$ & $3804(1)$ & $4114(1)$ & $3556(1)$ & $21(1)$ \\
\hline$C(22)$ & 0 & $-106(1)$ & 7500 & $44(1)$ \\
\hline $\mathrm{Cl}$ & $282(1)$ & $672(1)$ & $8673(1)$ & 55 (1) \\
\hline
\end{tabular}


Table 3. Bond lengths [Å] and angles $\left[{ }^{\circ}\right]$ for FENGWS10.

\begin{tabular}{|c|c|c|c|}
\hline$O(1)-C(1)$ & $1.2140(13)$ & $o(2)-C(15)$ & $1.4145(12)$ \\
\hline$O(2)-C(14)$ & $1.4417(11)$ & $O(3)-C(15)$ & $1.4015(13)$ \\
\hline$o(3)-c(16)$ & $1.4181(16)$ & $O(4)-C(18)$ & $1.3511(13)$ \\
\hline$O(4)-C(17)$ & $1.4431(12)$ & $O(5)-C(18)$ & $1.2107(12)$ \\
\hline$c(1)-C(21)$ & $1.5163(12)$ & $c(1)-c(2)$ & $1.5163(14)$ \\
\hline$C(2)-C(20)$ & $1.4931(14)$ & $c(2)-C(3)$ & $1.5553(14)$ \\
\hline$C(3)-C(4)$ & $1.5483(14)$ & $C(4)-C(5)$ & $1.5627(12)$ \\
\hline$C(5)-C(19)$ & $1.5348(12)$ & $c(5)-C(21)$ & $1.5521(12)$ \\
\hline$C(5)-C(6)$ & $1.5814(12)$ & $C(6)-C(17)$ & $1.5465(12)$ \\
\hline$C(6)-C(14)$ & $1.5591(12)$ & $C(6)-C(7)$ & $1.5929(12)$ \\
\hline$C(7)-C(8)$ & $1.5421(14)$ & $c(7)-C(9)$ & $1.5586(13)$ \\
\hline$c(9)-C(10)$ & $1.5340(14)$ & $C(9)-C(12)$ & $1.5347(14)$ \\
\hline$C(9)-C(11)$ & $1.5409(14)$ & $C(12)-C(13)$ & $1.5220(14)$ \\
\hline$C(13)-C(14)$ & $1.5289(13)$ & $C(18)-C(19)$ & 1.4767 (13) \\
\hline$C(19)-C(20)$ & $1.3405(13)$ & $C(22)-C 1 \# 1$ & $1.7639(11)$ \\
\hline$C(22)-C 1$ & $1.7639(11)$ & & \\
\hline$C(15)-O(2)-C(14)$ & $115.07(7)$ & $C(15)-0(3)-C(16)$ & $112.44(9)$ \\
\hline$C(18)-O(4)-C(17)$ & $116.56(7)$ & $O(1)-C(1)-C(21)$ & $122.54(9)$ \\
\hline$O(1)-C(1)-C(2)$ & $124.46(9)$ & $C(21)-C(1)-C(2)$ & $112.98(8)$ \\
\hline$C(20)-C(2)-C(1)$ & $106.02(8)$ & $C(20)-C(2)-C(3)$ & $107.57(8)$ \\
\hline$C(1)-C(2)-C(3)$ & $104.69(8)$ & $C(4)-C(3)-C(2)$ & $110.69(7)$ \\
\hline$C(3)-C(4)-C(5)$ & $111.52(7)$ & $C(19)-C(5)-C(21)$ & $103.91(7)$ \\
\hline$C(19)-C(5)-C(4)$ & $106.05(7)$ & $C(21)-C(5)-C(4)$ & $105.24(7)$ \\
\hline$C(19)-C(5)-C(6)$ & $109.57(7)$ & $C(21)-C(5)-C(6)$ & $116.97(7)$ \\
\hline$C(4)-C(5)-C(6)$ & $114.10(7)$ & $C(17)-C(6)-C(14)$ & $107.78(7)$ \\
\hline$C(17)-C(6)-C(5)$ & $104.79(7)$ & $C(14)-C(6)-C(5)$ & $112.76(7)$ \\
\hline$C(17)-C(6)-C(7)$ & $102.27(7)$ & $C(14)-C(6)-C(7)$ & $105.27(7)$ \\
\hline$C(5)-C(6)-C(7)$ & $122.73(7)$ & $C(8)-C(7)-C(9)$ & $111.78(8)$ \\
\hline$C(8)-C(7)-C(6)$ & $116.60(8)$ & $C(9)-C(7)-C(6)$ & $118.46(7)$ \\
\hline$C(10)-C(9)-C(12)$ & $111.46(9)$ & $C(10)-C(9)-C(11)$ & $106.74(8)$ \\
\hline$C(12)-C(9)-C(11)$ & $106.93(8)$ & $C(10)-C(9)-C(7)$ & $115.36(8)$ \\
\hline$C(12)-C(9)-C(7)$ & $107.96(7)$ & $C(11)-C(9)-C(7)$ & $108.01(8)$ \\
\hline$C(13)-C(12)-C(9)$ & $114.04(8)$ & $C(12)-C(13)-C(14)$ & $111.36(8)$ \\
\hline$O(2)-C(14)-C(13)$ & $109.57(8)$ & $O(2)-C(14)-C(6)$ & $110.43(7)$ \\
\hline$C(13)-C(14)-C(6)$ & $114.51(7)$ & $O(3)-C(15)-O(2)$ & $112.92(9)$ \\
\hline$O(4)-C(17)-C(6)$ & $114.97(8)$ & $O(5)-C(18)-O(4)$ & $118.84(9)$ \\
\hline$O(5)-C(18)-C(19)$ & $124.64(10)$ & $O(4)-C(18)-C(19)$ & $116.52(8)$ \\
\hline$C(20)-C(19)-C(18)$ & $119.24(8)$ & $C(20)-C(19)-C(5)$ & $116.06(8)$ \\
\hline$C(18)-C(19)-C(5)$ & $124.67(8)$ & $C(19)-C(20)-C(2)$ & $116.07(8)$ \\
\hline$C(1)-C(21)-C(5)$ & $111.21(7)$ & $\mathrm{CI} \# 1-\mathrm{C}(22)-\mathrm{Cl}$ & $111.77(10)$ \\
\hline
\end{tabular}

Symmetry transformations used to generate equivalent atoms:

$\# I-x, y,-z+3 / 2$ 
Table 4. Anisotropic displacement parameters $\left[\dot{A}^{2} \times 10^{3}\right]$ for FENGWS10.

The anisotropic displacement factor exponent takes the form:

$-2 \mathrm{~m}^{2}\left[\left(\mathrm{ha}^{*}\right)^{2} \mathrm{U}_{11}+\ldots+2 \mathrm{hka} \mathrm{b}^{*} \mathrm{v}_{12}\right]$

\begin{tabular}{|c|c|c|c|c|c|c|}
\hline & U11 & $\mathrm{U} 22$ & U33 & $\mathrm{U} 23$ & $\mathrm{U} 13$ & $\mathrm{U} 12$ \\
\hline$O(1)$ & $47(1)$ & $23(1)$ & $46(1)$ & $4(1)$ & $14(1)$ & $-2(1)$ \\
\hline$O(2)$ & $30(1)$ & $24(1)$ & $18(1)$ & $-3(1)$ & $-2(1)$ & $4(1)$ \\
\hline$O(3)$ & $51(1)$ & $27(1)$ & $24(1)$ & $-4(1)$ & $4(1)$ & $8(1)$ \\
\hline$O(4)$ & $22(1)$ & $29(1)$ & $32(1)$ & $-2(1)$ & $-5(1)$ & $-4(1)$ \\
\hline$O(5)$ & $30(1)$ & $44(1)$ & $32(1)$ & $-2(1)$ & $-11(1)$ & $2(1)$ \\
\hline$C(1)$ & $31(1)$ & $20(1)$ & $21(1)$ & $1(1)$ & $2(1)$ & $2(1)$ \\
\hline$C(2)$ & $31(1)$ & $21(1)$ & $26(1)$ & $-2(1)$ & $2(1)$ & $8(1)$ \\
\hline$c(3)$ & $37(1)$ & $26(1)$ & $24(1)$ & $-5(1)$ & $5(1)$ & $6(1)$ \\
\hline$C(4)$ & $28(1)$ & $23(1)$ & $18(1)$ & $-2(1)$ & $3(1)$ & $2(1)$ \\
\hline$C(5)$ & $18(I)$ & $18(1)$ & $17(1)$ & $0(1)$ & $1(1)$ & $2(1)$ \\
\hline$C(6)$ & $18(1)$ & $18(1)$ & $19(1)$ & $0(1)$ & $1(1)$ & $0(1)$ \\
\hline$C(7)$ & $24(1)$ & $19(1)$ & $21(1)$ & $2(1)$ & $0(1)$ & $1(1)$ \\
\hline$C(8)$ & $39(1)$ & $34(1)$ & $22(1)$ & $8(1)$ & $4(1)$ & $3(1)$ \\
\hline$c(9)$ & $24(1)$ & $22(1)$ & $26(1)$ & $0(1)$ & $-5(1)$ & $3(1)$ \\
\hline$C(10)$ & $28(1)$ & $27(1)$ & $36(1)$ & $-2(1)$ & $-7(1)$ & $-1(1)$ \\
\hline$C(11)$ & $38(1)$ & $29(1)$ & $37(1)$ & $5(1)$ & $-11(1)$ & $7(1)$ \\
\hline$C(12)$ & $24(1)$ & $28(1)$ & $34(1)$ & $-3(1)$ & $-1(1)$ & $8(1)$ \\
\hline$C(13)$ & $23(1)$ & $27(I)$ & $27(1)$ & $-3(1)$ & $5(1)$ & $5(1)$ \\
\hline$C(14)$ & $24(1)$ & $20(1)$ & $19(1)$ & $-2(1)$ & $O(1)$ & $2(1)$ \\
\hline$C(15)$ & $34(1)$ & 34 (1) & $22(1)$ & $-7(1)$ & $-2(1)$ & $0(1)$ \\
\hline$c(16)$ & $62(1)$ & $45(1)$ & $32(1)$ & $2(1)$ & $17(1)$ & $9(1)$ \\
\hline$c(17)$ & $21(1)$ & $26(1)$ & $27(1)$ & $1(1)$ & $1(1)$ & $-3(1)$ \\
\hline$C(18)$ & $20(1)$ & $31(1)$ & $26(1)$ & $-4(1)$ & $-1(1)$ & $2(1)$ \\
\hline$C(19)$ & $19(1)$ & $26(1)$ & $19(1)$ & $-1(1)$ & $0(1)$ & $3(1)$ \\
\hline$C(20)$ & $24(1)$ & $28(1)$ & $26(1)$ & $0(1)$ & $-1(1)$ & $8(1)$ \\
\hline$C(2 I)$ & $22(1)$ & $18(1)$ & $23(1)$ & $1(1)$ & $4(1)$ & $2(1)$ \\
\hline$C(22)$ & $39(1)$ & $31(1)$ & $64(1)$ & 0 & $17(1)$ & 0 \\
\hline$C I$ & 59 (1) & $50(1)$ & $61(1)$ & $-7(1)$ & $20(1)$ & $-8(1)$ \\
\hline
\end{tabular}




\section{Chapter 3 Synthesis of Maoecrystal V Pentacyclic Core Structure}

The undesired facial selectivity of the IMDA reaction is a serious problem to our first approach toward Maoecrystal V, since cycloadduct $\mathbf{2 . 8 2}$ or $\mathbf{2 . 8 4}$ could not be used to elaborate to the final natural product with the right relative stereochemistry. We were at a cross point whether to kill this project or to explore a new route to solve this problem. We decided to take this great challenge and develop a modified route to achieve a diastereoselective synthesis of the Maoecrystal V core structure.

\subsection{IMDA Using a Symmetric Precursor}

Although the IMDA reaction proceeded with the undesired sense of facial selectivity, it indeed afforded the bicyclo-[2.2.2] octane core substructure of Maoecrystal V. Since it was not quite clear what is the exact factor controlling the facial selectivity in our previous key IMDA reaction due to the rotamer character of the IMDA precursor, we envisioned that it would be very difficult to redesign a new IMDA precursor which would only afford us the desired facial adduct. ${ }^{1}$ We then turned our attention to the comparison of the stereochemistry of desired and undesired adducts, hoping to find some clues to solve this problem. 


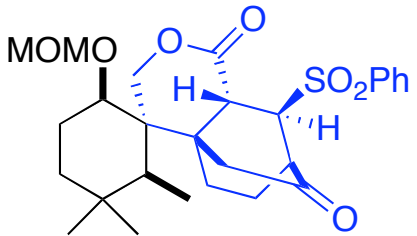

Undesired Facial Product 3.1

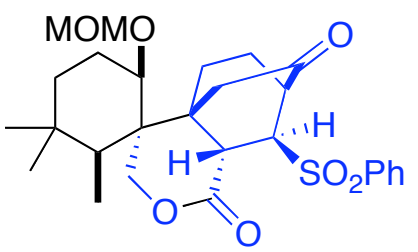

Desired Facial Product 3.2

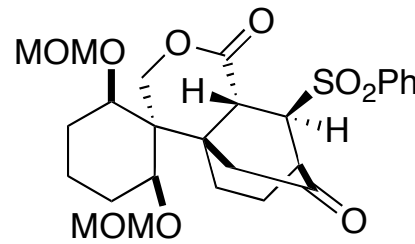

3.3

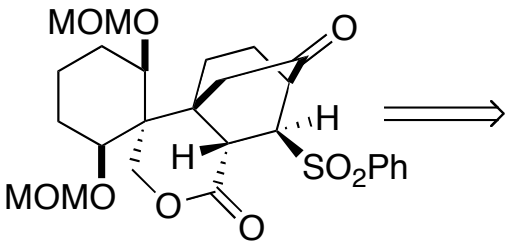

3.4

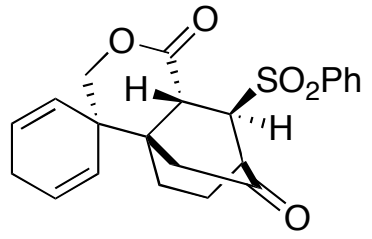

3.5

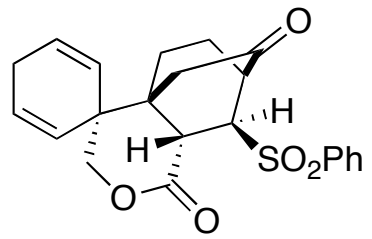

3.6

Figure 3-1 Symmetry logic to solve IMDA facial problem

As shown in the Figure 3-1, we soon noticed the right part substructure from both undesired facial product and the desired facial product, which has a lactone ring and a bicyclo-[2.2.2] octane motif (in blue color), shared mirror symmetry character. If the left A-ring was also a symmetric structure such as $\mathbf{3 . 3}$ and $\mathbf{3 . 4}$, then the cycloadducts would share a mirror symmetry character totally and they were enantiomers. ${ }^{2}$ We further envisioned that compounds 3.3 and 3.4 could be prepared from compounds $\mathbf{3 . 5}$ and $\mathbf{3 . 6}$ respectively by sequential oxidation of the alkenes.

From this analysis, we then designed a new route (Scheme 3-1), featuring using an IMDA reaction with a symmetric precursor 3.7. After IMDA reaction, a tetracyclic core 3.8 would be obtained. Selective hydration of the enone alkene would provide tertiary alcohol 3.9, in which the stereochemistry may be directed by C-16 ketone function group. 
At this stage, a key oxidative cyclization reaction through a 5-exo ring closure would afford compound $\mathbf{3 . 1 0},{ }^{3}$ which upon functional group transformation would provide 3.11. The C-4 ketone in $\mathbf{3 . 1 1}$ then would be used to prepare gem-dimethyl group, a concept was introduced by Oppolzer in his longifolene synthesis. ${ }^{4}$ With $\mathbf{3 . 1 2}$ in hand, an olefination reaction and the following oxidation state tuning would eventually afford Maoecrystal V.

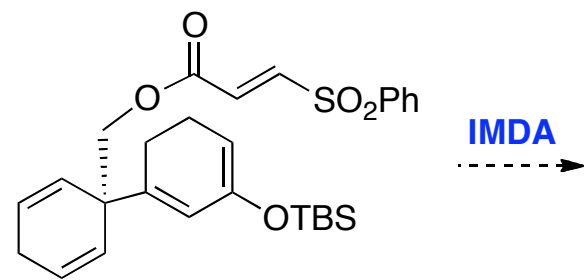

3.7

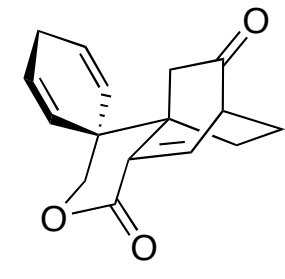

3.8

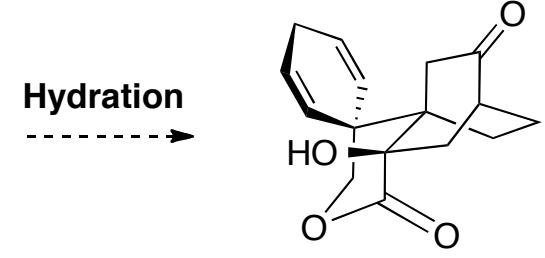

3.9

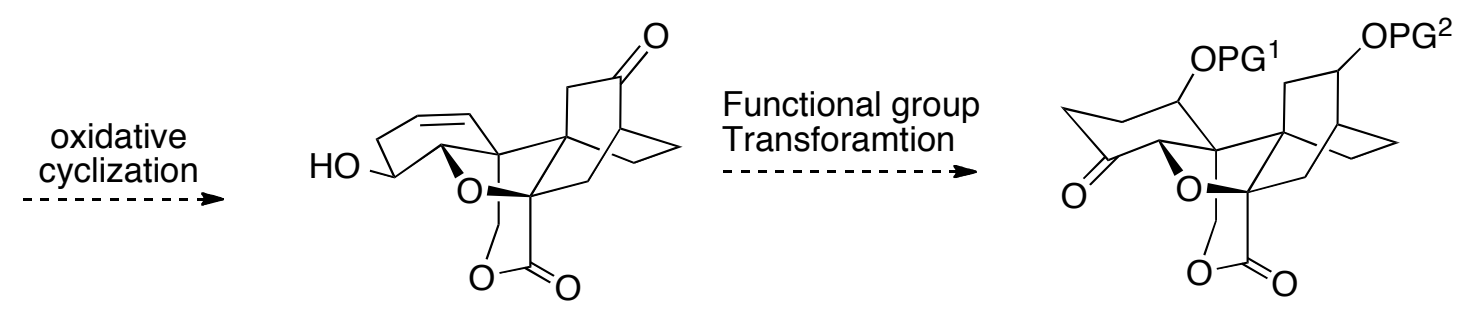

3.10

3.11

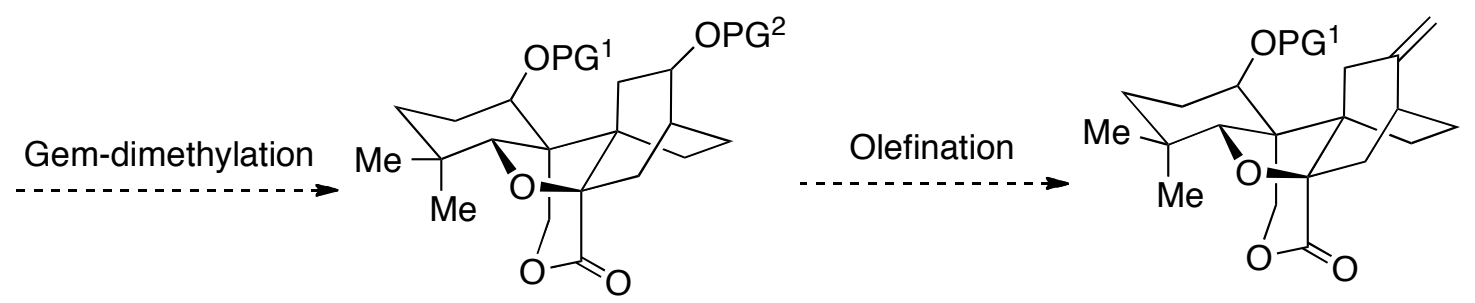

3.12

3.13

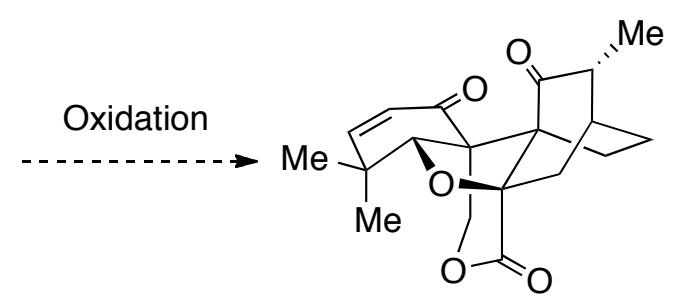

3.14

Scheme 3-1 Second generation synthetic plan toward Maoecrystal V 
In order to prove our new design, we began to explore the possibility to prepare a pentacyclic core model structure $\mathbf{3 . 1 5}$ with the appropriate relative stereochemistry (Scheme 3-2), which we envisioned it would be a big challenge in the Maoecrystal V synthesis.

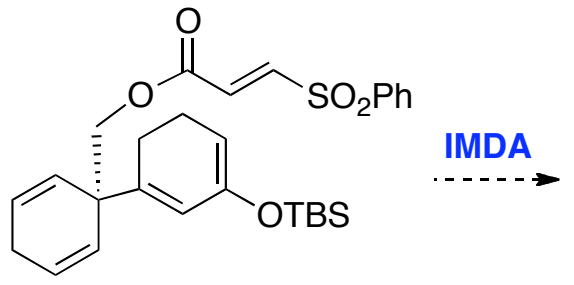

3.7

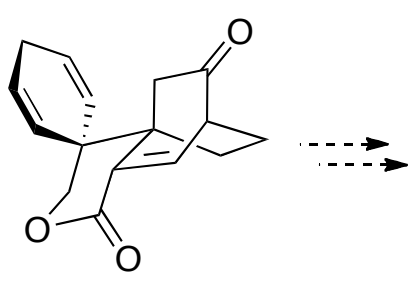

3.8

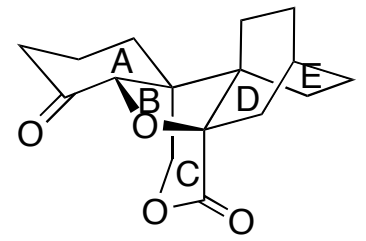

maoecrystal $\mathrm{V}$ core $\mathbf{3 . 1 5}$

Scheme 3-2 Plan to synthesize Maoecrystal V core structure

\subsection{Results and Discussions}

Our new route to the IMDA precursor 3.7 started with a Birch-type vinylogous acylation between substrate $\mathbf{3 . 1 6}$ and $\mathbf{3 . 1 7},{ }^{5}$ which afforded compound 3.18, possessing one of the requisite quaternary carbon centers. Intermediate $\mathbf{3 . 1 8}$ was subjected to global DIBAL-H reduction, followed by selective $\mathrm{MnO}_{2}$-mediated reoxidation of the resultant allylic alcohol, to provided 3.19. Esterification of compound 3.19 with acyl chloride $\mathbf{3 . 2 0}$

${ }^{6}$ followed by formation of the TBS enol ether, furnished our IMDA precursor 3.7. The thermo-promoted Diels-Alder reaction of compound 3.7 went on smoothly and without isolation, the cycloadduct was treated with TBAF, which furnished key intermediate $\mathbf{3 . 8}$ in $62 \%$ yield. 


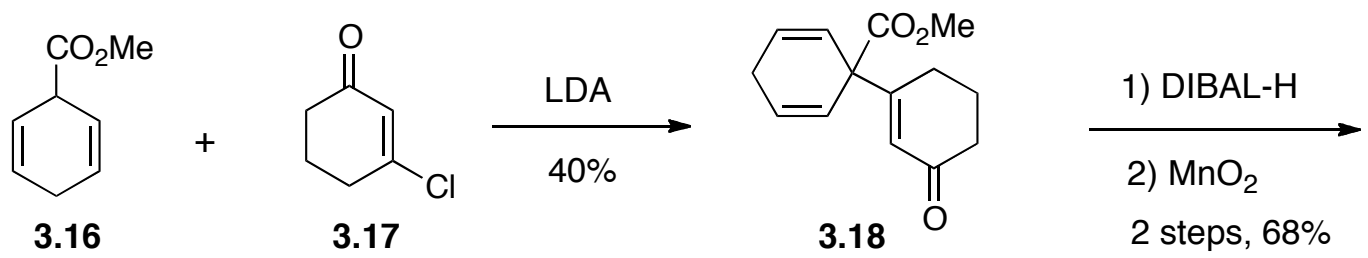<smiles>O=C1C=C(C2(CO)C=CCC=C2)CCC1</smiles>

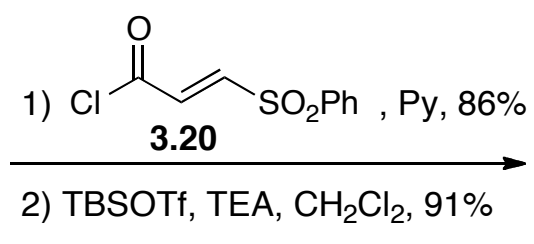<smiles>O=C(/C=C/S(=O)(=O)c1ccccc1)OCC1C=CCC=C1</smiles>

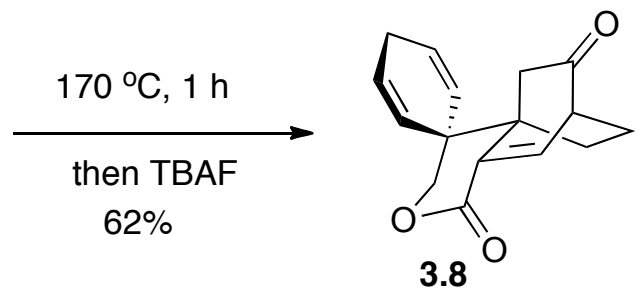

Scheme 3-3 Preparation of 3.8

We then turned to the emplacement of the requisite C-10 tertiary alcohol. We first tested our proposed hydration reaction, which took place through a cascade 1,4reduction/oxidation sequence, ${ }^{7}$ and we obtained the desired tertiary alcohol $\mathbf{3 . 2 1}$ as a 1.5:1 diastereomeric mixture of compound 3.21a and 3.21b.

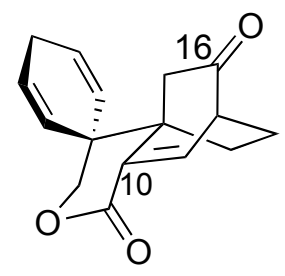

3.8

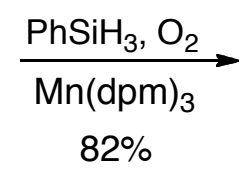

$82 \%$

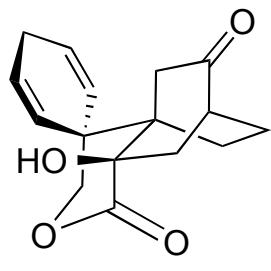

$3.21 a$

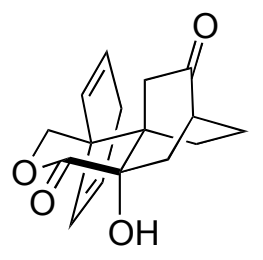

3.21b

Scheme 3-4 Hydration of alkene 3.8 
The poor diastereoselectivity probably arose from the enol oxidation step, in which the stereo-bias caused by C-11 and C-15 were not significant (Figure 3-2). ${ }^{8}$ Thus a larger difference between $\mathrm{C}-11$ and $\mathrm{C}-15$ might improve the diastereoselectivity of this hydration process. Based this hypothesis, we prepared compound $\mathbf{3 . 2 3}$ and subjected it to the hydration process.

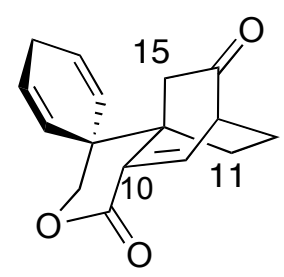

3.8

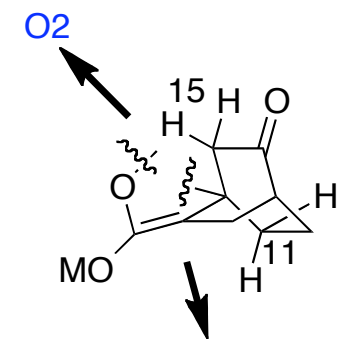

$3.22 \mathrm{O}_{2}$

Figure 3-2 Explanation of stereoselectivity of hydration

In this case, we did obtain a single diastereomer, however, it was the undesired isomer (3.25).
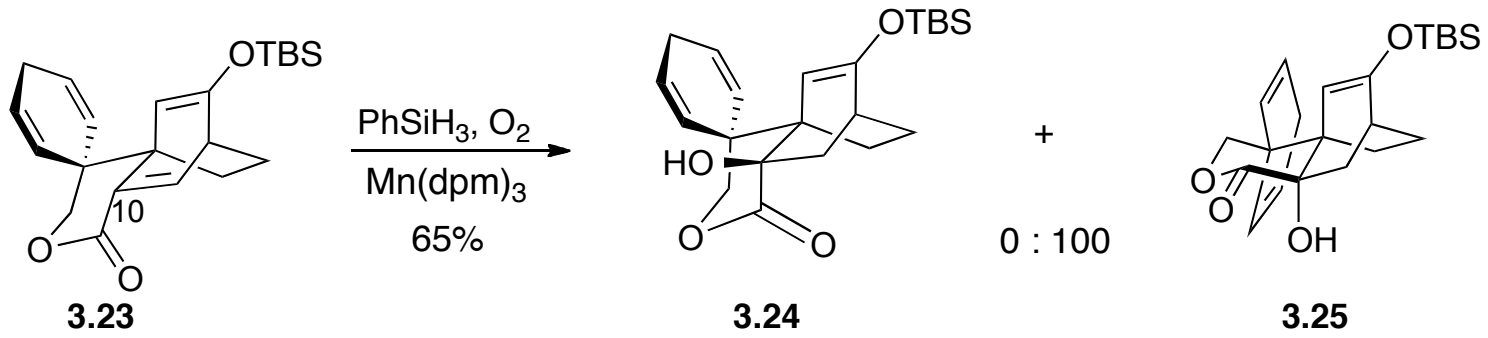

Scheme 3-5 Hydration reaction of 3.23 
While the direct hydration process met with difficulty to afford 3.21a as a single isomer, it was soon found that epoxidation of $\mathbf{3 . 8}$ with $\mathrm{H}_{2} \mathrm{O}_{2}$ provided epoxide $\mathbf{3 . 2 6}$ as a single isomer. Upon exposure to sequential epoxide opening and reduction of the resultant iodohydrin, epoxide $\mathbf{3 . 2 6}$ was converted to $\mathbf{3 . 2 1}$ a as a single diastereoisomer. ${ }^{9}$

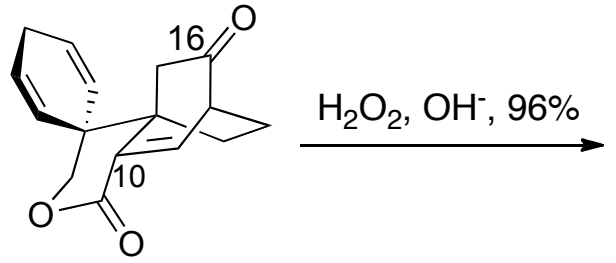

3.8

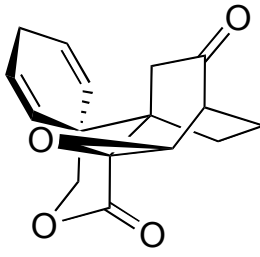

3.26

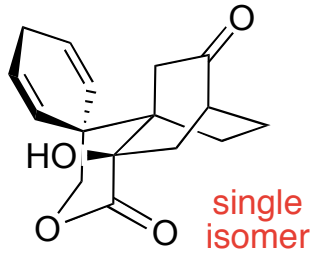

$3.21 a$

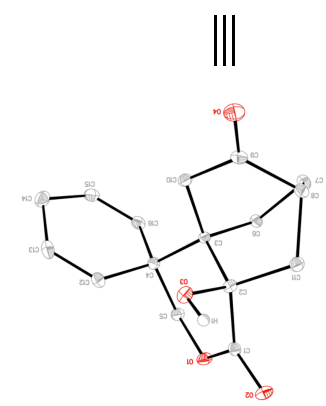

Scheme 3-6 Preparation 3.21a

We thought there might be two reasons to account for the stereoselective epoxidation of compound $\mathbf{3 . 8}$ (Figure 3-3). First, the stereo-bias between the C-12 and C16 now was significant and obvious the C-12 $\beta$-hydrogen atom probably blocked the $\beta$ facial nucleophilic attack. Second, $\sigma$ bond between C-12 and C-13 had more donating effect to the $\sigma^{*}$ of the new formed $\mathrm{C}-\mathrm{O}$ bond than $\sigma$ bond between $\mathrm{C}-16$ and $\mathrm{C}-13$, which is called Cieplak effect. ${ }^{10}$ 


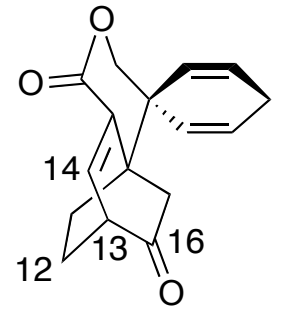

3.8

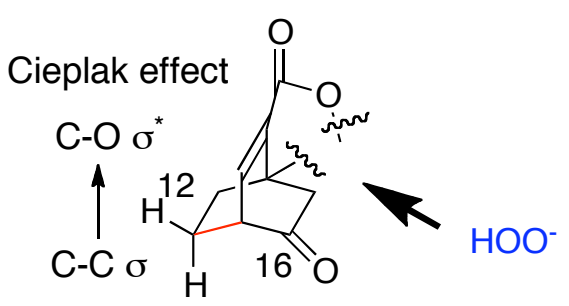

3.27

Figure 3-3 Explanation of stereoselectivity of epoxidation of $\mathbf{3 . 8}$

With compound 3.21a in hand, our effort now was focused on ring closure of the THF ring. Initially we screened a lot of electrophiles such as $\mathrm{I}_{2}, \mathrm{NIS}, \mathrm{PhSeCl}, \mathrm{NBS}, \mathrm{Br}_{2}$, $\mathrm{Hg}(\mathrm{TFA}), \mathrm{Pb}(\mathrm{OAc})_{4}$, and $\mathrm{Pd}(\mathrm{OAc})_{2}$, hoping to achieve a ring closure through the activation of the A-ring-5-exo alkene moiety. To our disappointment, none of these conditions afforded a desired ring closure product, either starting material was recovered (eg: $\mathrm{I}_{2}, \mathrm{NIS}, \mathrm{Pd}(\mathrm{OAc})_{2}$ ), or decomposition of material was observed (eg: $\mathrm{Hg}(\mathrm{TFA})_{2}$, $\left.\mathrm{Pb}(\mathrm{OAc})_{4}, \mathrm{PhSeBr}\right)$. Further screening revealed that $m$-CPBA reacted with 3.21a and provided epoxide 3.28 as a single isomer. Following treatment with $p-\mathrm{TsOH} \cdot \mathrm{H}_{2} \mathrm{O},{ }^{11}$ a cyclized product (3.29) was obtained as a single diastereomer. X-ray analysis indicated that the stereochemistry at $\mathrm{C} 5$ was opposite as that required for Maeocrystal V. 


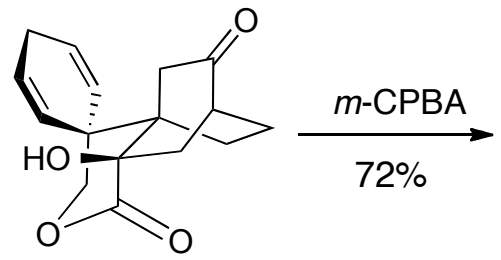

$3.21 a$

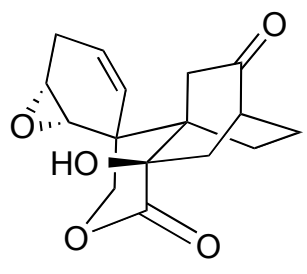

3.28

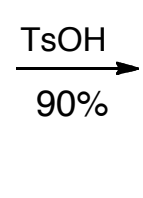

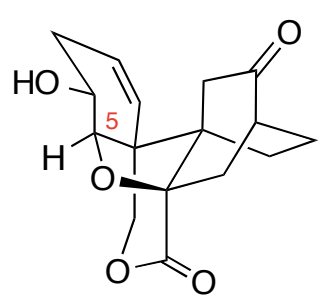

3.29

||

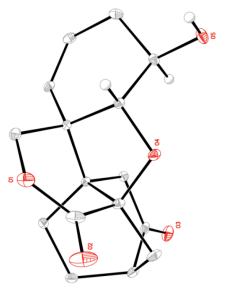

Scheme 3-7 Preparation of 3.29

To correct the C-5 stereocenter, our initial plan was to prepare $\beta$-epoxide 3.30, which a trans diaxial opening of epoxide would provide desired compound 3.31. We then screened several epoxidation conditions such as Payne epoxidation ${ }^{12}$ and hydroxyldirected epoxidation ${ }^{13}$; unfortunately, we only recovered our starting material. These results indicated that the $\beta$-face of the A-ring was very congested and reagents could not reach it.

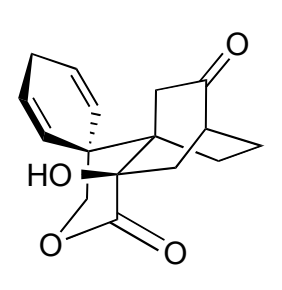

$3.21 a$

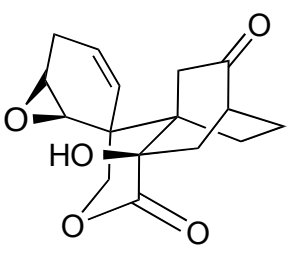

3.30

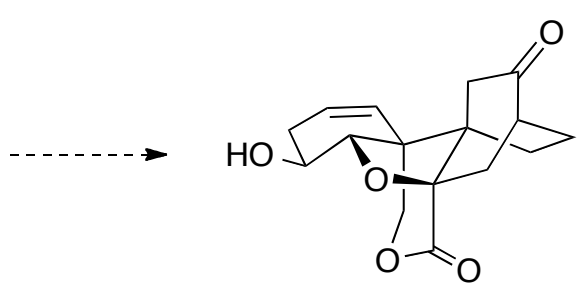

3.31

Scheme 3-8 $\beta$-Epoxide to form trans ring junction 
We then turned our efforts to correct the C-5 center on compound $\mathbf{3 . 2 9}$, hoping to achieve an epimerization reaction on C-5. We first prepared compound $\mathbf{3 . 3 2}$ through a hydrogenation and oxidation sequence on which we planed to test a base-promoted epimerization reaction. ${ }^{14}$ After careful degassing oxygen (with trace oxygen, hydroxylation of ketone $\alpha$ position was observed.), compound $\mathbf{3 . 3 2}$ was subjected to $\mathrm{NaOMe}$ condition and to our delight, a new compound 3.33 was isolated in about $80 \%$ yield. However, X-ray crystallography results revealed structure $\mathbf{3 . 3 3}$ as shown. Instead of epimerization, a transannular aldol reaction occurred.

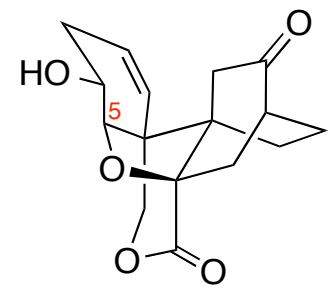

3.29
1) $\mathrm{Pd} / \mathrm{C}, \mathrm{H}_{2}$ $\mathrm{EtOH}, \mathrm{rt}, 12 \mathrm{~h}$

2) $\mathrm{DMP}, \mathrm{CH}_{2} \mathrm{Cl}_{2}$

2 steps, $75 \%$

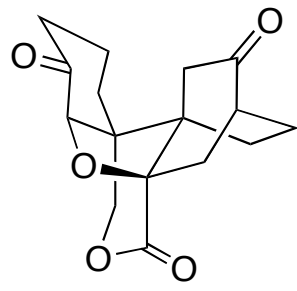

3.32

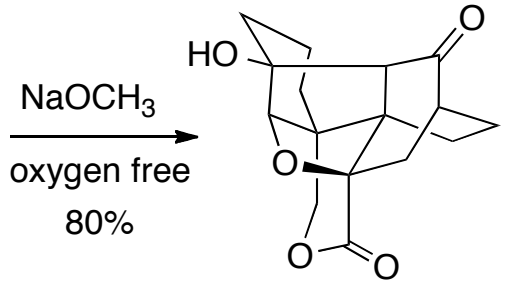

3.33

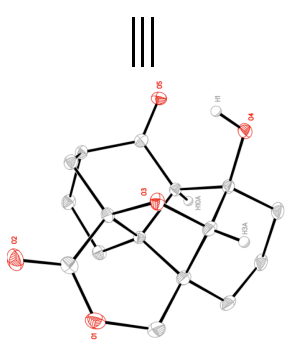

Scheme 3-9 Epimerization of $\mathbf{3 . 3 2}$

In order to circumvent the undesired aldol reaction, we then reduced the C-16 ketone functional group to a methylene through a dithioketal intermediate $\mathbf{3 . 3 4}$. Compound 3.35 and compound 3.36 then would serve as model compounds to explore the epimerization of C-5 center. In our real synthetic plan, the C-16 ketone functional group would be masked as a protected secondary alcohol. 


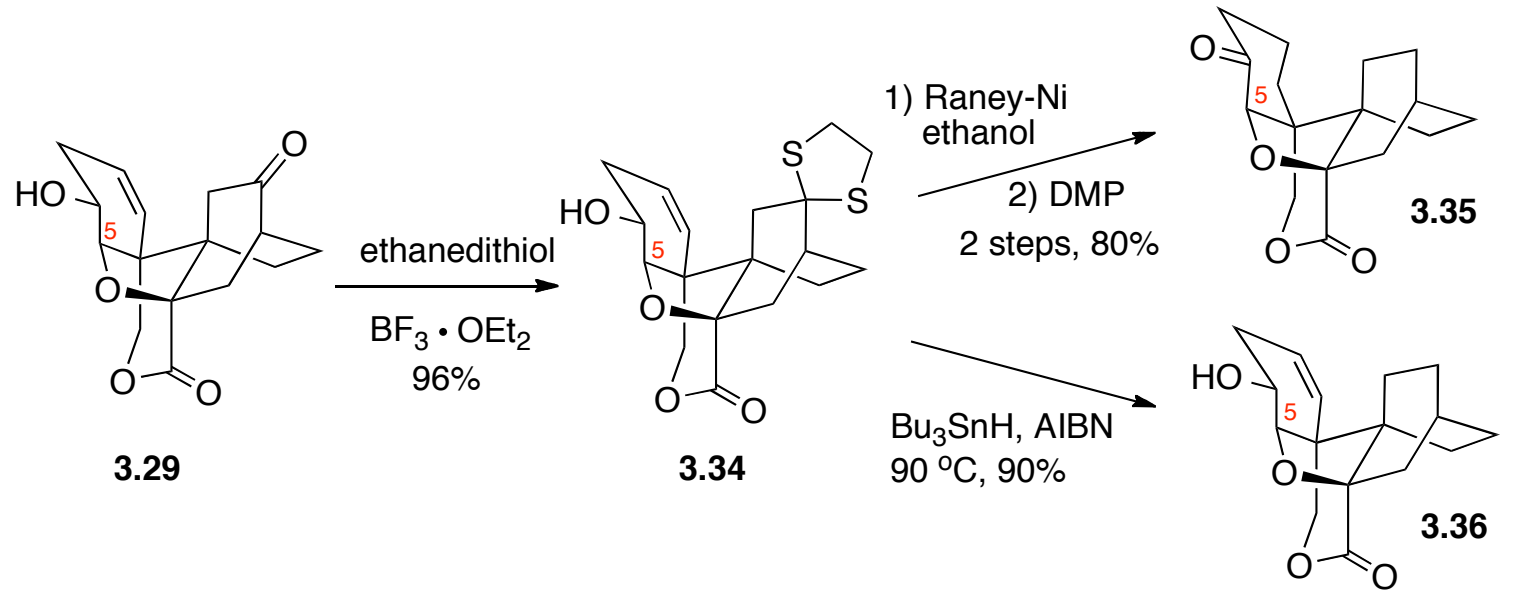

Scheme 3-10 Functionalization of C-16 ketone

We then subjected compound $\mathbf{3 . 3 5}$ to $\mathrm{NaOMe}$ condition and to our disappointment we only recovered starting material 3.35. During the time I was working on this problem, our group developed a trans-de-nitro-hydrogenation protocol to synthesis trans-fused hydro indane motifs. ${ }^{15}$ I wondered if I could use this method to solve the junction problem. We first prepared the bromo compound 3.37 and the following de-bromo-hydrogenation reaction again provided us with a cis-fused compound 3.38. This result indicated hydrogenation process happened on $\alpha$-face of Aring.

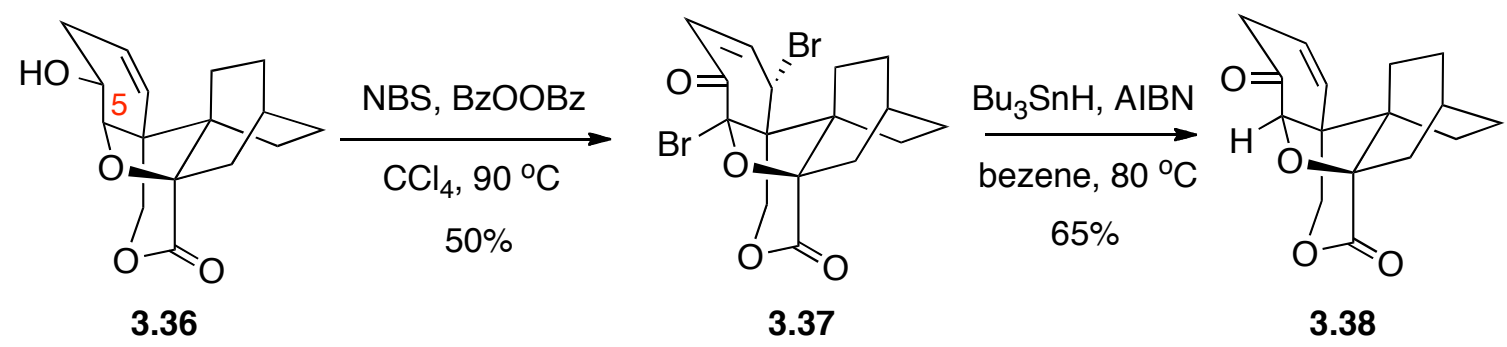

Scheme 3-11 Radical reduction approach to synthesis 3.38 
It seemed that we needed to answer a key question: how to deliver a hydrogen atom to a more congested $\beta$-face in order to solve this junction problem. We soon realized that a possible solution was to design an intramolecular hydrogen transfer process, which allowed for delivery of a hydrogen atom from more congested $\beta$-face of A-ring. As such, we designed a reaction termed "exo-glycal epoxide rearrangement". 16 We envisioned that the $\beta$-epoxidation reaction of $\mathbf{3 . 3 9}$ would provide exo-glycal epoxide 3.40 and our strategically important C-4 hydrogen was pushed into the $\beta$-face of A-ring. Subsequent rearrangement would provide the desired trans-fused structure 3.41. Since glycal epoxide 3.40 was not a stable species, we would take great care when handling it.

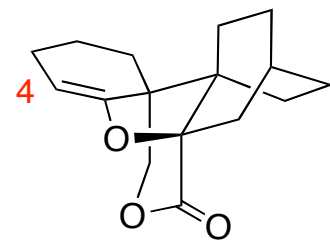

3.39
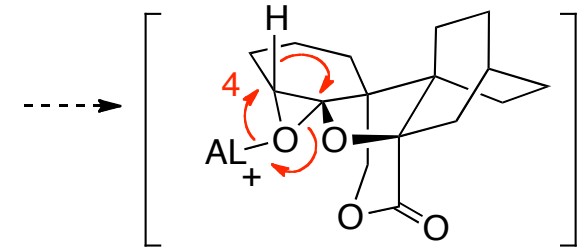

3.40

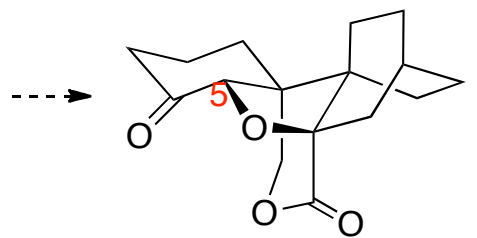

3.41

Scheme 3-12 Exo-glycal epoxide rearrangement design

Our initially approach to prepare junction enol ether 3.39, which involved dehydration of alcohol 3.29, didn't work well and only starting material was recovered with several conditions such as Burgess dehydration and Martin dehydration. This is probably due to the equatorial configuration of the hydroxyl group, which disfavored an E2 elimination. We then reduced compound 3.35 with $\mathrm{NaBH}_{4}$ and the resultant axial alcohol was subjected to the dehydration conditions, which provided the desire compound 3.39 in good yield. 


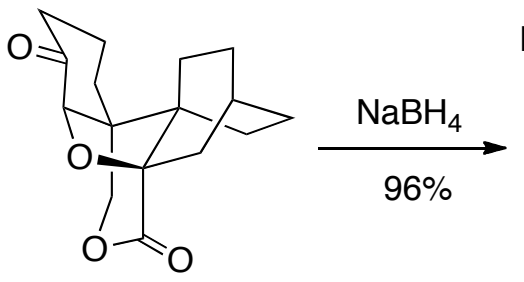

3.35

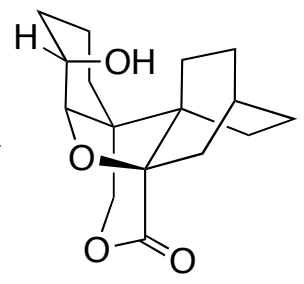

3.42

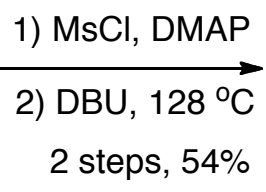

2 steps, $54 \%$

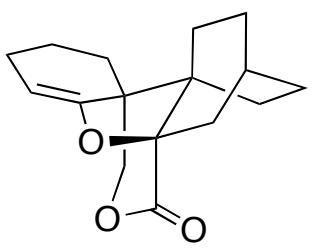

3.39

Scheme 3-13 Preparation of enol ether 3.39

With the key intermediate $\mathbf{3 . 3 9}$ in hand, we treated it first with DMDO, which furnished the exo-glycal expoxide 3.40. Without isolation, in situ treatment of $\mathrm{BF}_{3} \cdot \mathrm{OEt}_{2}{ }^{17}$ generated the target compound $\mathbf{3 . 4 1}$ as a single isomer, whose structure was verified by X-ray analysis of the reduced compound $\mathbf{3 . 4 3}$.

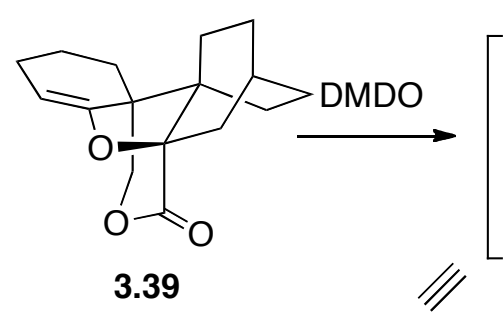

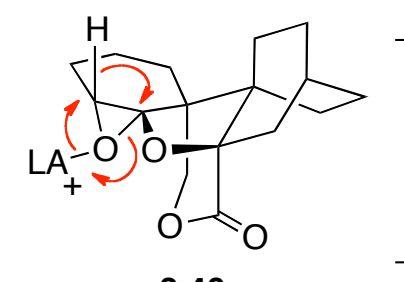

3.40

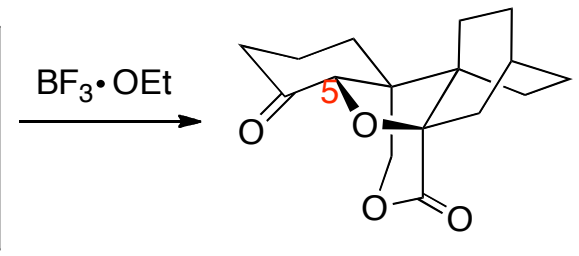

3.41

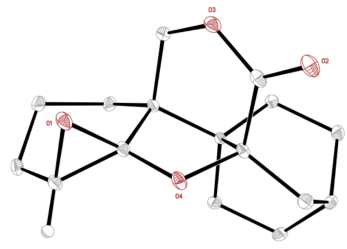

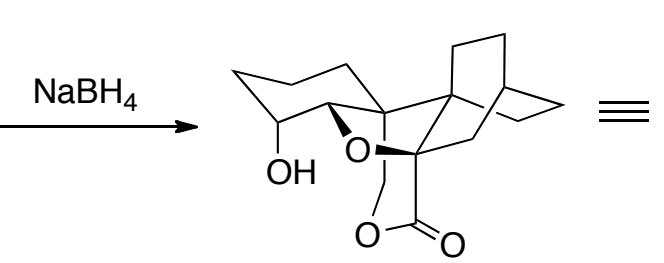

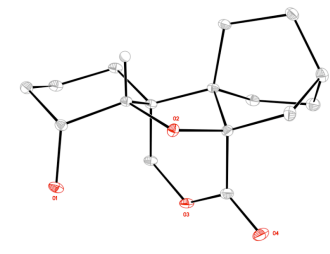

3.43

Scheme 3-14 Preparation of compound 3.43 
We then further installed the gem-dimethyl group using Oppolzer's sequence ${ }^{4}$ to compound 3.41. As such, olefination of compound 3.41 afforded exo-methylene 3.44 without epimerization of the junction proton. Cyclopropanation and the following hydrogenation furnished gem-dimethyl compound 3.46.

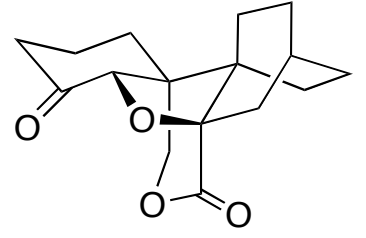

3.41

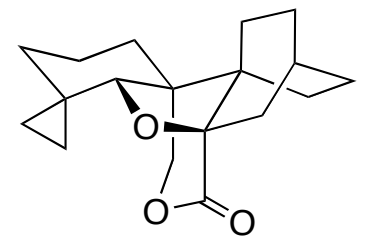

3.45

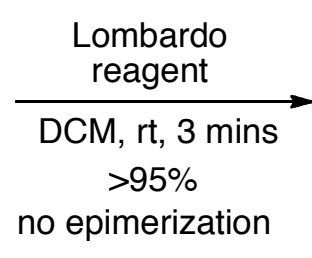

$$
\text { no epimerization }
$$

Scheme 3-15 Preparation of compound 3.46

\section{3 Experimental}

\section{General information}

Unless otherwise noted, all reactions were performed under an argon atmosphere using flame-dried glassware. $\mathrm{CH}_{2} \mathrm{Cl}_{2}(\mathrm{DCM})$ was distilled over $\mathrm{CaH}_{2}$ under argon atmosphere. THF was distilled over sodium/benzophenone ketyl under argon atmosphere. Anhydrous toluene, hexane, ether were purchased and used without further purification. All reagents 
were commercially available and used without further purification unless indicated otherwise. Thin layer chromatography (TLC) was performed on Silica Gel 60 F254 plates and was visualized with UV light and $\mathrm{KMnO}_{4}$ stain. Preparative thin layer chromatography was performed with Merck silica gel $60-\mathrm{F} 254$ coated $0.50 \mathrm{~mm}$ plates. Flash chromatography was performed with Sorbent Tech. silica gel 60. Yields reported are for isolated, spectroscopically pure compounds. NMR spectra were recorded on 300, 400 or $500 \mathrm{MHz}$ instruments. The residual solvent protons $\left({ }^{1} \mathrm{H}\right)$ or the solvent carbons $\left({ }^{13} \mathrm{C}\right)$ were used as internal standards. ${ }^{1} \mathrm{H}$ NMR data are presented as follows: chemical shift in ppm downfield from tetramethylsilane (multiplicity, coupling constant, integration). The following abbreviations are used in reporting NMR data: s, singlet; br $\mathrm{s}$, broad singlet; d, doublet; t, triplet; q, quartet; qt, quartet of triplets; dd, doublet of doublets; dt, doublet of triplets; $\mathrm{AB}, \mathrm{AB}$ quartet; m, multiplet. High-resolution mass spectra were recorded by the Columbia University Mass Spectrometry Core facility on a JEOL HX110 spectrometer.

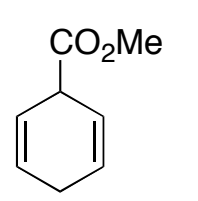

3.16<smiles>O=C1C=C(Cl)CCC1</smiles>

3.17

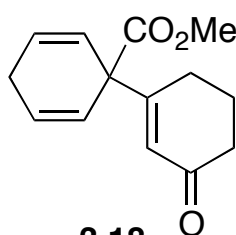

3.18

Compound 3.18: An oven-dried, $250 \mathrm{~mL}$ round bottom flask containing a stir bar was capped with a rubber septum. This flask was then charged with diisopropylamine (5.6 $\mathrm{mL}, 40 \mathrm{mmol})$ and $100 \mathrm{~mL}$ of anhydrous THF under argon. At $0{ }^{\circ} \mathrm{C}, \mathrm{n}-\mathrm{BuLi}(16 \mathrm{~mL}, 40$ mmol) was injected dropwise in 10 mins. The reaction mixture was stirred at this temperature for additional 10 mins before cooled down to $-78{ }^{\circ} \mathrm{C}$. After stirring at $-78{ }^{\circ} \mathrm{C}$ 
for 20 mins, a solution of $7(5.2 \mathrm{~g}, 38 \mathrm{mmol})$ in $10 \mathrm{~mL}$ of THF was injected slowly to the flask in 15 mins. After addition of compound 7, the reaction mixture was stirred at $-78{ }^{\circ} \mathrm{C}$ for additional 40 mins. Then a solution of $8(7.3 \mathrm{~g}, 57 \mathrm{mmol})$ in $15 \mathrm{~mL}$ of THF was injected in 5 mins. 30 mins later, the reaction was quenched by addition of $30 \mathrm{~mL}$ of water, extracted with ethyl acetate and dried over anhydrous $\mathrm{MgSO}_{4}$. The drying agent was filtered and solvents were removed under reduced pressure using rotavap. The residues were purified by flash chromatography to give a colourless oil. $(3.5 \mathrm{~g}, 40 \%$ yield). ${ }^{1} \mathrm{H}$ NMR $\left(\mathrm{CDCl}_{3}, 300 \mathrm{MHz}\right): \delta 6.03-5.97(\mathrm{~m}, 2 \mathrm{H}), 5.90(\mathrm{~s}, 1 \mathrm{H}), 5.87-5.82(\mathrm{~m}$, $2 \mathrm{H}), 3.73(\mathrm{~s}, 3 \mathrm{H}), 2.69-2.67(\mathrm{~m}, 2 \mathrm{H}), 2.38-2.34(\mathrm{~m}, 2 \mathrm{H}), 2.30-2.26(\mathrm{~m}, 2 \mathrm{H}), 2.00-1.92$ (m, 2H); ${ }^{13} \mathrm{C} \mathrm{NMR}\left(\mathrm{CDCl}_{3}, 75 \mathrm{MHz}\right): \delta 199.7,172.3,164.6,127.3,126.2,124.3,54.3$, 52.6, 37.4, 26.4, 25.9, 22.9; IR (neat): $\mathrm{cm}^{-1}$ 2951, 2869, 1734, 1671, 1250, 1225, 1040; HRMS (FAB, $m / z)$ calcd for $\mathrm{C}_{14} \mathrm{H}_{17} \mathrm{O}_{3}[\mathrm{M}+\mathrm{H}]^{+}$233.113, found 233.118.
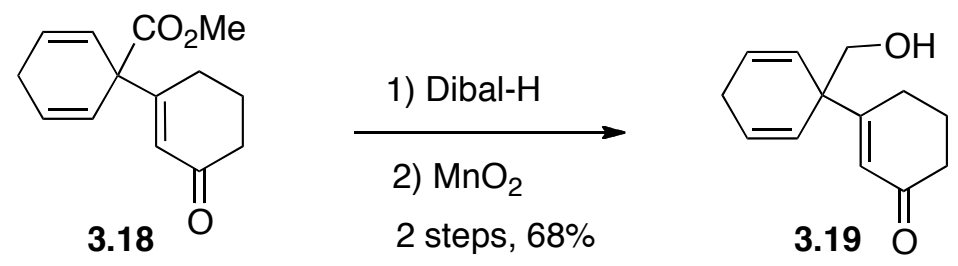

Compound 3.19: compound 3.18 (1.5 g, $6.4 \mathrm{mmol})$ was dissolved in anhydrous DCM under argon and cooled to $-78^{\circ} \mathrm{C} .25 .8 \mathrm{~mL}$ of DIBAL-H in hexane $(1 \mathrm{M})$ was injected into the solution slowly and the reaction mixture was stirred for 50 mins at this temperature. Then the reaction was quenched with $0.5 \mathrm{~mL}$ of ethyl acetate and $5 \mathrm{~g}$ of sodium sulfate decahydrate. After warming up to room temperature, the mixture was stirred for additional 1 hour, dried over $\mathrm{MgSO}_{4}$ and filtered. The solvent was removed 
using rotovap and the residue was used directly in the next step. The residue was dissolved in DCM in a round bottom flask and $30 \mathrm{~g}$ of $\mathrm{MnO}_{2}$ was added. The reaction mixture was stirred for $40 \mathrm{~min}$ and the solid was filtered (The reaction process was monitored carefully under TLC). After removing of the solvent with rotavap, the residues were purified with flash chromatography to give a colourless oil. ( $890 \mathrm{mg}, 68 \%$ yield). ${ }^{1} \mathrm{H} \mathrm{NMR}\left(\mathrm{CDCl}_{3}, 300 \mathrm{MHz}\right): \delta 6.03(\mathrm{ddd}, J=10.4,3.2,3.2 \mathrm{~Hz}, 2 \mathrm{H}), 5.91(\mathrm{~s}, 1 \mathrm{H}), 5.51$ (ddd, $J=10.4,2.0,2.0 \mathrm{~Hz}, 2 \mathrm{H}), 3.65(\mathrm{~d}, J=5.6 \mathrm{~Hz}, 2 \mathrm{H}), 2.70(\mathrm{dd}, J=3.2,3.2 \mathrm{~Hz}, 2 \mathrm{H})$, 2.35-2.30 (m, 4H), $\quad 1.95-1.85 \quad(\mathrm{~m}, \quad 3 \mathrm{H}) ; \quad{ }^{13} \mathrm{C} \quad \mathrm{NMR} \quad\left(\mathrm{CDCl}_{3}, \quad 75 \quad \mathrm{MHz}\right)$ : $\delta 200.2,167.1,128.2,126.7,125.2,67.1,48.6,37.4,26.7,26.2,22.9 ;$ IR (neat): $\mathrm{cm}^{-1}$ 3416, 2944, 2869, 1652, 1348, 1033; HRMS $(\mathrm{FAB}, \mathrm{m} / \mathrm{z})$ calcd for $\mathrm{C}_{13} \mathrm{H}_{17} \mathrm{O}_{2}[\mathrm{M}+\mathrm{H}]^{+}$ 205.123, found 205.122.
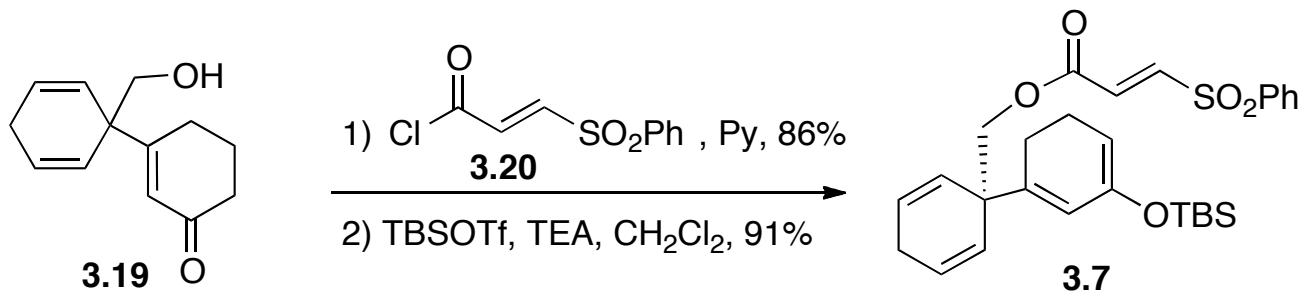

Compound 3.7: compound 3.19 (1.35 g, $6.6 \mathrm{mmol})$ and pyridine $(10.4 \mathrm{~mL}, 12 \mathrm{mmol})$ was dissolved in DCM under argon at $0{ }^{\circ} \mathrm{C}$. Acyl chloride $3.20(1.67 \mathrm{~g}, 7.2 \mathrm{mmol})$ in 5 $\mathrm{mL}$ of DCM was injected slowly. After addition of acyl chloride solution, the reaction mixture was stirred at this temperature for 1 hour. The reaction was then quenched by addition of methanol and the solvent was removed under the reduced pressure. The residue was purified using flash chromatography. The pure product and TEA $(1.6 \mathrm{~mL}, 12$ mmol) was dissolved in anhydrous DCM under argon at $-78{ }^{\circ} \mathrm{C}$, TBSOTf $(2.3 \mathrm{~mL}, 10$ 
mmol) was injected dropwise and the reaction mixture was stirred at this temperature for 12 hours. The reaction mixture was quenched with water, extracted with ether and dried over $\mathrm{MgSO}_{4}$. The solid was filtered and the solvent was removed with rotavap. Then the residues were purified by flash chromatography using TEA deactivated silica gel and afforded a colourless oil. (2,4 g, 72\% yield for two steps). ${ }^{1} \mathrm{H}$ NMR $\left(\mathrm{CDCl}_{3}, 300 \mathrm{MHz}\right): \delta$ $7.92(\mathrm{~d}, J=7.6 \mathrm{~Hz}, 2 \mathrm{H}), 7.71-7.67(\mathrm{~m}, 1 \mathrm{H}), 7.61-7.57(\mathrm{~m}, 2 \mathrm{H}), 7.29(\mathrm{~d}, J=15.2 \mathrm{~Hz}, 1 \mathrm{H})$, $6.82(\mathrm{~d}, J=15.2 \mathrm{~Hz}, 1 \mathrm{H}), 5.92-5.89(\mathrm{~m}, 2 \mathrm{H}), 5.49-5.46(\mathrm{~m}, 3 \mathrm{H}), 4.80-4.78(\mathrm{~m}, 1 \mathrm{H}), 4.27$ (s, 2H), 2.62 (br s, 2H), 2.12-1.99 (m, 4H), $0.91(\mathrm{~s}, 9 \mathrm{H}), 0.11(\mathrm{~s}, 6 \mathrm{H}) ;{ }^{13} \mathrm{C} \mathrm{NMR}\left(\mathrm{CDCl}_{3}\right.$, $75 \mathrm{MHz}): \delta 163.4,148.7,143.3,143.0,138.5,134.4,130.9,129.6,128.3,127.4,126.7$, 121.1, 101.1, 69.5, 45.1, 26.2, 25.7, 24.1, 22.4, 18.1, -4.5; IR (neat): $\mathrm{cm}^{-1} 2951,2858$, 1729, 1670, 1448, 1294; HRMS (FAB, $m / z$ ) calcd for $\mathrm{C}_{28} \mathrm{H}_{37} \mathrm{O}_{5} \mathrm{SiS}[\mathrm{M}+\mathrm{H}]^{+}$513.2131, found 513.2131.

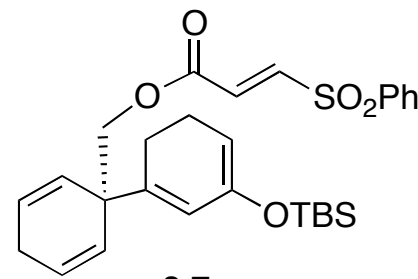

3.7

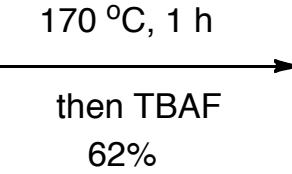

$62 \%$

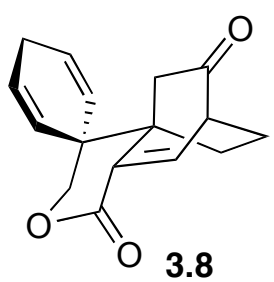

3.8

Compound 3.8: compound $3.7(513 \mathrm{mg}, 1 \mathrm{mmol})$ was dissolved in $50 \mathrm{~mL}$ of anhydrous toluene in a high-pressure tube. The reaction mixture was degassed with argon and then stirred at $166{ }^{\circ} \mathrm{C}$ for 1 hour. After cooling to room temperature and the mixture was transferred to a round bottom flask and the solvent was removed with rotavap. The residue was dissolved in THF and cooled to $0{ }^{\circ} \mathrm{C}$. Then TBAF in THF $(1.5 \mathrm{~mL}, 1.5$ mmol) was added. 2 hours later the reaction was quenched with saturated ammonium chloride solution, extracted with ethyl acetate $(3 \times 10 \mathrm{~mL})$ and dried over $\mathrm{MgSO}_{4}$. The 
drying agent was removed by filtration and the solvent was removed under reduced pressure. The residue was purified using flash chromatography (156 mg, 62\%) ${ }^{1} \mathrm{H}$ NMR $\left(\mathrm{CDCl}_{3}, 400 \mathrm{MHz}\right): \delta 7.55(\mathrm{~d}, J=6.8 \mathrm{~Hz}, 1 \mathrm{H}), 6.12-6.07(\mathrm{~m}, 2 \mathrm{H}), 5.68-5.60(\mathrm{~m}, 2 \mathrm{H})$, $4.30(\mathrm{~d}, J=10.0 \mathrm{~Hz}, 1 \mathrm{H}), 4.15(\mathrm{~d}, J=10.0 \mathrm{~Hz}, 1 \mathrm{H}), 3.49-3.47(\mathrm{~m}, 1 \mathrm{H}), 2.78-2.76(\mathrm{~m}$, 2H), $2.15(\mathrm{~d}, J=18 \mathrm{~Hz}, 1 \mathrm{H}), 2.08-1,99(\mathrm{~m}, 2 \mathrm{H}), 1.88-1.60(\mathrm{~m}, 3 \mathrm{H}) ;{ }^{13} \mathrm{C} \mathrm{NMR}\left(\mathrm{CDCl}_{3}\right.$, $100 \mathrm{MHz}): \delta 208.5,163.5,143.1,135.7,129.3,128.6,124.4,123.3,71.7,50.1,46.5$, 41.3, 38.9, 26.9, 25.9, 23.1; IR (neat): $\mathrm{cm}^{-1}$ 2946, 1720, 1616, 1242, 1075;

HRMS (FAB, $m / z$ ) calcd for $\mathrm{C}_{16} \mathrm{H}_{17} \mathrm{O}_{3}[\mathrm{M}+\mathrm{H}]^{+}$257.1178, found 257.1178.

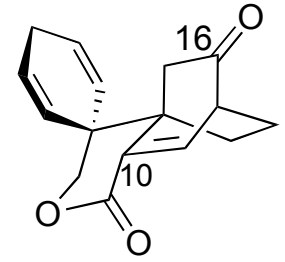

3.8

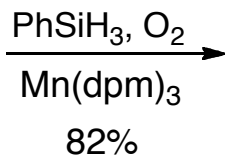

$82 \%$

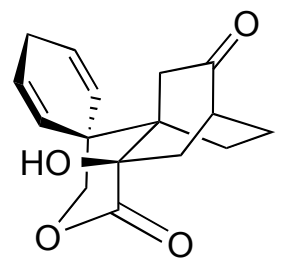

$3.21 a$

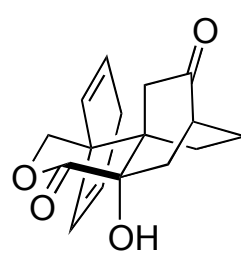

3.21b

Compound 3.21: An oven-dried, $50 \mathrm{~mL}$ round bottom flask containing a stir bar was capped with a rubber septum. This flask was then charged with 3.8 (125 mg, $0.5 \mathrm{mmol}$ ), $\mathrm{Mn}(\mathrm{dpm})_{3}(6 \mathrm{mg}, 0.01 \mathrm{mmol}), 8 \mathrm{~mL}$ of anhydrous DMC and $8 \mathrm{~mL}$ of anhydrous IPA under argon. This mixture was stirred until a homogenous solution formed. Then $\mathrm{PhSiH}_{3}$ $(150 \mu \mathrm{L}, 1.25 \mathrm{mmol})$ was injected and the reaction mixture was stirred under a balloon pressure of oxygen. 40 mins later, the ice-bath was removed and the reaction mixture was stirred at room temperature for $10 \mathrm{~min}$. Then the oxygen balloon was removed and the reaction mixture was stirred at $0{ }^{\circ} \mathrm{C} .5 \mathrm{~mL}$ of saturated $\mathrm{Na}_{2} \mathrm{~S}_{2} \mathrm{O}_{3}$ solution was added to the reaction mixture and the mixture was stirred at $0{ }^{\circ} \mathrm{C}$ for 1 hour. The mixture was extracted with Ethyl Acetate and the extract was dried over MgSO4. The drying agent 
was filtered and the solvent was removed with rotavap. The residue was purified with flash chromatography to afford a diastereomer mixture 13a and 13b. $(110 \mathrm{mg}, 21 \mathrm{a}: 21 \mathrm{~b}=$ 1.5:1, 80\%) 21a: ${ }^{1} \mathrm{H} \mathrm{NMR}\left(\mathrm{CDCl}_{3}, 400 \mathrm{MHz}\right): \delta$ 6.06-6.02 (m, 2H), 5.96-5.92 (m, $\left.1 \mathrm{H}\right)$, $5.60(\mathrm{dd}, J=10.4,2 \mathrm{~Hz}, 1 \mathrm{H}), 4.45(\mathrm{~d}, J=11.6 \mathrm{~Hz}, 1 \mathrm{H}), 4.28(\mathrm{~d}, J=11.6 \mathrm{~Hz}, 1 \mathrm{H}), 2.77$ (dd, $J=14.8,2.4 \mathrm{~Hz}, 1 \mathrm{H}), 2.70(\mathrm{dd}, J=18.8,3.2 \mathrm{~Hz}, 1 \mathrm{H}), 2.62$ (br s, 2H), 2.45 (br s, 1H), 2.29 (br s, $1 \mathrm{H}), 2.00(\mathrm{~d}, J=18.4 \mathrm{~Hz}, 1 \mathrm{H}), 1.95-1.60(\mathrm{~m}, 5 \mathrm{H}) ;{ }^{13} \mathrm{C} \mathrm{NMR}\left(\mathrm{CDCl}_{3}, 100\right.$ MHz): $\delta 213.6,172.9,128.9,128.3,126.3,125.7,76.1,73.9,45.8,41.9,39.8,39.7,39.4$, 26.2, 23.0, 22.1; IR (neat): $\mathrm{cm}^{-1}$ 3401, 2916, 2849,1728, 1075; HRMS (FAB, m/z) calcd for $\mathrm{C}_{16} \mathrm{H}_{19} \mathrm{O}_{4}[\mathrm{M}+\mathrm{H}]^{+} 275.1283$, found 275.1278 .

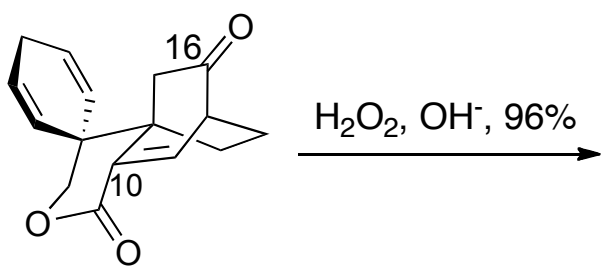

3.8

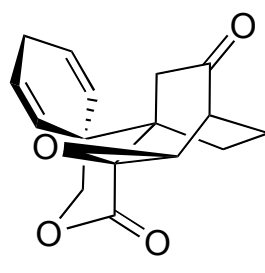

3.26

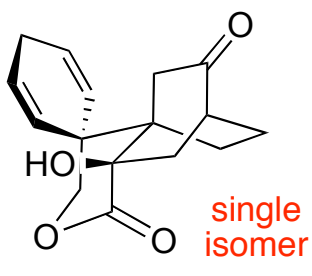

$3.21 \mathrm{a}$

Compound 3.21a: Compound 3.8 (64 mg, $0.25 \mathrm{mmol}$ ) was dissolved in $5 \mathrm{~mL}$ of methanol at $0{ }^{\circ} \mathrm{C}$ under argon. $30 \% \mathrm{H}_{2} \mathrm{O}_{2}(32 \mu \mathrm{L}, 0.28 \mathrm{mmol})$ was injected into the reaction flask followed by an addition of $3 \mathrm{~N} \mathrm{NaOH}$ aqueous solution $(20 \mu \mathrm{L}, 0.06$ mmol). 3 hours later, $3 \mathrm{~N} \mathrm{NaOH}$ solution $(20 \mu \mathrm{L}, 0.06 \mathrm{mmol})$ was added again to the reaction mixture and the reaction was stirred at $0{ }^{\circ} \mathrm{C}$ for additional 3 hours. The reaction was quenched with $200 \mu \mathrm{L}$ of $1 \mathrm{~N} \mathrm{HCl}$ solution, extracted with ethyl acetate and the extract was dried over $\mathrm{MgSO}_{4}$. After filtration of the drying agent and removing of the solvent, the residue was purified with flash chromatography to afford $\mathbf{3 . 2 6}$ (65 $\mathrm{mg}, 95 \%)$. Compound 3.26 (14 mg, $0.05 \mathrm{mmol})$ was dissolved in $5 \mathrm{~mL}$ of anhydrous DCM and 
magnesium diiodide (42 $\mathrm{mg}, 0.15 \mathrm{mmol}$ ) was added to this solution. The reaction mixture was stirred at $45{ }^{\circ} \mathrm{C}$ for about 40 mins (Monitored under TLC). After cooling down to room temperature, the reaction mixture was filtered and the solvent was removed using rotavap. The residue and $\mathrm{Bu}_{3} \mathrm{SnH}(43 \mu \mathrm{L}, 0.15 \mathrm{mmol})$ were dissolved in anhydrous toluene and the solution was degassed three times with argon. After addition of $5 \mathrm{mg}$ of AIBN, the reaction mixture was heated under reflux for 1 hour. Then the reaction mixture cooled down to room temperature and the toluene was removed with rotavap. The residue was purified with flash chromatography to afford $\mathbf{2 1 a}(7 \mathrm{mg}, 50 \%)$.

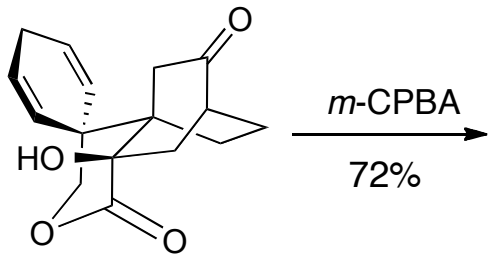

$3.21 a$

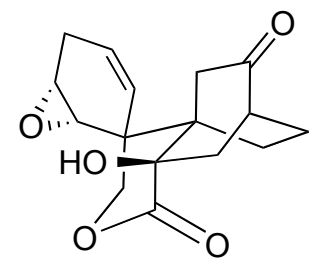

3.28

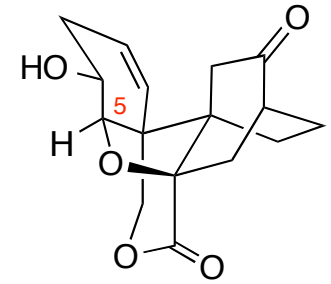

3.29

Compound 3.29: $\mathrm{m}$-CPBA (42 mg, $0.18 \mathrm{mmol}$ ) was added to a solution of 21a (33 $\mathrm{mg}$, $0.12 \mathrm{mmol}$ ) in $5 \mathrm{~mL}$ of anhydrous $\mathrm{DCM}$ at $0{ }^{\circ} \mathrm{C} .12$ hour later, the ice-bath was removed and the reaction mixture was stirred at room temperature for additional 12 hours. The reaction process was monitored under TLC until the disappearance of starting material. Then the reaction was quenched with $\mathrm{Na}_{2} \mathrm{~S}_{2} \mathrm{O}_{3}$ solution, extracted with ethyl acetate and the extract was dried over $\mathrm{MgSO}_{4}$. The drying agent was filtered and the solvent was removed under reduced pressure. The residue was purified using flash chromatography to afford $3.28(25 \mathrm{mg}, 72 \%) .{ }^{1} \mathrm{H}$ NMR $\left(\mathrm{CDCl}_{3}, 400 \mathrm{MHz}\right): \delta 5.72-5.67(\mathrm{~m}, 1 \mathrm{H}), 5.22(\mathrm{~d}, J=$ $10.8 \mathrm{~Hz} 1 \mathrm{H}), 4.72(\mathrm{~d}, J=11.6 \mathrm{~Hz}, 1 \mathrm{H}), 4.30(\mathrm{~d}, J=11.6 \mathrm{~Hz}, 1 \mathrm{H}), 4.15-4.13(\mathrm{~m}, 1 \mathrm{H})$, 3.40-3.39 (m, 1H), 2.82-2.72 (m, 2H), $2.62(\mathrm{dd} J=18.4,5.6 \mathrm{~Hz}, 1 \mathrm{H}), 2.51-2.41(\mathrm{~m}, 3 \mathrm{H})$, 
$2.10(\mathrm{~d}, J=18 \mathrm{~Hz}, 1 \mathrm{H}), 2.00-1.65(\mathrm{~m}, 5 \mathrm{H}) ;{ }^{13} \mathrm{C} \mathrm{NMR}\left(\mathrm{CDCl}_{3}, 100 \mathrm{MHz}\right)$ $\delta 212.5,172.5,125.8,124.5,73.5,65.8,60.4,54.5,51.9,45.0,41.6,40.0,38.9,25.1$, 23.5, 21.7; HRMS (FAB, $m / z$ ) calcd for $\mathrm{C}_{16} \mathrm{H}_{19} \mathrm{O}_{5}[\mathrm{M}+\mathrm{H}]^{+}$291.1219, found 291.1232.

Compound 3.29: compound 3.28 (12 mg, $0.04 \mathrm{mmol})$ dissolved in anhydrous DCM at room temperature and p-TSA monohydrate $(2 \mathrm{mg}, 0.01 \mathrm{mmol})$ was added to this solution. The reaction mixture was stirred at this temperature for about 12 hours. The solvent was removed under reduced pressure and the residue was purified using flash chromatography to afford compound $\mathbf{3 . 2 9}(11 \mathrm{mg}, 90 \%) .{ }^{1} \mathrm{H} \mathrm{NMR}\left(\mathrm{CDCl}_{3}, 400 \mathrm{MHz}\right): \delta 5.99-5,95(\mathrm{~m}$, 1H), 5.37 (dd, $J=9.6,2.8 \mathrm{~Hz}, 1 \mathrm{H}), 4.37$ (d, $J=11.2 \mathrm{~Hz}, 1 \mathrm{H}), 4.17$ (d, $J=9.2 \mathrm{~Hz}, 1 \mathrm{H})$, 4.00-3.97 (m, 2H), 3.09 (dd, $J=15.2,4 \mathrm{~Hz}, 1 \mathrm{H}), 2.48$ (ddd, $J=17.2,6.0,6.0 \mathrm{~Hz}, 1 \mathrm{H})$,

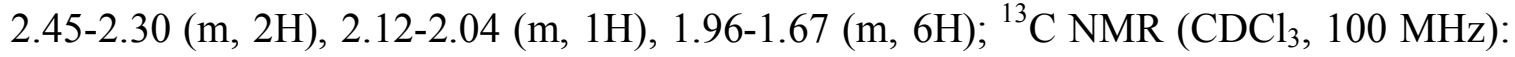
$\delta 211.4,169.1,131.1,121.1,88.3,83.4,76.1,69.8,49.2,46.5,42.9,41.6,30.7,29.2,21.4$ , 20.2; IR (neat): $\mathrm{cm}^{-1}$ 3401, 2916, 2849,1728, 1075; HRMS (FAB, m/z) calcd for $\mathrm{C}_{16} \mathrm{H}_{19} \mathrm{O}_{5}[\mathrm{M}+\mathrm{H}]^{+}$291.1219, found 291.1232.

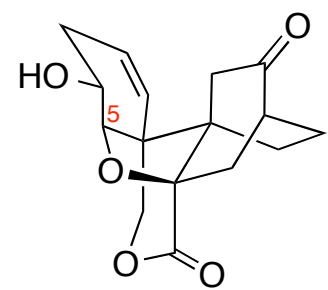

3.29

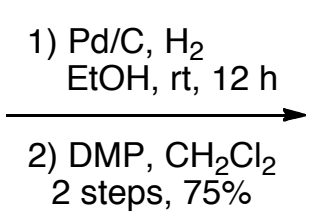

2 steps, $75 \%$

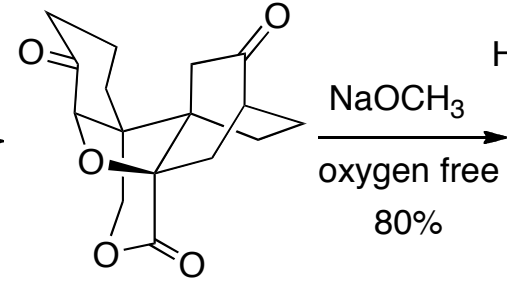

3.32

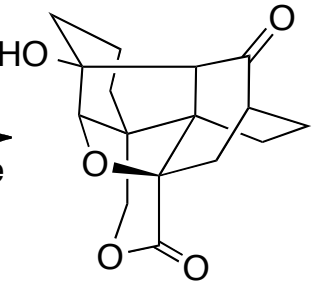

3.33

Compound 3.33: compound 3.29 (30 mg, $0.1 \mathrm{mmol}$ ) was dissolved in anhydrous ethanol at room temperature and palladium on charcoal $(3 \mathrm{mg})$ was added to this solution. The 
reaction mixture was degassed with hydrogen for three times before stirred under a balloon pressure of hydrogen for 8 hours at room temperature. The reaction mixture was then filtered with a short column of silica gel. The solvent was removed under reduced pressure and the residue was dissolved in $5 \mathrm{~mL}$ of anhydrous DCM. To this solution, Dess-Marin reagent $(130 \mathrm{mg}, 0.3 \mathrm{mmol})$ was added and the reaction was stirred at room temperature for 8 hours. Then the reaction was quenched with $\mathrm{Na}_{2} \mathrm{~S}_{2} \mathrm{O}_{3}$, extracted with ethyl acetate and the extract was dried over $\mathrm{MgSO}_{4}$. The drying agent was filtered and the solvent was removed under reduced pressure. The residue was purified using flash chromatography to afford compound $3.32(23 \mathrm{mg}, 75 \%) .{ }^{1} \mathrm{H} \mathrm{NMR}\left(\mathrm{CDCl}_{3}, 400 \mathrm{MHz}\right): \delta$ $4.53(\mathrm{~s}, 1 \mathrm{H}), 4.40(\mathrm{~d}, J=11.2 \mathrm{~Hz}, 1 \mathrm{H}), 4.32(\mathrm{~d}, J=11.2 \mathrm{~Hz}, 1 \mathrm{H}), 3.13(\mathrm{dd}, J=15.2,4.0$ $\mathrm{Hz}, 1 \mathrm{H}), 2.47-2.42(\mathrm{~m}, 2 \mathrm{H}), 2.22(\mathrm{~d}, J=18.0 \mathrm{~Hz}, 1 \mathrm{H}), 2.10(\mathrm{~d}, J=15.2,1 \mathrm{H}), 2.08-1.57$ $(\mathrm{m}, 10 \mathrm{H}) ;{ }^{13} \mathrm{C} \mathrm{NMR}\left(\mathrm{CDCl}_{3}, 100 \mathrm{MHz}\right): \delta 210.1,206.6,168.7,86.7,83.4,76.1,48.9$, 47.4, 41.5, 41.1, 37.9, 28.9, 24.1, 21.2, 20.1; IR (neat): $\mathrm{cm}^{-1}$ 2956, 1730, 1133; HRMS (FAB, $m / z$ ) calcd for $\mathrm{C}_{16} \mathrm{H}_{19} \mathrm{O}_{5}[\mathrm{M}+\mathrm{H}]^{+}$291.1232, found 291.1246.

Compound 3.33: compound 3.22 (5 mg, $17 \mu \mathrm{mol})$ was dissolved in anhydrous methanol at room temperature and sodium methoxide $(0.9 \mathrm{mg}, 17 \mu \mathrm{mol})$ was added to this solution. The reaction mixture was degassed using the freeze-pump-thaw technique and then stirred at $40{ }^{\circ} \mathrm{C}$ for 36 hours. After quenching with $1 \mathrm{~N} \mathrm{HCl}$, the mixture was extracted with ethyl acetate and the extract was dried over $\mathrm{MgSO}_{4}$. The drying agent was filtered and the solvent was removed under reduced pressure. The residue was purified using flash chromatography. ${ }^{1} \mathrm{H} \mathrm{NMR}\left(\mathrm{CDCl}_{3}, 400 \mathrm{MHz}\right): \delta 4.50(\mathrm{~d}, J=12.4 \mathrm{~Hz}, 1 \mathrm{H}), 4.10(\mathrm{~d}$, 
$J=12 \mathrm{~Hz}, 1 \mathrm{H}), 3.79(\mathrm{~s}, 1 \mathrm{H}), 3.22(\mathrm{~s}, 1 \mathrm{H}), 3.17(\mathrm{dd}, J=16,3.2 \mathrm{~Hz}, 1 \mathrm{H}), 2.57-2.54(\mathrm{~m}$, 2H), 2.20-2.16 (m, 1H), 1.95-1.65 (m, 8H); $\left.{ }^{13} \mathrm{C} \mathrm{NMR} \mathrm{(CDCl} 3,100 \mathrm{MHz}\right): \delta 214.0$,

169.2, 91.8, 81.1, 78.3, 72.0, 53.0, 52.9, 47.4, 43.4, 35.9, 33.1, 25.0, 21.5, 19.0, 17.4;

HRMS (FAB, $m / z$ ) calcd for $\mathrm{C}_{16} \mathrm{H}_{19} \mathrm{O}_{5}[\mathrm{M}+\mathrm{H}]^{+}$291.1232, found 291.1247.

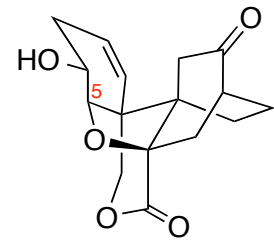

3.29

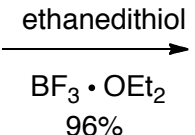

$96 \%$

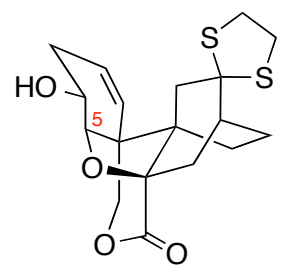

3.34

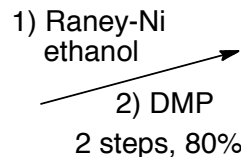

2 steps, $80 \%$

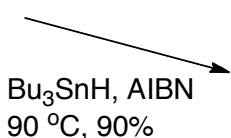

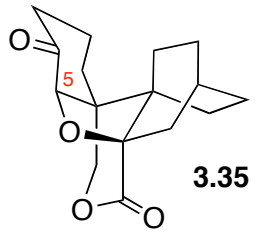

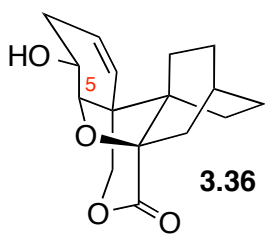

Compound 3.35: compound $3.29(29 \mathrm{mg}, 0.1 \mathrm{mmol})$ and ethanedithiol (27 $\mu \mathrm{L}, 0.3$ mmol) dissolved in anhydrous DCM at $0{ }^{\circ} \mathrm{C}$. To this solution, boron trifluoride etherate $(5 \mu \mathrm{L}, 50 \mu \mathrm{mol})$ was injected and the reaction mixture was stirred at $0{ }^{\circ} \mathrm{C}$ for 6 hours. After quenching with $\mathrm{NaHCO}_{3}$ solution, the mixture was extracted with ethyl acetate and the extract was dried over MgSO4. The drying agent was filtered and the solvent was removed under reduced pressure. The residue was purified using flash chromatography. The pure thioketal dissolved in anhydrous ethanol. To this solution, $150 \mathrm{mg}$ of Raney-Ni was added and the reaction mixture was stirred at $75{ }^{\circ} \mathrm{C}$ for 4 hours. Then the mixture was filtered through a short silica gel column using ethyl acetate as eluent. The solvent was removed under reduced pressure and the residue dissolved in anhydrous DCM. Dess Martin periodinane $(120 \mathrm{mg}, 0.28 \mathrm{mmol})$ was added and the reaction mixture was stirred at room temperature for 8 hours. Quenched with $\mathrm{Na}_{2} \mathrm{~S}_{2} \mathrm{O}_{3}$, the mixture was extracted with 
ethyl acetate, dried over anhydrous $\mathrm{MgSO}_{4}$. After filtration of the drying agent, the solvent was removed under reduced pressure and the residue was purified using flash chromatography. ${ }^{1} \mathrm{H} \mathrm{NMR}\left(\mathrm{CDCl}_{3}, 400 \mathrm{MHz}\right): \delta 4.39(\mathrm{~s}, 1 \mathrm{H}), 4.26(\mathrm{~s}, 2 \mathrm{H}), 2.80(\mathrm{ddd}, J=$ 14.4, 4.8, $2.4 \mathrm{~Hz}, 1 \mathrm{H}), 2.45-2.34(\mathrm{~m}, 2 \mathrm{H}), 2.04-1.75(\mathrm{~m}, 4 \mathrm{H}), 1.75-1.45-1.60(\mathrm{~m}, 10 \mathrm{H})$;

${ }^{13} \mathrm{C} \mathrm{NMR}\left(\mathrm{CDCl}_{3}, 100 \mathrm{MHz}\right): \delta 207.4,169.9,86.9,84.5,76.4,49.2,42.4,38.1,31.1$, 25.0, 24.4, 24.1, 23.9, 22.5, 21.5, 21.0; IR (neat): $\mathrm{cm}^{-1}$ 2940, 2868, 1742, 1270, 1203, 1158; HRMS (EI, $m / z)$ calcd for $\mathrm{C}_{16} \mathrm{H}_{20} \mathrm{O}_{4}[\mathrm{M}]^{+}$276.1362, found 276.1356.

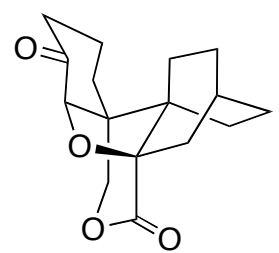

3.35

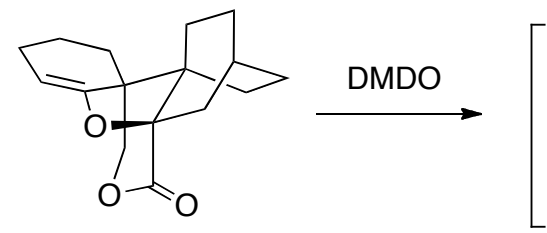

3.39

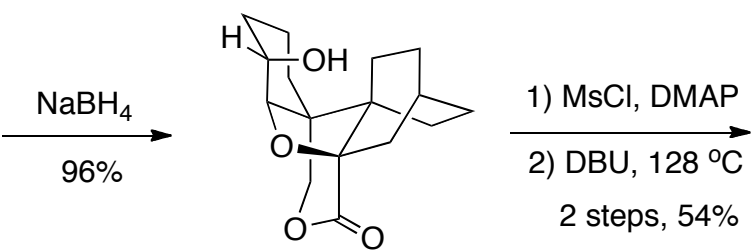

3.42

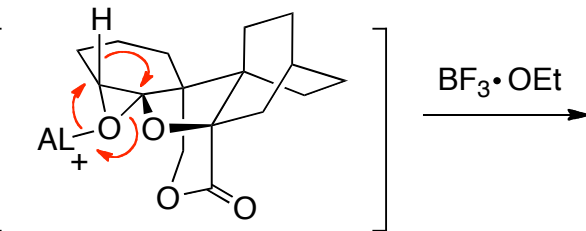

3.40

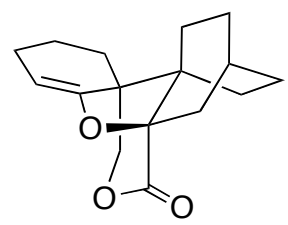

3.39

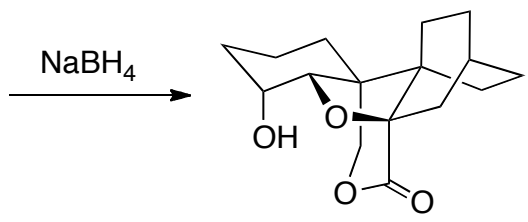

3.43

Compound 3.42: compound $3.35(27 \mathrm{mg}, 100 \mu \mathrm{mol})$ dissolved in a solution of anhydrous DCM and anhydrous methanol $(1: 1)$ at room temperature. The reaction was cooled down to $-78{ }^{\circ} \mathrm{C}$ and $\mathrm{NaBH}_{4}(11 \mathrm{mg}, 300 \mu \mathrm{mol})$ was added to this solution. The temperature was slowly warmed up to $-50{ }^{\circ} \mathrm{C}$ in 2 hour. The reaction was then quenched with saturated $\mathrm{NH}_{4} \mathrm{Cl}$ solution, extracted with ethyl acetate and dried over $\mathrm{MgSO}_{4}$. After 
filtration of the drying agent, the solvent was removed under reduced pressure and the residue was purified using flash chromatography. ${ }^{1} \mathrm{H}$ NMR $\left(\mathrm{CDCl}_{3}, 400 \mathrm{MHz}\right): \delta 4.19$ (ddd, $J=6.0,3.2,3.2 \mathrm{~Hz}, 1 \mathrm{H}), 4.13(\mathrm{~d}, J=10.8 \mathrm{~Hz}, 1 \mathrm{H}), 4.06(\mathrm{~d}, J=6.0 \mathrm{~Hz}, 1 \mathrm{H}), 3.77$ $(\mathrm{d}, J=10.4 \mathrm{~Hz}, 1 \mathrm{H}), 2.79(\mathrm{ddd}, J=14.0,4.8,2.4 \mathrm{~Hz}, 1 \mathrm{H}), 2.49-2.46(\mathrm{~m}, 2 \mathrm{H}), 2.10-1.95$ $(\mathrm{m}, 1 \mathrm{H}), 1.83-1.79(\mathrm{~m}, 1 \mathrm{H}), 1.76-1.71(\mathrm{~m}, 1 \mathrm{H}), 1.64-1.38(\mathrm{~m}, 9 \mathrm{H}), 1.32-1.23(\mathrm{~m}, 2 \mathrm{H})$ 0.94 (ddd, $J=14.0, \quad 14.0, \quad 5.6 \mathrm{~Hz}, 1 \mathrm{H}) ;{ }^{13} \mathrm{C} \quad \mathrm{NMR} \quad\left(\mathrm{CDCl}_{3}, 100 \mathrm{MHz}\right)$ $\delta 170.6,84.1,83.4,79.2,63.7,42.3,41.4,31.7,27.7,25.4,24.4,24.1,22.2,21.6,15.5$; IR (neat): $\mathrm{cm}^{-1} 3477,2923,2868,1742,1456,1155$; HRMS (EI, $\left.m / z\right)$ calcd for $\mathrm{C}_{16} \mathrm{H}_{23} \mathrm{O}_{4}$ $[\mathrm{M}+\mathrm{H}]^{+}$279.1596, found 279.1592.

Compound 3.39: At room temperature, compound $3.42(28 \mathrm{mg}, 0.1 \mathrm{mmol})$ and DMAP (30 mg, $0.3 \mathrm{mmol}$ ) dissolved in anhydrous DCM. To this solution, methanesulfonyl chloride $(24 \mu \mathrm{L}, 0.3 \mathrm{mmol})$ was injected and the reaction mixture was heated to $55{ }^{\circ} \mathrm{C}$ and stirred at this temperature for 12 hours. Then the mixture was directly purified using flash chromatography. The pure product dissolved in anhydrous toluene in a sealed tube and $50 \mu \mathrm{L}$ of DBU was injected into the solution. The reaction mixture was stirred at 130 ${ }^{\circ} \mathrm{C}$ for 2 hours. The reaction mixture was directly purified using flash chromatography. ${ }^{1} \mathrm{H}$ NMR $\left(\mathrm{CDCl}_{3}, 400 \mathrm{MHz}\right): \delta 4.96(\mathrm{dd}, J=3.6,3.6 \mathrm{~Hz}, 1 \mathrm{H}), 4.39(\mathrm{~d}, J=10.4 \mathrm{~Hz}, 1 \mathrm{H})$, $4.21(\mathrm{~d}, J=11.8 \mathrm{~Hz}, 1 \mathrm{H}), 2.85(\mathrm{dd}, J=14.0,3.2 \mathrm{~Hz}, 1 \mathrm{H}), 2.11-2.09$ (m, 2H), 1.85 (br s, 1H) $\quad 1.72-1.43 \quad(\mathrm{~m}, \quad 13 \mathrm{H}) ; \quad{ }^{13} \mathrm{C} \quad \mathrm{NMR} \quad\left(\mathrm{CDCl}_{3}, \quad 100 \quad \mathrm{MHz}\right)$ : $\delta 169.9,157.5,95.3,83.5,75.4,43.2,41.9,30.7,25.1,24.5,24.1,23.6,22.6,21.5,20.6$, 18.6; IR (neat): $\mathrm{cm}^{-1} 2947,2867,1745,1702,1210,1147$; HRMS (EI, $\mathrm{m} / \mathrm{z}$ ) calcd for $\mathrm{C}_{16} \mathrm{H}_{20} \mathrm{O}_{3}[\mathrm{M}]^{+}$260.1412, found 260.1418. 
Compound 3.41: At $0{ }^{\circ} \mathrm{C}$, compound $3.39(26 \mathrm{mg}, 100 \mu \mathrm{mol})$ dissolved in anhydrous DCM under argon. $50 \mathrm{mg}$ of $4 \mathrm{~A}$ molecular sieve was added to the solution and the mixture was stirred for 1 hour. Then fresh dimethyldioxirane in acetone (120 $\mu \mathrm{mol}, 1.2$ eq) was injected dropwise to the reaction mixture. 30 mins later, the solvent was blowed out using argon flow and $2 \mathrm{~mL}$ of anhydrous ether was injected to the residue. Boron trifluoride etherate ( $30 \mu \mathrm{L}, 3 \mathrm{eq})$ was added sequentially to the reaction mixture slowly and the temperature was slowly warmed up to room temperature in 3 hours. Then the reaction was quenched with saturated $\mathrm{NaHCO}_{3}$ when TLC indicated completion of the reaction, extracted with ethyl acetate and dried over $\mathrm{MgSO}_{4}$. After filtration of the drying agent, the solvent was removed under reduced pressure and the residue was purified using flash chromatography to afford a white solid (19 mg, 75\%). ${ }^{1} \mathrm{H}$ NMR $\left(\mathrm{CDCl}_{3}, 400\right.$ MHz): $\delta 5.48(\mathrm{~d}, J=10.8 \mathrm{~Hz}, 1 \mathrm{H}), 4.45$ (br s, $1 \mathrm{H}), 4.09$ (br s, $1 \mathrm{H}), 4.23(\mathrm{~d}, J=12.0 \mathrm{~Hz}$, 1H), $4.12(\mathrm{dd} J=12,2 \mathrm{~Hz}, 1 \mathrm{H}), 2.86(\mathrm{ddd}, J=14.0,3.2,2 \mathrm{~Hz}, 1 \mathrm{H}), 2.43-2.39$ (m, 2H), 2.13-1.59 (m, $\quad 14 \quad \mathrm{H}) ; \quad{ }^{13} \mathrm{C} \quad \mathrm{NMR} \quad\left(\mathrm{CDCl}_{3}, \quad 100 \quad \mathrm{MHz}\right)$ $\delta 204.9,169.6,88.2,85.3,70.3,50.6,42.8,38.5,30.7,25.4,24.8,24.5,23.7$, 21.9, 21.4, 21.3; IR (neat): $\mathrm{cm}^{-1}$ 2925, 2869, 1743, 1269, 1150; HRMS (FAB, m/z) calcd for $\mathrm{C}_{16} \mathrm{H}_{21} \mathrm{O}_{4}[\mathrm{M}+\mathrm{H}]^{+}$277.1440, found 277.1446.

Compound 3.43: compound 3.41 (19 $\mathrm{mg}$ ) dissolved in a solution of anhydrous DCM and anhydrous methanol $(1: 1)$ at room temperature. The reaction was cooled down to $-78{ }^{\circ} \mathrm{C}$ and $\mathrm{NaBH}_{4}(11 \mathrm{mg}, 300 \mu \mathrm{mol})$ was added to this solution. The temperature was slowly warmed up to $-50{ }^{\circ} \mathrm{C}$ in 2 hour. The reaction was then quenched with saturated $\mathrm{NH}_{4} \mathrm{Cl}$ solution, extracted with ethyl acetate and dried over $\mathrm{MgSO}_{4}$. After filtration of the drying 
agent, the solvent was removed under reduced pressure and the residue was purified using flash chromatography to afford a white solid (19 mg, 95\%). ${ }^{1} \mathrm{H}$ NMR $\left(\mathrm{CDCl}_{3}, 400\right.$ MHz): $\delta 5.48(\mathrm{~d}, J=10.8 \mathrm{~Hz}, 1 \mathrm{H}), 4.45($ br s, $1 \mathrm{H}), 4.09$ (br s, $1 \mathrm{H}), 4.07(\mathrm{~d}, J=10.8 \mathrm{~Hz}$, 1H), $2.86(\mathrm{dd}, J=14.0,5.2 \mathrm{~Hz}, 1 \mathrm{H}), 2.70(\mathrm{dd}, J=18.8,3.2 \mathrm{~Hz}, 1 \mathrm{H}), 2.62(\mathrm{br} \mathrm{s}, 2 \mathrm{H}), 2.45$ (br s, 1H), 2.29 (br s, 1H), $2.00(\mathrm{~d}, J=18.4 \mathrm{~Hz}, 1 \mathrm{H}), 1.95-1.60(\mathrm{~m}, 5 \mathrm{H}) ;{ }^{13} \mathrm{C} \mathrm{NMR}$ $\left(\mathrm{CDCl}_{3}, 100 \mathrm{MHz}\right): \delta 171.8,85.7,84.2,72.3,67.7,43.6,42.6,31.0,30.0,26.0,24.9$, 24.7, 24.1, 21.3, 20.8, 16.9; HRMS (FAB, $m / z$ ) calcd for $\mathrm{C}_{16} \mathrm{H}_{22} \mathrm{O}_{4}[\mathrm{M}]^{+}$278.1518, found 278.1512 .

\subsection{References}

(1) Baran's IMDA reaction afforded a single diastereoisomer.

(2) Lei, B.; Fallis, A. G. J. Am. Chem. Soc. 1990, 112, 4609-4610.

(3) Baldwin, J. E. J. C. S. Chem. Comm. 1976, 18, 734-736.

(4) Oppolzer, W.; Godel, T. J. Am. Chem. Soc. 1978, 100, 2583-2584.

(5) Elliott, M. C.; EI Sayed, N.; Paine, J. Eur. J. Org. Chem. 2007, 5, 792-803.

(6) (a) Strekowski, L.; Kong, S.; Battiste, M. A. J. Org. Chem. 1988, 53, 901-904; (b) Guan, Z. H.; Zuo, W.; Zhao, L. B.; Ren, Z. H.; Liang, Y. M. Synthesis. 2007, 1465-1470. (7) (a) Inoki, S.; Kato, K.; Isayama, S.; Mukaiyama, T. Chem. Lett. 1990, 1869-1872; (b) Magnus, P.; Payne, A. H.; Waring, M. J.; Scott, D. A.; Lynch, V. Tetrahedron Lett. 2000, 41, 9725-9730.

(8) H. C. Brown, J. Muzzio, J. Am. Chem. Soc. 1966, 88, 2811-2822.

(9) Otsubo, K.; Inanaga, J.; Yamaguchi, M. Tetrahedron Lett. 1987, 28, 4435-4436. 
(10) A. S. Cieplak, J. Am. Chem. Soc. 1981, 105, 4540-4552.

(11) Birman, V. B.; Danishefsky, S. J. J. Am. Chem. Soc. 2002, 124, 2080-2081.

(12) Payne, G. B.; Deming, P. H.; Williams, P. H. J. Org. Chem. 1961, 26, 659-663.

(13) Hoveyda, A. H.; Evans, D. A.; Fu, G. C. Chem. Rev. 1993, 93, 1307-1370.

(14) (a) Woodward, R. B.; Sondheimer, F.; Taub, D.; Heusler, K.; Maclamore, W. M. J. Am. Chem. Soc. 1952, 74, 4223-4251; (b) Fukuzawa, A.; Sato, H.; Masamune, T. Tetrahedron Lett. 1987, 28, 4303-4306; (c) Oballa, R. M.; Carson, R.; Lait, S.; Cadieux, J. A.; Robichaud, J. Tetrahedron. 2005, 61, 2761-2766.

(15) Kim, W. H.; Lee, J. H.; Danishefsky, S. J. J. Am. Chem. Soc. 2009, 131, 1257612578. (b) Kim, W. H.; Lee, J. H.; Aussedat, B.; Danishefsky, S. J. Tetrahedron 2010, $66,6391-6398$.

(16) (a) Halcomb, R. L.; Danishefsky, S. J. J. Am. Chem. Soc. 1989, 111, 6661-6666; (b) Link, J. T.; Danishefsky, S. J.; Schulte, G. Tetrahedron Lett. 1994, 35, 9131-9134.

(17) (a) Ireland, R. E.; Hengartner, U. J. Am. Chem. Soc, 1972, 94, 3652-3653; (b) Aloto, C. O.; Rainier, J. D. Angew. Chem. Int. Ed. 2008, 47, 8055-8058. 


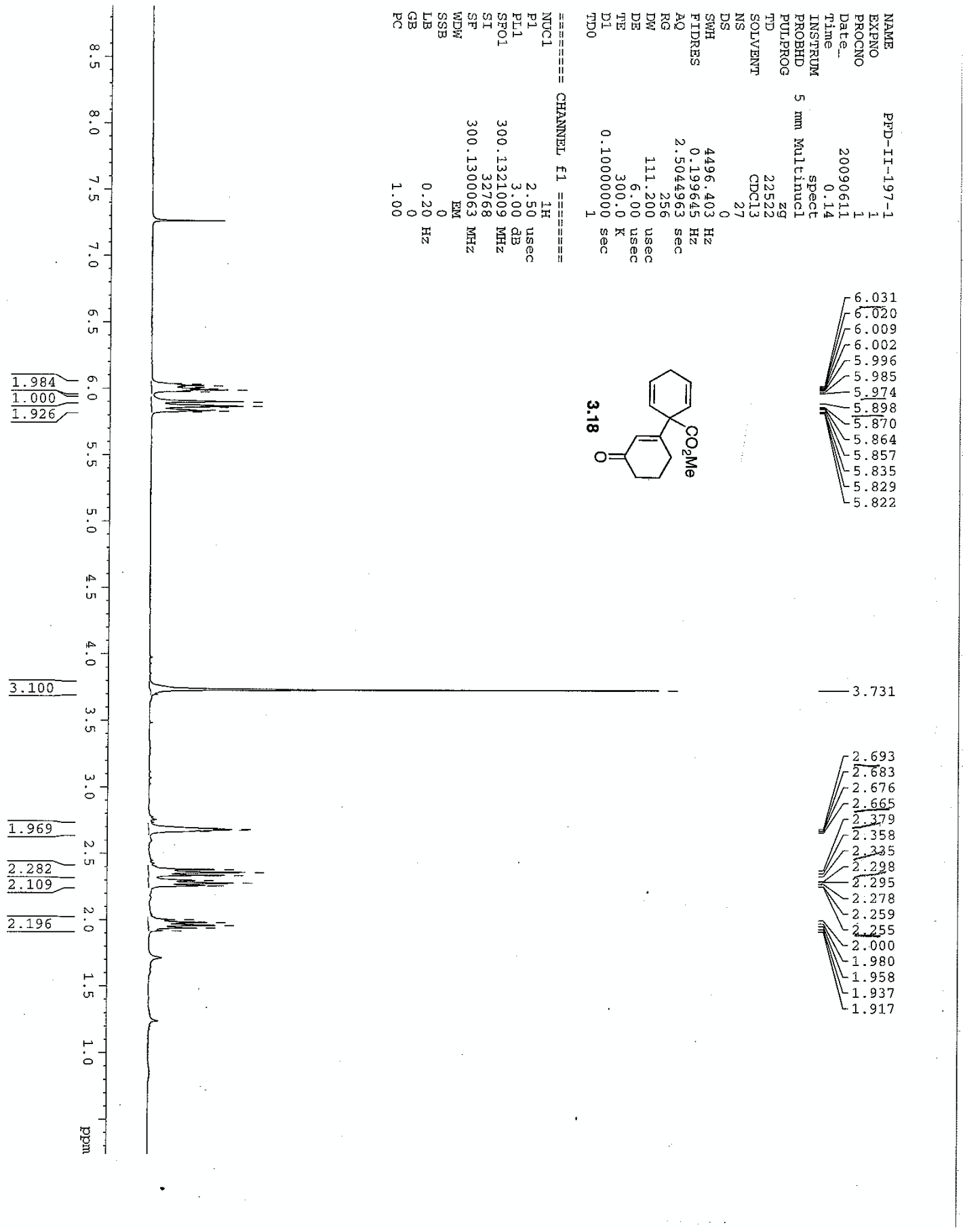




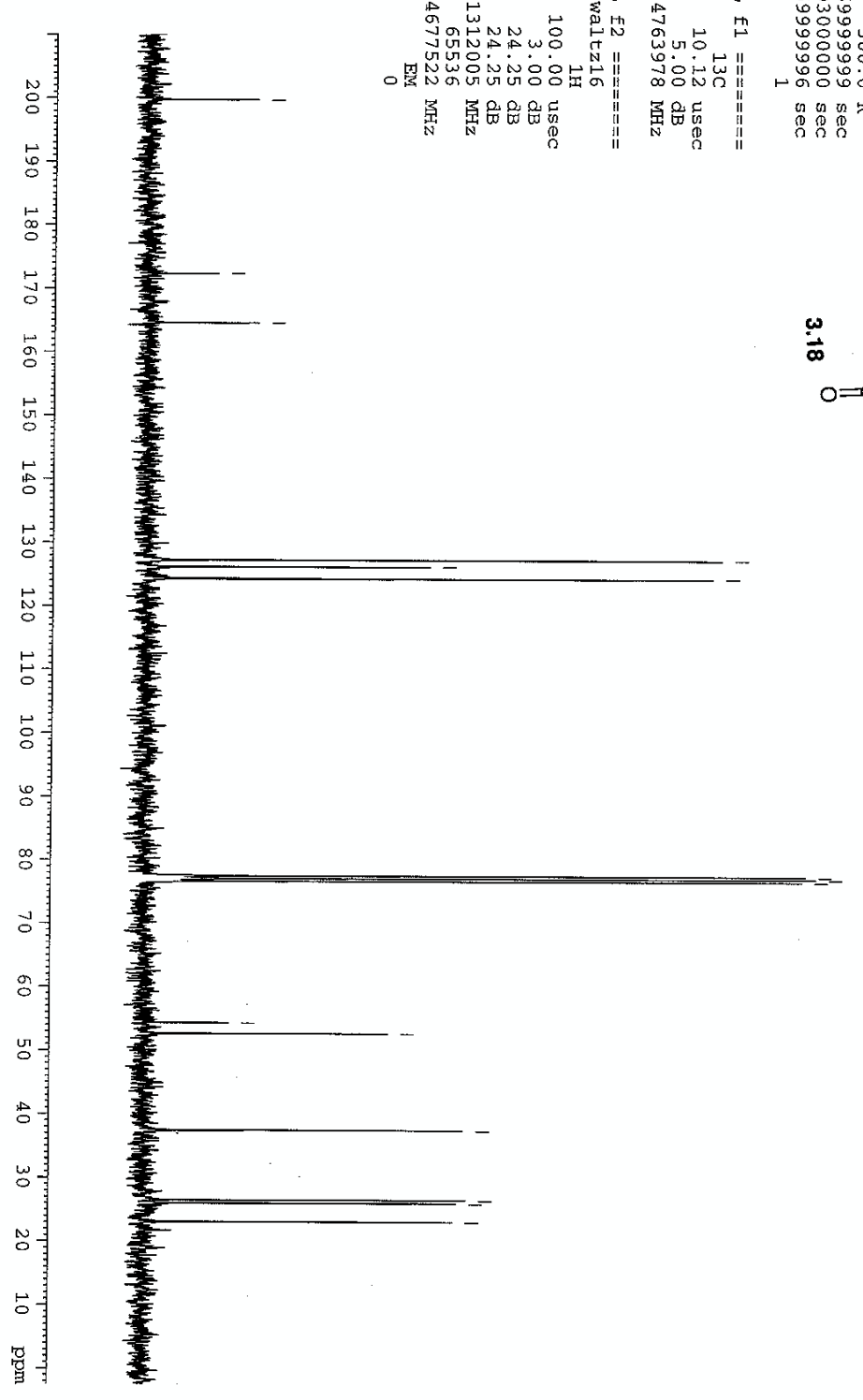

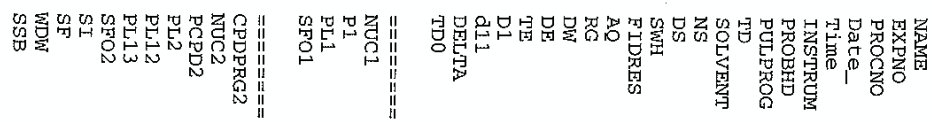
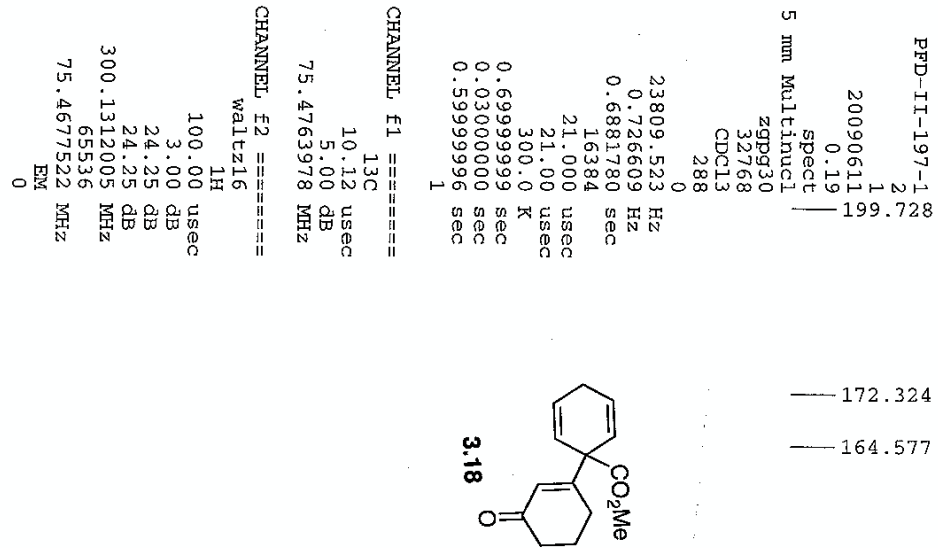

$-172.324$

$-164.577$

$-127.242$

$\simeq \quad 126.150$

$-77.425$

77.001
-76.577

-54.262
$=52.565$

$-37.354$

$-26.392$

$=25.877$
--22.947 


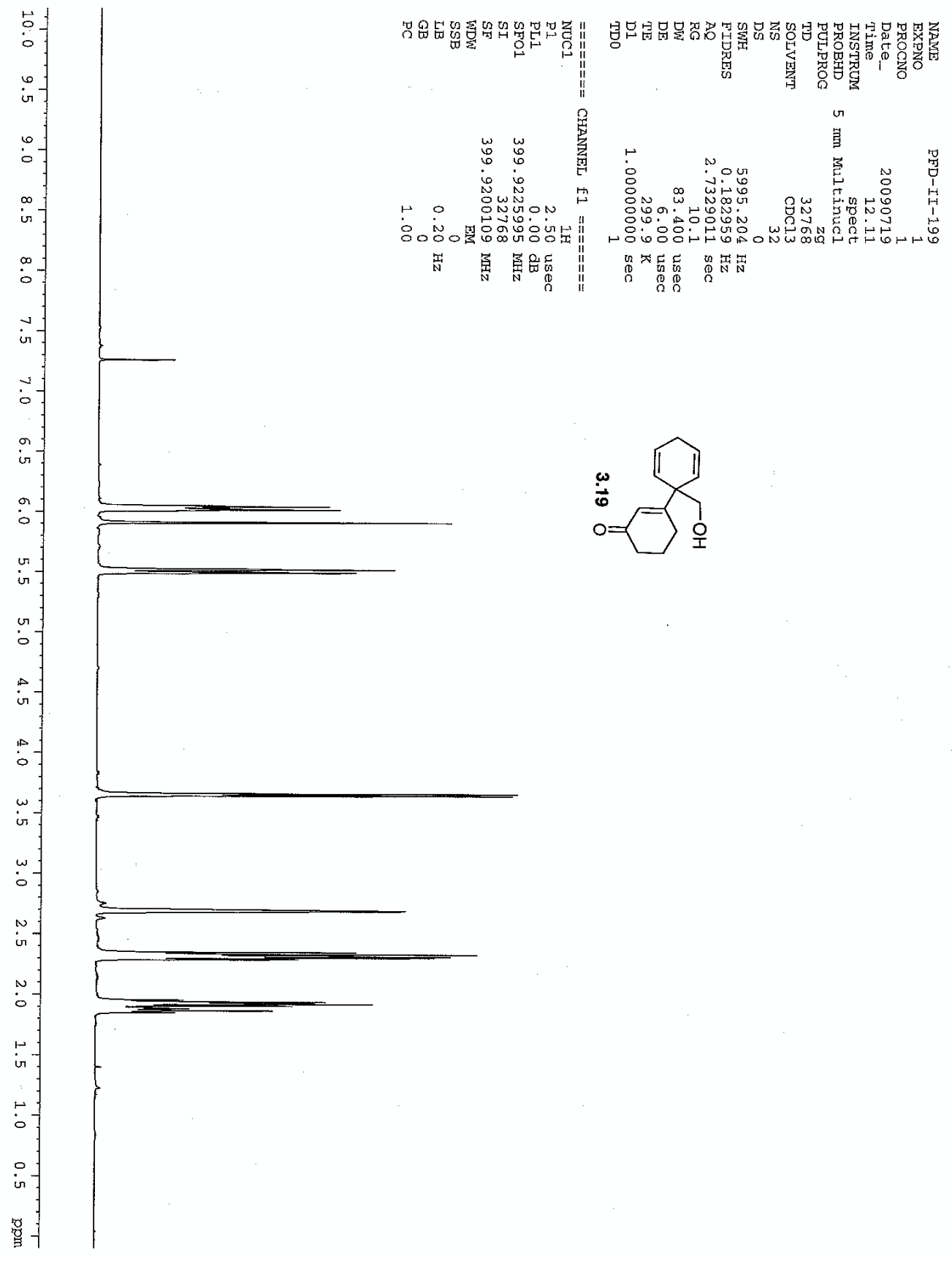



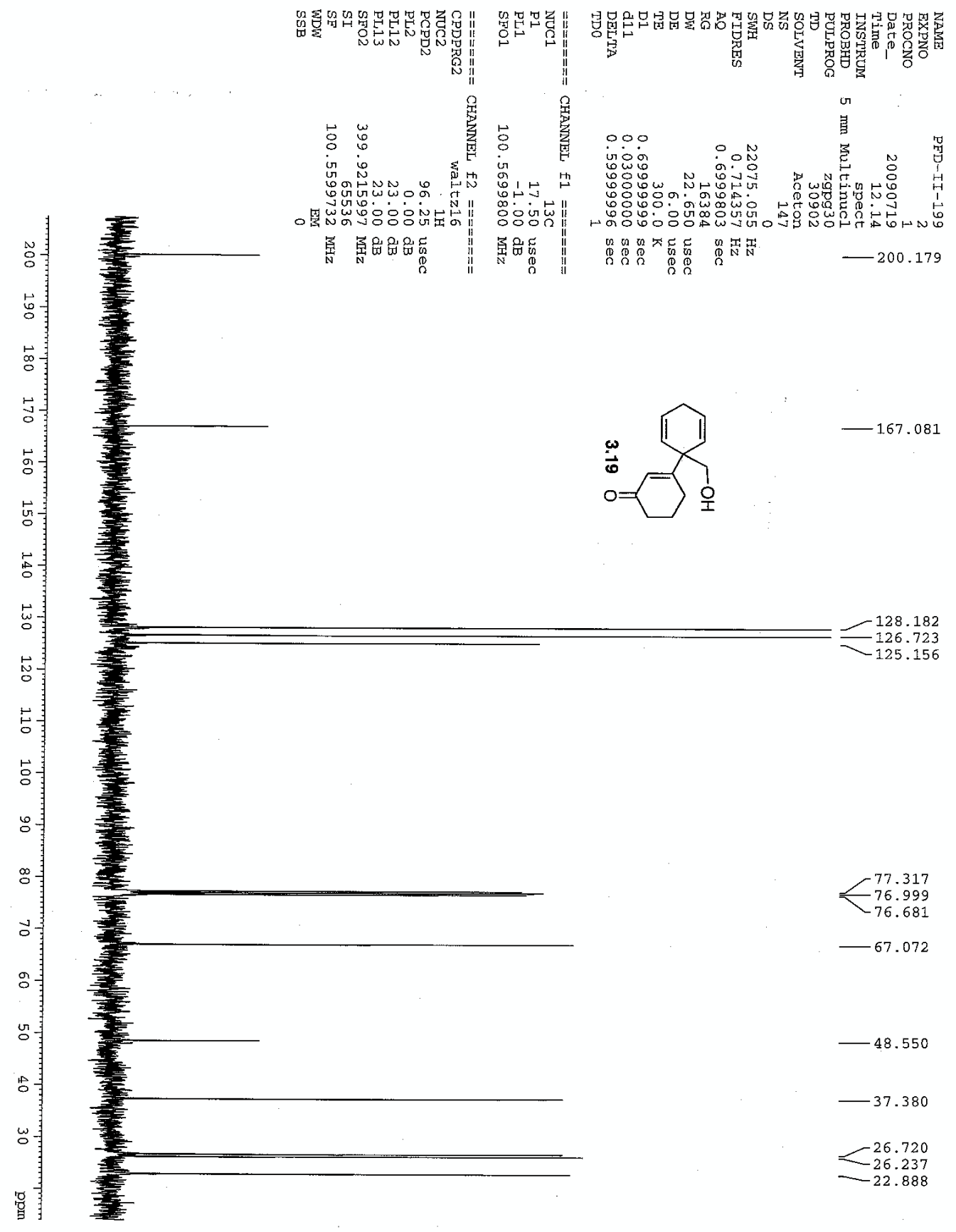


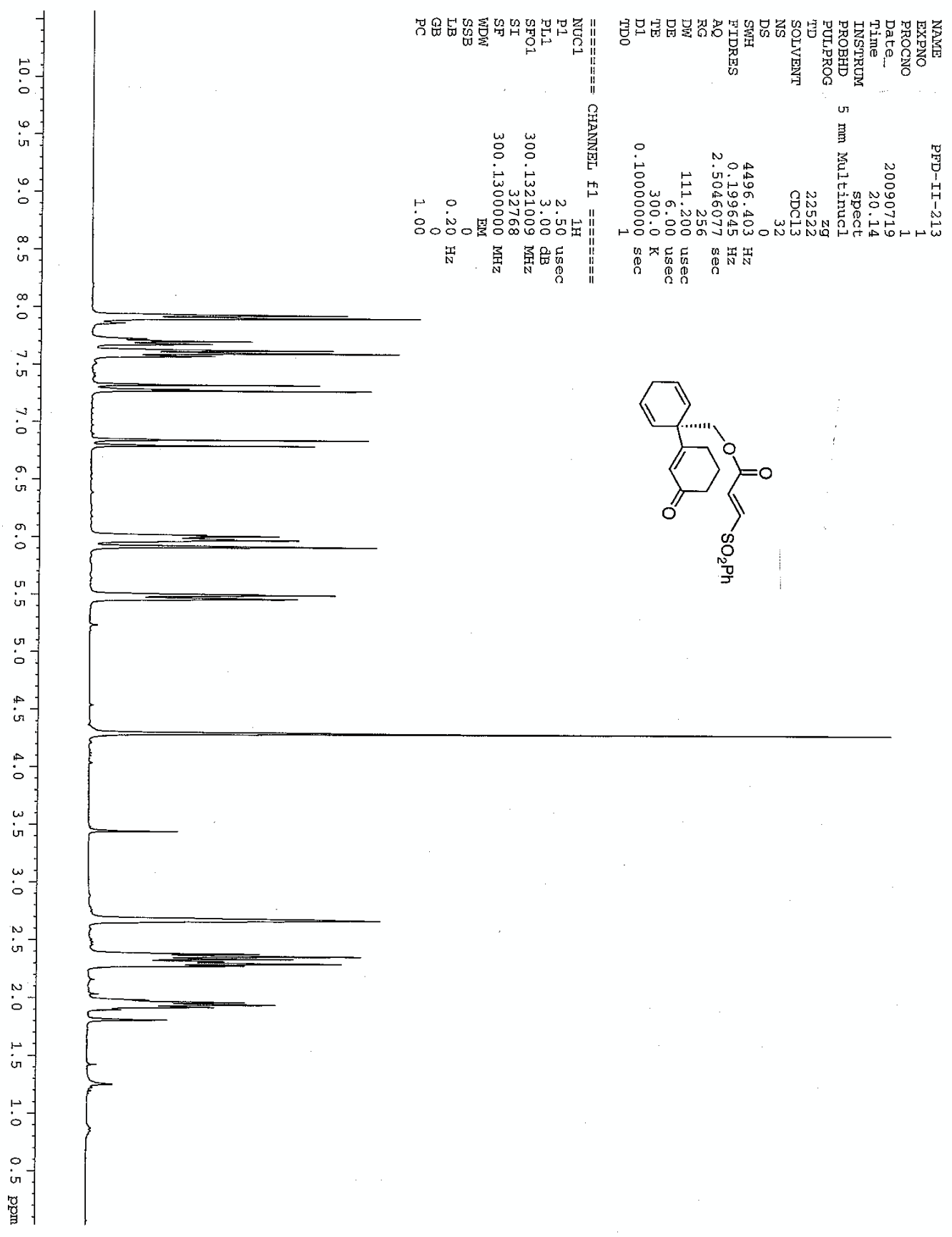




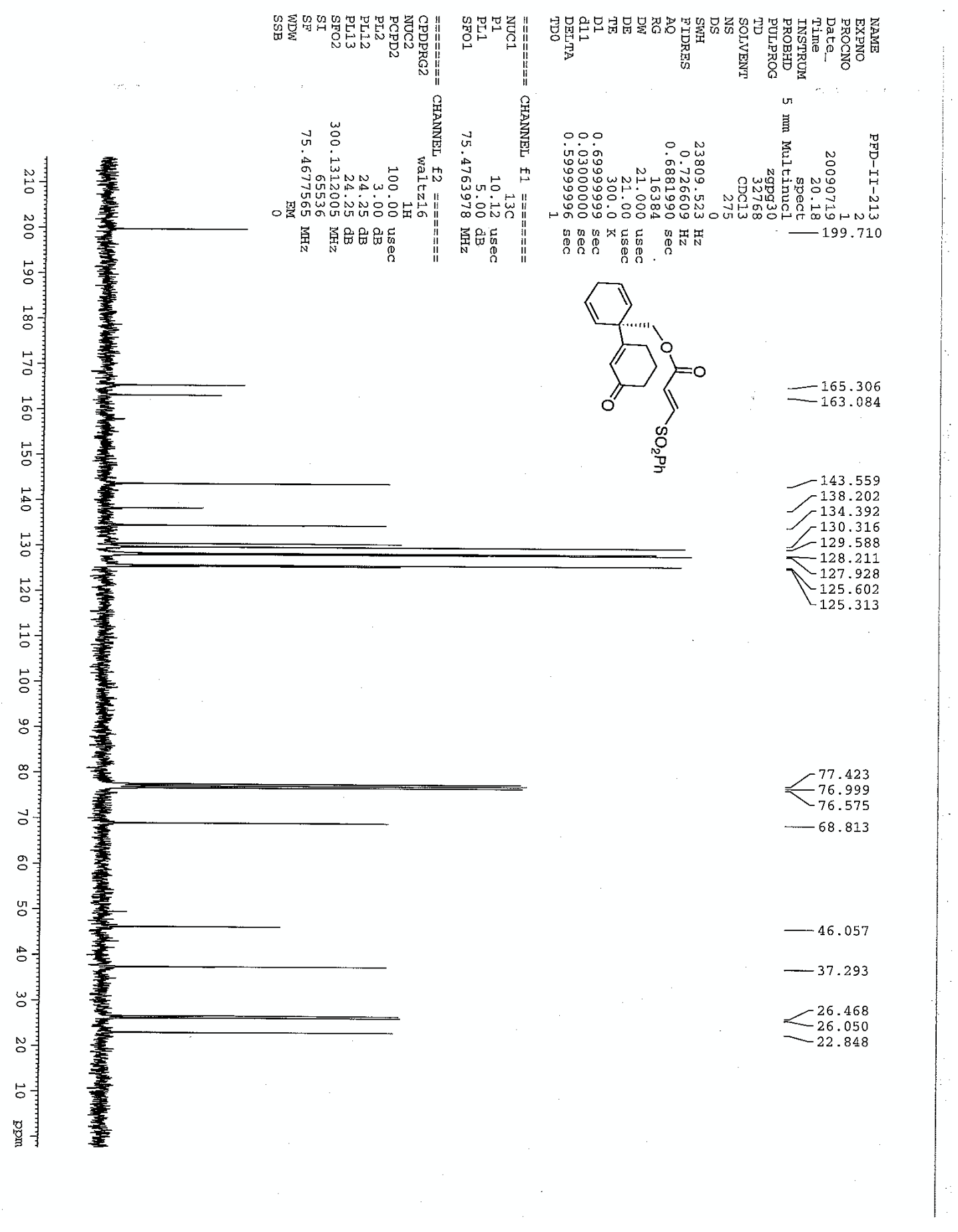




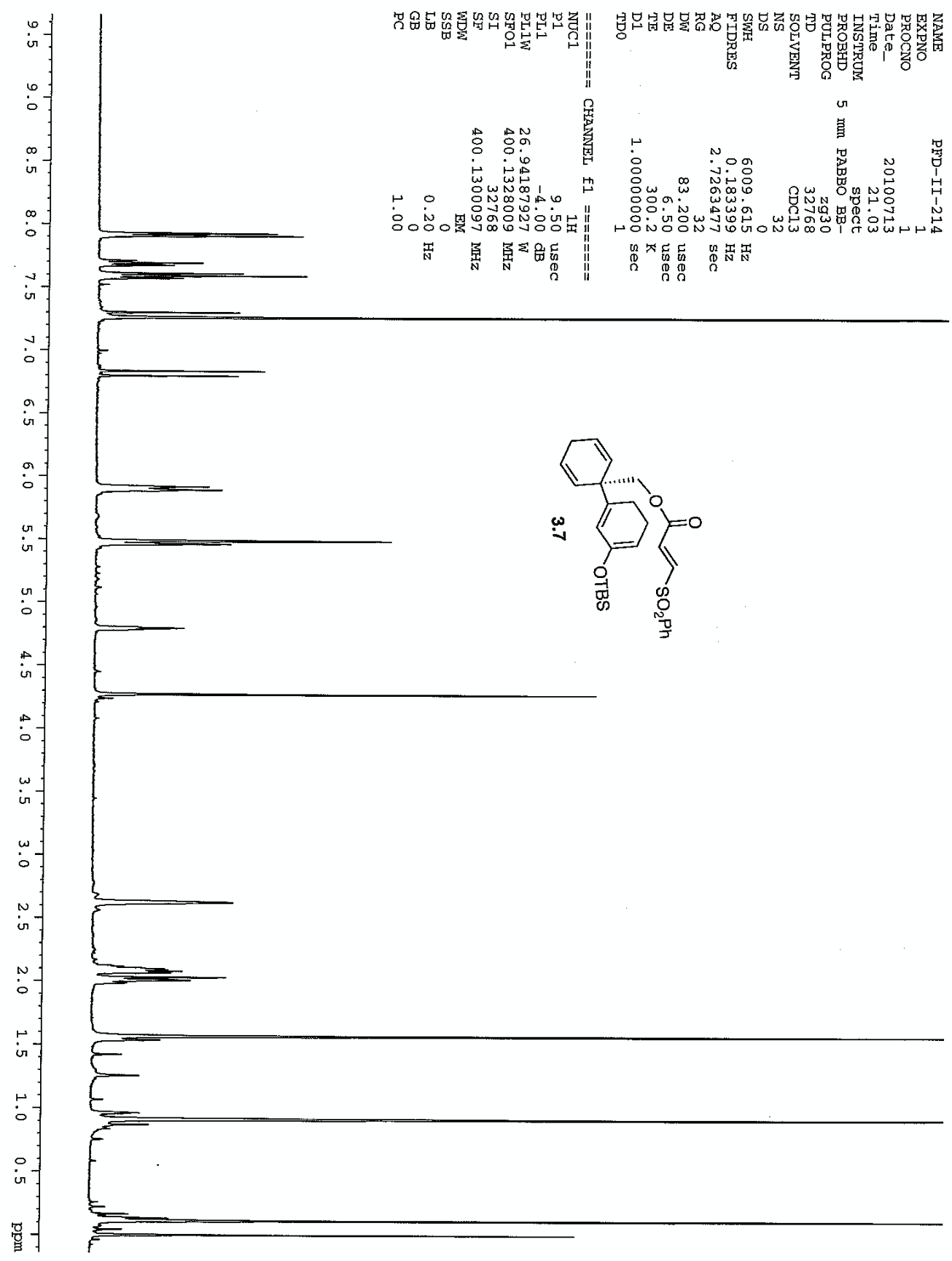




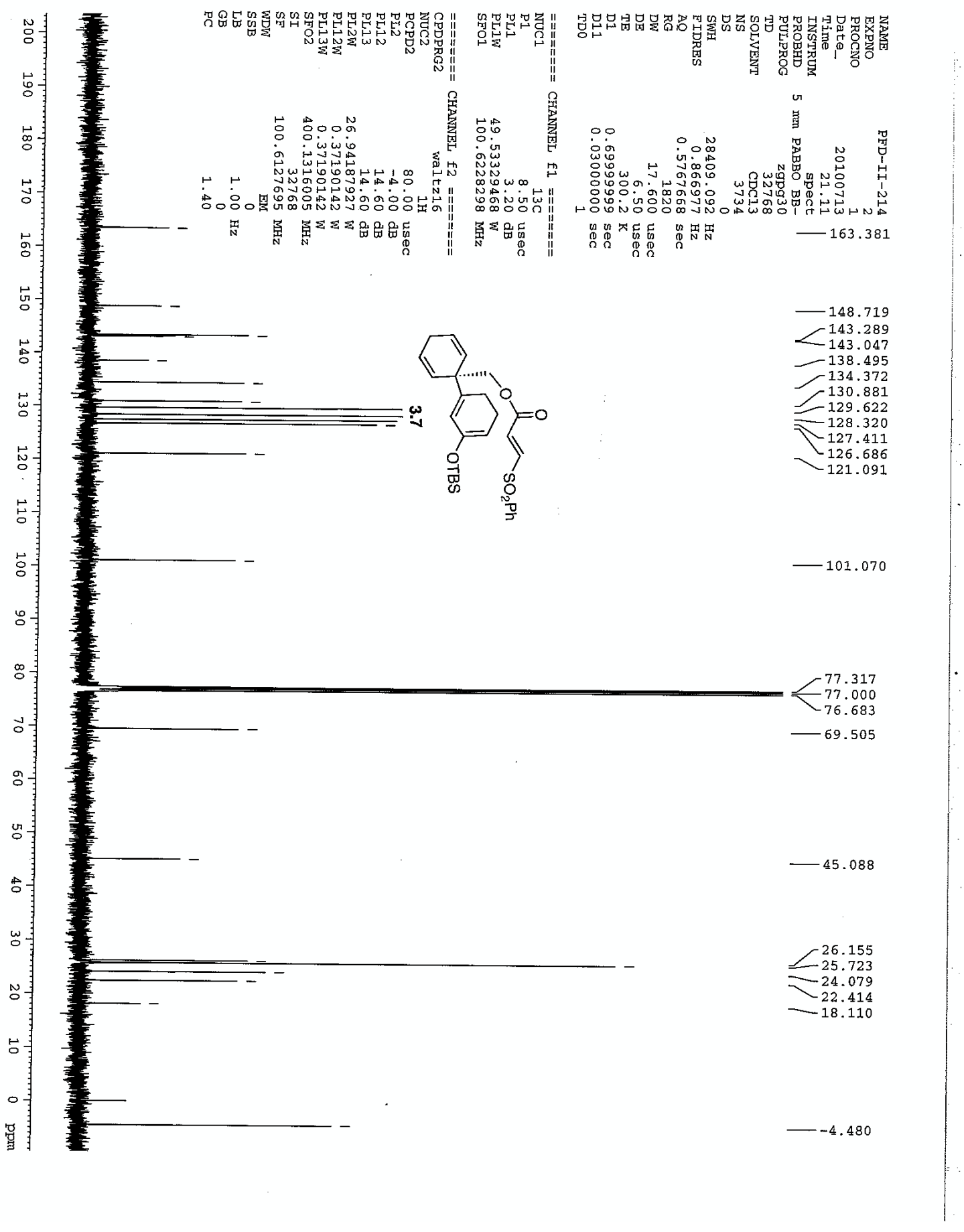




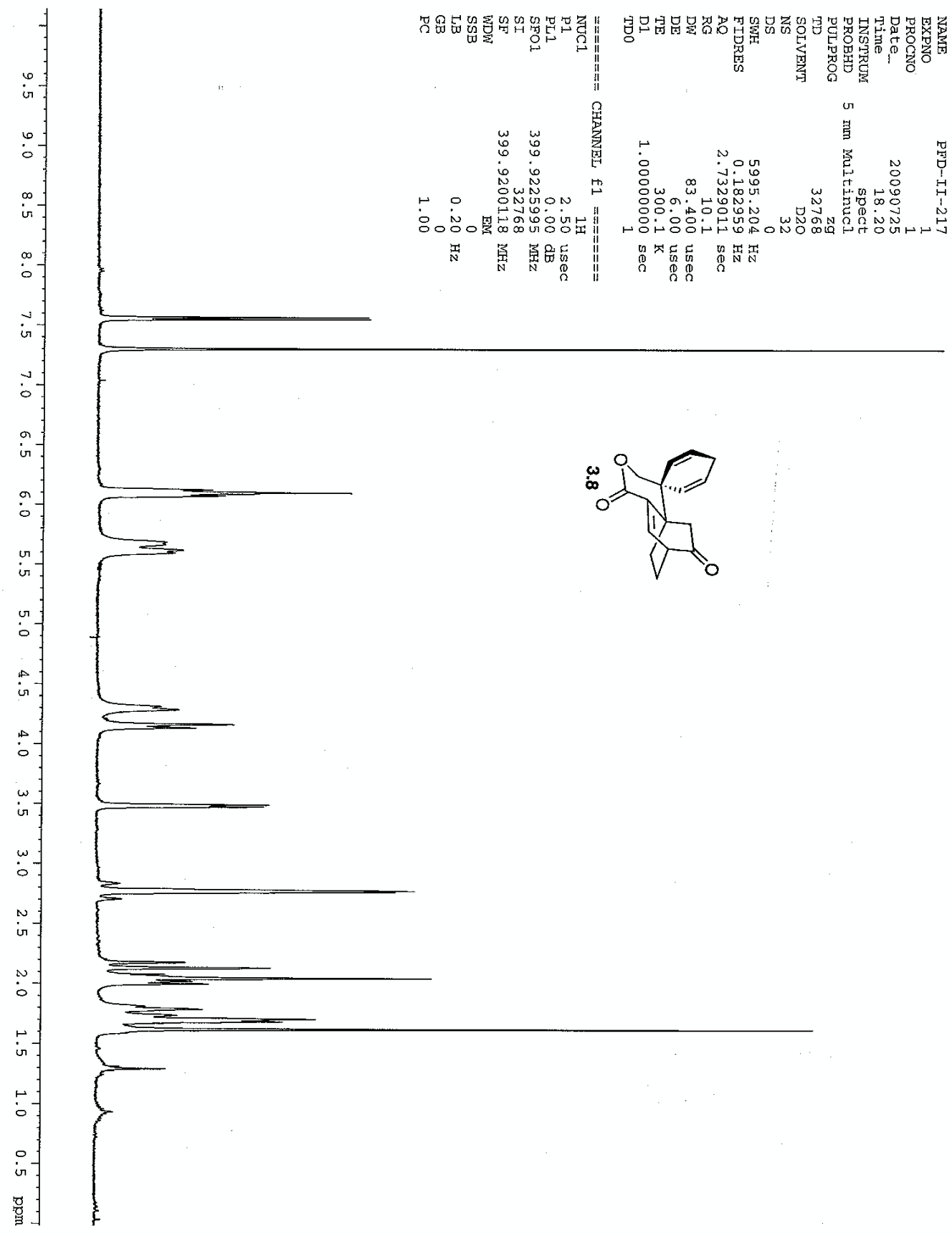



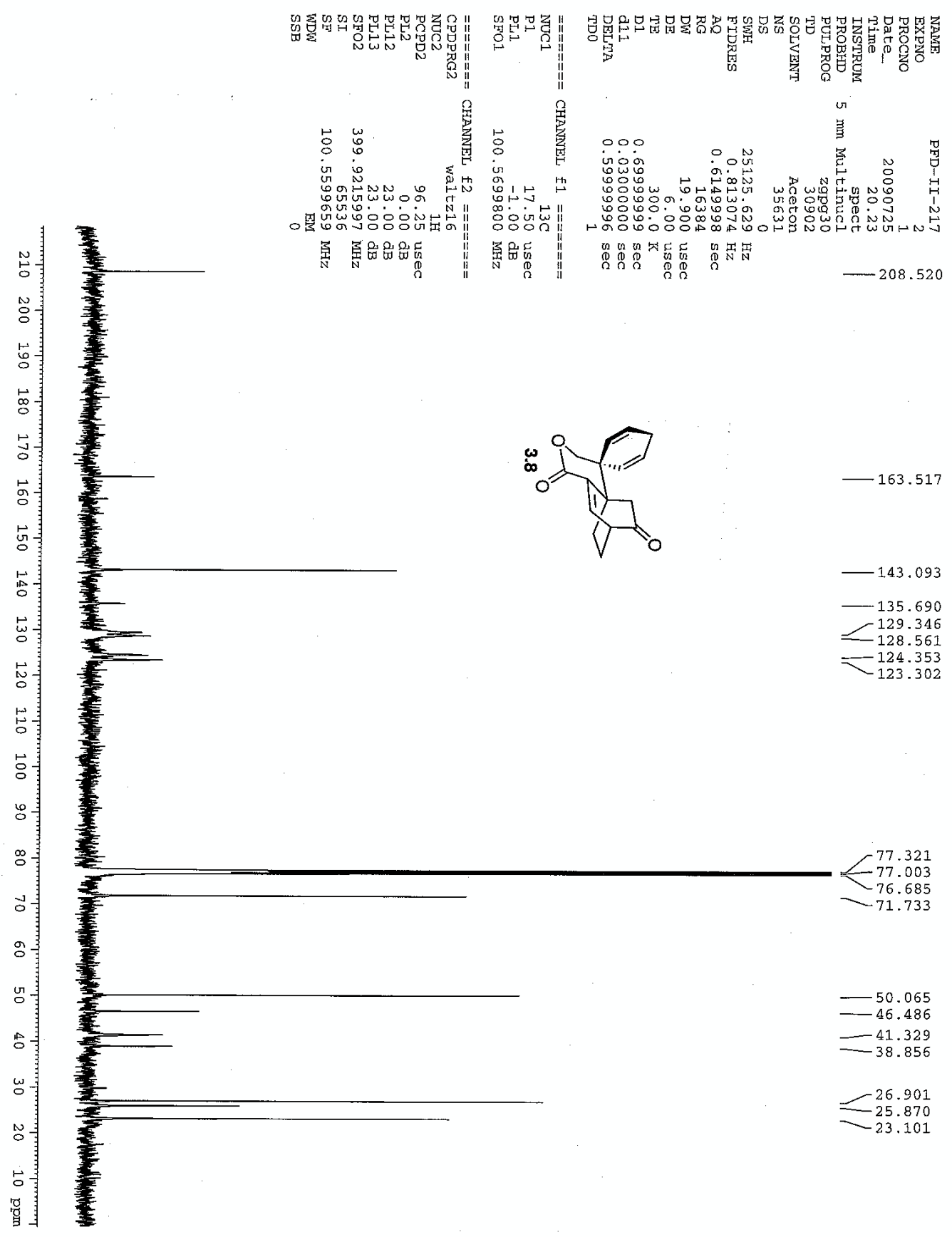


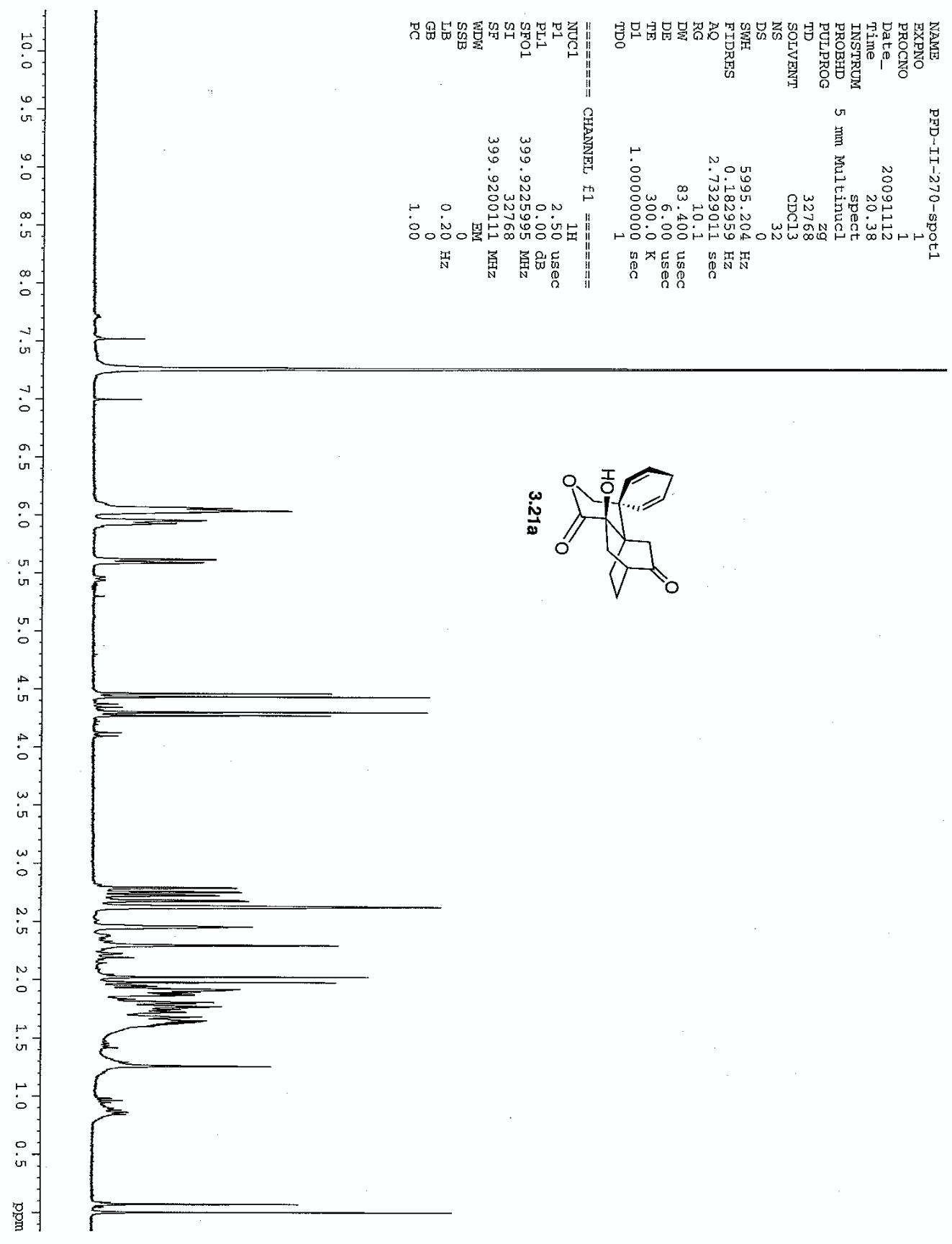




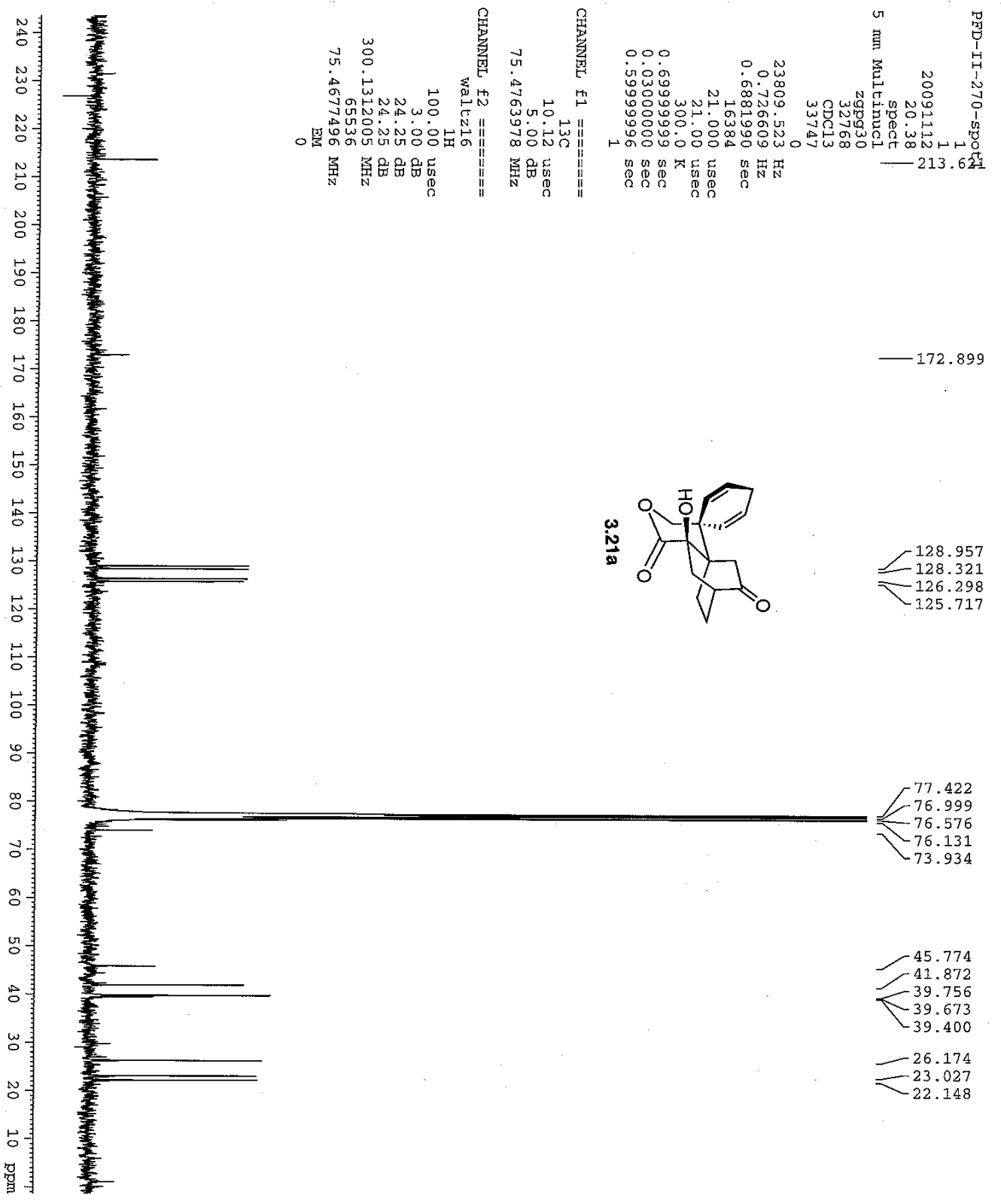




$$
E^{*}
$$



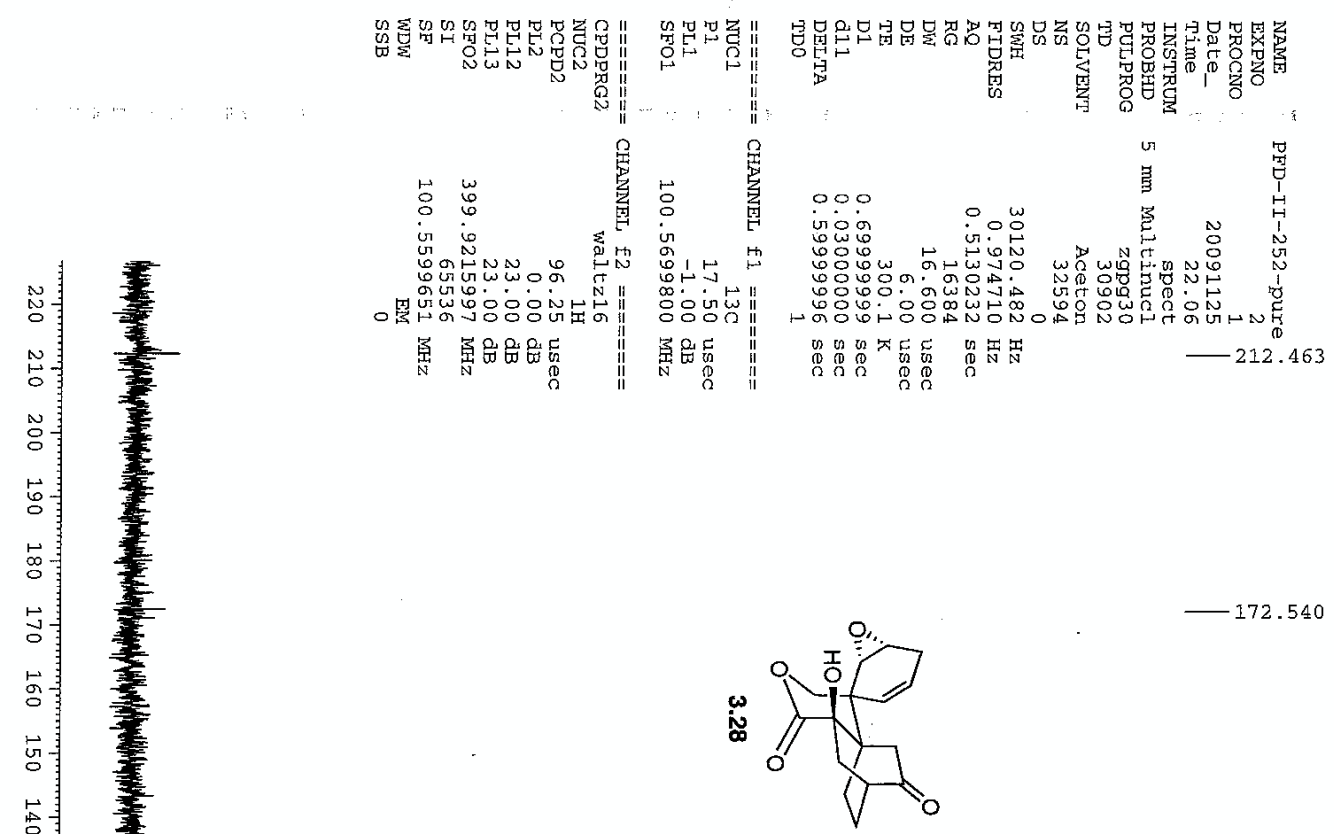

$-172.540$

$-125.800$

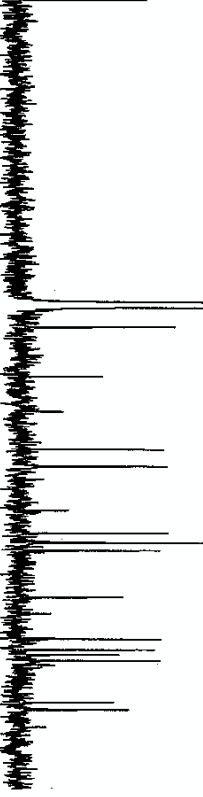

77.318

$=-77.000$

-73.542
-73.53

$-65.836$

$-60.390$

$-54.543$

$-51.901$

$-45.047$

$-41.554$

$-40.034$

-38.875
-31.575

31.575

23.458

$-22.638$

21.744

$-15.252$

14.186
14.093 


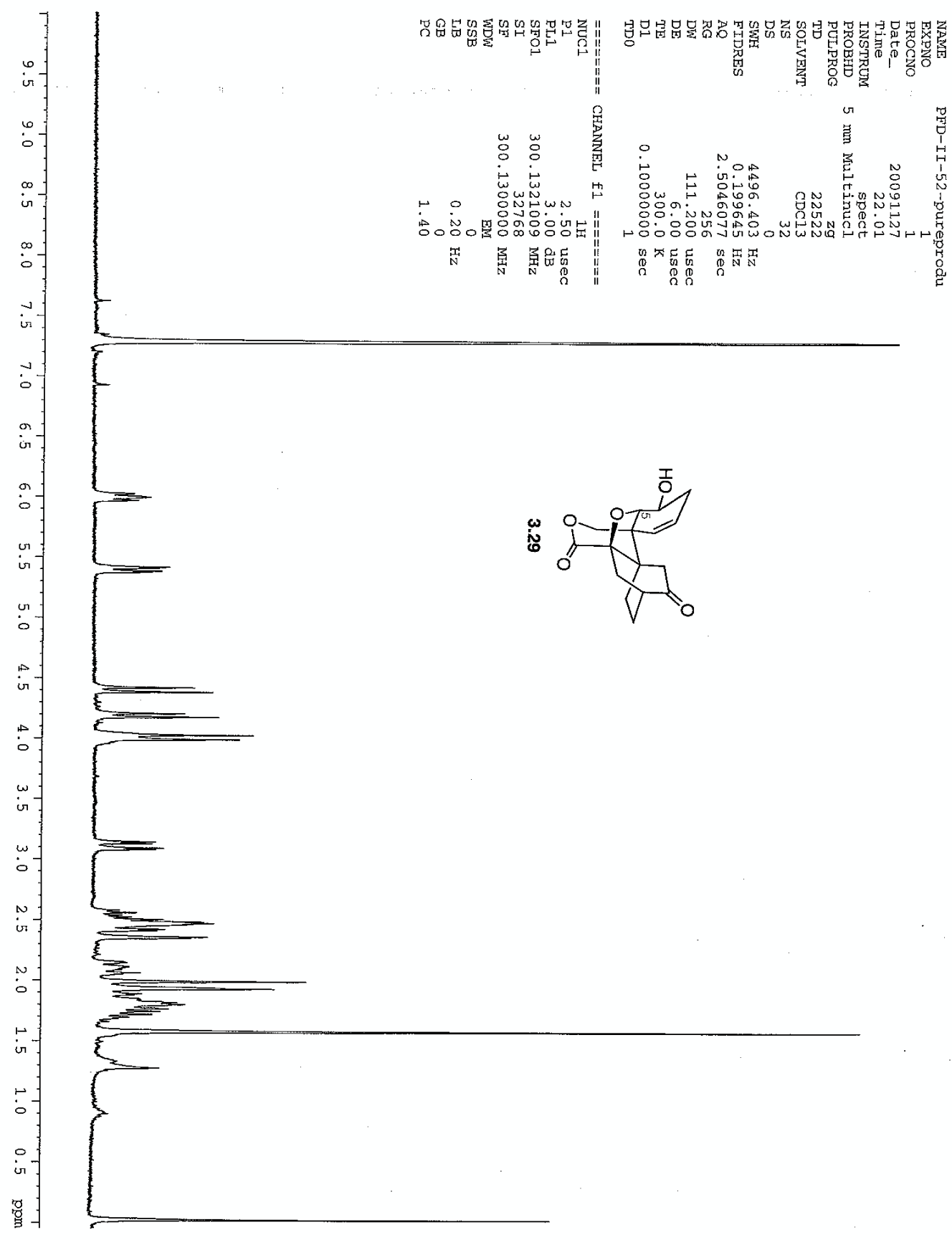



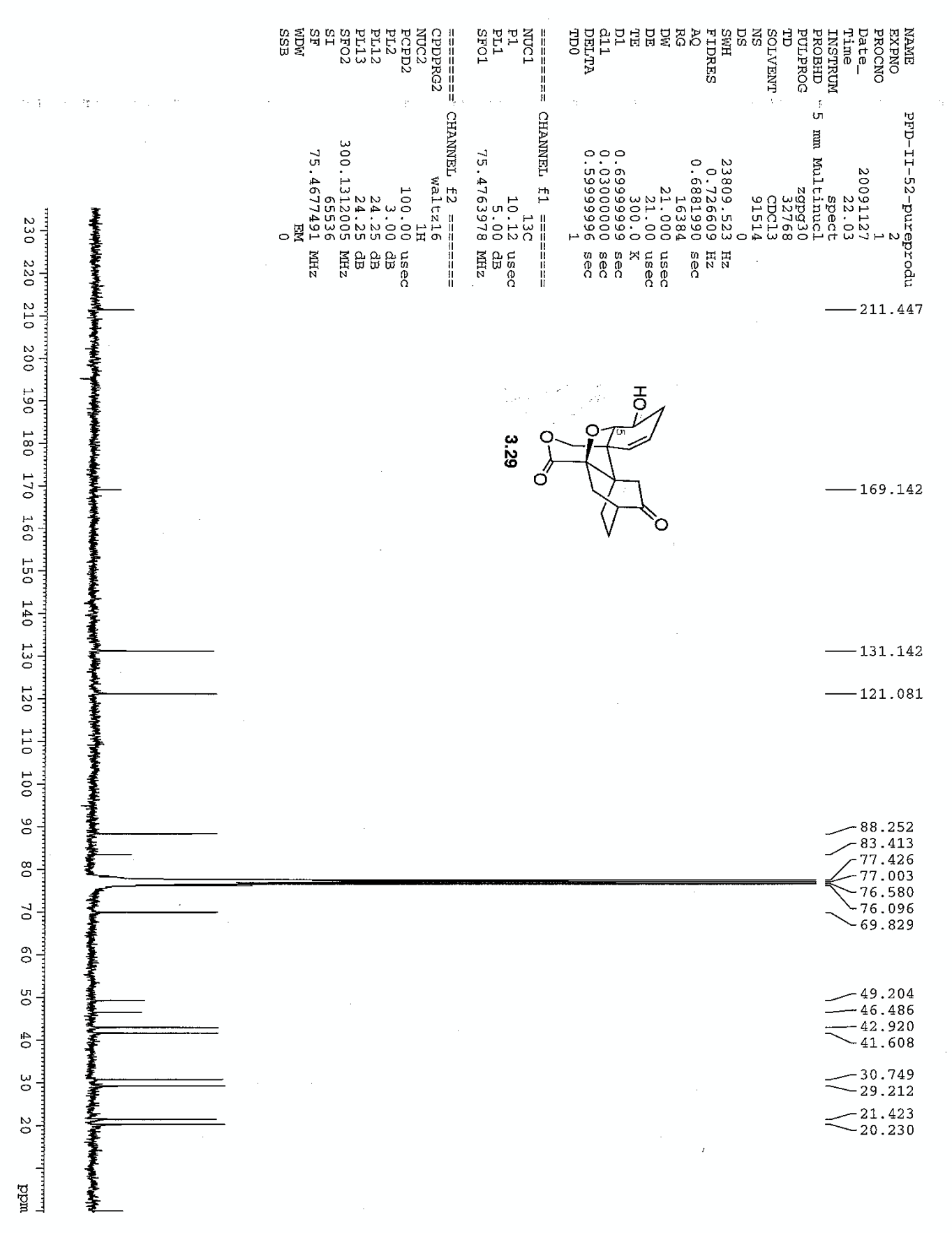

.




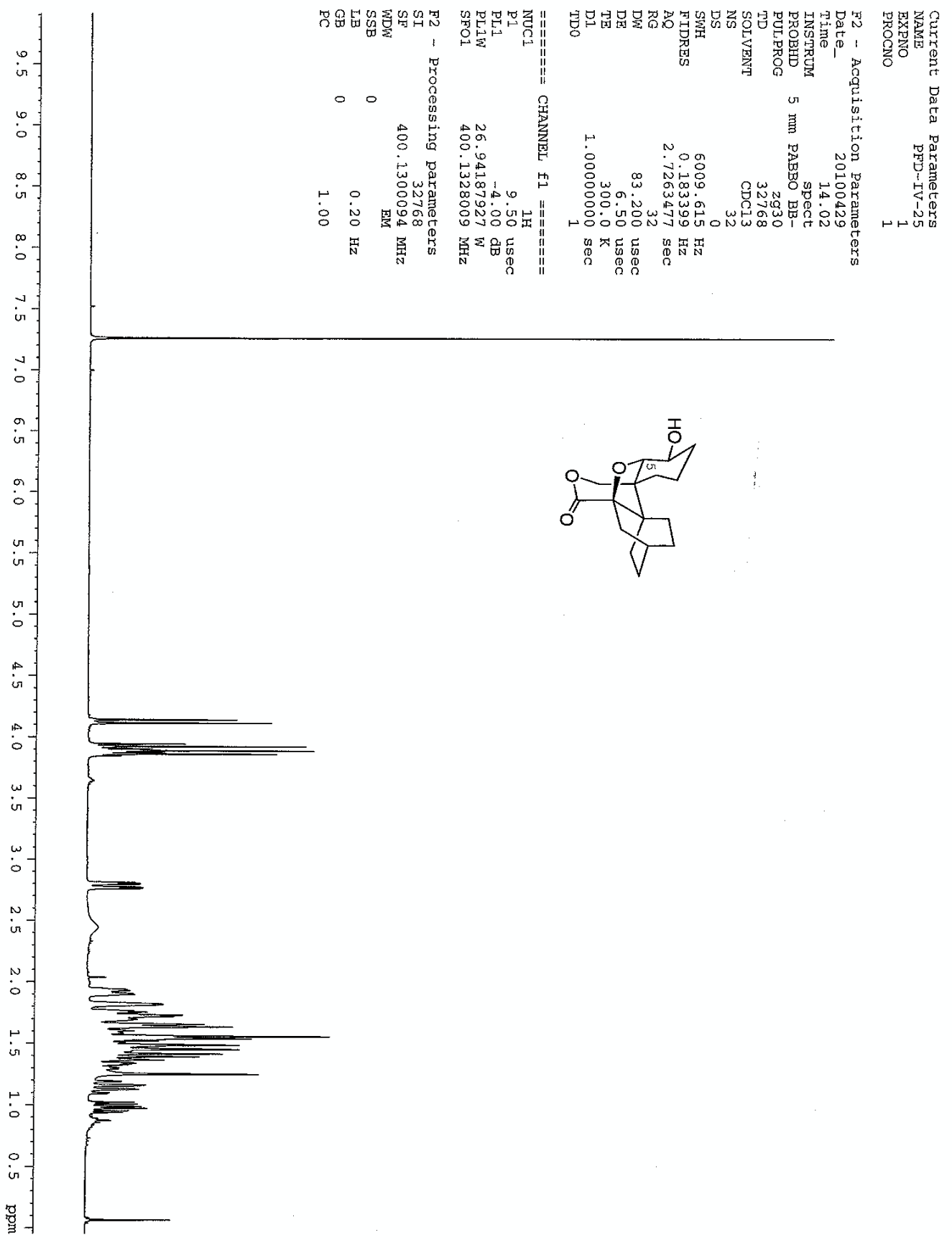



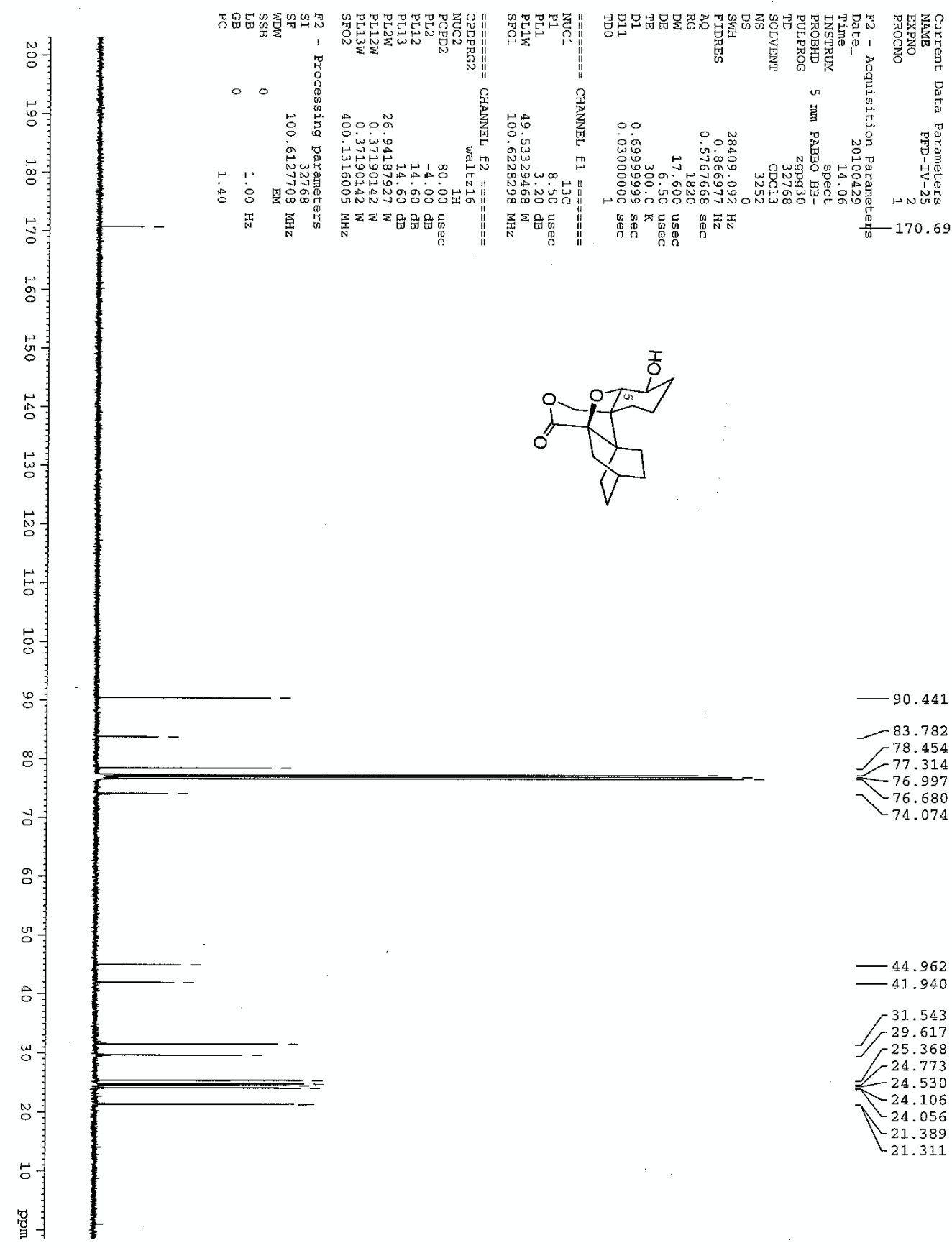


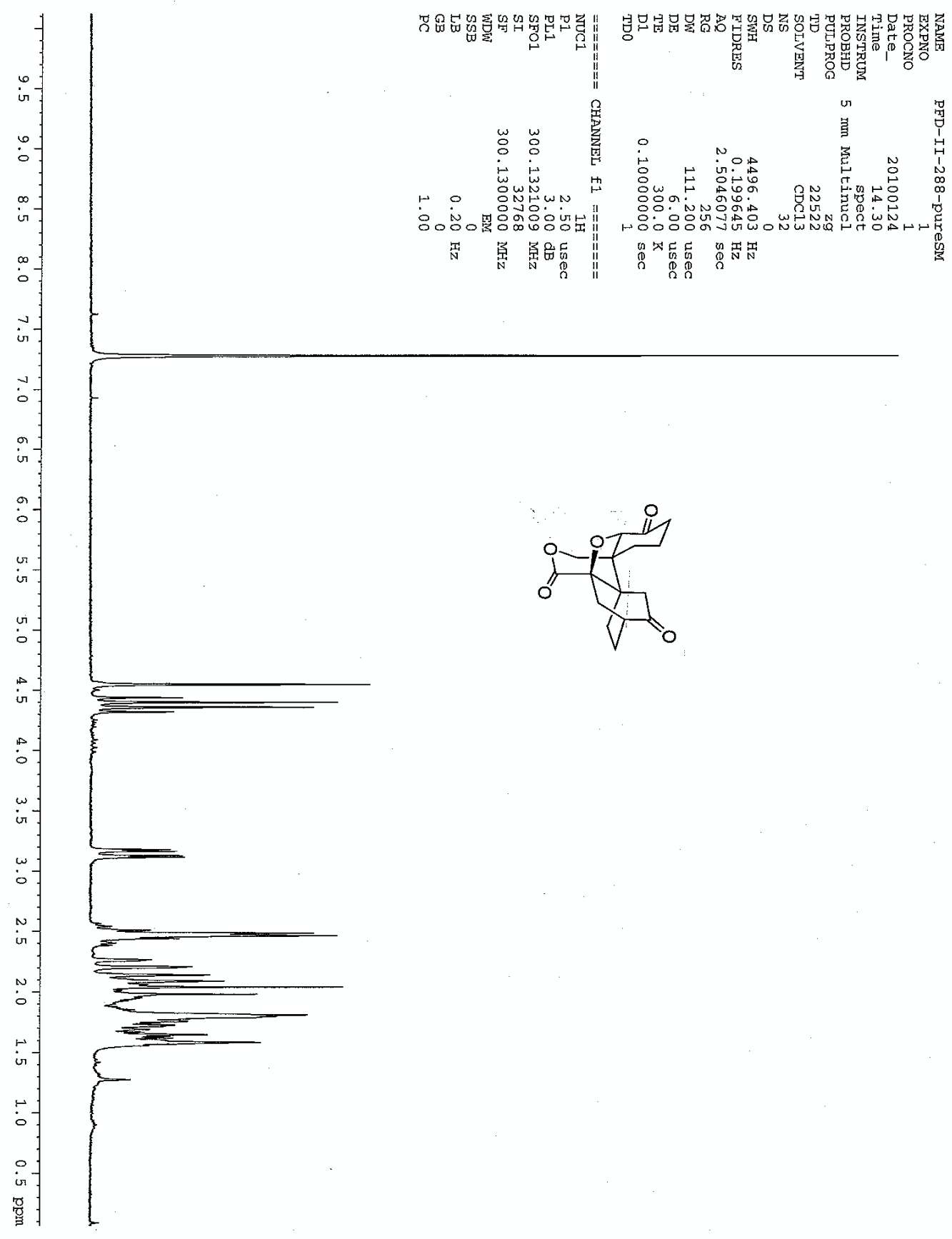



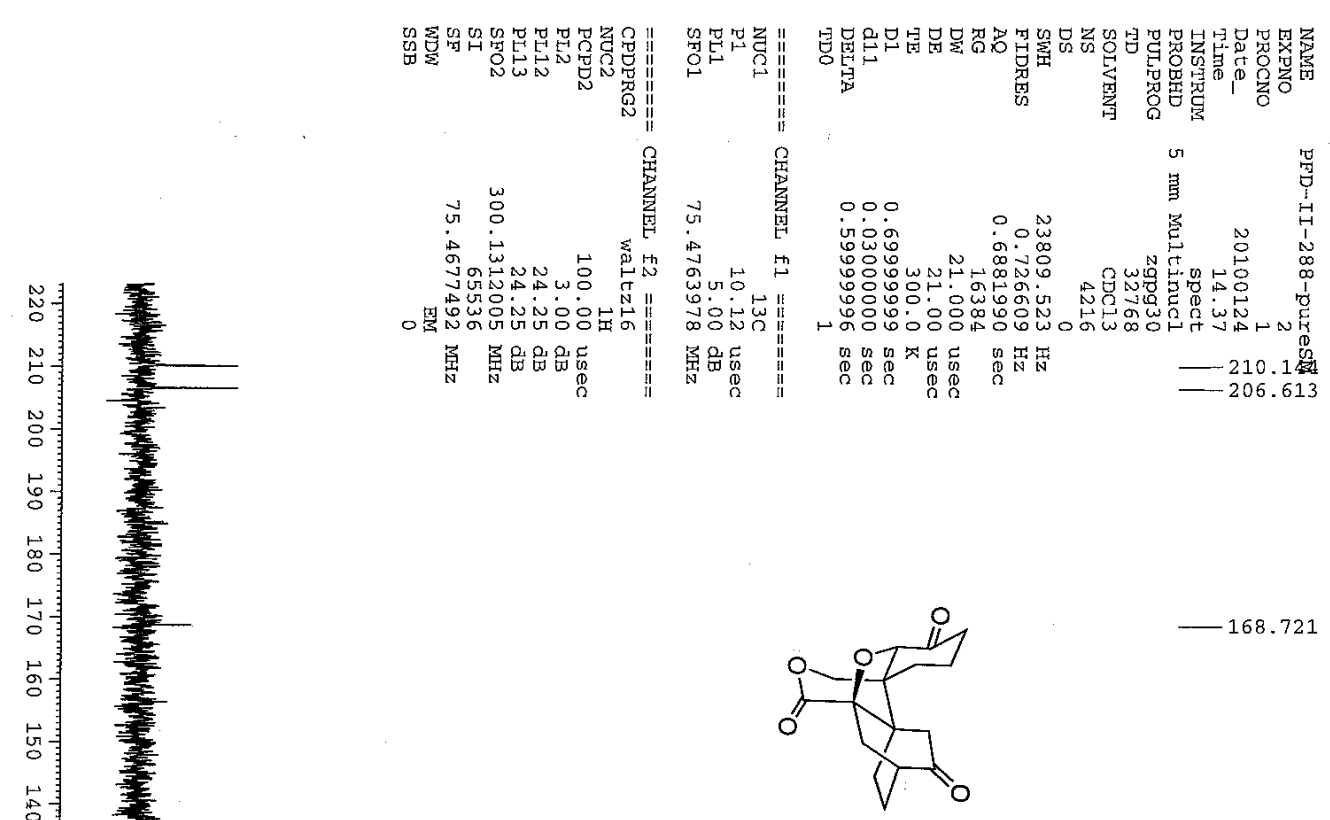

$-168.721$

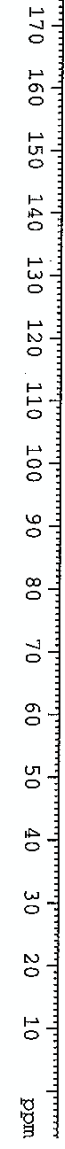

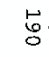

品

章 


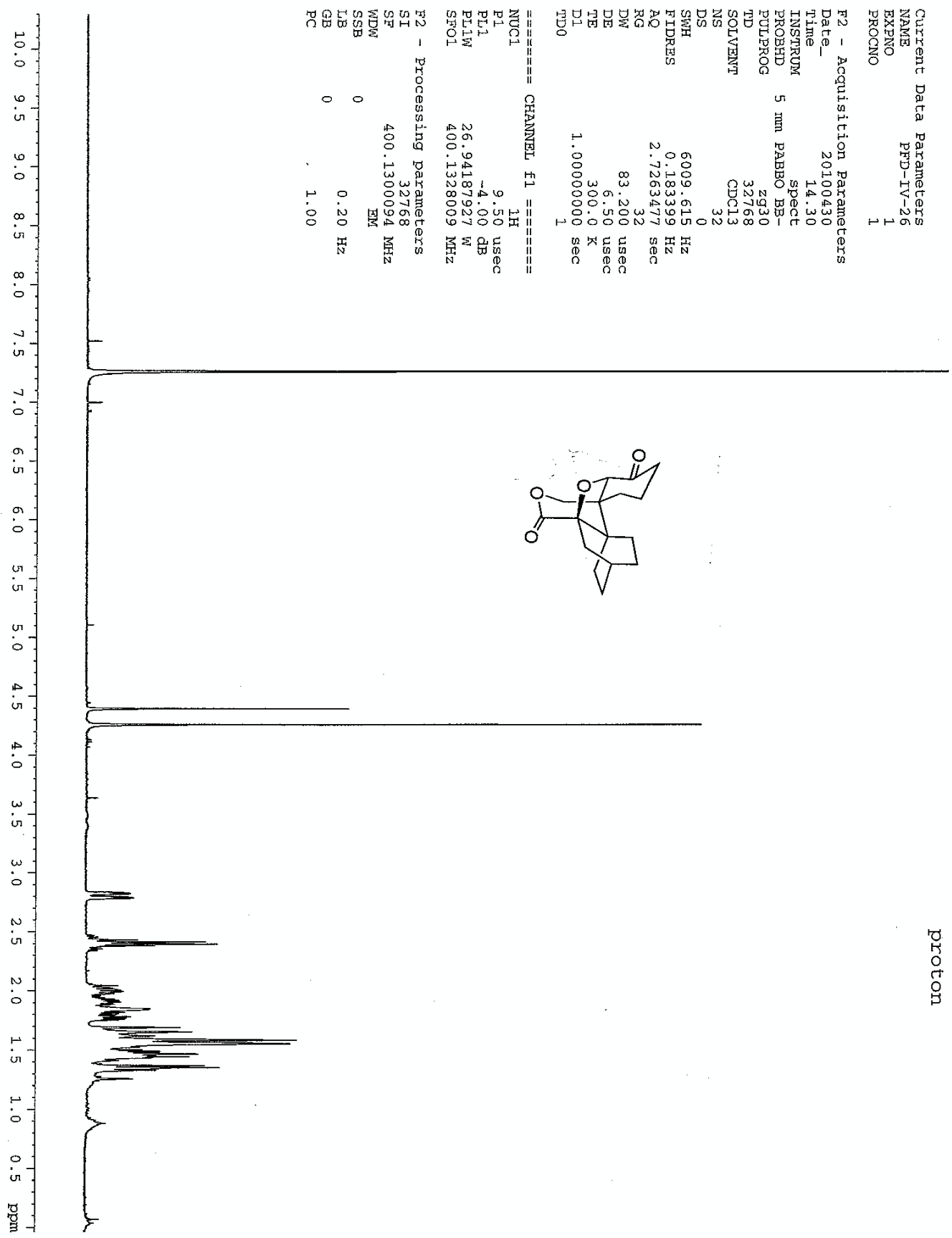



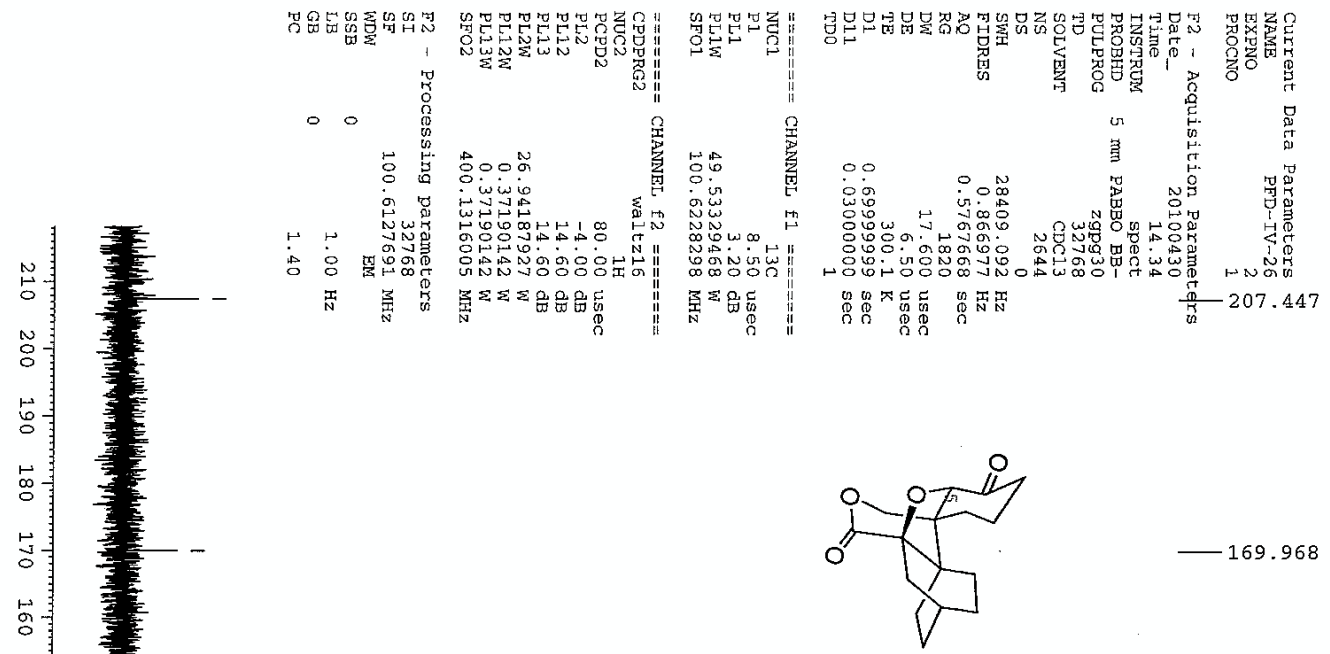

169.968

닝

茄

$\stackrel{b}{\omega}$

芯空

占

客

$\because$

$\stackrel{\infty}{\circ}$

$\because$

웅

ज

吕

w

N

号

骂事

$-49.195$

$-42.381$

$-38.127$

31.074

25.041
.2474

24.072

$-23.932$

22.530

$-21.541$

21.060 


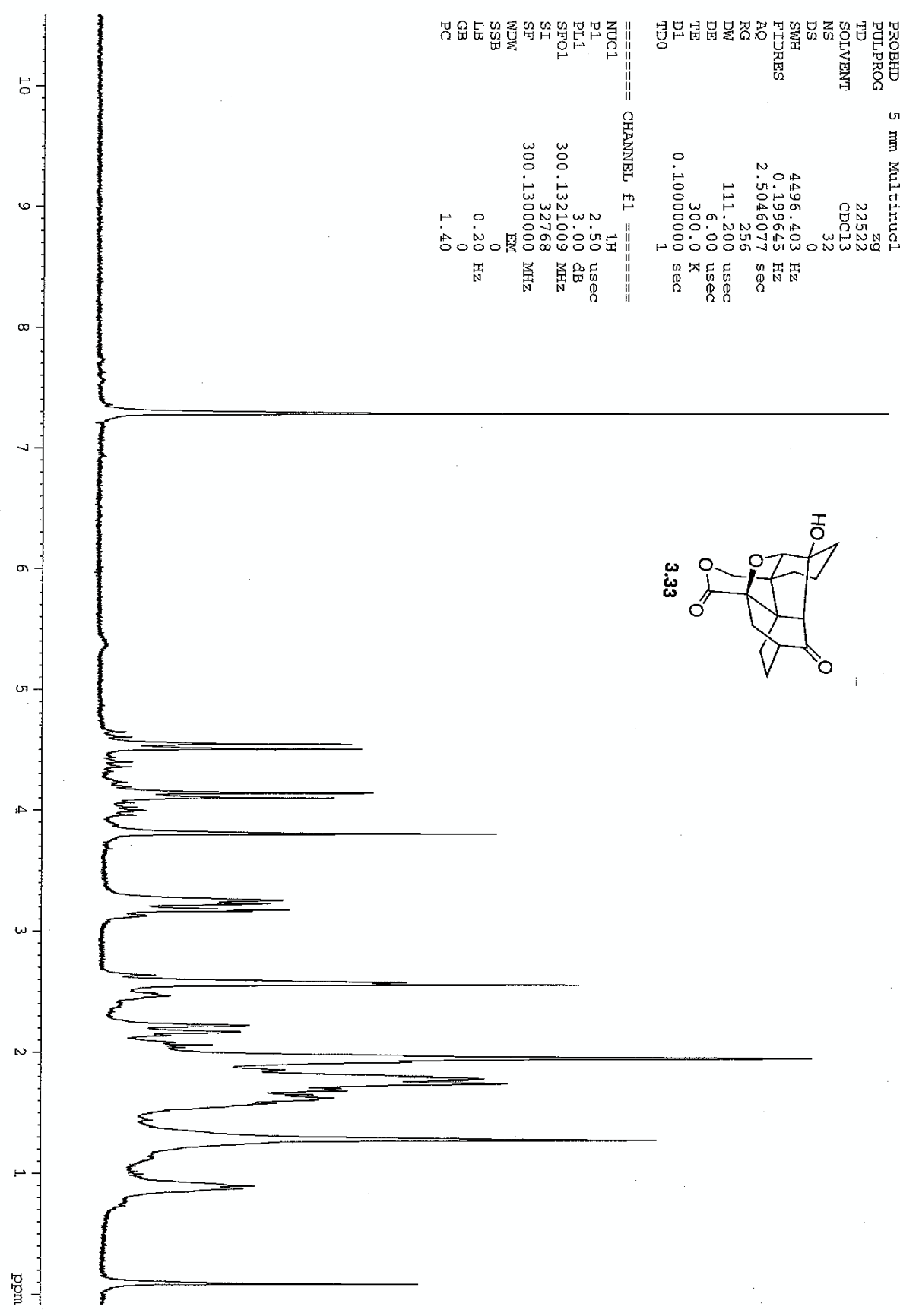




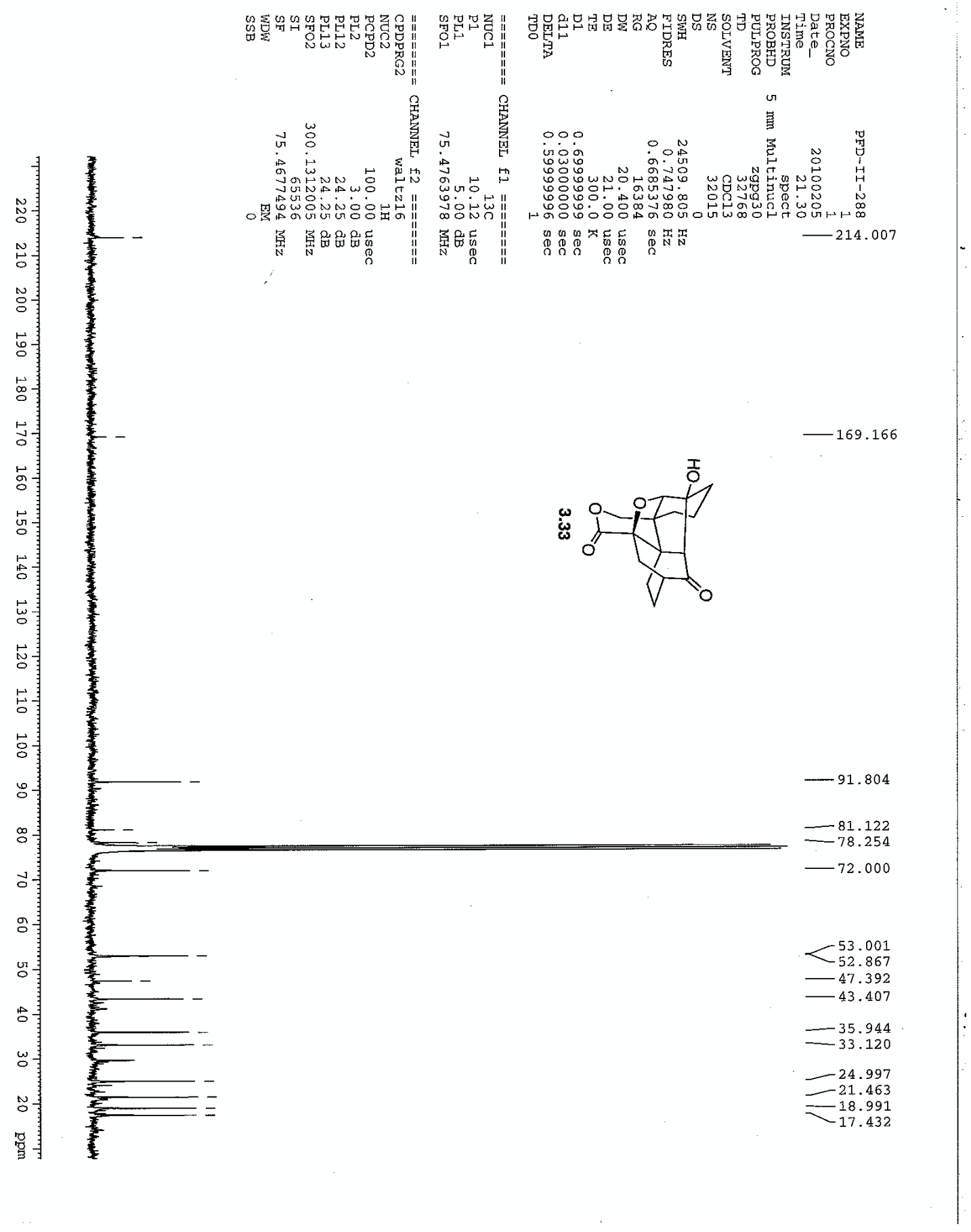




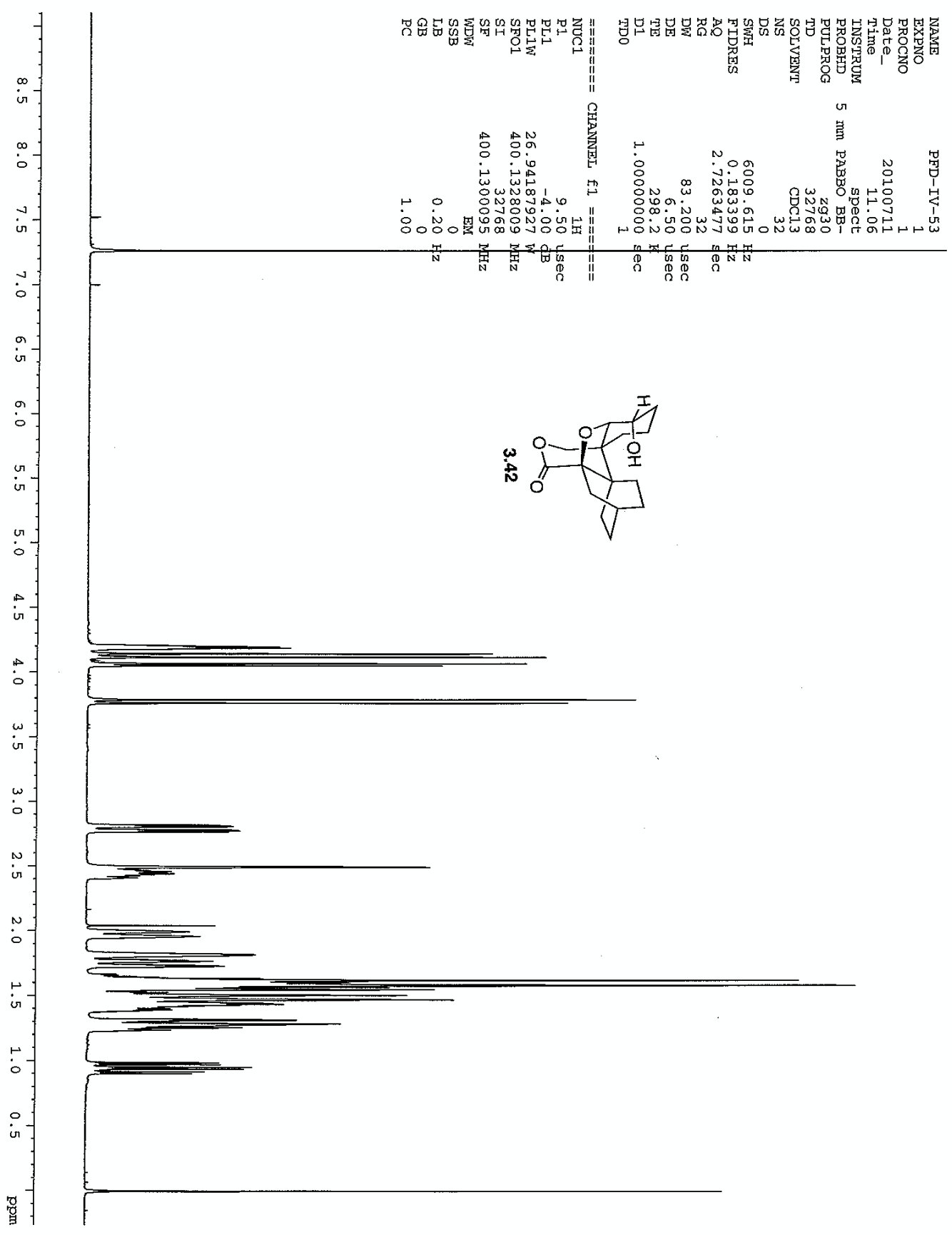




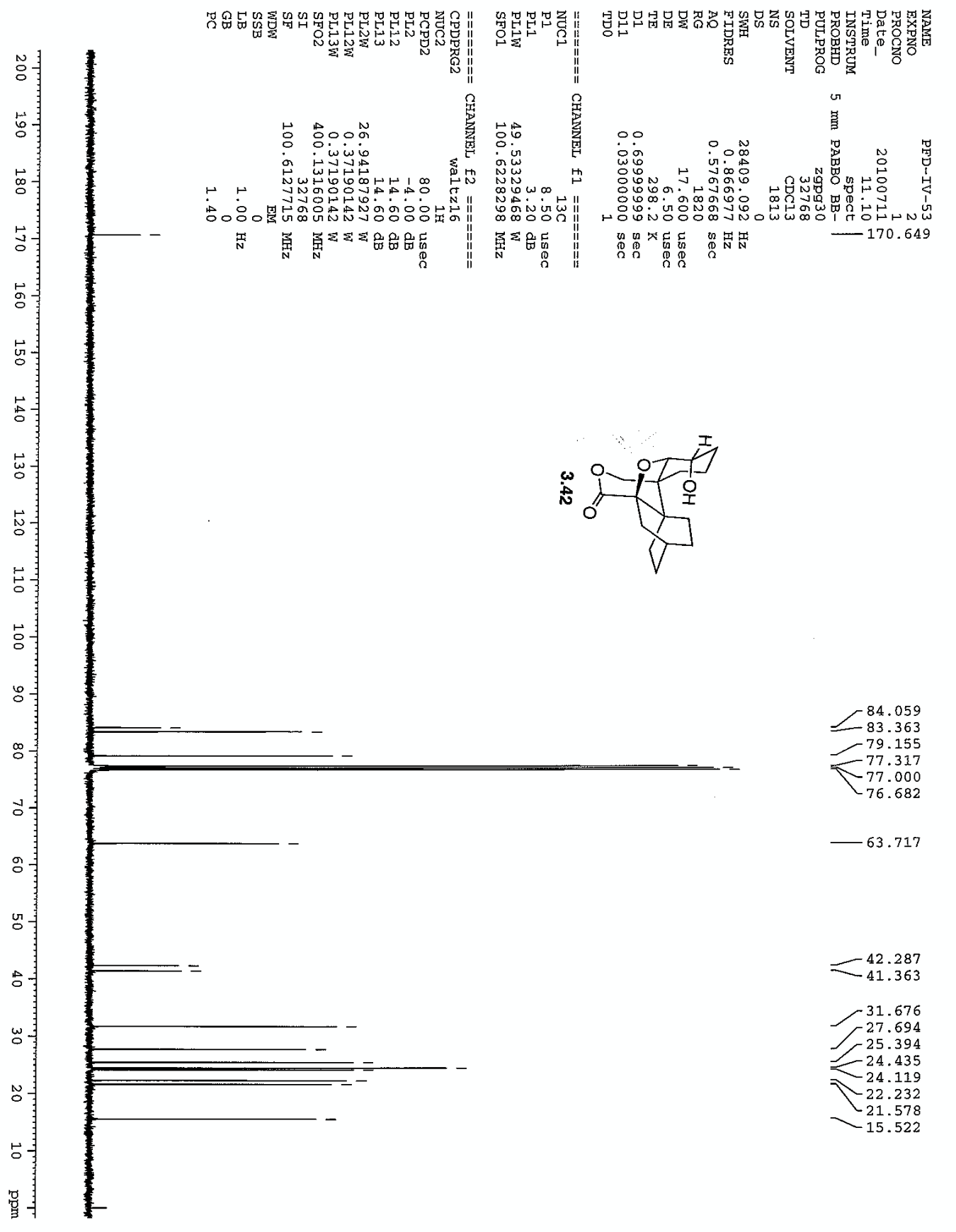




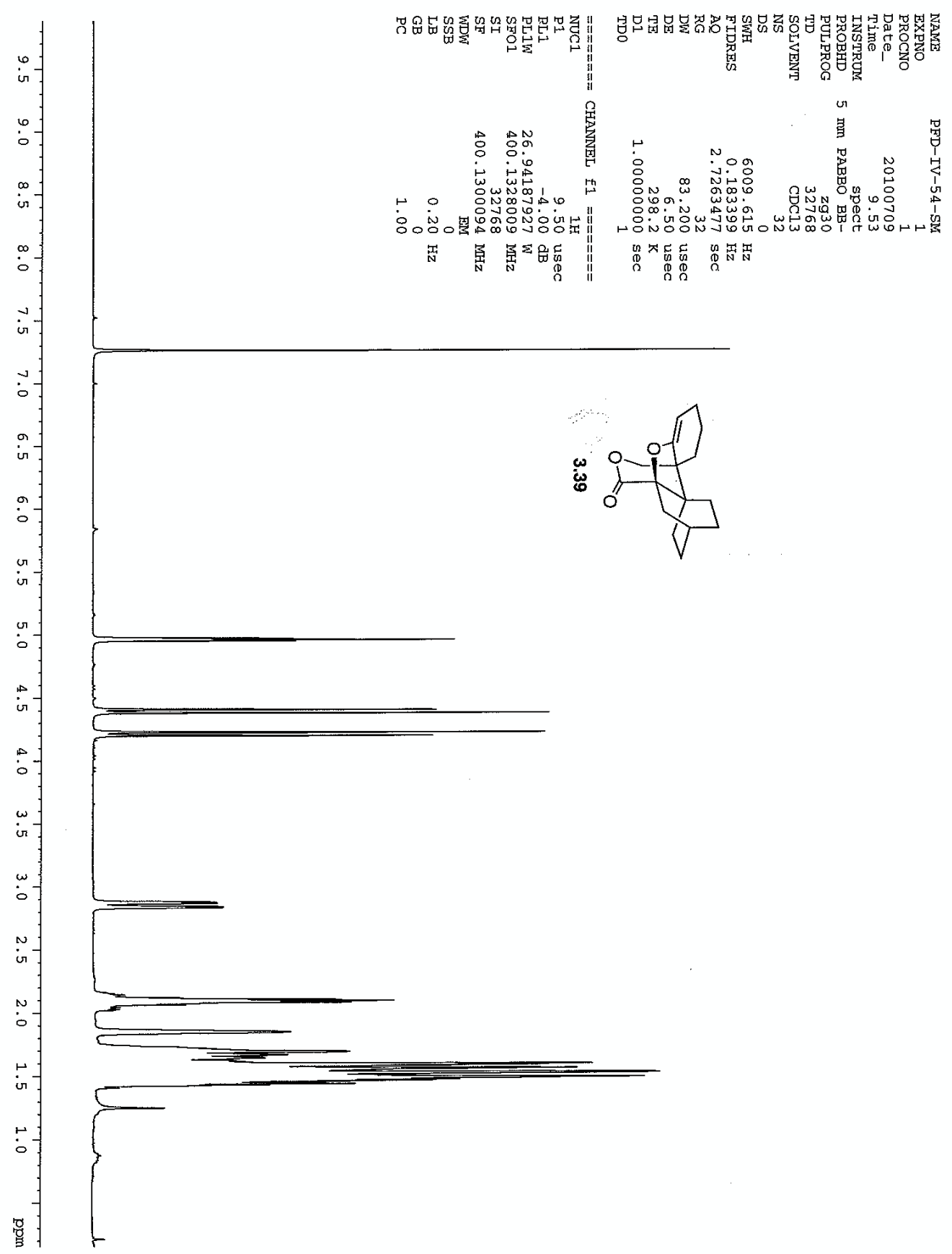




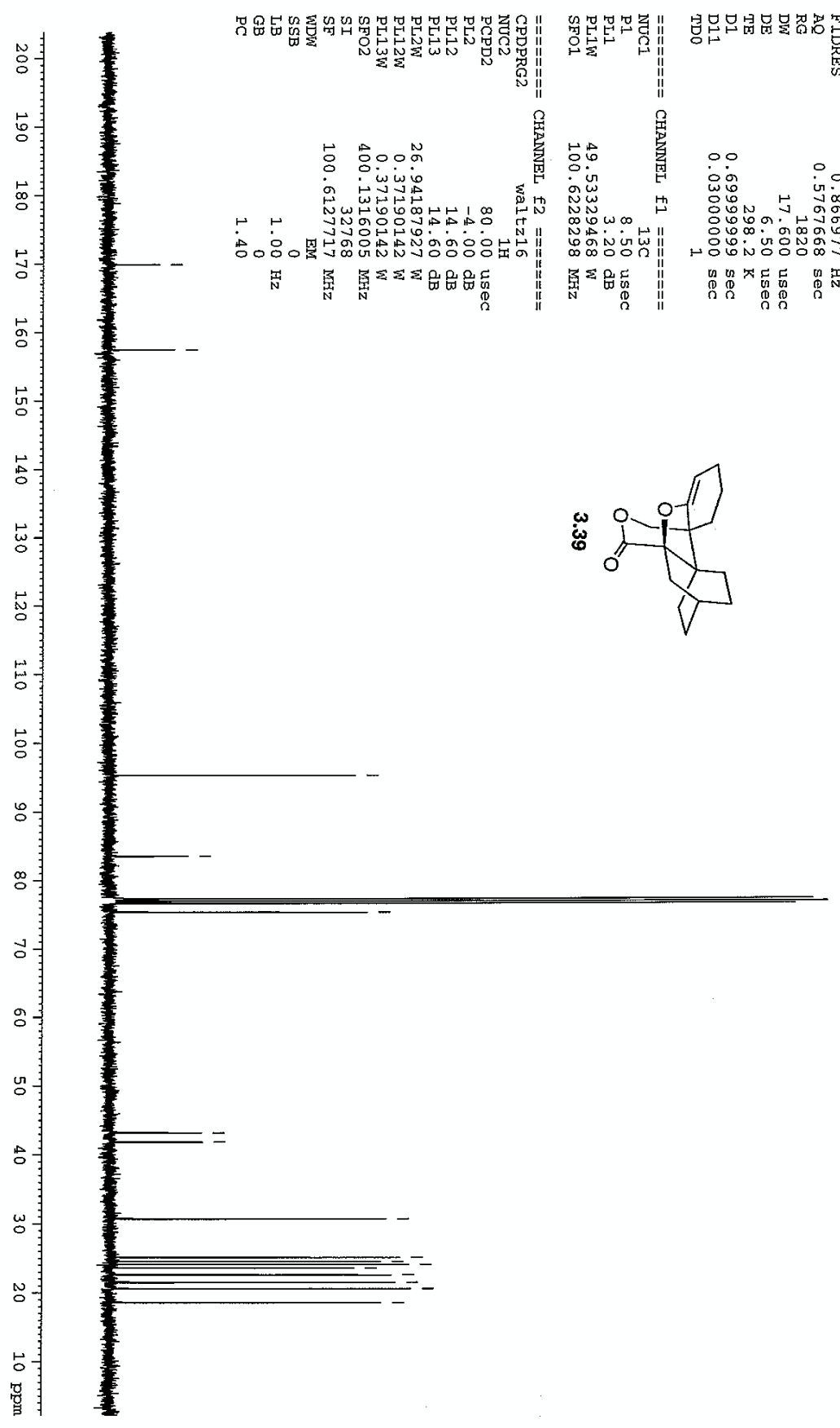

$-95.329$

$-83.523$

$-75.357$

-43.201
-41.853

30.720

25.119

24.539
-24.126 24.126
23.596 $-22.609$

$-21.525$

20.585
18.573 


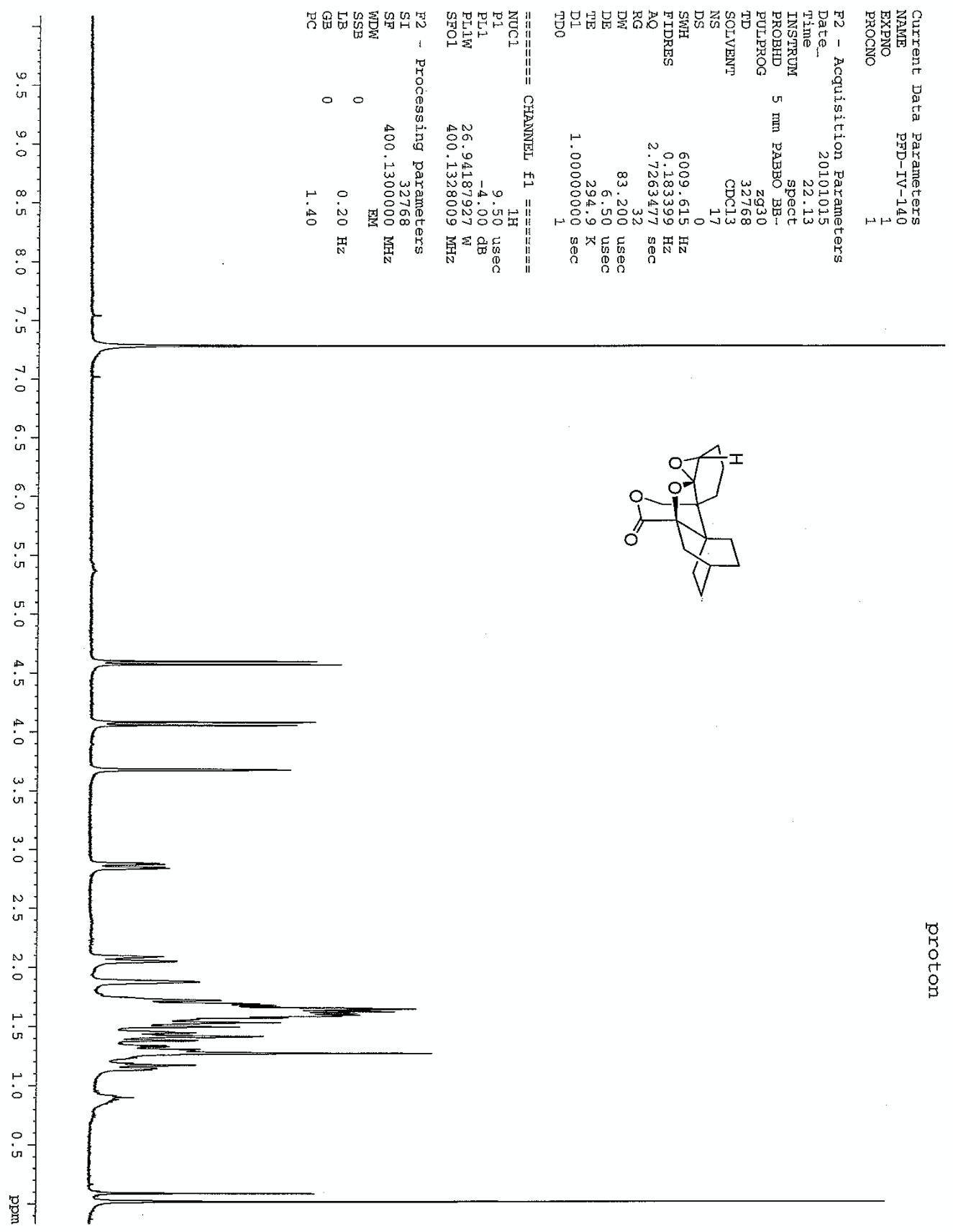




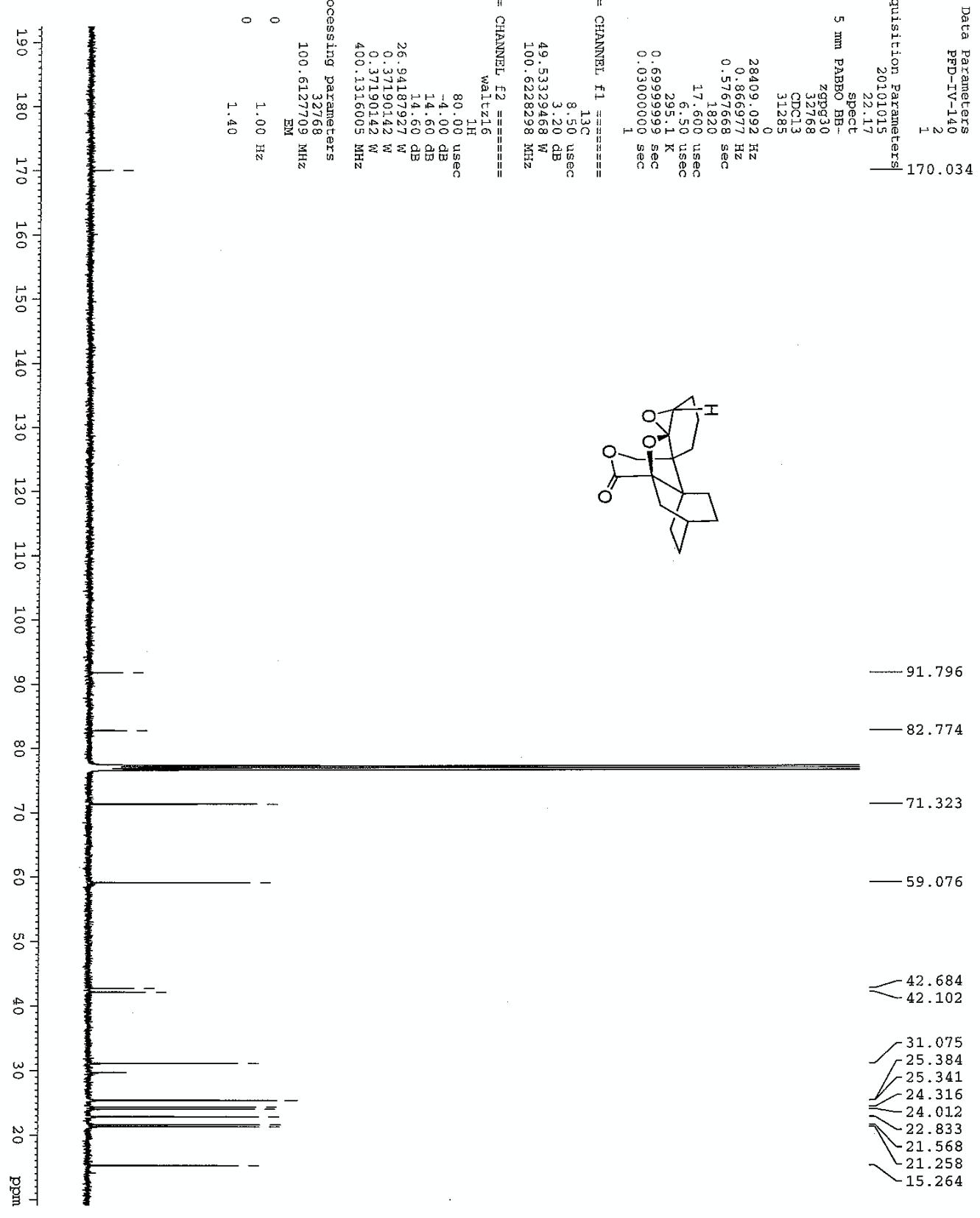




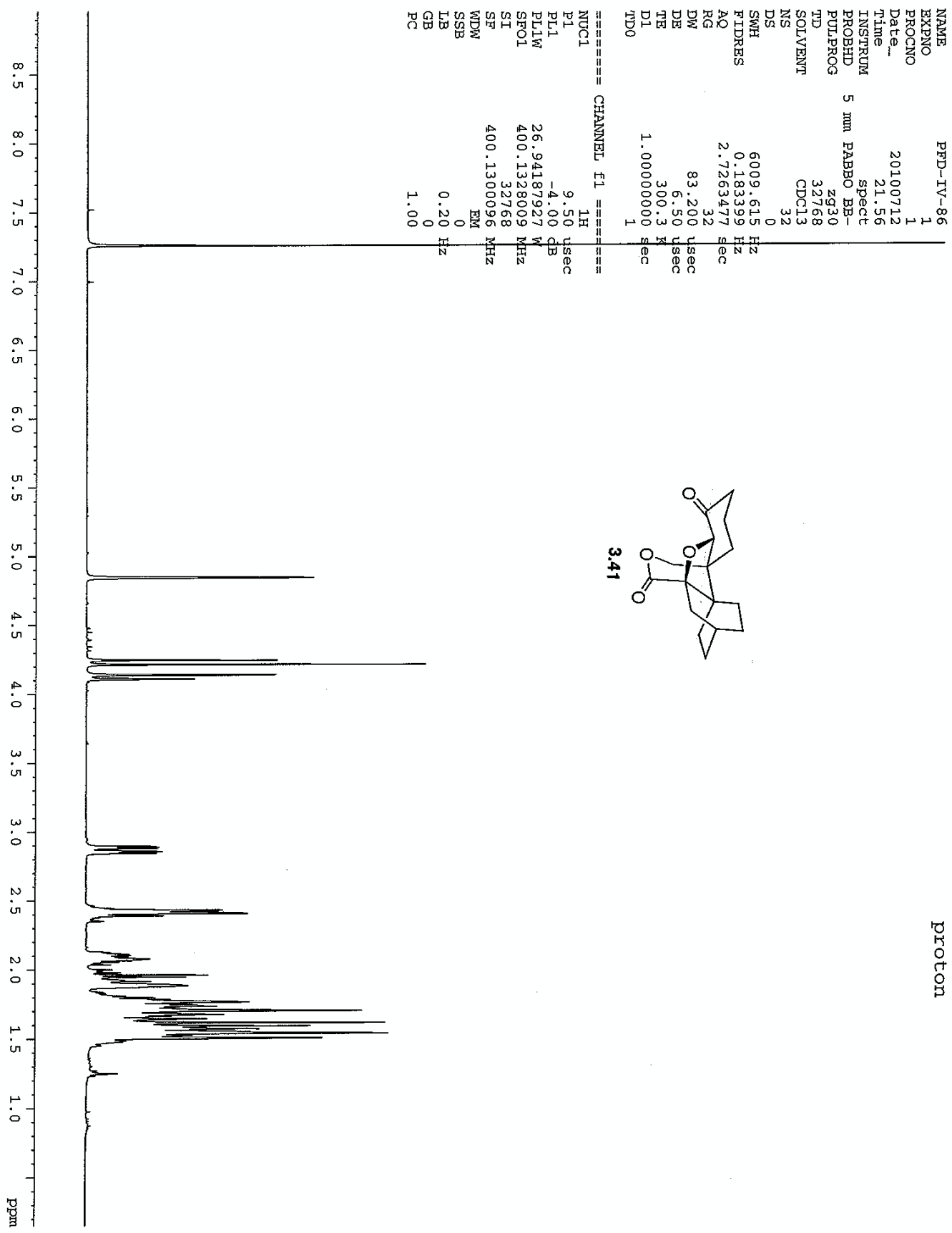




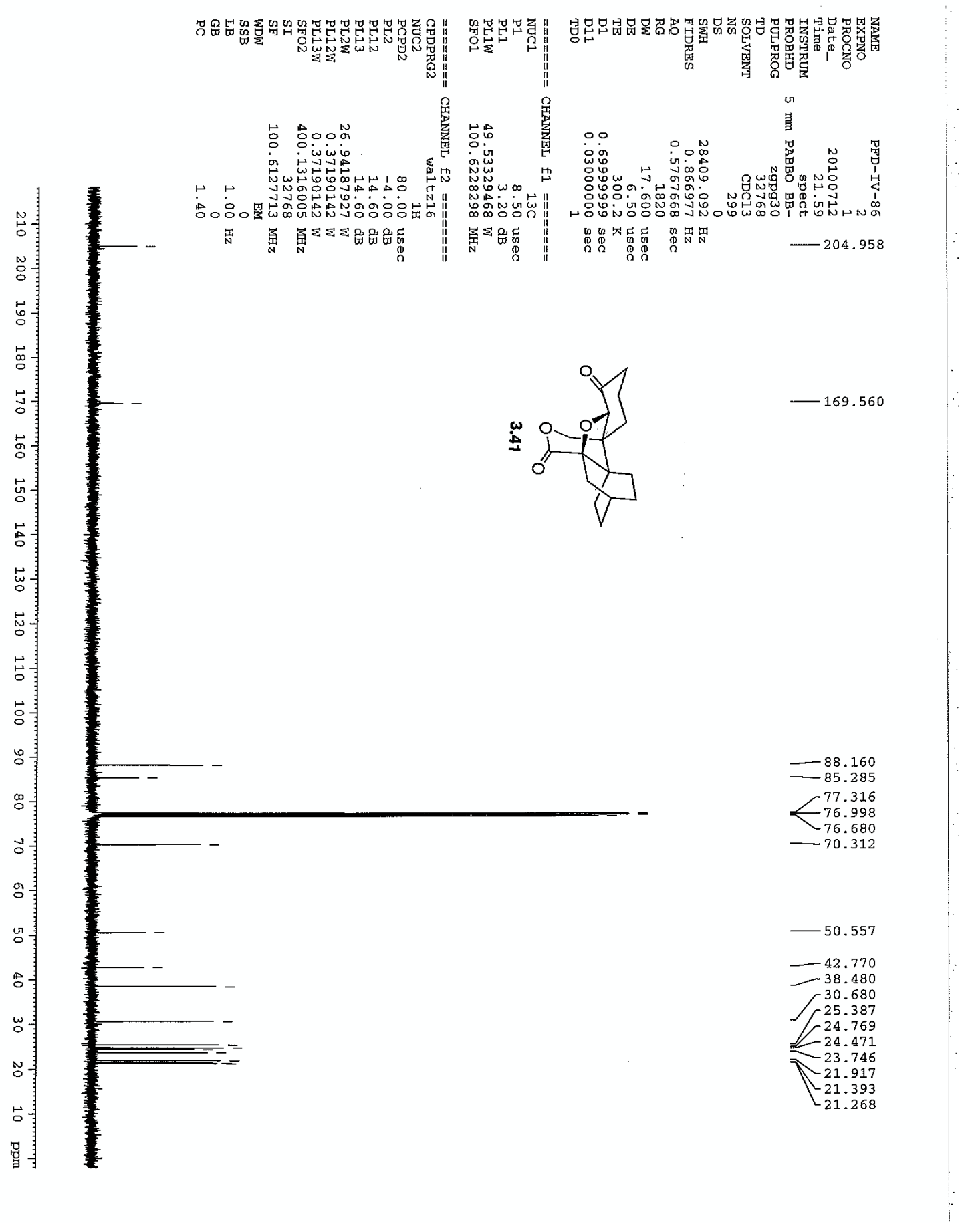




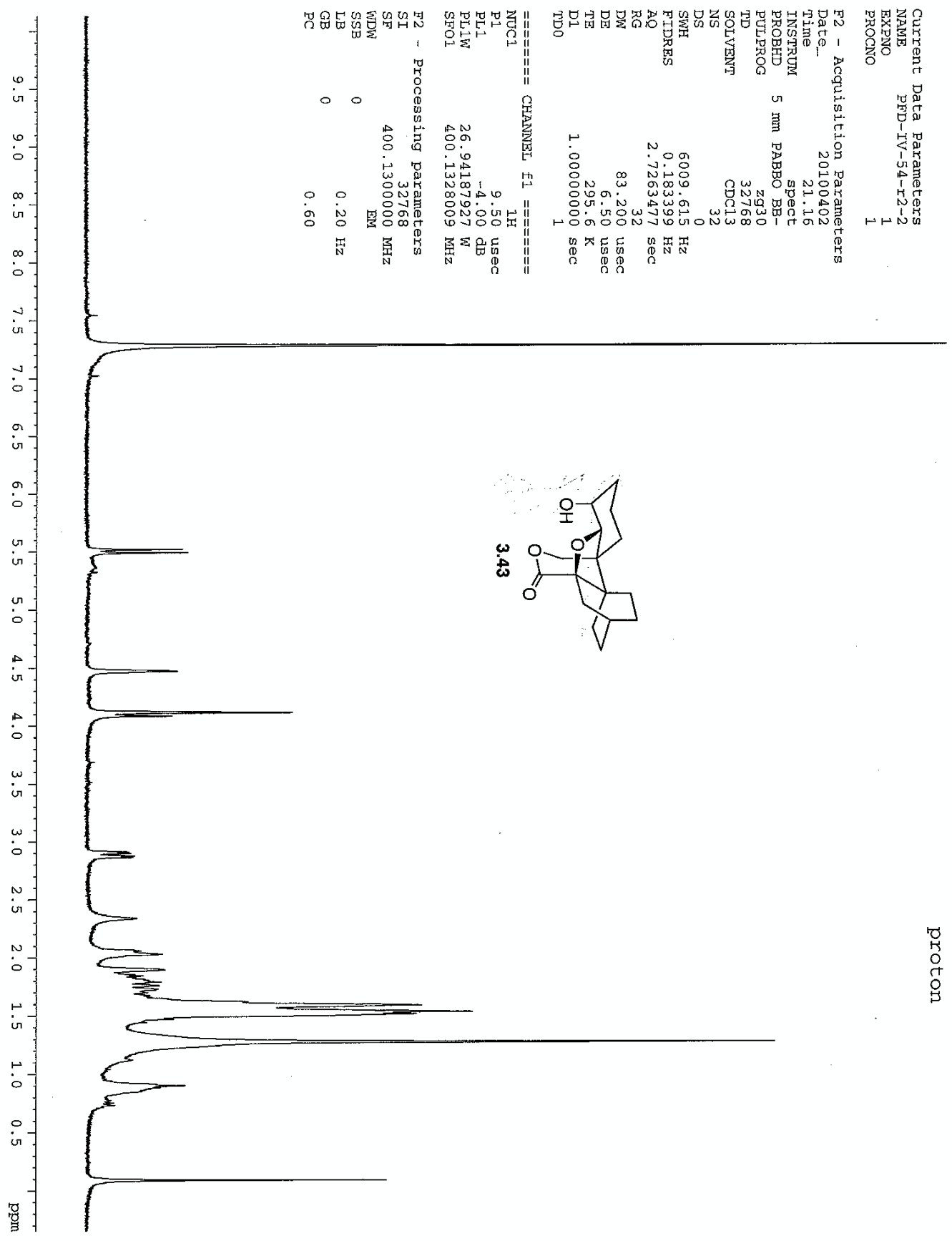




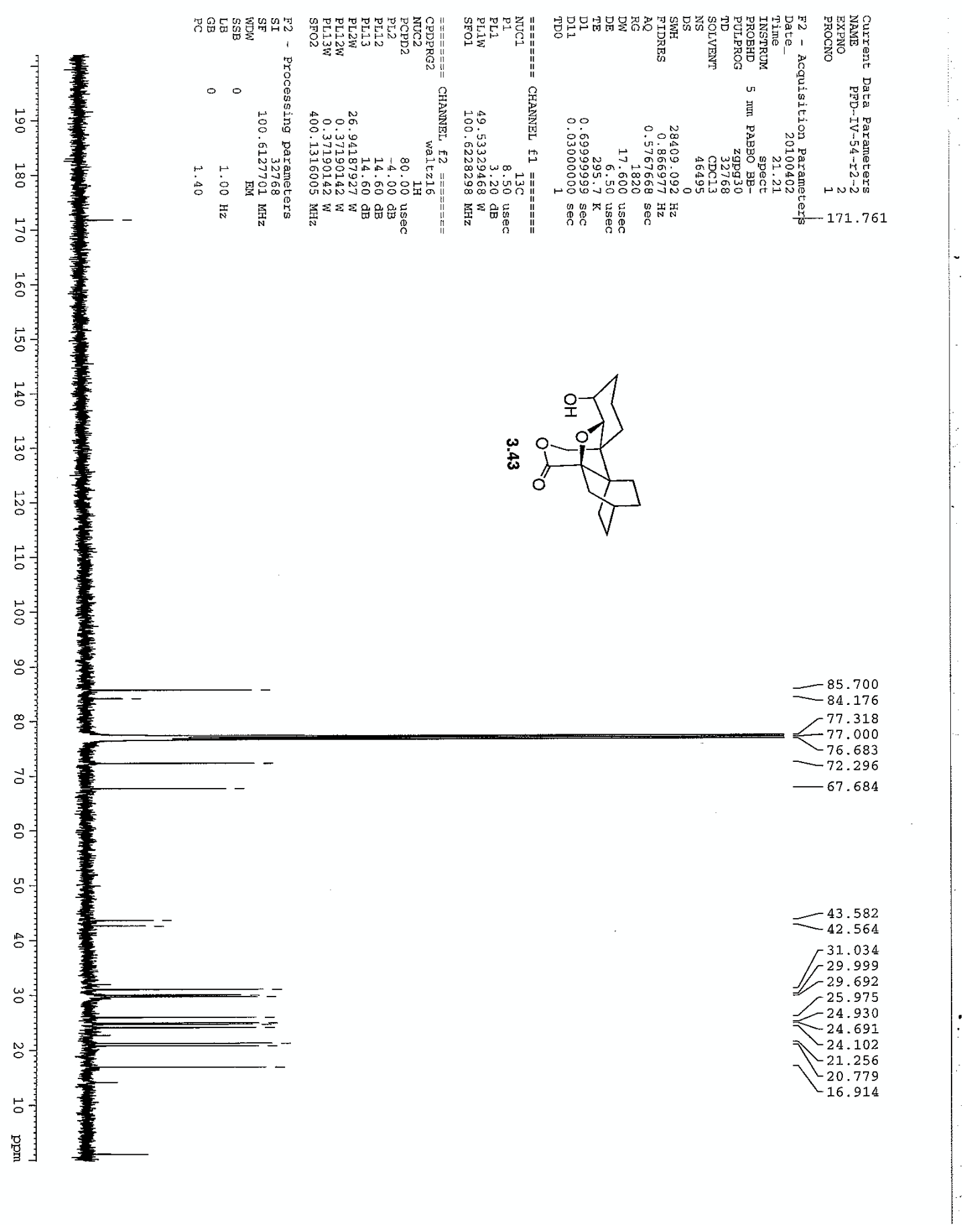




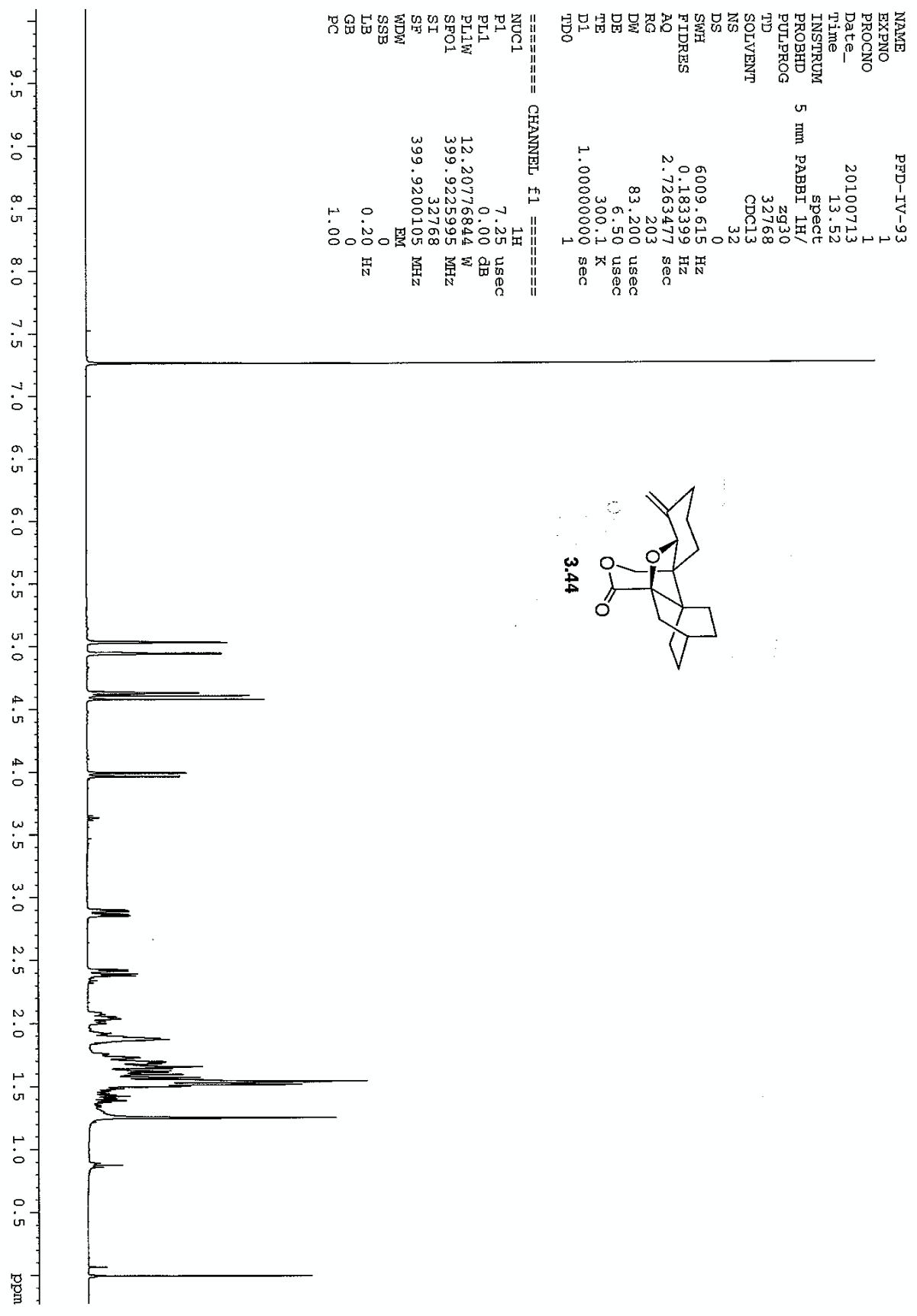




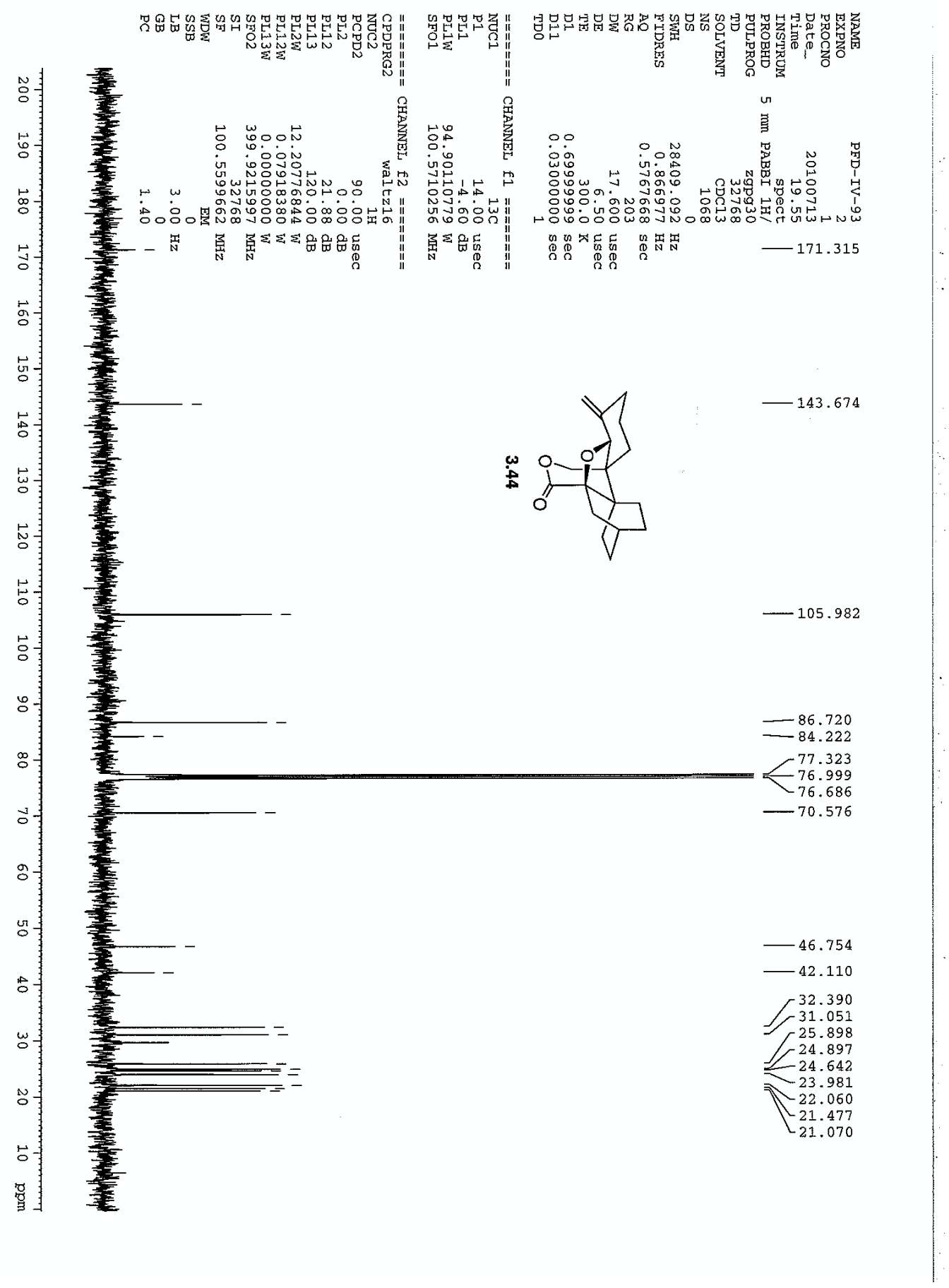




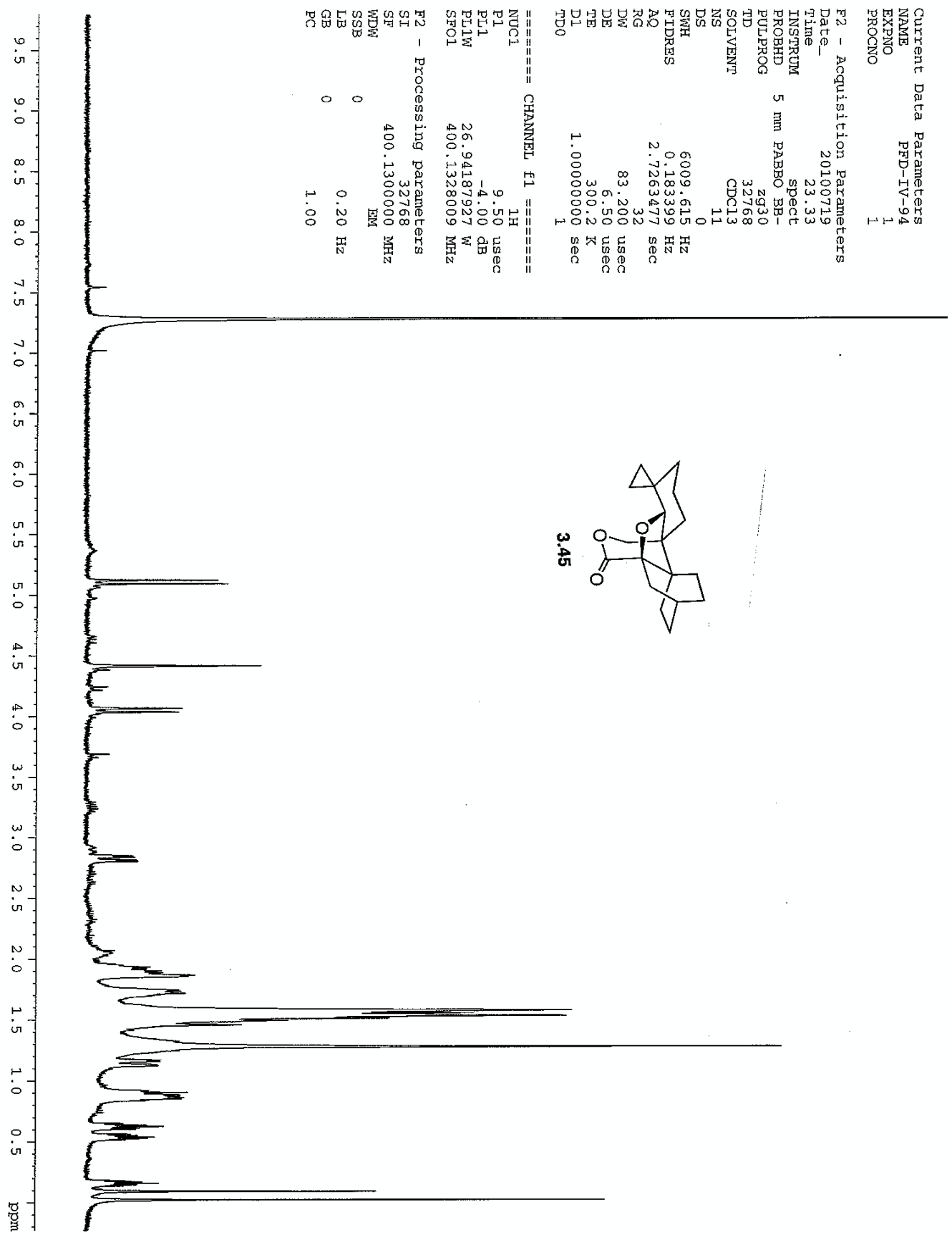




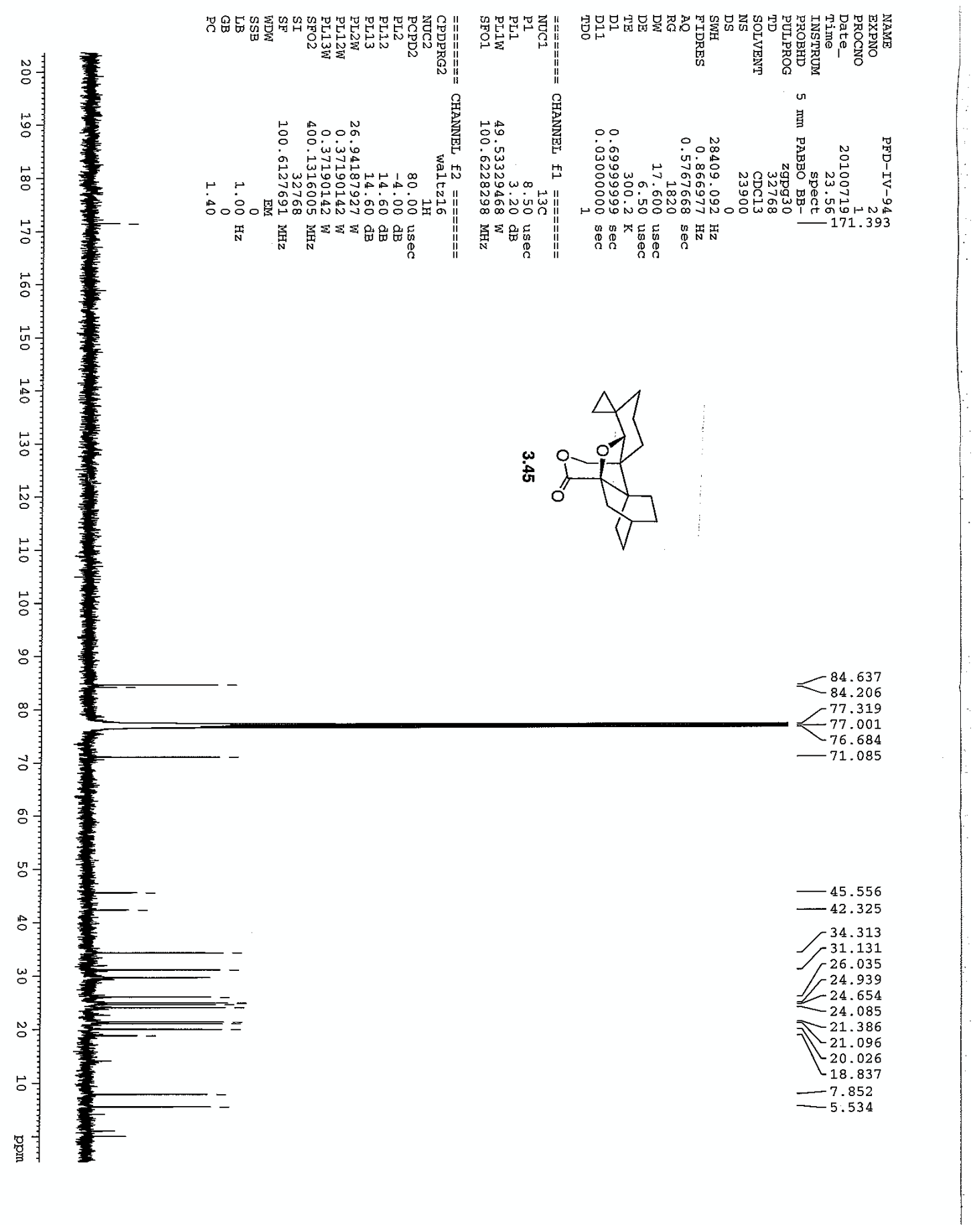




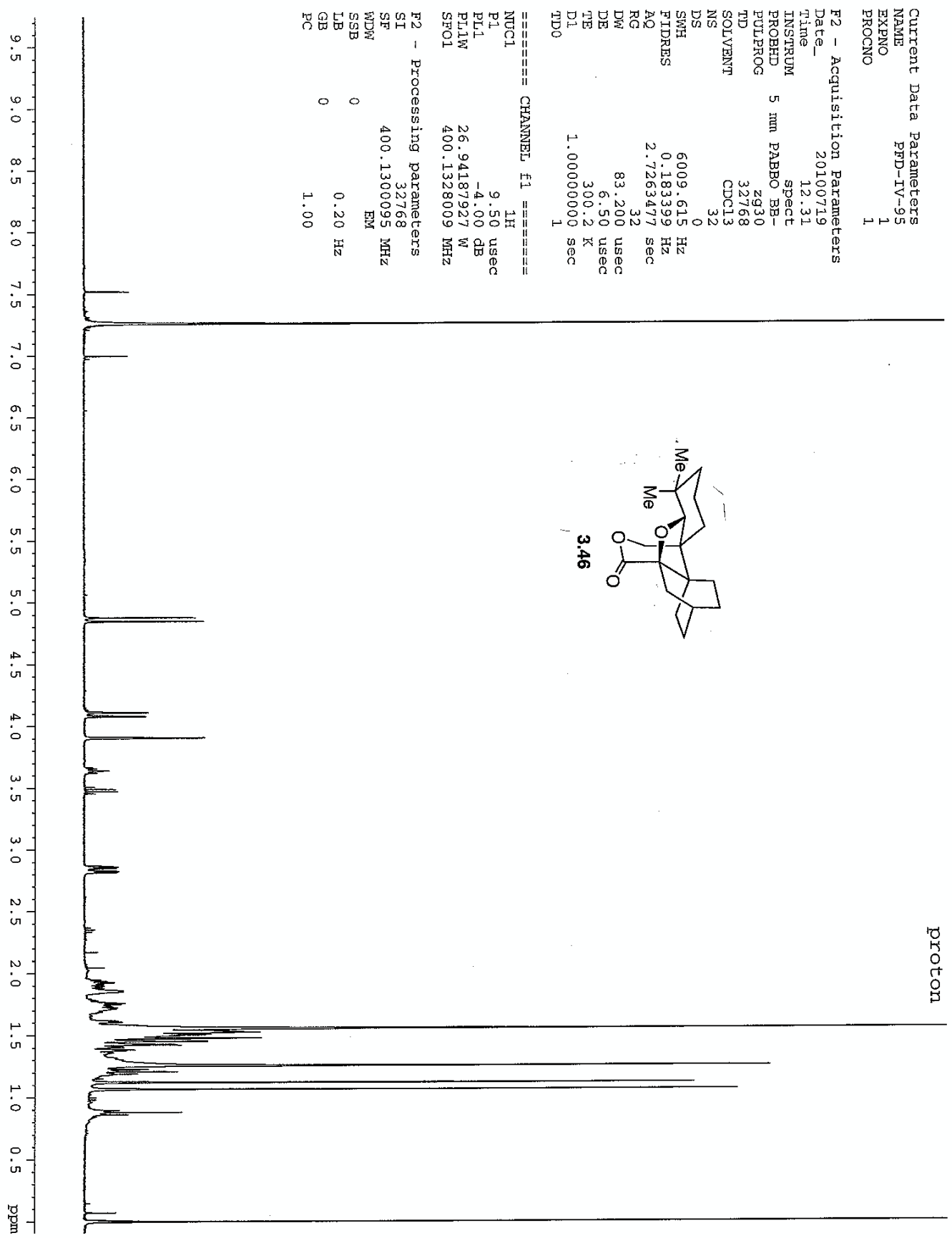




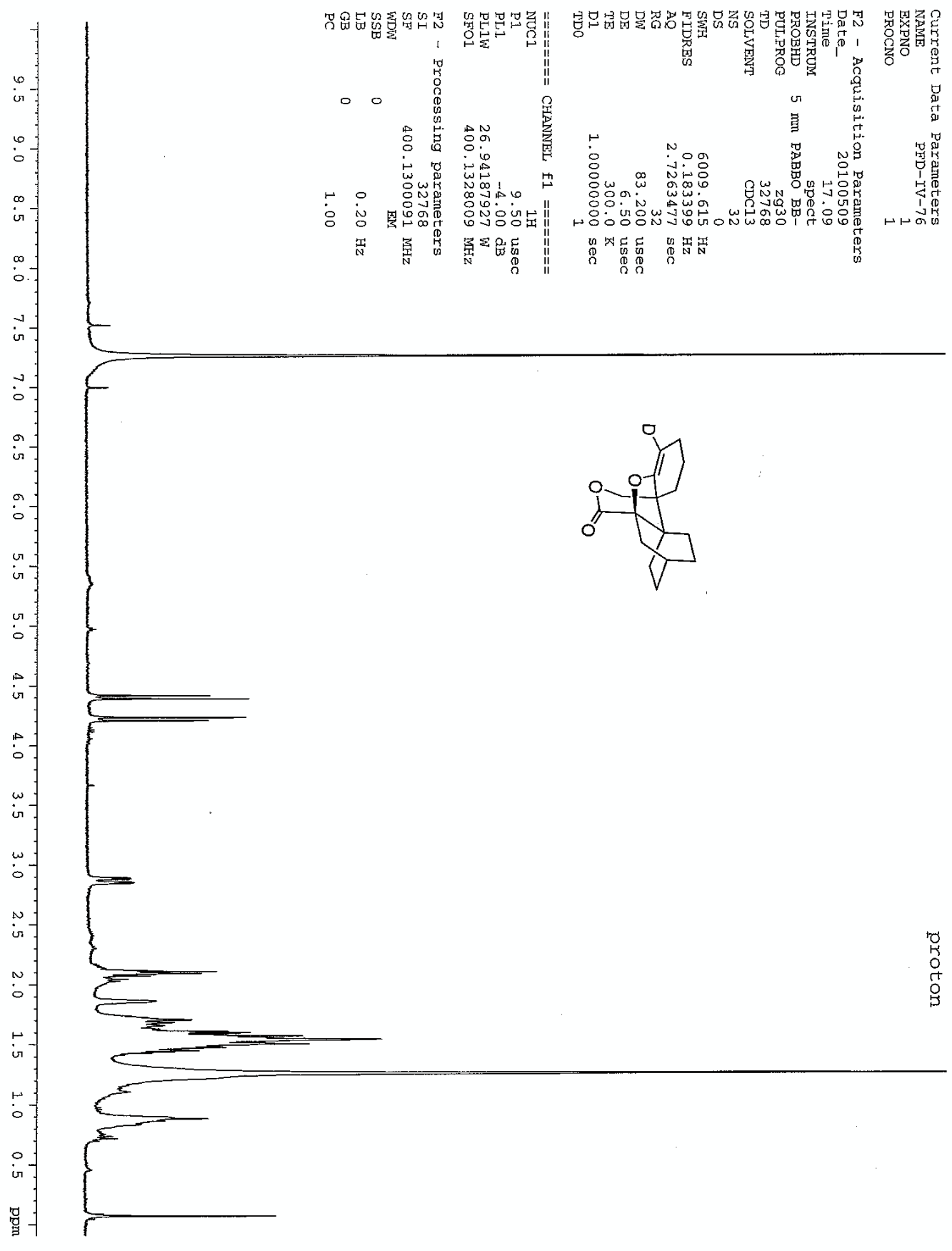




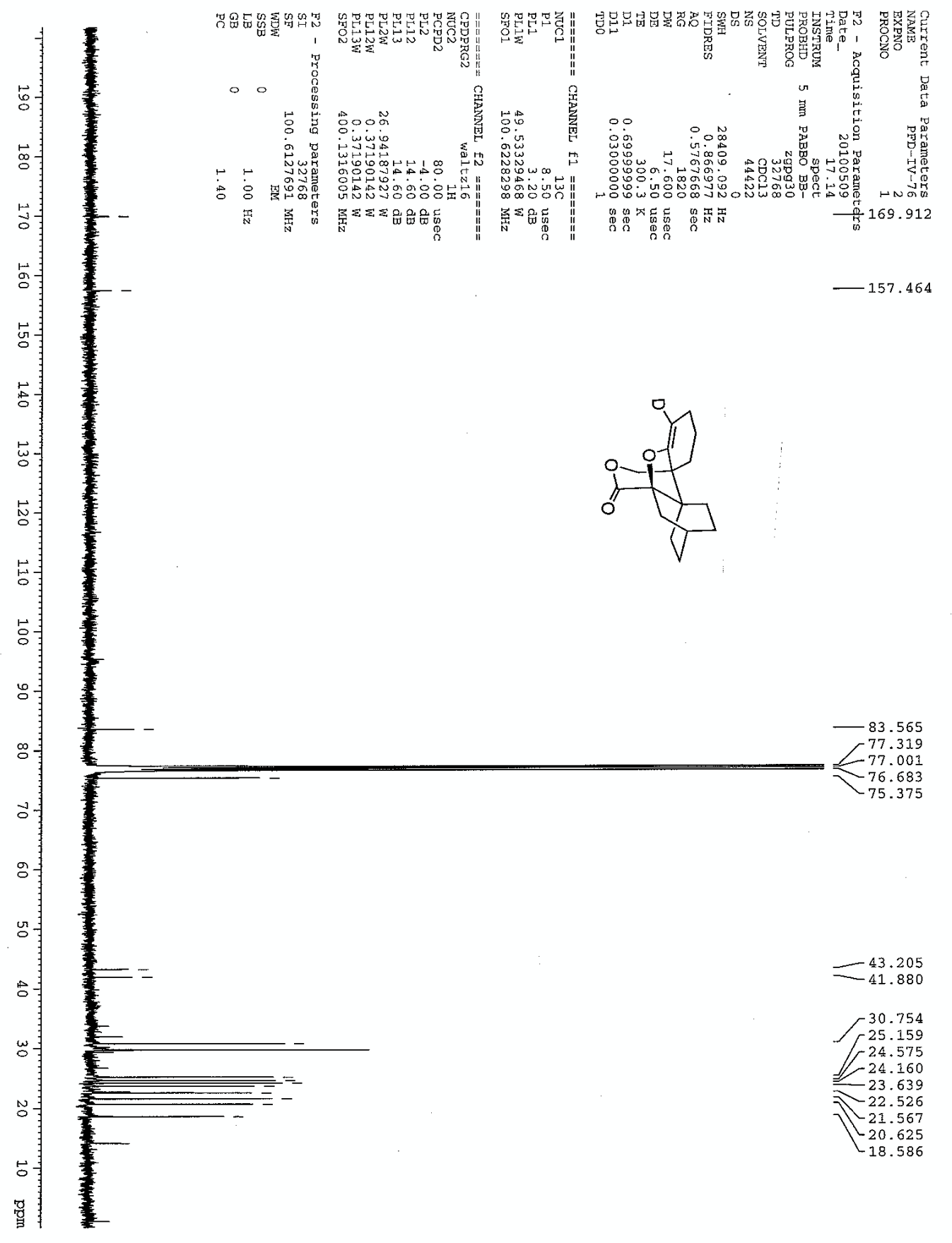




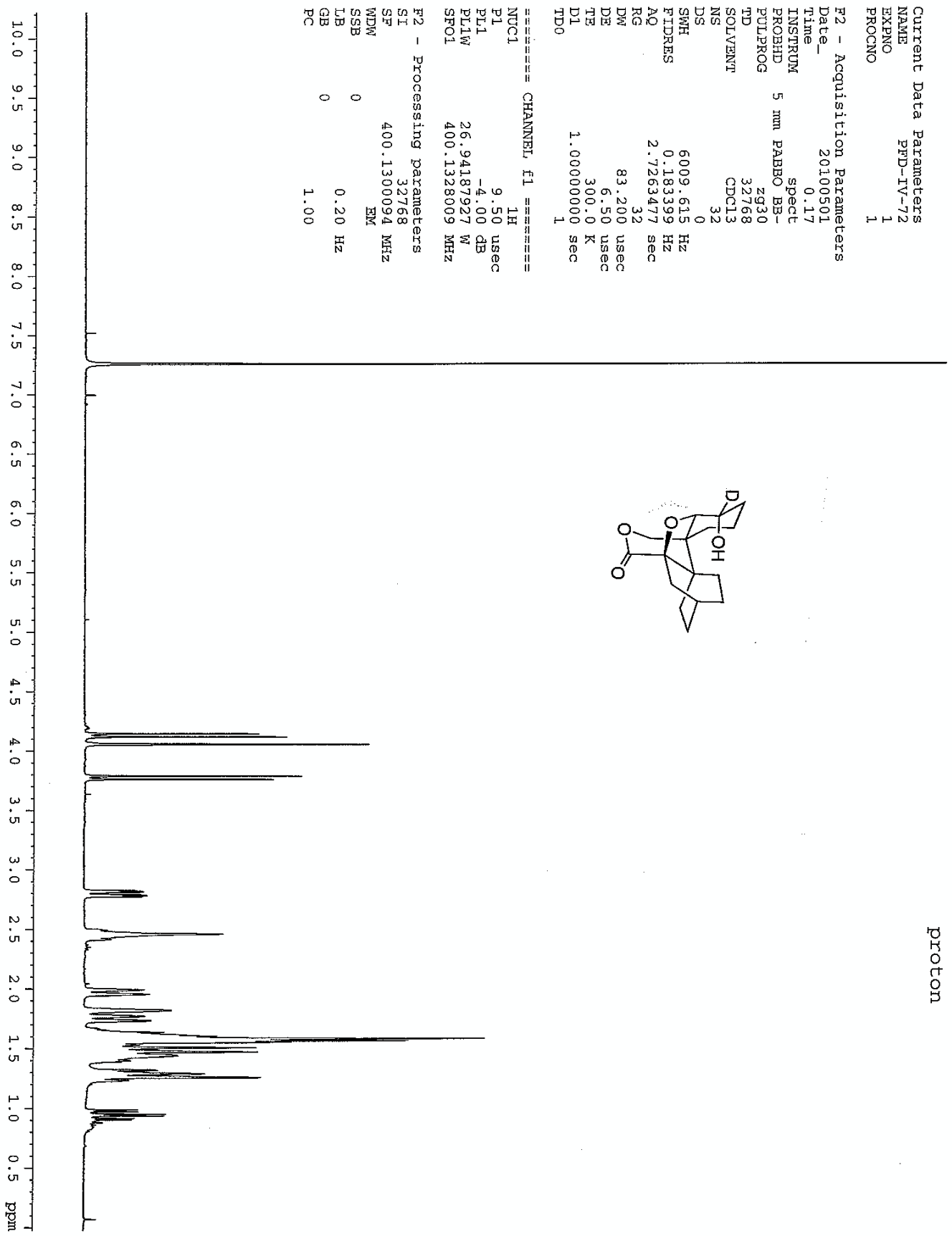




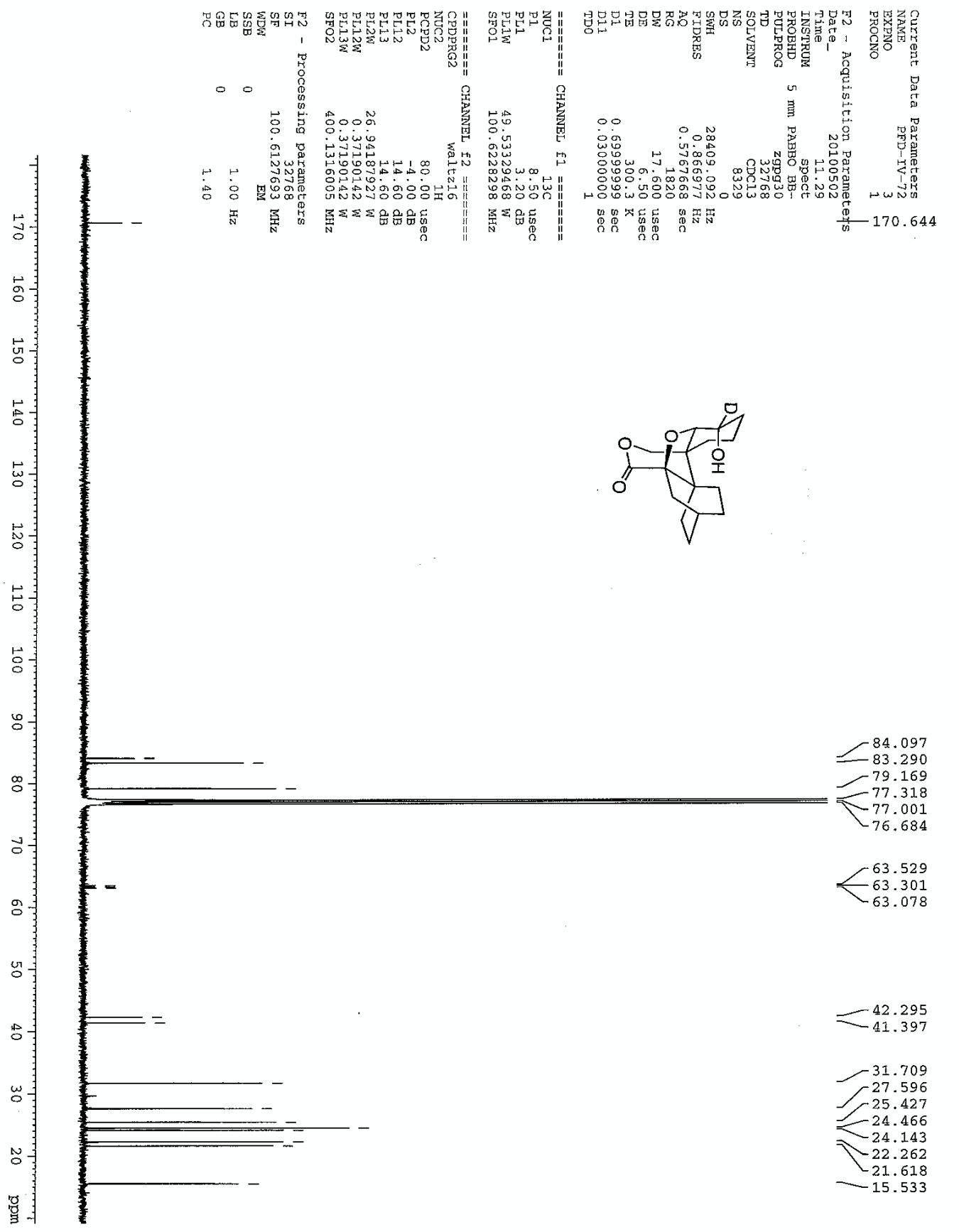




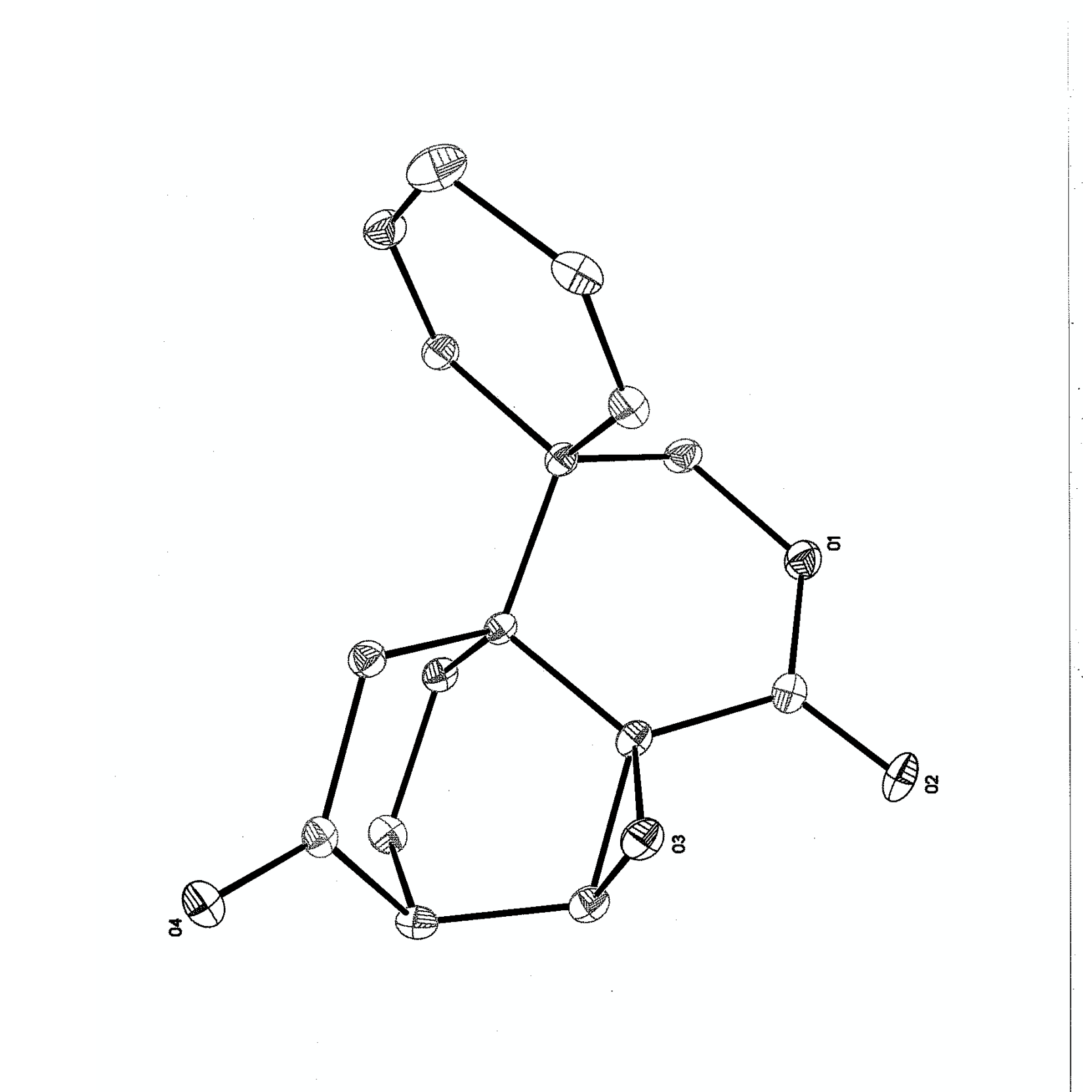


Table 1. Crystal data and structure refinement for FENG3WS10.

\begin{tabular}{|c|c|}
\hline Identification code & feng3ws 10 \\
\hline Empirical formula & $\mathrm{C}_{16^{\mathrm{H}}}{ }_{16^{\circ}}{ }^{\mathrm{O}}$ \\
\hline Formula weight & 272.29 \\
\hline Temperature & $150(2) \mathrm{K}$ \\
\hline Wavelength & $0.71073 \AA$ \\
\hline Crystal system & Monoclinic \\
\hline Space group & $\mathrm{P} 2{ }_{1} / \mathrm{c}$ \\
\hline Unit cell dimensions & $\begin{array}{lll}a=6.837(3) \AA & \text { alpha }=90^{\circ} \\
b=9.269(4) \AA & \text { beta }=93.680(7)^{\circ} \\
c=20.261(8) \AA & \text { gamma }=90^{\circ}\end{array}$ \\
\hline Volume, $\mathbf{z}$ & $1281.3(9) \AA^{3}, 4$ \\
\hline Density (calculated) & $1.412 \mathrm{Mg} / \mathrm{m}^{3}$ \\
\hline Absorption coefficient & $0.101 \mathrm{~mm}^{-1}$ \\
\hline$F(000)$ & 576 \\
\hline Crystal size & $0.12 \times 0.03 \times 0.03 \mathrm{~mm}$ \\
\hline (1) range for data collection & 2.01 to $28.28^{\circ}$ \\
\hline Limiting indices & $-9 \leq h \leq 9,-12 \leq k \leq 12,-26 \leq 1 \leq 26$ \\
\hline Reflections collected & 17277 \\
\hline Independent reflections & $3181\left(R_{\text {int }}=0.1389\right)$ \\
\hline Completeness to $\theta=28.28^{\circ}$ & $100.0 \%$ \\
\hline Absorption correction & EMPIRICAL \\
\hline Max. and min. transmission & 0.9970 and 0.9880 \\
\hline Refinement method & Ful1-matrix least-squares on $\mathrm{F}^{2}$ \\
\hline Data / restraints / parameters & $3181 / 0 / 181$ \\
\hline Goodness-of-fit on $F^{2}$ & 1.031 \\
\hline Final $R$ indices $[I>2 \sigma(I)]$ & $R 1=0.0730, w R 2=0.1549$ \\
\hline R indices (all data) & $\mathrm{R} 1=0.1497, \mathrm{wR2}=0.1846$ \\
\hline Largest diff. peak and $h$ & 0.297 and $-0.328 \mathrm{et}^{-3}$ \\
\hline
\end{tabular}


Table 2. Atomic coordinates $\left[\mathbf{x} 10^{4}\right]$ and equivalent isotropic displacement parameters $\left[\AA^{2} \times 10^{3}\right]$ for FENG3WS10. U(eq) is defined as one third of the trace of the orthogonalized $U_{i f}$ tensor.

\begin{tabular}{|c|c|c|c|c|}
\hline & $\mathrm{x}$ & $\mathrm{y}$ & $\mathbf{z}$ & $U(\mathrm{eq})$ \\
\hline$O(1)$ & $4434(3)$ & $5833(2)$ & $1112(1)$ & $31(1)$ \\
\hline$O(2)$ & $6850(3)$ & $4965(3)$ & $573(1)$ & $32(1)$ \\
\hline $0(3)$ & $5896(3)$ & $2123(2)$ & $938(1)$ & $27(1)$ \\
\hline$O(4)$ & $2687(3)$ & $-852(2)$ & $922(1)$ & $29(1)$ \\
\hline$c(1)$ & $2373(4)$ & $4158(3)$ & $1742(2)$ & $22(1)$ \\
\hline$c(2)$ & $375(5)$ & $4123(4)$ & $2023(2)$ & $27(1)$ \\
\hline$c(3)$ & $89(5)$ & $3815(4)$ & $2642(2)$ & $32(1)$ \\
\hline$C(4)$ & $1674(6)$ & $3479(5)$ & $3150(2)$ & $46(1)$ \\
\hline$c(5)$ & $3668(5)$ & $3598(4)$ & $2895(2)$ & $31(1)$ \\
\hline$C(6)$ & $3967(5)$ & $3881(3)$ & $2273(2)$ & $27(1)$ \\
\hline$C(7)$ & $2629(5)$ & $5659(4)$ & $1446(2)$ & $27(1)$ \\
\hline$C(8)$ & $5330(5)$ & $4731(3)$ & $832(2)$ & $24(1)$ \\
\hline$c(9)$ & $4433(4)$ & $3252(3)$ & $852(2)$ & $22(1)$ \\
\hline$C(10)$ & $4736(5)$ & $2240(3)$ & $316(2)$ & $26(1)$ \\
\hline$C(11)$ & $3047(5)$ & $1196(3)$ & $212(2)$ & $26(1)$ \\
\hline$C(12)$ & $2729(4)$ & $451(3)$ & $859(2)$ & $23(1)$ \\
\hline $\mathrm{c}(13)$ & $2378(5)$ & $1490(3)$ & $1412(2)$ & $24(1)$ \\
\hline$C(14)$ & $2500(4)$ & $3046(3)$ & $1170(1)$ & $18(1)$ \\
\hline$c(15)$ & $915(4)$ & $3235(3)$ & $601(2)$ & $24(1)$ \\
\hline$c(16)$ & $1183(5)$ & $2110(4)$ & $51(2)$ & $29(1)$ \\
\hline
\end{tabular}


Table 3. Bond lengths $[\AA \AA \cap]$ and angles $\left[{ }^{\circ}\right]$ for FENG 3 wS 10 .

\begin{tabular}{|c|c|c|c|}
\hline$o(1)-C(8)$ & $1.336(4)$ & $O(1)-C(7)$ & $1.454(4)$ \\
\hline$O(2)-C(8)$ & $1.213(4)$ & $o(3)-C(10)$ & $1.449(4)$ \\
\hline$o(3)-c(9)$ & $1.450(4)$ & $O(4)-C(12)$ & $1.215(4)$ \\
\hline$C(1)-C(6)$ & $1.503(4)$ & $C(1)-C(2)$ & $1.514(4)$ \\
\hline$C(1)-C(7)$ & $1.530(4)$ & $C(1)-C(14)$ & $1.558(4)$ \\
\hline$C(2)-C(3)$ & $1.313(5)$ & $C(3)-C(4)$ & $1.478(5)$ \\
\hline$c(4)-C(5)$ & $1.494(5)$ & $C(5)-C(6)$ & $1.316(5)$ \\
\hline$C(8)-C(9)$ & $1.504(4)$ & $C(9)-C(10)$ & $1.460(4)$ \\
\hline$c(9)-c(14)$ & $1.519(4)$ & $c(10)-c(11)$ & $1.511(5)$ \\
\hline$C(11)-C(12)$ & $1.510(4)$ & $C(11)-C(16)$ & $1.548(4)$ \\
\hline$C(12)-C(13)$ & $1.507(4)$ & $C(13)-C(14)$ & $1.527(4)$ \\
\hline$c(14)-C(15)$ & $1.541(4)$ & $C(15)-C(16)$ & $1.546(4)$ \\
\hline$C(8)-O(1)-C(7)$ & $122.6(3)$ & $C(10)-O(3)-C(9)$ & $60.47(19)$ \\
\hline$C(6)-C(1)-C(2)$ & $110.9(3)$ & $C(6)-C(1)-C(7)$ & $109.9(3)$ \\
\hline$C(2)-C(1)-C(7)$ & $107.2(3)$ & $C(6)-C(1)-C(14)$ & $110.3(2)$ \\
\hline$C(2)-C(1)-C(14)$ & $111.1(2)$ & $C(7)-C(1)-C(14)$ & $107.3(2)$ \\
\hline$C(3)-C(2)-C(1)$ & $123.9(3)$ & $C(2)-C(3)-C(4)$ & $124.3(3)$ \\
\hline$C(3)-C(4)-C(5)$ & $112.8(3)$ & $C(6)-C(5)-C(4)$ & $123.2(3)$ \\
\hline$C(5)-C(6)-C(1)$ & $124.7(3)$ & $O(1)-C(7)-C(1)$ & $113.9(3)$ \\
\hline$o(2)-C(8)-O(1)$ & $118.4(3)$ & $O(2)-C(8)-C(9)$ & $122.4(3)$ \\
\hline$o(1)-C(8)-C(9)$ & $119.1(3)$ & $O(3)-C(9)-C(10)$ & $59.75(19)$ \\
\hline$O(3)-C(9)-C(8)$ & $112.4(2)$ & $C(10)-C(9)-C(8)$ & $119.3(3)$ \\
\hline$O(3)-C(9)-C(14)$ & $118.1(2)$ & $C(10)-C(9)-C(14)$ & $113.8(3)$ \\
\hline$C(8)-C(9)-C(14)$ & $119.6(3)$ & $O(3)-C(10)-C(9)$ & $59.79(19)$ \\
\hline$o(3)-C(10)-C(11)$ & $116.3(3)$ & $C(9)-C(10)-C(11)$ & $111.8(3)$ \\
\hline$C(12)-C(11)-C(10)$ & $108.9(3)$ & $C(12)-C(11)-C(16)$ & $105.8(3)$ \\
\hline$C(10)-C(11)-C(16)$ & $106.9(3)$ & $O(4)-C(12)-C(13)$ & $123.4(3)$ \\
\hline$O(4)-C(12)-C(11)$ & $123.5(3)$ & $C(13)-C(12)-C(11)$ & $113.0(3)$ \\
\hline$C(12)-C(13)-C(14)$ & $110.5(2)$ & $C(9)-C(14)-C(13)$ & $108.8(2)$ \\
\hline$C(9)-C(14)-C(15)$ & $104.9(2)$ & $C(13)-C(14)-C(15)$ & $107.3(3)$ \\
\hline$C(9)-C(14)-C(1)$ & $108.9(2)$ & $C(13)-C(14)-C(1)$ & $112.3(2)$ \\
\hline$C(15)-c(14)-C(1)$ & $114.3(2)$ & $C(14)-C(15)-C(16)$ & $110.7(2)$ \\
\hline$C(15)-C(16)-C(11)$ & $110.5(3)$ & & \\
\hline
\end{tabular}

Symmetry transformations used to generate equivalent atoms: 
Table 4. Anisotropic displacement parameters $\left[\mathrm{A}^{2} \times 10^{3}\right.$ ] for FENG3WS10.

The anisotropic displacement factor exponent takes the form: $-2 \pi^{2}\left[\left(\mathrm{ha}^{*}\right)^{2} \mathrm{U}_{11}+\ldots+2 \mathrm{hka}{ }^{*} \mathrm{~b}^{*} \mathrm{v}_{12} 1\right.$

\begin{tabular}{|c|c|c|c|c|c|c|}
\hline & U11 & $\mathrm{U} 22$ & U33 & บ23 & U13 & $\mathrm{U} 12$ \\
\hline$O(1)$ & $32(1)$ & $25(1)$ & $37(1)$ & $1(1)$ & $8(1)$ & $-1(1)$ \\
\hline$O(2)$ & $18(1)$ & $35(1)$ & $44(2)$ & $11(1)$ & $5(1)$ & $-1(1)$ \\
\hline$O(3)$ & $19(1)$ & $30(1)$ & $32(1)$ & $2(1)$ & $1(1)$ & $6(1)$ \\
\hline$O(4)$ & $30(1)$ & $23(1)$ & $34(1)$ & $-2(1)$ & $-2(1)$ & $-1(1)$ \\
\hline$C(1)$ & $18(2)$ & $24(2)$ & $23(2)$ & $-1(1)$ & $3(1)$ & $1(1)$ \\
\hline$C(2)$ & $20(2)$ & $31(2)$ & $30(2)$ & $-5(2)$ & $4(1)$ & $I(1)$ \\
\hline$C(3)$ & $30(2)$ & $32(2)$ & $35(2)$ & $-2(2)$ & $10(2)$ & $2(2)$ \\
\hline$C(4)$ & $54(3)$ & $55(3)$ & $30(2)$ & $8(2)$ & $10(2)$ & $5(2)$ \\
\hline$c(5)$ & $39(2)$ & $27(2)$ & $27(2)$ & $-4(2)$ & $-6(2)$ & $7(2)$ \\
\hline$C(6)$ & $24(2)$ & $24(2)$ & $31(2)$ & $-2(1)$ & $-2(1)$ & $2(1)$ \\
\hline$C(7)$ & $26(2)$ & $26(2)$ & $31(2)$ & $-1(1)$ & $8(1)$ & $5(1)$ \\
\hline$c(8)$ & $22(2)$ & $26(2)$ & $25(2)$ & $5(1)$ & $-1(1)$ & $1(1)$ \\
\hline$C(9)$ & $16(2)$ & $24(2)$ & $24(2)$ & $4(1)$ & $I(1)$ & $5(1)$ \\
\hline$C(10)$ & $26(2)$ & $26(2)$ & $27(2)$ & $4(1)$ & $5(1)$ & $6(1)$ \\
\hline$C(11)$ & $30(2)$ & $26(2)$ & $21(2)$ & $-2(1)$ & $2(1)$ & $4(1)$ \\
\hline$C(12)$ & $14(2)$ & $25(2)$ & $29(2)$ & $-1(1)$ & $-4(1)$ & $0(1)$ \\
\hline$c(13)$ & $24(2)$ & $24(2)$ & $23(2)$ & $1(1)$ & $3(1)$ & $0(1)$ \\
\hline$c(14)$ & $14(2)$ & $22(2)$ & $19(2)$ & $2(1)$ & $1(1)$ & $3(1)$ \\
\hline$C(15)$ & $18(2)$ & $29(2)$ & $24(2)$ & $1(1)$ & $-1(1)$ & $4(1)$ \\
\hline$C(16)$ & $30(2)$ & $30(2)$ & $26(2)$ & $0(2)$ & $-3(1)$ & $0(2)$ \\
\hline
\end{tabular}


Table 5. Hydrogen coordinates $\left(x 10^{4}\right)$ and isotropic displacement parameters $\left(\AA^{2} \times 10^{3}\right)$ for FENG3WS10.

\begin{tabular}{lrrrr}
\hline & & & & \\
& $X$ & & & \\
& & & & \\
$H(2 A)$ & -738 & 4335 & 1735 & 33 \\
$H(3 A)$ & -1221 & 3808 & 2772 & 39 \\
$H(4 A)$ & 1487 & 2486 & 3315 & 55 \\
$H(4 B)$ & 1582 & 4148 & 3528 & 55 \\
$H(5 A)$ & 4776 & 3463 & 3196 & 37 \\
$H(6 A)$ & 5285 & 3911 & 2150 & 32 \\
$H(7 A)$ & 1504 & 5857 & 1126 & 33 \\
$H(7 B)$ & 2601 & 6384 & 1804 & 33 \\
$H(10 A)$ & 5386 & 2598 & -81 & 31 \\
$H(11 A)$ & 3273 & 487 & -147 & 31 \\
$H(13 A)$ & 3370 & 1331 & 1782 & 28 \\
$H(13 B)$ & 1067 & 1312 & 1576 & 28 \\
$H(15 A)$ & 1000 & 4219 & 415 & 28 \\
$H(15 B)$ & -399 & 3120 & 773 & 28 \\
$H(16 A)$ & 1292 & 2611 & -377 & 35 \\
$H(16 B)$ & 22 & 1470 & 9 & 35 \\
\hline
\end{tabular}




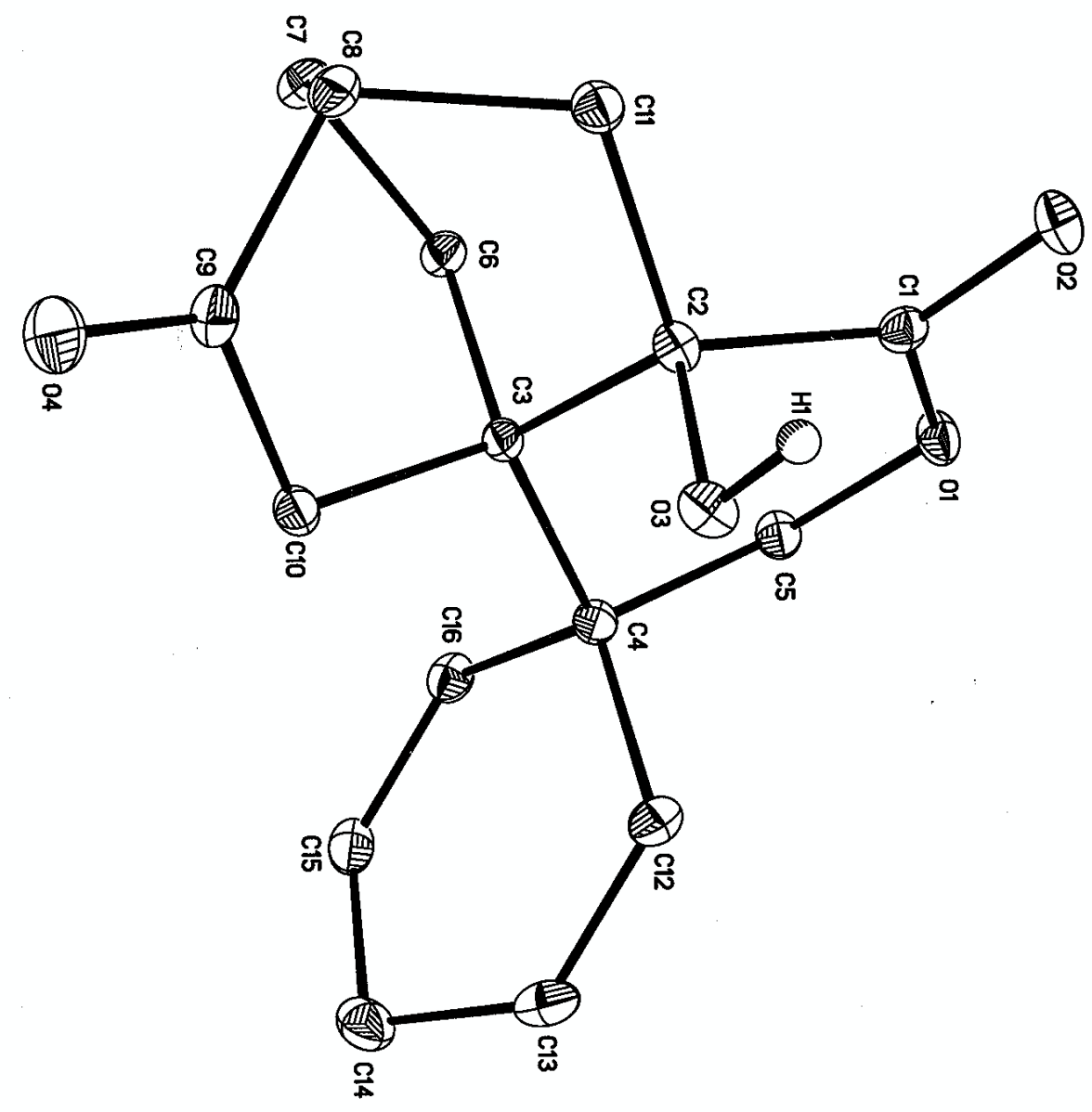


Table 1. Crystal data and structure refinement for fpta5.

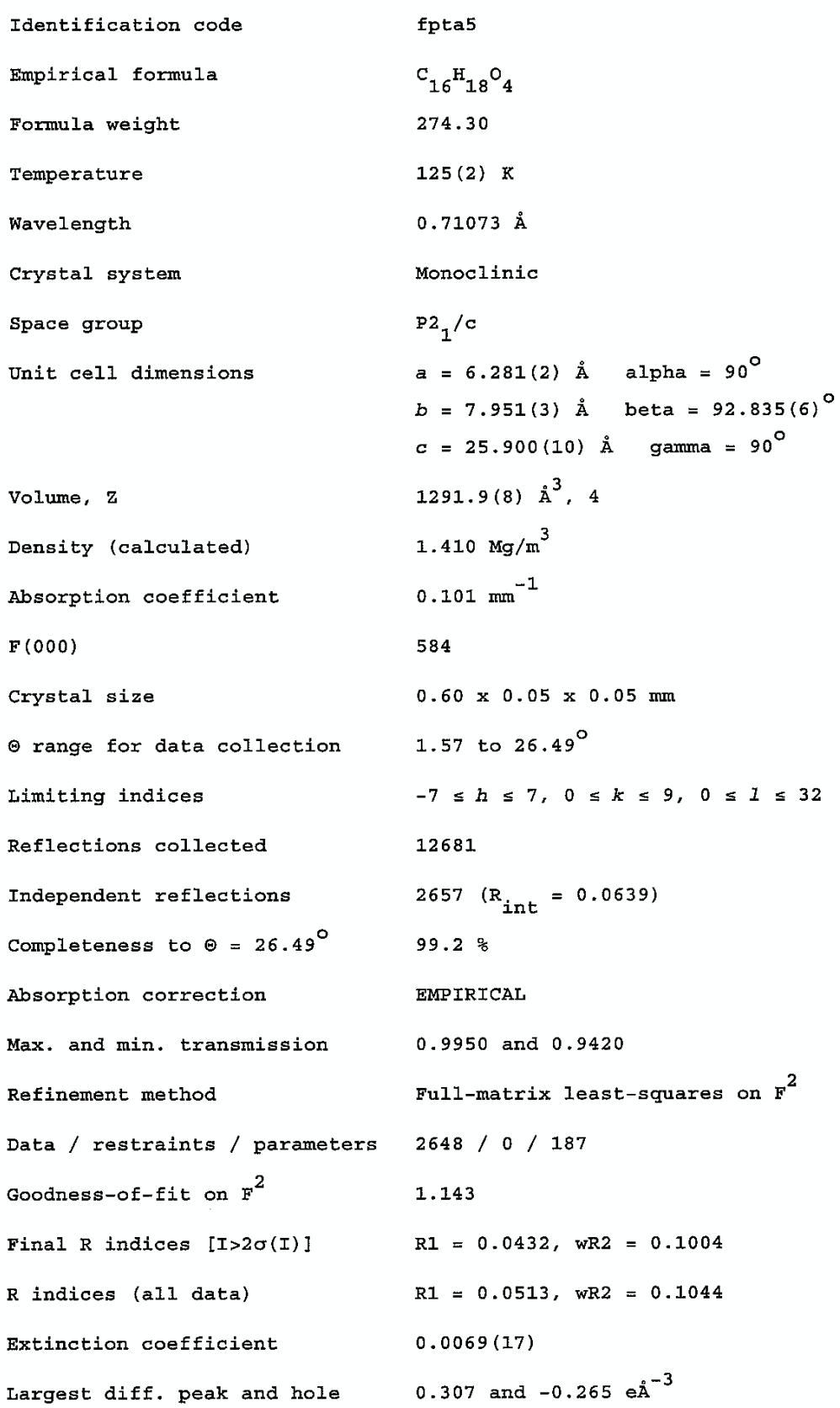


Table 2. Atomic coordinates $\left[\times 10^{4}\right]$ and equivalent isotropic displacement parameters $\left[\AA^{2} \times 10^{3}\right]$ for fpta5. $U(e q)$ is defined as one third of the trace of the orthogonalized $U_{i j}$ tensor.

\begin{tabular}{lrrrr}
\hline & & & \\
& & & & \\
& & & & \\
& & & & \\
$O(1)$ & & & & \\
$O(2)$ & $-4472(3)$ & $11340(2)$ & $4231(1)$ & $22(1)$ \\
$O(3)$ & $-2672(3)$ & $10819(2)$ & $4954(1)$ & $22(1)$ \\
$O(4)$ & $-127(3)$ & $9187(2)$ & $4178(1)$ & $20(1)$ \\
$C(1)$ & $183(3)$ & $4528(2)$ & $3872(1)$ & $31(1)$ \\
$C(2)$ & $-3202(4)$ & $10341(3)$ & $4519(1)$ & $17(1)$ \\
$C(3)$ & $-2261(4)$ & $8732(3)$ & $4297(1)$ & $16(1)$ \\
$C(4)$ & $-3458(4)$ & $8116(3)$ & $3792(1)$ & $15(1)$ \\
$C(5)$ & $-4051(4)$ & $9638(3)$ & $3425(1)$ & $17(1)$ \\
$C(6)$ & $-5425(4)$ & $10843(3)$ & $3729(1)$ & $19(1)$ \\
$C(7)$ & $-5484(4)$ & $7190(3)$ & $3965(1)$ & $18(1)$ \\
$C(8)$ & $-4882(4)$ & $5496(3)$ & $4226(1)$ & $22(1)$ \\
$C(9)$ & $-2517(4)$ & $5580(3)$ & $4423(1)$ & $20(1)$ \\
$C(10)$ & $-1235(4)$ & $5528(3)$ & $3941(1)$ & $21(1)$ \\
$C(11)$ & $-1993(4)$ & $6825(3)$ & $3541(1)$ & $18(1)$ \\
$C(12)$ & $-2205(4)$ & $7294(3)$ & $4702(1)$ & $20(1)$ \\
$C(13)$ & $-2081(4)$ & $10535(3)$ & $3243(1)$ & $21(1)$ \\
$C(14)$ & $-1433(4)$ & $10436(3)$ & $2764(1)$ & $25(1)$ \\
$C(15)$ & $-2597(5)$ & $9489(3)$ & $2337(1)$ & $30(1)$ \\
$C(16)$ & $-4773(4)$ & $8970(3)$ & $2482(1)$ & $25(1)$ \\
& $-5432(4)$ & $9058(3)$ & $2960(1)$ & $21(1)$ \\
\hline & & & & \\
\hline & & & &
\end{tabular}


Table 3. Bond lengths [̊] and angles $\left[{ }^{0}\right]$ for fpta5.

\begin{tabular}{|c|c|c|c|}
\hline$O(1)-C(1)$ & $1.329(3)$ & $O(1)-C(5)$ & $1.459(3)$ \\
\hline$O(2)-C(I)$ & $1.218(3)$ & $O(3)-C(2)$ & $1.436(3)$ \\
\hline$O(4)-C(9)$ & $1.214(3)$ & $C(1)-C(2)$ & $1.534(3)$ \\
\hline$C(2)-C(11)$ & $1.552(3)$ & $C(2)-c(3)$ & $1.554(3)$ \\
\hline$c(3)-C(10)$ & $1.543(3)$ & $c(3)-c(6)$ & $1.555(3)$ \\
\hline$C(3)-C(4)$ & $1.572(3)$ & $C(4)-C(16)$ & $1.520(3)$ \\
\hline$C(4)-C(12)$ & $1.524(3)$ & $C(4)-C(5)$ & $1.532(3)$ \\
\hline$C(6)-C(7)$ & $1.546(3)$ & $C(7)-C(8)$ & $1.547(3)$ \\
\hline$C(8)-C(9)$ & $1.518(3)$ & $C(8)-C(11)$ & $1.551(3)$ \\
\hline$C(9)-C(10)$ & $1.521(3)$ & $c(12)-c(13)$ & $1.326(3)$ \\
\hline$C(13)-C(14)$ & $1.497(4)$ & $C(14)-C(15)$ & $1.493(4)$ \\
\hline$C(15)-C(16)$ & $1.327(3)$ & & \\
\hline$C(1)-O(1)-C(5)$ & $123.17(18)$ & $O(2)-C(1)-O(1)$ & $117.5(2)$ \\
\hline$O(2)-C(1)-C(2)$ & $121.0(2)$ & $O(1)-C(1)-C(2)$ & $121.28(19)$ \\
\hline$O(3)-C(2)-C(1)$ & $104.58(18)$ & $O(3)-C(2)-C(11)$ & $109.93(18)$ \\
\hline$C(1)-C(2)-C(11)$ & $110.87(17)$ & $O(3)-C(2)-C(3)$ & $108.48(17)$ \\
\hline$C(1)-C(2)-C(3)$ & $113.54(18)$ & $C(11)-C(2)-C(3)$ & $109.30(18)$ \\
\hline$C(10)-C(3)-C(2)$ & $106.83(18)$ & $C(10)-C(3)-C(6)$ & $108.64(18)$ \\
\hline$C(2)-C(3)-C(6)$ & $106.08(17)$ & $C(10)-C(3)-C(4)$ & $112.67(18)$ \\
\hline$C(2)-C(3)-C(4)$ & $110.86(17)$ & $C(6)-C(3)-C(4)$ & $111.44(18)$ \\
\hline$C(16)-C(4)-C(12)$ & $109.64(18)$ & $C(16)-C(4)-C(5)$ & $106.41(19)$ \\
\hline$C(12)-C(4)-C(5)$ & $110.69(19)$ & $C(16)-C(4)-C(3)$ & $110.75(18)$ \\
\hline$C(12)-C(4)-C(3)$ & $112.10(18)$ & $C(5)-C(4)-C(3)$ & $107.08(17)$ \\
\hline$O(1)-C(5)-C(4)$ & $114.22(19)$ & $C(7)-C(6)-C(3)$ & $110.62(19)$ \\
\hline$C(6)-C(7)-C(8)$ & $108.49(19)$ & $C(9)-C(8)-C(7)$ & $105.55(18)$ \\
\hline$C(9)-C(8)-C(11)$ & $110.33(19)$ & $C(7)-C(8)-C(11)$ & $106.74(19)$ \\
\hline$O(4)-C(9)-C(8)$ & $124.2(2)$ & $O(4)-C(9)-C(10)$ & $123.4(2)$ \\
\hline$C(8)-C(9)-C(10)$ & $112.3(2)$ & $C(9)-C(10)-C(3)$ & $109.72(18)$ \\
\hline$C(8)-C(11)-C(2)$ & $109.49(17)$ & $C(13)-C(12)-C(4)$ & $123.7(2)$ \\
\hline$C(12)-C(13)-C(14)$ & $123.9(2)$ & $C(15)-C(14)-C(13)$ & $111.9(2)$ \\
\hline$C(16)-C(15)-C(14)$ & $123.5(2)$ & $C(15)-C(16)-C(4)$ & $124.3(2)$ \\
\hline
\end{tabular}

Symmetry transformations used to generate equivalent atoms: 
Table 4. Anisotropic displacement parameters $\left[\AA^{2} \times 10^{3}\right.$ ] for fpta5.

The anisotropic displacement factor exponent takes the form:

$-2 \pi^{2}\left[\left(\mathrm{ha}^{*}\right)^{2} \mathrm{U}_{11}+\ldots+2 \mathrm{hka}{ }^{*} \mathrm{~b}^{*} \mathrm{U}_{12}\right]$

\begin{tabular}{|c|c|c|c|c|c|c|}
\hline & U11 & U22 & U33 & U2 3 & $\mathrm{U} 13$ & $\mathrm{U} 12$ \\
\hline$O(1)$ & $29(1)$ & $18(1)$ & $19(1)$ & $-3(1)$ & $-1(1)$ & $6(1)$ \\
\hline$O(2)$ & $19(1)$ & $26(1)$ & $20(1)$ & $-8(1)$ & $-2(1)$ & $1(1)$ \\
\hline$o(3)$ & $13(1)$ & $27(1)$ & $18(1)$ & $-1(1)$ & $O(1)$ & $0(1)$ \\
\hline$O(4)$ & $28(1)$ & $27(1)$ & $38(1)$ & $-1(1)$ & $7(1)$ & $11(1)$ \\
\hline$C(1)$ & $14(1)$ & $18(1)$ & $19(1)$ & $1(1)$ & $1(1)$ & $-3(1)$ \\
\hline$C(2)$ & $14(1)$ & $19(1)$ & $16(1)$ & $-2(1)$ & $0(1)$ & $1(1)$ \\
\hline$c(3)$ & $18(1)$ & $14(1)$ & $13(1)$ & $-1(1)$ & $0(1)$ & $0(1)$ \\
\hline$C(4)$ & $19(1)$ & $17(1)$ & $15(1)$ & $I(1)$ & $-1(1)$ & $0(1)$ \\
\hline$C(5)$ & $22(1)$ & $18(1)$ & $17(1)$ & $0(1)$ & $-2(1)$ & $3(1)$ \\
\hline$C(6)$ & $17(1)$ & $16(1)$ & $20(1)$ & $I(1)$ & $2(1)$ & $0(1)$ \\
\hline$C(7)$ & 22 (I) & $18(1)$ & $26(1)$ & $4(1)$ & $3(1)$ & $0(1)$ \\
\hline$c(8)$ & $22(1)$ & $16(1)$ & $2 I(1)$ & $3(1)$ & $1(1)$ & $3(1)$ \\
\hline$C(9)$ & $20(1)$ & $17(1)$ & $25(1)$ & $-4(1)$ & $0(1)$ & $-1(1)$ \\
\hline$C(10)$ & $18(1)$ & $18(1)$ & $18(1)$ & $-3(1)$ & $3(1)$ & $0(1)$ \\
\hline$C(11)$ & $22(1)$ & $19(1)$ & $17(1)$ & $1(1)$ & $0(1)$ & $3(1)$ \\
\hline$C(12)$ & $20(1)$ & $20(1)$ & $22(1)$ & $4(1)$ & $-2(1)$ & $-2(1)$ \\
\hline$C(13)$ & $20(1)$ & $25(1)$ & $31(1)$ & $11(1)$ & $6(1)$ & $2(1)$ \\
\hline$C(14)$ & $41(2)$ & $29(1)$ & $21(1)$ & $4(1)$ & $6(1)$ & $8(1)$ \\
\hline$C(15)$ & $37(2)$ & $19(1)$ & $19(1)$ & $0(1)$ & $-6(1)$ & $2(1)$ \\
\hline$c(16)$ & $23(1)$ & $18(1)$ & $21(1)$ & $2(1)$ & $-4(1)$ & $0(1)$ \\
\hline
\end{tabular}


Table 5. Hydxogen coordinates $\left(\times 10^{4}\right)$ and isotropic displacement parameters $\left(\dot{A}^{2} \times 10^{3}\right)$ for Epta5.

\begin{tabular}{|c|c|c|c|c|}
\hline & $x$ & $y$ & $\mathbf{z}$ & $\mathrm{U}(\mathrm{eq})$ \\
\hline$H(1)$ & $600(50)$ & $9340(30)$ & 4468 (11) & $26(7)$ \\
\hline$H(5 A)$ & -5702 & 11867 & 3519 & 23 \\
\hline$H(5 B)$ & -6815 & 10299 & 3782 & 23 \\
\hline$H(6 A)$ & -6235 & 7906 & 4210 & 21 \\
\hline$H(6 B)$ & -6463 & 6983 & 3660 & 21 \\
\hline$E(7 A)$ & -5091 & 4565 & 3975 & 26 \\
\hline$F(7 B)$ & -5801 & 5288 & 4519 & 26 \\
\hline $\mathrm{F}(8 \mathrm{~A})$ & -2139 & 4620 & 4659 & 24 \\
\hline $\mathrm{H}(10 \mathrm{~A})$ & -2781 & 6259 & 3250 & 22 \\
\hline $\mathrm{H}(\mathrm{IOB})$ & -749 & 7409 & 3404 & 22 \\
\hline$H(11 A)$ & -821 & 7303 & 4902 & 24 \\
\hline $\mathrm{F}(11 \mathrm{~B})$ & -3351 & 7464 & 4946 & 24 \\
\hline$H(12 A)$ & -1272 & 11202 & 3485 & 25 \\
\hline $\mathrm{H}(13 \mathrm{~A})$ & -153 & 10999 & 2687 & 30 \\
\hline $\mathrm{H}(14 \mathrm{~A})$ & -2720 & 10207 & 2025 & 36 \\
\hline$H(14 B)$ & -1767 & 8476 & 2253 & 36 \\
\hline$H(15 A)$ & -5736 & 8555 & 2218 & 30 \\
\hline$H(16 A)$ & -6859 & 8738 & 3016 & 25 \\
\hline
\end{tabular}


Table 6. Hydrogen bonds for fpta 5 [Å and $\left.{ }^{\circ}\right]$.

\begin{tabular}{lllll}
\hline D-H...A & $\mathrm{d}(\mathrm{D}-\mathrm{H})$ & $\mathrm{d}(\mathrm{H} \ldots \mathrm{A})$ & $\mathrm{d}(\mathrm{D} \ldots \mathrm{A})$ & $<(\mathrm{DHA})$ \\
$\mathrm{O}(3)-\mathrm{H}(\mathrm{I}) \ldots \mathrm{O}(2) \# \mathrm{I}$ & $0.87(3)$ & $1.94(3)$ & $2.786(2)$ & $165(3)$ \\
\hline
\end{tabular}

symmetry transformations used to generate equivalent atoms:

$\# 1-x,-y+2,-z+1$ 


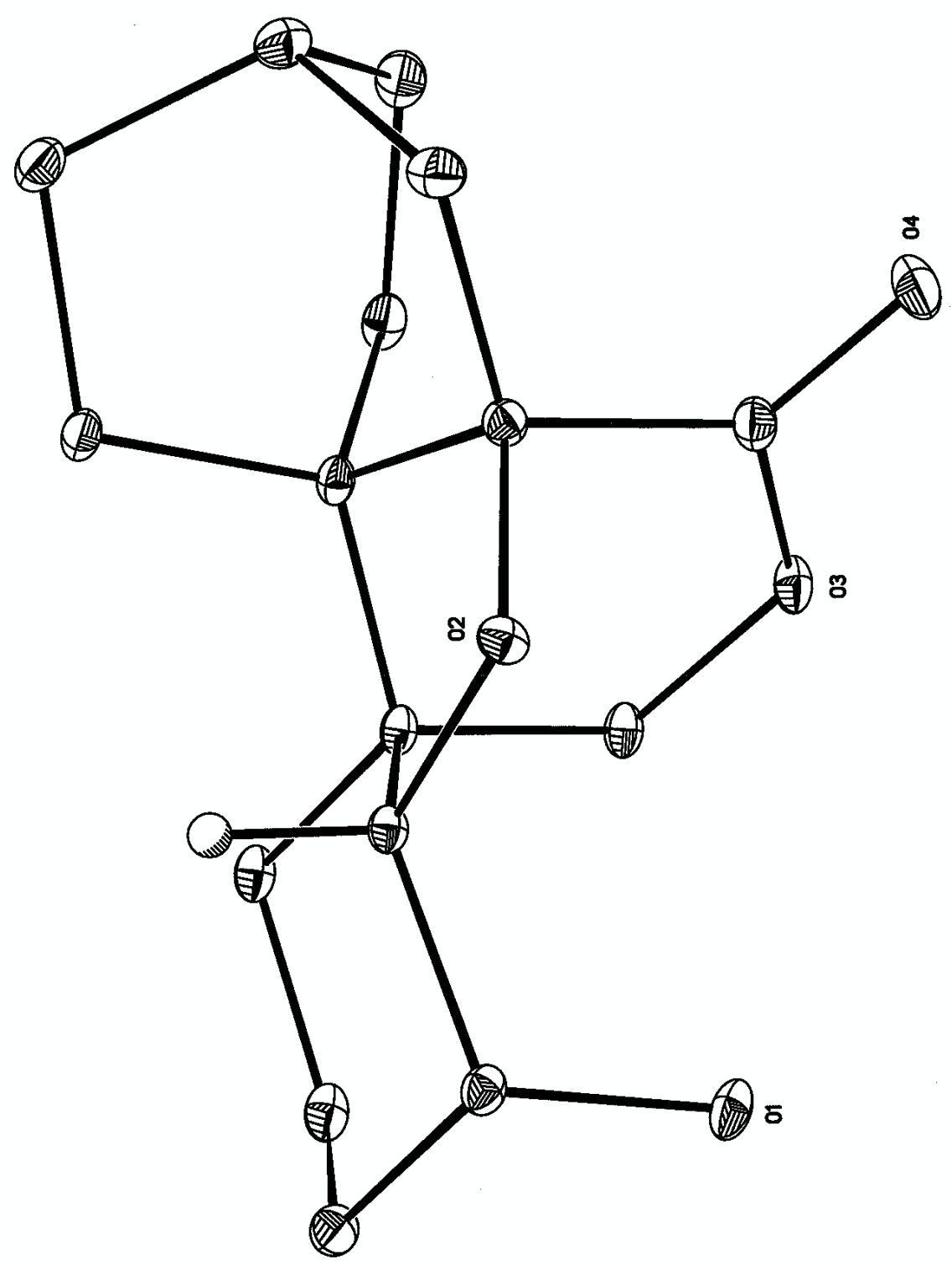


Table 1. Crystal data and structure refinement for FPEWS10.

\begin{tabular}{|c|c|}
\hline Identification code & fpews10 \\
\hline Empirical formula & $\mathrm{C}_{16} \mathrm{H}_{22}{ }^{\mathrm{O}} 4$ \\
\hline Formula weight & 278.34 \\
\hline Temperature & $123(2) \mathrm{K}$ \\
\hline Wavelength & $0.71073 \AA$ \\
\hline Cxystal system & Orthorhombic \\
\hline Space group & Pbca \\
\hline Unit cell dimensions & $\begin{array}{lll}a=11.2174(9) \AA & \text { alpha }=90^{\circ} \\
b=11.2661(9) \AA & \text { beta }=90^{\circ} \\
c=21.3465(17) \AA & \text { gamma }=90^{\circ}\end{array}$ \\
\hline Volume, $\mathrm{z}$ & $2697.7(4) \AA^{3}, 8$ \\
\hline Density (calculated) & $1.371 \mathrm{Mg} / \mathrm{m}^{3}$ \\
\hline Absorption coefficient & $0.097 \mathrm{~mm}^{-1}$ \\
\hline$F(000)$ & 1200 \\
\hline Crystal size & $0.25 \times 0.23 \times 0.16 \mathrm{~mm}$ \\
\hline (-) range for data collection & 1.91 to $31.66^{\circ}$ \\
\hline Limiting indices & $-16 \leq h \leq 16,-16 \leq k \leq 16,-31 \leq 1 \leq 31$ \\
\hline Reflections collected & 41822 \\
\hline Independent reflections & $4542\left(R_{\text {int }}=0.0393\right)$ \\
\hline Completeness to $\oplus=31.66^{\circ}$ & $99.5 \%$ \\
\hline Absorption correction & EMPIRICAL \\
\hline Max. and $\min$. transmission & 0.9846 and 0.9761 \\
\hline Refinement method & Ful1-matrix least-squares on $F^{2}$ \\
\hline Data / restraints / parameters & $4542 / 0 / 182$ \\
\hline Goodness-of-fit on $F^{2}$ & 1.081 \\
\hline Final $R$ indices $[I>2 \sigma(I)]$ & $\mathrm{R} 1=0.0407, w R 2=0.1007$ \\
\hline $\mathrm{R}$ indices (all data) & $\mathrm{R} 1=0.0568, \mathrm{wR2}=0.1 .137$ \\
\hline Largest diff, peak and hole & 0.517 and $-0.221 \mathrm{eA}^{-3}$ \\
\hline
\end{tabular}


Table 2. Atomic coordinates $\left[x 10^{4}\right]$ and equivalent isotropic displacement parameters $\left[\AA^{2} \times 10^{3}\right]$ for FREWS10. U(eq) is defined as one third of the trace of the orthogonalized $U_{i j}$ tensor.

\begin{tabular}{|c|c|c|c|c|}
\hline & $\mathbf{x}$ & $y$ & $\mathbf{z}$ & $\mathrm{U}(\mathrm{eq})$ \\
\hline$O(1)$ & $3478(1)$ & $1153(1)$ & $5360(1)$ & $21(1)$ \\
\hline$O(2)$ & $5403(1)$ & $1020(1)$ & $4366(1)$ & $16(1)$ \\
\hline $0(3)$ & $2997(1)$ & $963(1)$ & $3834(1)$ & $23(1)$ \\
\hline$O(4)$ & $3950(1)$ & $-476(1)$ & $3353(1)$ & $30(1)$ \\
\hline$c(1)$ & 4512 (1) & $1890(1)$ & $5342(1)$ & $17(1)$ \\
\hline$C(2)$ & $4211(1)$ & $3135(1)$ & 5595 (1) & $21(1)$ \\
\hline$C(3)$ & $3453(1)$ & $3895(1)$ & $5149(1)$ & $23(1)$ \\
\hline$C(4)$ & $3977(1)$ & $3978(1)$ & $4481(1)$ & $20(1)$ \\
\hline$C(5)$ & $4194(1)$ & $2728(1)$ & $4220(1)$ & $15(1)$ \\
\hline$c(6)$ & $5008(1)$ & $2084(1)$ & $4686(1)$ & $14(1)$ \\
\hline$C(7)$ & $5160(1)$ & $1195(1)$ & 3698 (1) & $17(1)$ \\
\hline$c(8)$ & $6175(1)$ & $777(1)$ & $3274(1)$ & $23(1)$ \\
\hline$C(9)$ & $6290(1)$ & $1687(1)$ & $2734(1)$ & $26(1)$ \\
\hline$C(10)$ & $6833(1)$ & $2838(1)$ & 2995 (1) & $24(1)$ \\
\hline$c(11)$ & $6134(1)$ & $3203(1)$ & $3592(1)$ & 19 (1) \\
\hline$C(12)$ & $4930(1)$ & $2538(1)$ & $3613(1)$ & $16(1)$ \\
\hline$C(13)$ & $4342(1)$ & $2726(1)$ & $2968(1)$ & $23(1)$ \\
\hline$c(14)$ & $5046(1)$ & $1996(1)$ & $2473(1)$ & $28(1)$ \\
\hline$C(15)$ & $2975(1)$ & $2138(1)$ & $4121(1)$ & $20(1)$ \\
\hline$C(16)$ & $4001(1)$ & $490(1)$ & $3599(1)$ & $20(1)$ \\
\hline
\end{tabular}


Table 3. Bond lengths [Å] and angles $\left[{ }^{\circ}\right]$ for FPEWS10.

\begin{tabular}{|c|c|c|c|}
\hline$o(1)-C(1)$ & $1.4270(13)$ & $O(2)-C(6)$ & $1.4488(12)$ \\
\hline$O(2)-C(7)$ & $1.4639(12)$ & $o(3)-C(16)$ & $1.3429(14)$ \\
\hline$o(3)-C(1.5)$ & $1.4587(13)$ & $O(4)-C(16)$ & $1.2102(14)$ \\
\hline$C(1)-C(6)$ & $1.5224(14)$ & $C(1)-C(2)$ & $1.5404(14)$ \\
\hline$c(2)-C(3)$ & $1.5358(17)$ & $c(3)-C(4)$ & $1.5459(16)$ \\
\hline$C(4)-C(5)$ & $1.5334(14)$ & $c(5)-C(6)$ & $1.5338(14)$ \\
\hline$c(5)-c(15)$ & $1.5354(14)$ & $C(5)-C(12)$ & $1.5504(14)$ \\
\hline$C(7)-C(8)$ & $1.5286(15)$ & $C(7)-C(16)$ & $1.5385(15)$ \\
\hline$c(7)-C(12)$ & $1.5457(14)$ & $C(8)-C(9)$ & $1.5479(17)$ \\
\hline$c(9)-c(10)$ & $1.5363(17)$ & $C(9)-C(14)$ & $1.5432(18)$ \\
\hline$C(10)-C(11)$ & $1.5519(15)$ & $c(11)-c(12)$ & $1.5453(14)$ \\
\hline$C(12)-C(13)$ & $1.5415(14)$ & $c(13)-C(14)$ & $1.5545(19)$ \\
\hline$C(6)-O(2)-C(7)$ & $106.93(7)$ & $c(16)-o(3)-c(15)$ & $122.07(8)$ \\
\hline$o(1)-C(1)-C(6)$ & $113.95(8)$ & $o(1)-C(1)-C(2)$ & $110.02(8)$ \\
\hline$C(6)-C(1)-C(2)$ & $105.76(8)$ & $C(3)-C(2)-C(1)$ & $114.31(9)$ \\
\hline$C(2)-C(3)-C(4)$ & $113.27(9)$ & $C(5)-C(4)-C(3)$ & $109.89(8)$ \\
\hline$C(4)-C(5)-C(6)$ & $107.07(8)$ & $C(4)-C(5)-C(15)$ & $107.80(8)$ \\
\hline$C(6)-C(5)-C(15)$ & $114.47(8)$ & $C(4)-C(5)-C(12)$ & $120.99(8)$ \\
\hline$C(6)-C(5)-C(12)$ & $99.21(8)$ & $c(15)-C(5)-C(12)$ & $107.45(8)$ \\
\hline$o(2)-C(6)-C(1)$ & $115.30(8)$ & $o(2)-C(6)-C(5)$ & $105.49(8)$ \\
\hline$c(1)-c(6)-c(5)$ & $116.54(8)$ & $O(2)-C(7)-C(8)$ & $113.36(9)$ \\
\hline$o(2)-C(7)-C(16)$ & $102.78(8)$ & $C(8)-C(7)-C(16)$ & $112.87(9)$ \\
\hline$O(2)-C(7)-C(12)$ & $106.13(8)$ & $C(8)-C(7)-C(12)$ & $110.90(8)$ \\
\hline$c(16)-c(7)-c(12)$ & $110.35(8)$ & $C(7)-C(8)-C(9)$ & $107.41(9)$ \\
\hline$C(10)-C(9)-C(14)$ & $107.35(10)$ & $C(10)-C(9)-C(8)$ & $108.83(9)$ \\
\hline$C(14)-C(9)-C(8)$ & $110.05(10)$ & $c(9)-c(10)-c(11)$ & $108.73(9)$ \\
\hline$c(12)-c(11)-c(10)$ & $109.69(9)$ & $C(13)-C(12)-C(11)$ & $106.31(8)$ \\
\hline$C(13)-C(12)-C(7)$ & $108.16(8)$ & $C(11)-C(12)-C(7)$ & $109.37(8)$ \\
\hline$C(13)-C(12)-C(5)$ & $119.99(9)$ & $C(11)-C(12)-C(5)$ & $115.00(8)$ \\
\hline$c(7)-c(12)-c(5)$ & $97.22(7)$ & $C(12)-C(13)-C(14)$ & $108.48(9)$ \\
\hline$C(9)-C(14)-C(13)$ & $109.48(9)$ & $o(3)-C(15)-C(5)$ & $115.82(8)$ \\
\hline$O(4)-C(16)-O(3)$ & $118.65(10)$ & $o(4)-C(16)-C(7)$ & $124.33(11)$ \\
\hline$o(3)-c(16)-c(7)$ & $116.93(9)$ & & \\
\hline
\end{tabular}

Symmetry transformations used to generate equivalent atoms: 
Table 4. Anisotropic displacement parameters $\left[\AA^{2} \times 10^{3}\right]$ for FPEWS10.

The anisotropic displacement factor exponent takes the form: $-2{ }^{2}\left[\left(h a^{*}\right)^{2} v_{11}+\ldots+2 h k a{ }^{*} b^{*} v_{12}\right]$

\begin{tabular}{lrrrrrr}
\hline & & & & & \\
& U11 & U22 & U33 & U23 & U13 & U12 \\
\hline & & & & & & \\
$O(1)$ & $20(1)$ & $13(1)$ & $29(1)$ & $4(1)$ & $6(1)$ & $-1(1)$ \\
$O(2)$ & $20(1)$ & $12(1)$ & $16(1)$ & $0(1)$ & $-1(1)$ & $3(1)$ \\
$O(3)$ & $19(1)$ & $20(1)$ & $31(1)$ & $0(1)$ & $-1(1)$ & $-8(1)$ \\
$O(4)$ & $37(1)$ & $22(1)$ & $31(1)$ & $-6(1)$ & $-3(1)$ & $-9(1)$ \\
$C(1)$ & $18(1)$ & $13(1)$ & $19(1)$ & $1(1)$ & $2(1)$ & $0(1)$ \\
$C(2)$ & $25(1)$ & $15(1)$ & $24(1)$ & $-3(1)$ & $6(1)$ & $-1(1)$ \\
$C(3)$ & $19(1)$ & $14(1)$ & $35(1)$ & $-2(1)$ & $5(1)$ & $2(1)$ \\
$C(4)$ & $16(1)$ & $12(1)$ & $32(1)$ & $3(1)$ & $0(1)$ & $2(1)$ \\
$C(5)$ & $12(1)$ & $12(1)$ & $21(1)$ & $3(1)$ & $-1(1)$ & $0(1)$ \\
$C(6)$ & $13(1)$ & $10(1)$ & $18(1)$ & $1(1)$ & $0(1)$ & $1(1)$ \\
$C(7)$ & $18(1)$ & $16(1)$ & $16(1)$ & $1(1)$ & $-1(1)$ & $-2(1)$ \\
$C(8)$ & $25(1)$ & $23(1)$ & $21(1)$ & $-3(1)$ & $4(1)$ & $1(1)$ \\
$C(9)$ & $27(1)$ & $32(1)$ & $18(1)$ & $-1(1)$ & $4(1)$ & $-5(1)$ \\
$C(10)$ & $20(1)$ & $31(1)$ & $19(1)$ & $4(1)$ & $2(1)$ & $-6(1)$ \\
$C(11)$ & $17(1)$ & $20(1)$ & $20(1)$ & $3(1)$ & $0(1)$ & $-5(1)$ \\
$C(12)$ & $15(1)$ & $15(1)$ & $17(1)$ & $4(1)$ & $-3(1)$ & $-3(1)$ \\
$C(13)$ & $23(1)$ & $27(1)$ & $20(1)$ & $8(1)$ & $-6(1)$ & $-6(1)$ \\
$C(14)$ & $32(1)$ & $35(1)$ & $18(1)$ & $3(1)$ & $-5(1)$ & $-10(1)$ \\
$C(15)$ & $14(1)$ & $17(1)$ & $28(1)$ & $3(1)$ & $-2(1)$ & $-1(1)$ \\
$C(16)$ & $24(1)$ & $18(1)$ & $19(1)$ & $2(1)$ & $-3(1)$ & $-5(1)$ \\
\hline
\end{tabular}


Table 5. Hydrogen coordinates $\left(x 10^{4}\right)$ and isotropic displacement parameters $\left(\AA^{2} \times 10^{3}\right)$ for FPEWS10.

\begin{tabular}{|c|c|c|c|c|}
\hline & $x$ & $y$ & z & $\mathrm{U}(\in \mathrm{q})$ \\
\hline $\mathrm{H}(1 \mathrm{~A})$ & 3686 & 438 & 5380 & 31 \\
\hline $\mathrm{H}(1 \mathrm{~B})$ & 5145 & 1528 & 5611 & 20 \\
\hline $\mathrm{H}(2 \mathrm{~A})$ & 3779 & 3048 & 5996 & 26 \\
\hline $\mathrm{H}(2 \mathrm{~B})$ & 4965 & 3561 & 5682 & 26 \\
\hline$H(3 A)$ & 3382 & 4705 & 5325 & 27 \\
\hline$H(3 B)$ & 2642 & 3552 & 5125 & 27 \\
\hline$H(4 A)$ & 3415 & 4410 & 4205 & 24 \\
\hline $\mathrm{H}(4 \mathrm{~B})$ & 4737 & 4423 & 4491 & 24 \\
\hline $\mathrm{H}(6 \mathrm{~A})$ & 5731 & 2595 & 4737 & 17 \\
\hline Н $(8 A)$ & 5995 & -21 & 3104 & 28 \\
\hline H (8B) & 6929 & 733 & 3514 & 28 \\
\hline$H(9 A)$ & 6807 & 1359 & 2394 & 31 \\
\hline$H(10 A)$ & 6781 & 3475 & 2677 & 28 \\
\hline$H(10 B)$ & 7683 & 2711 & 3099 & 28 \\
\hline $\mathrm{H}(11 \mathrm{~A})$ & 6608 & 3007 & 3969 & 23 \\
\hline H (IIB) & 5991 & 4070 & 3588 & 23 \\
\hline$H(13 A)$ & 4356 & 3579 & 2856 & 28 \\
\hline$H(13 B)$ & 3501 & 2461 & 2980 & 28 \\
\hline$H(14 A)$ & 4607 & 1258 & 2373 & 34 \\
\hline$H(14 B)$ & 5130 & 2464 & 2083 & 34 \\
\hline$H(15 A)$ & 2574 & 2073 & 4533 & 23 \\
\hline $\mathrm{H}(15 \mathrm{~B})$ & 2483 & 2666 & 3856 & 23 \\
\hline
\end{tabular}


Table 6. Hydrogen bonds for FPEWS10 [Å and ${ }^{\circ}$ ].

\begin{tabular}{lllll}
\hline D-H...A & $d(D-H)$ & $d(H \ldots A)$ & $d(D . \ldots A)$ & $<(D H A)$ \\
$O(1)-H(1 A) \ldots O(2) \# 1$ & 0.84 & 2.01 & $2.8132(10)$ & 159.9
\end{tabular}

Symmetry transformations used to generate equivalent atoms:

$\# 1-x+1,-y,-z+1$ 


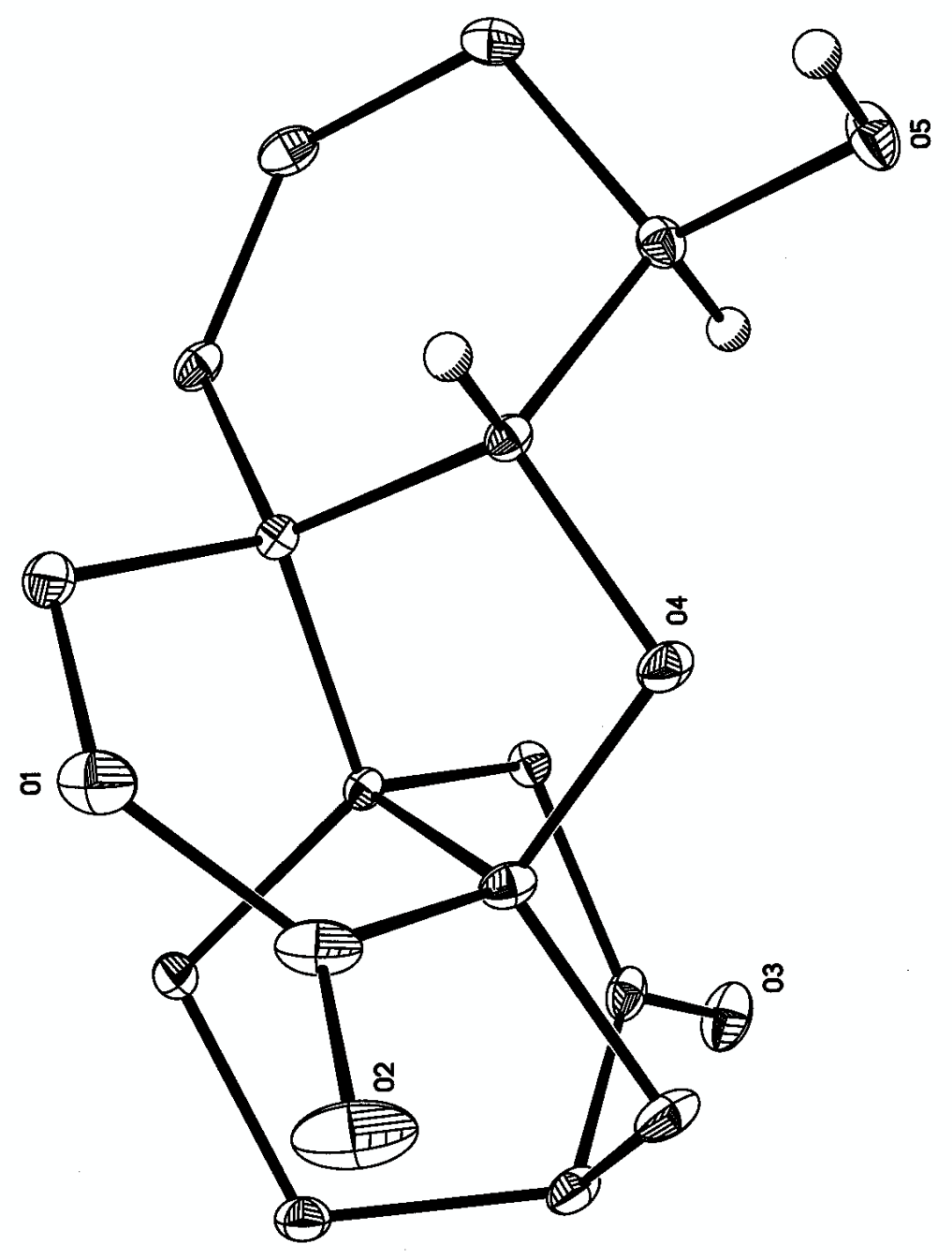


Table 1. Crystal data and structure refinement for FPAS10.

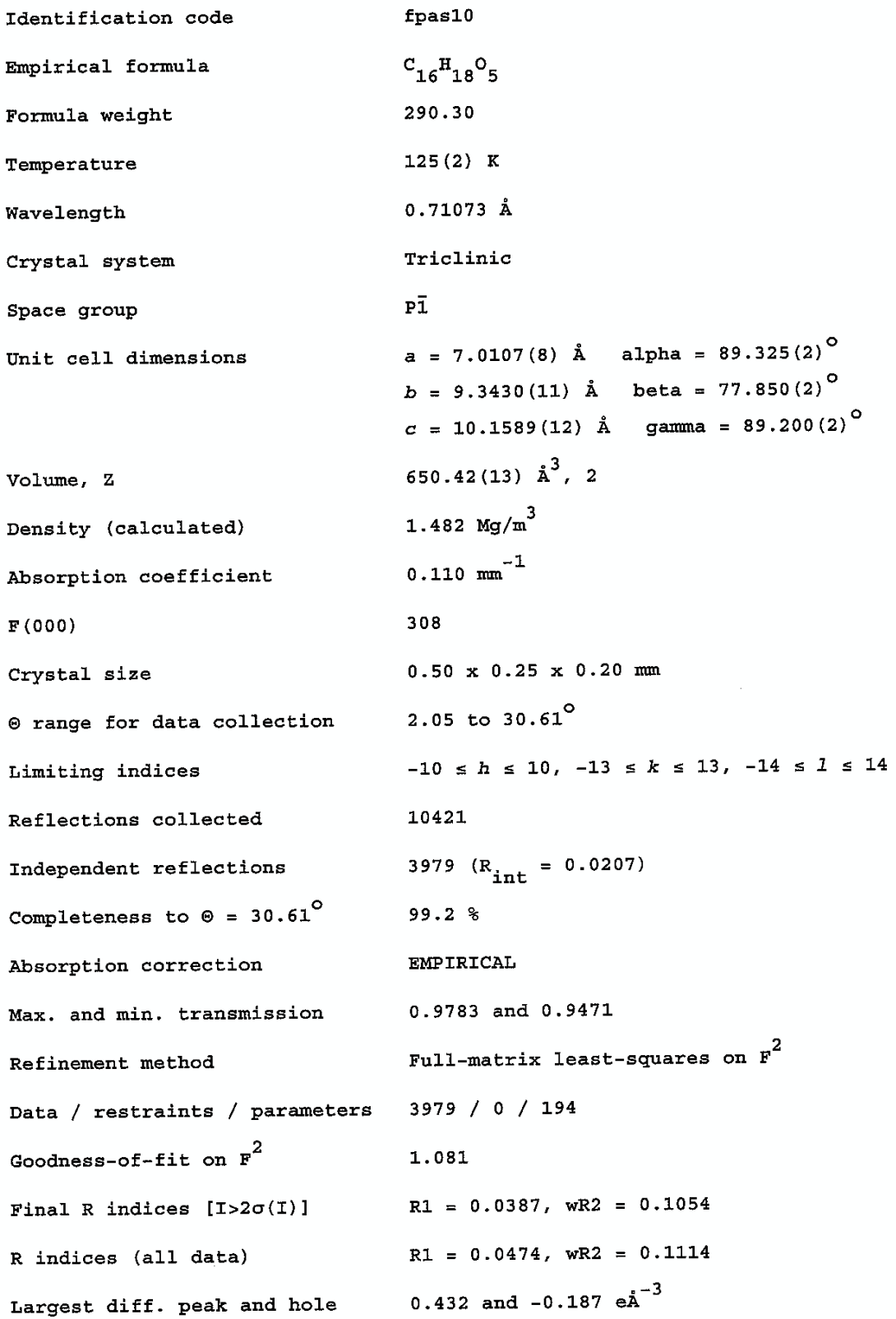


Table 2. Atomic coordinates [ $x \quad 10^{4}$ ] and equivalent isotropic displacement parameters $\left[\AA^{2} \times 10^{3}\right]$ for FPAS10. U(eq) is defined as one third of the trace of the orthogonalized $\sigma_{i j}$ tensor.

\begin{tabular}{|c|c|c|c|c|}
\hline & $\mathbf{x}$ & $y$ & $\mathbf{z}$ & $\mathbf{U}(\mathrm{eq})$ \\
\hline$O(1)$ & $2159(1)$ & $10509(1)$ & $2954(1)$ & $28(1)$ \\
\hline$O(2)$ & $-301(2)$ & $10499(1)$ & $1948(1)$ & $44(1)$ \\
\hline$O(3)$ & $2702(1)$ & $4376(1)$ & $352(1)$ & $30(1)$ \\
\hline$O(4)$ & $70(1)$ & $7702(1)$ & $3660(1)$ & $20(1)$ \\
\hline$O(5)$ & $-438(1)$ & $6456(1)$ & $6359(1)$ & $25(1)$ \\
\hline$c(1)$ & $918(2)$ & $9801(1)$ & $2353(1)$ & $26(1)$ \\
\hline $\mathrm{C}(2)$ & $1036(1)$ & $8151(1)$ & $2330(1)$ & $17(1)$ \\
\hline$C(3)$ & $111(2)$ & $7519(1)$ & $1234(1)$ & $23(1)$ \\
\hline$C(4)$ & $1737(2)$ & $6842(1)$ & $144(1)$ & $21(1)$ \\
\hline$C(5)$ & $3415(2)$ & $7915(1)$ & $-296(1)$ & $22(1)$ \\
\hline$C(6)$ & $4393(2)$ & $8273(I)$ & $898(1)$ & $17(1)$ \\
\hline$C(7)$ & $3147(1)$ & $7638(1)$ & $2194(1)$ & $13(1)$ \\
\hline$C(8)$ & $3238(2)$ & $6004(1)$ & $2050(1)$ & $15(1)$ \\
\hline$C(9)$ & $2569(1)$ & $5587(I)$ & $783(1)$ & $19(1)$ \\
\hline$C(10)$ & $3456(1)$ & $8161(1)$ & $3570(1)$ & $14(1)$ \\
\hline$C(11)$ & $5162(1)$ & $7527(1)$ & $4059(1)$ & $18(1)$ \\
\hline$C(12)$ & $5003(2)$ & $6881(1)$ & $5252(1)$ & $21(1)$ \\
\hline$c(13)$ & $3078(2)$ & $6651(1)$ & $6200(1)$ & $22(1)$ \\
\hline$C(14)$ & $1410(2)$ & $6569(1)$ & $5455(1)$ & $17(1)$ \\
\hline$c(15)$ & $1452(1)$ & $7862(1)$ & $4526(1)$ & $14(1)$ \\
\hline$c(16)$ & $3697(2)$ & $9782(1)$ & $3477(1)$ & $21(1)$ \\
\hline
\end{tabular}


Table 3. Bond lengths [Å] and angles $\left[{ }^{\circ}\right]$ for FPAS10.

\begin{tabular}{|c|c|c|c|}
\hline$O(1)-C(1)$ & $1.3480(15)$ & $O(1)-C(16)$ & $1.4550(14)$ \\
\hline$O(2)-C(1)$ & $1.2052(14)$ & $o(3)-C(9)$ & $1.2137(13)$ \\
\hline$O(4)-C(2)$ & $1.4378(12)$ & $O(4)-C(15)$ & $1.4498(11)$ \\
\hline$O(5)-C(14)$ & $1.4259(12)$ & $C(1)-C(2)$ & $1.5430(16)$ \\
\hline$c(2)-c(7)$ & $1.5283(13)$ & $C(2)-C(3)$ & $1.5287(14)$ \\
\hline$C(3)-C(4)$ & $1.5465(15)$ & $C(4)-C(9)$ & $1.5027(16)$ \\
\hline$C(4)-C(5)$ & $1.5461(16)$ & $C(5)-C(6)$ & $1.5557(14)$ \\
\hline$C(6)-C(7)$ & $1.5361(13)$ & $C(7)-C(8)$ & $1.5336(13)$ \\
\hline$C(7)-C(10)$ & $1.5451(13)$ & $C(8)-C(9)$ & $1.5167(14)$ \\
\hline$c(10)-c(11)$ & $1.5007(13)$ & $C(10)-C(16)$ & $1.5258(14)$ \\
\hline$C(10)-C(15)$ & $1.5573(13)$ & $c(11)-c(12)$ & $1.3315(15)$ \\
\hline$C(12)-C(13)$ & $1.4997(16)$ & $c(13)-c(14)$ & $1.5243(15)$ \\
\hline$C(14)-C(15)$ & $1.5206(14)$ & & \\
\hline$c(1)-o(1)-c(16)$ & $122.34(8)$ & $c(2)-0(4)-c(15)$ & $107.07(7)$ \\
\hline$O(2)-C(1)-O(1)$ & 117.39 (11) & $o(2)-C(1)-C(2)$ & $124.12(12)$ \\
\hline$O(1)-C(1)-C(2)$ & $118.30(9)$ & $O(4)-C(2)-C(7)$ & $104.74(8)$ \\
\hline$O(4)-C(2)-C(3)$ & $112.41(9)$ & $C(7)-C(2)-C(3)$ & $111.36(8)$ \\
\hline$O(4)-C(2)-C(1)$ & $105.00(8)$ & $C(7)-C(2)-C(1)$ & $110.47(9)$ \\
\hline$C(3)-C(2)-C(1)$ & $112.42(9)$ & $C(2)-C(3)-C(4)$ & $108.86(8)$ \\
\hline$C(9)-C(4)-C(5)$ & $106.80(8)$ & $C(9)-C(4)-C(3)$ & $107.38(9)$ \\
\hline$C(5)-C(4)-C(3)$ & $109.94(9)$ & $C(4)-C(5)-C(6)$ & $111.02(8)$ \\
\hline$c(7)-C(6)-C(5)$ & $108.39(8)$ & $C(2)-C(7)-C(8)$ & $109.17(8)$ \\
\hline$C(2)-C(7)-C(6)$ & $109.09(8)$ & $C(8)-C(7)-C(6)$ & $107.23(8)$ \\
\hline$C(2)-C(7)-C(10)$ & $97.83(7)$ & $C(8)-C(7)-C(10)$ & $113.76(8)$ \\
\hline$C(6)-C(7)-C(10)$ & $119.10(8)$ & $C(9)-C(8)-C(7)$ & $109.70(8)$ \\
\hline$O(3)-C(9)-C(4)$ & $124.88(10)$ & $o(3)-C(9)-C(8)$ & $123.10(10)$ \\
\hline$C(4)-C(9)-C(8)$ & $112.02(8)$ & $C(11)-C(10)-C(16)$ & $108.21(8)$ \\
\hline$C(11)-C(10)-C(7)$ & $116.59(8)$ & $C(16)-C(10)-C(7)$ & $107.79(8)$ \\
\hline$c(11)-c(10)-c(15)$ & $113.60(8)$ & $C(16)-C(10)-C(15)$ & $107.21(8)$ \\
\hline$C(7)-C(10)-C(15)$ & $102.94(7)$ & $C(12)-C(11)-C(10)$ & $123.35(9)$ \\
\hline$C(11)-C(12)-C(13)$ & $122.79(9)$ & $C(12)-C(13)-C(14)$ & $111.85(9)$ \\
\hline$O(5)-C(14)-C(15)$ & $110.99(8)$ & $O(5)-C(14)-C(13)$ & $111.92(8)$ \\
\hline$C(15)-C(14)-C(13)$ & $109.77(8)$ & $O(4)-C(15)-C(14)$ & $110.76(8)$ \\
\hline$O(4)-C(15)-C(10)$ & $105.88(7)$ & $C(14)-C(15)-C(10)$ & $115.45(8)$ \\
\hline$O(1)-C(16)-C(10)$ & $113.03(8)$ & & \\
\hline
\end{tabular}

Symmetry transformations used to generate equivalent atoms: 
Table 4. Anisotropic displacement parameters $\left[\AA^{2} \times 10^{3}\right]$ for FPAs10.

The anisotropic displacement factor exponent takes the form:

$-2 \mathrm{rI}^{2}\left[\left(\mathrm{ha}^{*}\right)^{2} \mathrm{v}_{11}+\cdots+2 \mathrm{hka}{ }^{*} \mathrm{~b}^{*} \mathrm{v}_{12}\right]$

\begin{tabular}{|c|c|c|c|c|c|c|}
\hline & U11 & U22 & U33 & บ23 & $\mathrm{v} 13$ & $\mathrm{U} 12$ \\
\hline$O(1)$ & $44(1)$ & $15(1)$ & $26(1)$ & $-2(1)$ & $-12(1)$ & $8(1)$ \\
\hline$O(2)$ & $59(1)$ & $42(1)$ & $38(1)$ & $-8(1)$ & $-24(1)$ & 34 (1) \\
\hline$O(3)$ & $25(1)$ & $28(1)$ & $35(1)$ & $-16(1)$ & $-3(1)$ & $-3(1)$ \\
\hline$O(4)$ & $11(1)$ & $34(1)$ & $16(1)$ & $-2(1)$ & $-3(1)$ & $0(1)$ \\
\hline$O(5)$ & $25(1)$ & $21(1)$ & $23(1)$ & $-3(1)$ & $7(1)$ & $-5(1)$ \\
\hline$c(1)$ & $35(1)$ & $25(1)$ & $19(1)$ & $-3(1)$ & $-8(1)$ & $14(1)$ \\
\hline$c(2)$ & $14(1)$ & $22(1)$ & $15(1)$ & $-3(1)$ & $-4(1)$ & $5(1)$ \\
\hline$c(3)$ & $15(1)$ & $37(1)$ & $20(1)$ & $-8(1)$ & $-8(1)$ & $5(1)$ \\
\hline$C(4)$ & $16(1)$ & $3 I(1)$ & $16(1)$ & $-5(1)$ & $-6(1)$ & $0(1)$ \\
\hline$C(5)$ & $23(1)$ & $27(1)$ & $15(1)$ & $1(1)$ & $-6(1)$ & $0(1)$ \\
\hline$C(6)$ & $19(1)$ & $18(1)$ & $15(1)$ & $1(1)$ & $-3(1)$ & $-3(1)$ \\
\hline$C(7)$ & $11(1)$ & $13(1)$ & $14(1)$ & $-1(1)$ & $-3(1)$ & $0(1)$ \\
\hline$C(8)$ & $16(1)$ & $14(1)$ & $16(1)$ & $-2(1)$ & $-2(1)$ & $O(1)$ \\
\hline$C(9)$ & $12(1)$ & $23(1)$ & $19(1)$ & $-7(1)$ & $O(I)$ & $-3(1)$ \\
\hline$C(10)$ & $13(1)$ & $14(1)$ & $14(1)$ & $-1(1)$ & $-4(1)$ & $O(1)$ \\
\hline$c(1 I)$ & $12(1)$ & $22(1)$ & $20(1)$ & $-4(1)$ & $-6(1)$ & $0(1)$ \\
\hline$C(12)$ & $20(1)$ & $23(1)$ & $23(1)$ & $-4(1)$ & $-11(1)$ & $6(1)$ \\
\hline$C(13)$ & $28(1)$ & $22(1)$ & $17(1)$ & $I(1)$ & $-6(1)$ & $4(1)$ \\
\hline$C(14)$ & $18(1)$ & $16(1)$ & $16(1)$ & $-2(1)$ & $0(1)$ & $-1(1)$ \\
\hline$C(15)$ & $12(1)$ & $16(1)$ & $15(1)$ & $-2(1)$ & $-4(1)$ & $1(1)$ \\
\hline$C(16)$ & $28(1)$ & $15(1)$ & $21(1)$ & $-1(1)$ & $-8(1)$ & $-3(1)$ \\
\hline
\end{tabular}


Table 5. Hydrogen coordinates $\left(x 0^{4}\right)$ and isotropic displacement parameters $\left(\AA^{2} \times 10^{3}\right)$ for FPAs10.

\begin{tabular}{|c|c|c|c|c|}
\hline & $\boldsymbol{x}$ & $Y$ & $\mathbf{z}$ & $\mathrm{U}\left(\mathrm{eq}_{\mathrm{q}}\right)$ \\
\hline $\mathbf{H}(1)$ & $-650(30)$ & $7240(20)$ & $6827(18)$ & $47(5)$ \\
\hline$H(3 A)$ & -580 & 8281 & 827 & 28 \\
\hline $\mathrm{H}(3 \mathrm{~B})$ & -848 & 6782 & 1630 & 28 \\
\hline $\mathrm{H}(4 \mathrm{~A})$ & 1206 & 6532 & -646 & 25 \\
\hline$H(5 B)$ & 2895 & 8806 & -633 & 26 \\
\hline $\mathrm{H}(5 \mathrm{C})$ & 4403 & 7501 & -1040 & 26 \\
\hline $\mathrm{H}(6 \mathrm{~A})$ & 5729 & 7860 & 739 & 21 \\
\hline$H(6 B)$ & 4482 & 9323 & 982 & 21 \\
\hline $\mathrm{H}(\mathrm{8A})$ & 4592 & 5658 & 2002 & 19 \\
\hline $\mathrm{H}(8 \mathrm{~B})$ & 2389 & 5555 & 2845 & 19 \\
\hline H $(11 \mathrm{~A})$ & 6420 & 7589 & 3489 & 21 \\
\hline H (12A) & 6157 & 6552 & 5515 & 26 \\
\hline $\mathbf{H}(13 \mathrm{~A})$ & 2819 & 7447 & 6850 & 26 \\
\hline $\mathrm{H}(13 \mathrm{~B})$ & 3141 & 5751 & 6714 & 26 \\
\hline $\mathrm{H}(14 \mathrm{~A})$ & 1617 & 5691 & 4885 & 21 \\
\hline$H(15 A)$ & 1063 & 8726 & 5096 & 17 \\
\hline$H(16 A)$ & 3706 & 10155 & 4384 & 25 \\
\hline$H(16 B)$ & 4973 & 10004 & 2885 & 25 \\
\hline
\end{tabular}




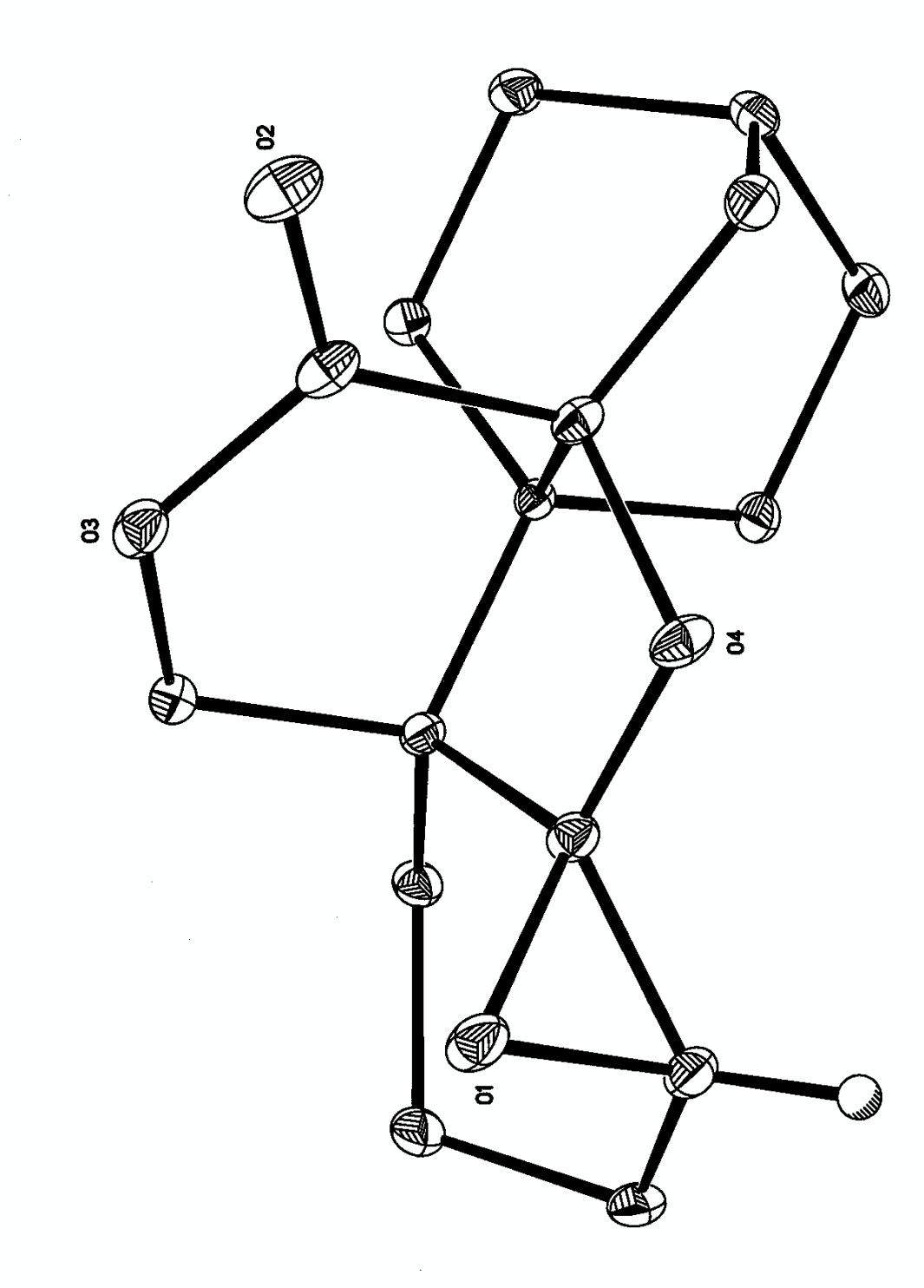


Table 1. Cxystal data and structure refinement for F4WS10.

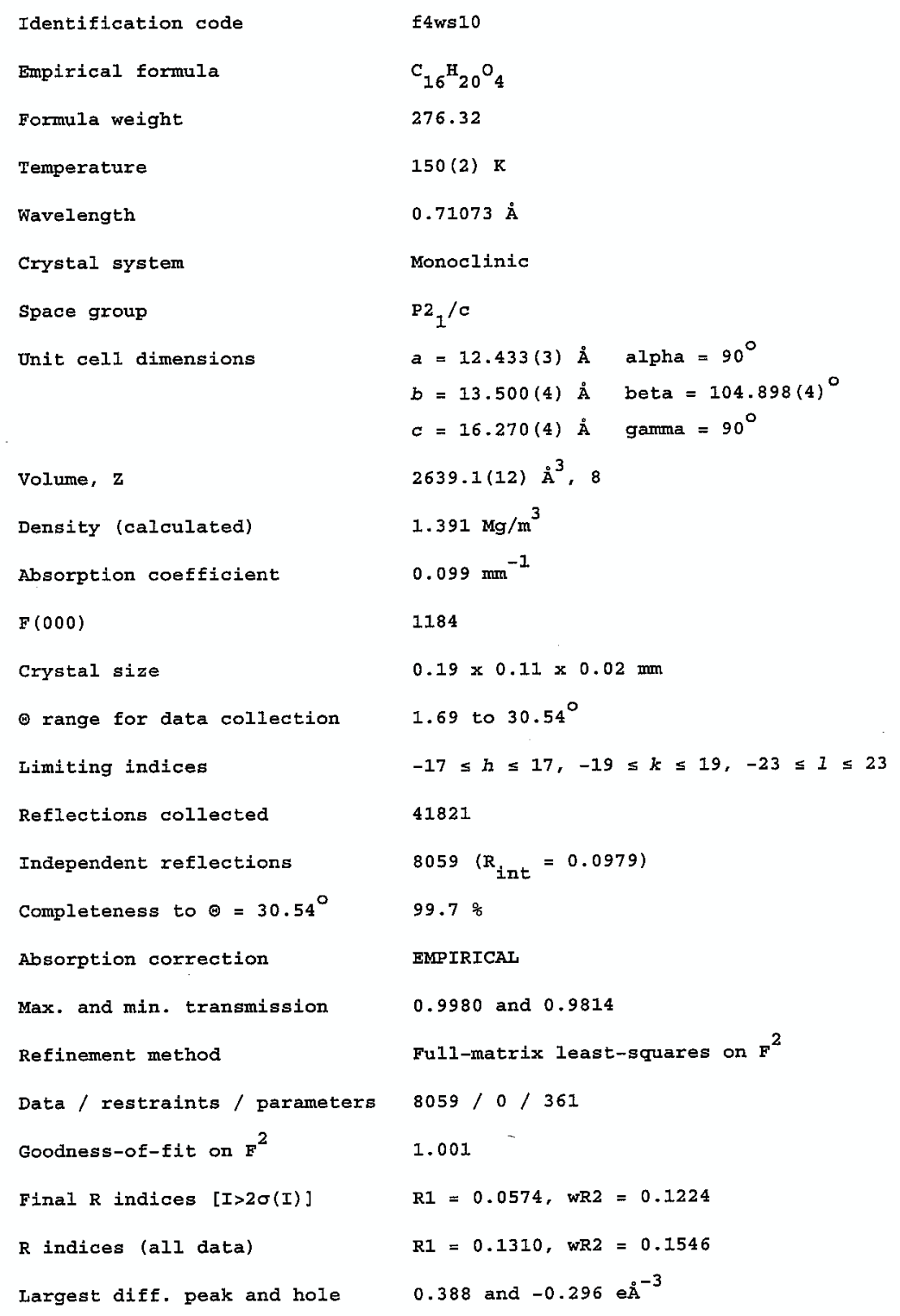


Table 2. Atomic coordinates $\left[\times 10^{4}\right]$ and equivalent isotropic displacement parameters $\left[\AA^{2} \times 10^{3}\right]$ for F4WS10. U(eq) is defined as one third of the trace of the orthogonalized $v_{i f}$ tensor.

\begin{tabular}{|c|c|c|c|c|}
\hline & $x$ & $y$ & $\mathbf{z}$ & $\mathrm{u}(\mathrm{eq})$ \\
\hline$o(1)$ & $8420(1)$ & $3711(1)$ & $1584(1)$ & $28(1)$ \\
\hline$O(2)$ & $11694(1)$ & $4364(1)$ & $3548(1)$ & $28(1)$ \\
\hline$O(3)$ & $11141(1)$ & $3872(1)$ & $2220(1)$ & $24(1)$ \\
\hline$O(4)$ & $9334(1)$ & $3237(1)$ & $2998(1)$ & $23(1)$ \\
\hline$C(1)$ & $7462(2)$ & $2137(2)$ & $976(1)$ & $30(1)$ \\
\hline$c(2)$ & $11407(2)$ & $1702(2)$ & $4391(1)$ & $24(1)$ \\
\hline$C(3)$ & $7797(2)$ & $2820(2)$ & $1725(1)$ & $25(1)$ \\
\hline$C(4)$ & $12312(2)$ & $1668(2)$ & $3897(1)$ & $25(1)$ \\
\hline$c(5)$ & $9940(2)$ & $1138(1)$ & $3112(1)$ & $22(1)$ \\
\hline$C(6)$ & $10642(2)$ & $3129(1)$ & $1589(1)$ & $21(1)$ \\
\hline$C(7)$ & $10560(2)$ & $886(2)$ & $4042(1)$ & $25(1)$ \\
\hline$C(8)$ & $10807(2)$ & $2708(2)$ & $4248(1)$ & $24(1)$ \\
\hline$C(9)$ & $8403(2)$ & $1987(2)$ & $541(1)$ & $29(1)$ \\
\hline$C(10)$ & $9459(2)$ & $1621(2)$ & $1186(1)$ & $23(1)$ \\
\hline$C(11)$ & $8978(2)$ & $2955(1)$ & $2138(1)$ & $20(1)$ \\
\hline$C(12)$ & $11173(2)$ & $3764(1)$ & $3050(1)$ & $21(1)$ \\
\hline$C(13)$ & $11774(2)$ & $1668(1)$ & $2926(1)$ & $19(1)$ \\
\hline$C(14)$ & $9911(2)$ & $2380(1)$ & $1894(1)$ & $18(1)$ \\
\hline$C(15)$ & $10552(2)$ & $1984(1)$ & $2777(1)$ & $15(1)$ \\
\hline$c(16)$ & $10487(2)$ & $2918(1)$ & $3296(1)$ & $18(1)$ \\
\hline$O(5)$ & $6587(1)$ & $3762(1)$ & $3567(1)$ & $24(1)$ \\
\hline$O(6)$ & $3292(1)$ & 2985 (1) & $1557(1)$ & $26(1)$ \\
\hline$O(7)$ & $3854(1)$ & $3502(1)$ & $2877(1)$ & $22(1)$ \\
\hline$O(8)$ & $5665(1)$ & $4118(I)$ & $2125(1)$ & $20(1)$ \\
\hline$C(17)$ & $7473(2)$ & $5406(2)$ & $4076(1)$ & 24 (1) \\
\hline$C(18)$ & $6003(2)$ & $4467(1)$ & $2967(1)$ & $18(1)$ \\
\hline$C(19)$ & $6526(2)$ & $5557(2)$ & $4507(1)$ & $24(1)^{\circ}$ \\
\hline$C(20)$ & $4320(2)$ & $4279(1)$ & $3486(1)$ & $20(1)$ \\
\hline$C(21)$ & $5016(2)$ & $6210(1)$ & $1918(1)$ & $22(1)$ \\
\hline$C(22)$ & $4401(2)$ & $6432(2)$ & $980(1)$ & $25(1)$ \\
\hline$C(23)$ & $5441(2)$ & $5830(1)$ & $3855(1)$ & $22(1)$ \\
\hline$C(24)$ & $7175(2)$ & $4654(1)$ & $3373(1)$ & $21(1)$ \\
\hline$C(25)$ & $3814(2)$ & $3591(1)$ & 2045 (1) & $19(1)$ \\
\hline$C(26)$ & $4233(2)$ & 4591 (I) & $833(1)$ & $22(1)$ \\
\hline$C(27)$ & $3591(2)$ & $5583(2)$ & $639(1)$ & $22(1)$ \\
\hline$C(28)$ & $2664(2)$ & $5609(2)$ & $1109(1)$ & $24(1)$ \\
\hline$C(29)$ & $3176(2)$ & $5671(1)$ & $2084(1)$ & $19(1)$ \\
\hline$C(30)$ & $4509(2)$ & $4424(1)$ & $1789(1)$ & $16(1)$ \\
\hline$C(31)$ & $5037(2)$ & $5033(1)$ & $3172(1)$ & $16(1)$ \\
\hline$C(32)$ & $4409(2)$ & $5382(1)$ & $2270(1)$ & $15(1)$ \\
\hline
\end{tabular}


Table 3. Bond lengths $[\AA \AA a]$ and angles $\left[{ }^{\circ}\right]$ for F4WS10.

\begin{tabular}{|c|c|c|c|}
\hline$O(1)-C(11)$ & $1.419(2)$ & $O(1)-C(3)$ & $1.481(2)$ \\
\hline$O(2)-C(12)$ & $1.209(2)$ & $O(3)-C(12)$ & $1.349(2)$ \\
\hline$O(3)-C(6)$ & $1.455(2)$ & $O(4)-C(11)$ & $1.408(2)$ \\
\hline$O(4)-C(16)$ & $1.455(2)$ & $C(1)-C(3)$ & $1.499(3)$ \\
\hline$C(1)-C(9)$ & $1.529(3)$ & $c(2)-C(7)$ & $1.529(3)$ \\
\hline$c(2)-C(8)$ & $1.539(3)$ & $c(2)-C(4)$ & $1.543(3)$ \\
\hline$c(3)-C(11)$ & $1.460(3)$ & $C(4)-C(13)$ & $1.549(3)$ \\
\hline$C(5)-C(15)$ & $1.547(3)$ & $c(5)-C(7)$ & $1.550(3)$ \\
\hline$C(6)-C(14)$ & $1.526(3)$ & $c(8)-C(16)$ & $1.522(3)$ \\
\hline$C(9)-C(10)$ & $1.536(3)$ & $C(10)-C(14)$ & $1.536(3)$ \\
\hline$C(11)-C(14)$ & $1.531(3)$ & $C(12)-C(16)$ & $1.540(3)$ \\
\hline$C(13)-C(15)$ & $1.537(2)$ & $C(14)-C(15)$ & $1.548(2)$ \\
\hline$C(15)-C(16)$ & $1.531(2)$ & $O(5)-C(18)$ & $1.423(2)$ \\
\hline$O(5)-C(24)$ & $1.484(2)$ & $O(6)-C(25)$ & $1.207(2)$ \\
\hline$O(7)-C(25)$ & $1.347(2)$ & $O(7)-C(20)$ & $1.457(2)$ \\
\hline$O(8)-C(18)$ & $1.407(2)$ & $O(8)-C(30)$ & $1.459(2)$ \\
\hline$C(17)-C(24)$ & $1.502(3)$ & $C(17)-C(19)$ & $1.531(3)$ \\
\hline$C(18)-C(24)$ & $1.459(3)$ & $C(18)-C(31)$ & $1.531(3)$ \\
\hline$C(19)-C(23)$ & $1.531(3)$ & $c(20)-C(31)$ & $1.526(3)$ \\
\hline$C(21)-C(32)$ & $1.539(3)$ & $C(21)-C(22)$ & $1.550(3)$ \\
\hline$C(22)-C(27)$ & $1.533(3)$ & $c(23)-c(31)$ & $1.536(3)$ \\
\hline$C(25)-C(30)$ & $1.539(3)$ & $C(26)-C(30)$ & $1.521(3)$ \\
\hline$C(26)-C(27)$ & $1.550(3)$ & $C(27)-C(28)$ & $1.538(3)$ \\
\hline$C(28)-C(29)$ & $1.552(3)$ & $C(29)-C(32)$ & $1.535(3)$ \\
\hline$C(30)-C(32)$ & $1.534(3)$ & $C(31)-C(32)$ & $1.546(2)$ \\
\hline$C(11)-0(1)-C(3)$ & $60.40(12)$ & $C(12)-0(3)-C(6)$ & $121.97(15)$ \\
\hline$C(11)-0(4)-C(16)$ & $106.44(14)$ & $C(3)-C(1)-C(9)$ & $111.84(17)$ \\
\hline$C(7)-C(2)-C(8)$ & $108.63(16)$ & $C(7)-C(2)-C(4)$ & $108.07(16)$ \\
\hline$C(8)-C(2)-C(4)$ & $109.68(16)$ & $C(11)-C(3)-O(1)$ & $57.70(11)$ \\
\hline$C(11)-C(3)-C(1)$ & $119.14(18)$ & $O(1)-C(3)-C(1)$ & $114.86(17)$ \\
\hline$C(2)-C(4)-C(13)$ & $110.49(16)$ & $C(15)-C(5)-C(7)$ & $109.86(15)$ \\
\hline$O(3)-C(6)-C(14)$ & $114.14(15)$ & $C(2)-C(7)-C(5)$ & $109.07(15)$ \\
\hline$C(16)-C(8)-C(2)$ & $107.94(15)$ & $C(1)-C(9)-C(10)$ & $110.35(17)$ \\
\hline$C(14)-C(10)-C(9)$ & $112.44(17)$ & $O(4)-C(11)-O(1)$ & $114.47(15)$ \\
\hline$O(4)-C(11)-C(3)$ & $120.88(17)$ & $O(1)-C(11)-C(3)$ & $61.90(12)$ \\
\hline$O(4)-C(11)-C(14)$ & $109.20(15)$ & $O(I)-C(11)-C(14)$ & $118.63(16)$ \\
\hline$C(3)-C(11)-C(14)$ & $123.87(17)$ & $O(2)-C(12)-o(3)$ & $118.15(18)$ \\
\hline$O(2)-C(12)-C(16)$ & $124.02(19)$ & $O(3)-C(12)-C(16)$ & $117.73(16)$ \\
\hline$C(15)-C(13)-C(4)$ & $108.11(15)$ & $C(6)-C(14)-C(11)$ & $107.66(15)$ \\
\hline$C(6)-C(14)-C(10)$ & $109.41(15)$ & $C(11)-C(14)-C(10)$ & $112.12(15)$ \\
\hline$C(6)-C(14)-C(15)$ & $108.85(15)$ & $C(11)-C(14)-C(15)$ & $100.25(14)$ \\
\hline$C(10)-C(14)-C(15)$ & $117.87(15)$ & $C(16)-C(15)-C(13)$ & $109.39(15)$ \\
\hline$C(16)-C(15)-C(5)$ & $108.22(15)$ & $C(13)-C(15)-C(5)$ & $107.20(15)$ \\
\hline$C(16)-C(15)-C(14)$ & $98.08(14)$ & $C(13)-C(15)-C(14)$ & $119.64(15)$ \\
\hline$C(5)-C(15)-C(14)$ & 113.51 (15) & $O(4)-C(16)-C(8)$ & $111.79(15)$ \\
\hline$O(4)-C(16)-C(15)$ & $104.29(14)$ & $C(8)-C(16)-C(15)$ & $111.79(15)$ \\
\hline$O(4)-C(16)-C(12)$ & $104.93(15)$ & $C(8)-C(16)-C(12)$ & $112.68(16)$ \\
\hline$C(15)-C(16)-C(12)$ & $110.84(15)$ & $C(18)-O(5)-C(24)$ & $60.20(11)$ \\
\hline$C(25)-O(7)-C(20)$ & $121.66(15)$ & $C(18)-0(8)-C(30)$ & $106.77(13)$ \\
\hline$c(24)-c(17)-c(19)$ & $111.68(16)$ & $O(8)-C(18)-O(5)$ & 114.64 (15) \\
\hline$O(8)-C(18)-C(24)$ & $120.86(16)$ & $O(5)-C(18)-C(24)$ & $62.00(12)$ \\
\hline$O(8)-C(18)-C(31)$ & $108.95(14)$ & $O(5)-C(18)-C(31)$ & $118.36(15)$ \\
\hline$C(24)-C(18)-C(31)$ & $124.23(16)$ & $C(23)-C(19)-C(17)$ & $111.07(16)$ \\
\hline$O(7)-C(20)-C(31)$ & $114.67(15)$ & $C(32)-C(21)-C(22)$ & $110.02(15)$ \\
\hline
\end{tabular}




$\begin{array}{lrlr}C(27)-C(22)-C(21) & 108.89(15) & C(19)-C(23)-C(31) & 113.49(16) \\ C(18)-C(24)-O(5) & 57.80(11) & C(18)-C(24)-C(17) & 119.00(17) \\ O(5)-C(24)-C(17) & 115.20(16) & O(6)-C(25)-O(7) & 118.41(17) \\ O(6)-C(25)-C(30) & 124.17(18) & O(7)-C(25)-C(30) & 117.27(16) \\ C(30)-C(26)-C(27) & 107.79(15) & C(22)-C(27)-C(28) & 108.02(16) \\ C(22)-C(27)-C(26) & 108.43(16) & C(28)-C(27)-C(26) & 109.34(16) \\ C(27)-C(28)-C(29) & 110.28(16) & C(32)-C(29)-C(28) & 108.34(15) \\ O(8)-C(30)-C(26) & 111.46(14) & O(8)-C(30)-C(32) & 104.38(14) \\ C(26)-C(30)-C(32) & 111.73(15) & O(8)-C(30)-C(25) & 105.11(14) \\ C(26)-C(30)-C(25) & 112.96(15) & C(32)-C(30)-C(25) & 110.69(15) \\ C(20)-C(31)-C(18) & 107.37(15) & C(20)-C(31)-C(23) & 109.58(15) \\ C(18)-C(31)-C(23) & 112.26(15) & C(20)-C(31)-C(32) & 108.93(15) \\ C(18)-C(31)-C(32) & 100.29(14) & C(23)-C(31)-C(32) & 117.70(15) \\ C(30)-C(32)-C(29) & 108.75(14) & C(30)-C(32)-C(21) & 108.09(15) \\ C(29)-C(32)-C(21) & 107.54(15) & C(30)-C(32)-C(31) & 98.03(14) \\ C(29)-C(32)-C(31) & 120.01(15) & C(21)-C(32)-C(31) & 113.47(15) \\ & & & \end{array}$

Symmetry transformations used to generate equivalent atoms: 
Table 4. Anisotropic displacement parameters $\left[\AA^{2} \times 10^{3}\right]$ for F4WS10. The anisotropic displacement factor exponent takes the form: $-2 \mathrm{II}^{2}\left[\left(\mathrm{ha}^{*}\right)^{2} \mathrm{U}_{11}+\ldots+2 \mathrm{hka}{ }^{*} \mathrm{~b}^{*} \mathrm{U}_{12}\right]$

\begin{tabular}{|c|c|c|c|c|c|c|}
\hline & U11 & บ22 & บ33 & U23 & V13 & $\mathrm{v} 12$ \\
\hline$O(1)$ & $18(1)$ & $23(1)$ & $43(1)$ & $12(1)$ & $4(1)$ & $1(1)$ \\
\hline$O(2)$ & $23(1)$ & $22(1)$ & $39(1)$ & $-7(1)$ & $4(1)$ & $-3(1)$ \\
\hline$O(3)$ & $21(1)$ & $20(1)$ & $30(1)$ & $2(1)$ & $5(1)$ & $-4(1)$ \\
\hline$O(4)$ & $14(1)$ & $26(1)$ & $28(1)$ & $-6(1)$ & $4(1)$ & $4(1)$ \\
\hline$c(1)$ & $19(1)$ & $40(1)$ & $26(1)$ & $7(1)$ & $-4(1)$ & $-7(1)$ \\
\hline$C(2)$ & $23(1)$ & $32(1)$ & $15(1)$ & $2(1)$ & $4(1)$ & $2(1)$ \\
\hline$c(3)$ & $16(1)$ & $26(1)$ & $32(1)$ & $10(1)$ & $4(1)$ & $-1(1)$ \\
\hline$C(4)$ & $17(1)$ & $33(1)$ & $23(1)$ & $2(1)$ & $2(1)$ & $4(1)$ \\
\hline$c(5)$ & $22(1)$ & $20(1)$ & $23(1)$ & $1(1)$ & $6(1)$ & $-4(1)$ \\
\hline$c(6)$ & $19(1)$ & $22(1)$ & $21(1)$ & $2(1)$ & $5(1)$ & $-2(1)$ \\
\hline$C(7)$ & $30(1)$ & $24(1)$ & $22(1)$ & $5(1)$ & $8(1)$ & $-I(1)$ \\
\hline$C(8)$ & $23(1)$ & $27(1)$ & $22(1)$ & $-7(1)$ & $8(1)$ & $-4(1)$ \\
\hline$c(9)$ & $28(1)$ & $36(1)$ & $20(1)$ & $2(1)$ & $O(I)$ & $-6(1)$ \\
\hline$C(10)$ & $26(1)$ & $24(1)$ & $18(1)$ & $-1(1)$ & $2(1)$ & $-4(1)$ \\
\hline$c(11)$ & $17(1)$ & $17(1)$ & $25(1)$ & $3(1)$ & $4(1)$ & $1(1)$ \\
\hline$C(12)$ & $15(1)$ & $16(1)$ & $32(1)$ & $-2(1)$ & $4(1)$ & $2(1)$ \\
\hline $\mathrm{C}(13)$ & $19(1)$ & $18(1)$ & $21(1)$ & $1(1)$ & $6(1)$ & $3(1)$ \\
\hline$C(14)$ & $14(1)$ & $18(1)$ & $20(1)$ & $2(1)$ & $3(1)$ & $0(1)$ \\
\hline$c(15)$ & $14(1)$ & $16(1)$ & $16(1)$ & $-1(1)$ & $3(1)$ & $-1(1)$ \\
\hline$c(16)$ & $13(1)$ & $19(1)$ & $22(1)$ & $-3(1)$ & $4(1)$ & $1(1)$ \\
\hline$o(5)$ & $18(1)$ & $23(1)$ & $28(1)$ & $6(1)$ & $1(1)$ & $0(1)$ \\
\hline$O(6)$ & $23(1)$ & $21(1)$ & $32(1)$ & $-6(1)$ & $5(1)$ & $-3(1)$ \\
\hline $0(7)$ & $24(1)$ & $20(1)$ & $23(1)$ & $O(1)$ & $7(1)$ & $-4(1)$ \\
\hline$O(8)$ & $14(1)$ & $26(1)$ & $20(1)$ & $-6(1)$ & $3(1)$ & $2(1)$ \\
\hline$c(17)$ & $18(1)$ & $30(1)$ & $23(1)$ & $0(1)$ & $O(1)$ & $-6(1)$ \\
\hline$C(18)$ & $16(1)$ & $19(1)$ & $18(1)$ & $1(1)$ & $4(1)$ & $-1(1)$ \\
\hline$C(19)$ & $20(1)$ & $34(1)$ & $17(1)$ & $-3(1)$ & $2(1)$ & $-7(1)$ \\
\hline$C(20)$ & $20(1)$ & $22(1)$ & 19 (1) & $-1(1)$ & $6(1)$ & $-1(1)$ \\
\hline$c(21)$ & $22(1)$ & $21(1)$ & $20(1)$ & $0(1)$ & $5(1)$ & $-6(1)$ \\
\hline$c(22)$ & $31(1)$ & $24(1)$ & 21 (I) & $3(1)$ & $9(1)$ & $-1(1)$ \\
\hline$C(23)$ & $20(1)$ & $25(1)$ & $19(1)$ & $-4(1)$ & $4(1)$ & $-2(1)$ \\
\hline$C(24)$ & $15(1)$ & $23(1)$ & $24(1)$ & $3(1)$ & $3(1)$ & $-3(1)$ \\
\hline$C(25)$ & $15(1)$ & $17(1)$ & $24(1)$ & $O(1)$ & $5(1)$ & $3(1)$ \\
\hline$C(26)$ & $24(1)$ & $22(1)$ & $18(1)$ & $-4(1)$ & $6(1)$ & $-2(1)$ \\
\hline$C(27)$ & $25(1)$ & $27(1)$ & $15(1)$ & $1(1)$ & $4(1)$ & $0(1)$ \\
\hline$C(28)$ & $20(1)$ & $27(1)$ & $23(1)$ & $3(1)$ & $3(1)$ & $3(1)$ \\
\hline$C(29)$ & $17(1)$ & $18(1)$ & $23(1)$ & $0(1)$ & $6(1)$ & $3(1)$ \\
\hline$C(30)$ & $12(1)$ & 19 (1) & $18(1)$ & $-2(1)$ & $4(1)$ & $1(1)$ \\
\hline$C(31)$ & $16(1)$ & $17(1)$ & $16(1)$ & $-1(1)$ & $4(1)$ & $-2(1)$ \\
\hline$c(32)$ & $14(1)$ & $17(1)$ & $15(1)$ & $-1(1)$ & $5(1)$ & $-1(1)$ \\
\hline
\end{tabular}


Table 5. Hydrogen coordinates $\left(x 0^{4}\right)$ and isotropic displacement parameters $\left(\AA^{2} \times 10^{3}\right)$ for F4WS10.

\begin{tabular}{|c|c|c|c|c|}
\hline & $x$ & $y$ & $\mathbf{z}$ & $\mathrm{U}(\mathrm{eq})$ \\
\hline$H(1 \mathrm{~A})$ & 6805 & 2415 & 561 & 36 \\
\hline $\mathrm{H}(1 \mathrm{~B})$ & 7246 & 1488 & 1168 & 36 \\
\hline$H(2 A)$ & 11748 & 1594 & 5011 & 28 \\
\hline$H(3 A)$ & 7263 & 2899 & 2087 & 30 \\
\hline $\mathrm{H}(4 \mathrm{~A})$ & 12769 & 1064 & 4056 & 30 \\
\hline $\mathrm{H}(4 \mathrm{~B})$ & 12807 & 2251 & 4050 & 30 \\
\hline $\mathrm{H}(5 \mathrm{~A})$ & 9907 & 544 & 2749 & 26 \\
\hline $\mathrm{H}(5 \mathrm{~B})$ & 9168 & 1345 & 3086 & 26 \\
\hline $\mathrm{H}(6 \mathrm{~A})$ & 11244 & 2768 & 1419 & 25 \\
\hline $\mathrm{H}(6 \mathrm{~B})$ & 10188 & 3469 & 1077 & 25 \\
\hline $\mathrm{H}(7 \mathrm{~A})$ & 10021 & 834 & 4394 & 30 \\
\hline $\mathrm{H}(7 \mathrm{~B})$ & 10946 & 241 & 4061 & 30 \\
\hline $\mathrm{H}(8 \mathrm{~A})$ & 10133 & 2688 & 4465 & 28 \\
\hline $\mathrm{H}(\mathrm{BB})$ & 11304 & 3236 & 4554 & 28 \\
\hline $\mathrm{H}(9 \mathrm{~A})$ & 8561 & 2620 & 288 & 34 \\
\hline $\mathrm{H}(9 \mathrm{~B})$ & 8172 & 1496 & 77 & 34 \\
\hline$H(10 A)$ & 9290 & 995 & 1445 & 28 \\
\hline $\mathrm{H}(10 \mathrm{~B})$ & 10039 & 1480 & 884 & 28 \\
\hline$F(13 A)$ & 12173 & 2135 & 2639 & 23 \\
\hline$H(13 B)$ & 11818 & 997 & 2690 & 23 \\
\hline $\mathrm{H}(17 \mathrm{~A})$ & 7645 & 6046 & 3840 & 29 \\
\hline $\mathrm{H}(17 \mathrm{~B})$ & 8149 & 5184 & 4504 & 29 \\
\hline $\mathrm{H}(19 \mathrm{~A})$ & 6415 & 4941 & 4804 & 29 \\
\hline $\mathrm{H}(19 \mathrm{~B})$ & 6729 & 6091 & 4936 & 29 \\
\hline$H(20 A)$ & 4776 & 3967 & 4010 & 24 \\
\hline $\mathrm{H}(2 \mathrm{OB})$ & 3701 & 4634 & 3637 & 24 \\
\hline$H(21 A)$ & 5790 & 6003 & 1952 & 26 \\
\hline $\mathrm{H}(21 \mathrm{~B})$ & 5042 & 6817 & 2264 & 26 \\
\hline$H(22 A)$ & 3987 & 7064 & 945 & 30 \\
\hline $\mathrm{H}(22 \mathrm{~B})$ & 4945 & 6495 & 633 & 30 \\
\hline $\mathrm{H}(23 \mathrm{~A})$ & 5552 & 6458 & 3574 & 26 \\
\hline $\mathrm{H}(23 \mathrm{~B})$ & 4856 & 5944 & 4158 & 26 \\
\hline $\mathrm{H}(24 \mathrm{~A})$ & 7715 & 4557 & 3019 & 25 \\
\hline$H(26 A)$ & 3770 & 4040 & 532 & 26 \\
\hline $\mathrm{H}(26 \mathrm{~B})$ & 4926 & 4621 & 640 & 26 \\
\hline $\mathrm{H}(27 \mathrm{~A})$ & 3267 & 5658 & 13 & 27 \\
\hline H $(28 \mathrm{~A})$ & 2202 & 5004 & 973 & 29 \\
\hline $\mathrm{H}(28 \mathrm{~B})$ & 2179 & 6190 & 917 & 29 \\
\hline $\mathrm{H}(29 \mathrm{~A})$ & 3104 & 6353 & 2287 & 23 \\
\hline $\mathrm{H}(29 \mathrm{~B})$ & 2780 & 5216 & 2382 & 23 \\
\hline
\end{tabular}




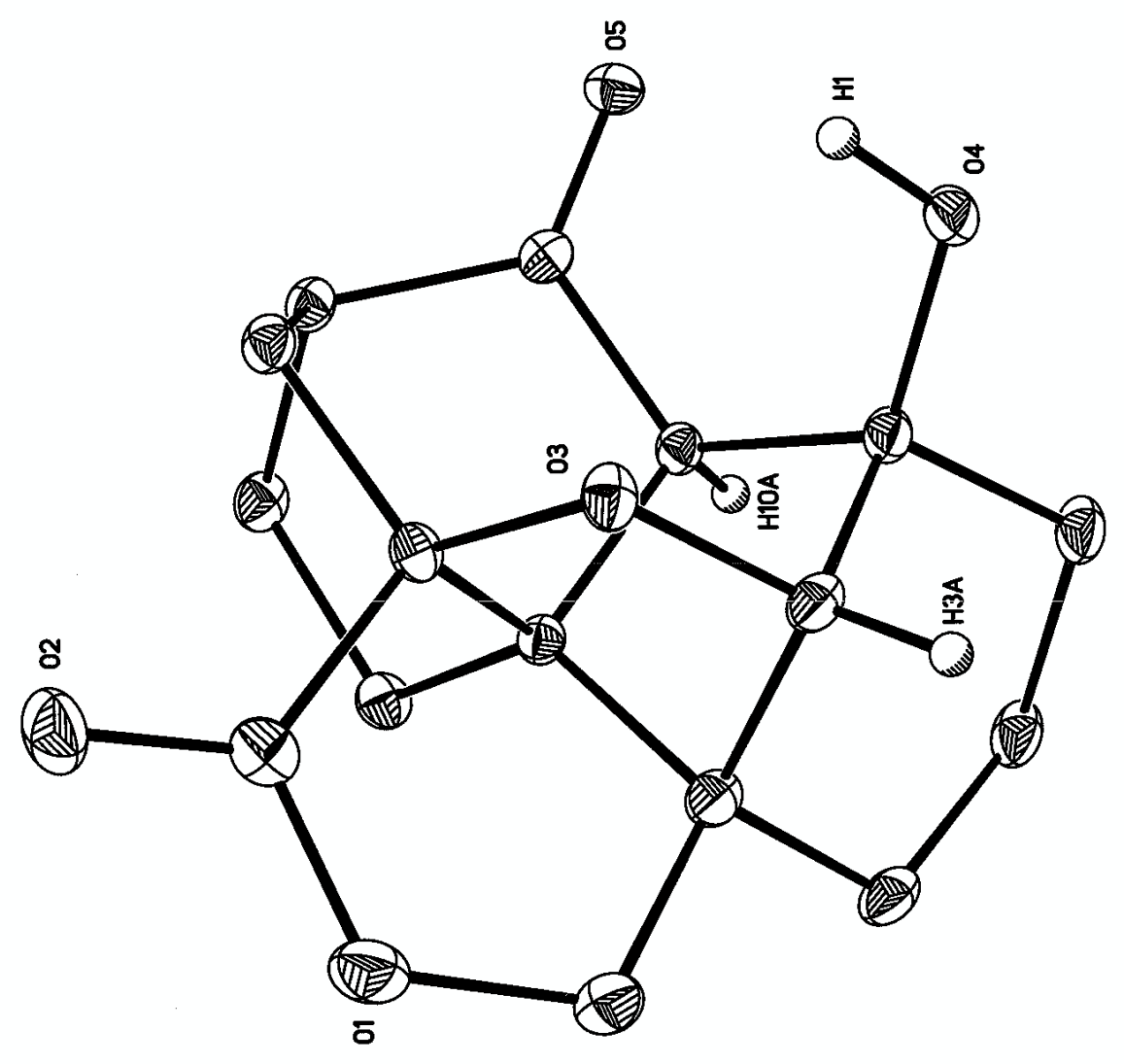


Table 1. Crystal data and structure refinement for FPA5sio.

\begin{tabular}{|c|c|}
\hline Identification code & fpass10 \\
\hline Empirical formula & $\mathrm{C}_{16}{ }^{\mathrm{H}} 18^{\mathrm{O}} 5.20$ \\
\hline Formula weight & 293.42 \\
\hline Temperature & $125(2) \mathrm{K}$ \\
\hline Wavelength & $0.71073 \AA$ \\
\hline Crystal system & Monoclinic \\
\hline Space group & $\mathrm{P}{ }_{1} / \mathrm{C}$ \\
\hline Unit cell dimensions & $\begin{array}{l}a=6.4057(10) \AA \quad \text { alpha }=90^{\circ} \\
b=24.970(4) \AA \quad \text { beta }=109.779(2)^{\circ} \\
c=8.6806(13) \AA \quad \text { gamma }=90^{\circ}\end{array}$ \\
\hline Volume, $\mathrm{z}$ & $1306.5(3) \AA^{3}, 4$ \\
\hline Density (calculated) & $1.492 \mathrm{Mg} / \mathrm{m}^{3}$ \\
\hline Absorption coefficient & $0.111 \mathrm{~mm}^{-1}$ \\
\hline$F(000)$ & 622 \\
\hline Crystal size & $0.20 \times 0.10 \times 0.04 \mathrm{~mm}$ \\
\hline (4) range for data collection & 1.63 to $30.53^{\circ}$ \\
\hline Limiting indices & $-9 \leq h \leq 9,-35 \leq k \leq 35,-12 \leq I \leq 12$ \\
\hline Reflections collected & 20658 \\
\hline Independent reflections & $3987\left(R_{\text {Int }}=0.0831\right)$ \\
\hline Completeness to $\odot=30.53^{\circ}$ & $99.6 \%$ \\
\hline Absorption correction & EMPIRICAL \\
\hline Max. and min. transmission & 0.9956 and 0.9781 \\
\hline Refinement method & Full-matrix least-squares on $F^{2}$ \\
\hline Data / restraints / parameters & $3987 / 2 / 205$ \\
\hline Goodness-of-fit on $F^{2}$ & 1.027 \\
\hline Final $R$ indices $[I>2 \sigma(I)]$ & $R 1=0.0596, \quad w R 2=0.1395$ \\
\hline $\mathrm{R}$ indices (all data) & $\mathrm{R} 1=0.1027, \mathrm{wR2}=0.1601$ \\
\hline Largest diff. peak and hole & 0.434 and $-0.257 \mathrm{e}^{-3}$ \\
\hline
\end{tabular}


Table 2. Atomic coordinates $\left[x 0^{4}\right]$ and equivalent isotropic displacement parameters $\left[\mathrm{A}^{2} \times 10^{3}\right.$ ] for FPA5S10. U(eq) is defined as one third of the trace of the orthogonalized $u_{i j}$ tensor.

\begin{tabular}{lcccc}
\hline & & & & \\
& & & & \\
& & & & \\
& & & & \\
$O$ & & & & \\
\hline & & & & \\
$O(2)$ & $5634(3)$ & $2667(1)$ & $3403(2)$ & $30(1)$ \\
$O(3)$ & $3024(3)$ & $3071(1)$ & $1431(2)$ & $33(1)$ \\
$O(4)$ & $4777(2)$ & $3611(1)$ & $5099(2)$ & $20(1)$ \\
$O(5)$ & $6727(2)$ & $4434(1)$ & $7415(2)$ & $24(1)$ \\
$C(1)$ & $7308(2)$ & $5119(1)$ & $4758(2)$ & $22(1)$ \\
$C(2)$ & $4546(3)$ & $3113(1)$ & $2707(3)$ & $23(1)$ \\
$C(3 A)$ & $5340(3)$ & $3641(1)$ & $3608(2)$ & $17(1)$ \\
$C(3 B)$ & $6871(3)$ & $3519(1)$ & $6422(2)$ & $20(1)$ \\
$O(6)$ & $6871(3)$ & $3519(1)$ & $6422(2)$ & $20(1)$ \\
$C(4)$ & $6635(11)$ & $3333(3)$ & $7837(7)$ & $20(2)$ \\
$C(5)$ & $8353(3)$ & $3236(1)$ & $5603(2)$ & $21(1)$ \\
$C(6)$ & $7488(4)$ & $2685(1)$ & $4930(3)$ & $27(1)$ \\
$C(7)$ & $10734(3)$ & $3155(1)$ & $6803(3)$ & $25(1)$ \\
$C(8)$ & $11836(3)$ & $3647(1)$ & $7790(3)$ & $27(1)$ \\
$C(9)$ & $10198(3)$ & $3988(1)$ & $8311(2)$ & $26(1)$ \\
$C(10)$ & $8011(3)$ & $4063(1)$ & $6901(2)$ & $20(1)$ \\
$C(11)$ & $8461(3)$ & $4200(1)$ & $5271(2)$ & $17(1)$ \\
$C(12)$ & $7893(3)$ & $3673(1)$ & $4261(2)$ & $16(1)$ \\
$C(13)$ & $8791(3)$ & $3689(1)$ & $2853(2)$ & $21(1)$ \\
$C(14)$ & $7856(3)$ & $4206(1)$ & $1845(2)$ & $23(1)$ \\
$C(15)$ & $6184(3)$ & $4503(1)$ & $2474(2)$ & $19(1)$ \\
$C(16)$ & $7303(3)$ & $4666(1)$ & $4241(2)$ & $18(1)$ \\
\hline & $4307(3)$ & $4123(1)$ & $2535(2)$ & $19(1)$ \\
\hline & & & & \\
\hline
\end{tabular}


Table 3. Bond lengths [Å] and angles $\left[{ }^{\circ}\right]$ for FPA5S10.

\begin{tabular}{|c|c|c|c|}
\hline$o(1)-C(1)$ & $1.344(3)$ & $O(1)-C(5)$ & $1.451(3)$ \\
\hline$o(2)-C(1)$ & $1.207(2)$ & $O(3)-C(2)$ & $1.458(2)$ \\
\hline$O(3)-C(3 A)$ & $1.459(2)$ & $O(4)-H(1)$ & $0.92(3)$ \\
\hline$O(4)-C(9)$ & $1.408(2)$ & $O(5)-C(15)$ & $1.217(2)$ \\
\hline$c(1)-c(2)$ & $1.530(3)$ & $C(2)-C(16)$ & $1.529(3)$ \\
\hline$C(2)-C(11)$ & $1.541(3)$ & $C(3 A)-C(9)$ & $1.530(3)$ \\
\hline$C(3 A)-C(4)$ & $1.538(3)$ & $C(4)-C(5)$ & $1.523(3)$ \\
\hline$c(4)-c(6)$ & $1.541(3)$ & $C(4)-C(11)$ & $1.551(3)$ \\
\hline$C(6)-C(7)$ & $1.528(3)$ & $C(7)-C(8)$ & $1.533(3)$ \\
\hline$C(8)-C(9)$ & $1.528(3)$ & $C(9)-C(10)$ & $1.574(3)$ \\
\hline$C(10)-C(15)$ & $1.502(3)$ & $c(10)-c(11)$ & $1.553(3)$ \\
\hline$C(11)-C(12)$ & $1.517(3)$ & $C(12)-C(13)$ & $1.561(3)$ \\
\hline$C(13)-C(14)$ & $1.547(3)$ & $C(14)-C(15)$ & $1.513(3)$ \\
\hline$C(14)-C(16)$ & $1.546(3)$ & & \\
\hline$C(1)-O(1)-C(5)$ & $121.65(16)$ & $C(2)-O(3)-C(3 A)$ & $105.61(13)$ \\
\hline$H(1)-O(4)-C(9)$ & $113.1(16)$ & $O(2)-C(1)-O(1)$ & $118.38(18)$ \\
\hline$O(2)-C(1)-C(2)$ & $124.73(18)$ & $O(1)-C(1)-C(2)$ & $116.89(17)$ \\
\hline$O(3)-C(2)-C(16)$ & $113.26(14)$ & $O(3)-C(2)-C(1)$ & $105.70(14)$ \\
\hline$C(16)-C(2)-C(1)$ & $111.52(16)$ & $O(3)-C(2)-C(11)$ & $103.10(14)$ \\
\hline$C(16)-C(2)-C(11)$ & $111.67(15)$ & $C(1)-C(2)-C(11)$ & $111.17(15)$ \\
\hline$O(3)-C(3 A)-C(9)$ & $107.56(15)$ & $O(3)-C(3 A)-C(4)$ & $104.79(15)$ \\
\hline$C(9)-C(3 A)-C(4)$ & $102.83(15)$ & $C(5)-C(4)-C(3 A)$ & $113.09(17)$ \\
\hline$C(5)-C(4)-C(6)$ & $106.80(16)$ & $C(3 A)-C(4)-C(6)$ & $111.86(16)$ \\
\hline$C(5)-C(4)-C(11)$ & $113.34(17)$ & $C(3 A)-C(4)-C(11)$ & $91.86(14)$ \\
\hline$C(6)-C(4)-C(11)$ & $119.49(16)$ & $O(1)-C(5)-C(4)$ & $117.22(16)$ \\
\hline$C(7)-C(6)-C(4)$ & $115.93(16)$ & $C(6)-C(7)-C(8)$ & $112.29(17)$ \\
\hline$C(9)-C(8)-C(7)$ & $111.39(16)$ & $O(4)-C(9)-C(8)$ & $107.58(15)$ \\
\hline$O(4)-C(9)-C(3 A)$ & $113.20(16)$ & $C(8)-C(9)-C(3 A)$ & $109.45(16)$ \\
\hline$O(4)-C(9)-C(10)$ & $116.47(15)$ & $c(8)-c(9)-c(10)$ & $110.45(16)$ \\
\hline$C(3 A)-C(9)-C(10)$ & $99.44(14)$ & $C(15)-C(10)-C(11)$ & $110.26(15)$ \\
\hline$C(15)-C(10)-C(9)$ & $119.96(15)$ & $C(11)-C(10)-C(9)$ & $104.14(14)$ \\
\hline$C(12)-C(11)-C(2)$ & $110.46(15)$ & $C(12)-C(11)-C(4)$ & $126.54(16)$ \\
\hline$c(2)-c(11)-c(4)$ & $98.46(15)$ & $C(12)-C(11)-C(10)$ & $110.69(15)$ \\
\hline$C(2)-C(11)-C(10)$ & $105.57(14)$ & $C(4)-C(11)-C(10)$ & $102.93(15)$ \\
\hline$C(11)-C(12)-C(13)$ & $107.10(15)$ & $C(14)-C(13)-C(12)$ & $112.30(15)$ \\
\hline$C(15)-C(14)-C(16)$ & $103.76(15)$ & $C(15)-C(14)-C(13)$ & $109.88(16)$ \\
\hline$C(16)-C(14)-C(13)$ & $110.91(16)$ & $O(5)-C(15)-C(10)$ & $124.22(18)$ \\
\hline$O(5)-C(15)-C(14)$ & $124.46(17)$ & $C(10)-C(15)-C(14)$ & $111.30(16)$ \\
\hline$C(2)-C(16)-C(14)$ & $108.82(15)$ & & \\
\hline
\end{tabular}

Symmetry transfoxmations used to generate equivalent atoms: 
Table 4. Anisotropic displacement parameters $\left[\mathrm{A}^{2} \times 10^{3}\right.$ ] for FPA5S10.

The anisotropic displacement factor exponent takes the form:

$-2 \mathrm{~m}^{2}\left[\left(\mathrm{ha}^{*}\right)^{2} \mathrm{U}_{11}+\ldots+2 \mathrm{hka}{ }^{*} \mathrm{~b}^{*} \mathrm{U}_{12}\right]$

\begin{tabular}{|c|c|c|c|c|c|c|}
\hline & $\mathrm{U} 11$ & $\mathrm{U} 22$ & U33 & U23 & U13 & $\mathrm{U} 12$ \\
\hline$o(1)$ & $34(1)$ & $19(1)$ & $35(1)$ & $-3(1)$ & $11(1)$ & $-2(1)$ \\
\hline$o(2)$ & $36(1)$ & $30(1)$ & $26(1)$ & $-5(1)$ & $2(1)$ & $-11(1)$ \\
\hline$O(3)$ & $16(1)$ & $27(1)$ & $17(1)$ & $O(1)$ & $7(1)$ & $0(1)$ \\
\hline$O(4)$ & $22(1)$ & $31(1)$ & $18(1)$ & $-3(1)$ & $6(1)$ & $7(1)$ \\
\hline$O(5)$ & $20(1)$ & $19(1)$ & $27(1)$ & $-3(1)$ & $7(1)$ & $0(1)$ \\
\hline$c(1)$ & $26(1)$ & $22(1)$ & 25 (I) & $-1(1)$ & $12(1)$ & $-5(1)$ \\
\hline$c(2)$ & $18(1)$ & $20(1)$ & $16(1)$ & $-1(1)$ & $7(1)$ & $-2(1)$ \\
\hline$C(3 A)$ & $18(1)$ & $24(1)$ & $18(1)$ & $6(1)$ & $7(1)$ & $6(1)$ \\
\hline$C(3 B)$ & $18(1)$ & $24(1)$ & $18(1)$ & $6(1)$ & $7(1)$ & $6(1)$ \\
\hline$O(6)$ & $21(4)$ & $21(4)$ & $22(4)$ & $8(3)$ & $12(3)$ & $1(3)$ \\
\hline$C(4)$ & $21(1)$ & $22(1)$ & $20(1)$ & $3(1)$ & $8(1)$ & $4(1)$ \\
\hline$C(5)$ & $33(1)$ & $21(1)$ & $27(1)$ & $4(1)$ & $10(1)$ & $4(1)$ \\
\hline$c(6)$ & $23(1)$ & $28(1)$ & $24(1)$ & $8(1)$ & $9(1)$ & $11(1)$ \\
\hline$C(7)$ & $20(1)$ & $40(1)$ & $19(1)$ & $4(1)$ & $3(1)$ & $9(1)$ \\
\hline$C(8)$ & $20(1)$ & $39(1)$ & $16(1)$ & $O(I)$ & $2(1)$ & $7(1)$ \\
\hline$C(9)$ & $18(1)$ & $26(1)$ & $15(1)$ & $1(1)$ & $4(1)$ & $6(1)$ \\
\hline$C(10)$ & $14(1)$ & $20(1)$ & $16(1)$ & $-1(1)$ & $3(1)$ & $0(1)$ \\
\hline$c(11)$ & $14(1)$ & $18(1)$ & $15(1)$ & $1(1)$ & $5(1)$ & $2(1)$ \\
\hline$C(12)$ & $21(1)$ & $24(1)$ & $21(1)$ & $-1(1)$ & $10(1)$ & $O(1)$ \\
\hline$c(13)$ & $25(1)$ & $28(1)$ & $20(1)$ & $4(1)$ & $11(1)$ & $1(1)$ \\
\hline$C(14)$ & $20(1)$ & $20(1)$ & $16(1)$ & $3(1)$ & $6(1)$ & $1(1)$ \\
\hline$c(15)$ & $13(1)$ & $20(1)$ & $21(1)$ & $2(1)$ & $6(1)$ & $O(1)$ \\
\hline$c(16)$ & $15(1)$ & $23(1)$ & $18(1)$ & $-1(1)$ & $3(1)$ & $O(1)$ \\
\hline
\end{tabular}


Table 5. Hydrogen coordinates $\left(x \quad 10^{4}\right)$ and isotropic displacement parameters $\left(\dot{\AA}^{2} \times 10^{3}\right)$ for FPA5S10.

\begin{tabular}{|c|c|c|c|c|}
\hline & $\mathrm{x}$ & $y$ & $\mathbf{z}$ & $\mathrm{U}(\mathrm{eq})$ \\
\hline $\mathrm{H}(1)$ & $5360(50)$ & $4494(10)$ & $6640(30)$ & $41(7)$ \\
\hline$H(3 A)$ & 6704 & 3317 & 7366 & 24 \\
\hline $\mathrm{H}(6 \mathrm{C})$ & 5649 & 3095 & 7611 & 30 \\
\hline $\mathrm{H}(5 \mathrm{~A})$ & 8732 & 2482 & 4778 & 33 \\
\hline $\mathrm{H}(5 \mathrm{~B})$ & 7046 & 2494 & 5772 & 33 \\
\hline $\mathrm{H}(6 \mathrm{~A})$ & 10707 & 2867 & 7579 & 30 \\
\hline $\mathrm{H}(6 \mathrm{~B})$ & 11670 & 3029 & 6172 & 30 \\
\hline $\mathrm{H}(7 \mathrm{~A})$ & 12486 & 3869 & 7121 & 33 \\
\hline$H(7 B)$ & 13059 & 3530 & 8778 & 33 \\
\hline$H(8 A)$ & 9905 & 3812 & 9240 & 31 \\
\hline $\mathrm{H}(\mathrm{BB})$ & 10865 & 4343 & 8687 & 31 \\
\hline $\mathrm{H}(10 \mathrm{~A})$ & 10092 & 4261 & 5565 & 21 \\
\hline$H(12 A)$ & 8308 & 3367 & 2156 & 25 \\
\hline $\mathrm{H}(12 \mathrm{~B})$ & 10433 & 3698 & 3275 & 25 \\
\hline $\mathrm{H}(13 \mathrm{~A})$ & 9102 & 4449 & 1907 & 28 \\
\hline $\mathrm{H}(13 \mathrm{~B})$ & 7114 & 4108 & 682 & 28 \\
\hline $\mathrm{H}(14 \mathrm{~A})$ & 5558 & 4823 & 1778 & 23 \\
\hline $\mathrm{H}(16 \mathrm{~A})$ & 3296 & 4314 & 2995 & 23 \\
\hline $\mathrm{H}(16 \mathrm{~B})$ & 3436 & 4002 & 1416 & 23 \\
\hline
\end{tabular}


Table 6. Hydrogen bonds for FPA5S10 [Å and $\left.{ }^{\circ}\right]$.

\begin{tabular}{lllll}
\hline D-H...A & $d(D-H)$ & $d(H \ldots A)$ & $d(D \ldots A)$ & $<(D H A)$ \\
$O(4)-H(1) \ldots O(5) \# 1$ & $0.92(3)$ & $1.98(3)$ & $2.861(2)$ & $160(2)$ \\
\hline
\end{tabular}

Symmetry transformations used to generate equivalent atoms:

$\# 1-x+1,-y+1,-z+1$ 


\section{Chapter 4 Total Synthesis of Maoecrystal V}

\subsection{Early Exploration toward the Total Synthesis of Maoecrystal V}

In previous chapter, I described in detail how we developed the chemistry to synthesize the core structure of Maoecrystal $\mathrm{V}$ with the appropriate relative stereochemistry. In this chapter, I'd like to talk about the chemistry we developed to achieve the total synthesis of Maoecrystal V.
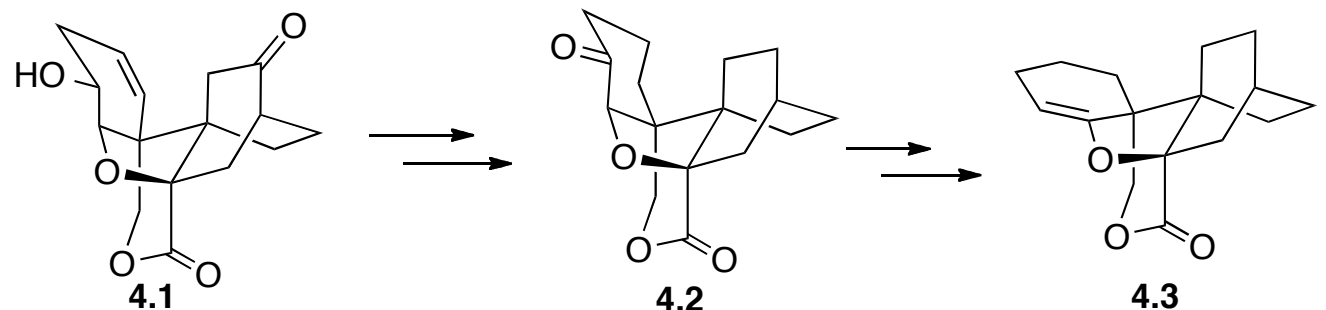

4.2

4.3
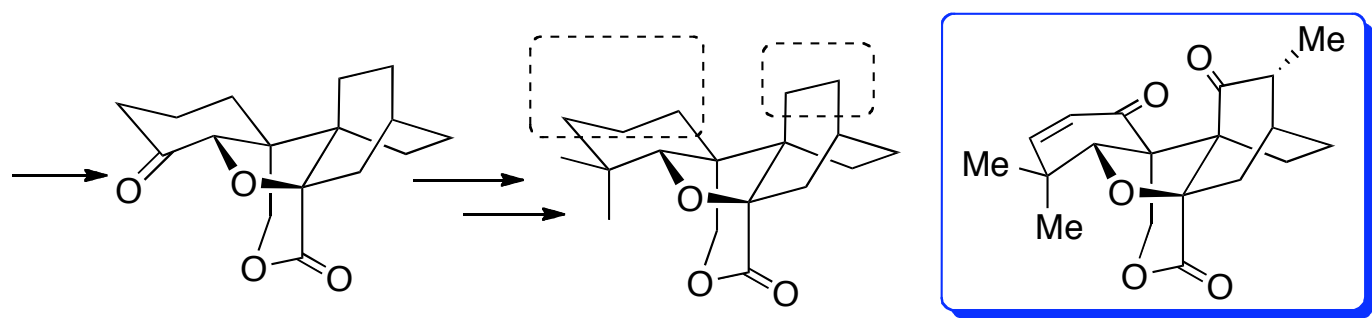

4.4

4.5

Scheme 4-1 Summary of model study toward Maoecrystal V

By a simple comparison of intermediate 4.5 and Maoecrystal V (Scheme 4-1), we realized that the chemistry required for a total synthesis of Maoecrystal V is to synthesize A-ring enone and the $\alpha$-methyl ketone which was corresponding $\mathrm{C}-15$ and $\mathrm{C}-16$. To be 
more specific, we needed to develop sequences to achieve the following transformation (Scheme 4-2).<smiles>O=C1CCCCC1</smiles>

4.6
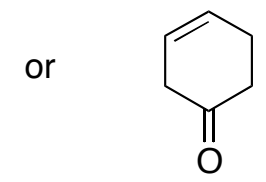

4.7

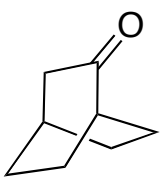

4.9
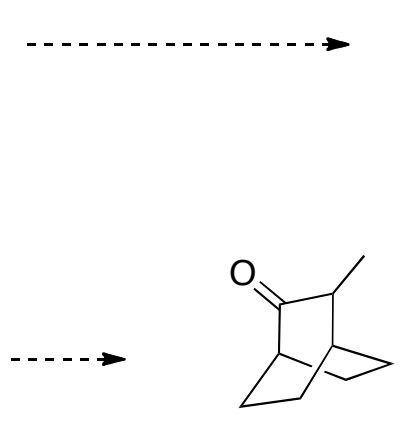

4.10

Scheme 4-2 Functional Group transformations required for the total synthesis

We first explored the chemistry to synthesize the A-ring enone moiety. Our first plan was shown in Scheme 4-3, in which we wanted to start with a C-4 ketone function group. Olefination of ketone 4.11 would afford us an exo-methylene 4.12, which upon

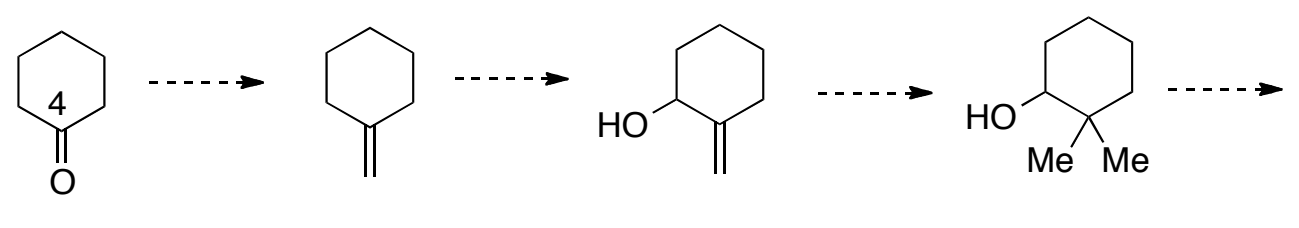
4.11 4.12

4.13

4.14

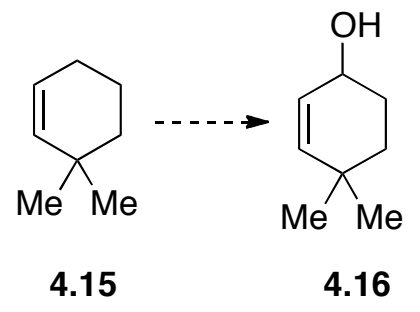

Scheme 4-3 First plan for A ring enone synthesis 
allylic oxidation would provide allylic alcohol 4.13. A cyclopropanation / hydrogenation sequence would furnish gem-dimethyl structure 4.14. Dehydration of $\mathbf{4 . 1 4}$ would give olefin 4.15, which would eventually be transformed to 4.16 by an allylic oxidation. To test this design, we used 4.17 as model compound to try the allylic oxidation first. To our disappointment, the allylic oxidation ${ }^{1}$ did not provide the desired allylic alcohol. Compound 4.18 was isolated instead.

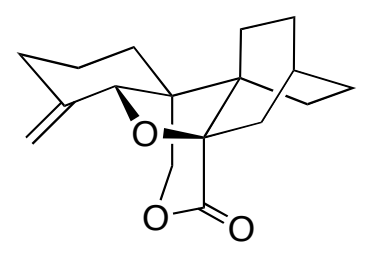

4.17

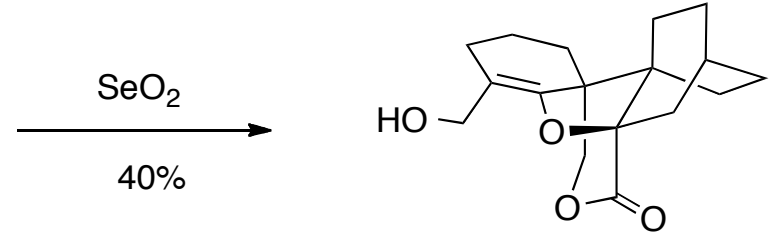

4.18

Scheme 4-4 Allylic oxidation of exo-methylene 4.17

To circumvent this allylic oxidation problem, we then tried a direct $\mathrm{C}-\mathrm{H}$ oxidation $^{2}$ reaction on substrate 4.19 , hoping the cyclopropane ring could activate the $\alpha$ hydrogen. Unfortunately, this reaction only gave starting material.

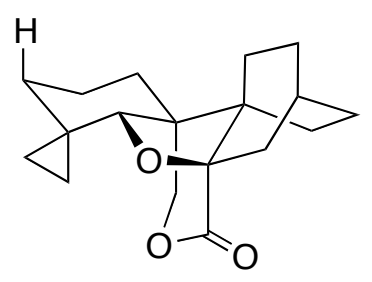

4.19
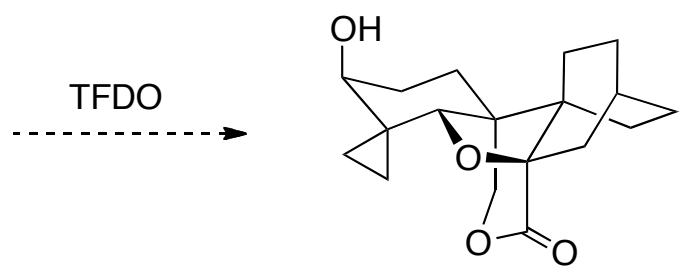

4.20

Scheme 4-5 C-H activation approach 
We then tried installing the of hydroxyl group at an earlier stage, such as substrate 4.21, which required an $\alpha$-hydroxylation reaction of the corresponding ketone 4.21. To our delight, we did obtain the desired $\alpha$-hydroxyl ketone 4.23 in good yield when we subjected ketone 4.21 to Davis oxaziridine reagent 4.22. ${ }^{3}$ Further protection of 4.23 with $\mathrm{MOMCl}$, however, afforded $\mathbf{4 . 2 4}$ in $20 \%$ yield. Optimization of protecting group conditions did not improve the chemical yield.

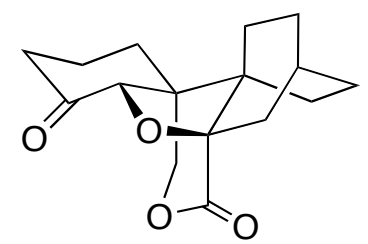

4.21

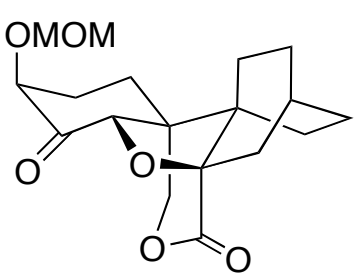

4.24

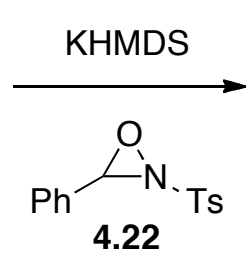

$60 \%$

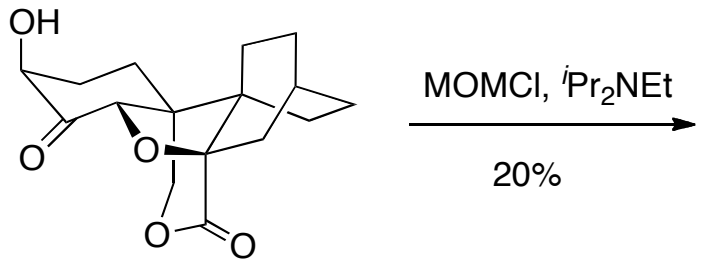

4.23

Scheme 4-6 $\alpha$-Hydroxylation of ketone 4.21

Since we met problem to prepare compounds with 4.14-type structures, we then changed our design. The new design (Scheme 4-7) featured a deconjugated alkylation reaction with compound $4.27{ }^{4}$ Compound 4.27 could be synthesized from 4.26 by a direct oxidation reaction. Allylic alcohol $\mathbf{4 . 2 6}$ could be prepared through a base-catalyzed isomerization of epoxide 4.25. We then turned our attention to the isomerization of epoxide 4.30. 


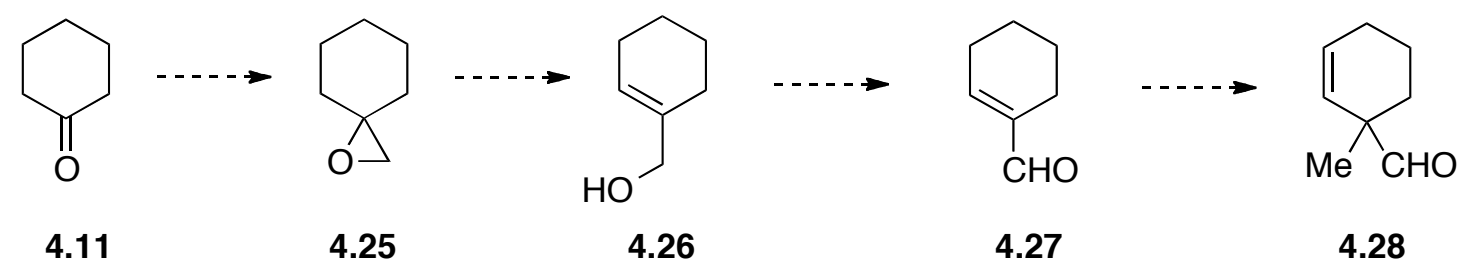

Scheme 4-7 Second plan for A-ring enone synthesis

We screened many conditions and were surprised to find that this epoxide $\mathbf{4 . 3 0}$ was quite inert to the isomerization conditions ${ }^{5}$ and we did not obtain the desired allylic alcohol.

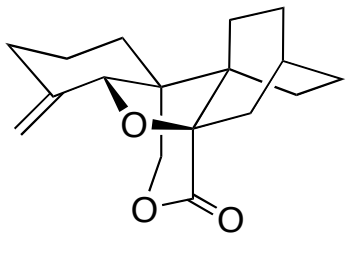

4.29

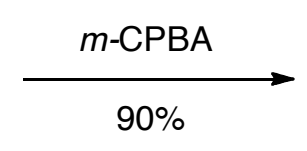

Scheme 4-8 Epoxidation of 4.29

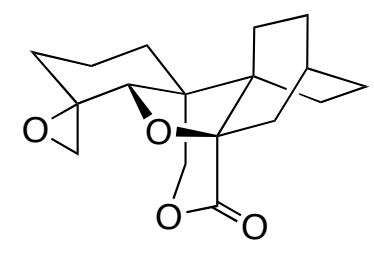

4.30

At this stage, we turned our attention to compound 4.31, in which there was a $\mathrm{C}$ 1-C 2 double bond. We envisioned that we could take advantage of this double bond to install the C-1 oxygen functional group. Based on this hypothesis, we subjected compound 4.31 to an epoxidation reaction using $m$-CPBA as oxidative reagent. Although the reaction rate was quite slow, we did obtain the desired epoxide 4.32 as a single diastereomer in excellent yield. We then treated this epoxide with DMP reagent through 
which we hoped to get a $\beta$-epoxide ketone. To our delight, we actually isolated $\gamma$ hydroxyl enone 4.33, a little bit in low yield. A side product, dienone 4.34, was also isolated. We rationalized that the DMP oxidation reaction condition was a little bit acidic, which isomerized the $\beta$-epoxide ketone to the $\gamma$-hydroxyl enone 4.33 and further oxidation of 4.33 provided us the dienone 4.34. In order to increase the yield of this $\gamma$ hydroxyl enone 4.33 , we used a mild oxidation condition $\left(0{ }^{\circ} \mathrm{C}, \mathrm{NaHCO}_{3}\right.$ buffer $)$, which did increase the yield of $\gamma$-hydroxyl enone $\mathbf{4 . 3 3}$ to $85 \%$.

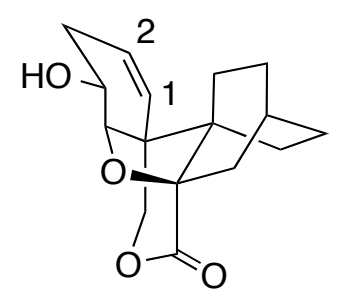

4.31

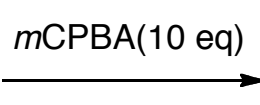

$\mathrm{rt}, 7 \mathrm{~d}, 95 \%$
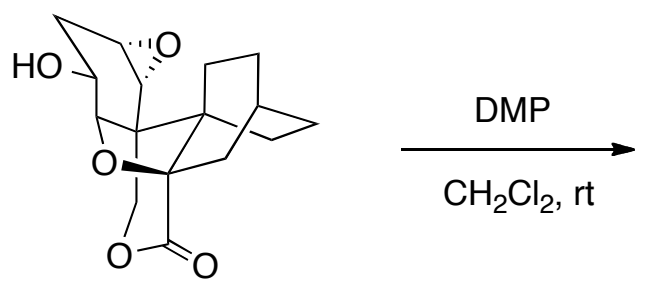

4.33

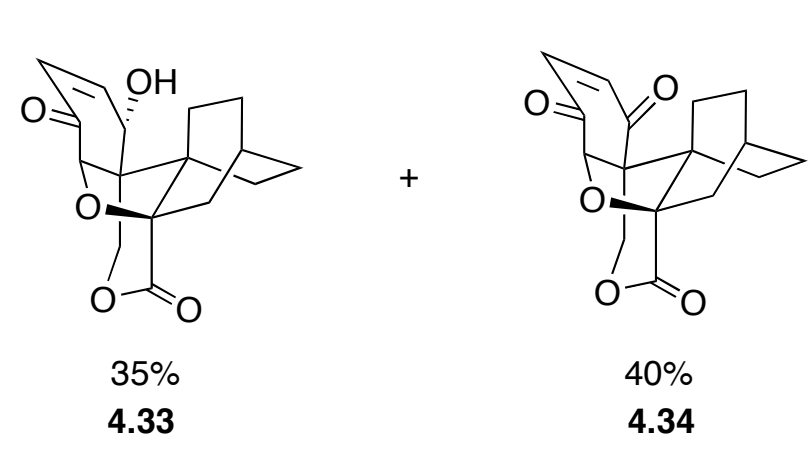

4.32 did inctease the yield of $\gamma$-hydroxyl enone 4.33 to $85 \%$

Scheme 4-9 Epoxidation of 4.31

With this $\gamma$-hydroxyl enone $\mathbf{4 . 3 3}$ in hand, we then explored the protecting group on this $\gamma$-hydroxyl group. Initially, we tried MOM ether formation condition and we only recovered the starting material, probably due to the neopentyl character of this alcohol. 
Using acetic anhydride and DMAP condition, we obtained enol acetate 4.35, which indicated the condition is a little bit harsh. Finally, when using acetic anhydride and pyridine condition, we isolated $\mathbf{4 . 3 6}$ in excellent yield.

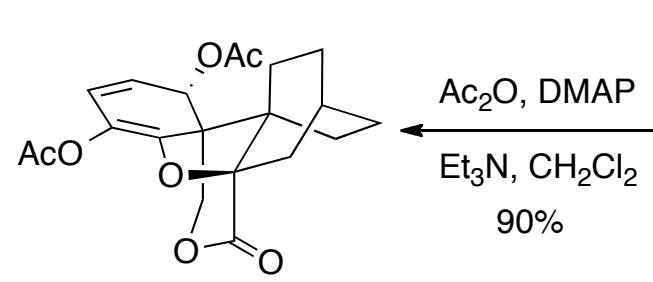

4.35

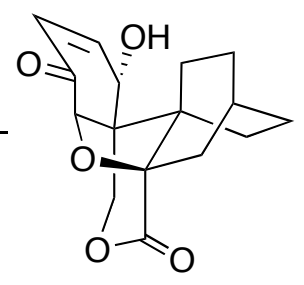

4.33

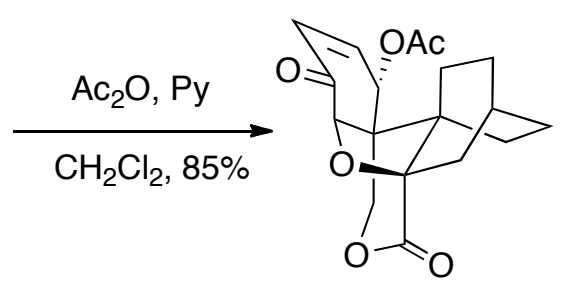

4.36

Scheme 4-10 Protection of $\gamma$-hydroxyl group

We then wanted to apply the chemistry we developed to epimerize the C-5 stereogenic center to compound $\mathbf{4 . 3 6}$, hoping to synthesize compound $\mathbf{4 . 3 8}$. To synthesize the junction enol ether $\mathbf{4 . 3 7}$, we wanted to reduce the enone $\mathbf{4 . 3 6}$ to a ketone via a $1,4-$ reduction fashion, which was well-documented using Stryker's reagent. ${ }^{6}$

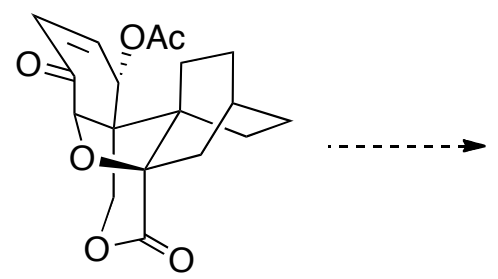

4.36

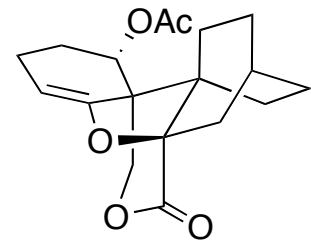

4.37

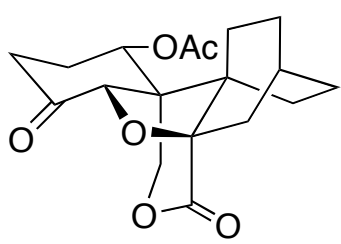

4.38

Scheme 4-11 Third plan for the A ring functionalization 
Herein, we first treated enone 4.36 with Raney-Ni hydrogenation condition, which was reported to reduce the enone double bond. ${ }^{7}$ To our surprise, we obtained a mixture of 4.39 and 4.40 as 3:2 ratio. Since these two compounds were not separable, we still needed to develop a clean sequence to synthesize the dehydration precursor 4.39.

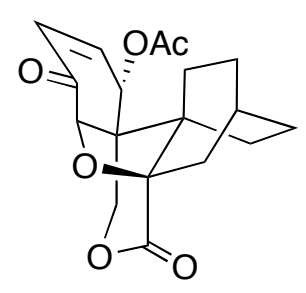

4.36

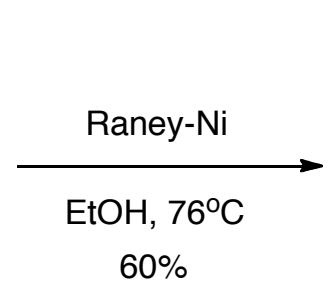

$60 \%$

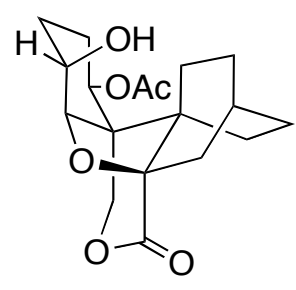

4.39

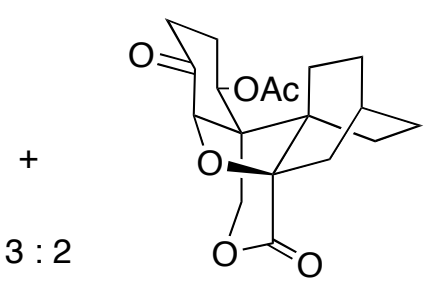

4.40

Scheme 4-12 Raney-Ni reduction of enone 4.36

Instead of reduction of the double bond in compound 4.36, we also considered temporary masking this enone olefin functional group, which would regenerate the double bond after we epimerized the $\mathrm{C}-5$ stereocenter. ${ }^{8}$ We then thought of using a sulfur atom to achieve this goal. Thus, we subjected compound $\mathbf{4 . 3 6}$ to $\mathrm{PhSH}$ condition, which afforded us the desired 1,4-addition product 4.41. Without isolation of this unstable ketone 4.41, we treated the reaction mixture with $\mathrm{EtOH}$ and $\mathrm{NaBH}_{4}$ and we eventually isolated compound 4.42 in excellent yield. With compound 4.42 in hand, we treated it with the dehydration condition, hoping to synthesize the junction enol ether. To our disappointment, we only observed the decomposition of material. Subsequently, we subjected 4.42 to Raney-Ni, which provided compound $\mathbf{4 . 4 3}$ in $80 \%$ yield. 


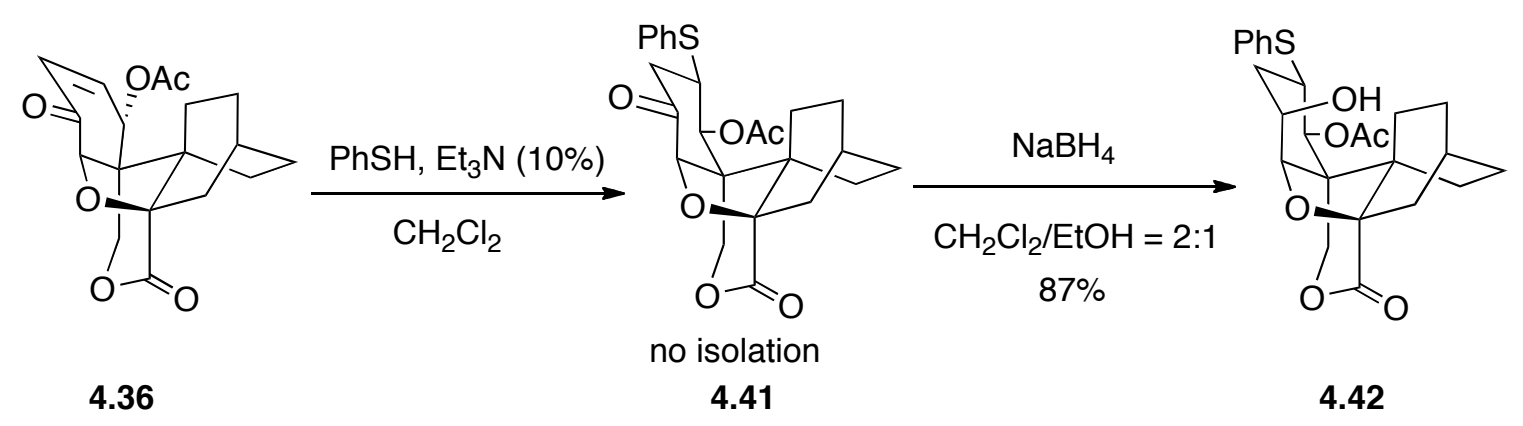

Scheme 4-13 Reduction of enone 4.36

Further dehydration reaction and exo-glycal epoxide rearrangement sequence worked extremely well on compound 4.46 and we isolated compound 4.45 in good yield as a single diastereomer. These results provided us with important information that we could take advantage of the C 1-C 2 double bond to install oxygen function group on C-1.

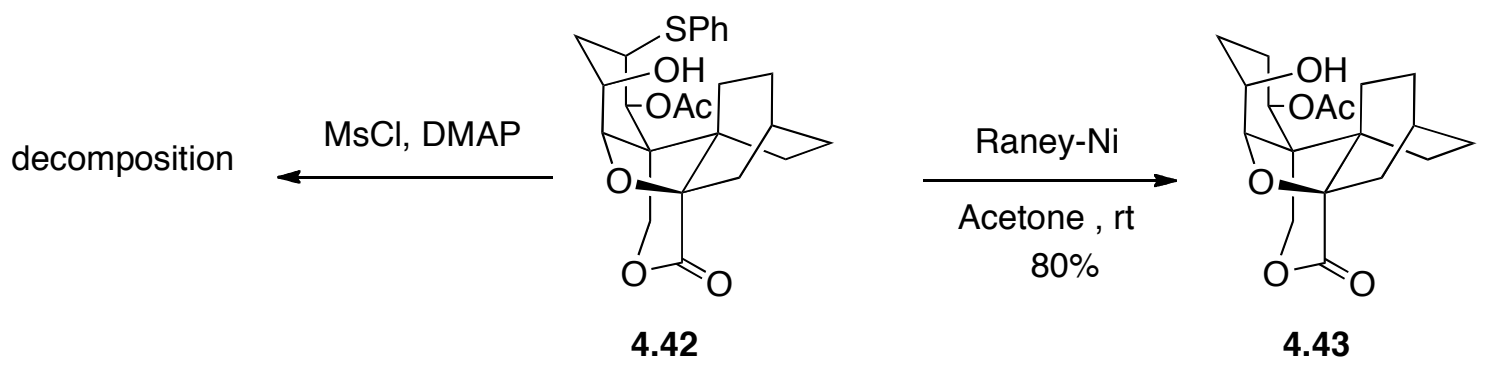

Scheme 4-14 Hydrodesulfurization of 4.42 


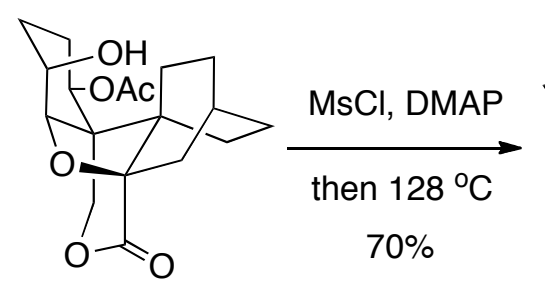

4.43

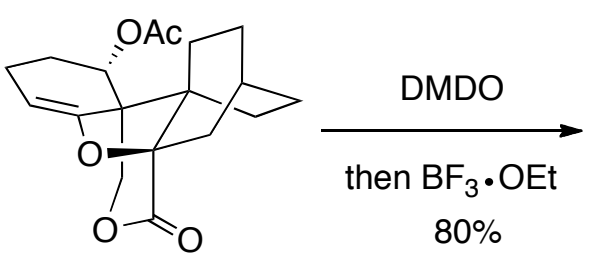

4.44

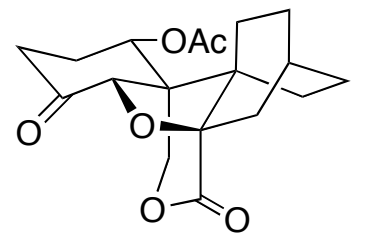

4.45

Scheme 4-15 Exo-gycal epoxide rearrangement of 4.40

Based on the above results, we envisioned that the A ring enone could be prepared through ketone $\mathbf{4 . 4 6}$ through a Saegusa oxidation protocol ${ }^{9}$ (Scheme 4-16).

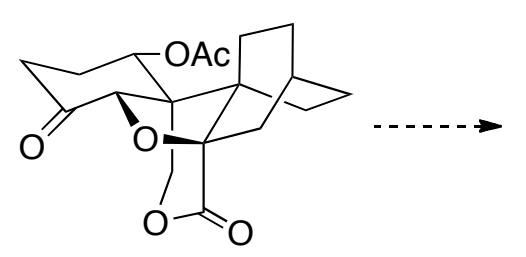

4.45

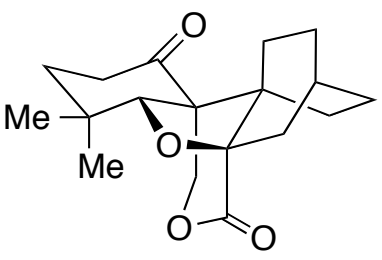

4.46

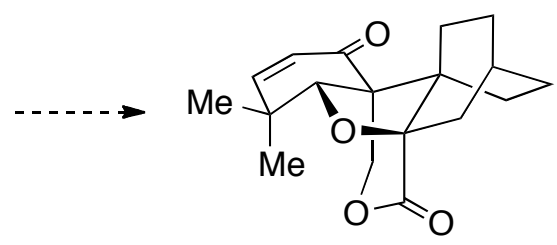

4.47

Scheme 4-16 Third plan for A-ring enone synthesis

We did not further explore how to transform compound 4.45 to the enone 4.47, instead, we were planning the chemistry of C 15 and C 16 so it could be compatible with the A ring functionalization during our total synthesis. Considering we used hydrogenation and $\mathrm{NaBH}_{4}$ reduction, we thought the function group we put on $\mathrm{C}-16$ should tolerate these conditions and an ether function group such as 4.48 or 4.49 might be our choice. 


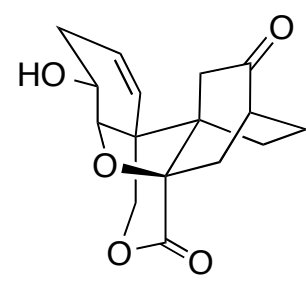

4.1

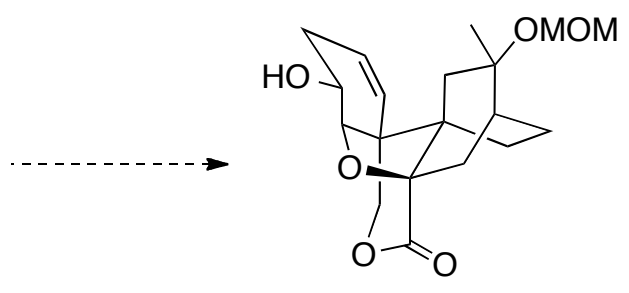

4.48

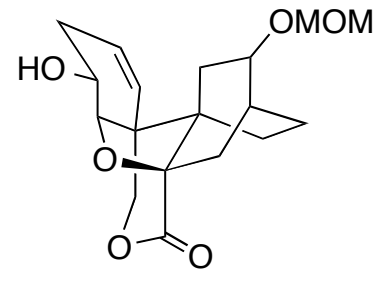

4.49

Scheme 4-17 Functionalization of C 16

The preparation of compound 4.50 turned out to be straightforward. We treated compound 4.1 with methyl cerium chloride reagent at $0{ }^{\circ} \mathrm{C}$ and the desired tertiary alcohol 4.50 was isolated in about $80 \%$ yield. The diastereoselectivity of this ketone addition reaction proved to be quite poor, which a 1.2:1 ratio of diastereoisomer 4.50a and 4.50b was isolated. The poor diastereoselectivity might due to the fact that both $\mathrm{C}-14$ and C-12 are methylene groups, which created a similar stereo environment on both side of the C 16 ketone. Nevertheless, these two diastereomers were then subjected to the $m$ CPBA-mediated epoxidation reaction and afforded epoxides $\mathbf{4 . 5 1}$ as diastereomers only at $\mathrm{C}-16$ center, which indicated that the epoxidation only happened at $\alpha$-face of A-ring. Dess-Martin oxidation of the secondary alcohol in the presence of the C-16 tertiary alcohol provided a ketone, which gave the desired $\gamma$-hydroxyl enone 4.52 upon purification with silica gel column. The enone olefin was then reduced to give compound 4.53 via a thiophenol addition/Raney-Ni reduction sequence, which was already discussed in Scheme 4-14. 


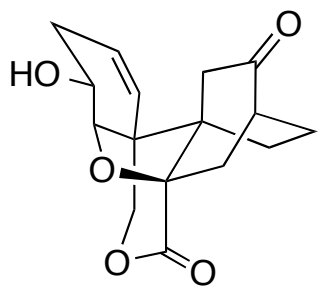

4.1

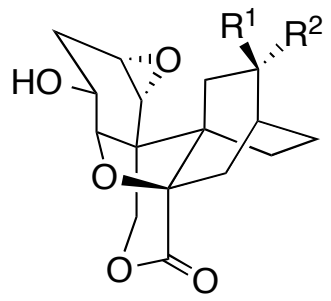

4.51

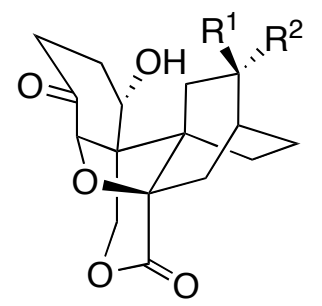

4.53

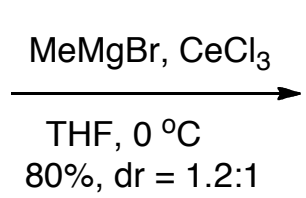

$80 \%, d r=1.2: 1$

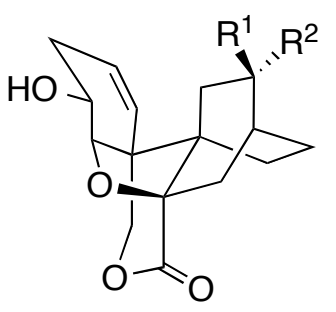

4.50

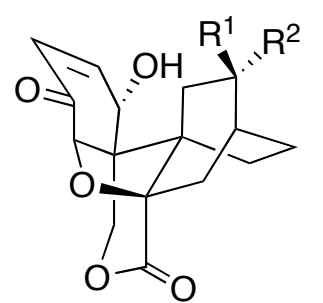

4.52
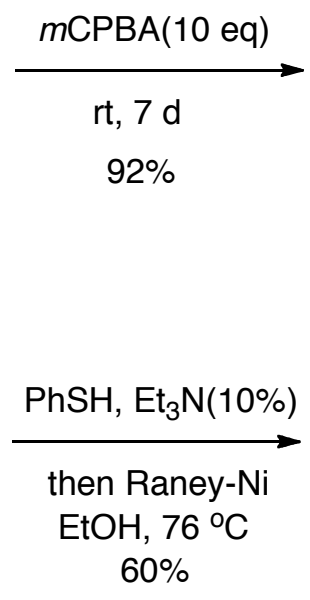

Scheme 4-18 Preparation of 4.53

Now it was a time for us to selectively protect the C-1 and C-16 hydroxyl group. We thought the C-1 hydroxyl group was more hindered since it was next to two quaternary centers. Therefore we envisioned that protection of C 16 hydroxyl group in the presence of C-1 hydroxyl group was possible.

This was indeed the case. Compound 4.53 was subjected to MOM ether protecting condition and afforded us only compound 4.54. Further protection of C-1 hydroxyl group proved to be problematic, which gave the desired compound $\mathbf{4 . 5 5}$ in about $10 \%$ yield. A large amount of material was isolated as decomposition product. 


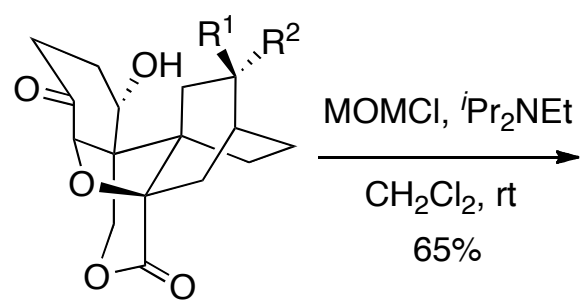

4.53

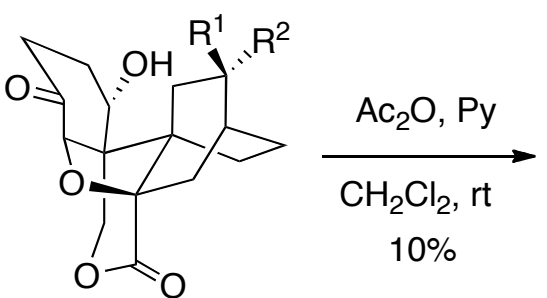

4.54

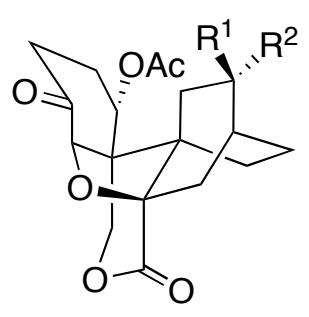

4.55

Scheme 4-19 Protection of $\mathrm{C} 1$ and $\mathrm{C} 16$ alcohol

Since further optimization of this step did not improve the yield, we then turned to compound 4.49, in which C-16 was a secondary alcohol. We hoped that a less hindered C-16 secondary alcohol would facilitate our synthesis. Our synthesis of compound $\mathbf{4 . 4 9}$ started with an alcohol protection of compound 4.1. The resultant ketone was then subjected to a $\mathrm{NaBH}_{4}$ reduction condition and gave compound 4.56 in about $85 \%$. The $\mathrm{NaBH}_{4}$ reduction proved to proceed without stereoselectivity, since compound 4.56 was isolated as a 1:1 diastereomers. Since in our synthesis plan, these secondary alcohols would eventually undergo oxidation reaction to regenerate a ketone, the optimization of the diastereoselective reduction of this C-16 was not explored. These secondary alcohols were then treated with $\mathrm{MOMCl}$ and the following deprotection of acetyl group furnished compound 4.49 in excellent yield. 


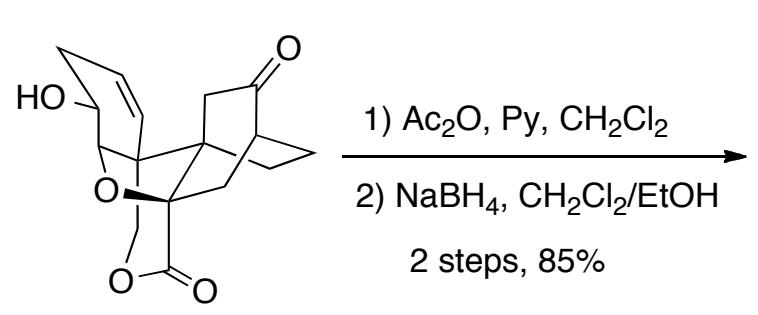

4.1

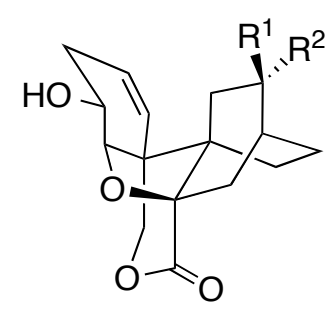

4.49

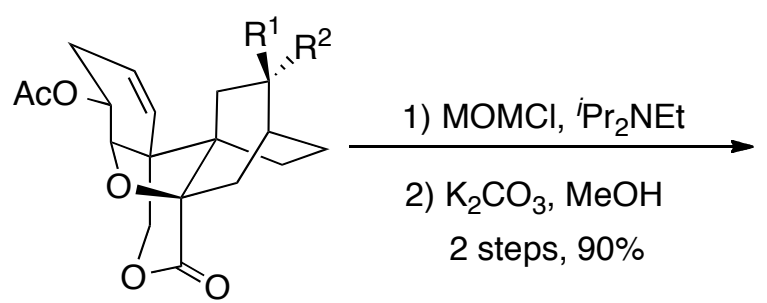

4.56

Scheme 4-20 Preparation of 4.49

With compound 4.49 in hand, we then wanted to test chemistry that we developed to synthesize 4.45 . Thus, compound 4.49 was subjected to $m$-CPBA and gave only $\alpha$ epoxide 4.57 in about $95 \%$ yield. Subsequent Dess-Martin oxidation of the secondary alcohol and upon purification with silica gel column furnished the $\gamma$-hydroxyl enone $\mathbf{4 . 5 8}$ in $85 \%$ yield. The resultant enone $\mathbf{4 . 5 8}$ was then treated with acetic anhydride in the presence of pyridine, which gave the protected enone 4.59 in good yield. A thiophenol addition and the following one-pot $\mathrm{NaBH}_{4}$ reduction furnished alcohol 4.60. The resultant alcohol 4.60 was then subjected to the Raney-Ni and dehydration sequence and afforded the desired junction enol ether $\mathbf{4 . 6 1}$ in good yield. 


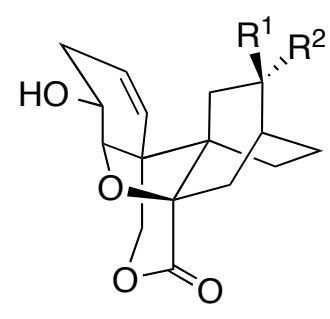

4.49

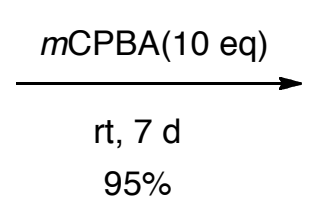

$95 \%$

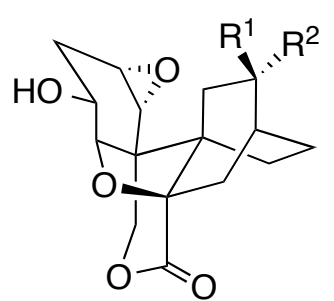

4.57

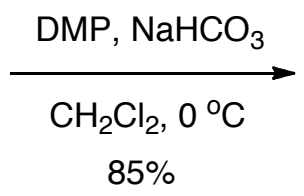

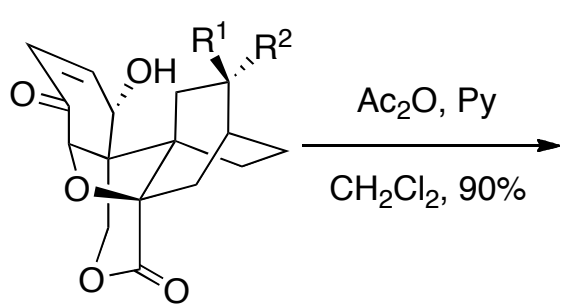

4.58

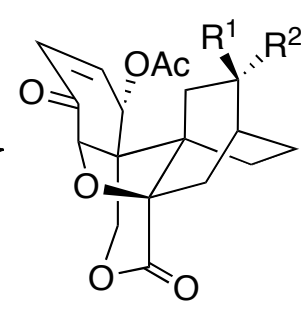

4.59

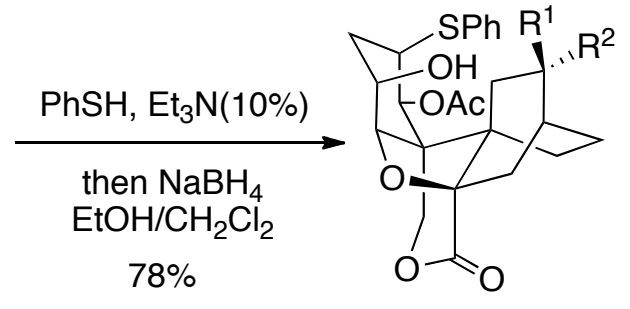

4.60

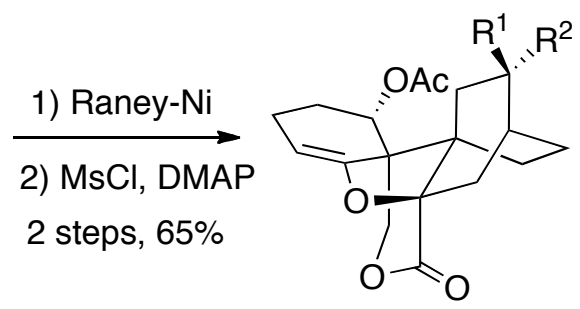

4.61

Scheme 4-21 Preparation of enol ether 4.61

A key exo-glycal epoxide rearrangement reaction was then tested on compound 4.61. To our great happiness, this rearrangement reaction did work on the fully protected compound 4.61 and afforded ketone $\mathbf{4 . 6 2}$ in 78\% yield, in which the C-5 stereochemistry is the same. The rearrangement was stereospecific, and only delivered the hydrogen on $\beta$ face to furnish a trans-5,6-AB ring junction. With these results, we now executed our total synthesis plan. 


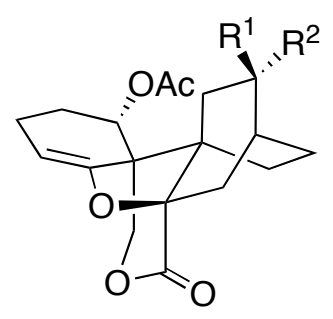

4.61

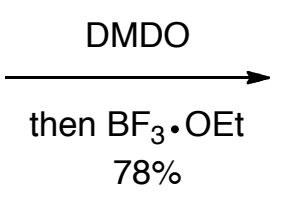

$78 \%$

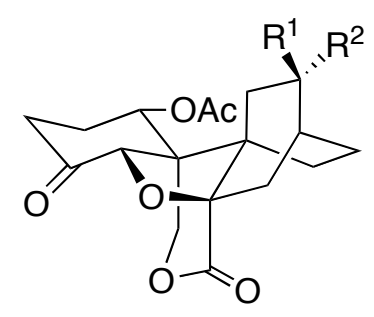

4.62

Scheme 4-22 Exo-glycal epoxide rearrangement

\subsection{Total Synthesis of Maoecrystal V}

After careful exploration of functionalization of A-ring and C 16 carbon, we then started the study toward total synthesis of Maoecrystal V. As shown in Scheme 4-23, our first plan started with compound 4.62, in which the ketone would be used to install gemdimethyl group and provide substrate 4.63. ${ }^{10}$ Cleavage of the MOM ether from the C-16 secondary alcohol and subsequent oxidation of the resultant free alcohol would give ketone 4.64. Olefination of ketone 4.64 would provide exo-methylene 4.65. The C-1 hydroxyl group was then freed and oxidized to ketone 4.66, which upon Saegusa oxidation protocol to give enone 4.67. Isomerization of exo-methylene would afford internal alkene 4.68, which would be transformed to epoxide 4.69. Finally, a key epoxide rearrangement of $\mathbf{4 . 6 9}$ would provide Maoecrystal V. 

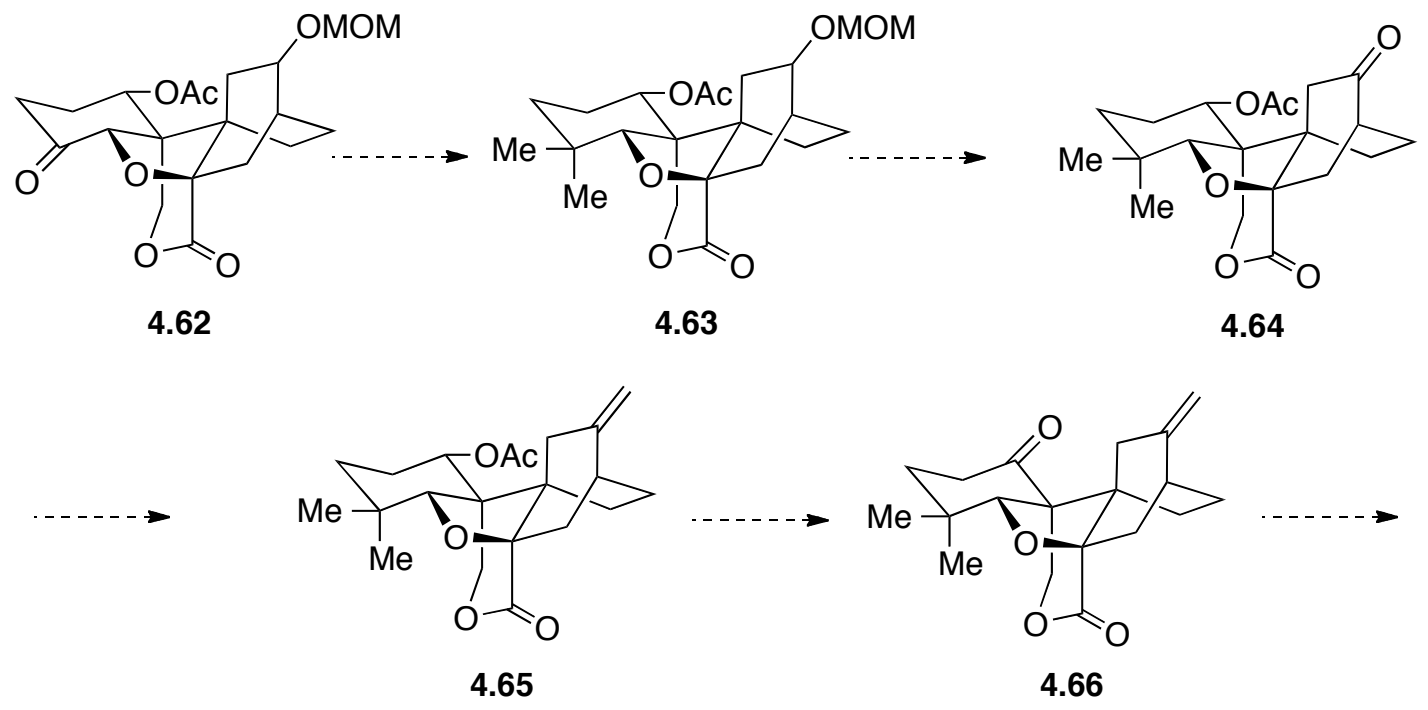

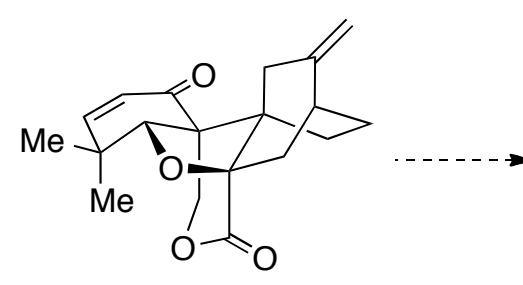

4.67

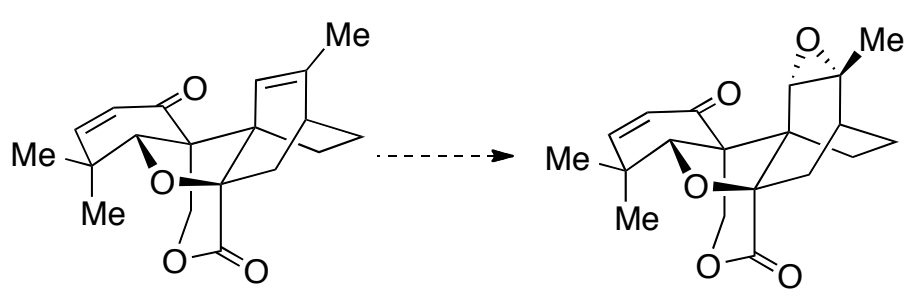

4.68

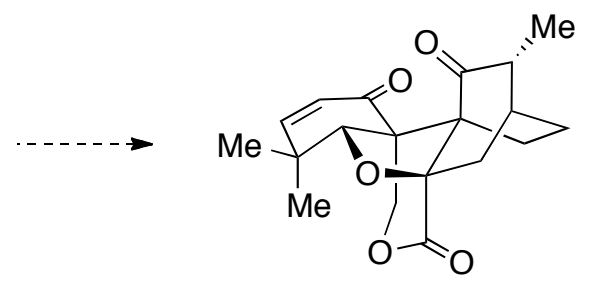

4.70

Scheme 4-23 Total synthesis plan

We then started to prepare compound 4.71, which required an olefination reaction on ketone 4.62. 


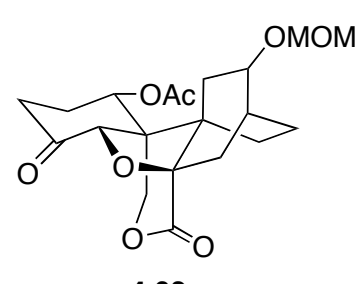

4.62

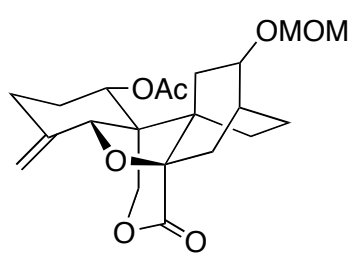

4.71

Scheme 4-24 Preparation of olefin 4.71

To our great surprise, when ketone 4.62 was subjected to Lombardo reagent ${ }^{11}$ for more than 30 mins, we only observed recovery of ketone 4.62. Since we already observed the smooth olefination reaction of compound 3.41, we suspected that our Lombardo reagent turned bad. Three batches of fresh Lombardo reagent were soon prepared, however, no improvement was observed on the conversion of our olefination reaction. We then realized this would be a big problem to our plan. If we could not prepare the exo-methylene 4.71, then we could not prepare the gem-dimethyl compound 4.63 using the chemistry we developed on compound 3.41. At this point, we did not have much choice but to make the olefination reaction work! To optimize this reaction, we first extended the reaction time to $1 \mathrm{~h}$ and also increased the reaction temperature to $40{ }^{\circ} \mathrm{C}$, hoping more harsh conditions would help the conversion of this reaction. To our disappointment, we only observed the decomposition of ketone 4.62 during our close monitoring the reaction process. We then turned our attention to more reactive reagent such as Takai reagent ${ }^{12}$ and Tebbe reagent ${ }^{13}$, however, the reactions using these two reagent only provided ketone $\mathbf{4 . 6 2}$ back. 
Considering the fact the $\mathbf{3 . 4 1}$ reacted smoothly with Lombardo reagent in 5 mins, we thought that compound $\mathbf{4 . 6 2}$ might not be the right substrate for this olefination reaction thought compound 3.41 and compound 4.62 are so close to each other on structure. In order to find the right substrate for the olefination reaction, we first analyzed the reaction mechanism. ${ }^{14}$ The first step of this olefination reaction involved a ketone addition reaction, which would afford a tertiary alkoxide intermedated 4.72. A subsequent 1, 2 elimination reaction would provide the exo-methylene 4.71. The first step probably was a rate-determining step. Since we observed recovery of ketone $\mathbf{4 . 6 2}$ during this reaction, we thought probably the ketone addition reaction did not occur for compound 4.62. The more congested structure of 4.62 might account for the difficulty of this addition reaction because the increased 1,3 diaxial interaction in 4.72a, which caused by an A 1, 2 interaction, might increase the activation energy significantly during the addition process.

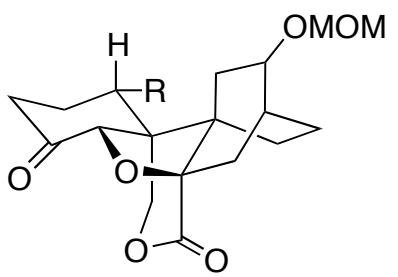

4.62

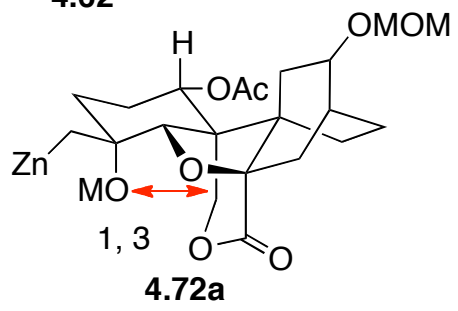

Poor Conversion
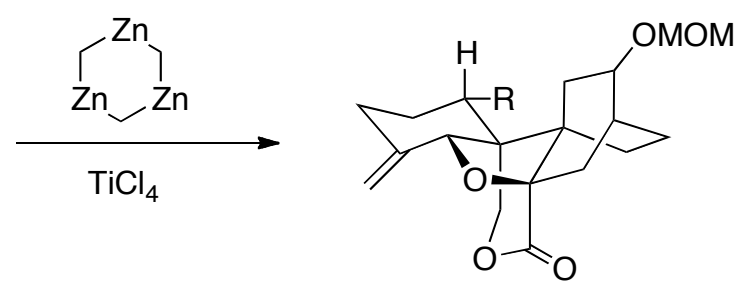

4.71

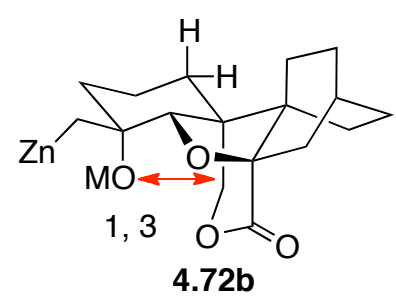

Full Conversion 
This analysis provided us with the hypothesis that a less congested compound such as $\mathbf{4 . 7 3}$ would probably improve the conversion of the olefination reaction. We then decided to prepare compound 4.73. We thought that a simple deprotection of compound 4.62 would afford 4.73. Compound 4.62 was then subjected to $\mathrm{K}_{2} \mathrm{CO}_{3} / \mathrm{MeOH}$ condition and the deprotection process was indeed observed. Unfortunately, the C-5 carbon center was complete epimerized during this reaction. Milder condition such as $\mathrm{KCN} / \mathrm{EtOH}^{15}$ did not provide the desired product.

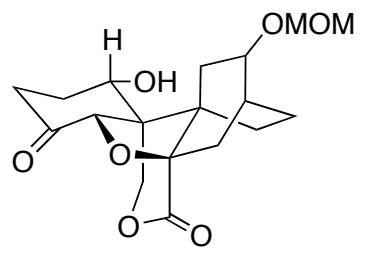

4.73

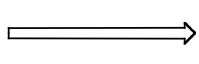

Figure 4-1 Compound 4.73
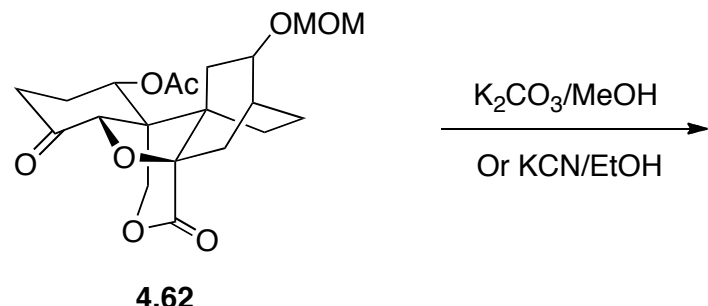

4.62

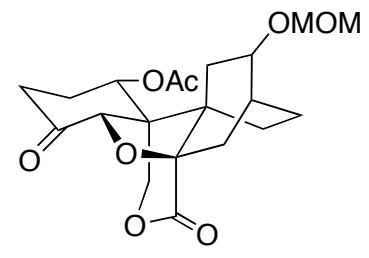

4.62

Scheme 4-26 Deprotection of 4.62

We then thought compound $\mathbf{4 . 7 3}$ might be prepared directly through an exo-glycal epoxide rearrangement. The free alcohol at $\mathrm{C}-1$ might cause problems in this 
rearrangement since there would be proton species present. Nevertheless, we subjected compound 4.75 to the exo-glycal epoxide rearrangement condition and to our delight we did isolate the desired compound $\mathbf{4 . 7 3}$ in about $82 \%$ yield. We observed that the reaction was completed in 10 min, while compound 4.61 took more than 5 hours to complete.

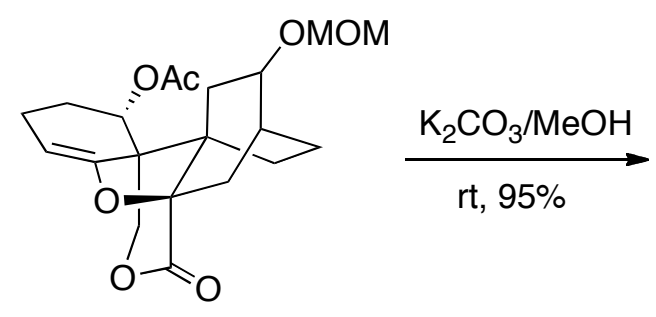

4.61
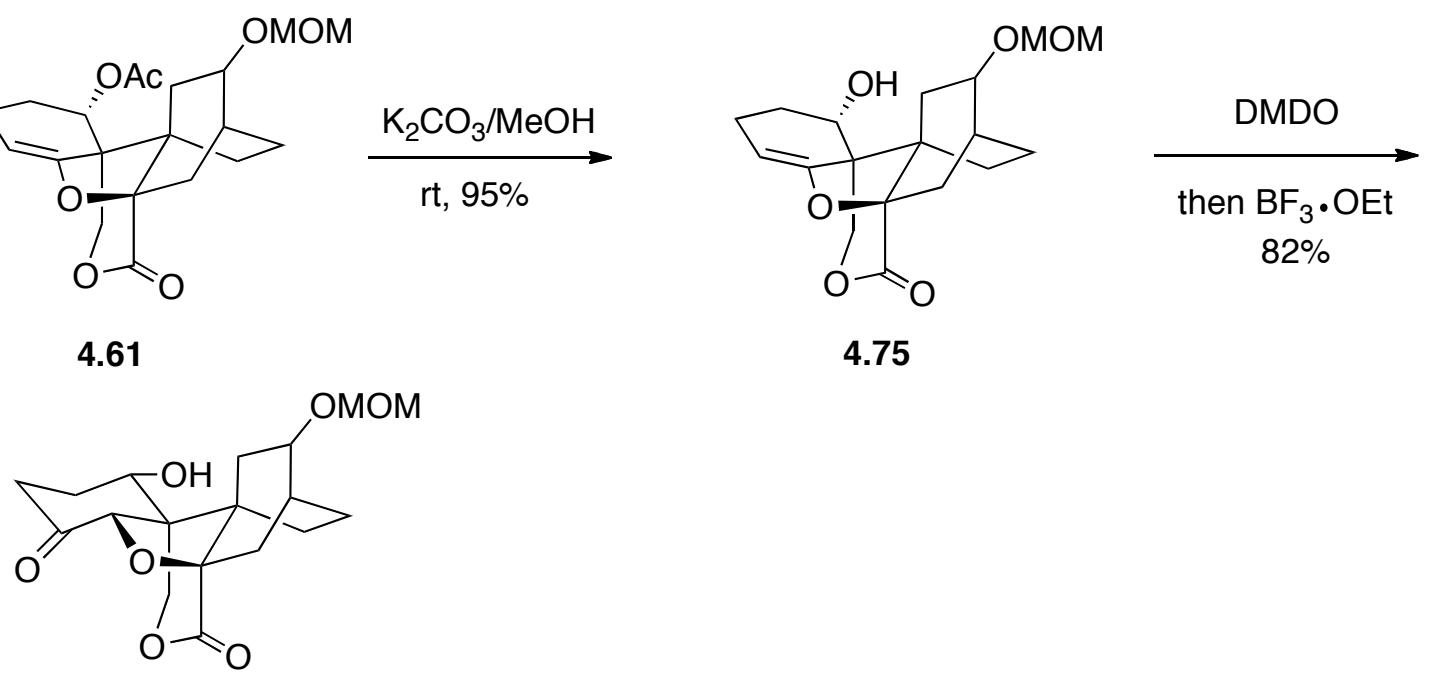

4.75

4.73

Scheme 4-27 Exo-glycal epoxide rearrangement

With compound 4.73 in hand, we did not hesitate to test the olefination reaction. Luckily, with the Lombardo-Takai reagent, the olefination reaction was completed in 8 mins and the exo-methylene was isolated in $85 \%$ yield. 


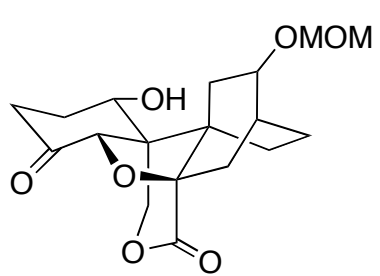

4.76

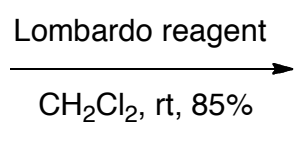

Scheme 4-28 Olefination of $\mathbf{4 . 7 6}$

Cyclopropanation of the exo-methylene in compound 4.77 did not proceed using previously $\mathrm{Cu} / \mathrm{Zn}$ couple condition. After screening other conditions, we were pleased to find that the $\mathrm{Zn} / \mathrm{Ag}^{16}$ couple reacted well with the exo-methylene in compound 4.77 and provided cyclopropane $\mathbf{4 . 7 8}$ in about $88 \%$ yield. We also observed that a carbene insertion to the methyl group of the MOM ether happened, presumably $\mathrm{Zn} / \mathrm{Ag}$ couple is more reactive then $\mathrm{Zn} / \mathrm{Cu}$ couple in the Simmon-Smith cyclopropanation.

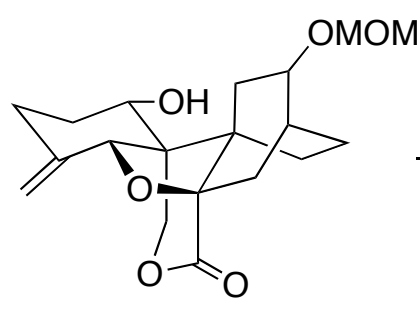

4.77

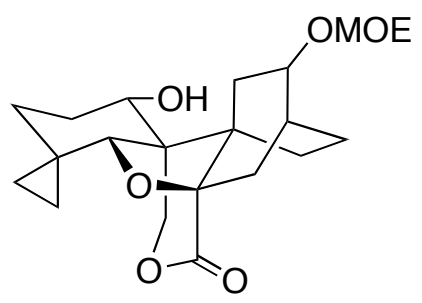

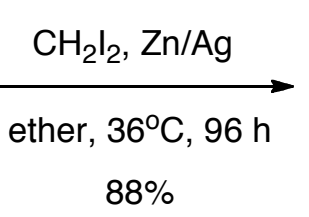

$88 \%$

\subsection{8}

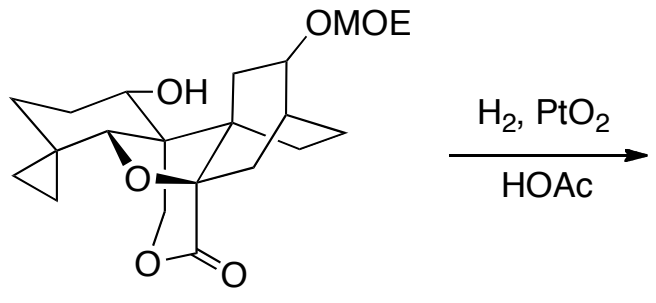

4.78

Scheme 4-29 Cyclopropanation of $\mathbf{4 . 7 7}$ 
The opening of this cyclopropane also turned out to be problematic. With the same hydrogenation condition as compound 3.45, compound 4.78 did not give any conversion during this hydrogenation reaction. Considering the experience we gained on the olefination reaction of compound $\mathbf{4 . 6 2}$, we thought in this case it might be the substrate problem. Since we did not have a clue which substrate was the right one, we prepared several derivatives of compound $\mathbf{4 . 7 8}$ and subjected all these substrate to the hydrogenation conditions. ${ }^{17}$ luckily, we found that compound 4.83 proceeded upon hydrogenation condition. Now, we finally obtained the gem-dimethyl compound $\mathbf{4 . 8 4}$, which took about 8 months for us to find the right conditions to prepare 4.84 from compound 4.49.

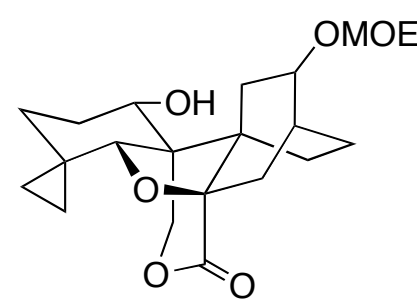

4.78

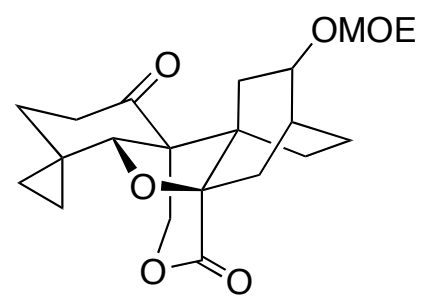

4.81

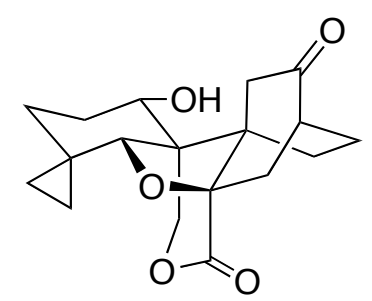

4.79

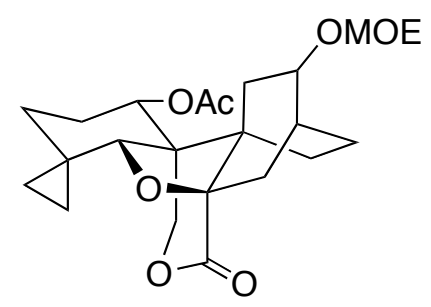

4.82

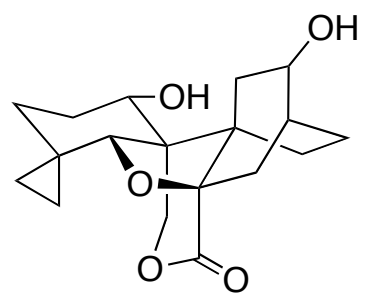

4.80

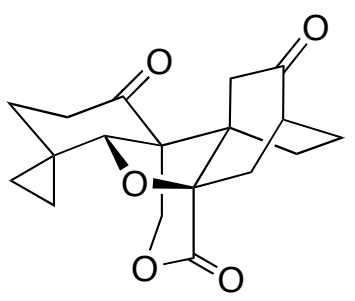

4.83

Figure 4-2 Derivatives of 4.78 


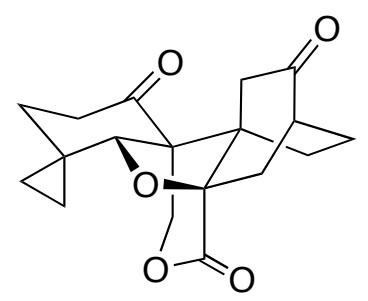

4.83

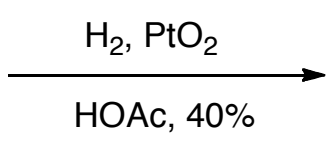

HOAc, $40 \%$

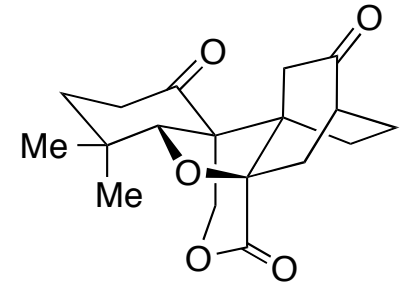

4.84

Scheme 4-30 Hydrogenation of 4.83

With compound 4.84 in hand, we were at the end game stage of the synthesis now. Treatment of compound 4.84 with the Lombardo-Takai reagent at $0{ }^{\circ} \mathrm{C}$, a regio-selective olefination reaction occurred and gave C 16 methylene in about $80 \%$ yield. Subsequent isomerization of the $\mathrm{C}-16$ exo-methylene was achieved upon treatment with $p$ toluenesulfonic acid ${ }^{18}$ and ketone 4.86 was obtained in about $85 \%$ yield.

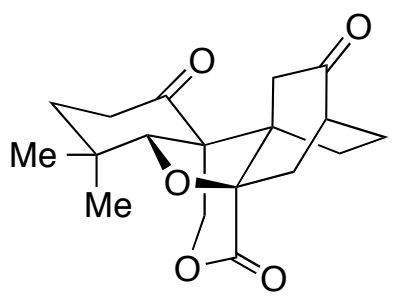

4.84

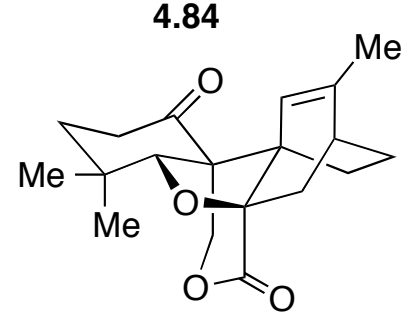

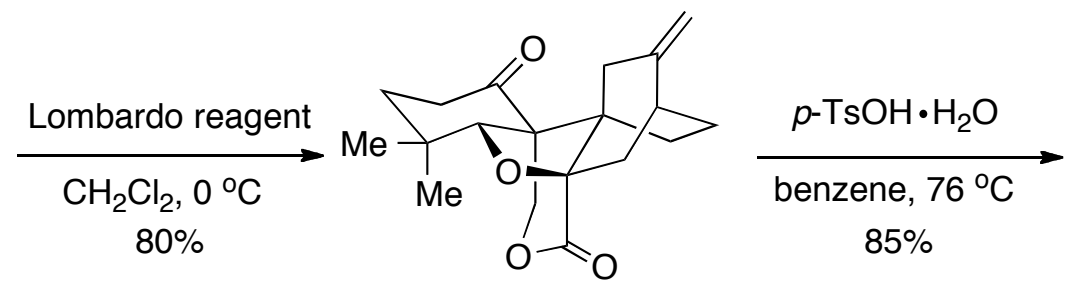

4.85

4.86

Scheme 4-31 Preparation of 4.86 
Dehydrogenation of ketone $\mathbf{4 . 8 6}$ proved to be problematic probably due to the neopentyl character of this ketone. Initially we tried the direct $\alpha$-selenylation of this ketone $^{19}$ and we did not observe the formation of the desired product. We then focused on the preparation of the trimethyl silyl enol ether 4.87, hoping a classic Saegusa type oxidation of this silyl enol ether would afford us the enone 4.88. After screening several silyl enol ether formation conditions, we were pleased to find that under LDA/TMSCl condition, the desire silyl enol ether was obtained in about $90 \%$ yield. This trimethyl silyl enol ether was very stable and could be purified via silica gel column. This silyl enol ether was then subjected to $\mathrm{Pd}(\mathrm{OAc})_{2}$ at $60{ }^{\circ} \mathrm{C}$, a well documented condition for Saegusa oxidation reaction. To our great surprise, we only recovered the silyl enol ether, which indicated we need harsh reaction condition. A systematic screening revealed that $\operatorname{Pd}(\mathrm{TFA})_{2}$ could oxidize this silyl enol ether to enone $\mathbf{4 . 8 8}$ in about $80 \%$ isolated yield.

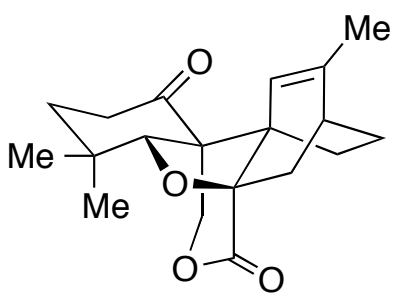

4.86

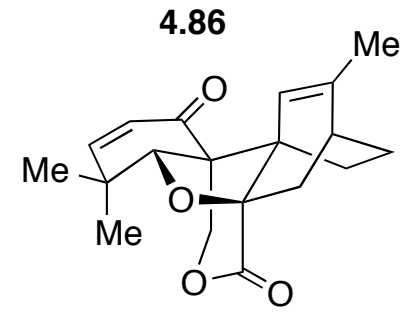

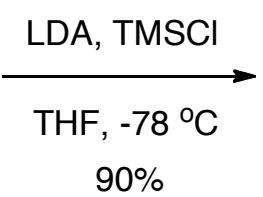

$90 \%$

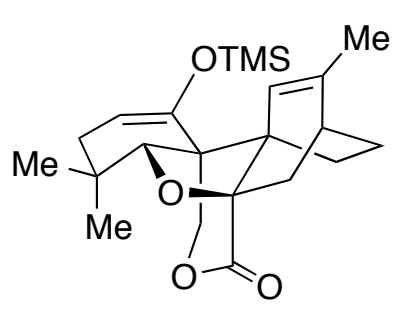

4.87

\subsection{8}


Following epoxidation of $\mathbf{4 . 8 8}$ using TFDO afforded a distereomer mixture $\mathbf{4 . 8 9}$ and 4.90, which could be separated through silica gel chromatography.

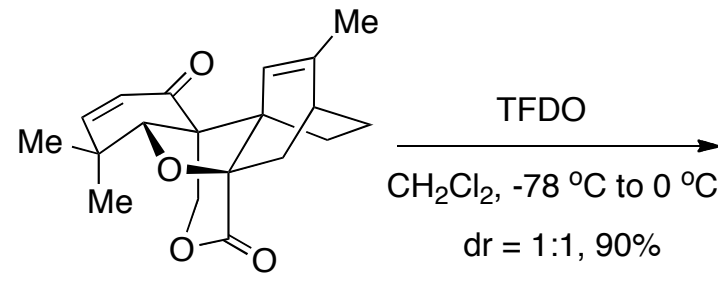

4.88

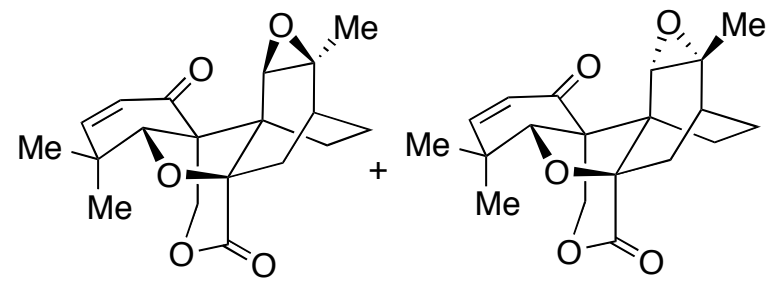

4.89

4.90

\section{Scheme 4-33 Epoxidation of 4.88}

Now the most important moment of Maoecrystal V project arrived. It was May $16^{\text {th }}$, 2012. Compound 4.89 was treated with $\mathrm{BF}_{3}$ etherate for $1 \mathrm{~h}$ and subsequent crude ${ }^{1} \mathrm{H}$ NMR showed that a single product, 16-epi-Maoecrystal V, was obtained. Encouraged by this result, I treated 4.90 with $\mathrm{BF}_{3}$ for $1 \mathrm{~h}$. After purification, Maoecyrstal $\mathrm{V}$ was isolated as a single isomer in excellent yield. The spectrum matched the isolation data.

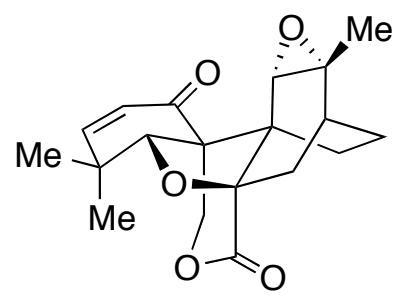

4.90

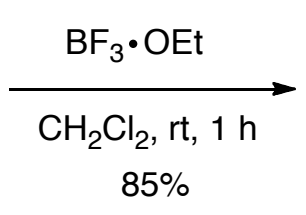

$85 \%$

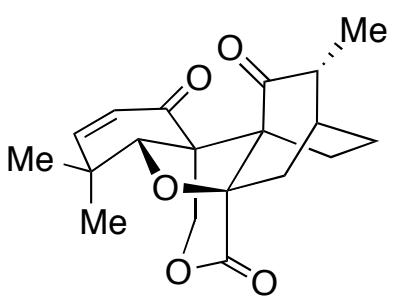

4.91

Scheme 4-34 Total synthesis of Maoecrystal V 


\subsection{Experimental}

\section{General information}

Unless otherwise noted, all reactions were performed under an argon atmosphere using flame-dried glassware. $\mathrm{CH}_{2} \mathrm{Cl}_{2}$ (DCM) was distilled over $\mathrm{CaH}_{2}$ under argon atmosphere. THF was distilled over sodium/benzophenone ketyl under argon atmosphere. Anhyrous toluene, hexane, ether were purchased and use without further purification. All reagents were commercially available and used without further purification unless indicated otherwise. Thin layer chromatography (TLC) was performed on Silica Gel 60 F254 plates and was visualized with UV light and $\mathrm{KMnO}_{4}$ stain. Preparative thin layer chromatography was performed with Merck silica gel 60-F254 coated $0.50 \mathrm{~mm}$ plates. Flash chromatography was performed with Sorbent Tech. silica gel 60. Yields reported are for isolated, spectroscopically pure compounds. NMR spectra were recorded on 300, 400 or $500 \mathrm{MHz}$ instruments. The residual solvent protons $\left({ }^{1} \mathrm{H}\right)$ or the solvent carbons $\left({ }^{13} \mathrm{C}\right)$ were used as internal standards. ${ }^{1} \mathrm{H}$ NMR data are presented as follows: chemical shift in ppm downfield from tetramethylsilane (multiplicity, coupling constant, integration). The following abbreviations are used in reporting NMR data: s, singlet; br s, broad singlet; d, doublet; t, triplet; q, quartet; qt, quartet of triplets; dd, doublet of doublets; $\mathrm{dt}$, doublet of triplets; $\mathrm{AB}, \mathrm{AB}$ quartet; $\mathrm{m}$, multiplet. High-resolution mass spectra were recorded by the Columbia University Mass Spectrometry Core facility on a JEOL HX110 spectrometer. 

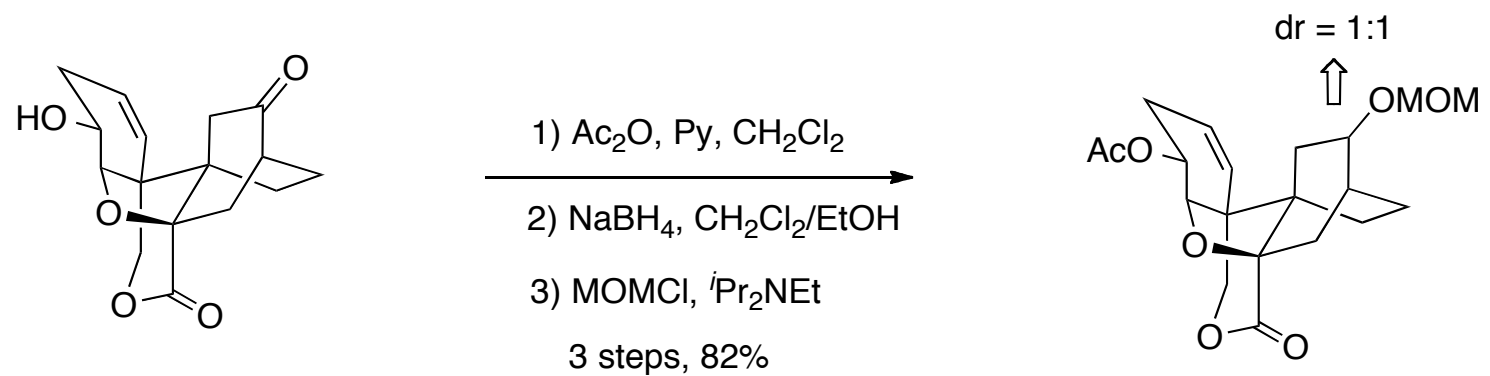

Compound 4.56: To a solution of ketone $4.1(1 \mathrm{~g}, 3.4 \mathrm{mmol})$ in anhydrous $\mathrm{CH}_{2} \mathrm{Cl}_{2}$ was added acetic anhydride $(1.38 \mathrm{~mL}, 13.8 \mathrm{mmol})$, pyridine $(1.2 \mathrm{~mL}, 15 \mathrm{mmol})$ and DMAP (24.4 mg, $0.2 \mathrm{mmol}$ ). The reaction mixture was stirred for $2 \mathrm{~h}$ at room temperature and the solvent was removed under vacuum. The residue was then subjected to flash chromatography purification. The resultant acetate was dissolved in a mixture solvent of $\mathrm{CH}_{2} \mathrm{Cl}_{2} / \mathrm{EtOH}(2: 1)$. To this solution at $-78{ }^{\circ} \mathrm{C}$ was added $\mathrm{NaBH}_{4}$ and the reaction was slowly warmed up to $-20{ }^{\circ} \mathrm{C}$ in $3 \mathrm{~h}$. The reaction was then quenched with acetone and water, extracted with ethyl acetate, and dried over $\mathrm{MgSO}_{4}$. The drying agent was filtered and the solution was concentrated under vacuum. The residue was purified using flash chromatography. The resultant pure secondary alcohol was dissolved in anhydrous $\mathrm{CH}_{2} \mathrm{Cl}_{2}$ and $\mathrm{MOMCl}$ and ${ }^{i} \mathrm{Pr}_{2} \mathrm{NEt}$ was injected. The reaction was stirred at room temperature for $24 \mathrm{~h}$ before quenched with water. The solvent was removed under vacuum and the residue was directly purified via flash chromatography to give a colorless oil. (1.05 g, 82\% yield, 1:1 diastereoisomers). ${ }^{1} \mathrm{H}$ NMR ( $\left.\mathrm{CDCl}_{3}, 400 \mathrm{MHz}\right): \delta 5.87$ (ddd, $J$ $=9.9,6.6,2.0 \mathrm{~Hz}, 1 \mathrm{H}), 5.38(\mathrm{dd}, J=9.9,3.2 \mathrm{~Hz}, 1 \mathrm{H}), 5.10(\mathrm{td}, J=10.1,5.8 \mathrm{~Hz}, 1 \mathrm{H})$, $4.62(\mathrm{~s}, 2 \mathrm{H}), 4.34(\mathrm{~d}, J=10.7 \mathrm{~Hz}, 1 \mathrm{H}), 4.24(\mathrm{~d}, J=9.7 \mathrm{~Hz}, 1 \mathrm{H}), 3.89(\mathrm{~d}, J=10.7 \mathrm{~Hz}$, 2H), $3.35(\mathrm{~s}, 3 \mathrm{H}), 2.80(\mathrm{dd}, J=14.7,5.0 \mathrm{~Hz}, 1 \mathrm{H}), 2.62(\mathrm{dt}, J=16.8,6.2 \mathrm{~Hz}, 1 \mathrm{H}), 2.09(\mathrm{~s}$, 
$3 \mathrm{H}), 2.05-1.90(\mathrm{~m}, 4 \mathrm{H}), 1.77-1.55(\mathrm{~m}, 3 \mathrm{H}), 1.40(\mathrm{dt}, J=14.8,1.9 \mathrm{~Hz}, 1 \mathrm{H}), 1.36-1.24(\mathrm{~m}$, 1H), 1.23-1.18 (m, 1H).; ${ }^{13} \mathrm{C}$ NMR (CDCl $\left.3,75 \mathrm{MHz}\right): \delta 170.7,169.9$, $129.1,122.3,94.8,84.1,76.0,72.9,71.9,55.5,49.8,42.6,33.7,29.8,28.6,28.5,21.2$, 20.9, 17.9; IR (neat): $\mathrm{cm}^{-1}$ 2942, 1745, 1370, 1038; HRMS (FAB, $\mathrm{m} / \mathrm{z}$ ) calcd for $\mathrm{C}_{20} \mathrm{H}_{27} \mathrm{O}_{7}[\mathrm{M}+\mathrm{H}]^{+}$379.1757, found 379.1758.
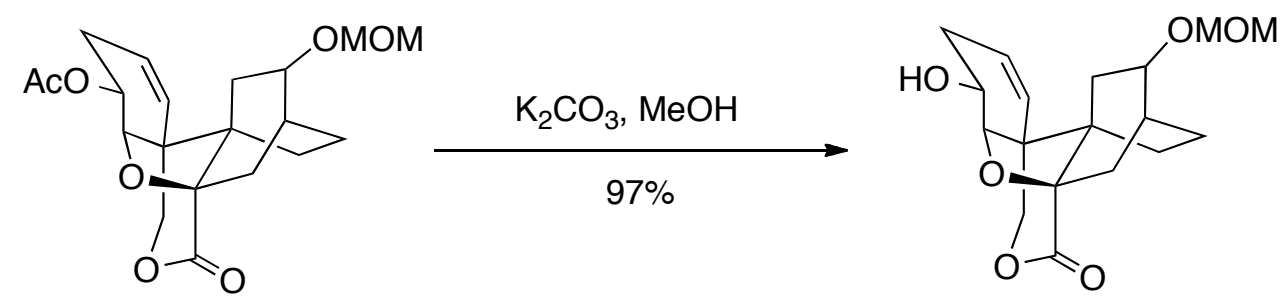

Compound 4.49: To a solution of compound $4.56(1 \mathrm{~g}, 2.6 \mathrm{mmol})$ in $5 \mathrm{~mL}$ of $\mathrm{MeOH}$ was added $20 \mathrm{mg}$ of $\mathrm{K}_{2} \mathrm{CO}_{3}$. The reaction mixture was quenched with water 30 min later and extracted with ethyl acetate. The solvent was removed under vacuum and the residue was purified directly using flash chromatography to afford alcohol $\mathbf{4 . 4 9}$ (845 $\mathrm{mg}, 97 \%$ yield). ${ }^{1} \mathrm{H}$ NMR (CDCl $\left.3,400 \mathrm{MHz}\right): \delta 5.91(\mathrm{ddd}, J=9.9,6.6,2.0 \mathrm{~Hz}, 1 \mathrm{H}), 5.32(\mathrm{dd}, J=9.9,3.1$ Hz, 1H), 4.63 (s, 2H), 4.29 (d, $J=10.7 \mathrm{~Hz}, 1 \mathrm{H}), 4.17-3.99$ (m, 2H), 3.89 (d, $J=10.7 \mathrm{~Hz}$, 1H), 3.74 (ddd, $J=9.6,5.7,2.3 \mathrm{~Hz}, 1 \mathrm{H}), 3.36(\mathrm{~s}, 3 \mathrm{H}), 2.67-2.57$ (m, $1 \mathrm{H}), 2.53-2.38$ (m, 1H), 2.17-1.91 (m, 3H), 1.77-1.42 (m, 7H); $\left.{ }^{13} \mathrm{C} \mathrm{NMR} \mathrm{(} \mathrm{CDCl}_{3}, 75 \mathrm{MHz}\right): \delta 170.1,130.3$, $121.8,94.9,88.2,84.3,77.2,76.3,73.3,69.5,55.4,49.3,42.5,33.4,30.7,30.0,25.3$, 20.9, 20.7; IR (neat): $\mathrm{cm}^{-1} 3500,2970,1750,1030$; HRMS (FAB, $\mathrm{m} / \mathrm{z}$ ) calcd for $\mathrm{C}_{18} \mathrm{H}_{25} \mathrm{O}_{6}[\mathrm{M}+\mathrm{H}]^{+}$337.1651, found 337.1647. 

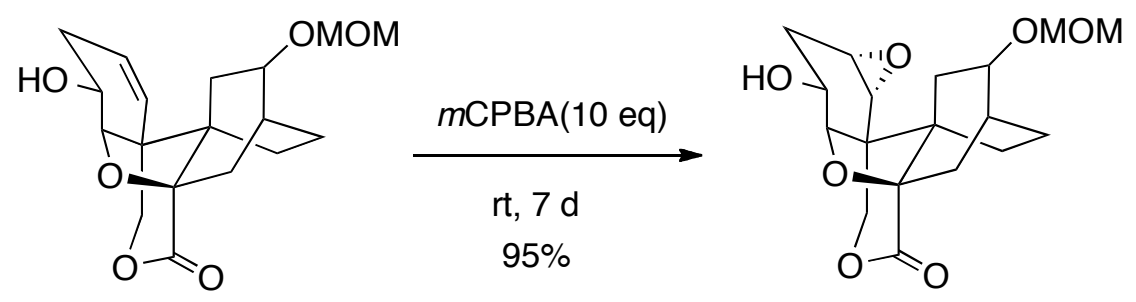

Compound 4.57: To a solution of compound $4.49(700 \mathrm{mg}, 2.1 \mathrm{mmol})$ in anhydrous $\mathrm{CH}_{2} \mathrm{Cl}_{2}$ was added $m$-CPBA $(4.8 \mathrm{~g}, 21 \mathrm{mmol})$. The reaction mixture was stirred at room temperature for about 7 days and nights. Then the solvent was removed under vacuum and the residue was purified via flash chromatography to give epoxide 4.57 (702 mg, 95\% yield). ${ }^{1} \mathrm{H}$ NMR (CDCl3, $\left.400 \mathrm{MHz}\right) \delta 4.71-4.58(\mathrm{~m}, 1 \mathrm{H}), 4.51(\mathrm{~d}, J=11.1 \mathrm{~Hz}, 1 \mathrm{H})$, $4.24(\mathrm{~d}, J=11.1 \mathrm{~Hz}, 1 \mathrm{H}), 4.14-3.99(\mathrm{~m}, 1 \mathrm{H}), 3.85(\mathrm{~d}, J=8.8 \mathrm{~Hz}, 1 \mathrm{H}), 3.81-3.70(\mathrm{~m}, 1 \mathrm{H})$, $3.38(\mathrm{~s}, 3 \mathrm{H}), 3.32$ (ddd, $J=6.1,3.7,1.6 \mathrm{~Hz}, 1 \mathrm{H}), 2.95$ (d, $J=3.7 \mathrm{~Hz}, 1 \mathrm{H}), 2.64$ (ddd, $J=$ 14.3, 4.4, 1.6 Hz, 1H), $2.46(\mathrm{dt}, J=15.2,6.2 \mathrm{~Hz}, 1 \mathrm{H}), 2.33$ (d, $J=26.1 \mathrm{~Hz}, 1 \mathrm{H}), 2.09-$ $1.85(\mathrm{~m}, 3 \mathrm{H}), 1.79-1.62(\mathrm{~m}, 1 \mathrm{H}) ;{ }^{13} \mathrm{C} \mathrm{NMR}\left(\mathrm{CDCl}_{3}, 75 \mathrm{MHz}\right): \delta 169.7,95.1,87.6$, 84.2, 74.6, 73.2, 69.3, 55.5, 51.1, 51.0, 45.3, 42.5, 32.3, 30.5, 29.8, 25.1, 20.9, 20.8; HRMS (FAB, $m / z$ ) calcd for $\mathrm{C}_{18} \mathrm{H}_{25} \mathrm{O}_{7}[\mathrm{M}+\mathrm{H}]^{+} 353.1600$, found 353.1610 .
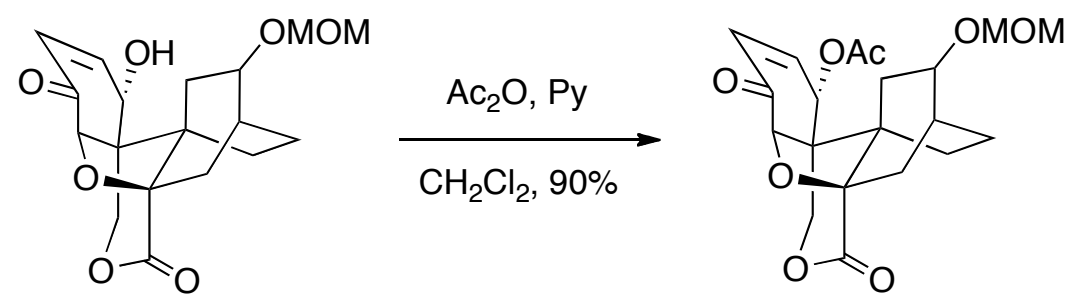
Compound 4.59: To a solution of compound $4.58(590 \mathrm{mg}, 1.7 \mathrm{mmol})$ in anhydrous DCM was added acetic anhydride and pyridine. The reaction mixture was then stirred under argon at room temperature for additional $12 \mathrm{~h}$. The solvent was removed under vacuum and the residue was purified via flash chromatography (600 mg, 90\% yield). ${ }^{1} \mathrm{H}$ NMR (400 MHz, $\left.\mathrm{CDCl}_{3}\right) \delta 6.79(\mathrm{dd}, J=10.3,4.8 \mathrm{~Hz}, 1 \mathrm{H}), 6.32(\mathrm{dd}, J=10.3,1.0 \mathrm{~Hz}$, $1 \mathrm{H}), 5.58(\mathrm{dd}, J=4.8,1.0 \mathrm{~Hz}, 1 \mathrm{H}), 4.60(\mathrm{~d}, J=7.0 \mathrm{~Hz}, 1 \mathrm{H}), 4.56-4.52(\mathrm{~m}, 2 \mathrm{H}), 4.46(\mathrm{~d}, J$ $=11.5 \mathrm{~Hz}, 1 \mathrm{H}), 4.25(\mathrm{~d}, J=11.5 \mathrm{~Hz}, 1 \mathrm{H}), 3.75-3.60(\mathrm{~m}, 1 \mathrm{H}), 3.29(\mathrm{~s}, 3 \mathrm{H}), 2.68(\mathrm{ddd}, J=$ 14.6, 4.7, $1.6 \mathrm{~Hz}, 1 \mathrm{H}), 2.13$ (s, 3H), 2.08-1.93 (m, 2H), 1.77-1.62 (m, 1H), 1.21-1.05 (m, 1H). ${ }^{13} \mathrm{C}$ NMR $\left(101 \mathrm{MHz}, \mathrm{CDCl}_{3}\right) \delta 192.95,169.50,168.86,141.88,131.28,95.37$, $82.68,80.96,73.37,72.61,64.02,55.52,46.85,43.82,33.70,29.87,24.75,20.66,20.56$, 20.03 .
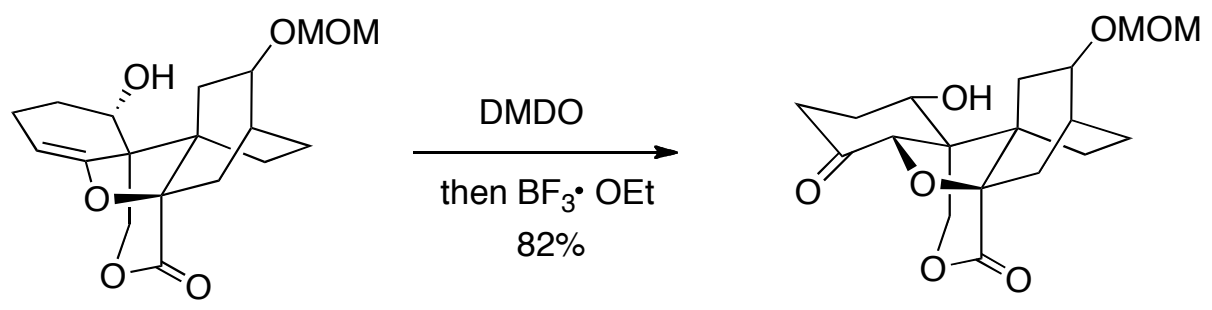

Compound 4.73: At $0{ }^{\circ} \mathrm{C}$, compound $4.75(26 \mathrm{mg}, 100 \mu \mathrm{mol})$ dissolved in anhydrous DCM under argon. $50 \mathrm{mg}$ of $4 \mathrm{~A}$ molecular sieve was added to the solution and the mixture was stirred for 1 hour. Then fresh-prepared dimethyldioxirane in acetone (120 $\mu \mathrm{mol}, 1.2 \mathrm{eq})$ was injected dropwise to the reaction mixture. 10 mins later, the solvent was blowed out using argon flow and $2 \mathrm{~mL}$ of anhydrous ether was injected to the residue. Boron trifluoride etherate ( $30 \mu \mathrm{L}, 3 \mathrm{eq}$ ) was added to the reaction mixture slowly. 15 min later the reaction was quenched with saturated $\mathrm{NaHCO}_{3}$, extracted with 
ethyl acetate, and dried over $\mathrm{MgSO}_{4}$. After filtration of the drying agent, the solvent was removed under reduced pressure and the residue was purified using flash chromatography to afford a white solid (22 mg, 82\% yield). ${ }^{1} \mathrm{H}$ NMR (400 MHz, $\left.\mathrm{CDCl}_{3}\right) \delta 4.71$ (d, $J=2.2$ Hz, 1H), 4.67 (s, 2H), 4.65-4.59 (m, 1H), 4.51 (ddd, $J=14.7,7.1,4.0 \mathrm{~Hz}, 1 \mathrm{H}), 4.20$ (dd, $J=12.2,2.6 \mathrm{~Hz}, 1 \mathrm{H}), 3.87(\mathrm{ddt}, J=9.8,5.9,2.1 \mathrm{~Hz}, 1 \mathrm{H}), 3.38(\mathrm{~s}, 3 \mathrm{H}), 2.70$ (ddd, $J=$ 14.2, 4.7, $1.6 \mathrm{~Hz}, 1 \mathrm{H}), 2.48-2.37$ (m, 2H), 2.32-2.18 (m, 2H), 2.06-1.90 (m, 3H), 1.86$1.62(\mathrm{~m}, 4 \mathrm{H}), 1.53(\mathrm{ddd}, J=9.7,5.2,1.9 \mathrm{~Hz}, 2 \mathrm{H}) ;{ }^{13} \mathrm{C}$ NMR $\left(101 \mathrm{MHz}, \mathrm{CDCl}_{3}\right) \delta$ $203.61,169.18,95.06,85.67,85.12,72.64,68.03,66.68,55.52,52.55,43.85,35.49$, 30.72, 30.24, 24.59, 22.19, 20.92; IR (neat): $\mathrm{cm}^{-1} 3480,2946,1740,1039$; HRMS (FAB, $m / z$ ) calcd for $\mathrm{C}_{18} \mathrm{H}_{25} \mathrm{O}_{7}[\mathrm{M}+\mathrm{H}]^{+} 353.1600$, found 353.1588.
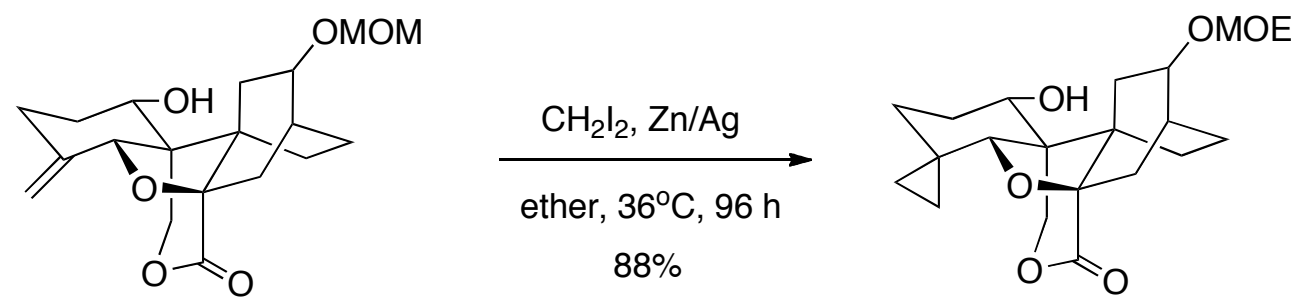

Compound 4.78: To a solution of compound $4.77(40 \mathrm{mg}, 0.11 \mathrm{mmol})$ in anhydrous ether was added $\mathrm{Zn} / \mathrm{Ag}(300 \mathrm{mg}, 4.6 \mathrm{mmol})$ couple. Then a solution of $\mathrm{CH}_{2} \mathrm{I}_{2}(200 \mu \mathrm{L}, 4.2$ mmol) in ether was injected in $1 \mathrm{~h}$ via syringe pump. Then the reaction mixture was stirred at $42{ }^{\circ} \mathrm{C}$ for $96 \mathrm{~h}$. Then the reaction mixture was cooled down to $\mathrm{rt}$ and quenched with $\mathrm{NaHCO} 3$ solution. The mixture was extracted with ethyl acetate and dried over MgSO4. After filtration, the liquid mixture was concentrated under vacuum and the residue was purified via flash chromatography to give cyclopropane $\mathbf{4 . 7 8}$ as an oil (36 $\mathrm{mg}, 88 \%)$. 

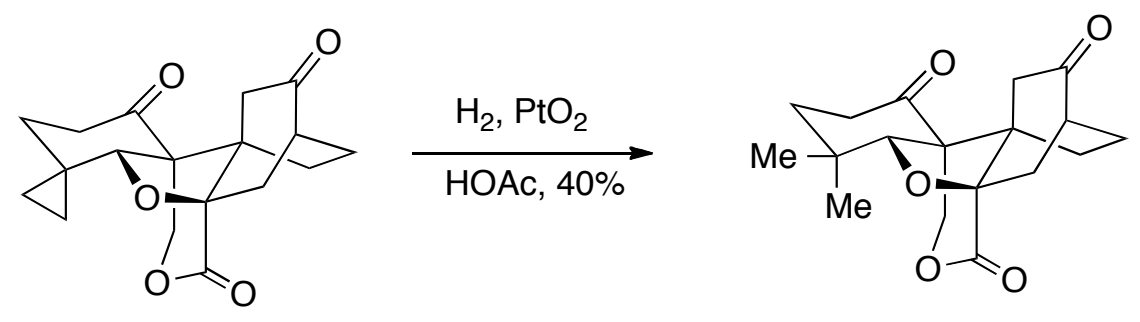

Compound 4.84: To a solution of compound $4.83(10 \mathrm{mg}, 0.027 \mathrm{mmol})$ in acetic acid was added $\mathrm{PtO}_{2}$. The reaction mixture was first degassed with $\mathrm{H}_{2}$ for about 30 min. Then the reaction was stirred at $40{ }^{\circ} \mathrm{C}$ for about 40 mins, filtered, and concentrated. The residue was purified via flash chromatography $(4.1 \mathrm{mg}, 40 \%) .{ }^{1} \mathrm{H}$ NMR $(\mathrm{CDCl} 3,400 \mathrm{MHz}) \delta$ $4.90(\mathrm{~d}, J=12.2 \mathrm{~Hz}, 1 \mathrm{H}), 4.30-4.25(\mathrm{~m}, 2 \mathrm{H}), 3.14(\mathrm{dd}, J=15.0,4.5 \mathrm{~Hz}, 1 \mathrm{H}), 2.63-2.53$ (m, 1H), 2.50-2.34 (m, 4H), 2.06-1.91 (m, 4H), 1.86-1.69 (m, 2H), $1.28(\mathrm{~s}, 3 \mathrm{H}), 1.23(\mathrm{~s}$, $3 \mathrm{H}) ;{ }^{13} \mathrm{C}$ NMR $\left(101 \mathrm{MHz}, \mathrm{CDCl}_{3}\right) \delta 169.4,155.8,94.9,94.8,83.3,72.7,72.2,68.3$, 55.4, 47.7, 42.8, 30. 5, 30.0, 27.5, 24. 2, 21.5, 21. 5, 21.3; HRMS (FAB, $m / z)$ calcd for $\mathrm{C}_{18} \mathrm{H}_{23} \mathrm{O}_{5}[\mathrm{M}+\mathrm{H}]^{+} 319.1545$, found 319.1559 . 

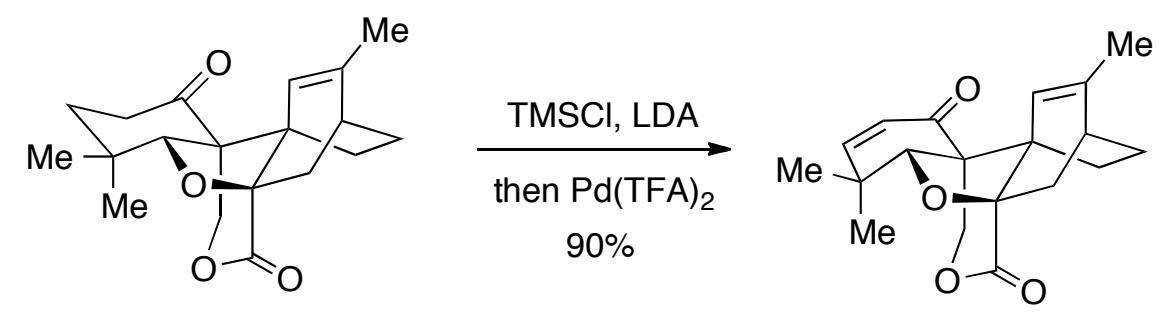

Compound 4.88: To a fresh prepared LDA solution in THF was added a solution of compound $4.86(8 \mathrm{mg}, 0.025 \mathrm{mmol})$ in $\mathrm{THF}$ at $-78{ }^{\circ} \mathrm{C} .1 \mathrm{~h}$ later, $\mathrm{TMSCl}$ was injected via syringe and the reaction mixture was stirred for additional $1 \mathrm{~h}$. Then the reaction mixture was quenched with $\mathrm{NaHCO}_{3}$ solution. The reaction mixture was then extracted with ether and dried over $\mathrm{MgSO}_{4}$. After filtration of the drying agent, the liquid mixture was concentrated and the residue dissolved in anhydrous $\mathrm{CH}_{3} \mathrm{CN}$. $\mathrm{Pd}(\mathrm{TFA})_{2}$ was added and the reaction mixture was then heated to $70{ }^{\circ} \mathrm{C}$ and stirred at this temperature for about 5 h. The solvent was removed via rotovap and the residue was purified via direct flash chromatography to afford enone 4.88 (7.2 $\mathrm{mg}, 90 \%) .{ }^{1} \mathrm{H}$ NMR (400 MHz, CDCl3) $\delta 6.70$ (d, $J=10.1 \mathrm{~Hz}, 1 \mathrm{H}), 5.92(\mathrm{~d}, J=10.1 \mathrm{~Hz}, 1 \mathrm{H}), 5.48(\mathrm{~d}, J=2.0 \mathrm{~Hz}, 1 \mathrm{H}), 4.65(\mathrm{~d}, J=11.8$ Hz, 1H), 4.29 (d, $J=1.5 \mathrm{~Hz}, 1 \mathrm{H}), 4.26(\mathrm{dd}, J=11.8,1.6 \mathrm{~Hz}, 1 \mathrm{H}), 2.79$ (dd, $J=13.8,3.5$ $\mathrm{Hz}, 1 \mathrm{H}), 2.50(\mathrm{dt}, J=3.9,2.0 \mathrm{~Hz}, 1 \mathrm{H}), 2.46-2.28(\mathrm{~m}, 1 \mathrm{H}), 1.84(\mathrm{dd}, J=4.5,1.6 \mathrm{~Hz}, 3 \mathrm{H})$, $1.82-1.75(\mathrm{~m}, 2 \mathrm{H}), 1.50(\mathrm{td}, J=6.2,3.0 \mathrm{~Hz}, 1 \mathrm{H}), 1.34(\mathrm{~s}, 3 \mathrm{H}), 1.27(\mathrm{~s}, 3 \mathrm{H}) ;{ }^{13} \mathrm{C} \mathrm{NMR}$ $\left(101 \mathrm{MHz}, \mathrm{CDCl}_{3}\right) \delta 196.81,170.44,157.72,145.79,126.84,122.47,86.69,86.62$, $68.59,54.42,47.76,38.63,35.65,35.21,30.71,23.14,22.06,19.83,18.59$; IR (neat): $\mathrm{cm}^{-}$ ${ }^{1} 2918,2850,1755,1691,1265,1154$; HRMS $\left(\mathrm{FAB}, \mathrm{m} / \mathrm{z}\right.$ ) calcd for $\mathrm{C}_{19} \mathrm{H}_{22} \mathrm{O}_{4}[\mathrm{M}+\mathrm{H}]^{+}$ 314.1518, found 314.1518. 

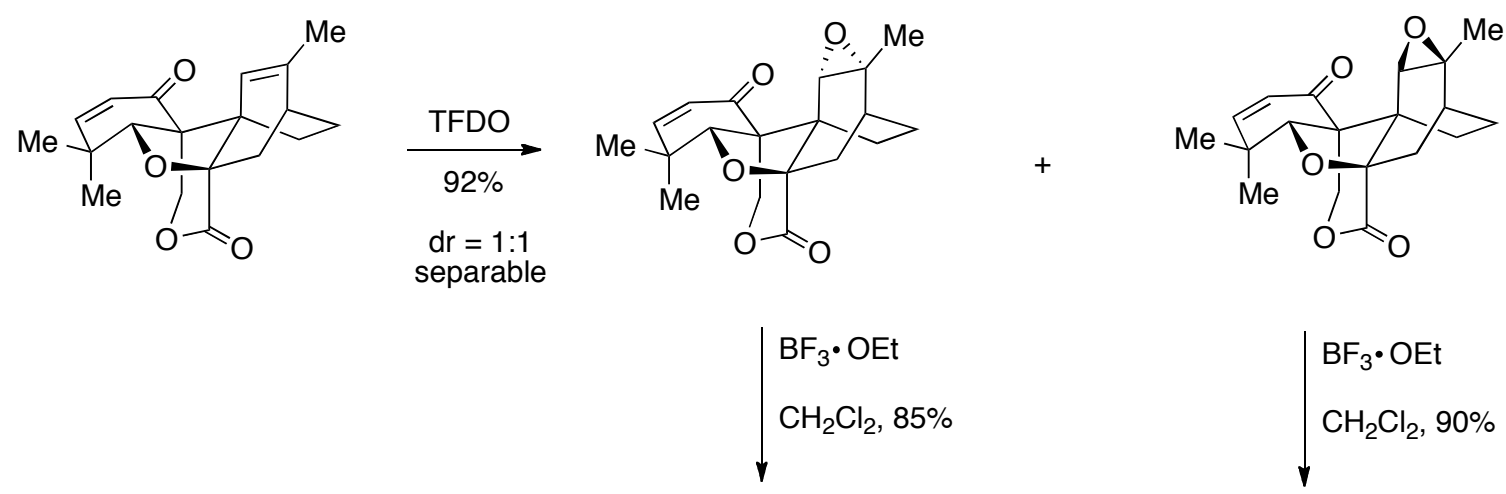

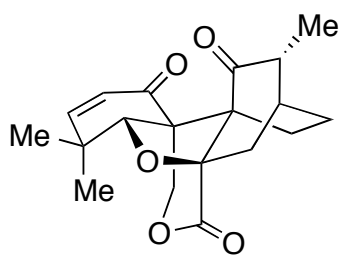

Maoecrystal V

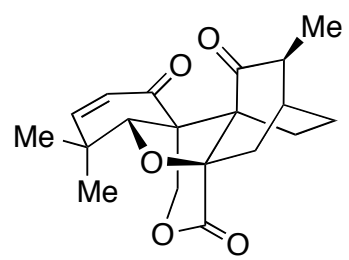

16-epi-Maoecrystal V

Maoecrystal V and 16-epi-maoecrystal V

To a solution of epoxide $\mathrm{XX}$ in $\mathrm{CH}_{2} \mathrm{Cl}_{2}$ at $0{ }^{\circ} \mathrm{C}$ was added molecular sieve. The reaction was stirred at this temperature for additional $1 \mathrm{~h}$. Then $\mathrm{BF}_{3}$ etherate was added. 20 mins later, the reaction temperature was warmed up to room temperature and additional $\mathrm{BF}_{3}$ etherate was added and the reaction was stirred for further $1 \mathrm{~h}$. Then it was quenched with $\mathrm{NaHCO}_{3}$ and extracted with ethyl acetate. The liquid mixture was dried over $\mathrm{MgSO}_{4}$, filtered, concentrated and the residue was purified using preparative TLC.

Maoecrystal V: $2 \mathrm{mg}, 85 \%$ yield. ${ }^{1} \mathrm{H}$ NMR (500 MHz, CDCl3) $\delta 6.66(\mathrm{~d}, J=10.2 \mathrm{~Hz}$, 1H), $5.95(\mathrm{~d}, J=10.2 \mathrm{~Hz}, 1 \mathrm{H}), 4.63(\mathrm{~d}, J=12.2 \mathrm{~Hz}, 1 \mathrm{H}), 4.43(\mathrm{~d}, J=1.6 \mathrm{~Hz}, 1 \mathrm{H}), 4.13$ (dd, $J=12.1,1.7 \mathrm{~Hz}, 1 \mathrm{H}), 3.19(\mathrm{dd}, J=14.6,4.7 \mathrm{~Hz}, 1 \mathrm{H}), 2.42-2.25$ (m, 2H), 2.21-2.06 
(m, 3H), 1.98 (dddd, $J=13.9,11.5,6.1,3.1 \mathrm{~Hz}, 1 \mathrm{H}), 1.70(\mathrm{dt}, J=14.6,2.1 \mathrm{~Hz}, 1 \mathrm{H})$, 1.67-1.61 (m, 1H), 1.30 (s, 3H), $1.25(\mathrm{~d}, J=7.5 \mathrm{~Hz}, 3 \mathrm{H}), 1.23(\mathrm{~s}, 3 \mathrm{H}) ;{ }^{13} \mathrm{C}$ NMR $(101$ $\left.\mathrm{MHz}, \mathrm{CDCl}_{3}\right) \delta 211.5,194.8,169.0,156.7,127.1,84.9,84.1,69.2,56.6,51.9,48.3,38.3$, 34.6, 32.7, 30.6, 18.6, 18.5, 18.0, 15.1; ${ }^{13} \mathrm{C}$ NMR (101 MHz, C5D5N) $\delta 211.8,194.8$, $169.6,156.8,127.3,85.6,84.7,69.6,57.0,52.5,48.4,38.4,34.9,33.0,30.5,30.1,18.8$, 18.4, 18.3, 15.1;IR (neat): $\mathrm{cm}^{-1} 2920,1754,1719,1688,1264,1155$; HRMS (FAB, $\left.m / z\right)$ calcd for $\mathrm{C}_{19} \mathrm{H}_{22} \mathrm{O}_{5}[\mathrm{M}+\mathrm{H}]^{+} 330.1467$, found 330.1472 .

16-epi-Maoecrystal V: $2.8 \mathrm{mg}, 90 \%$ yield. ${ }^{1} \mathrm{H}$ NMR (500 MHz, CDCl3) $\delta 6.66(\mathrm{~d}, J=$ $10.1 \mathrm{~Hz}, 1 \mathrm{H}), 5.94(\mathrm{~d}, J=10.1 \mathrm{~Hz}, 1 \mathrm{H}), 4.64(\mathrm{~d}, J=12.2 \mathrm{~Hz}, 1 \mathrm{H}), 4.52(\mathrm{~d}, J=1.5 \mathrm{~Hz}$, 1H), 4.13 (dd, $J=12.1,1.7 \mathrm{~Hz}, 1 \mathrm{H}), 2.94$ (ddd, $J=14.8,4.6,2.0 \mathrm{~Hz}, 1 \mathrm{H}), 2.47$ (qt, $J=$ 7.2, $2.1 \mathrm{~Hz}, 1 \mathrm{H}), 2.21$ (ddd, $J=14.8,10.8,8.3 \mathrm{~Hz}, 1 \mathrm{H}), 2.13-2.03(\mathrm{~m}, 2 \mathrm{H}), 1.93-1.73(\mathrm{~m}$, $3 \mathrm{H}), 1.30(\mathrm{~s}, 3 \mathrm{H}), 1.24(\mathrm{~s}, 3 \mathrm{H}), 1.21(\mathrm{~d}, J=7.2 \mathrm{~Hz}, 3 \mathrm{H}) ;{ }^{13} \mathrm{C} \mathrm{NMR}\left(101 \mathrm{MHz}, \mathrm{CDCl}_{3}\right) \delta$ 211.4, 194.9, 169.0, 156.8, 126.9, 85.2, 85.0, 69.3, 55.3, 52.1, 46.0, 38.4, 33.2, 30.8, 27.7, 23.3, 18.5, 18.3, 12.6; IR (neat): $\mathrm{cm}^{-1} 2924,1755,1725,1692,1154$; HRMS (FAB, $\left.m / z\right)$ calcd for $\mathrm{C}_{19} \mathrm{H}_{22} \mathrm{O}_{5}[\mathrm{M}+\mathrm{H}]^{+} 330.1467$, found 330.1476 .

\subsection{Reference}

(1) Bhalerao, U. t.; Rapaport, H. J. Am. Chem. Soc. 1971, 93, 4835-4838.

(2) (a) D'Accolti, L.; Dinoi, A.; Fusco, C.; Russo, A.; Curci, R. J. Org. Chem. 2003, 68, 7806-7810. (b) Chen, M.S.; White, M. C. Science 2010, 327, 566-571. 
(3) Davis, F. A.; Chattopadhyay, S.; Towson, J. C.; Lai, S.; Reddy, T. J. Org. Chem. 1988, 53, 2087-2089.

(4) Burnell, R. H.; Cote, C.; Theberge, N. J. Nat. Prod. 1993, 56, 1459-1467.

(5) Crandall, J.; Apparu, M. Organic Reactions, 2005, 345-443.

(6) Mahoney, W. S.; Brestensky, D. M.; Stryker, J. M. J. Am. Chem. Soc. 1988, 110, 291-293.

(7) Barrero, A. F.; Alvarez-Manzaneda, E. J.; Chahboun, R.; Meneses, R. Synlett 1999, 1663-1665.

(8) Trost, B. M.; Aponick, A. J. Am. Chem. Soc. 2006, 128, 3931-3933.

(9) Ito, Y.; Hirao, T.; Saegusa, T. J. Org. Chem. 1978, 43, 1011-1013.

(10) Oppolzer, W.; Godel, T. J. Am. Chem. Soc. 1978, 100, 2583-2584.

(11) (a) Lombardo, L. Tetrahedron Lett. 1982, 23, 4293-4294. (b) Lombardo, L. Organic Syntheses, 1987, 65, 81-83.

(12) Takai, K.; Hotta, Y.; Oshima, K.; Nozaki, H. Tetrahedron Lett. 1978, 27, 2417 2420.

(13) Tebbe, F. N.; Parshall, G. W.; Reddy, G. S. J. Am. Chem. Soc. 1978, 100, 36113612.

(14) (a) Beadham, I,; Micklefield, J. Current Organic Synthesis 2005, 2, 231-259. (b) Hartley, R. C.; Li, J.; Main, C. A.; McKiernan, G. J. Tetrahedron 2007, 63, 48254864.

(15) Mori, K.; Sasaki, M. Tetrahedron Lett. 1979, 20, 1979-1982. 
(16) (a) Rousseau, G.; Conia, J. M. Tetrahedron Lett. 1981, 22, 649-652. (b)

Oppolzer, W.; Godel, T. J. Am. Chem. Soc. 1978, 100, 2583-2584. (c) Denis, J. M.;

Girard, C.; Conia, J. M. Synthesis 1972, 549-551.

(17) (a) Wender, P. A.; Eck, S. L. Tetrahedron Lett. 1982, 23, 1871-1874. (b) Trost, B. M.; Hiemstra, H. J. Am. Chem. Soc. 1982, 104, 886-887. (c) Trost, B. M.; Curran, D. P. J. Am. Chem. Soc. 1981, 103, 7380-7381. (d) Pattenden, G.; Roberts, L.; Blake, A. J. J. Chem. Soc. Perkin Trans. 1. 1998, 863-867.

(18) Birman, V. B.; Danishefsky, S. J. J. Am. Chem. Soc. 2002, 124, 2080-2081.

(19) (a) Sharpless, K. B.; Lauer, R. F.; Teranishi, A. Y. J. Am. Chem. Soc. 1973, 95, 6137-6140. (b) Reich, H. J.; Renga, J. M.; Reich, I.L. J. Am. Chem. Soc. 1975, 97, 5434-5439. 


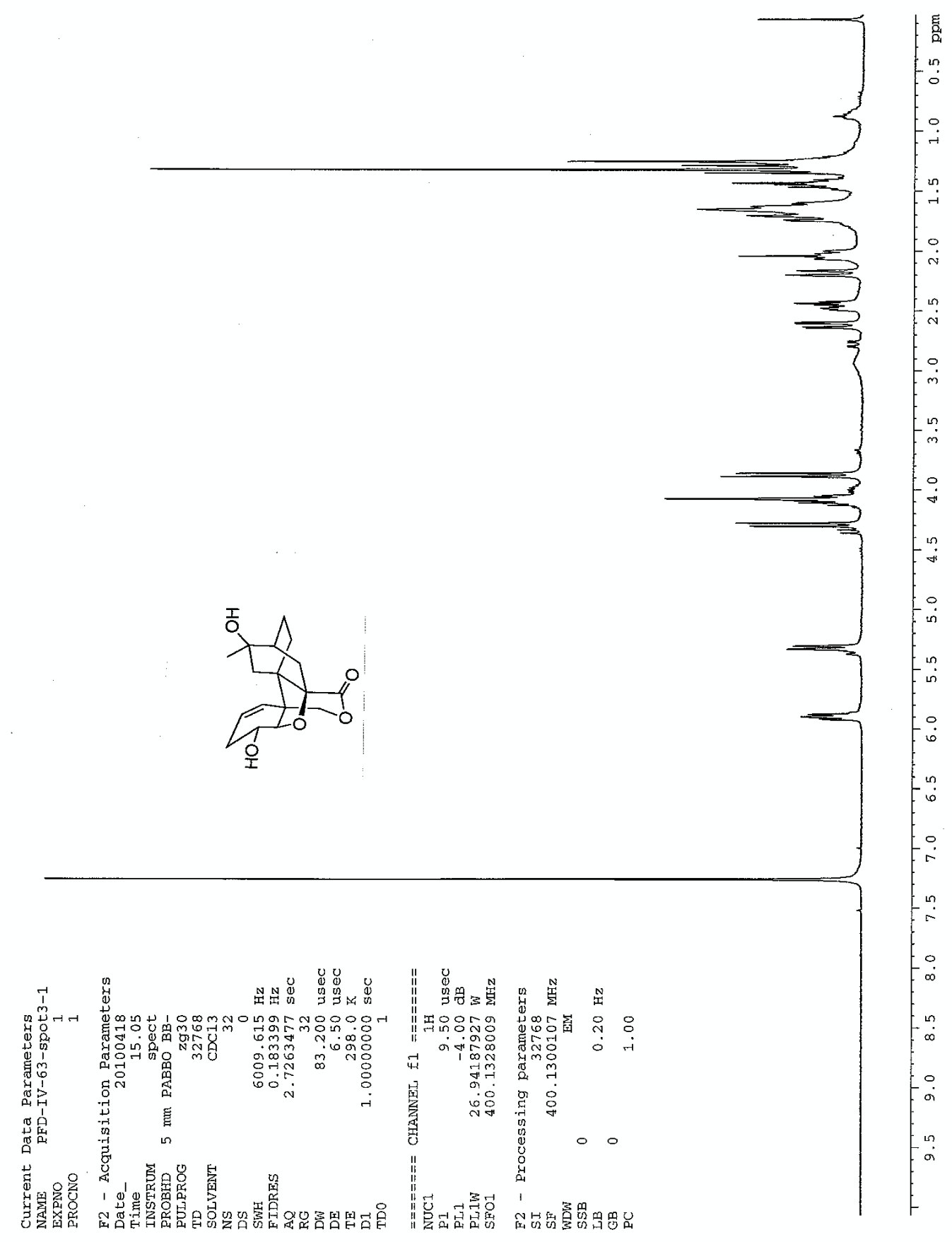



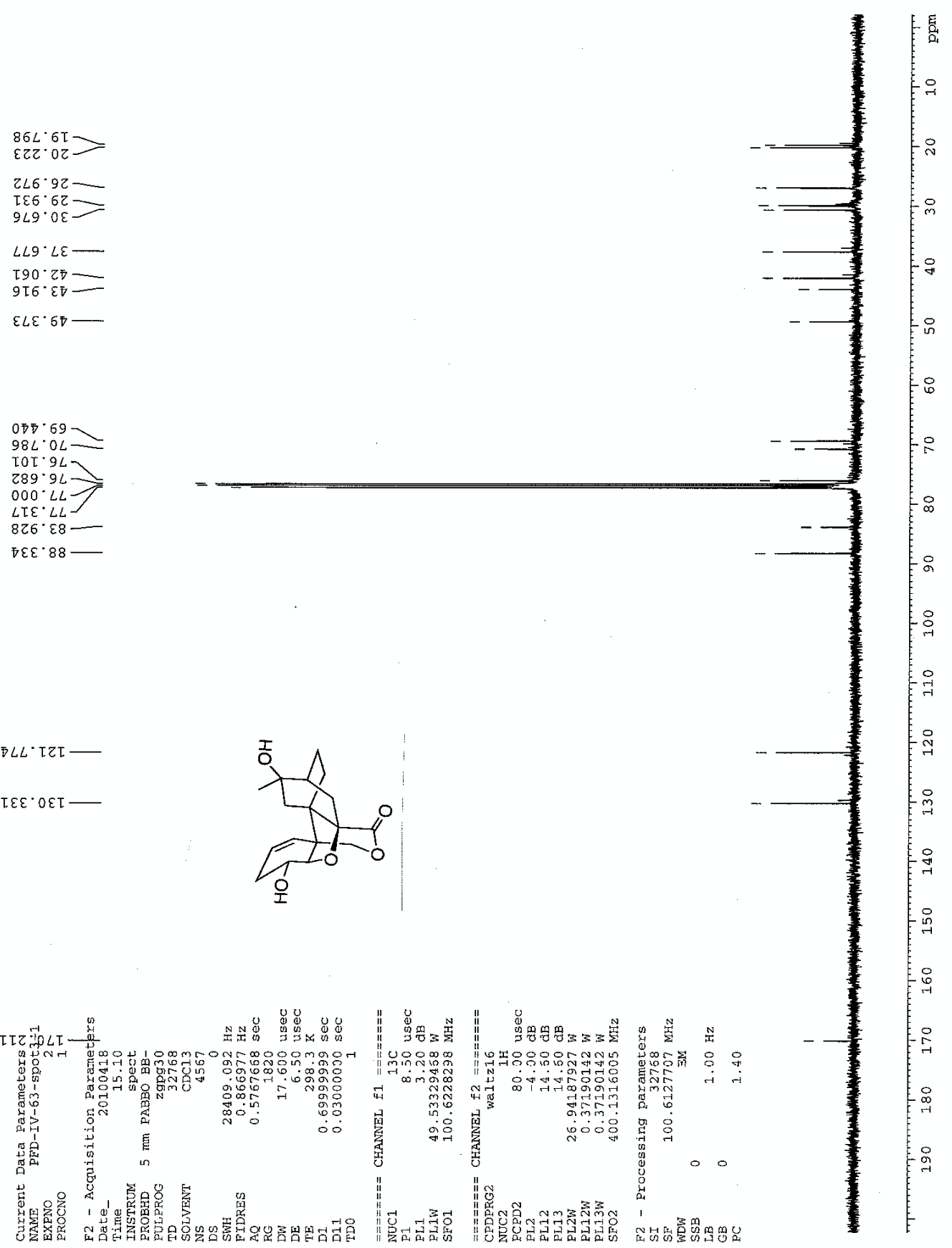

โรE. $0 \varepsilon \tau-$

075.69

$98 L^{\circ} 0 L-$

TOT.9L

$000 \cdot L L Y$

$826 \cdot \varepsilon 8$

จ $\varepsilon \cdot 88$ 


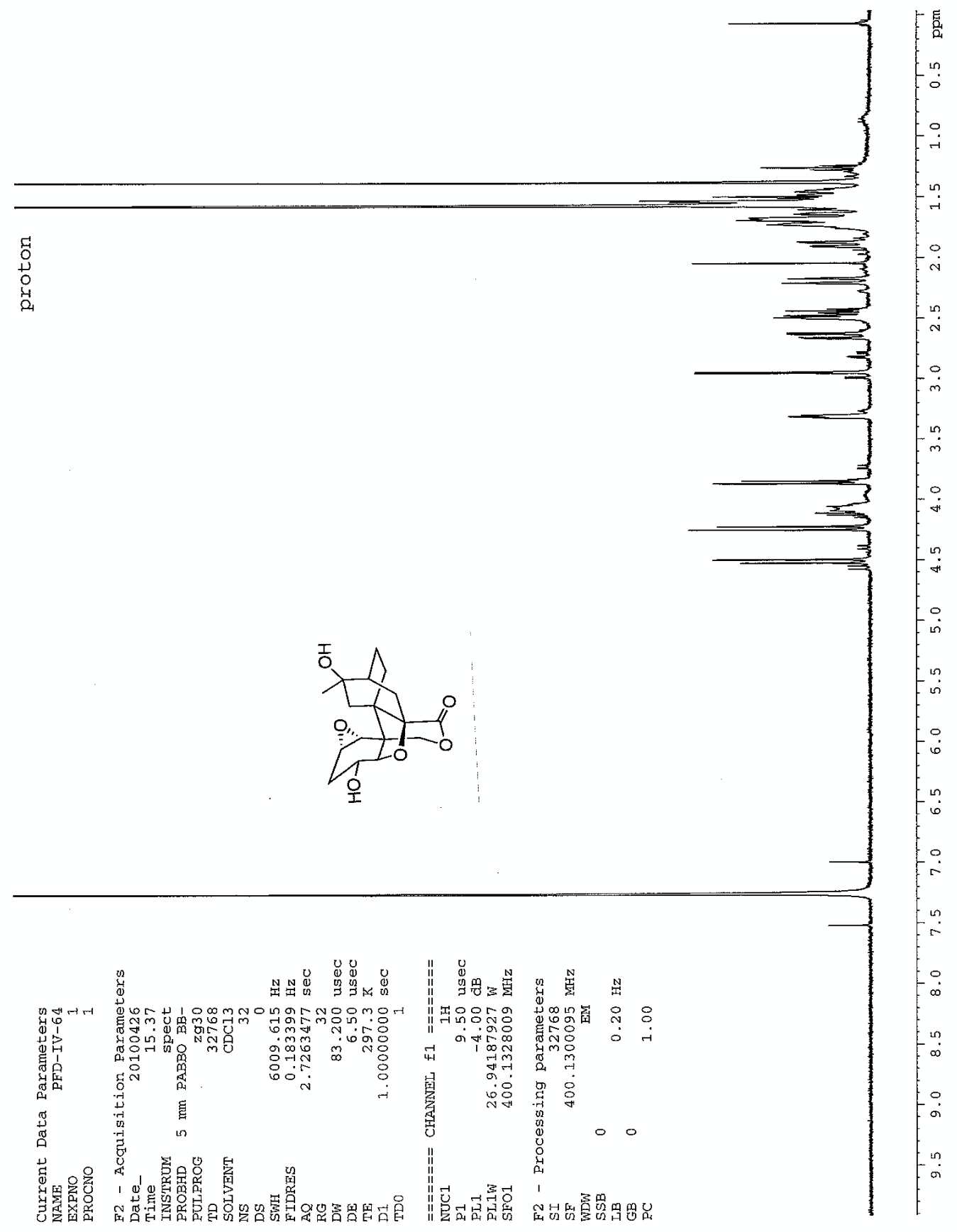




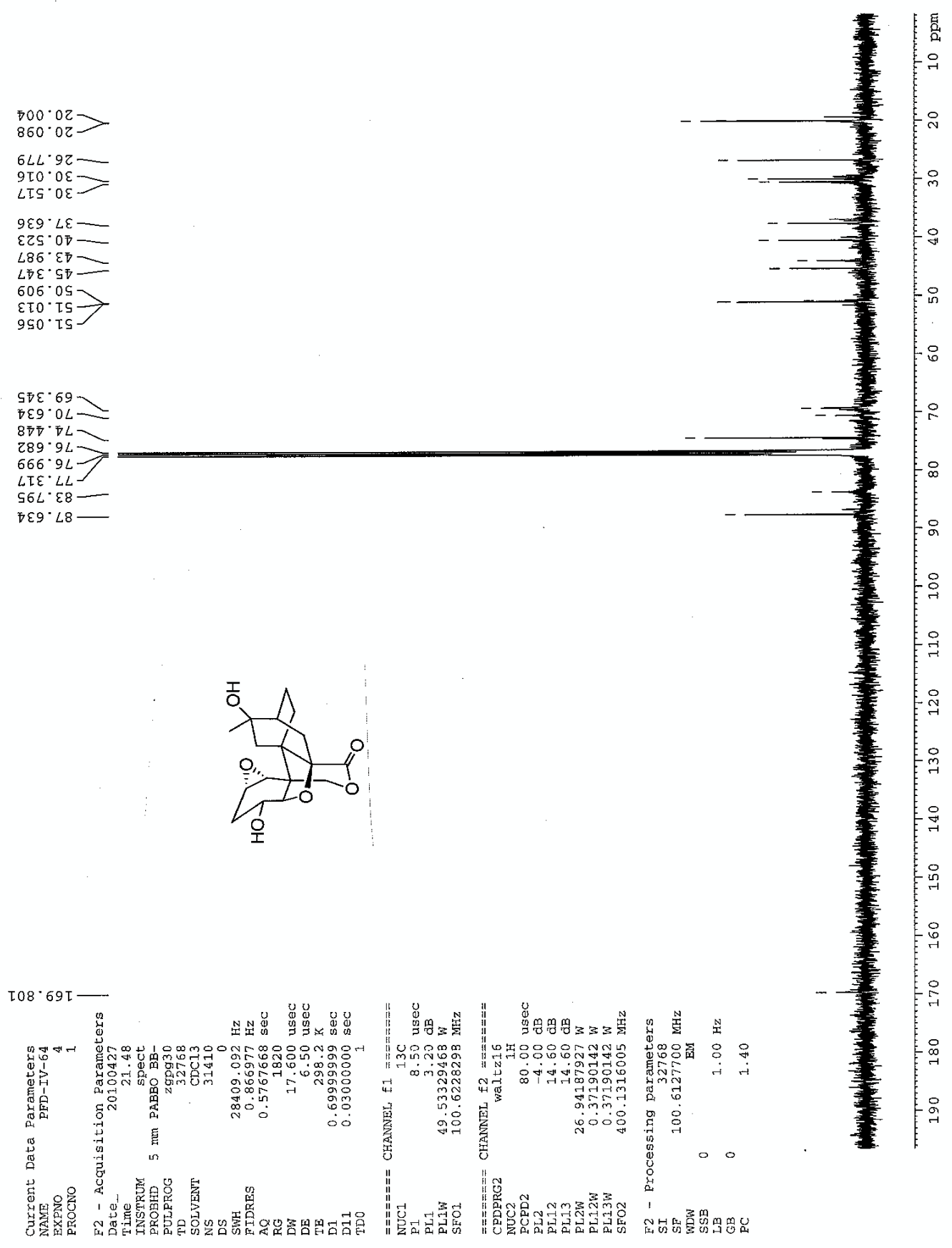




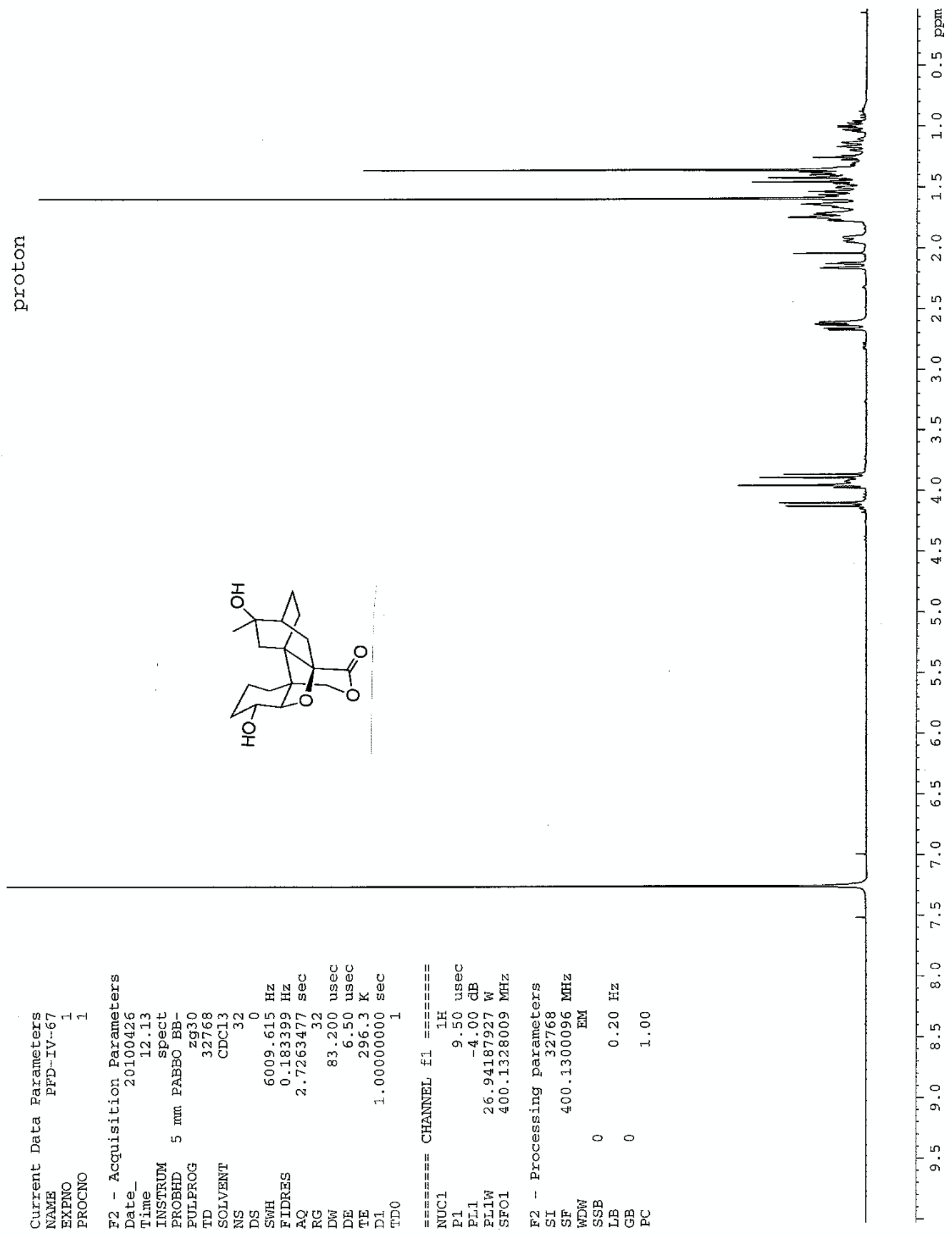




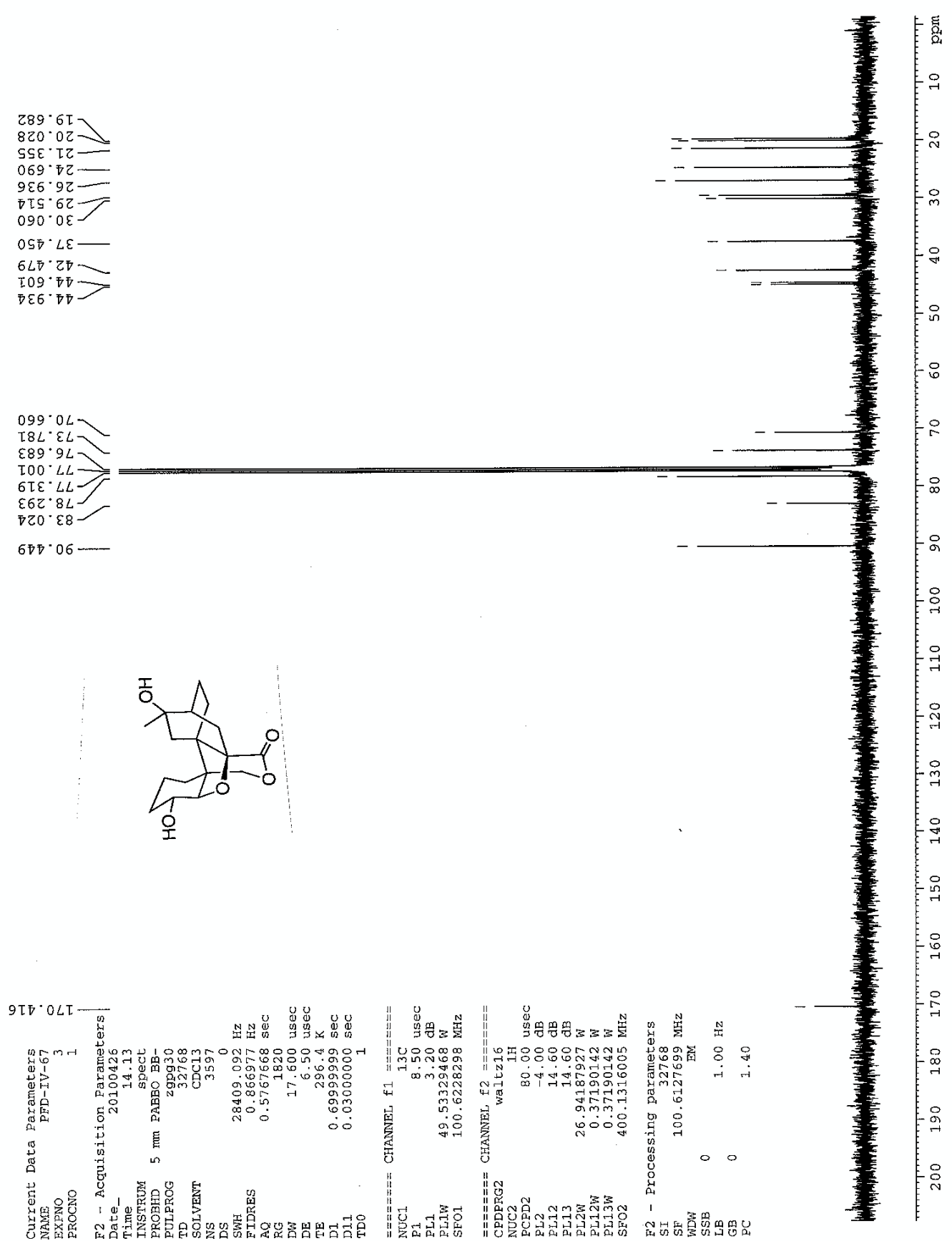


\begin{tabular}{l}
$\Xi$ \\
0 \\
+ \\
0 \\
\multirow{1}{1}{} \\
01
\end{tabular}

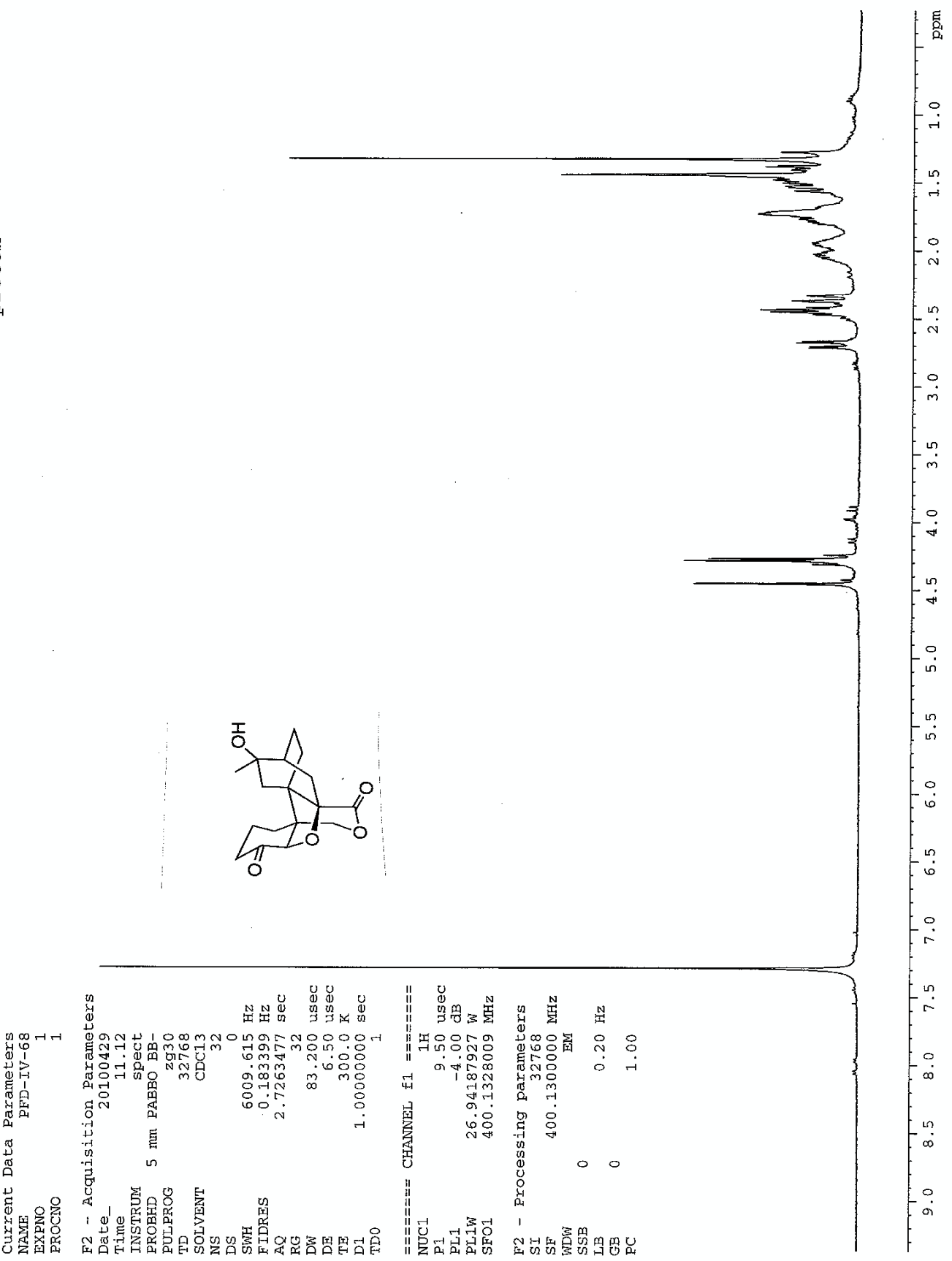



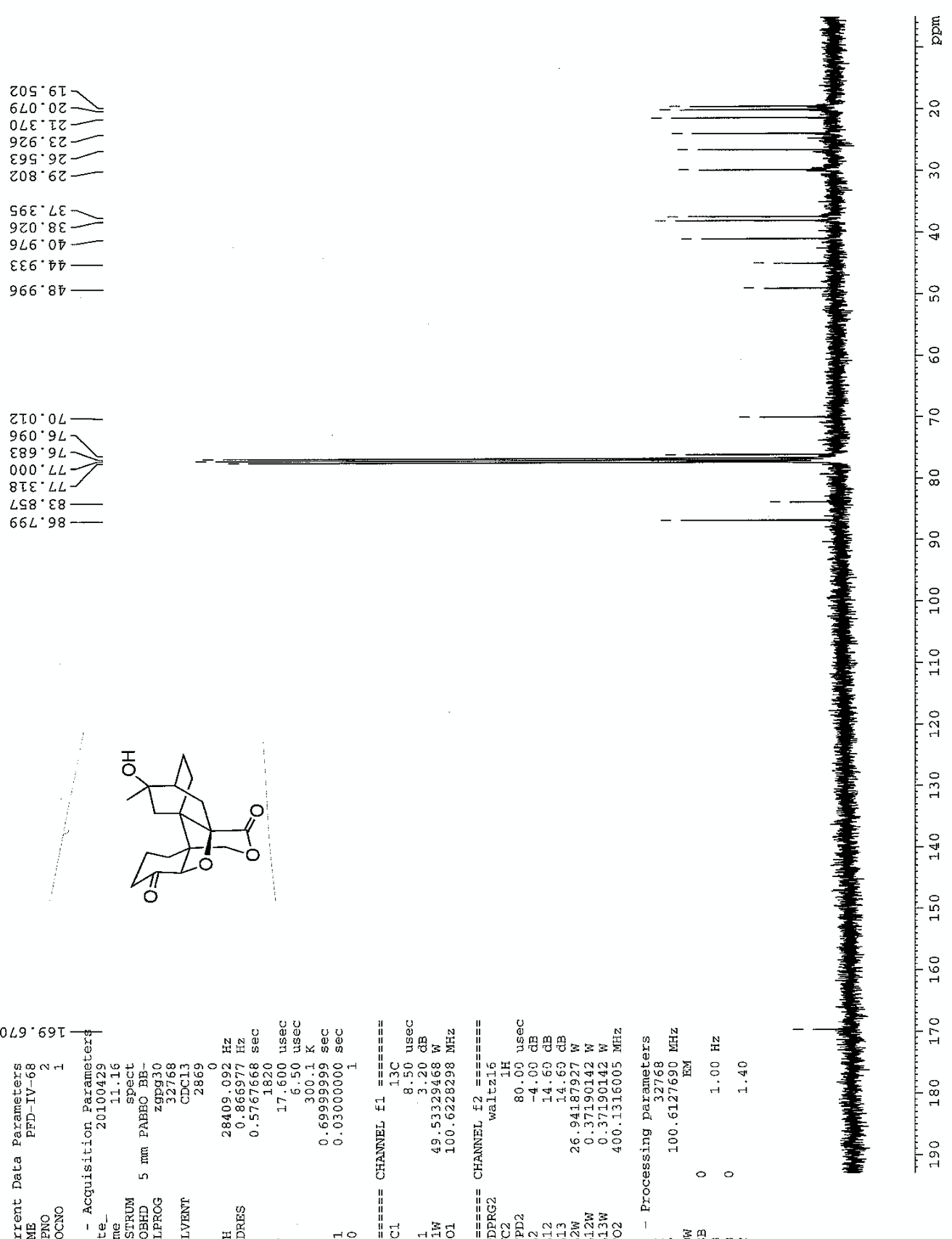

$2 T 0^{\circ} 0 L-$ $960^{\circ} 9 L>$ $889^{\circ} 9 L$

$8 \mathrm{TE} L L$

$\angle 58 \cdot 88-$
$66 L \cdot 98$
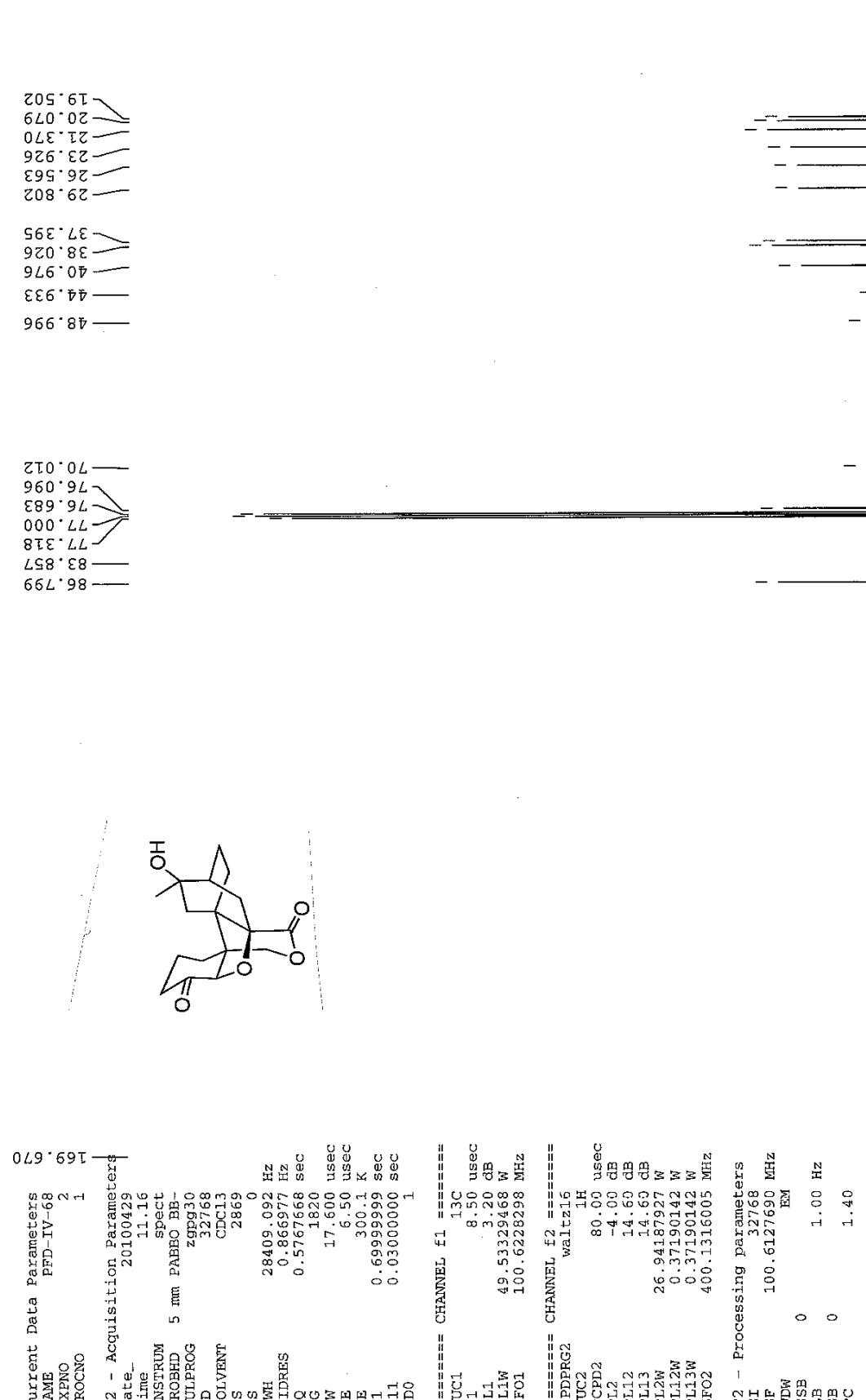

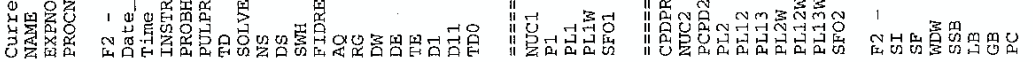




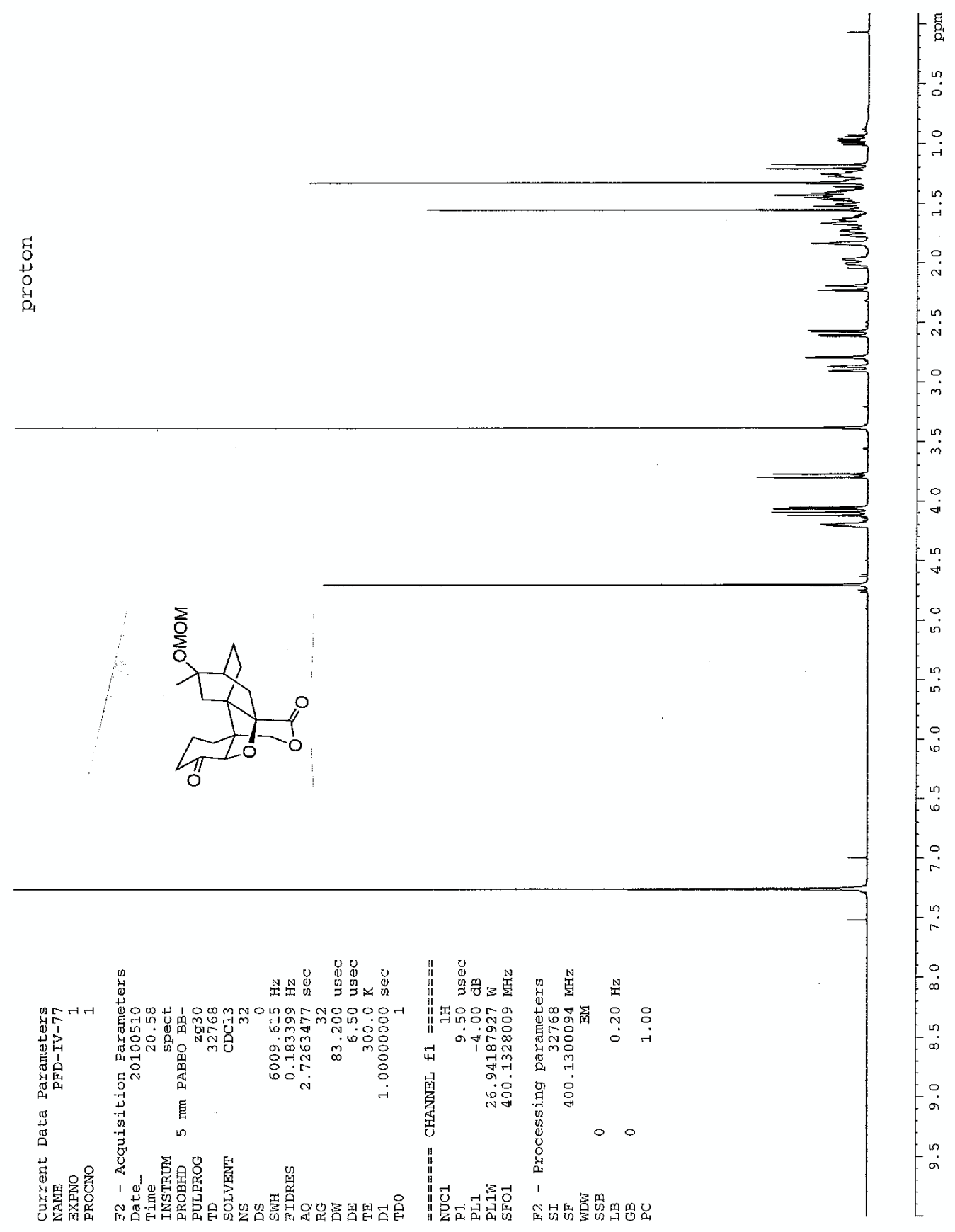




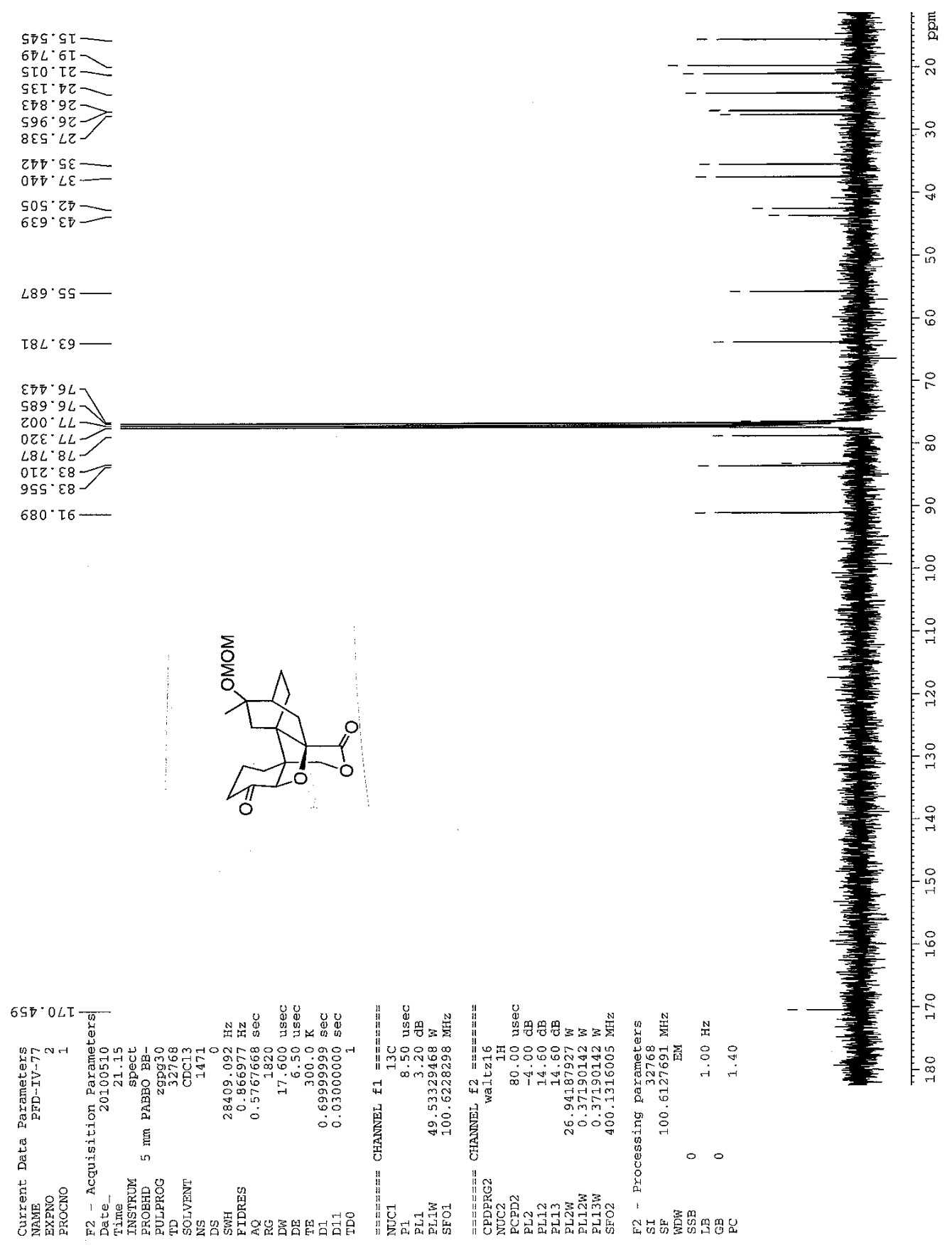




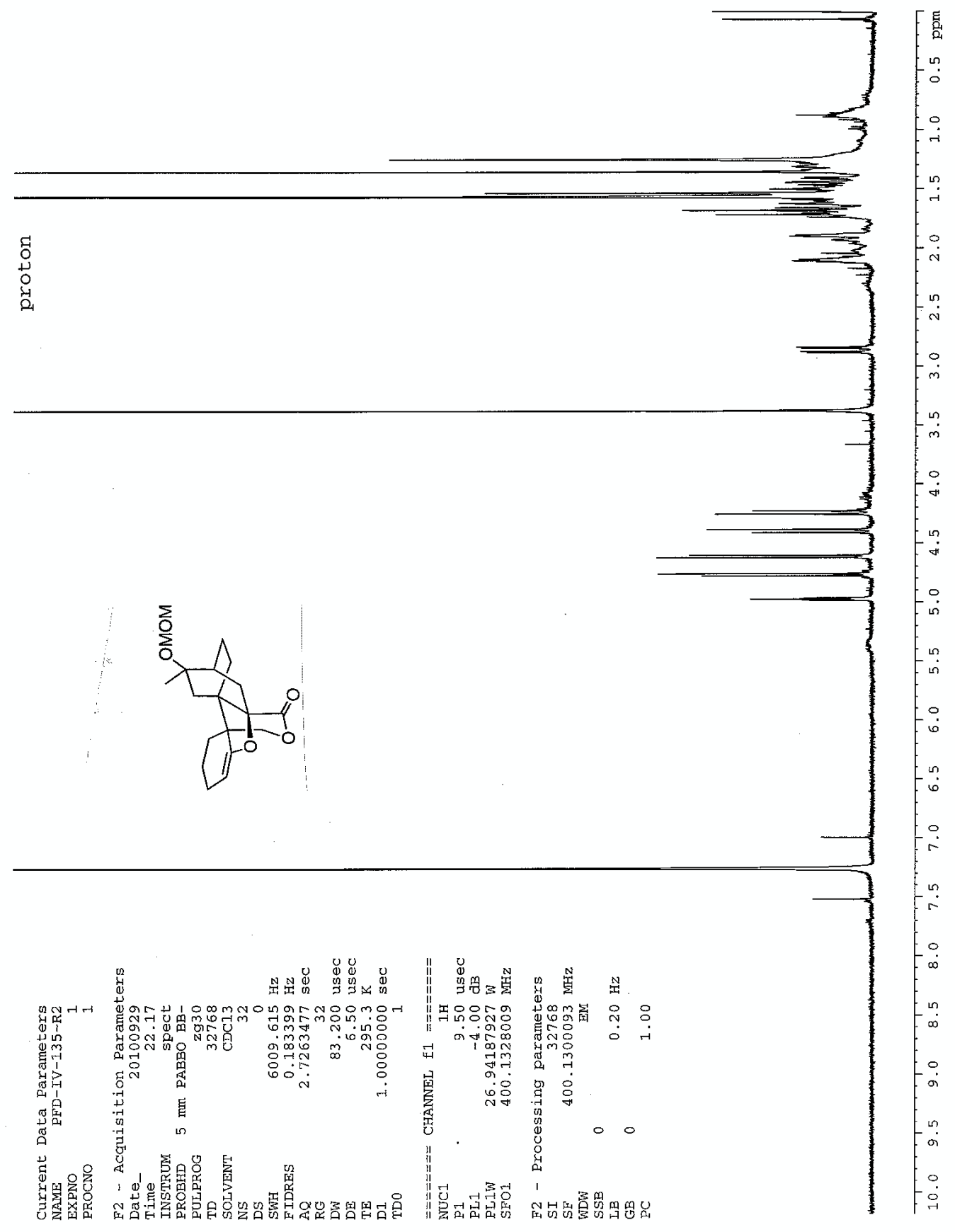




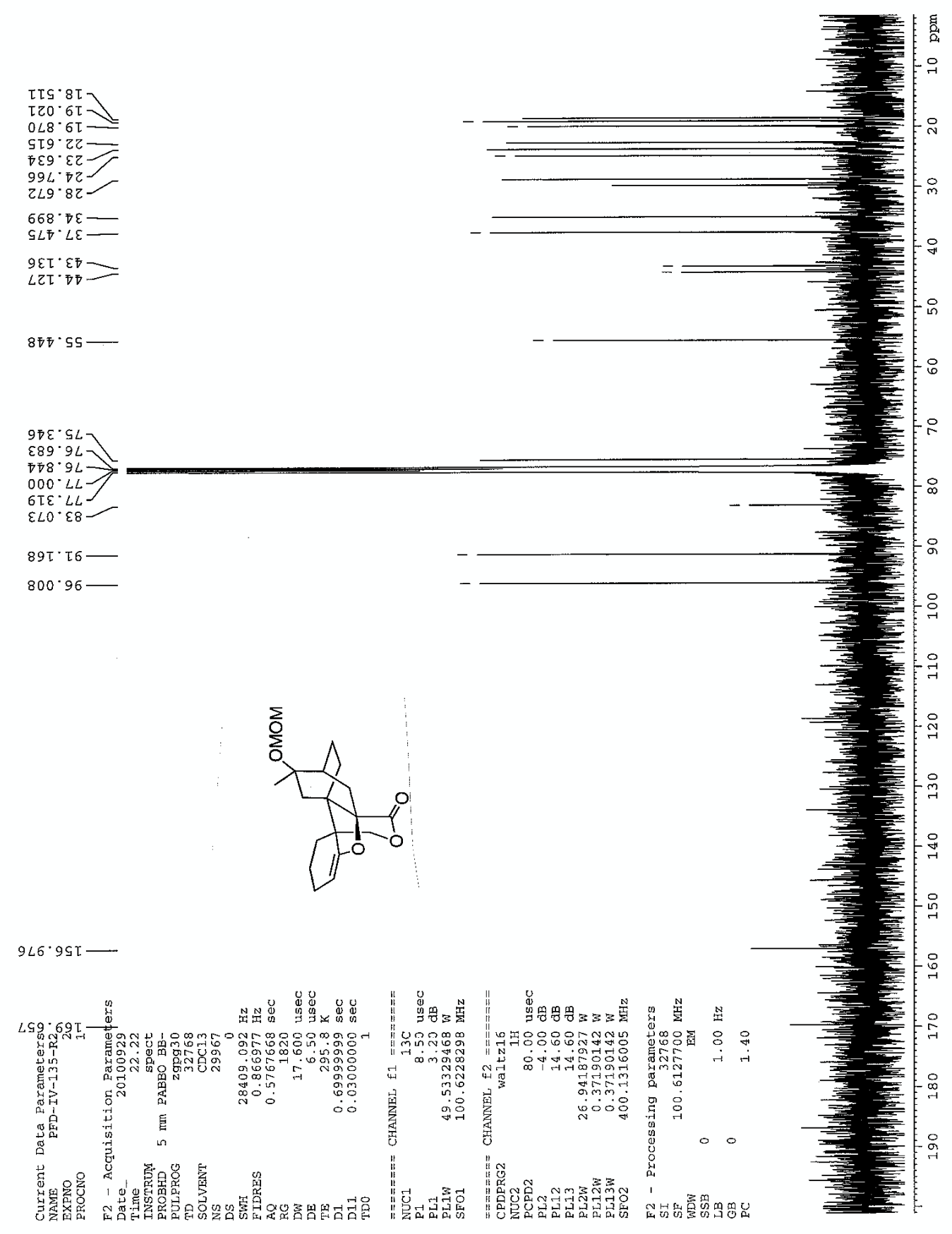




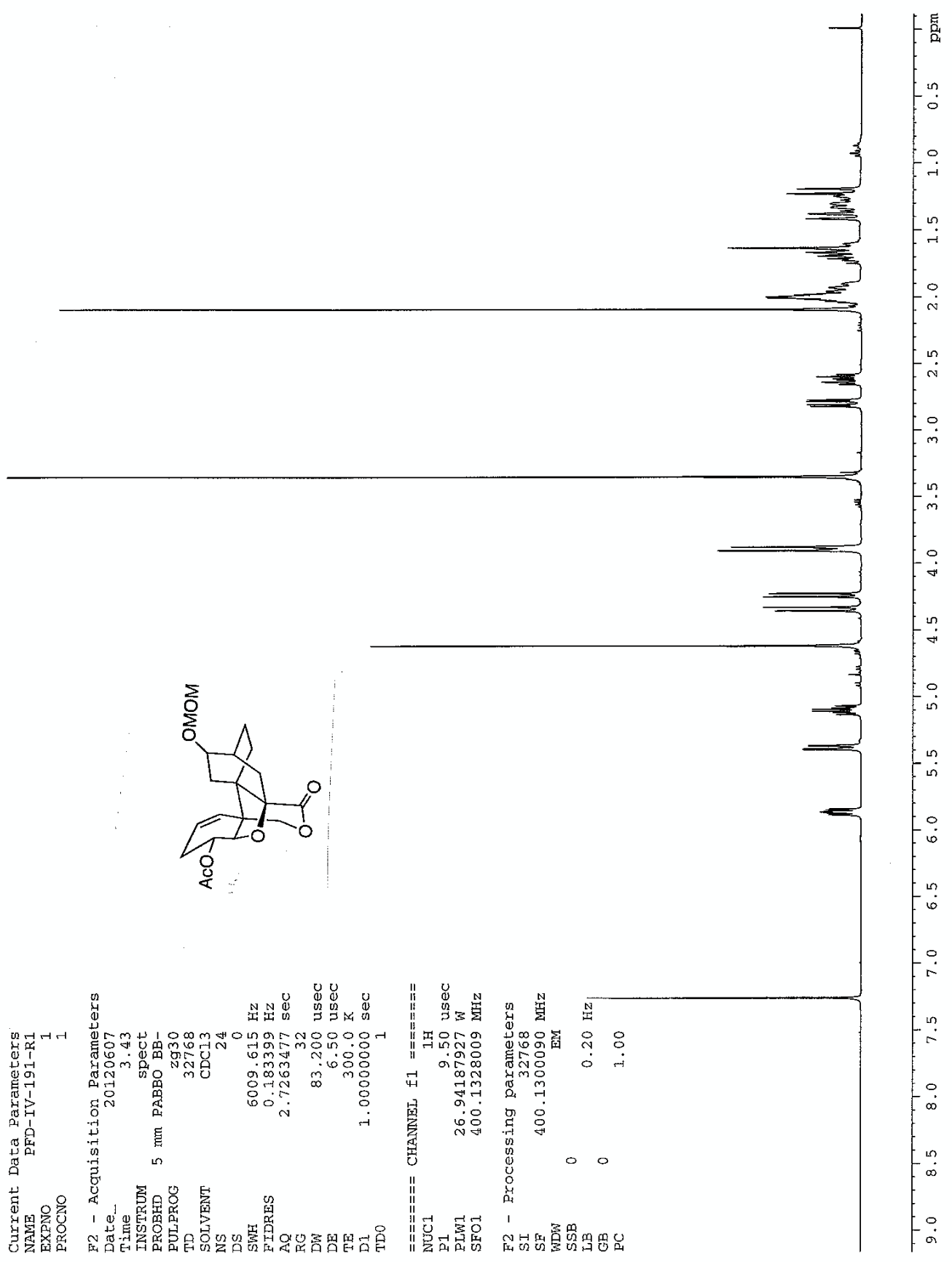




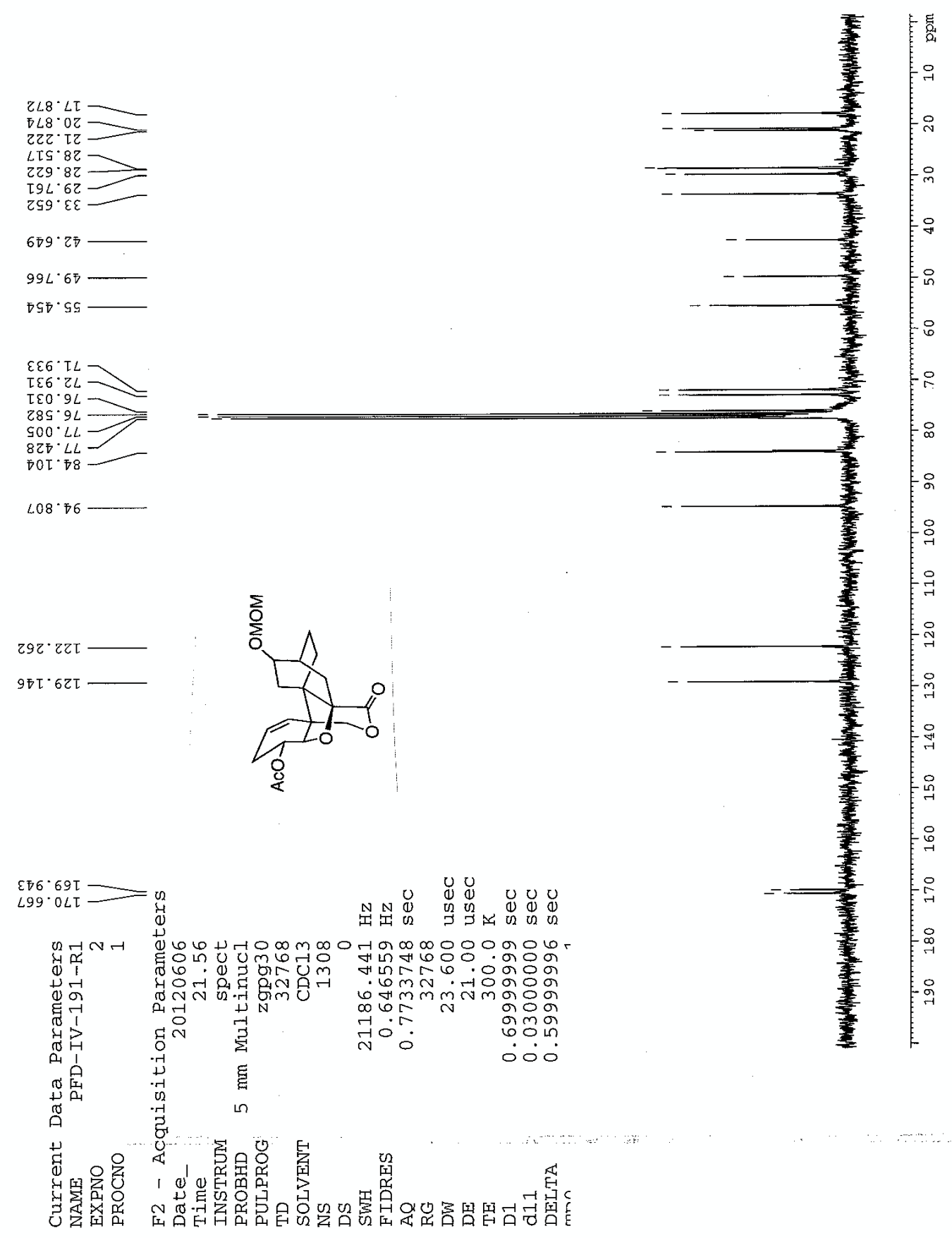




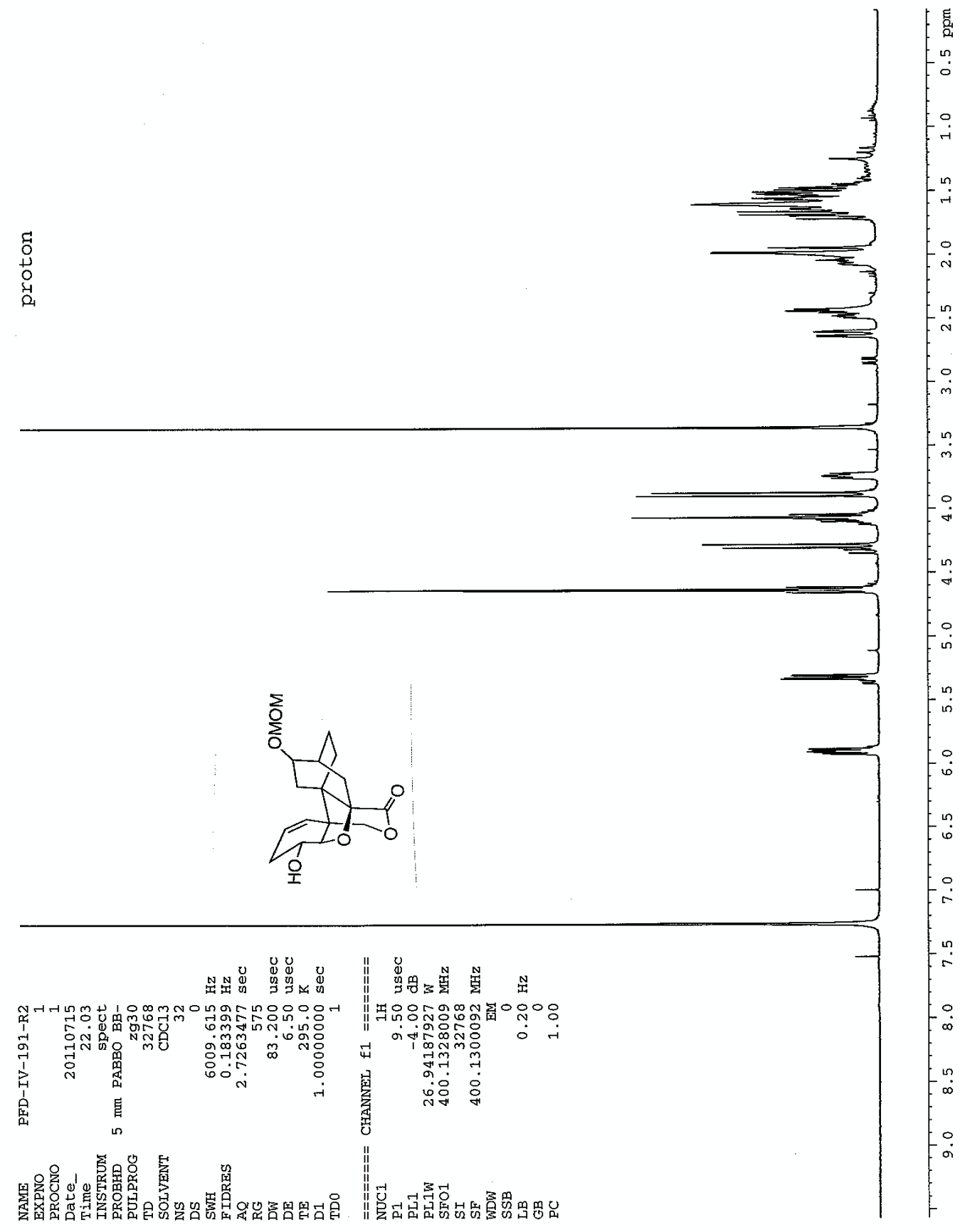




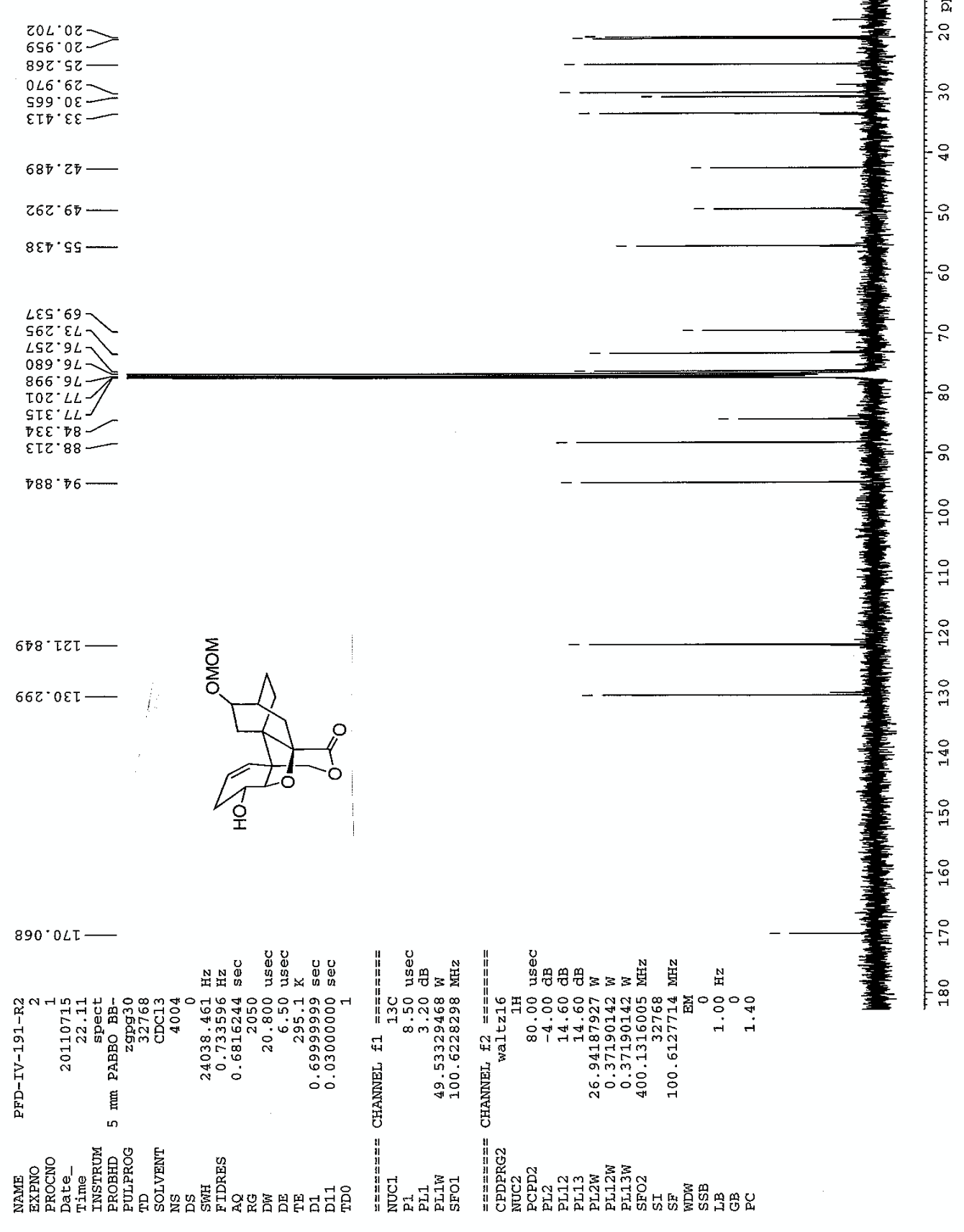




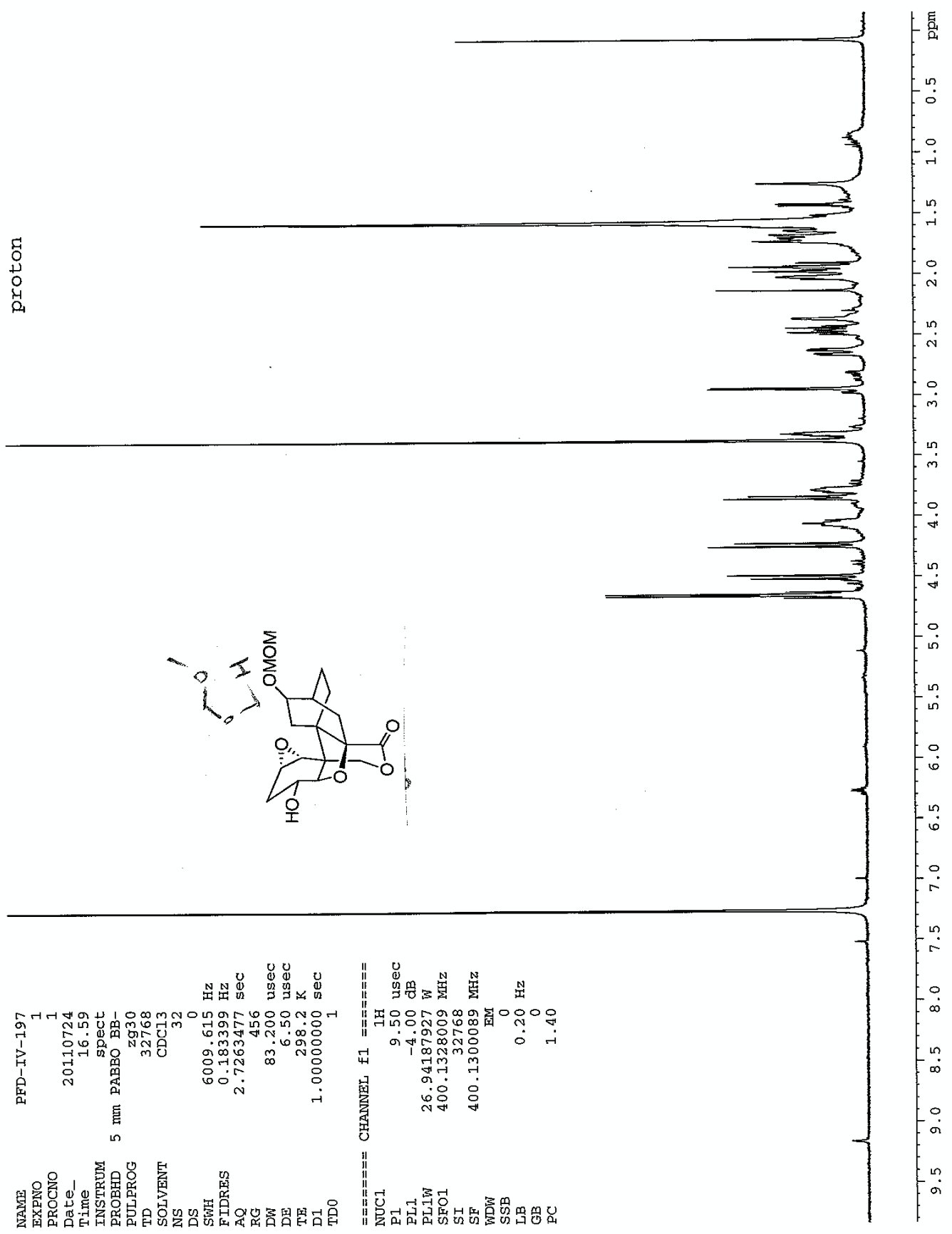




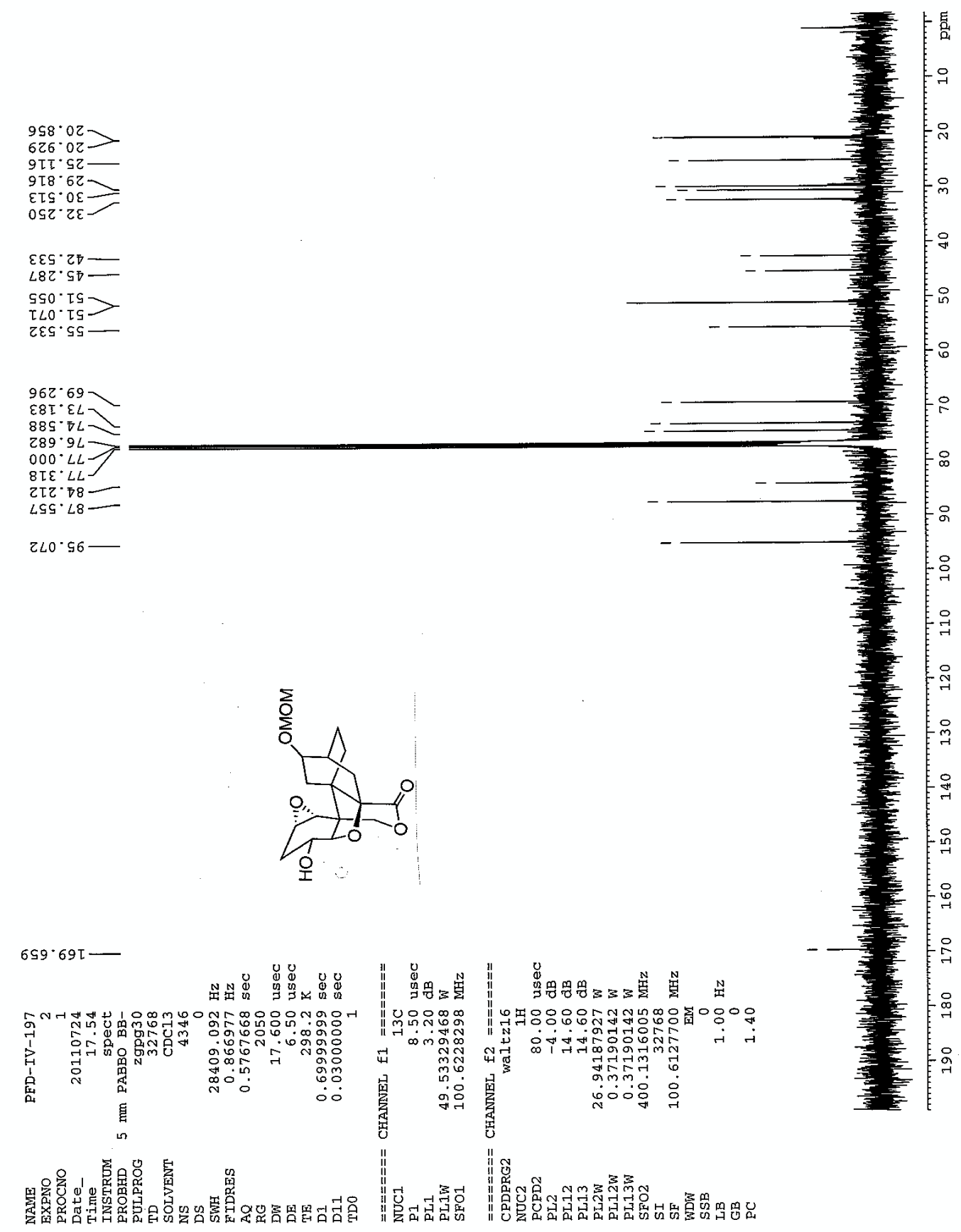




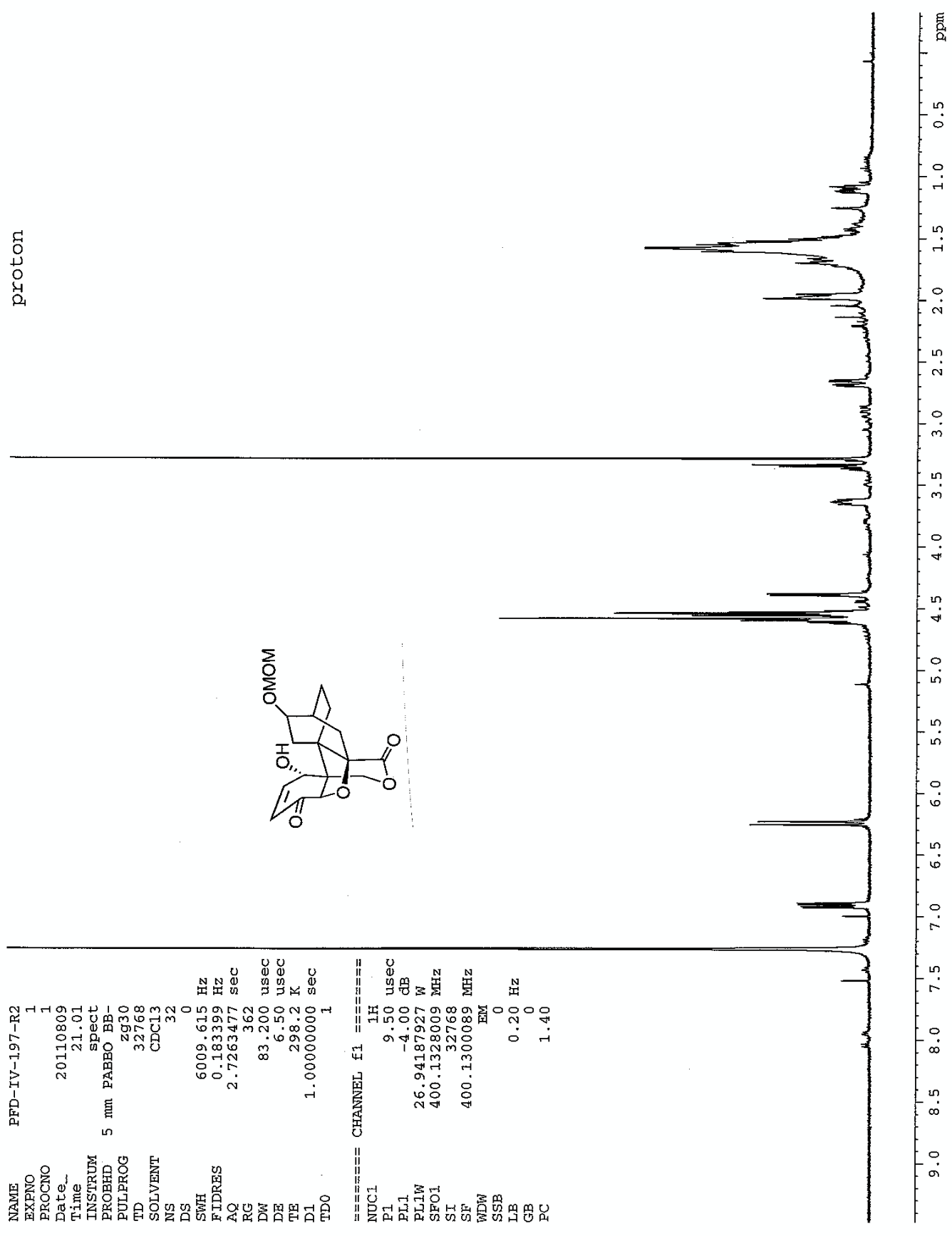




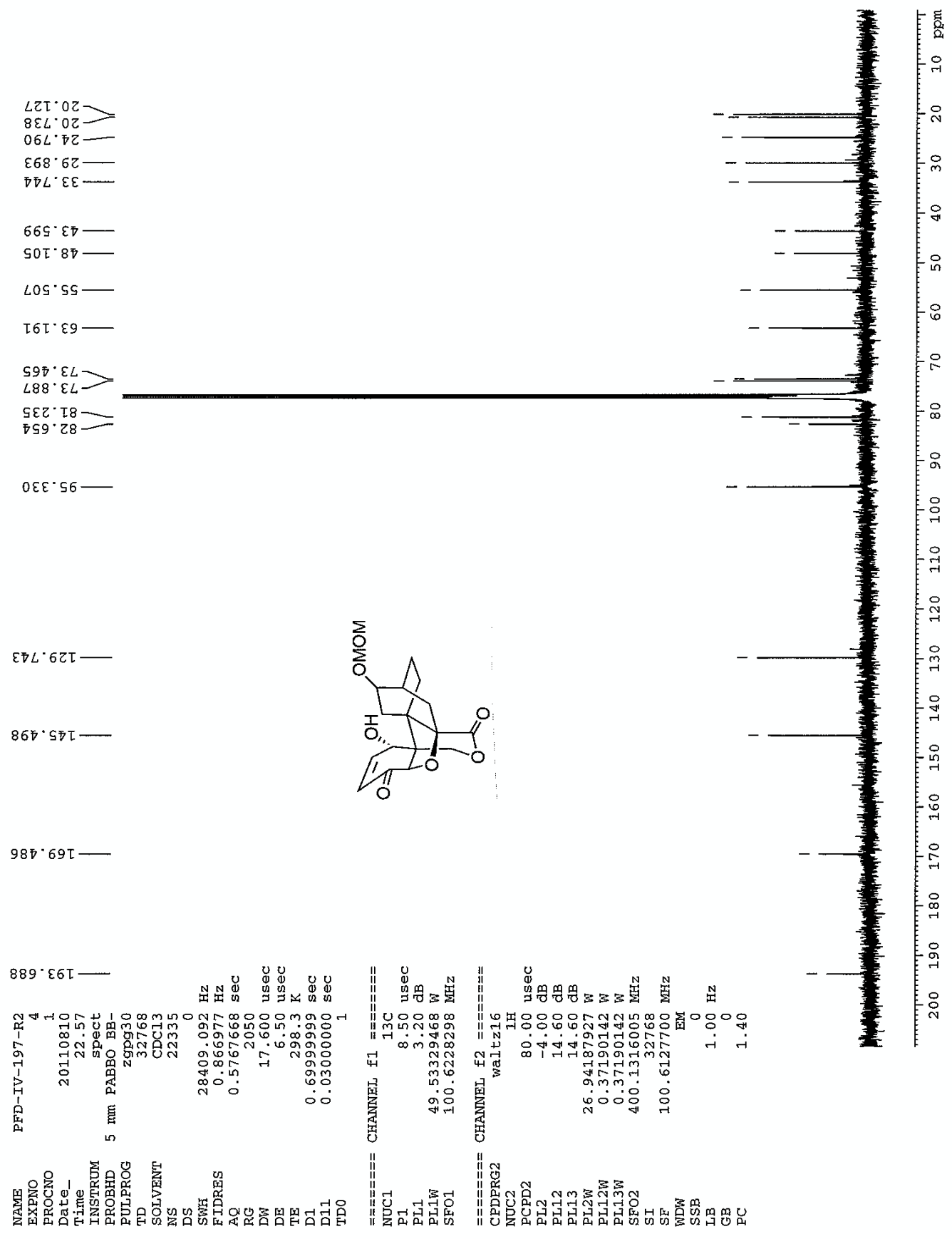




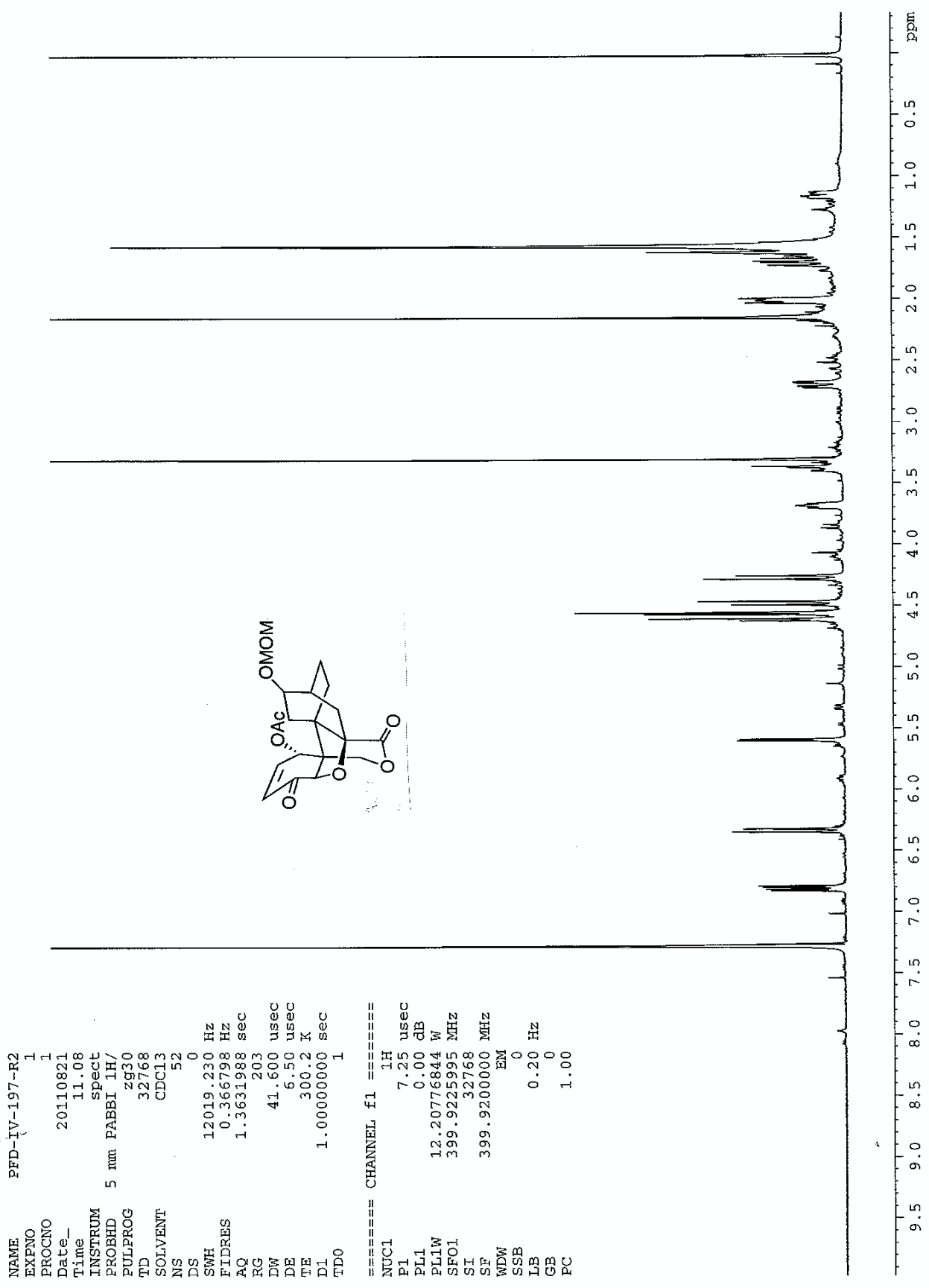




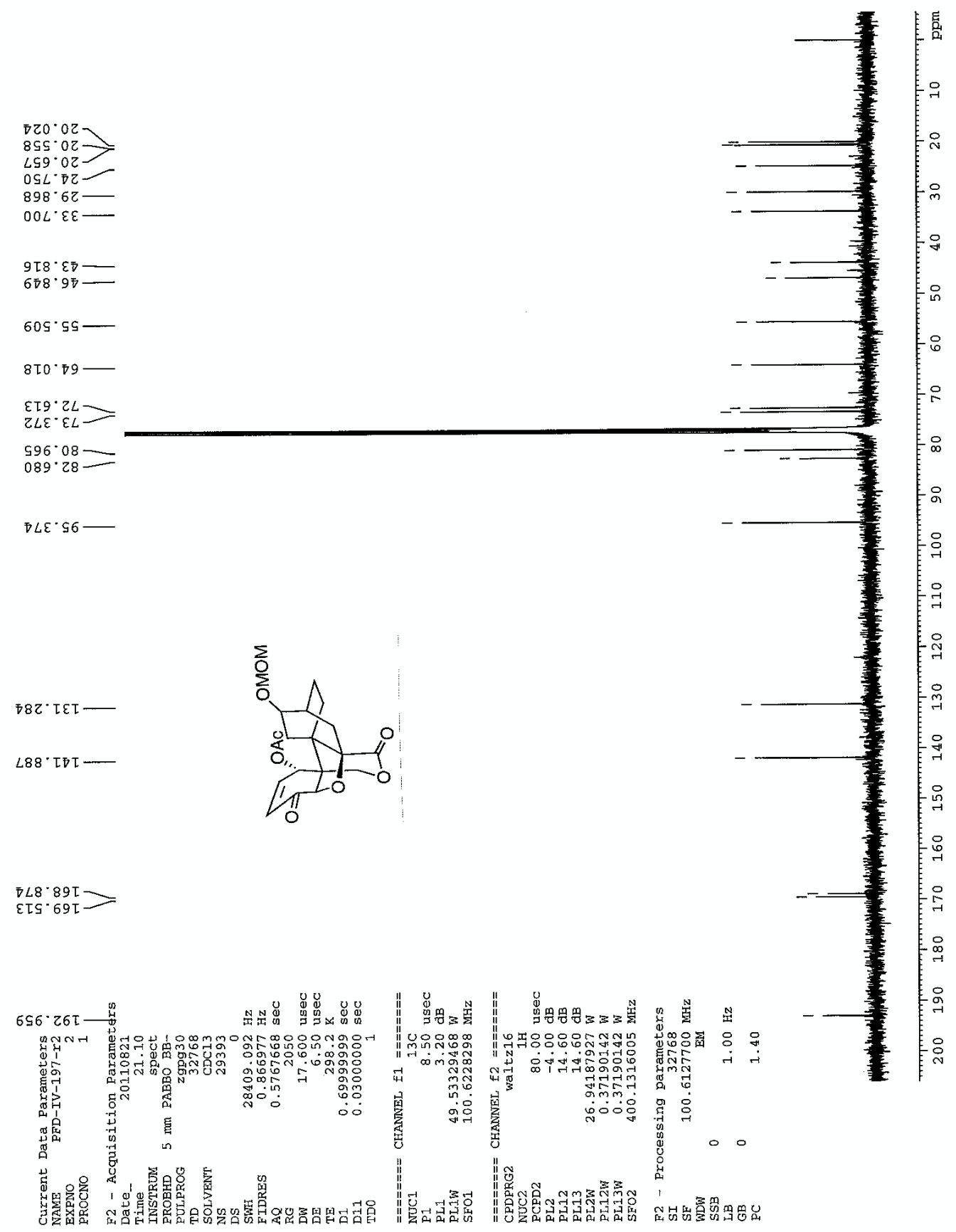




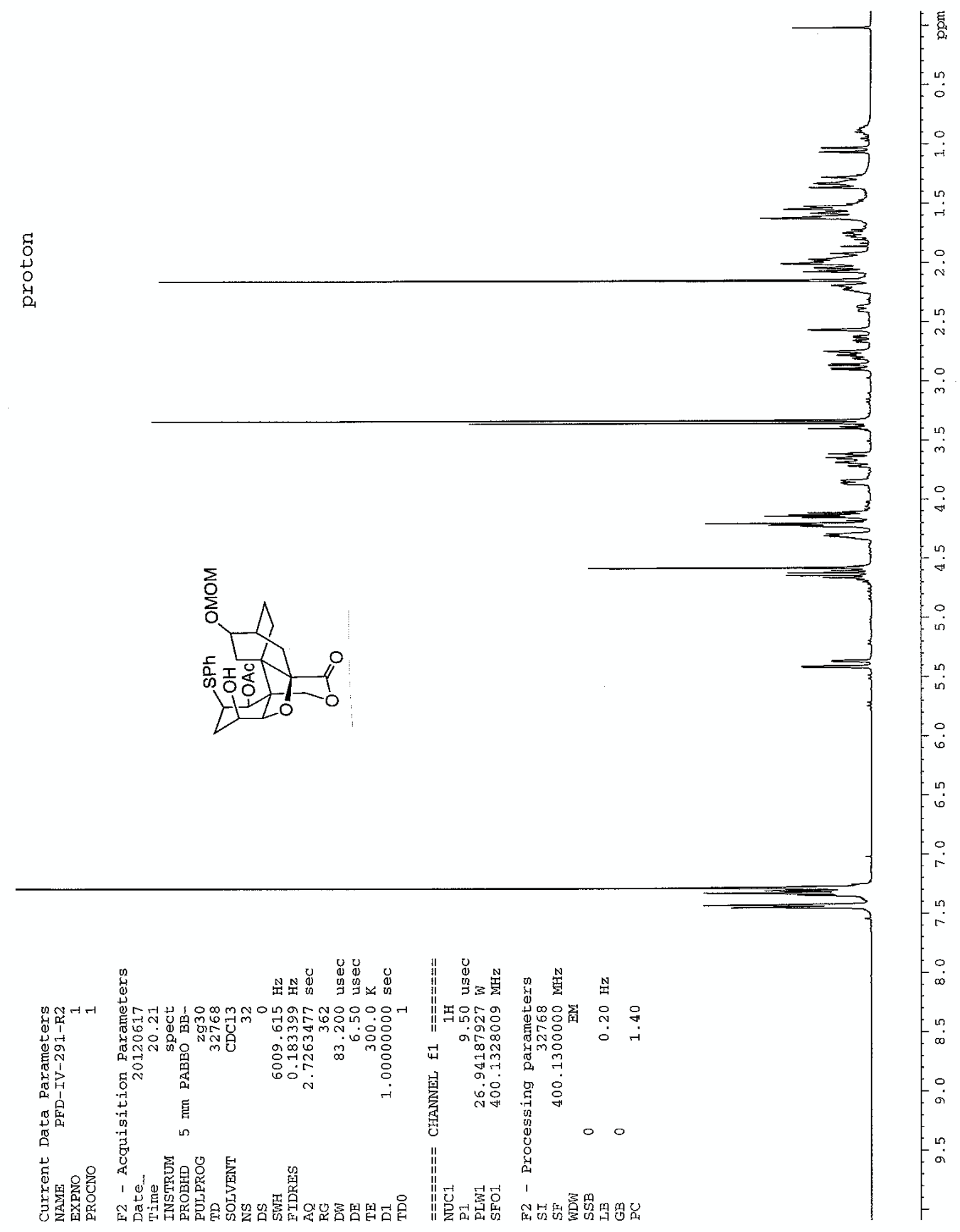




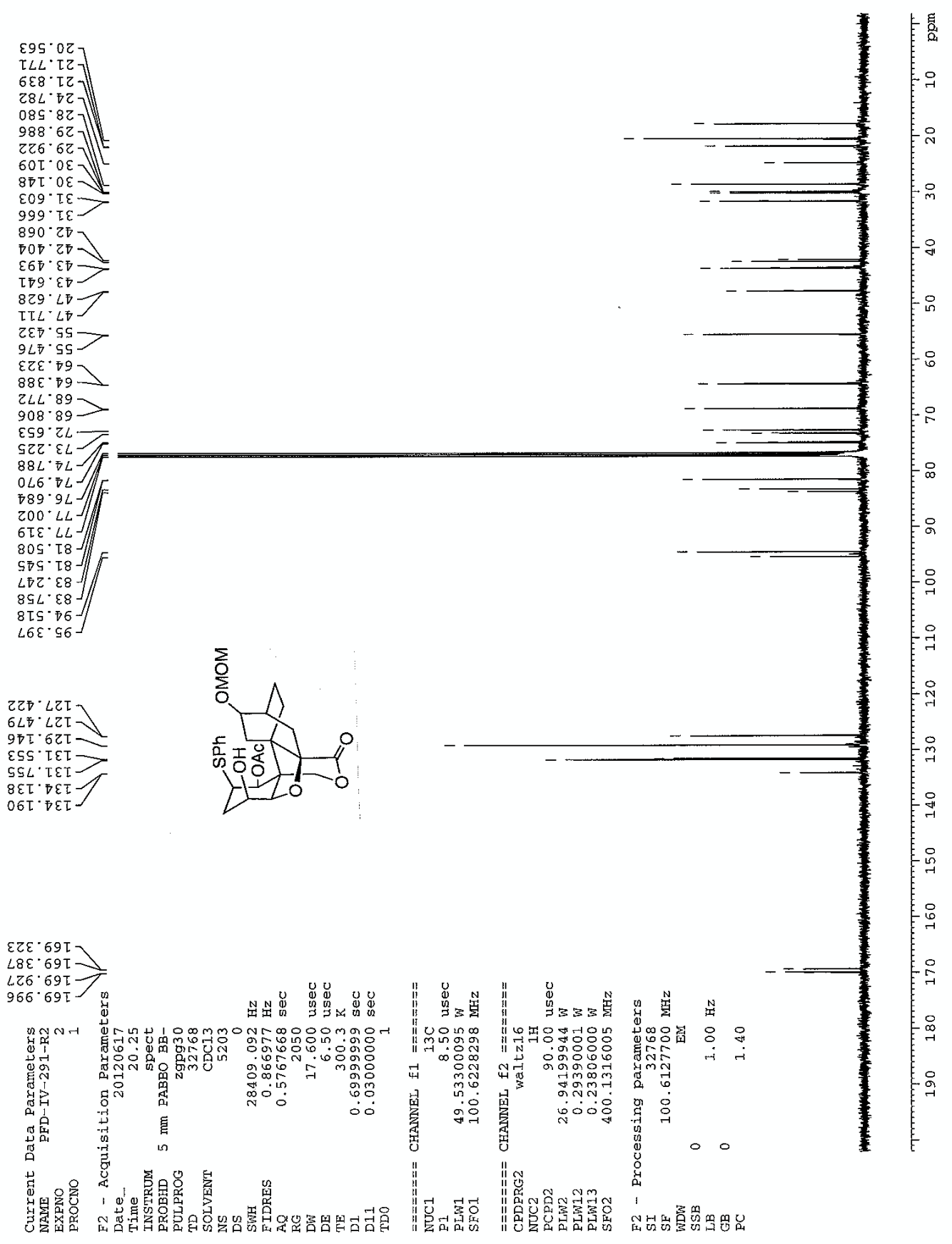




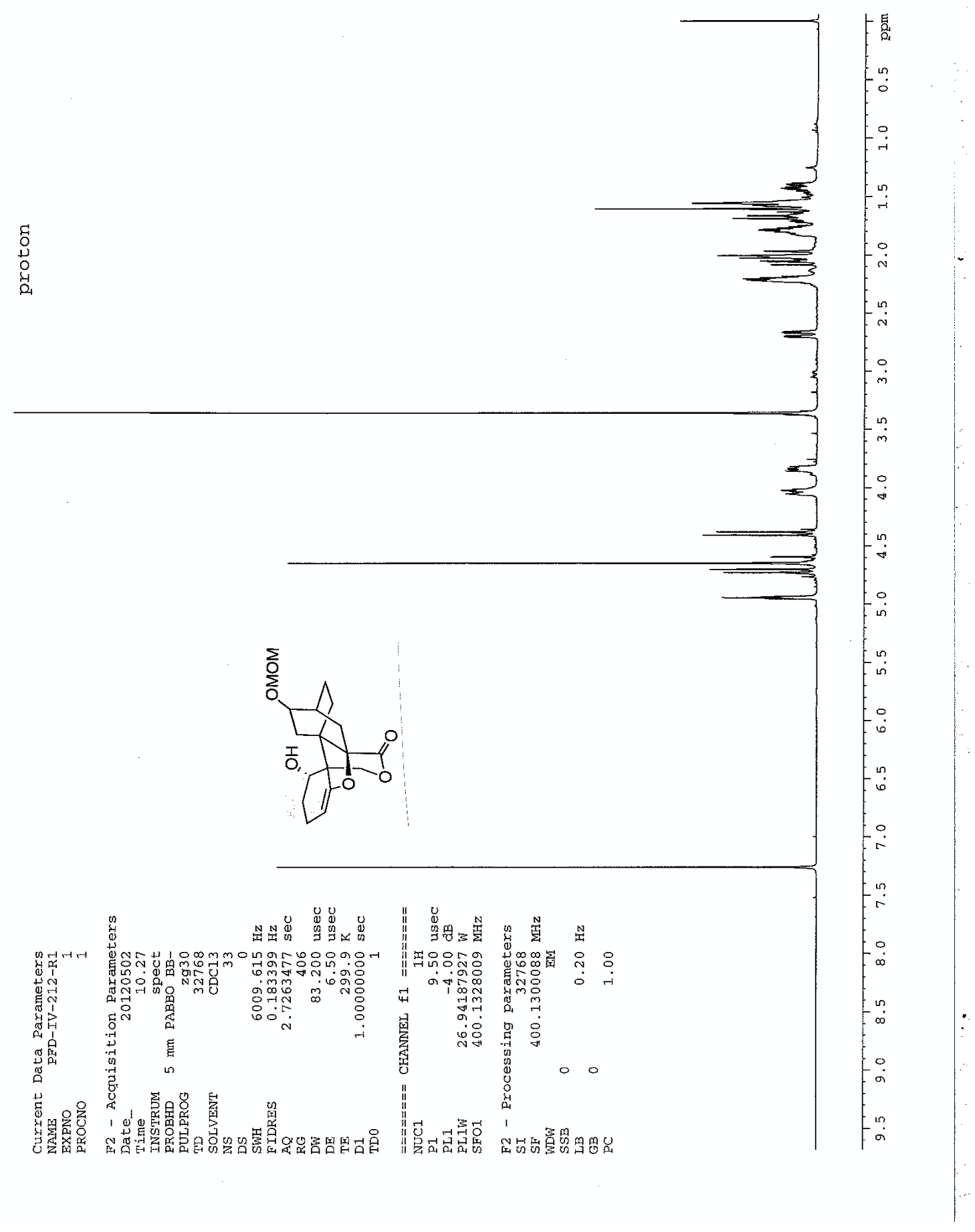




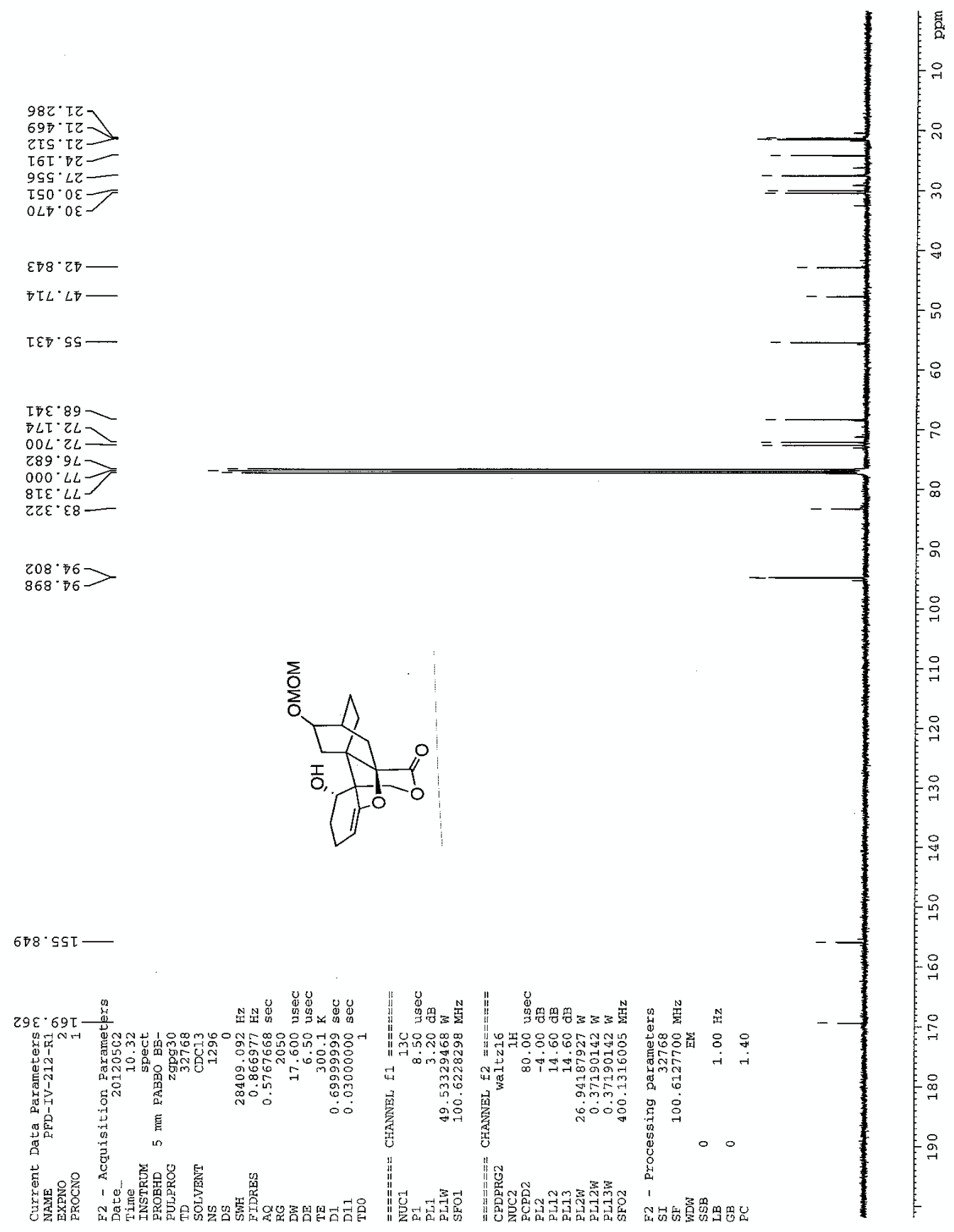




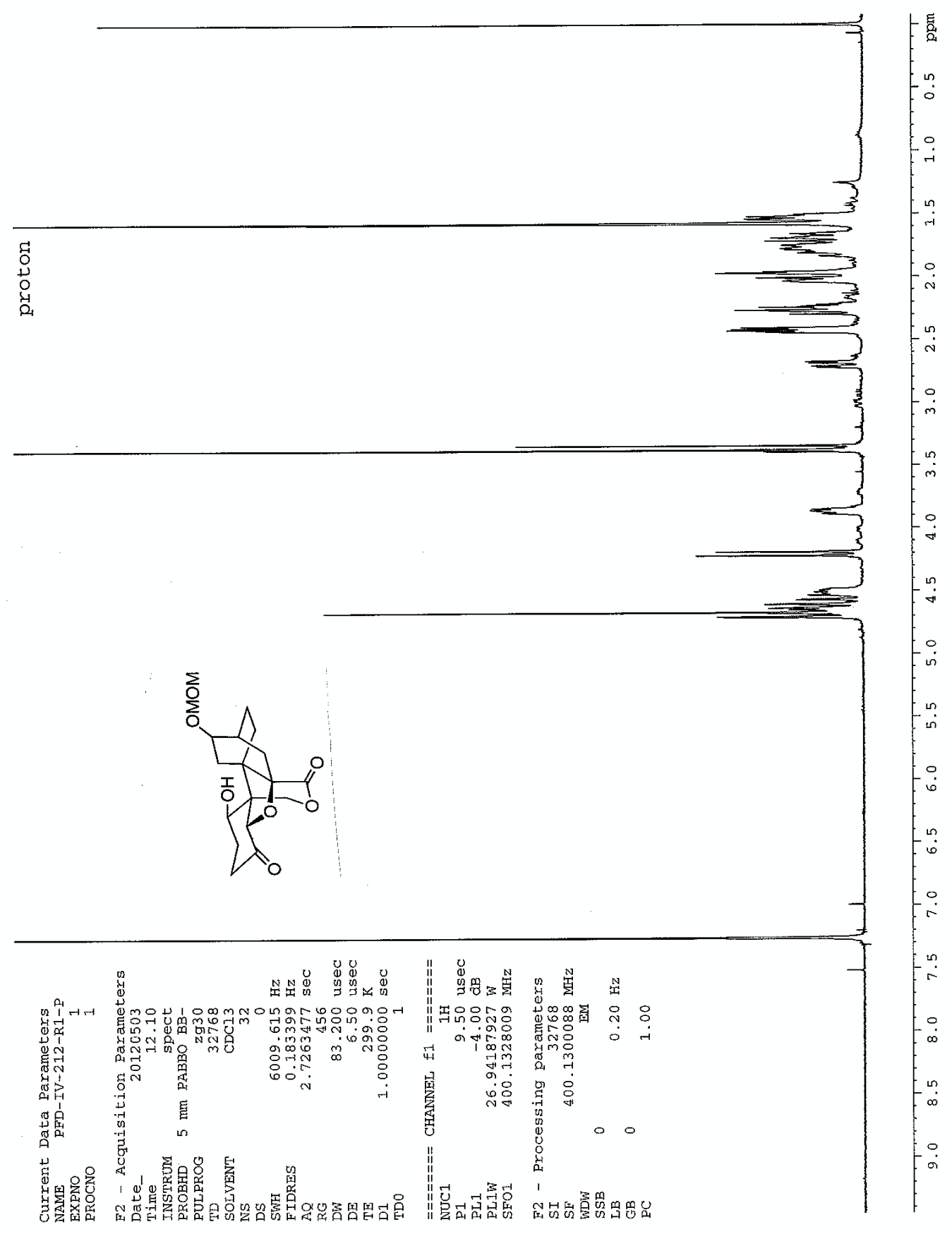




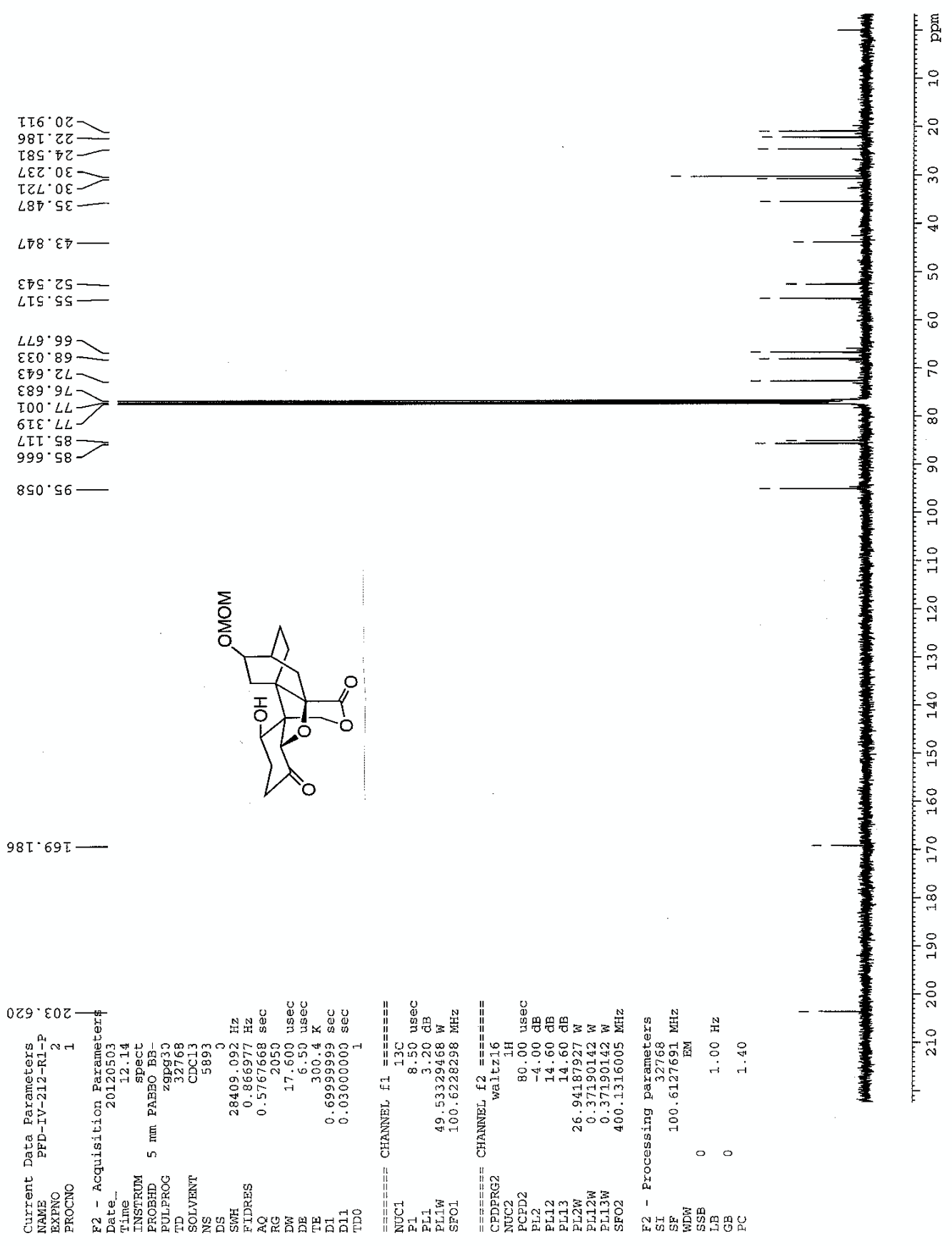




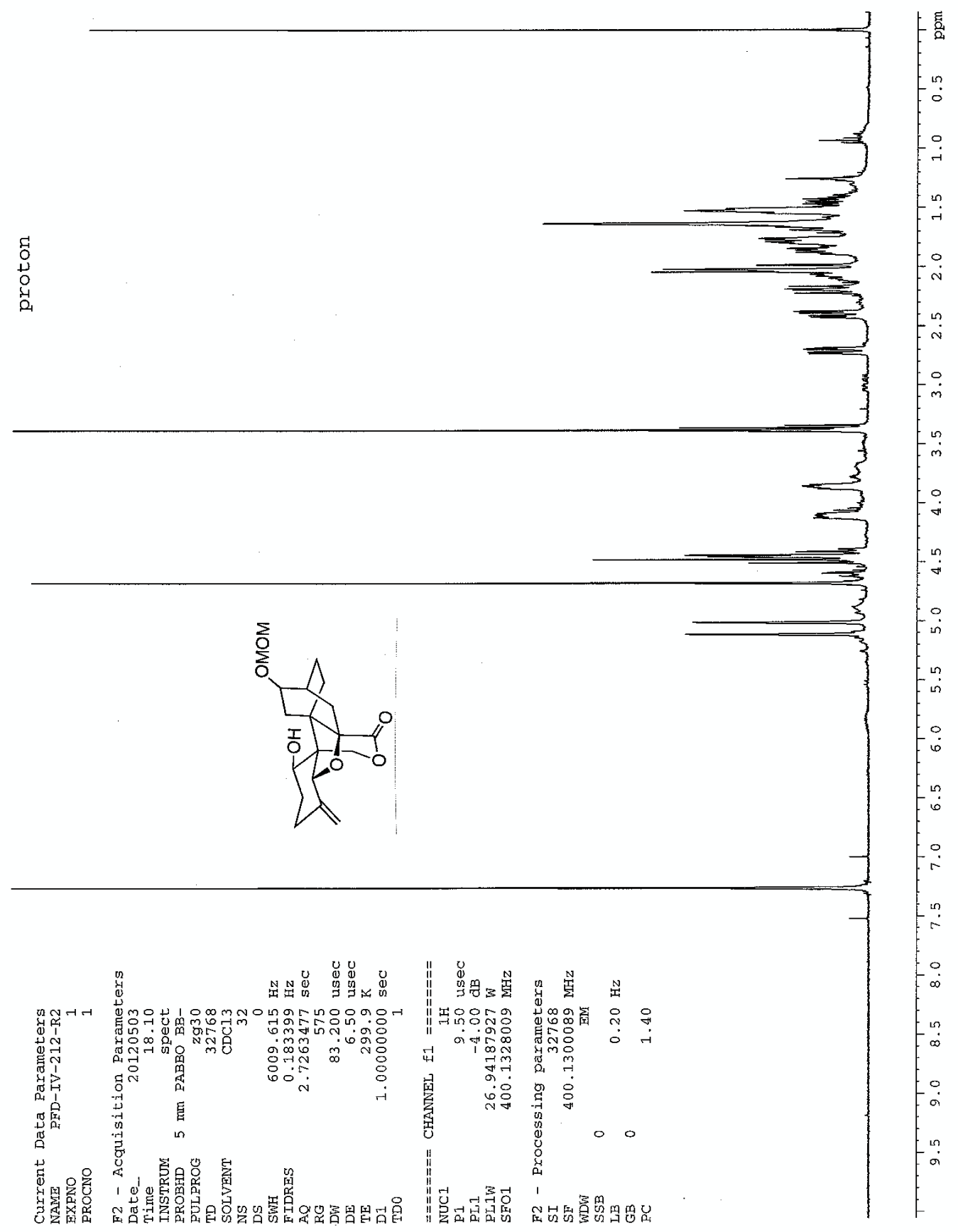




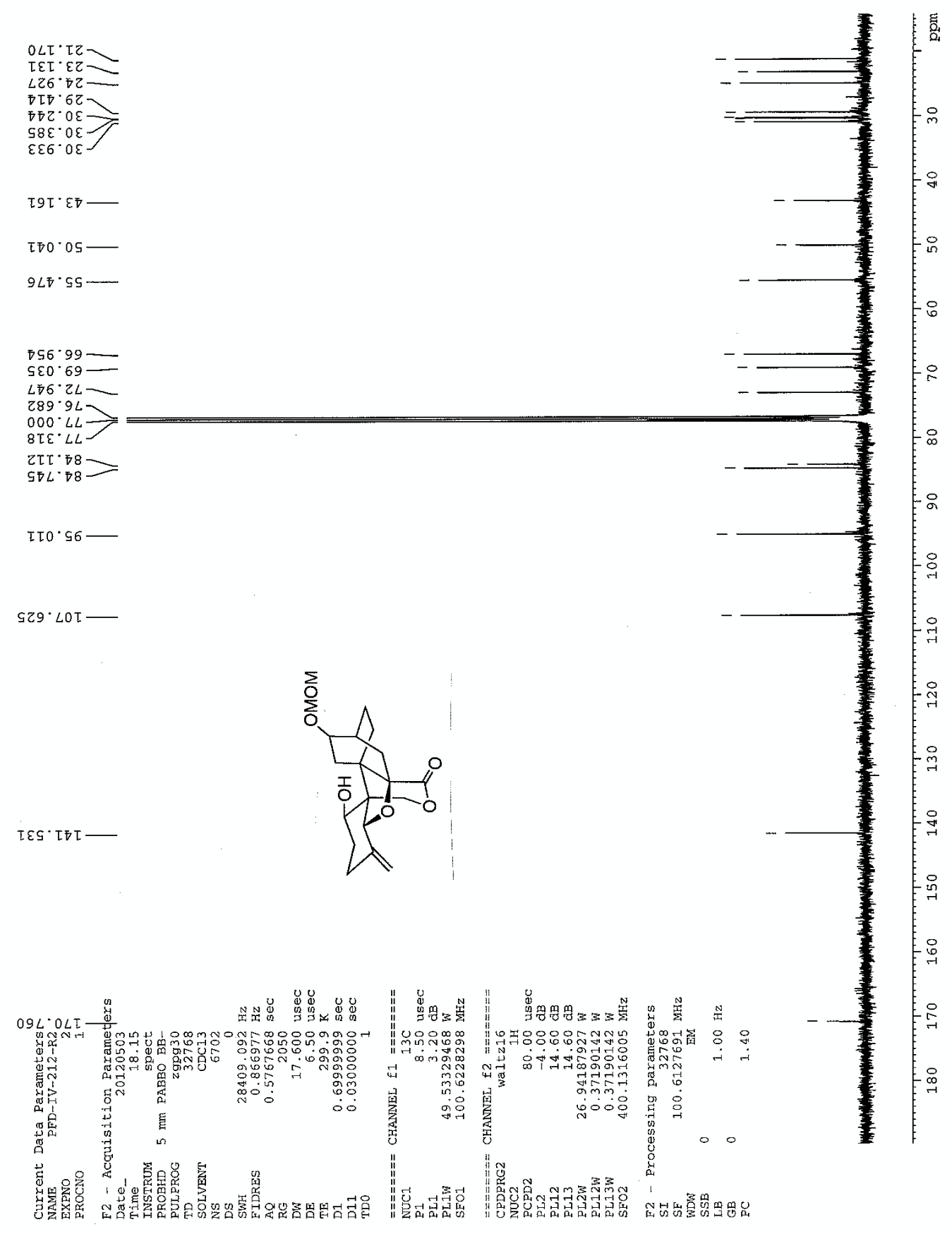




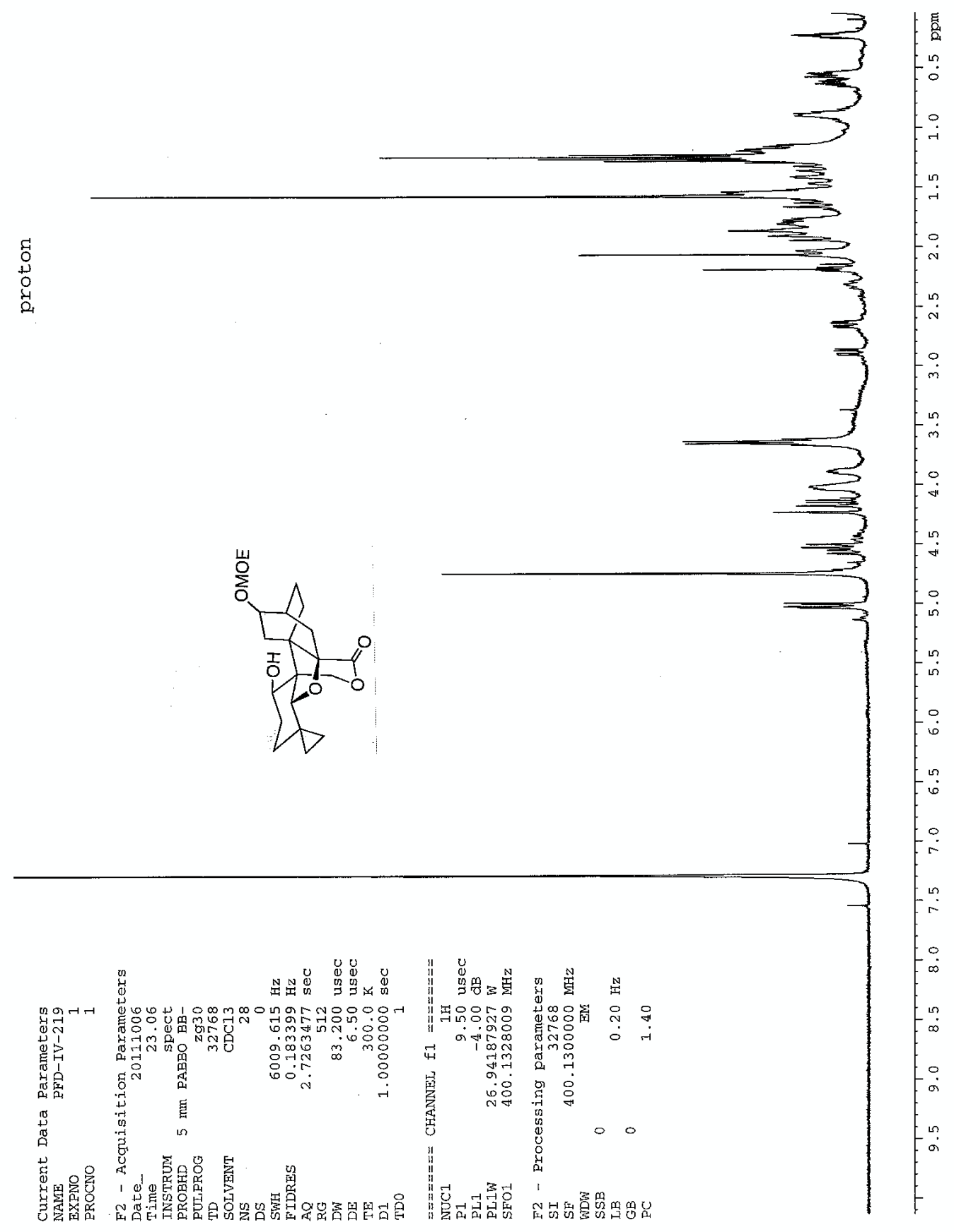



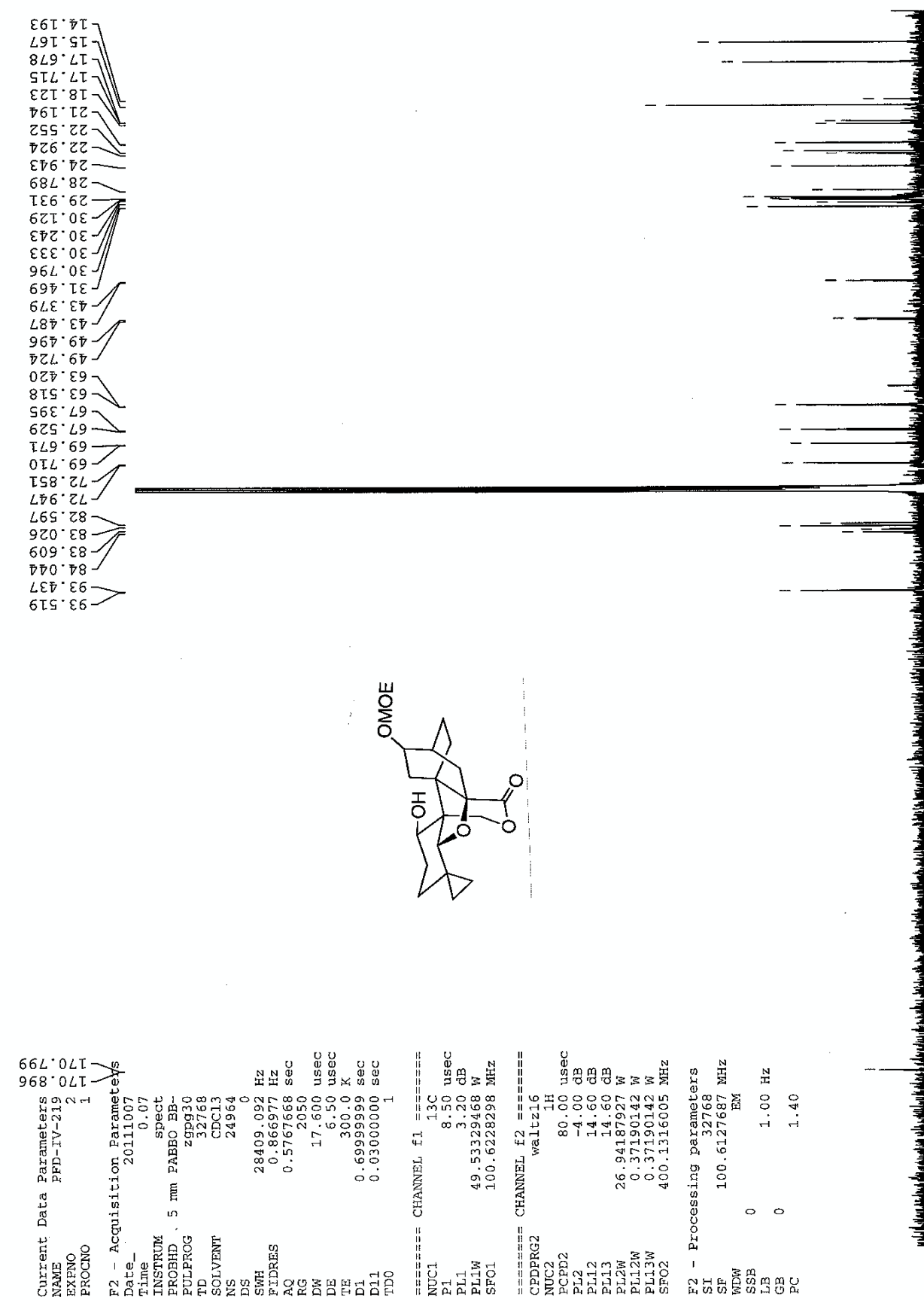


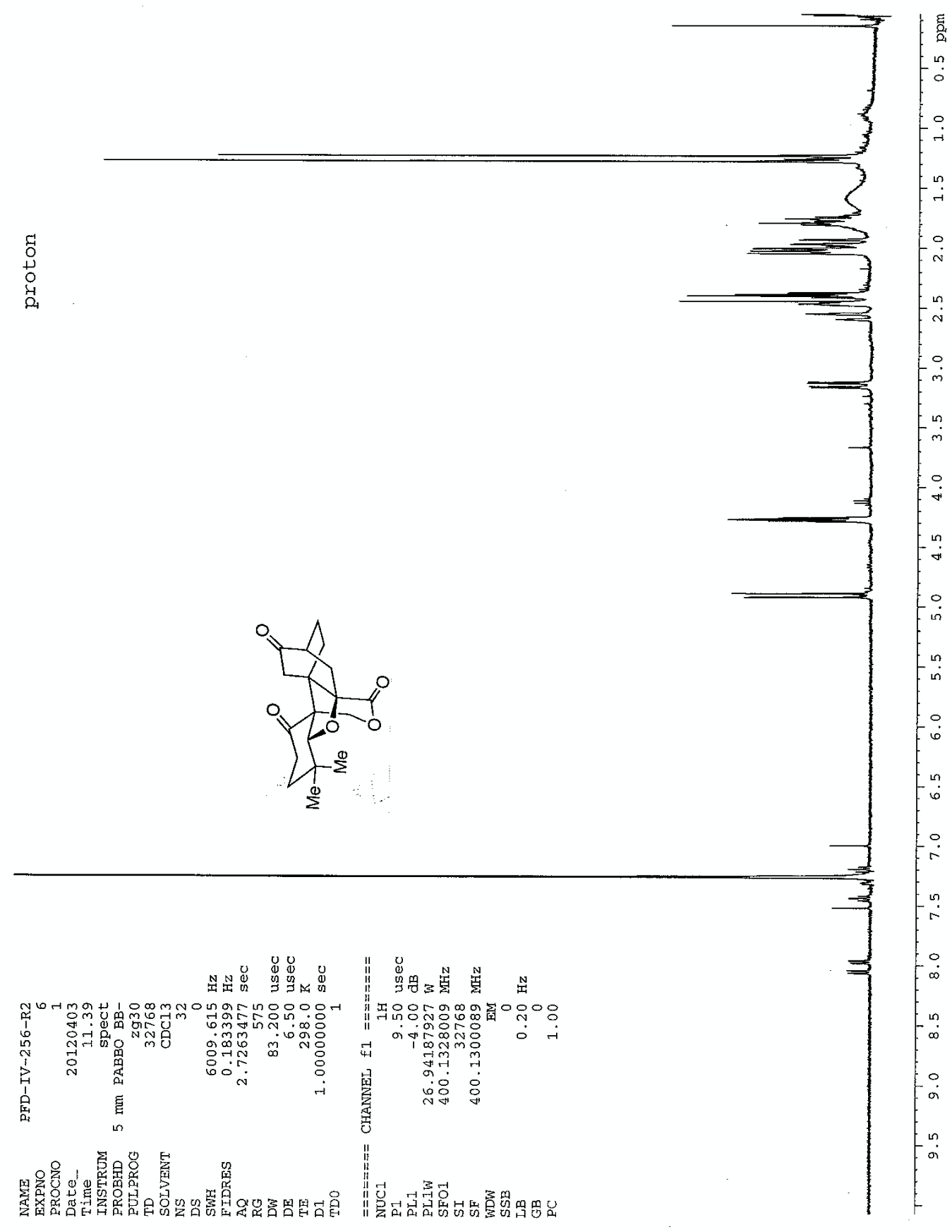




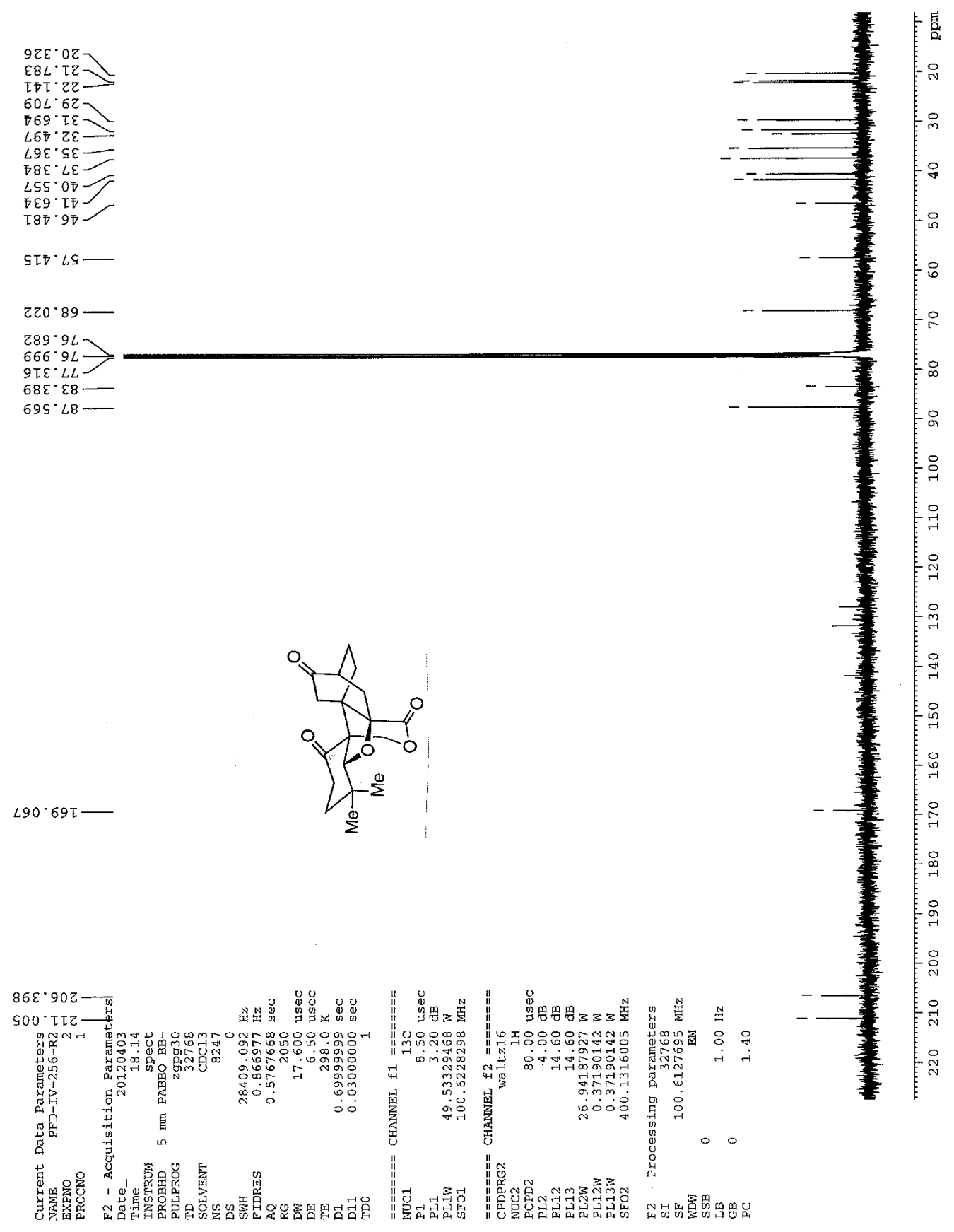




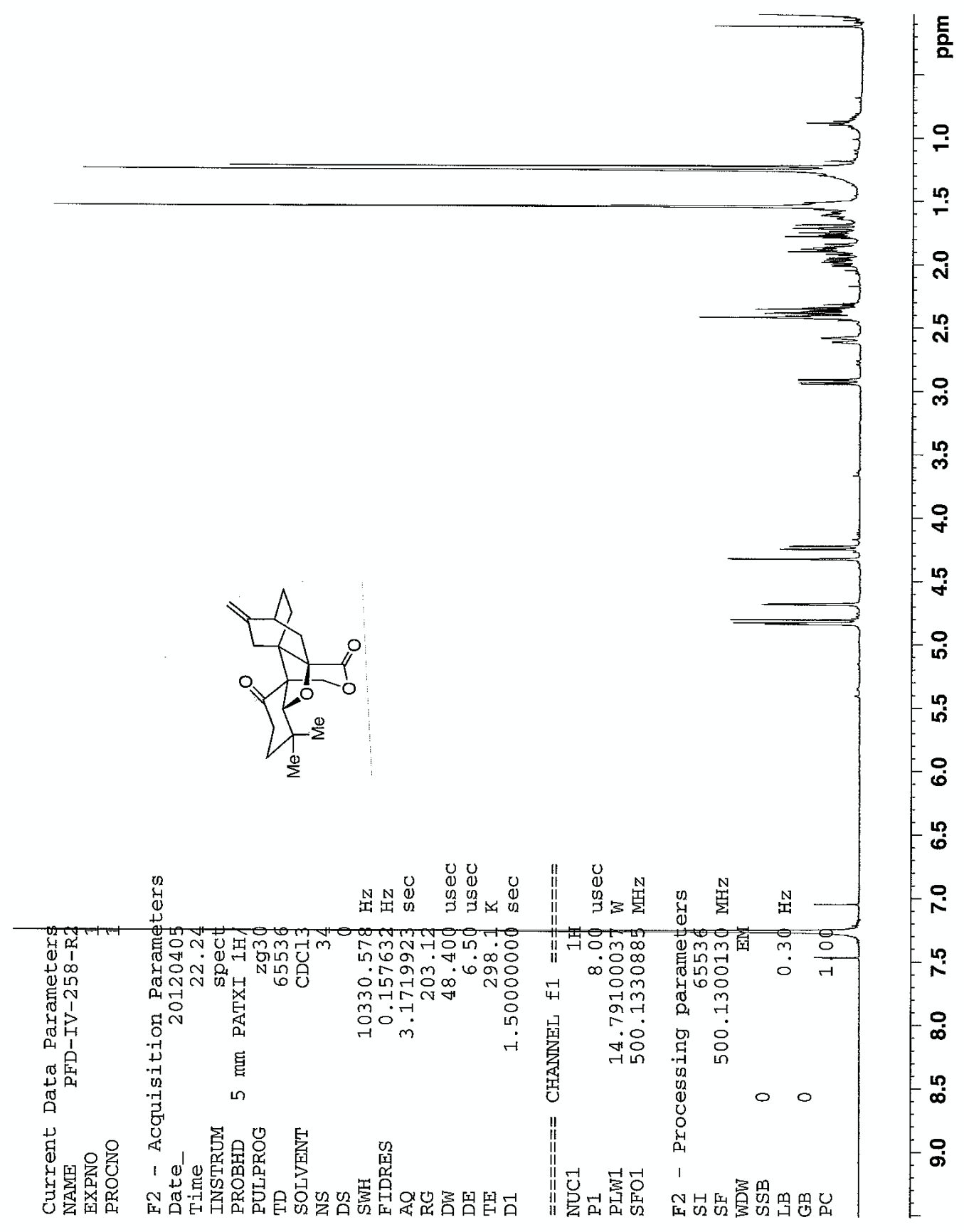




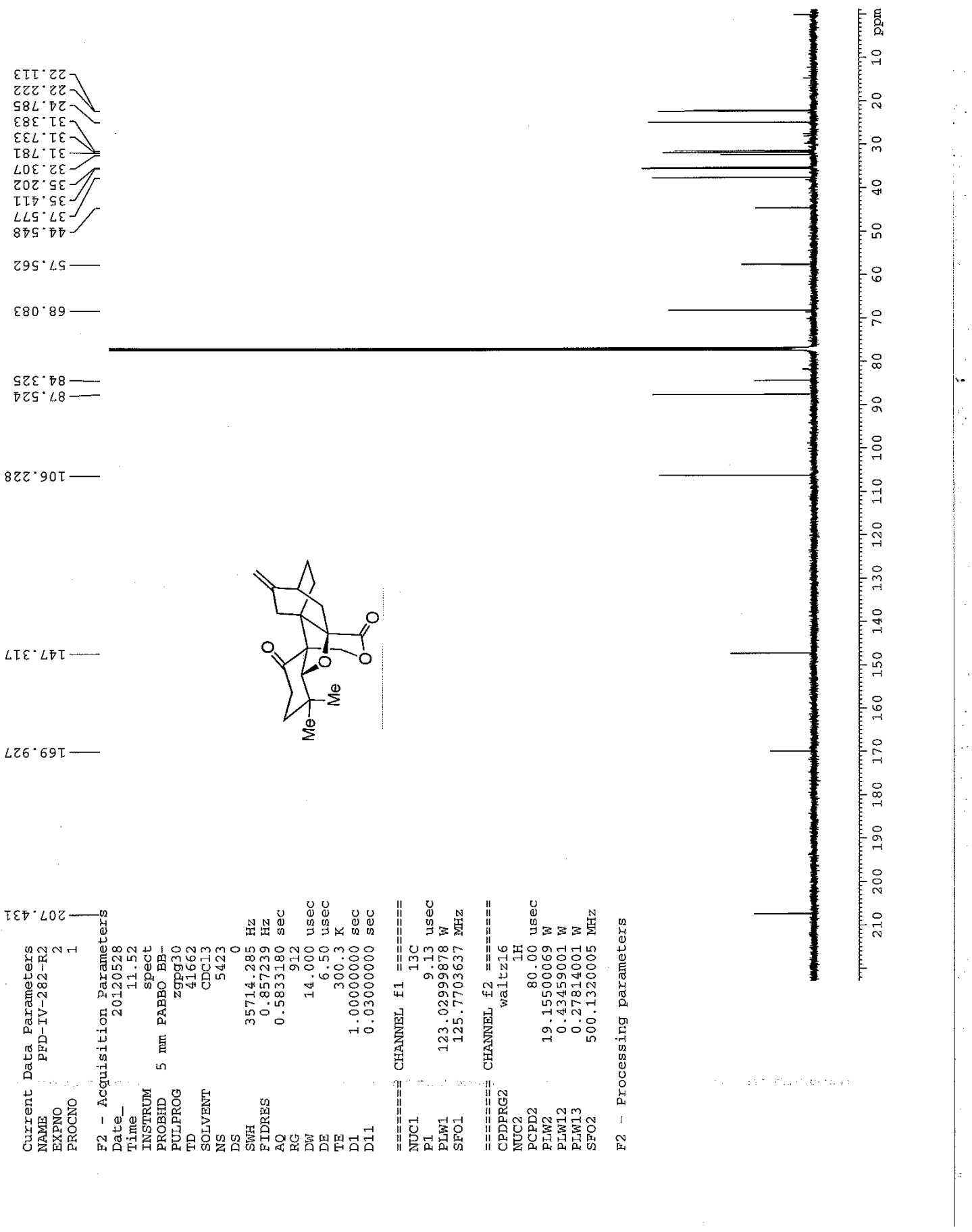




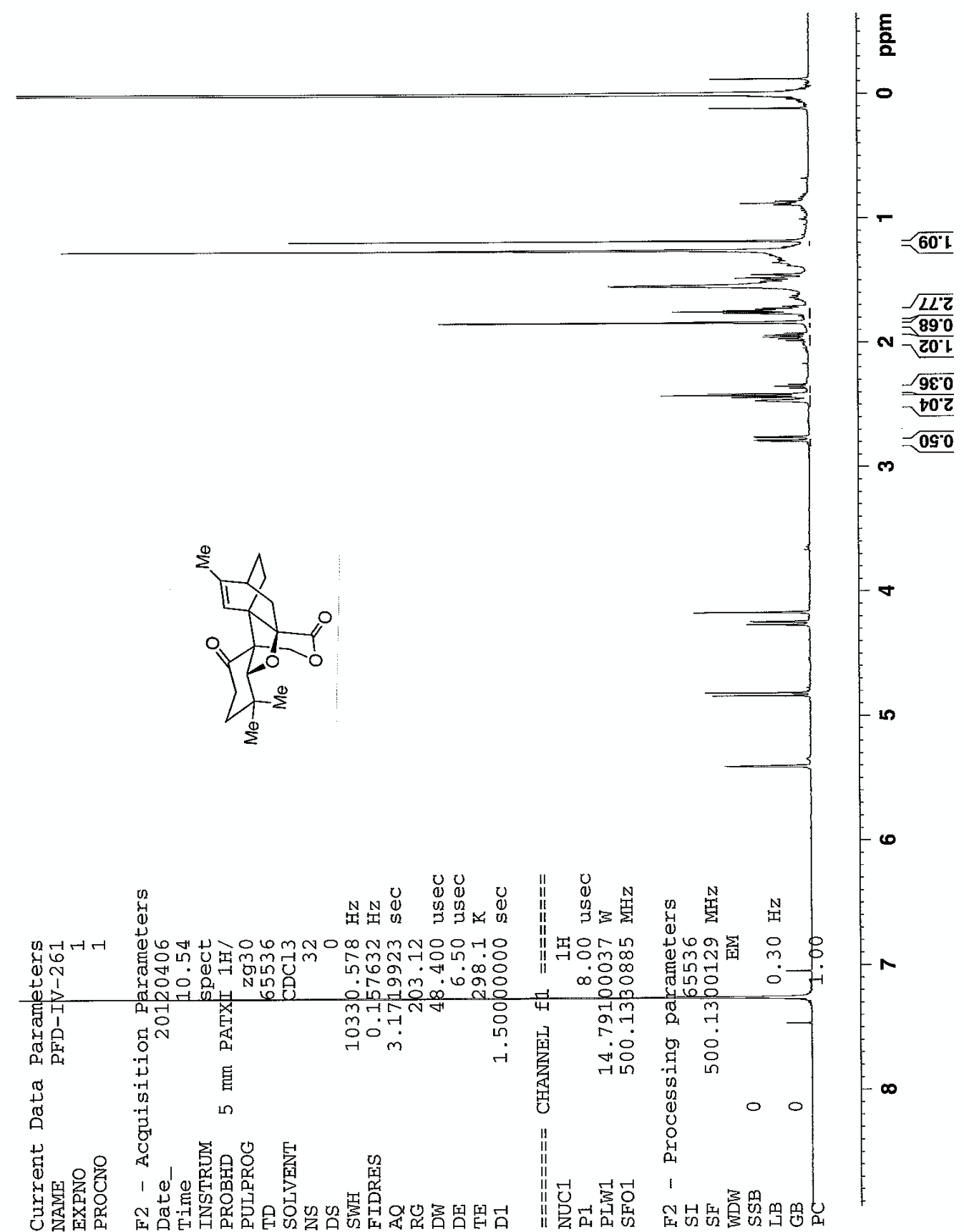




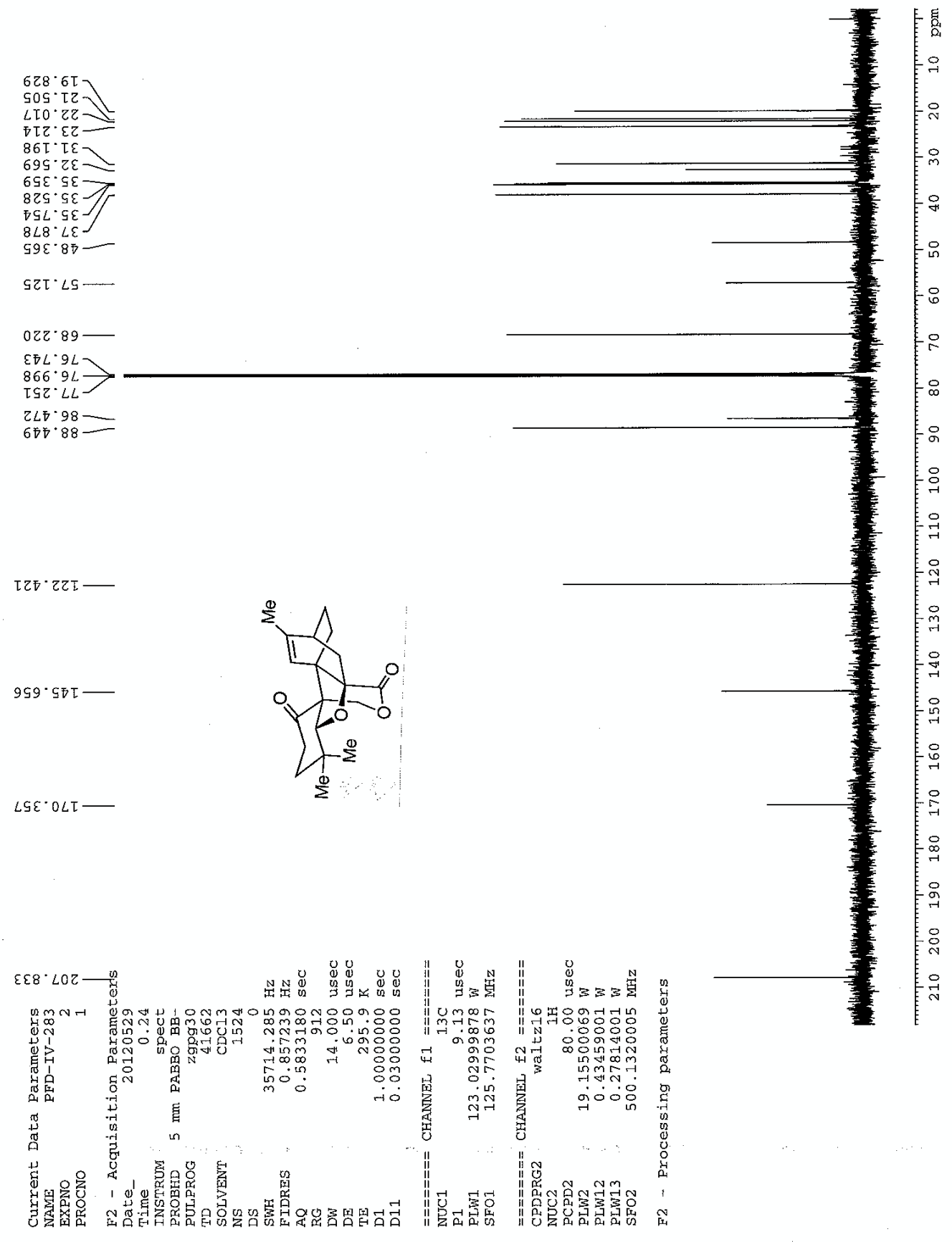




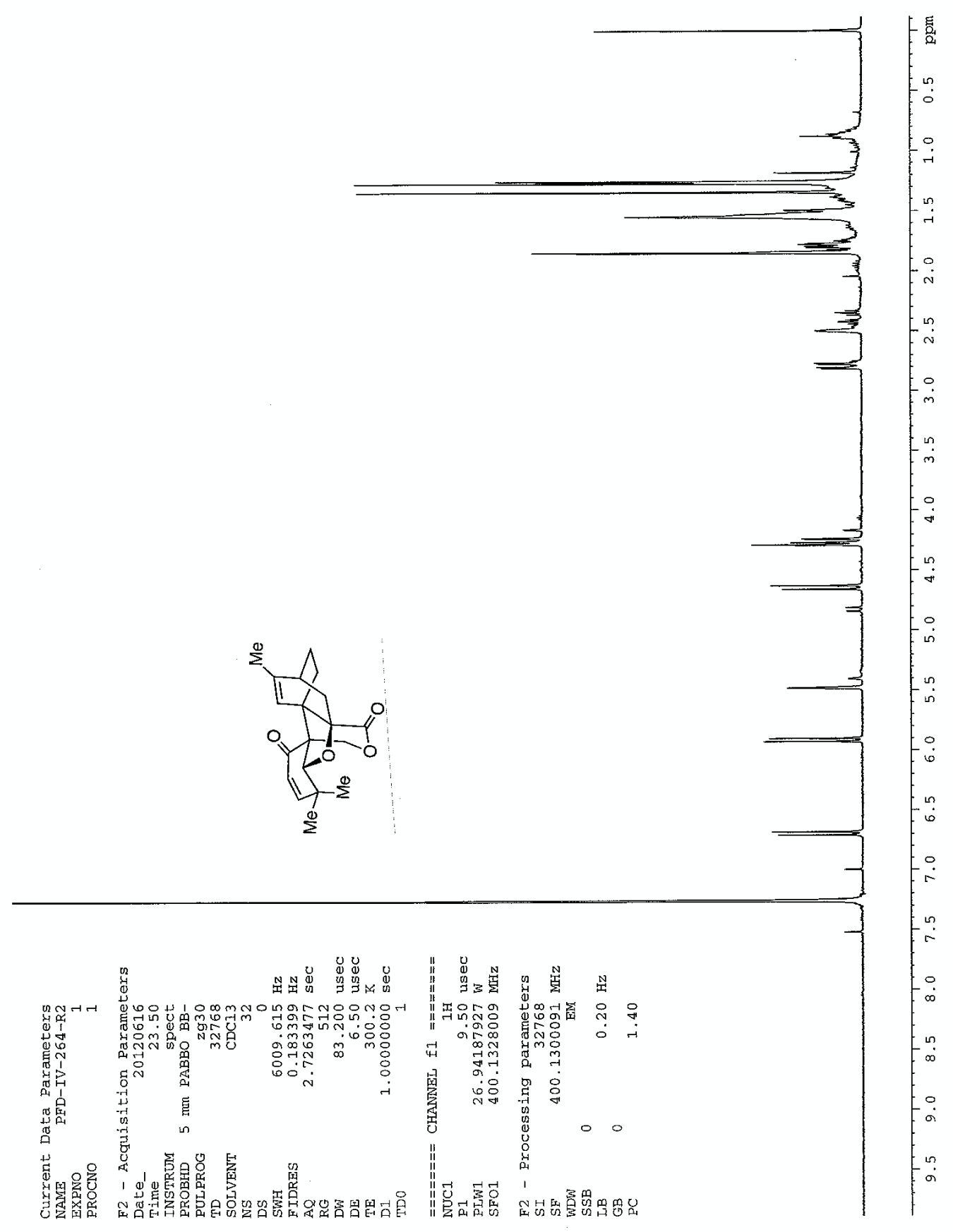




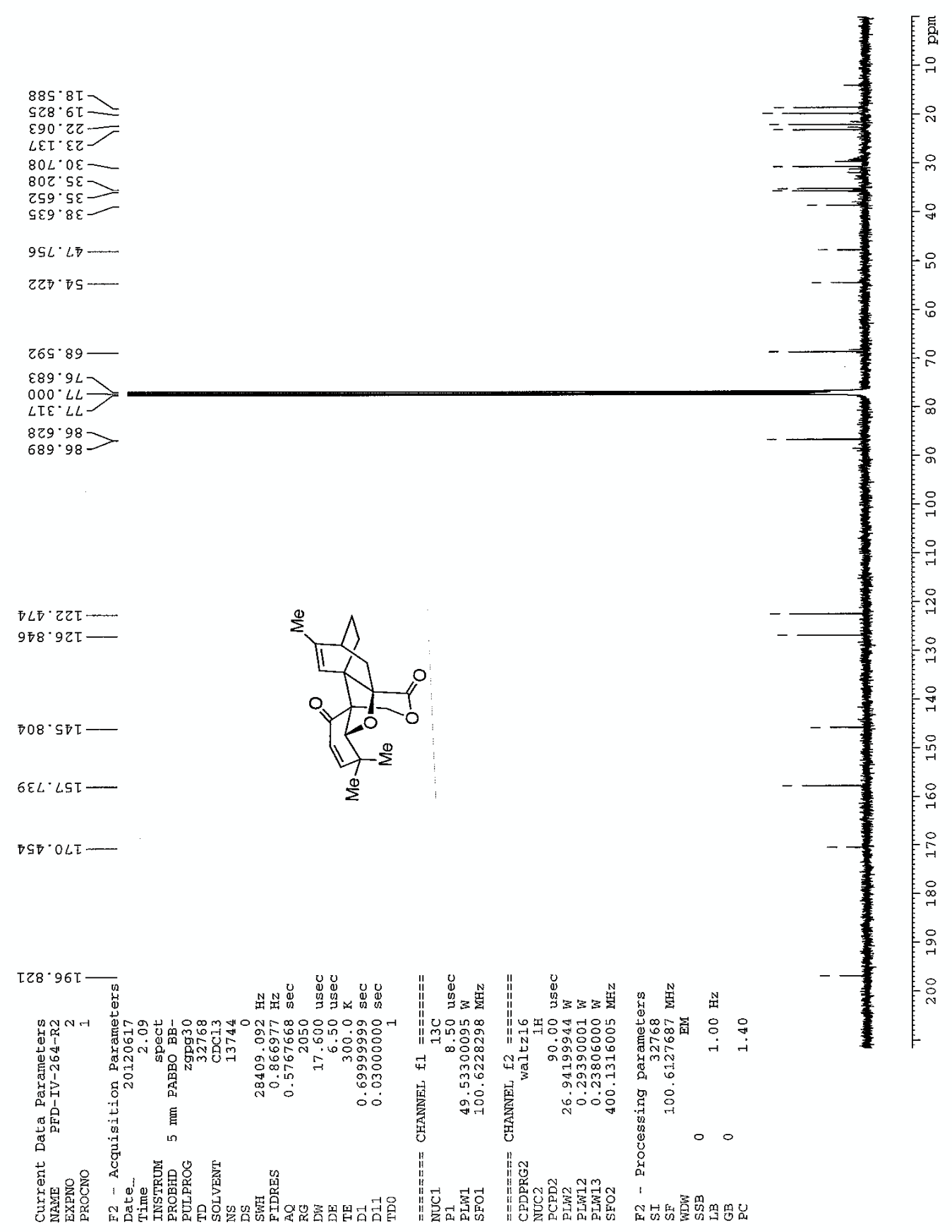




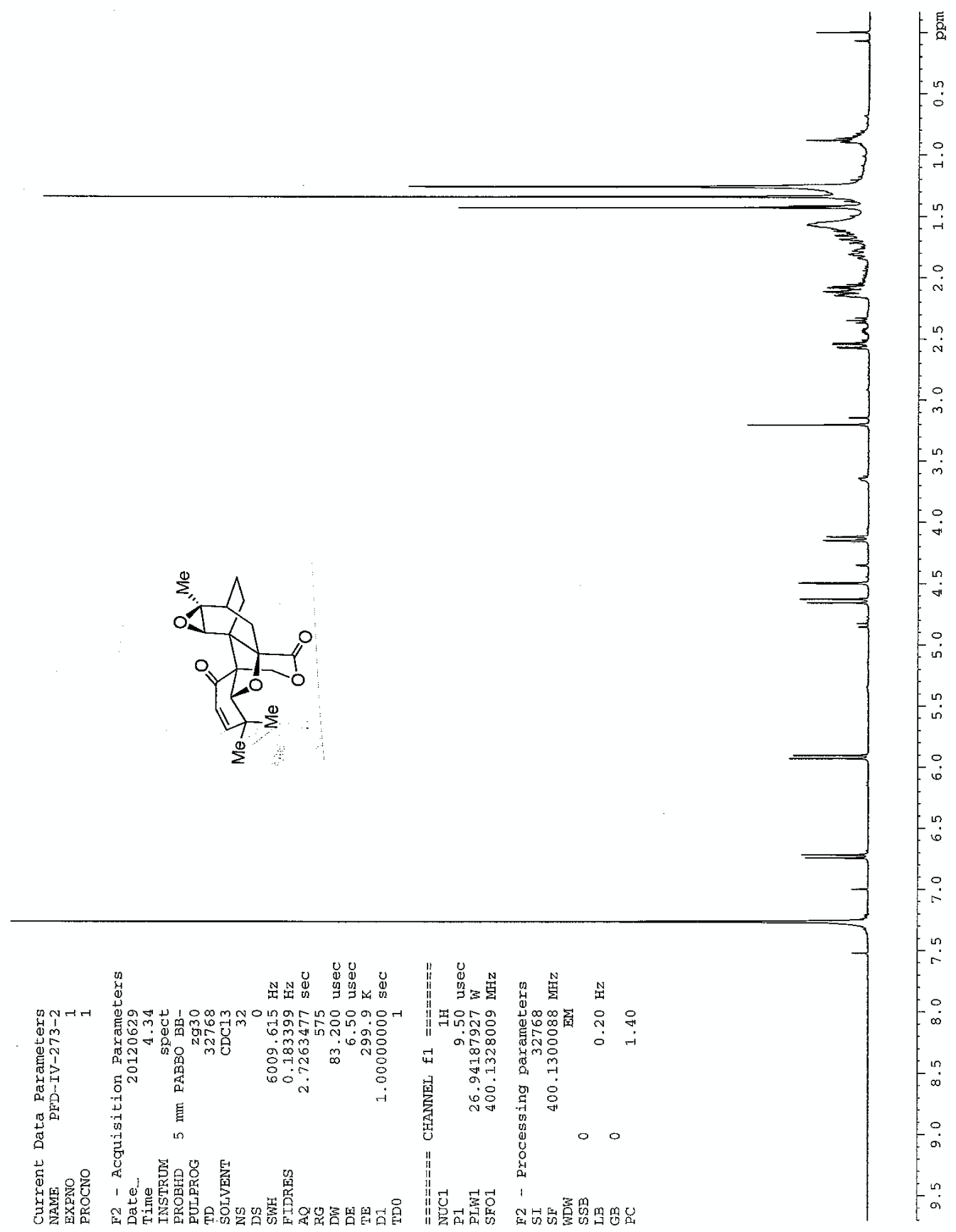




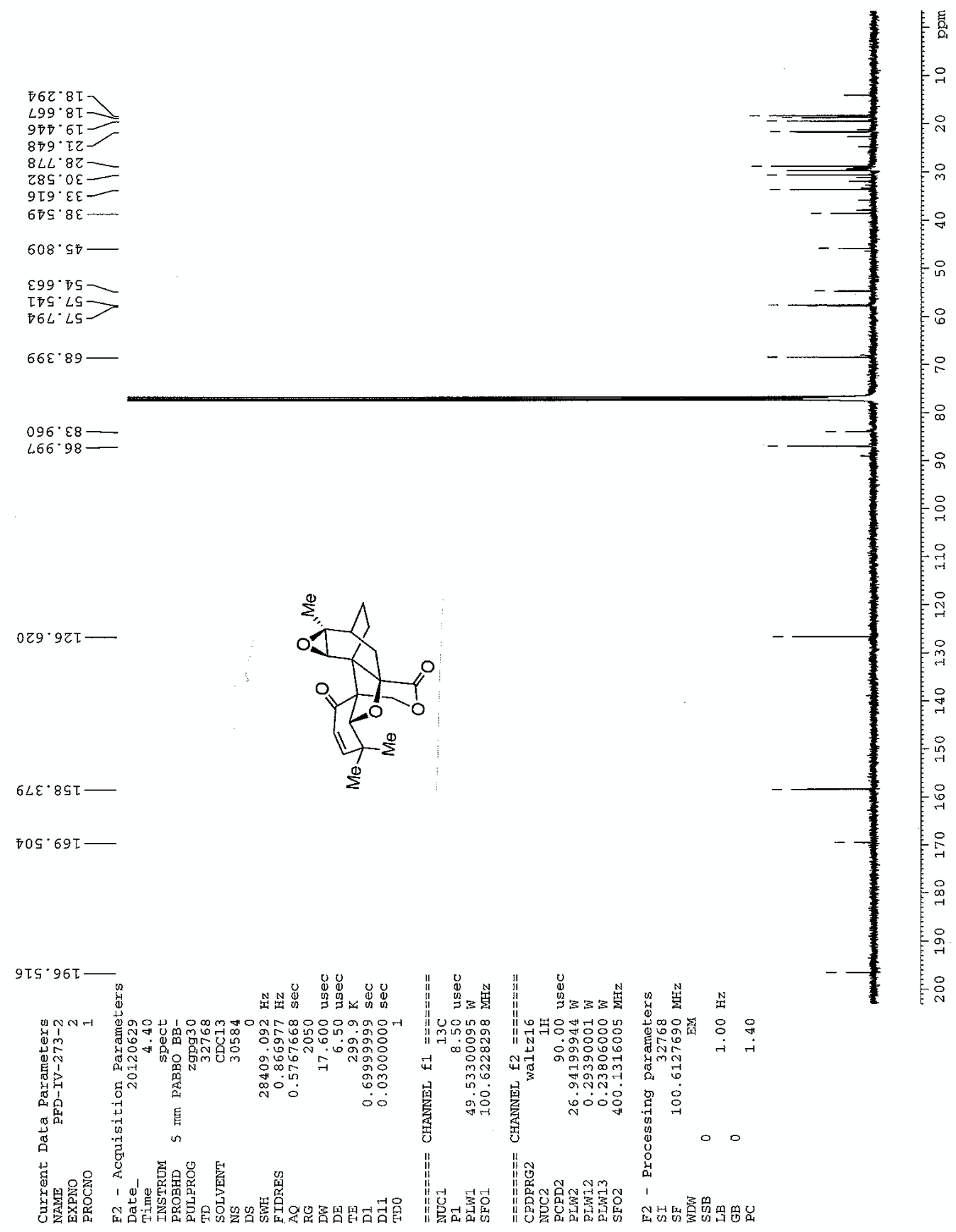




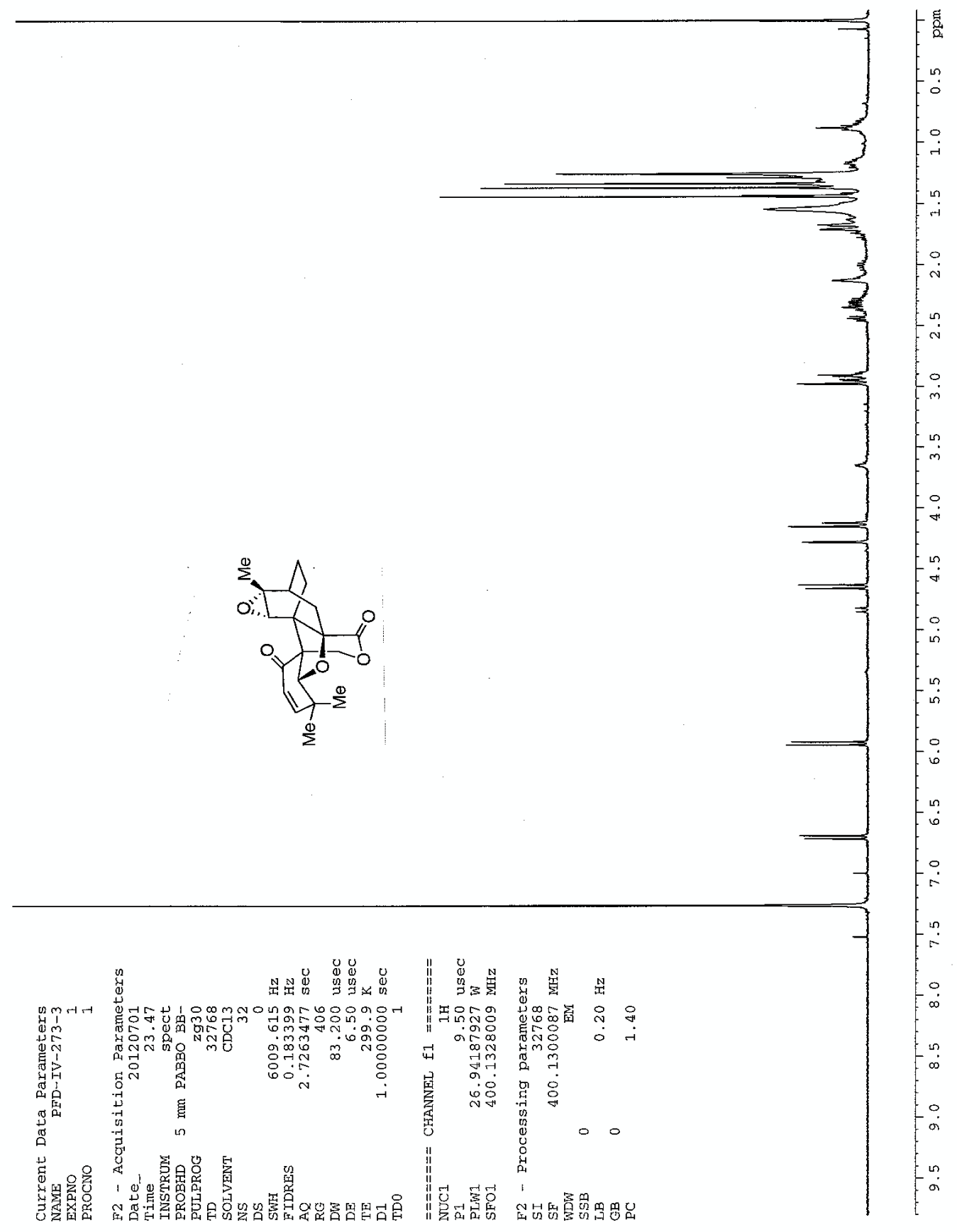




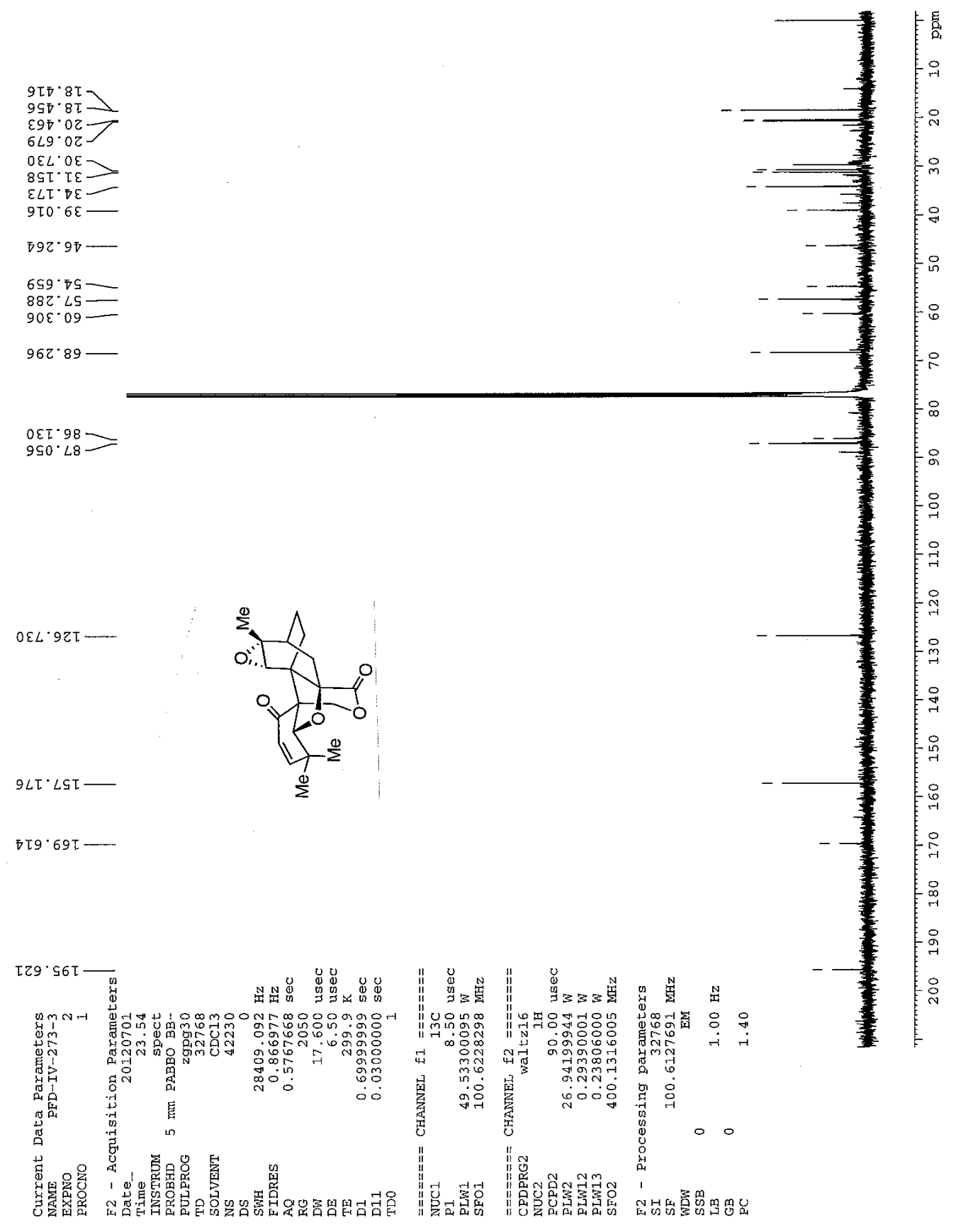




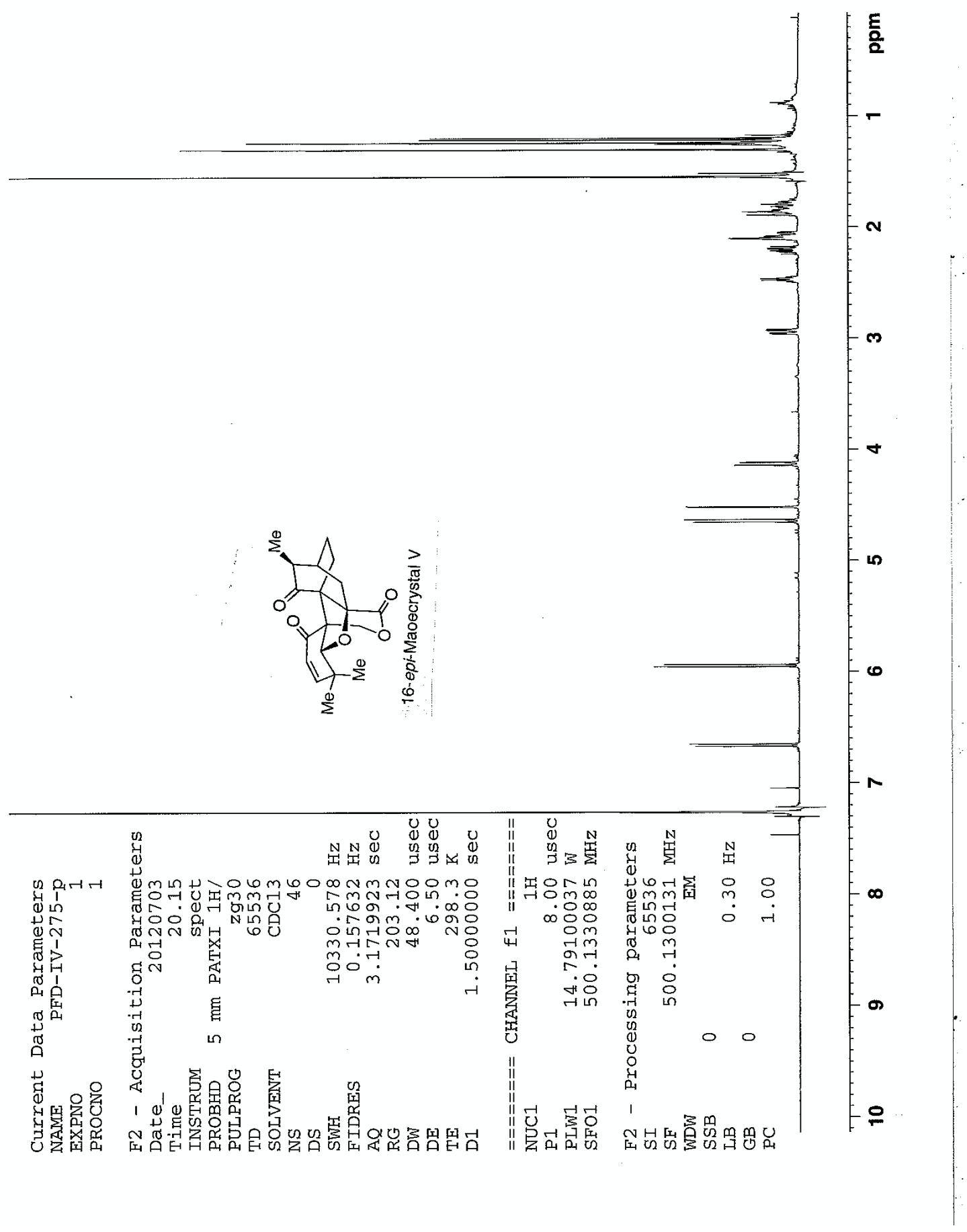




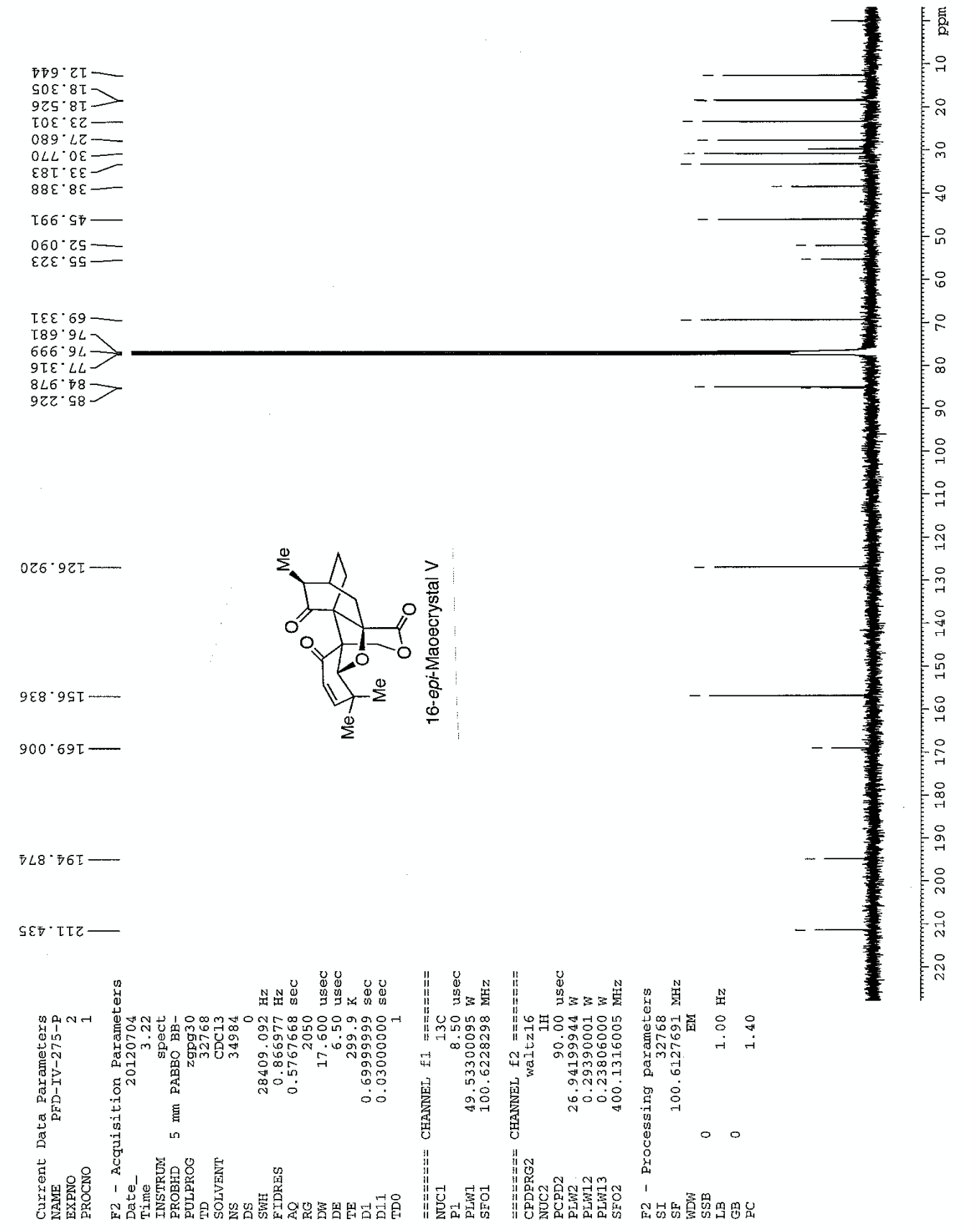




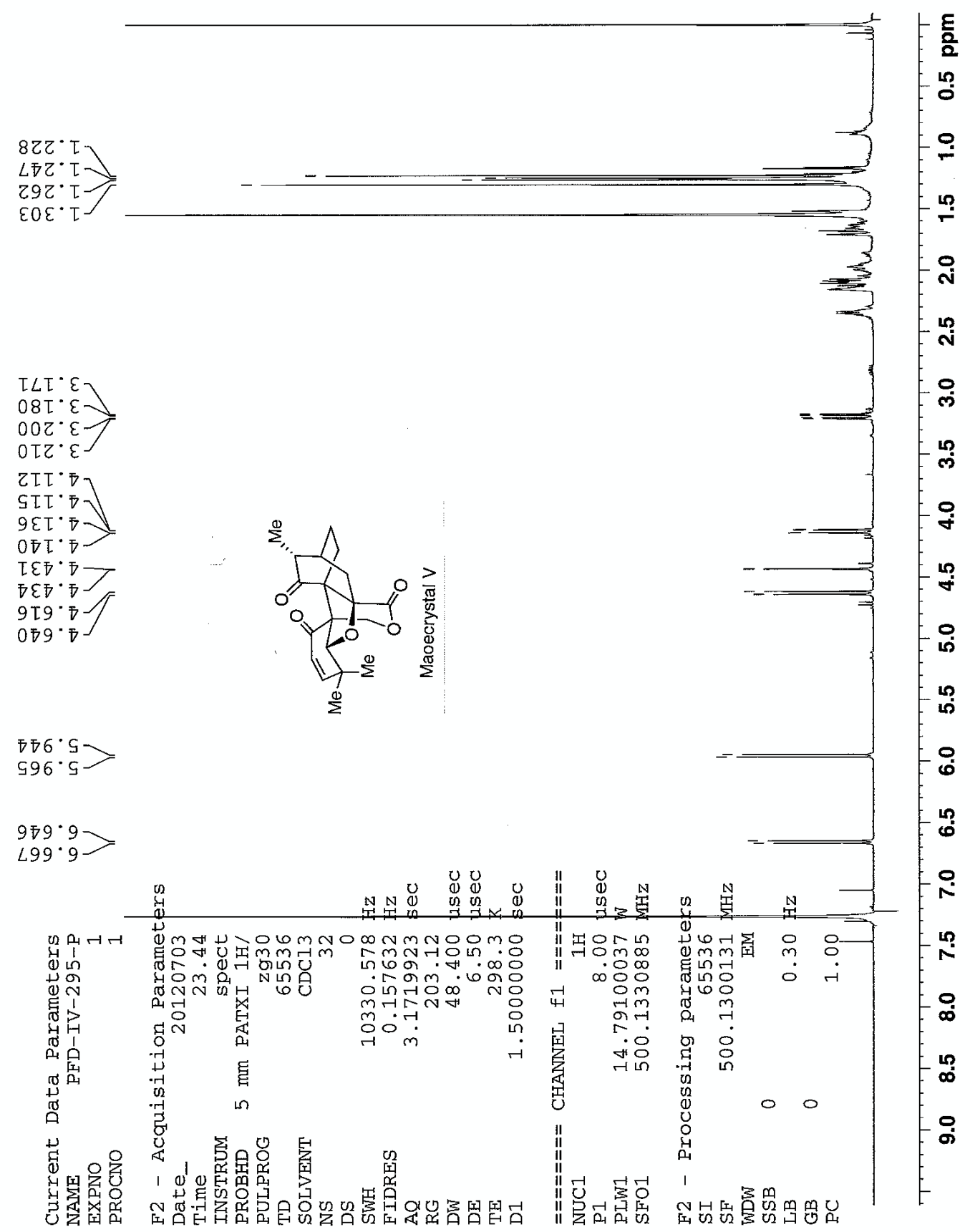




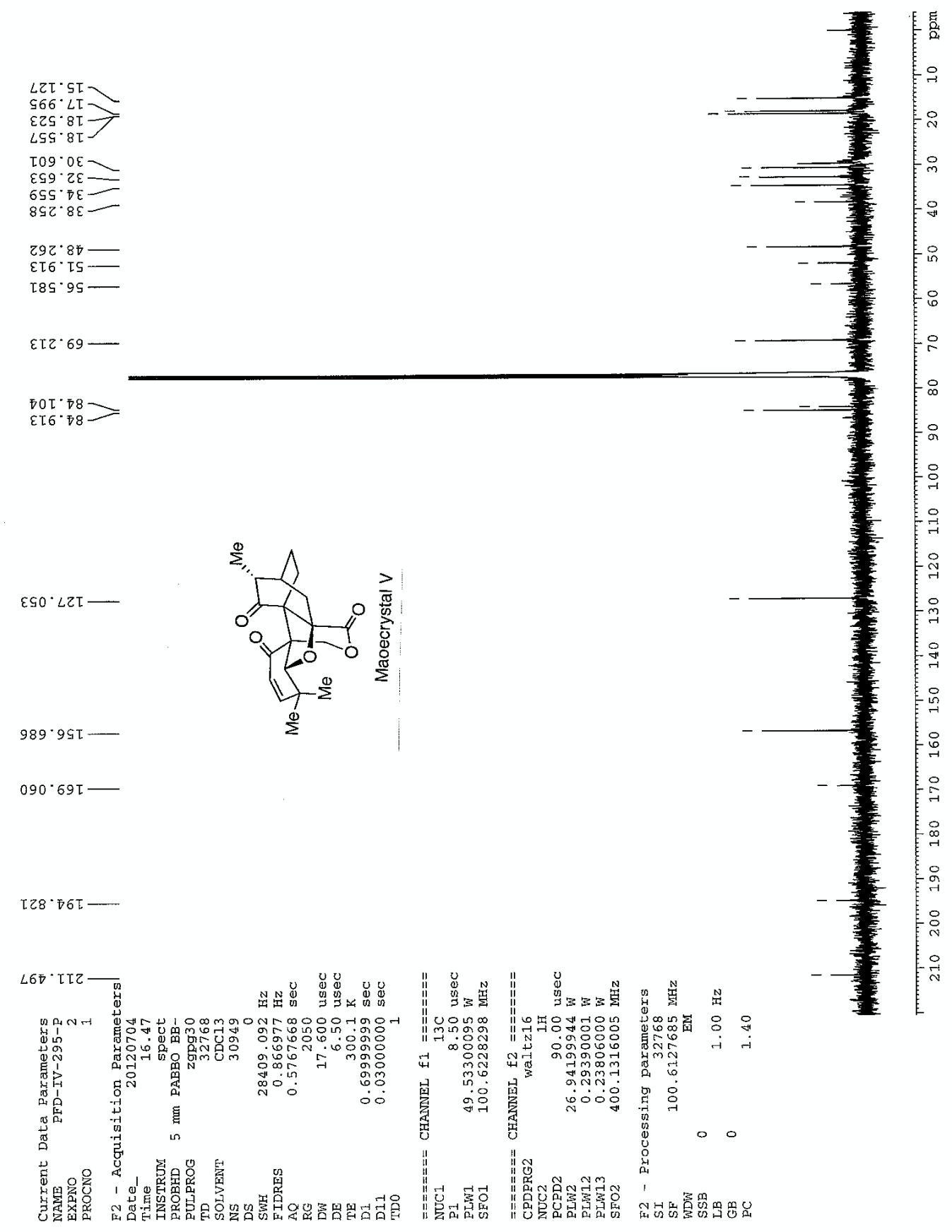



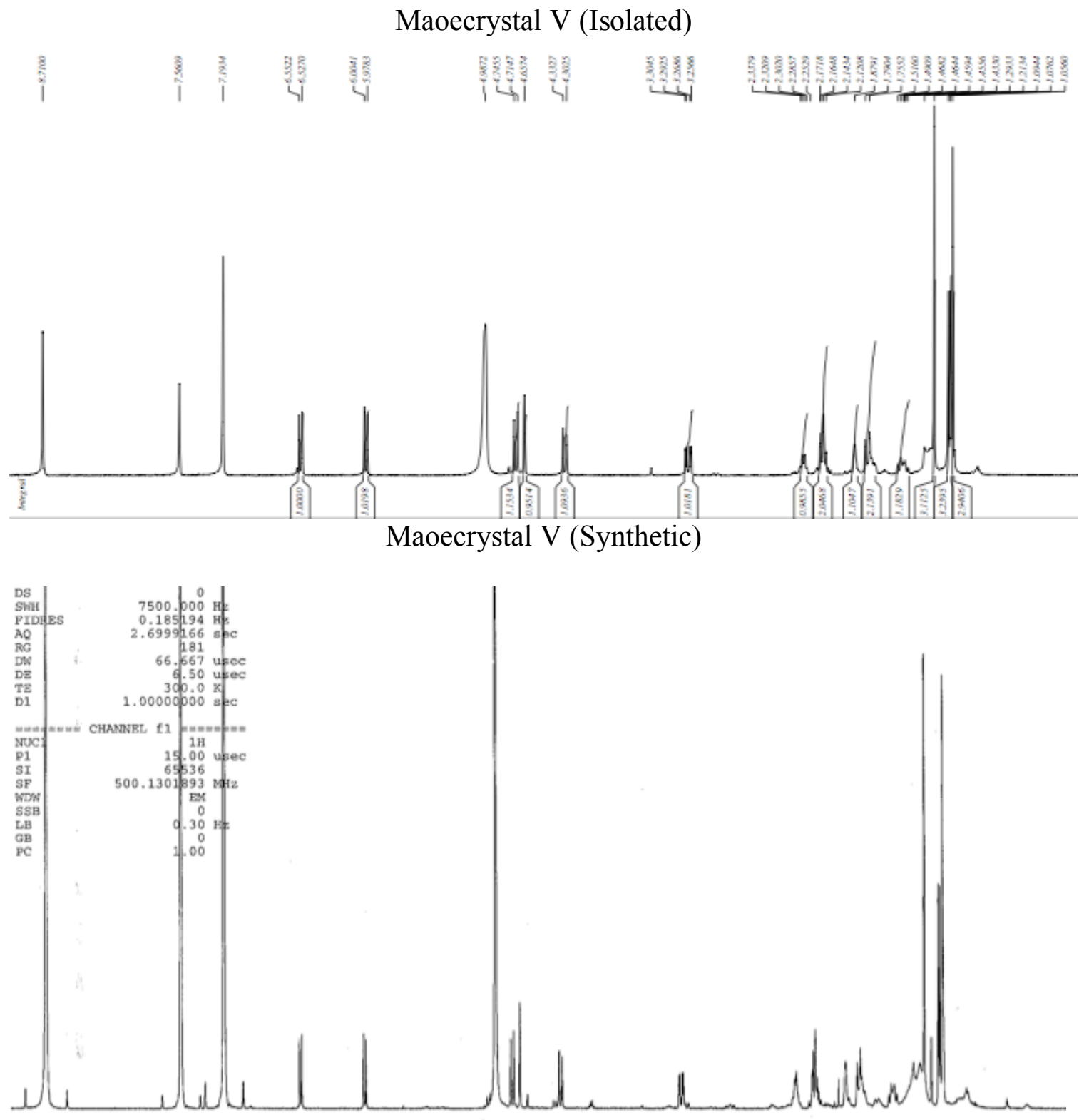


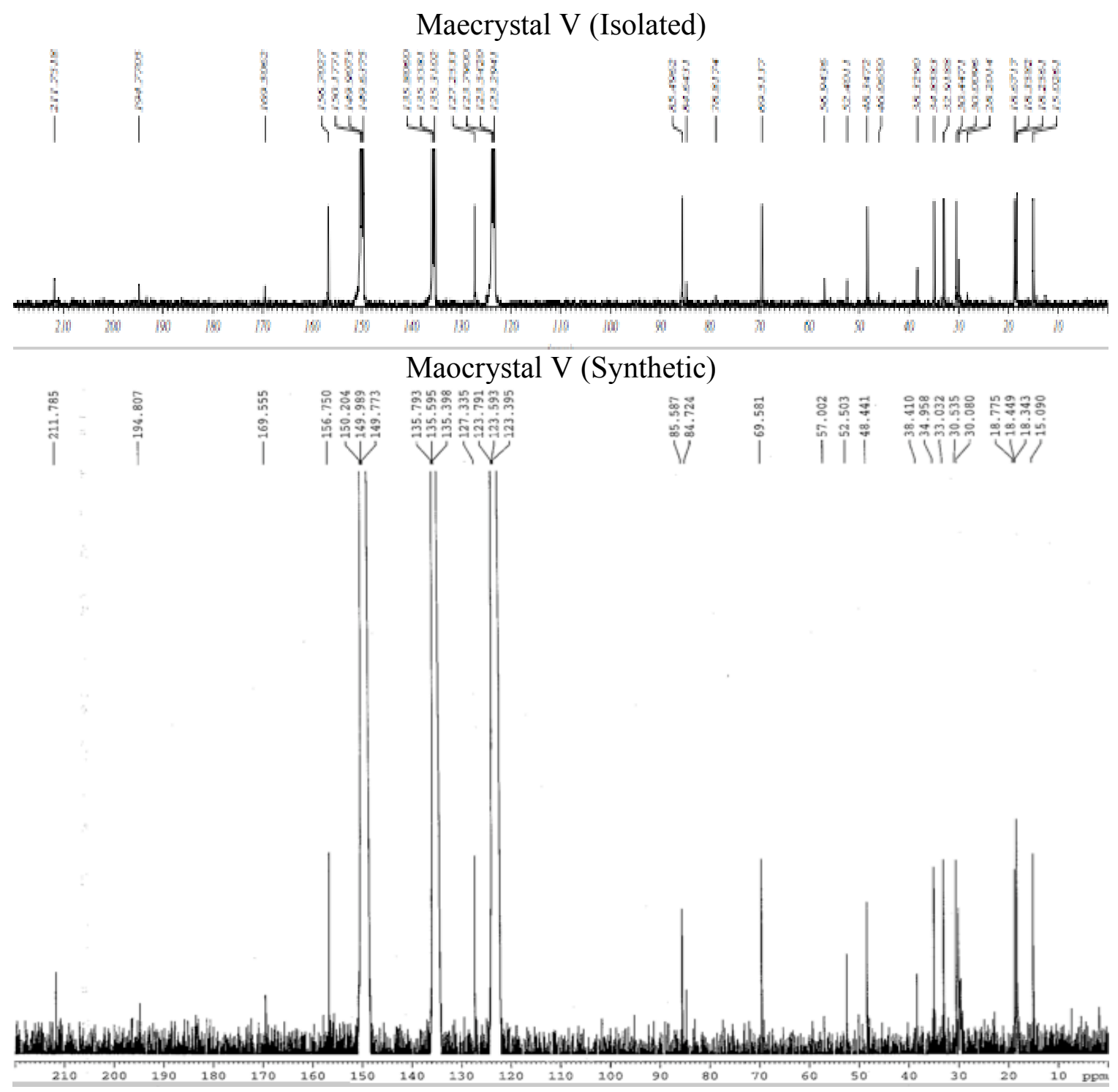

